\title{
Approaching Consciousness with Masked Priming
}

\section{A Comparison of Perceptual and Semantic Priming}

\author{
Dissertation \\ zur Erlangung des mathematisch-naturwissenschaftlichen Doktorgrades \\ "Doctor rerum naturalium" \\ der Georg-August-Universität Göttingen \\ im Promotionsprogramm Biologie \\ der Georg-August University School of Science (GAUSS)

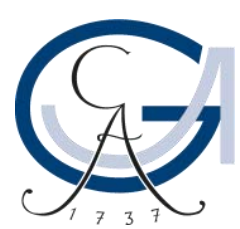 \\ vorgelegt von \\ Martina Wernicke \\ aus Erfurt, Thüringen
}

Göttingen, 2014 



\section{Betreuungsausschuss}

Prof. Dr. Uwe Mattler (Abt. Experimentelle Psychologie, GEMI für Psychologie)

Prof. Dr. Uta Lass (Abt. Experimentelle Psychologie, GEMI für Psychologie)

\section{Mitglieder der Prüfungskommission:}

Referent: Prof. Dr. Uwe Mattler (Abt. Experimentelle Psychologie, GEMI für Psychologie)

Koreferentin: Prof. Dr. Uta Lass (Abt. Experimentelle Psychologie, GEMI für Psychologie)

\section{Weitere Mitglieder der Prüfungskommission:}

Prof. Dr. Andreas Glöckner (Abt. Psychologische Diagnostik, Urteilen und Entscheiden, GEMI für Psychologie)

Prof. Dr. Nivedita Mani (Free Floater Nachwuchsgruppe - Spracherwerb)

Prof. Dr. Lars Penke (Abt. Biologische Persönlichkeitspsychologie und Diagnostik, GEMI für Psychologie)

Prof. Dr. Hannes Rakoczy (Abt. Biologische Entwicklungspsychologie, GEMI für Psychologie)

Tag der mündlichen Prüfung: 29.09.2014 


\section{Acknowledgements}

I am very thankful for the valuable support of several people. Doubts and problems characterize the work on a dissertation as much as hopefully inspiration, success and pleasant as well as productive cooperation. I was very lucky the good colleagues, supervisors and friends provided directive and moral support.

Uwe Mattler provided supervision and advice concerning scientific proceedings. I am glad that he shared his knowledge and ideas. His concern for a pleasant working atmosphere is fruitful and appreciated.

Uta Lass reviewed the current work. Her accumulated wealth of experience provided new perspectives throughout the whole process. She always found the right moments for words of encouragement.

Fortunately, all of my colleagues were exceptionally pleasant to work with. Daniel Krüger introduced me to the proceedings and answered every question readily. No problem missed a solution when Thorsten Albrecht was around. Simon Palmer provided inspiration and new perspectives in several discussions. Mareen Berndt offered great support during the final stages of this work.

Furthermore, several student assistants were involved in the collection of data. I like to mention Diemut Regel, Lisa Eilert and Nadja Arnold. It was easy to confide in the quality of the data. 


\section{Summary}

Masked Priming is an established paradigm to investigate consciousness. The impact of prime visibility on specific priming effects determines whether associated processes can occur independently of consciousness or might rely on consciousness. A meta-analytic review of 84 studies was conducted to assess the impact of prime visibility on effect sizes in interaction with various moderators (Chapter 2). A psychological model concerning the emergence of priming effects was proposed. Two main confoundings between moderators were identified. Specific task levels were predominantly applied with specific masking methods. Furthermore, temporal parameters were chosen differently between effect types. Subsequent experiments (Chapter 3) revealed that priming effects increase with increasing prime visibility when pattern masks are applied. Priming effects were independent of prime visibility with metacontrast masks. Pattern masks are thought to reduce prime visibility on an early level whereas metacontrast masks disturb recurrent processing. Lamme and Roelfsema (2000) previously proposed that recurrent processing characterizes consciousness. Perceptual and semantic priming effects were equally affected by variations in prime visibility. However, perceptual priming effects were generally larger than semantic priming effects. Further experiments were conducted to determine the courses of perceptual and semantic priming effects with increasing prime-mask SOA and mask-target SOA (Chapter 4). Priming effects generally increase with increasing prime-mask SOA and decline at long mask-target SOA. This decline seems to be based on a simple decay and active mechanisms of inhibition (e.g. Klapp, 2005). However, courses of perceptual and semantic priming effects with increasing mask-target SOA differed. This seems to be due to an additional mechanism of object updating that acts only with relevant masks (Lleras \& Enns, 2004). In the current design, masks only activated the incongruent category (i.e. relevant masks) with perceptual congruency. An electrophysiological study was conducted to compare perceptual and 
semantic priming with identical stimulation (Chapter 5). The applied design allowed the estimation of behavioral priming effects without response association as well as effects on event-related potentials without response execution. Perceptual priming effects without response association were larger than semantic priming effects without response association. Furthermore, the P2 component and the P3(b) component were modulated through perceptual congruency. Semantic congruency was reflected in an N400-like effect with a delayed latency. Perceptual ERP effects were observed earlier and with different distributions than semantic ERP effects. Perceptual and semantic priming effects seem to be based on different mechanisms that share several attributes. However, the current work supports the idea of approaching consciousness through the comparison of different masking methods (cf. Breitmeyer, 2014). 


\section{Contents}

1 Introduction ........................................................................................................................................ 1

\section{The Role of Visibility in Masked Priming:}

a Model and a Meta-Analysis................................................................................................ 7

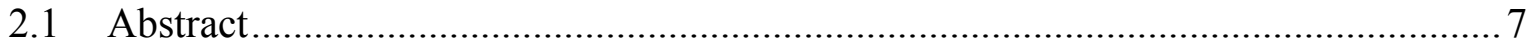

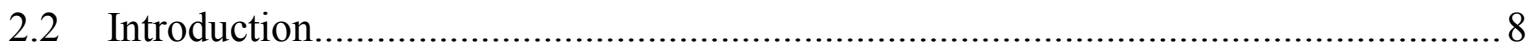

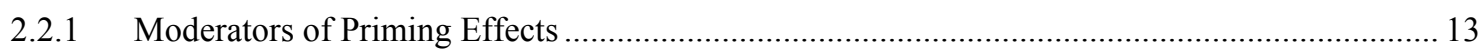

2.2.2 Moderators of Priming Effects and Prime Visibility ……….................................................. 19

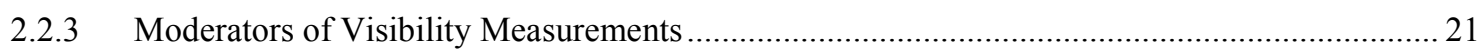

2.2.4 A Psychological Model for the Interplay of Moderators affecting Priming Effects .................... 23

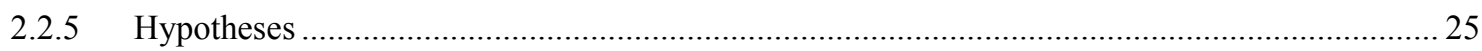

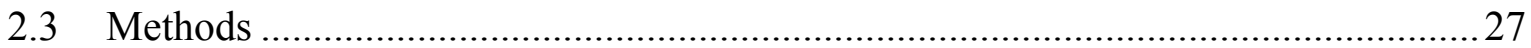

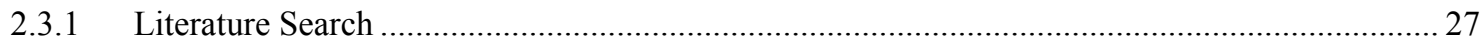

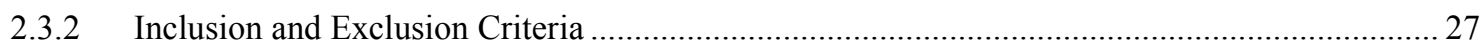

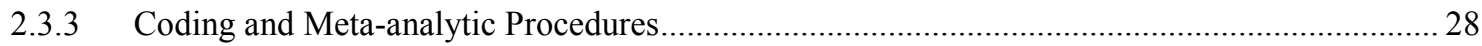

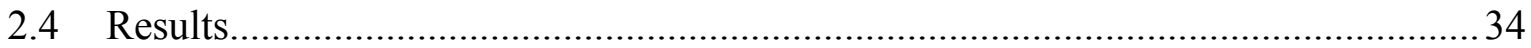

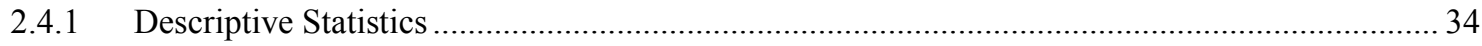

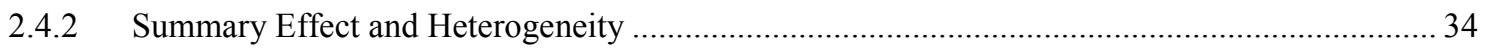

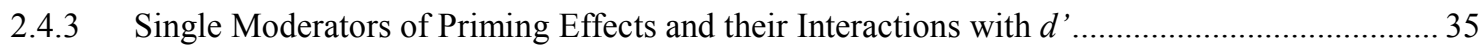

2.4.4 Meta-analytic Models for the Interplay of Moderators affecting Priming Effects ....................... 42

2.4.5 Influences on Prime Visibility and Visibility Measurements ..................................................... 54

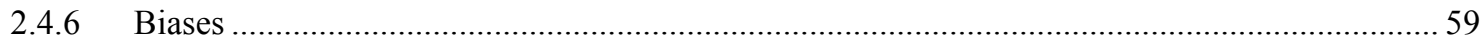

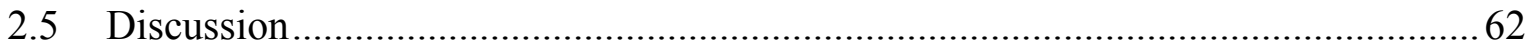

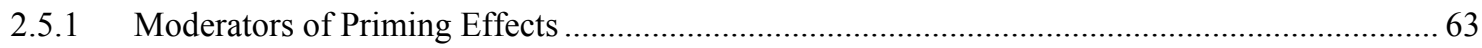

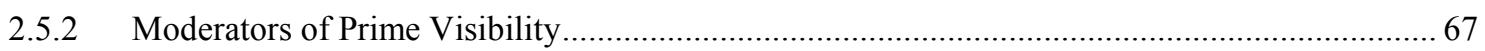

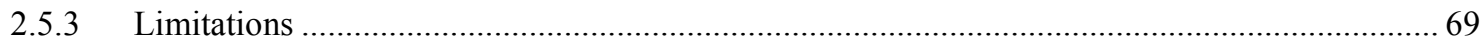

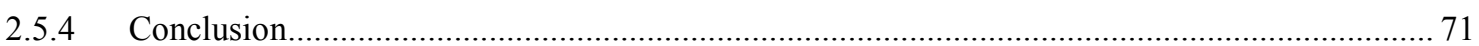




\section{Prime Visibility in Perceptual and Semantic Priming:}

Differences based on Masking Method ....................................................................73

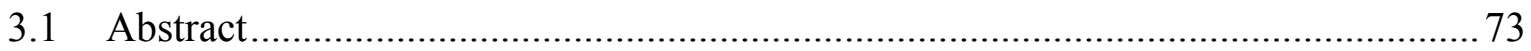

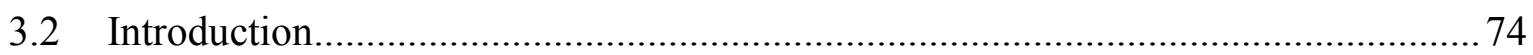

3.3 Experiment 3.1: Priming on a Perceptual and Semantic Level ............................... 79

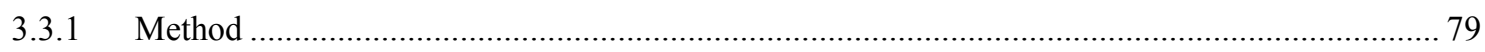

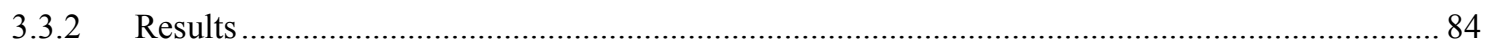

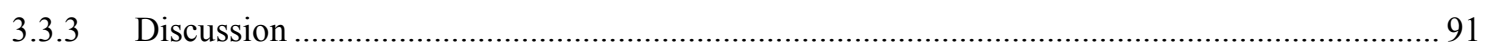

3.4 Experiment 3.2: Priming with Metacontrast Masking........................................... 93

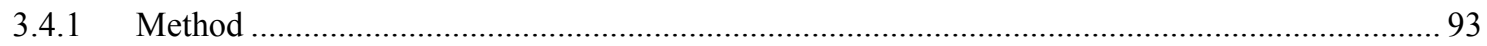

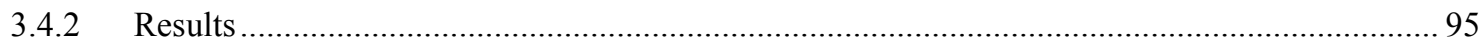

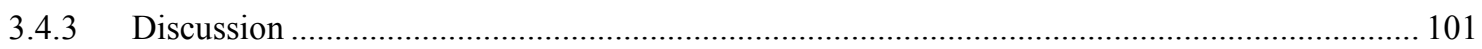

3.5 Experiment 3.3: Priming with Pattern Masking …................................................ 102

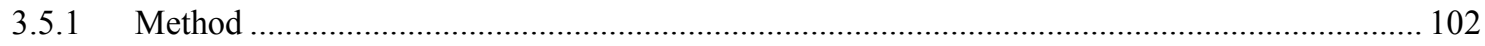

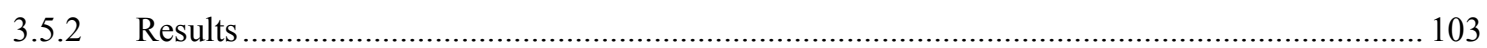

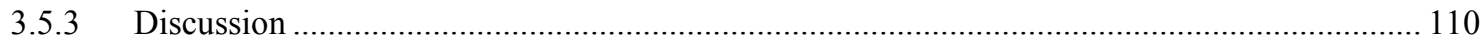

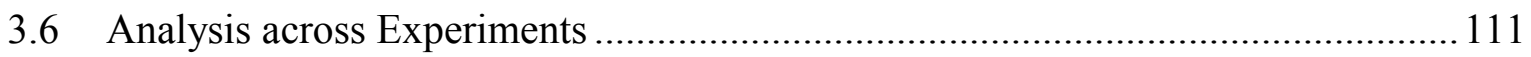

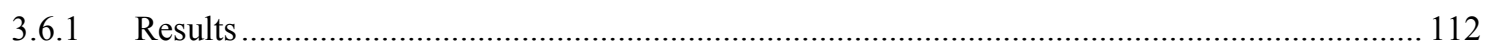

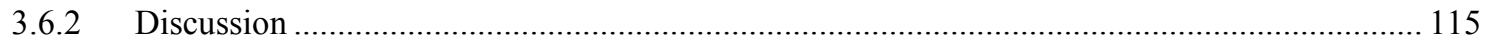

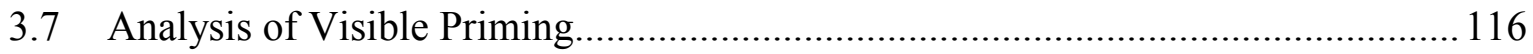

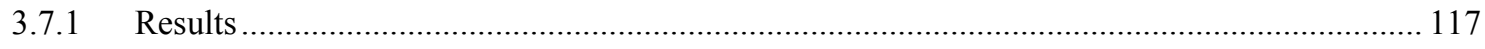

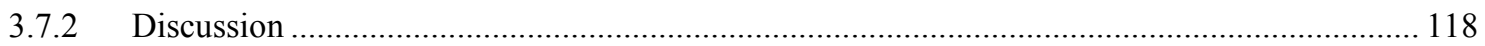

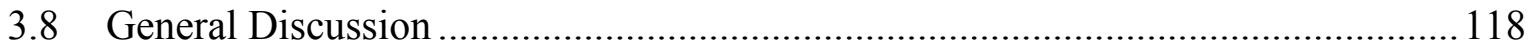

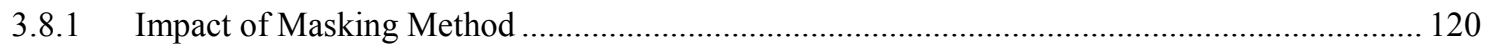

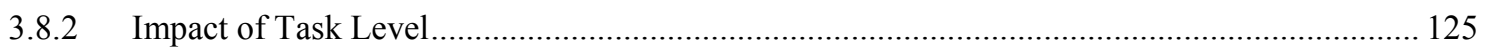

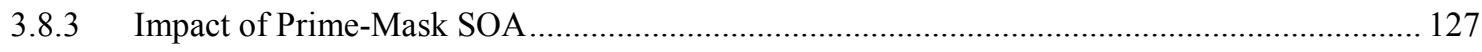

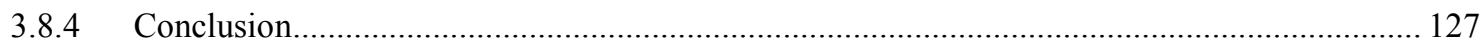




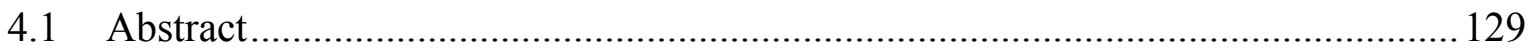

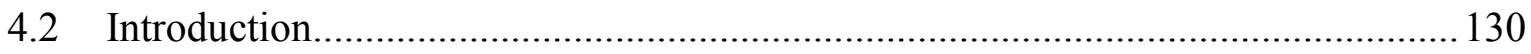

4.3 Experiment 4.1: SOA-Variation with Metacontrast Masking ................................. 134

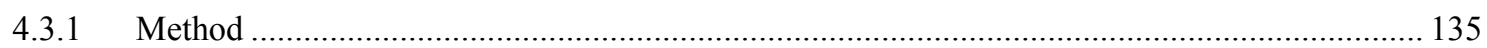

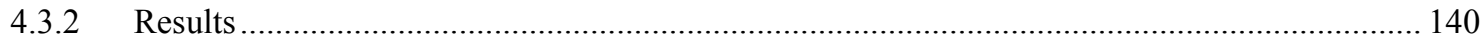

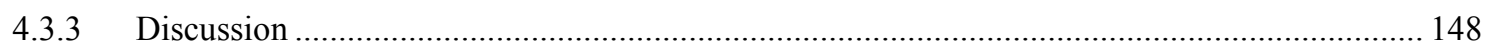

4.4 Experiment 4.2: SOA-Variation with Pattern Masking ........................................ 152

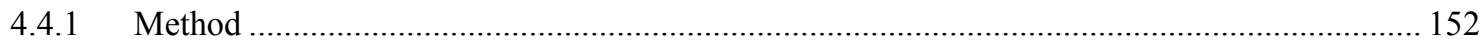

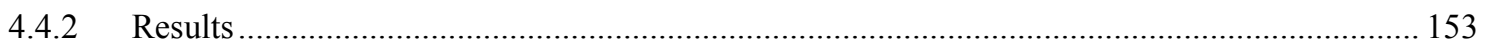

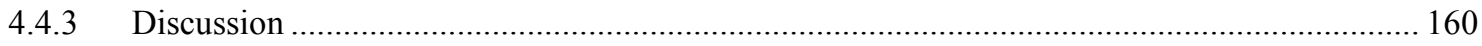

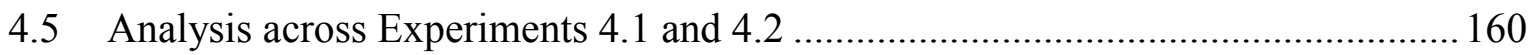

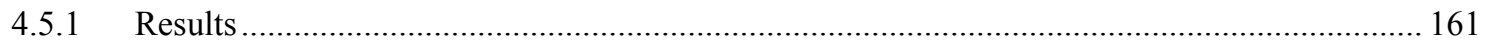

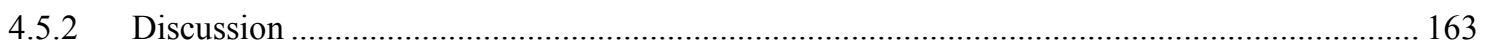

4.6 Experiment 4.3: Pattern Masking without Premasks.............................................. 166

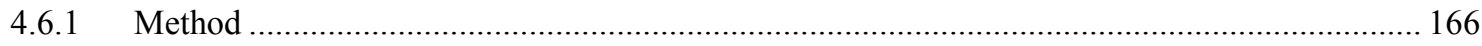

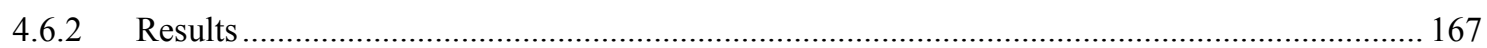

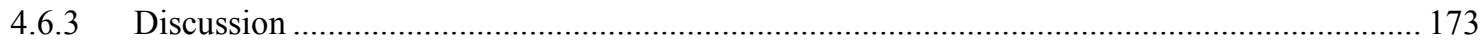

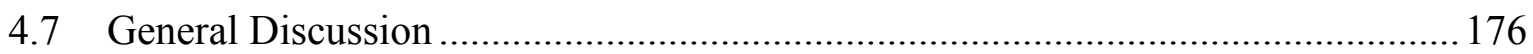

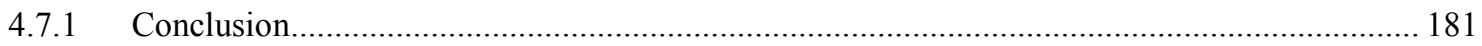

\section{Perceptual and Semantic Primingwithout ResponseAssociation:}

Electrophysiological Differences ........................................................................................183

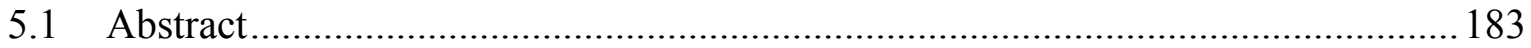

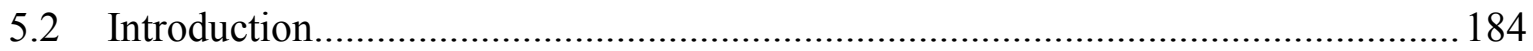

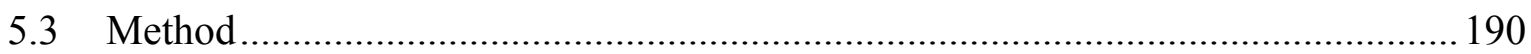




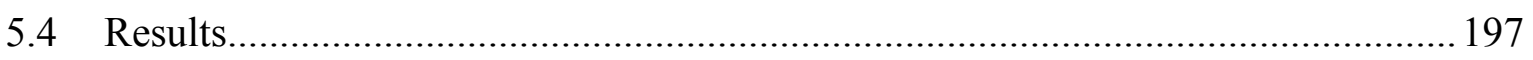

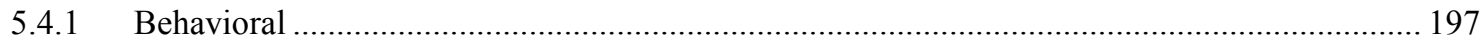

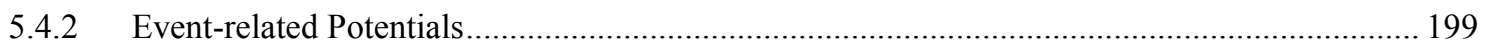

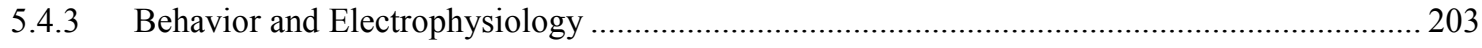

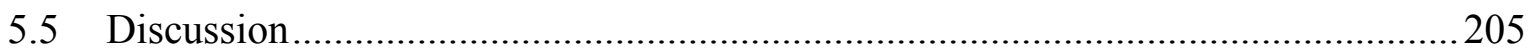

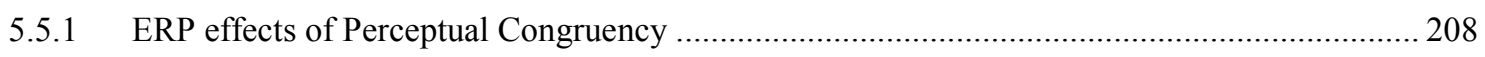

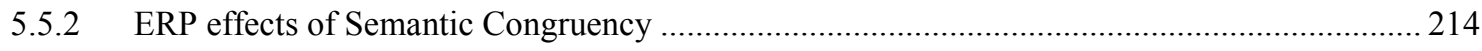

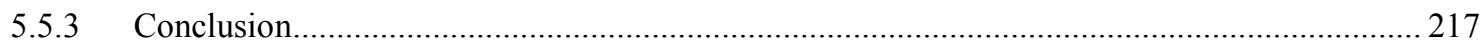

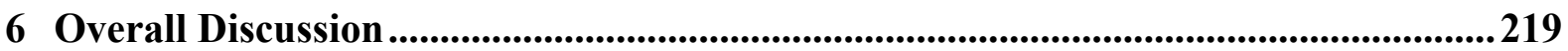

6.1 Manipulation and Measurement of Prime Visibility …........................................221

6.2 Perceptual and Semantic Priming - Similarities and Differences ..........................225

6.3 Masking Method - a Key to the Structure of Consciousness .................................2230

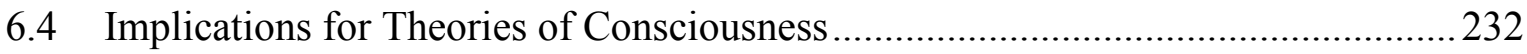

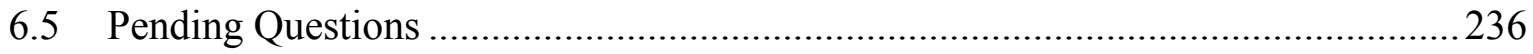

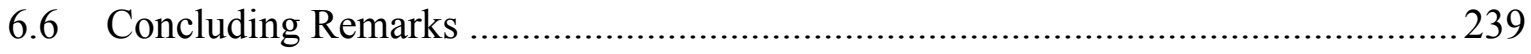

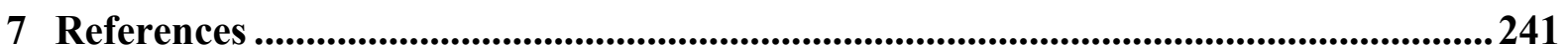

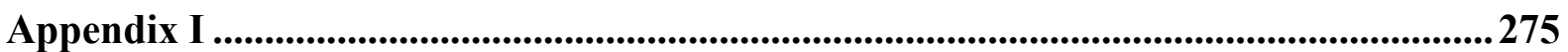

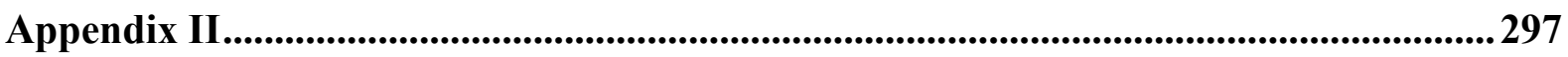

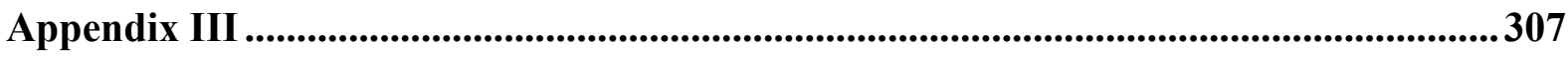

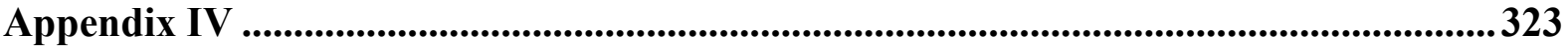

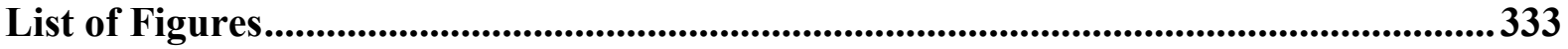

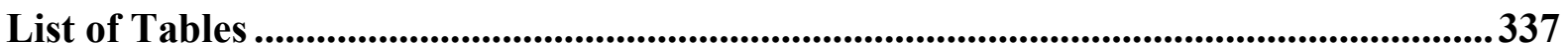

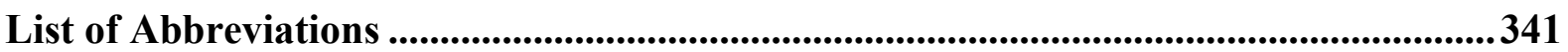




\section{Introduction}

Psychologists are interested in consciousness since psychology evolved as an independent discipline (Kouider \& Dehaene, 2007). The examination of unconscious processing provides an established approach to this topic. Several different theories were developed by psychologists and philosophers concerning the function and structure of consciousness (Blackmore, 2003). For instance, the Multiple Drafts Model by Dennett (1991), the Dynamic Core Hypothesis by Tononi and Edelman (1998), the Global Neuronal Workspace Theory by Dehaene, Kerszberg and Changeux (1998) and the model of Recurrent Processing by Lamme and Roelfsema (2000) are popular and extensively discussed conceptions of consciousness. Dennett (1991) proclaims that consciousness as an entity is an illusion. Stimulus information is processed in multiple drafts and as soon as relevant aspects are processed behavior is triggered. The illusion of consciousness as an entity is generated through memory. Tononi and Edelman (1998) describe consciousness as a dynamic core. Dynamic core refers to a functional cluster of different areas. These areas are connected and exchange and integrate information. Continuity is preserved as the dynamic core changes fluidly. Not all active areas are thought to be a part of the dynamic core. Dehaene and colleagues propose a derivative of Baars (1989) Global Workspace Theory (Dehaene \& Changeux, 2011). The global neuronal workspace is based on the integration of different automatic and nonconscious processes through cortical pyramidal cells. These processes reach global accessibility whereas other processes are inhibited. Lamme and Roelfsema (2000) distinguish two stages of (visual) processing. The authors review evidence that neuronal activity spreads rapidly to several areas. However, recurrent processing seems to be necessary for conscious access. Although experimental validation is generally desired and partially provided, these theories are framed on a high level. Specific predictions can hardly be derived and some statements seem not to be crucial for these theories. For instance, some 
authors proclaim that consciousness is dichotomous (e.g. Sergent \& Dehaene, 2004a). However, assumed mechanisms are conceivable with or without this characteristic. Furthermore, some theories are similar in certain aspects and seem partially integrable. For instance, recurrent and horizontal connectivity is widely assumed to be essential for consciousness. However, different theories emphasize different functions or characteristics of consciousness and one might argue that one or the other touches the very core of consciousness. These theories provide a broad framework for experiments that were conducted as a part of this dissertation. These experiments are described in following chapters. This work is mainly focused on masked priming as a method for examining unconscious processing of stimuli. However, besides singular insights into characteristics of consciousness, it was aimed to validate or invalidate aspects of established theories.

Initially, a meta-analysis was conducted to obtain an overview of previous research. The next chapter is dedicated to this meta-analysis. On the one hand, this was a first attempt to approach this topic and Chapter 2 should be regarded as an extensive introduction. On the other hand, integration of various studies through meta-regression revealed insights which exceed the sum of partial results.

Masked priming is a frequently applied method to examine the impact of unconsciously processed stimuli. A design that is comparable to contemporary applications was first used by Foster and Davis (1984). The unconsciously processed stimulus serves as a prime, i.e. it is presented prior to a target stimulus that usually indicates a response. In some studies the target served as a cue that indicated a specific task (Mattler, 2003). Priming effects are assessed by comparing congruent and incongruent trials. In congruent trials, primes and targets match with regards to a specific attribute. In incongruent trials, primes and targets differ with regards to this attribute. Faster or more accurate reactions to targets in congruent trials indicate an effect of the prime that is based on the processing of the specific 
attribute. Therefore, one might infer that the attribute was processed unconsciously. Several different relations between primes and targets (i.e. effect types) were investigated. For instance, semantic (e.g., Dell'Acqua \& Grainger, 1999), phonological (e.g., Rastle \& Brysbaert, 2006), orthographical (e.g., Bonin, Fayol \& Peereman, 1998) and response priming (e.g., Vorberg, Mattler, Heinecke, Schmidt \& Schwarzbach, 2003) were examined. The awareness of the prime is reduced by a mask. Several different masking techniques were developed and are frequently applied (reviewed in Breitmeyer \& Ögmen, 2006). The standard paradigm allows various modifications. Besides different effect types (Chapter 2 - 5) and masking methods (Chapter 2 - 3), studies also differ with regards to temporal parameters (Chapter $2 \& 4$ ), task attributes (Chapter 5) and several stimulus parameters (Chapter 2). The meta-analysis was conducted with the intention to include these factors in a comprehensive model. On the one hand, impacts of specific factors can be estimated and this provides insights into the acting mechanisms. On the other hand, confounding factors can be identified and addressed in subsequent experiments. The impact of one factor is of particular interest: prime visibility (Chapter $2 \& 3$ ). Low prime visibility indicates effective masking and establishes unconscious processing. If certain priming effects increase with increasing prime visibility one might assume that associated processes are not independent of awareness. Meta-regression model estimations might reveal informative interactions with prime visibility. Identifying processes that rely on awareness and processes that are independent of awareness provides insights into the structure of consciousness. For instance, the Global Neuronal Workspace Theory emphasizes the importance of consciousness for integration of information (Dehaene \& Changeux, 2011). In line with this model, low level processing of stimulus attributes might occur without consciousness whereas higher level processing should depend on awareness. Prime visibility is therefore an important factor and only studies that report prime visibility were included in the meta-analysis. Unfortunately, measurements of 
prime visibility also differ between studies. For instance, subjective or objective visibility measurements can be applied (Merikle \& Reingold, 1992). Subjective visibility measurements are usually based on an assessment of visibility by participants. Objective visibility is often measured with detection tasks or discrimination tasks. Signal Detection Theory provides a framework for calculating a specific value for stimulus visibility $(d$ '; Macmillan \& Creelman, 1991). Visibility measurements also differ concerning their exclusiveness and exhaustiveness (Khalid, König \& Ansorge, 2011). The use of different visibility measurements limits comparability of studies. However, a meta-analytic comparison of visibility measurements might suggest a hierarchical order. It was aimed to include a broad spectrum of masked priming studies across different fields of research. To minimize biases, it was essential to perform an open literature search that is based on a previously defined search string. We refrained wittingly from examining reference lists. However, only studies with subliminal primes were of interest. Prime visibility should have been reduced through short presentation, low contrast and masking. These methods should be distinguished from withdrawal of top-down attention that leads to preconscious processing (Kouider \& Dehaene, 2007).

Variance inflations factors can be calculated to reveal possible confounding factors in a meta-analysis (O'Brien, 2007). The search for confounding factors can also be driven by specific hypotheses. Two interesting confoundings were revealed. First, tasks on certain levels were applied predominantly with certain masking methods. Different masking methods might interfere with different stages of prime processing. This means that different levels of processing were examined with different residual activations induced by primes. Subsequently, six experiments were designed and conducted to solve this confounding (Chapter 3). Second, stimulus onset asynchronies (SOA) between primes, masks and targets can be applied differently. It was revealed that prime-mask SOA was varied predominantly in 
perceptual priming studies whereas mask-target SOA was varied predominantly in semantic priming studies. A systematic comparison of perceptual and semantic priming with different temporal parameters was conducted subsequently (Chapter 4). On the basis of these experiments, an experimental design was developed to examine perceptual and semantic priming with identical stimulation using electroencephalography (EEG; Chapter 5).

In an overall discussion, the results of the meta-analysis and subsequent experiments are summarized and discussed with regards to theories of consciousness. However, first of all the meta-analysis serves as an extensive introduction to masked priming. 
1 | Approaching Consciousness with Masked Priming 


\section{The Role of Visibility in Masked Priming: a Model and a Meta-Analysis}

\subsection{Abstract}

A great number of studies, using masked stimuli, provide evidence for unconsciously perceived stimuli to have an influence on mental processes. To specify the role of visibility in these processes, we analyzed 84 studies that reported the priming effects as well as a measurement of the prime visibility. Following a model-driven approach, we included the prime visibility, as an interacting moderator with several features of the different studies, in a meta-regression model. The model assumes primes to (a) influence target processing and (b) prepare the response. An effect of target-supporting is reduced, when prime and target are associated only through a common meaning and the primes never appear as targets. Under these circumstances visible primes could achieve stronger priming effects than unconscious perceived primes. Response preparation through primes is only reflected in priming effects, when primes trigger the same response as targets in congruent conditions and a different one in incongruent conditions. When this is the case and the task level is semantic, priming effects increase with increasing prime visibility. Due to a confounding between task level and masking method, this pattern might be generated by an actual impact of prime visibility on priming effect in cases using pattern masks. The results of the meta-analysis attest the viability of the developed model and highlight several moderators of priming effects, prime visibility and visibility measurements. 


\subsection{Introduction}

A wealth of studies in the literature has addressed the question whether conscious awareness of visual stimuli is a necessity of processing. Several of these studies employed masked priming paradigms to examine the extent to which unconscious stimuli can be processed, and whether or not certain processes do require stimulus awareness. In priming paradigms participants respond to consciously accessible stimuli (targets) while they are exposed to additional stimuli (primes) with reduced visibility due to some kind of masking. The processing of unconscious stimuli is accessed in these paradigms by the effects of the masked stimuli on the processing of the target. Here we report results of a meta-analytic approach on the role of consciousness for visual stimulus processing. To this end we examined priming effects of studies that employed the priming paradigm and reported measures of prime visibility.

Masked priming paradigms differ in various aspects because this method is used to address various questions. For instance, one group of studies addressed characteristics of the language processing system and employed semantic priming (e.g., Dell'Acqua \& Grainger, 1999), phonological priming (e.g., Rastle \& Brysbaert, 2006) or orthographical priming (e.g., Bonin, Fayol \& Peereman, 1998). Other studies addressed properties of the motor system (e.g., Mattler, 2003). The current meta-analysis was conducted with the intention to integrate results from all these fields of research. Within these different branches of research, scientists rely on different standard paradigms. In consequence, studies differ in respect of several experimental variables, including the kind of primes, masks, and targets, the relation between prime and target, the kind of the task that participants have to perform with the target stimuli, and the type of response that participants execute. In addition, studies vary in respect of temporal variables like the stimulus onset asynchrony (SOA) between prime and target. 
Beyond this, studies differ in respect of the methods that were used to measure participants visibility of the primes and also in respect of how effective the masking method was. Here we focus on the contribution of prime visibility to the priming effects to shed more light on the role of consciousness in visual processing. According to various theories of consciousness, conscious access is required for an integration of information, which is achieved by a recurrent connectivity and top-down processing (Tononi \& Edelman, 1998; Lamme \& Roelfsema; 2000; Dehaene \& Changeux, 2011; Dehaene \& Naccache, 2001). According to these views the processing of unconscious stimuli is severely restricted because the integration of information requires conscious access to the effective stimuli. Therefore, priming effects should increase with prime visibility, when consciousness is necessary for the priming effects. In consequence, our meta-analysis focusses on the impact of the prime visibility on priming effects.

A previous meta-analysis of Van den Bussche, Notebaert and Reynvoet (2009) examined variables that moderate semantic priming effects in semantic categorization and lexical decision as well as naming tasks. In semantic categorization tasks, authors found that priming effects were moderated by prime novelty, category size, SOA and prime visibility. In lexical decision tasks priming effects were moderated by sample size, target set size, prime duration, and whether or not prime visibility was assessed. We include moderators used by Van den Bussche and colleagues (2009) in our analyses of priming effects.

In addition to moderators of priming effects, we provide a first meta-analysis to determine moderators of performance in visibility tests. Khalid, König and Ansorge (2011) provided a literature overview on methods that have been used to test the visibility of prime stimuli. These authors suggest that performance in these visibility tests might have been confounded because participants might have confused prime and target stimuli in some studies, or because primes were previously associated with a response alternative that differs 
from the response that was required in the visibility test. In contrast to Khalid and colleagues, we conducted statistical analyses to examine the effect of moderators on performance in visibility tests.

For an overview, we first introduce the moderators that we identified in our literature search, both for priming effects on reaction times and performance in visibility tests. Then, we propose a psychological model which suggests three groups of moderators of priming effects with specific interactions. Third, we report results of meta-regression analyses of each moderator on priming effects and examine the effects and interactions of the moderators on priming effects as predicted by our model including their interaction with prime visibility. Fourth, we conducted regression analyses to of each moderator on performance in visibility tests. Finally, we report analyses of the effects of possible publication biases on our results.

In this paper, we distinguish between "conditions" which refers to one type of trials. Priming effects have been determined in all of the included studies by a comparison between congruent and incongruent trials of a specific type. We identified the effect size of priming effects by such pairs of congruent and incongruent conditions, and refer to such pairs as a "case". It is important to note that the congruency of a pair of prime and target stimuli results from the fact that both stimuli share certain features in the congruent condition and they differ in respect of these features in the incongruent condition. Congruency has to be distinguished from response association. In the lexical decision task, for instance, prime and target are semantically related on congruent trials but unrelated on incongruent trials, but participants have to respond "word" in both cases instead of "non-word" (e.g., Kouider \& Dupoux, 2001). In this task, response preparation cannot contribute to the priming effect, because the prime prepares the same response in both conditions. In contrast, in motor priming tasks, the prime is associated with the same response as the target on congruent 
trials, but with a different response on incongruent trials. In this task, response preparation contributes to the priming effects.

In the following section we introduce the moderators that we identified during the inspection of the studies that qualified for the present meta-analysis. Note that these moderators describe the characteristics of a broad range of different studies in an exploratory way. Therefore, moderators are not mutually exclusive. Table 2.1 gives all 23 moderators of this meta-analysis with their levels, a brief description and corresponding descriptive statistics.

Table 2.1

Moderators with a potential Influence on the Effect sizes or/and the visibility measures with their Operationalization, Description and Descriptive Statistics

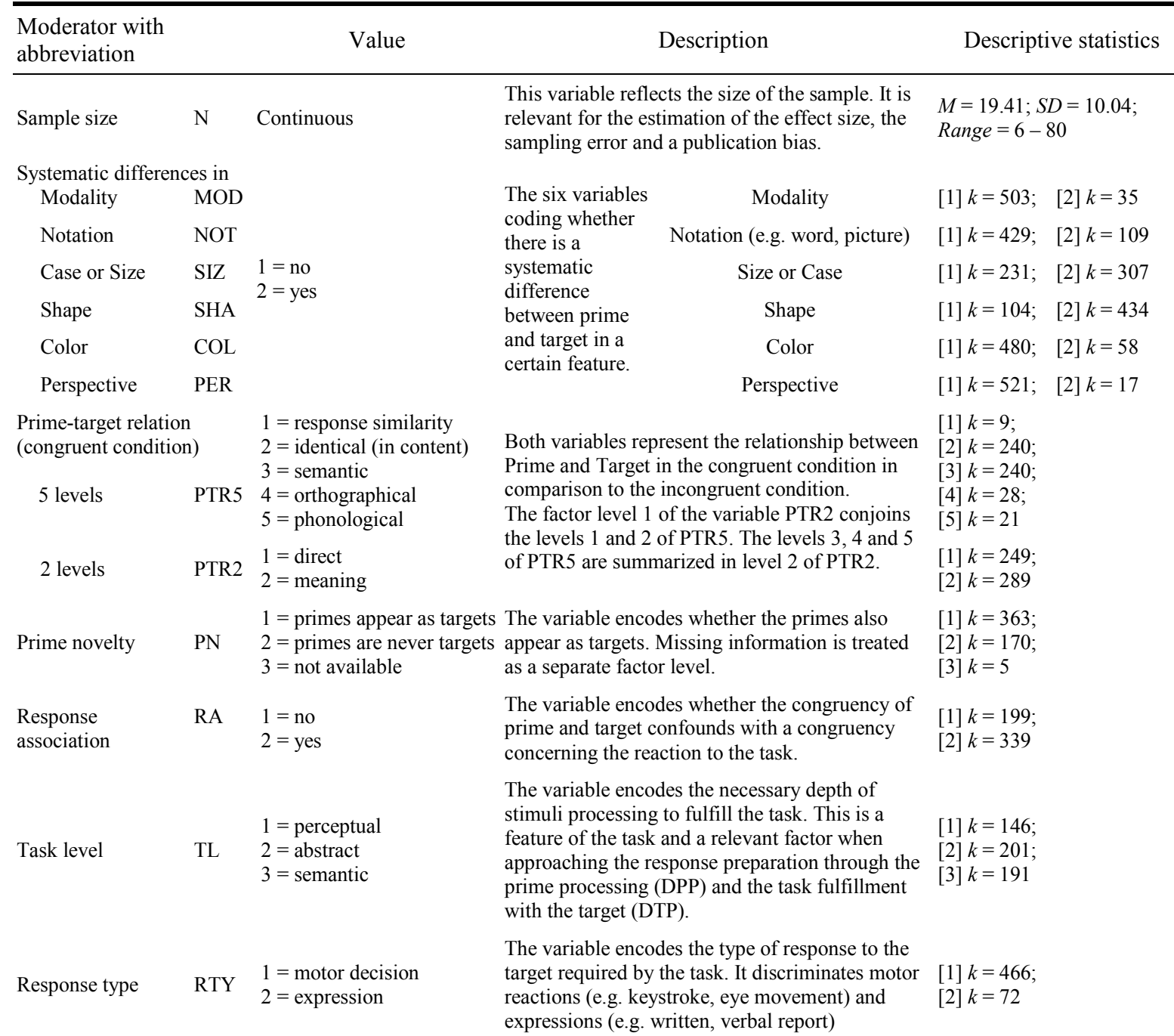


Table 2.1 (continued)

Moderators with a potential Influence on the Effect sizes or/and the visibility measures with their Operationalization, Description and Descriptive Statistics

\begin{tabular}{|c|c|c|c|c|}
\hline $\begin{array}{l}\text { Moderator with } \\
\text { abbreviation }\end{array}$ & & Value & Description & Descriptive statistics \\
\hline Prime duration & PDU & Continuous & $\begin{array}{l}\text { The variable represents the duration of the prime } \\
\text { presentation per trial and is expressed in } \\
\text { milliseconds. }\end{array}$ & $\begin{array}{l}\text { with one } d \text { '-value } \\
\qquad \begin{array}{l}M=44.02 ; S D=62.64 ; \\
\text { Range }=8-540 \\
\text { with two } d \text { '-values } \\
\quad M=43.49 ; S D=59.16 ; \\
\quad \text { Range }=8-540\end{array}\end{array}$ \\
\hline Prime-mask SOA & PMS & Continuous & $\begin{array}{l}\text { The variable represents the time interval between } \\
\text { prime and mask onset and is expressed in } \\
\text { milliseconds. }\end{array}$ & $\begin{array}{l}\text { with one } d \text { '-value } \\
\qquad M=58.40 ; S D=87.07 \\
\text { Range }=0-1000 \\
\text { with two } d \text { '-values } \\
\qquad \begin{array}{l}M=56.97 ; S D=81.81 ; \\
\text { Range }=0-1000\end{array}\end{array}$ \\
\hline Mask-target SOA & MTS & Continuous & $\begin{array}{l}\text { The variable represents the time interval between } \\
\text { mask and target onset and is expressed in } \\
\text { milliseconds. }\end{array}$ & $\begin{array}{l}\text { with one } d \text { '-value } \\
\qquad \begin{array}{l}M=103.45 ; S D=201.14 ; \\
\text { Range }=0-1486.43\end{array} \\
\text { with two } d \text { '-values } \\
\qquad \begin{array}{l}M=119.98 ; S D=208.39 \\
\text { Range }=0-1486.43\end{array}\end{array}$ \\
\hline Masking method & MAM & $\begin{aligned} & 1 \mathrm{a}= \text { Metacontrast } \\
& 1 \mathrm{~b}= \text { Flanker } \\
& 2 \mathrm{a}= \text { Target } \\
& 2 \mathrm{~b}= \text { Backward } \\
& 2 \mathrm{c}= \text { Forward \& Target } \\
& 2 \mathrm{~d}=\text { Forward \& Backward } \\
& 2 \mathrm{e}=\text { Interocular } \\
& \text { Suppression }\end{aligned}$ & $\begin{array}{l}\text { The variable encodes the masking method, which } \\
\text { is applied in the specific case. It differentiates } \\
\text { seven types of masking. Classic metacontrast } \\
\text { masking and masking with flankers can be } \\
\text { subsumed in one category ([1] Metacontrast } \\
\text { Masking). The last masking methods can be } \\
\text { subsumed under the label [2] "Pattern Masking". }\end{array}$ & $\begin{array}{l}{[1 \mathrm{a}] k=95} \\
{[1 \mathrm{~b}] k=4} \\
{[2 \mathrm{a}] k=20} \\
{[2 \mathrm{~b}] k=64} \\
{[2 \mathrm{c}] k=22\left(9 \text { with } 2 . d^{\prime}\right)} \\
{[2 \mathrm{~d}] k=326\left(69 \text { with } 2 . d^{\prime}\right)} \\
{[2 \mathrm{e}] k=7}\end{array}$ \\
\hline Visibility task & VIS & $\begin{aligned} 1 & =\text { detection } \\
2 & =\text { discrimination } \\
3 & =\text { prime-target } \\
& \text { discrimination } \\
4= & \text { identification of } \\
& \text { alternatives } \\
5 & =\text { identification }\end{aligned}$ & $\begin{array}{l}\text { This variable differentiates five tasks that serve } \\
\text { as visibility measurements. Task } 1 \text { requires a } \\
\text { present-absent decision. In task } 2 \text { subjects are } \\
\text { asked to discriminate between two previous } \\
\text { known alternatives. Task } 3 \text { is the discrimination } \\
\text { between prime and target. Task } 4 \text { requires the } \\
\text { identification of the prime out of a set of } \\
\text { alternatives, presented afterwards. Task } 5 \text { refers } \\
\text { to the free identification of the prime. }\end{array}$ & $\begin{array}{l}\text { [1] } k=55 \\
\text { [2] } k=369\left(13 \text { with } 2 . d^{\prime}\right) \\
\text { [3] } k=46 \\
\text { [4] } k=23\left(35 \text { with } 2 . d^{\prime}\right) \\
\text { [5] } k=45\left(30 \text { with } 2 . d^{\prime}\right)\end{array}$ \\
\hline $\begin{array}{l}\text { Prime-Target } \\
\text { Similarity }\end{array}$ & PTS & $\begin{array}{l}1=\text { no } \\
2=\text { yes }\end{array}$ & $\begin{array}{l}\text { Referring to Khalid, König and Ansorge (2011), } \\
\text { this variable encodes whether prime and target } \\
\text { are similar and therefore confusable. }\end{array}$ & $\begin{array}{l}\text { [1] } k=344\left(68 \text { with } 2 . d^{\prime}\right) \\
\text { [2] } k=194\left(10 \text { with } 2 . d^{\prime}\right)\end{array}$ \\
\hline $\begin{array}{l}\text { Prime-Response } \\
\text { Similarity }\end{array}$ & PRS & $\begin{array}{l}1=\text { no } \\
2=\text { yes }\end{array}$ & $\begin{array}{l}\text { Referring to Khalid, König and Ansorge (2011), } \\
\text { this variable encodes whether the required } \\
\text { response to the prime is consistent with a natural } \\
\text { response to the prime. }\end{array}$ & $\begin{array}{l}\text { [1] } k=446\left(13 \text { with } 2 . d^{\prime}\right) \\
\text { [2] } k=92\end{array}$ \\
\hline $\begin{array}{l}\text { Prime-Response } \\
\text { Mapping }\end{array}$ & PRM & $\begin{array}{l}1=\text { variable } \\
2=\text { fixed per block } \\
3=\text { fixed }\end{array}$ & $\begin{array}{l}\text { Referring to Khalid, König and Ansorge (2011), } \\
\text { this variable encodes whether the response } \\
\text { mapping is variable on a trial by trial basis, } \\
\text { which might be most confusing, fixed in every } \\
\text { block or fixed during the whole task. }\end{array}$ & $\begin{array}{l}{[1] k=54} \\
\text { [2] } k=4 \\
\text { [3] } k=480\left(78 \text { with } 2 . d^{\prime}\right)\end{array}$ \\
\hline $\begin{array}{l}\text { Target-Target } \\
\text { Similarity }\end{array}$ & TTS & $\begin{array}{l}1=\text { no } \\
2=\text { yes }\end{array}$ & $\begin{array}{l}\text { The variable encodes whether the target is } \\
\text { similar to the target, used in the priming task. }\end{array}$ & $\begin{array}{l}\text { [1] } k=139\left(42 \text { with } 2 . d^{\prime}\right) \\
\text { [2] } k=399\left(36 \text { with } 2 . d^{\prime}\right)\end{array}$ \\
\hline$d$ ' & $\mathrm{D}$ & Continuous & $\begin{array}{l}\text { The variable is a prime visibility measure based } \\
\text { on the signal detection theory. It is bias-free } \\
\text { measure of the ability to discriminate two or } \\
\text { more stimuli (e.g. in a prime discrimination task). } \\
\text { A value of } 0 \text { indicates a discriminatory ability at } \\
\text { chance-level. }\end{array}$ & $\begin{array}{l}\text { First visibility measure } \\
\qquad \begin{array}{l}M=0.48 ; S D=0.74 \\
\text { Range }=-0.24-3.83 \\
\text { Last visibility measure } \\
\quad M=0.47 ; S D=0.71 \\
\text { Range }=-0.24-3.83\end{array}\end{array}$ \\
\hline
\end{tabular}

Note. $k$ is the number of cases in the category, which is specified the second column. 


\subsubsection{Moderators of Priming Effects}

Systematic differences between primes and targets. Systematic differences between primes and targets can be found in many studies. It is reasonable to suspect a reduction of the priming effects based on a difference between prime and target, which is equally present in congruent and incongruent trials. Based on the data set we identified six different features that could be similar or different in prime and target.

Modality. While only including studies with a visual prime, we decided not to impose this restriction on the target (see "Inclusion and Exclusion Criteria", page 26). Therefore, a difference in the modality of prime and target is a possible moderator of the priming effects. In the majority of the cases a visual target was used (see Table 2.1). In the remaining cases an auditory target was applied (Grainger, Diependaele, Spinelli, Ferrand \& Farioli, 2003; Kouider \& Dehaene, 2009; Kouider \& Dupoux, 2001; Kiyonaga, Grainger, Midgley \& Holcomb, 2007).

Notation. A change in notation is to be indicated, when primes and targets are depicted in different formats. One example is the usage of a written word as prime and a picture as target (e.g., Ferrand, Grainger \& Segui, 1994), or the other way around (e.g., Carr, McCauley, Sperber \& Parmelee, 1982). Another frequently used change in notation is obtained by presenting a number with arabic digits and as a written word (e.g., Dehaene et al., 1998).

Size and case. The moderator "systematic differences between prime and target in size and case" indicates an often applied variation between primes and targets, even though different sizes are known to minimize priming effects at early, but not later, stages of vision (Clifford \& Rhodes, 2005; Zhou \& Davis, 2012). A reason for this is the aim to reduce the visibility of the prime, often pursued in masked priming paradigms (e.g., De Gardelle, 
Charles \& Kouider, 2011; and see "Influences on prime visibility" concerning masking with metacontrast masks). Therefore, the prime was usually smaller than the target (e.g., Fischer, Kiesel, Kunde \& Schubert, 2011; Huang, Zhou \& Chen, 2011) or written in lower case, while the target was written in upper case (e.g., Bodner \& Masson, 2003; Finkbeiner \& Caramazza, 2008), when this variation was applied. In only a few cases, the primes were presented in upper case, while the targets were displayed in lower case (e.g., Fisk \& Haase, 2011, Ortells; Frings \& Plaza-Ayllon, 2012). Another declared reason was the reduction of physical overlap between prime and target (e.g., Barbot \& Kouider, 2012; Eckstein \& Henson, 2012; Henson, Mouchlianitis, Matthews, Kouider, 2008; Ratinckx, Brysbaert \& Fias, 2005).

Shape. In many cases prime and target are not of the same shape (see Table 2.1). Nevertheless, it is a visual feature both stimuli can share in the congruent as well as the incongruent condition (e.g., Ansorge, 2003).

Color. In the field of masked priming, most studies use stimuli in black and white (e.g., Dell'Acqua \& Grainger, 1999; Mattler, 2003) or shades of gray (e.g., Di Luca \& Pesenti, 2008; Finkbeiner \& Caramazza, 2008; Van den Bussche, Notebaert \& Reynvoet, 2009), because masking a colored prime is attached to some difficulties (see Breitmeyer, Ro \& Singhal, 2004). However, we identified cases, in which systematic differences in color occurred, usually as a part of an experiment with more than one feature that could define a trial as congruent or incongruent (e.g., Enns \& Oriet, 2008; Tapia, Breitmeyer \& Shooner, 2010).

Perspective. The effects of prime and target that differ in their orientation and therefore are perceived from different perspectives are subject of several studies. Some of these studies focus on orientation as a congruency defining feature. In a subgroup of this type of experiments a second congruency defining feature is applied, causing cases with 
systematic differences between prime and target in orientation to occur (e.g., Huang et al., 2011). Other studies focus directly on these systematic differences, irrespective of the congruency (e.g., Elsner, Kunde \& Kiesel, 2008).

Prime-target relation. The similarity in the congruent condition is often in the focus of a study and directly named in the title or abstact (e.g. "semantic priming" or "repetition priming" in the title and abstract of Carr \& Dagenbach, 1990). We identified five different levels of the prime-target-relation, which can be summarized to two levels on a basis of the understanding of these levels and the data we assembled. Relationships between prime and target, that we classified as direct relationships in the congruent condition, are characterized in a way that no further abstract representation than the abstract representation of the stimulus itself is involved in constructing the relationship. Within this category we can distinguish the relations through response similarity and the identical relations. We categorized cases as cases with a congruent prime-target-relationship through response similarity, when the prime is associated with the same or a similar response as the target (e.g., Heinemann, Kiesel, Pohl \& Kunde, 2010; Reynvoet, Gevers \& Caessens, 2005). In this meta-analysis cases are considered as containing an identical relationship between prime and target, when prime and target have the exact same meaning in the congruent condition. This applies, when prime and target consist of the same physical stimulation regardless of the stimulus duration (e.g., Bodner \& Mulji, 2010), but it also applies when prime and target are stimuli of different modality, notation or any other physical feature with the content remaining the same (e.g., Kouider \& Dupoux, 2001). The following three kinds of congruent relations between prime and target are implemented through an associated meaning. In a semantic relation prime and target are linked through being part of at least one category. In a broader sense prime and target could be linked by sharing certain features on a contentual basis, for example appearing in the same context like "bread" and "butter" (see Carr et al., 1982). More 
specifically, the relation of prime and target could be through a superordinate category which is for both defining, like for example being a plant or an animal (see Fabre, Lemaire \& Grainger, 2007). Another kind of relation between prime and target is based on orthography and is achieved when they are similar in spelling. In most cases, one letter is different in prime and target (e.g., Bonin et al., 1998) to still maintain this relationship, without falling into the category of an identical relationship. A phonological relation appears to be analogous to the orthographical one. Here prime and target share a similar phonology. This is applied through similar pronunciations of different letter sting. Usually, more than on letter is different between prime and target (e.g., Rastle \& Brysbaert, 2006) with a focus on maintaining the same sound, when reading it out loud.

Prime novelty. In accordance with Van den Bussche, Van den Noortgate and Reynvoet (2009), we included prime novelty as a possible moderator. Primes can appear as targets (e.g., Pratte \& Rouder, 2009) or can be of an entirely different set of stimuli than the targets (e.g., Van den Bussche, Segers \& Reynvoet, 2008). This might contribute to the priming effects and is to be considered in connection with the prime-target relation.

Response association. Mechanisms of response preparation can only contribute to the priming effect, when they deliver an advantage in the congruent condition compared to the incongruent one. This is most likely the case, when the task is associated with the similarity in the congruent condition (e.g., Dehaene et al. 1998). Certain tasks, like the lexical decision task, are especially constructed to avoid a mechanism of response preparation (e.g., Kouider \& Dupoux, 2001). To fulfill this task the subject has to decide whether the target is a word or a nonword. In the congruent as well as the incongruent condition, a word is presented as prime and one as target. The nonword trials are discarded, usually. The congruency of prime and target is independent of the task. It is to be noted, that a response association, meaning that prime and target trigger identical reactions in the congruent condition and different 
reactions in the incongruent one, is necessary for a response preparation contributing to the priming effect. The depth of prime processing is only relevant in this context, when a response association is present.

Task level (depth of prime processing). This moderator describes the necessary depth of stimuli processing to fulfill the task with the prime. Three levels of this moderator are psychological meaningful and nonetheless economical, regarding the meta-analytic calculations. A perceptual level of stimulus processing is necessary to fulfill the task, when visual features of the stimuli have to be identified, for example shape (e.g., Huang et al., 2011), color (e.g., Kunde, 2004) or orientation (e.g., Mattler, 2003). An abstract task level is deeper than a perceptual one and includes the gathering of all information relevant to form an abstract representation, which is, for example, relevant in a naming task (e.g. Ferrand et al., 1994). An activation of a semantic network is the highest level of stimulus processing. Typically, this level of processing is demanded by semantic categorization tasks, where the target has to be identified as part of one or another specific category (e.g., Kouider, Eger, Dolan \& Henson, 2009). It is important to mind, that this moderator is a feature of the task. Thereby it is very closely related to the following moderator. Nevertheless it is important to distinguish prime and target processing, based on psychological considerations and especially regarding the role of visibility, which will be discussed below.

Task level (depth of target processing). This moderator corresponds to the depth of prime processing. Depth of target processing refers to the task fulfillment with the target and, as being a characteristic of the task, it contains the exact same levels with the exact same expression for each case. The absolute mean reaction times were not available in every included study, but, exemplarily shown, reactions to tasks only demanding perceptual stimuli processing (e.g., Kunde, 2004) are often faster than reactions to tasks demanding abstract stimuli processing (e.g. Ferrand et al., 1994) or stimuli processing (e.g., Kouider, Eger, Dolan 
\& Henson, 2009). One importance of separating the processes of task fulfillment triggered by prime and target lies in the role of the visibility, which is crucial to the present meta-analysis. While the visibility varies and is constrained with regards to the prime, the target is fully visible in all included paradigms. This is taken into account in the statistical modeling.

Response type. While only including studies measuring reaction times, we opened our meta-analysis to studies with different types of demanded responses (see "Inclusion and Exclusion Criteria"). Response types, that were present in those studies, were responses through button press (e.g., Dehaene et al., 1998), through movements of the eyes (Hermens, Sumner \& Walker, 2010), by naming the target (e.g., Ferrand et al., 1994) or writing it down (Bonin et al., 1998). Using those categories as levels of the moderator turned out as impractical, because only one included study adopted movements of the eyes as a method of response and only four cases used writing. Therefore, we combined reactions through button press und eye movement under the label of "motor decisions" and naming and writing under the label of "expressions", acknowledging shared underlying processes.

$\boldsymbol{d}^{\prime}$. The impact of the prime visibility on the priming effect is the main focus of the present meta-analysis and so we embedded the moderator, reflecting the prime visibility, in the psychological model. We relied on $d^{\prime}$ 'as a measure of the ability to discriminate primes that is widely used and approved (Macmillan \& Creelman, 1991). This measure is calculated as the difference between hit rate and false-alarms rate, both transformed by the inverse of the normal distribution function. One advantage of this calculation is that it is independent of a response bias. About a fifth of the studies of our meta-analysis ran a second visibility test after the first one. To capture these data, we decided to conduct always two analyses: one with $d$ ' from all studies' first tests and one in which the $d$ ' from the first test was replaced by the $d$ ' from the second test, if available. To prevent confusion we refer them as an estimation with the first visibility measurement (M1) and the last visibility measurement (M2). 


\subsubsection{Moderators of Priming Effects and Prime Visibility}

Prime duration. Besides the moderators describe above, we took in prime duration and stimulus onset asynchronies (SOA), in line with Van den Bussche, Van den Noortgate and Reynvort (2009), to investigate the impact of these timing parameters on the priming effect. An increasing prime duration is thought to be accompanied by an increasing priming effect (e.g., Carr et al., 1982). Beyond this, prime duration is also a moderator of prime visibility (e.g., Enns \& Di Lollo, 2000; Breitmeyer \& Ögmen, 2006).

Prime-mask SOA. In contrast to Van den Bussche, Van den Noortgate and Reynvoet (2009) we differentiated the prime-target SOA in prime-mask and mask-target SOA, as there is no consensus throughout different paradigms in varying one or the other to obtain different prime-target SOA. Van den Bussche, Van den Noortgate and Reynvoet (2009) noted a different effect of the prime-target SOA in different paradigms. In response priming paradigms, the priming effect typically increases with increasing prime-target SOA (e.g., Mattler, 2003; Vorberg, Mattler, Heinecke, Schmidt \& Schwarzbach, 2003). In contrast, the semantic priming effect decreases with increasing prime-target SOA (e.g., Greenwald, Draine \& Abrams, 1996). Prime-mask SOA is also a moderator of prime visibility (e.g., Enns \& Di Lollo, 2000; Breitmeyer \& Ögmen, 2006).

Mask-target SOA. The SOA between the mask and the target can produce inverse priming effects with faster and more correct responses on incongruent rather than congruent trials (e.g., Mattler, 2003; 2006; 2007; Krüger \& Mattler, 2012). Therefore, it seems worthwhile to distinguish this time parameter from the prime-mask SOA. Mask-target SOA is also a potential moderator of prime visibility, although few researches have addressed this moderator. 
Masking method. The type of masking method that has been used in a study to reduce the visibility of the prime might modulate the prime visibility. Among the studies included in the meta-analysis, we identified seven types of masking methods. (1a) A popular masking method is the Metacontrast Masking (e.g. Ansorge, 2003; Kunde, 2004; Mattler, 2003; Breitmeyer, Ogmen \& Chen, 2004). Adopting this method, a mask or a masking target is used, that is larger than the prime and has a cut-out, tightly enclosing the prime. (1b) The usage of flanking stimuli as masks is very similar to the usage of metacontrast masking, as the prime stimuli are not covert by the masks, but surrounded by them (Faivre \& Kouider, 2011). (2a) A simple way to decrease the prime visibility is implemented by presenting the target shortly after the prime (e.g., Enns \& Oriet, 2008; Kouider \& Dupoux, 2001). In this method, the masking effect depends on a short prime presentation, quickly followed by the target. These timing parameters allow the target to serve as a mask. (2b) To enhance the masking effect, several authors additionally apply a forward mask (e.g., Bodner \& Masson, 2003). The construction of masks strongly depends on the kind of prime that has to be masked. Therefore various kinds of masks are used. As examples, pattern masks consisting of lines (e.g., Ferrand et al., 1994) or random dots (e.g., Van den Bussche, Notebaert \& Reynvoet, 2009) as well as masks of letter strings (e.g., Elsner et al. 2008) or other character strings (e.g. Kouider \& Dupoux, 2001) or masks with parts of different prime versions (e.g. Eckstein \& Henson, 2012) can be mentioned. (2c) Alternatively, masks are presented after the prime and before, after or simultaneously with the target as backward masks, to enhance the masking effect and create a wider scope with regards to the timing parameters (e.g. Cheesman \& Merikle, 1984). (2d) A combination of a forward and a backward mask is also used in many designs (e.g., Elsner et al. 2008; Ferrand et al., 1994; Kouider \& Dupoux, 2001; Van den Bussche, Notebaert \& Reynvoet, 2009). (2e) Another rarely applied way of preventing a stimulus to be detected is the utilization of interocular suppression (Barbot \& 
Kouider, 2012). Using this method, the masking is achieved by presenting the prime to the nondominant eye, while presenting a special kind of morphing mask to the dominant one. This way the duration of prime presentation can be very long without subjects noticing the prime. The last masking methods (2) can be subsumed under the category of pattern masking as an overlapping stimulus is applied to reduce the visibility.

\subsubsection{Moderators of Visibility Measurements}

Visibility test. The moderators, introduced previously, are thought to have an influence on the participants' actual prime visibility. Other moderators might have an influence on how well participants' prime visibility can be measured. One characteristic of the visibility measurement is the general method applied. The used procedures are diverse and their pros and cons are debated for a long time without reaching consensus (for a review, see Merikle \& Reingold, 1992). Within the included studies, we identified 5 different types of visibility tests. Most of them are applied in an additional session after determining the priming effects (e.g., Mattler, 2003), but there are also studies in which a visibility measurement is used to identify a desired threshold before an effect is measured (e.g., Eckstein, Kubat \& Perrig, 2011). (1) One way to examine the visibility of the prime is to present trials with and without primes in a randomized and counterbalanced way (e.g., Ansorge, 2003). In these detection tasks the subjects have to decide, on a trial by trial basis, whether a prime was displayed or not. With these responses objective measures, like percent correct and $d$ ' (see "Impact of prime visibility and hypotheses"), can be calculated based on assumptions of the signal detection theory (Macmillan \& Creelman, 1991). (2) The results, compiled with a discrimination task, can be analyzed the same way. This method of measuring the visibility of the prime is widely used (e.g., Fabre et al., 2007; Zhou \& Davis, 2012). In contrast to the detection task, the prime is present in every trial of the discrimination task and can be one of, at least, two dissimilar versions. The subjects have to 
decide, which prime variant was displayed. (3) Another visibility test, which is very similar to the discrimination of (at least) two previously known prime versions, is the discrimination of prime and target (e.g., Ferrand et al., 1994). In this paradigm the subjects have to detect a change between prime and target to decide whether they are similar or not. The three described methods differ from the following two by allowing the subject to focus on a specific feature during the trial presentation. (4) This is not possible or beneficial in an identification task, when the subjects have to name the prime without a manageable set of alternatives (e.g., Bonin et al., 1998). (5) A set of, at least two, alternatives is given in the last type of visibility measurement. In contrast to the discrimination task, the set of alternatives differs from trial to trial and is given after the prime presentation (e.g., Kouider, Dehaene, Jobert \& Bihan, 2007).

Prime-target similarity. We also collected data concerning three factors introduced by Khalid and colleagues (2011). The prime-target similarity is documented with regards to a possible confusion of prime and target, which might distort the visibility measurement. A reason for applying a target, that is very similar to the prime, is the attempt to keep the priming task and visibility measurement alike (e.g., Schmidt \& Vorberg, 2006).

Prime-response similarity. Another factor that has been addressed by Khalid and colleagues (2011) is the prime-response similarity, which implies the possibility of confusing a natural response to the prime with the claimed one.

Prime-response mapping. The third factor is the variability of the prime-response mapping across trials. A variable response mapping is applied to prevent unaware response activation distorting the visibility measure. A side effect of this method is an increased risk of confusing the response mapping. 
Target-target similarity. Another debated aspect is the attitude towards the role of the target during the visibility measurement. While it is often aspired to keep priming and prime identification tasks very similar (e.g., Schmidt \& Vorberg, 2006), the relevance of the target is not always recognized. In some studies different target stimuli were used during the visibility measurement (e.g., Van den Bussche et al., 2009). Other studies applied no targets (e.g., O’Connor \& Neill, 2011) when prime visibility was measured. The possibility of an additional masking effect of targets was not considered in these studies. Therefore, we included the similarity of the targets, used in the priming sessions and the prime identification sessions, as a possible moderator.

\subsubsection{A Psychological Model for the Interplay of Moderators affecting Priming Effects}

The aim of the present meta-analysis is to specify the role of the prime visibility in masked priming without restrictions to certain paradigms. Therefore, we analyzed the effects of each moderator alone and in an interaction with prime visibility. Beyond this, the inspection of the literature suggested that a psychological model to us, which relates moderators to mechanisms which contribute to priming effects (see Figure 2.1). The psychological considerations that are reflected in the model suggest four groups of moderators that operate on the four mechanisms. Beyond this, the model suggests specific interactions between moderators, which could be tested statistically by meta-regression models.

Prime Processing. Before a Prime can trigger an effect on a reaction time by supporting the Target Processing or preparing a Response, the prime has to be processed itself. This regular course can be affected by an applied mask and therefore the kind of masking method that was used in the experiment. 


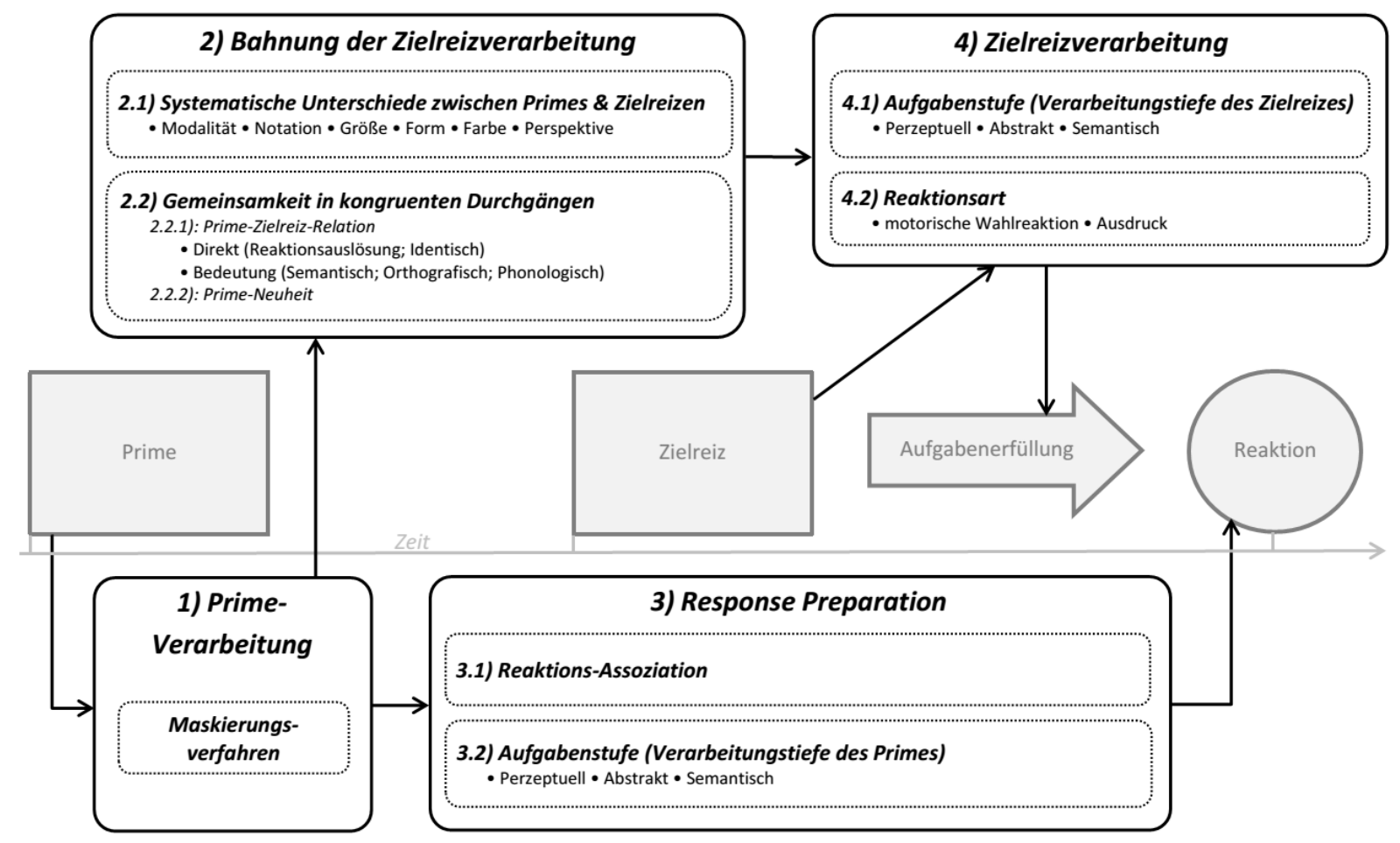

Figure 2.1. Psychological model of moderators with possible mechanisms of their influences on the priming effects.

Support of Target Processing. The first mechanism, which might contribute to fast and accurate responses on congruent trials as compared to incongruent trials results from a facilitation of target processing in consequence of the preceding prime. On the one hand, this facilitation depends on physical similarities between prime and target stimuli. On the other hand, the communalities between prime and target stimuli which constitute a congruent trial might also contribute to the facilitation of target processing. Note again, that these moderators might be confounded in some studies. Physical dissimilarities between prime and target stimuli can be found in many studies. Based on the data set we identified six different attributes on which prime and target may differ: modality, notation, size \& case, shape, color, and perspective. Communalities that constitute congruent trials are captured by the moderators prime-target relation and prime novelty. 
Response Preparation. Besides facilitating the target processing, a prime might prepare the response to the target. This mechanism of response preparation could be achieved by late levels of motor preparation and also by a preparation at earlier levels of processing like response selection. This effect of the prime is affected by the moderator response association and also by the task level.

Target Processing. This mechanism comprises the operations that are required to fulfill the task with the target. Different tasks, with different handling times, paths of processing, and brain areas involved, might have an influence on both, the congruent and incongruent condition. Beyond this, task differences might affect the effect sizes of priming effects due to a decay of information that depends on prime target congruency. One effect of task differences consists in different absolute reaction times. In this meta-analysis we identified two moderators, which might affect this mechanism: Task level and response type.

The role of prime visibility. According to our psychological model it seems plausible that prime visibility interacts with the Prime Processing as well as with the Support of Target Processing-mechanism and the Response Preparation-mechanism. The Target Processing is not directly related with the Prime processing and therefore might not depend on the visibility of the prime.

\subsubsection{Hypotheses}

On the basis of the psychological model, the following eleven hypotheses can be formulated. (1) Physical differences between primes and targets decrease the ability of the prime to support target processing and therefore decrease priming effects. (2) When the prime-target relation on congruent trials refers to early (i.e. direct) rather than high levels (i.e. meaning) of processing priming effects should be larger. This hypothesis is based on the assumption that processing of the relevant prime feature might be further progressed in the 
same time when a low level of processing is adequate. Activations on higher levels might also be more extended, less intensive and more variable. (3) When a high level of processing is necessary, more brain areas are involved. The integration of information is thought to be attached to conscious access (Tononi \& Edelman, 1998; Lamme \& Roelfsema; 2000; Dehaene \& Changeux, 2011; Dehaene \& Naccache, 2001). Therefore, prime visibility should increase priming effects when the prime-target relation refers to a high rather than a low level of processing. (4) The response preparation mechanism should only operate when primes are associated with the adequate response (cf. response association). In this case this mechanism should increase priming effects. (5) Tasks, that demand a deeper processing of the stimuli (cf. task level), are thought to reduce the priming effects, because the decay of a priming effect is further progressed when the stimuli processing takes more time. Perceptual processing is thought to occur on a low level followed by abstract and semantic processing. (6) Within the mechanism of response preparation, priming effects should depend on prime visibility in studies with a response association of the primes. We speculate that prime visibility increases priming effects due to response preparation when the task requires deep stimulus processing (e.g. semantic), because integration of information is demanded. (7) Temporal parameter of the design should influence priming effects and prime visibility. Prime duration and primemask SOA are thought to increase priming effects and prime visibility (e.g., Carr et al., 1982; Enns \& Di Lollo, 2000; Breitmeyer \& Ögmen, 2006). Prime visibility might also increase with increasing mask-target SOA as the target could also act as a weak mask. In line with theories of inverse priming, priming effects should decrease with increasing prime-mask SOA (e.g., Mattler, 2003; 2006; 2007; Krüger \& Mattler, 2012). (8) We expected results in line with Khalid and colleagues (2011), with regards to the three moderators of the visibility measurements, they introduced. The effect of prime-target similarity should be reflected in a more pronounced response bias towards the target in line with a lower general performance in 
the visibility test. (9) A match between long-term meaning of the prime (real-life context) and response mapping in the experimental task (prime-response similarity) should increase the performance in the visibility test as confusions of response mapping are reduced. (10) Another source of confusion and performance decrease could be changes in the response mapping during the experiment (variable prime-response mapping). (11) As an additional characteristic of visibility measurements, we included the target-target similarity, encoding whether the same target was used in the priming session and in the visibility task. Targets might have additional masking effects and changing or eliminating them might lead to an overestimation of prime visibility with regards to the priming session.

\subsection{Methods}

\subsubsection{Literature Search}

The aim of the present meta-analysis is to examine the role of the prime visibility in various priming paradigms. We restrained the literature search by using only PubMed as a database and applying the search string [('priming' or 'prime*') and ('mask*' or 'subliminal' or 'unconscious*' or 'nonconscious*' or 'conscious*' or 'invisib*' or 'visib*' or 'aware*' or 'unaware*')]. To prevent a bias, caused by the research history and tradition of our own research group, we set no limitations concerning the publication years and we included no unpublished data, neither of our own nor associated research groups. The search was conducted on 13th January 2012 and we identified 353 studies, of which only those published in English or German were inspected with regards to the inclusion and exclusion criteria.

\subsubsection{Inclusion and Exclusion Criteria}

In most studies, which were identified with the search, a visual prime was used. We required this as an inclusion criterion and excluded the few studies with auditory primes (e.g., 
Freyman, Balakrishnan \& Helfer, 2004; Kouider \& Dupoux, 2005). This restriction was not applied concerning the targets. Nevertheless, only studies with a target and a reaction to the target with a recorded reaction time were included. A distinct categorization with regards to the relation between prime and target had to be possible, for the study to be included (see "Complex 1.2.1: prime-target relation"). The task, the subjects had to fulfill, should concern the target and the subject should be encouraged to address the full attention to the target. As being the main focus of the present meta-analysis, the visibility had to be accessed with a task that required a reaction to the prime and allowed a calculation of $d^{\prime}$. Reaction times had to be reported in a way that made estimating an effect size of a congruent-incongruent comparison possible. Inverse priming paradigms were excluded (e.g., Eimer \& Schlaghecken, 2002; Lleras \& Enns, 2004). Only studies with healthy participants remained in the data set. In the case of missing information we tried to contract the corresponding authors. The process of study selection is illustrated in Figure 2.2. In total 84 studies remained in the analysis, providing 538 different cases. In 78 of these cases a second visibility measurement, yielding another $d$ '-value, was applied. This constitutes a problem, we address within the next section.

\subsubsection{Coding and Meta-analytic Procedures}

The applied coding system is based on the previously described psychological model, which evolved in line with the examination of the studies of interest. The data collection was conducted by the first author in permanent consultation with the second author. The different moderators are listed in Table 2.1 with their levels, a brief description and corresponding descriptive statistics. The integration of the second visibility measurement in the descriptive statistics is in line with the way it is considered in further meta-analytic calculations.

For our analysis we used the open-source package "metafor", which allows conducting meta-analyses with $\mathrm{R}$ (Viechtbauer, 2010). It is especially useful, when the 


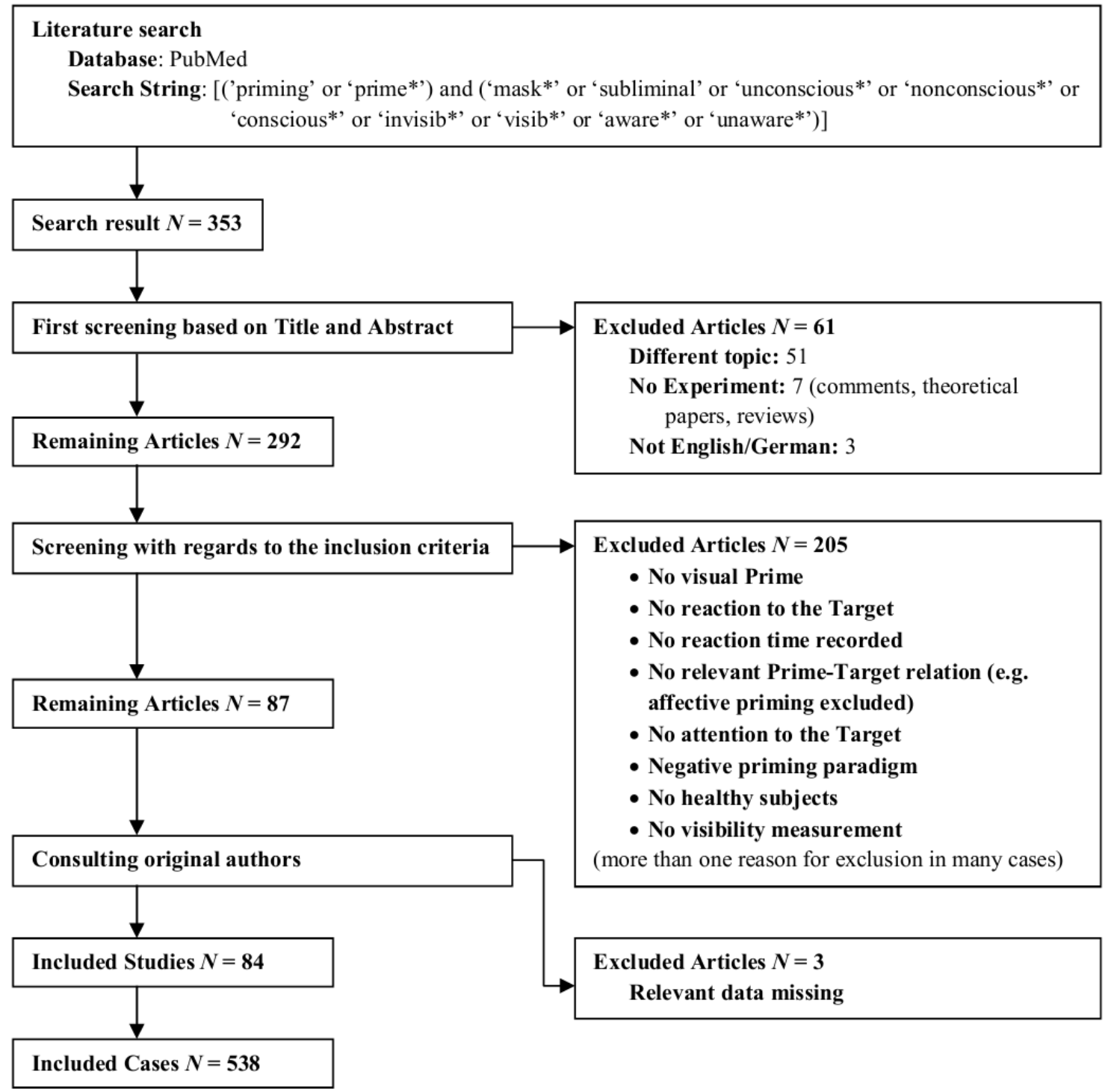

Figure 2.2. Flow diagram of study selection.

impact of certain moderators on the effect size is of interest, rather than the extent of the summary effect. Those meta-regression analyses can be conducted with continuous and categorical moderators, as described above, on the condition that an effect size (ES) and a sampling variance $\left(\mathrm{SE}^{2}\right)$ is given in every case. In a single-group repeated measures design, which is typically used in priming paradigms, the effect size is defined as the mean of the differences set in relation to the standard deviation of the differences (Gibbons, Hedeker \& Davis, 1993; Van den Bussche, Van den Noortgate \& Reynvoet, 2009). Generally, these 
values are not reported directly and are therefore to be estimated, if enough information is given. Test statistics are often reported and it is possible to estimate the effect size by dividing the t-value by the square of the sample size or by extracting the root of the F-value, divided by the sample size (Rosenthal, 1991; Van den Bussche, Van den Noortgate \& Reynvoet, 2009). In some cases it was necessary to infer these values from corresponding pvalues. If they were not reported in a more precise way, we took the required statistical values from diagrams by applying close-meshed grids to achieve a great accuracy. In line with Van den Bussche, Van den Noortgate and Reynvoet (2009), we used the following formula to estimate the sampling variance (Morris \& DeShon, 2002; Hedges, 1982):

$$
S E^{2}=\left(\frac{1}{n}\right)\left(\frac{n-1}{n-3}\right)\left(1+n \times E S^{2}\right)-\left(\frac{E S^{2}}{[c(d f)]^{2}}\right), \text { with } c(d f)=1-\frac{3}{4(n-2)}
$$

In a first examination of the data set, we applied a random-effects model, estimating the amount of heterogeneity with the restricted maximum likelihood (REML) approach. In a random-effects model it is taken into account that the included studies are not exactly alike (Borenstein, Hedges, Higgins \& Rothstein, 2009). Therefore, the underlying true effect sizes, from which the estimated ones differ by an error term, are not the same for each study. The differences between the true effect sizes, namely the heterogeneity, can be estimated with different approaches. Following Viechtbauers (2005) recommendation, we used the REML estimator as being preferable with regards to unbiasedness and efficiency. The presence of heterogeneity is a premise for further meta-regression analysis, as it is tested whether certain moderators can explain parts of the heterogeneity. Within these mixed-effect models, combinations of different moderators can be tested and it was in our interest to fit a complex model with several moderators to specify the role of the visibility in moderating the priming effect. To approach this aim in a structured way, we examined single moderators and their 
interactions with prime visibility, first. Direct comparisons of two alternative models with likelihood ratio tests are possible, when models are estimated with maximum likelihood (ML) estimation (Viechtbauer, 2010). As multicollinearity might be a problem in more complex regression models, we compiled an overview of the Pearson correlations among the moderators. Variance inflation factors (VIF) were computed for possible moderators of the effect size. Afterwards we relied on the introduced psychological model and addressed the moderators in small groups. In a fitting process insignificant moderators were excluded, beginning with the one with the highest p-value, until every remaining moderator was significant (Van den Bussche, Van den Noortgate \& Reynvoet, 2009). As a next step we included the visibility with regards to a possible improvement of the model, as indicated by a reduction of the amount of heterogeneity $\tau^{2}$ and a smaller Akaike information criterion (AIC). By combining the four groups of moderators and fitting the best model, with regards to both $\tau^{2}$ and the AIC, moderators with an influence on the priming effect can be identified. The hypothesis, concerning the moderator visibility and its interaction with other moderators, can be evaluated with this model.

As mentioned in the previous section, in 78 out of the 538 cases a second measure of $d$ ' is provided because some studies measured $d$ ' for a second time (M2) sometime after the first $d$ ' test (M1). To capture these data we decided to build two sets of data and run every analysis in which visibility is a moderator with both sets. Note, in both sets the same number of cases are included because the first data set includes the $d$ ' values of the first test and the second set includes $d$ ' values of the second test, in cases in which such a second test was performed. The other cases kept the $d^{\text {'}}$-values of the first test.

Moderators were excluded only when they seemed to be insignificant with both sets. The assignment of the visibility measurements to one set or the other was achieved quasi- 
random by them being addressed or mentioned as the first or the second measurement in the original studies.

As an estimation of the prime visibility, $d$ ' was reported in many studies. Nevertheless, in some cases it had to be calculated with the hit rate $(\mathrm{H})$ and false-alarms rate (F), if both were available:

$$
d^{\prime}=z(H)-z(F)
$$

with $\mathrm{z}$ as the inverse of the normal distribution function (Macmillan \& Creelman, 1991). In 214 of the 616 visibility measurements only percent correct was reported. We decided not to exclude these studies, but to estimate the $d^{\prime}$-values under the assumption that the responses were given unbiased. The package "psyphy" provides a function for these calculations, which requires the percent-correct-value and the number of alternatives, within a forces choice design (Green \& Dai, 1991; Knoblauch, 2012; Stanislaw \& Todorov, 1999). As the identification task cannot be accounted as a forced choice task, we added the percentfalse-value, divided by the number of alternatives, to the percent-correct-value as a component of guessing. If the subjects would have been aware of the alternatives, their performance would have been higher by this component of guessing. This way we transformed the performance in an identification task to a comparable performance in a forced choice task.

Some of the relevant moderators, we introduced previously, have a potential influence on the prime visibility or the prime visibility measurement. It is not possible to distinguish both aspects on a statistical basis. As a value of variance for each $d^{\prime}$-value is not available in most cases, applying meta-analytic models is not possible. Nevertheless, we wanted to examine the influences of certain design characteristics on the $d^{\prime}$-value and therefore we 
relied on linear regression models. We also estimated Pearson correlations among the moderators and VIF values.

One important issue, when conducting a meta-analysis, is the publication bias. The summary effect as well as all model estimations might be biased by a tendency to publish certain studies, while other results maintain inaccessible for other researchers (Borenstein et al., 2009). The most prominent bias is to publish mainly significant results. A reason for this might be that non-significant results are considered as less conclusive and replications of studies as less innovative. The consequential loss of studies is in a dependence of the sample size: Studies with large sample sizes are likely to be published regardless of their significance, as they are conducted with a lot of effort and considered as more conclusive. Furthermore, only large effects are likely to be significant in studies with a small sample size. Therefore, this bias is thought to be more present in studies with small sample sizes. Hence, a correlation between sample size and effect size is an indication, that this bias might have an influence on the effect. Examining a funnel plot, with the sample sizes plotted against the effect sizes, is insightful. The expected pyramid, with more variance between studies with small sample sizes than with large ones, is thought to be upright and isosceles in an unbiased data set. A tilted triangle gives an impression, where effect sizes might be missing. A publication bias would lead to an overestimation of the summary effect. Although there are methods of adjusting the summary effect with regards of this publication bias, these are less relevant in the present meta-analysis, as the focus is mainly on the influence of the moderators than the summary effect itself. Whether a publication bias has also a distorting influence on the meta-regression, is tested by examining whether the publication bias has a different influence on the single levels of each moderator. Further limitations of the results by other sources of a bias are addressed in the General Discussion. 


\subsection{Results}

\subsubsection{Descriptive Statistics}

Altogether, 538 cases out of 84 studies were analyzed with 78 effect sizes being associated with more than one $d^{\prime}$-value. The number of relevant moderators, we gathered, were 23, all listed in Table 2.1 with a short description and the descriptive statistics. The sample sizes ranged between 6 and 80 with approximately 19 subjects on average. The span of prime durations between 8 and $540 \mathrm{~ms}$ is wide, but with an average of $44 \mathrm{~ms}$ and a standard deviation of $63 \mathrm{~ms}$ the prime was presented for a short time, below $50 \mathrm{~ms}$, in most cases. The mean prime-mask SOA was $58 \mathrm{~ms}$ and the mean mask-target SOA $103 \mathrm{~ms}$, both also ranging widely between 0 and $1000 \mathrm{~ms}$, and between 0 and $1486 \mathrm{~ms}$, respectively. The described masking methods were all applied in different cases. Nevertheless, results concerning masking with flankers or interocular suppression must be interpreted with caution, as both methods are used in less than 10 cases each. Another level of a moderator with less than 10 cases is the prime-target relation through response similarity. Further analyses suggested, reducing the levels of the moderator by combining certain categories.

\subsubsection{Summary Effect and Heterogeneity}

Before including moderators, an empty random-effects model was calculated. The summary effect was significant with $0.61(S E=0.02,95 \% \mathrm{CI}=0.57-0.65, p<.0001)$. Considering the large number of included cases, a forest plot would be of little informational value. Appendix I contains a Table with the effect size, sampling variance and moderator levels for each case. The total amount of heterogeneity $\tau^{2}$ was 0.094 with $56.9 \%$ of the variability due to heterogeneity $\left(\mathrm{I}^{2}\right)$. The test for heterogeneity was significant $(p<.0001)$, suggesting the assumption of moderators. The AIC of this empty model is an important reference for all models with moderators and was set with 786.5. 


\subsubsection{Single Moderators of Priming Effects and their Interaction with $d$ '}

Table 2.2 contains the different models with only one potential moderator and their relevant test values. The residual heterogeneity $\tau^{2}$, compared to 0.094 ( $\tau^{2}$ of the empty model), indicates the amount of heterogeneity that is accounted for by the moderators. The test of moderators reveals the significance of their contribution to the estimated heterogeneity. Table 2.3 and 2.4 contain these models including an interaction with prime visibility based on estimation with the first (M1) or the last visibility measurement (M2), respectively. Test statistics of a likelihood ratio test indicate whether a model including prime visibility as an interacting moderator is preferable to a model with only one potential moderator. To apply these tests, models were estimated with the maximum likelihood approach.

Only with the last visibility measurement $d$ ' seems to be a single moderator of priming effects (regression coefficient $\beta_{M 2}=0.06, p<.05$ ). Systematic differences between primes and targets reduce the priming effects with modality $(\beta=-0.17)$, notation $(\beta=-0.11)$, shape $(\beta=-0.18)$ and color $(\beta=-0.17)$ having a significant influence, $p s<.05$. Including an interaction with prime visibility the pattern remains across both estimations with the first and the last visibility measurement in case of notation $\left(\beta_{M 1}=-0.21 ; \beta_{M 2}=-0.15\right)$, shape $\left(\beta_{M 1}=-\right.$ $\left.0.12 ; \beta_{M 2}=-0.17\right)$ and color $\left(\beta_{M 1}=-0.19 ; \beta_{M 2}=-0.16\right.$; all $\left.p s<.05\right)$. In these three models a positive interaction occurs with a difference in notation $\left(\beta_{M 1}=0.18 ; \beta_{M 2}=0.12\right)$, without a difference in Shape $\left(\beta_{M 1}=0.23\right)$ with the first visibility measurement and with a difference in shape $\left(\beta_{M 2}=0.06\right)$ with the last visibility measurement, $p s<.05$. Concerning the moderator difference in modality, the pattern remains when the new model is estimated with the first visibility measurement $\left(\beta_{M 1}=-0.26, p<.05\right)$. The single moderator has no significant influence when the model is estimated with the last visibility measurement, but $d$ ' has a 
Table 2.2

Test Statistics for estimated Models with Single Moderators of Priming-Effects

\begin{tabular}{|c|c|c|c|c|c|c|c|c|c|}
\hline Moderator & Level & $\beta$ & & & & $95 \% \mathrm{CI}$ & $\tau^{2}$ & AIC & $\begin{array}{c}\text { Test of Moderators } \\
\text { p-value }\end{array}$ \\
\hline \multirow[t]{2}{*}{ MOD } & 1 & 0.62 & $* * *$ & & & {$[0.58 ; 0.66]$} & 0.0921 & 785.54 & 0.0104 \\
\hline & 2 & 0.45 & $* * *$ & -0.17 & * & {$[0.32 ; 0.58]$} & & & \\
\hline \multirow[t]{2}{*}{ NOT } & 1 & 0.63 & $* * *$ & & & {$[0.59 ; 0.67]$} & 0.0931 & 787.30 & 0.0178 \\
\hline & 2 & 0.53 & $* * *$ & -0.11 & $*$ & {$[0.45 ; 0.60]$} & & & \\
\hline \multirow[t]{2}{*}{ SIZ } & 1 & 0.64 & $* * *$ & & & {$[0.59 ; 0.70]$} & 0.0937 & 791.02 & 0.1342 \\
\hline & 2 & 0.59 & $* * *$ & -0.06 & & {$[0.54 ; 0.63]$} & & & \\
\hline \multirow[t]{2}{*}{ SHA } & 1 & 0.75 & $* * *$ & & & {$[0.67 ; 0.83]$} & 0.0883 & 778.30 & 0.0001 \\
\hline & 2 & 0.57 & $* * *$ & -0.18 & $* * *$ & {$[0.53 ; 0.61]$} & & & \\
\hline \multirow[t]{2}{*}{$\mathrm{COL}$} & 1 & 0.63 & $* * *$ & & & {$[0.59 ; 0.66]$} & 0.0926 & 784.30 & 0.0045 \\
\hline & 2 & 0.46 & $* * *$ & -0.17 & $* *$ & {$[0.35 ; 0.57]$} & & & \\
\hline \multirow[t]{2}{*}{ PER } & 1 & 0.61 & $* * *$ & & & {$[0.57 ; 0.65]$} & 0.0946 & 790.75 & 0.5593 \\
\hline & 2 & 0.55 & $* * *$ & -0.07 & & {$[0.33 ; 0.76]$} & & & \\
\hline \multirow[t]{5}{*}{ PTR5 } & 1 & 0.75 & $* * *$ & & & {$[0.49 ; 1.01]$} & 0.0822 & 767.72 & $<0.0001$ \\
\hline & 2 & 0.73 & $* * *$ & -0.02 & & $[0.67 ; 0.78]]$ & & & \\
\hline & 3 & 0.52 & $* * *$ & -0.22 & . & {$[0.47 ; 0.58]$} & & & \\
\hline & 4 & 0.50 & $* * *$ & -0.24 & & {$[0.36 ; 0.65]$} & & & \\
\hline & 5 & 0.37 & $* * *$ & -0.37 & $*$ & {$[0.22 ; 0.52]$} & & & \\
\hline \multirow[t]{2}{*}{ PTR2 } & 1 & 0.73 & & & & {$[0.68 ; 0.78]$} & 0.0822 & 756.61 & $<0.0001$ \\
\hline & 2 & 0.51 & & -0.22 & $* * *$ & {$[0.46 ; 0.55]$} & & & \\
\hline \multirow[t]{3}{*}{ PN } & 1 & 0.68 & $* * *$ & & & {$[0.64 ; 0.72]$} & 0.0828 & 762.52 & $<0.0001$ \\
\hline & 2 & 0.46 & $* * *$ & -0.23 & $* * *$ & {$[0.19 ; 0.89]$} & & & \\
\hline & 3 & 0.54 & $* * *$ & -0.14 & & {$[0.39 ; 0.52]$} & & & \\
\hline \multirow[t]{2}{*}{ RA } & 1 & 0.52 & $* * *$ & & & {$[0.46 ; 0.57]$} & 0.0868 & 777.19 & $<0.0001$ \\
\hline & 2 & 0.67 & $* * *$ & 0.15 & $* * *$ & {$[0.62 ; 0.71]$} & & & \\
\hline \multirow[t]{3}{*}{ TL } & 1 & 0.82 & $* * *$ & & & {$[0.74 ; 0.90]$} & 0.0821 & 759.71 & $<0.0001$ \\
\hline & 2 & 0.53 & $* * *$ & -0.29 & $* * *$ & {$[0.47 ; 0.58]$} & & & \\
\hline & 3 & 0.56 & $* * *$ & -0.26 & $* * *$ & {$[0.51 ; 0.62]$} & & & \\
\hline \multirow[t]{2}{*}{ RTY } & 1 & 0.59 & $* * *$ & & & {$[0.56 ; 0.63]$} & 0.0927 & 788.20 & 0.0395 \\
\hline & 2 & 0.71 & $* * *$ & 0.12 & $*$ & {$[0.61 ; 0.82]$} & & & \\
\hline PDU & & 0.001 & $* *$ & & & {$[0.0003 ; 0.0018]$} & 0.0925 & 795.19 & 0.007 \\
\hline PMS & & 0.0002 & & & & {$[-0.0002 ; 0.0006]$} & 0.0951 & 802.38 & 0.263 \\
\hline MTS & & -0.0003 & $* * *$ & & & {$[-0.0005 ;-0.0001]$} & 0.0902 & 793.57 & 0.0005 \\
\hline \multirow[t]{2}{*}{ MAM } & 1 & 0.91 & $* * *$ & & & {$[0.82 ; 1.01]$} & 0.0798 & 748.73 & $<0.0001$ \\
\hline & 2 & 0.56 & $* * *$ & -0.36 & $* * *$ & {$[0.52 ; 0.59]$} & & & \\
\hline
\end{tabular}


significant impact with no difference in modality between prime and target $\left(\beta_{M 2}=0.07\right.$, $p<.05)$. The difference in size is only relevant in interaction with prime visibility. The model estimated with the first measurement revealed a positive impact of prime visibility with primes and targets of same size $\left(\beta_{M 1}=0.11, p<.05\right)$ and the model estimated with the first measurement yielded a positive impact of prime visibility with primes and targets of different size $\left(\beta_{M 2}=0.06, p<.05\right)$. A change in perspective between primes and targets is also only relevant in interaction with prime visibility. In case of primes and targets being presented in the same perspective, a positive impact of prime visibility is revealed when the model is estimated with the last visibility measurement $\left(\beta_{M 2}=0.06, p<.05\right)$. Regarding moderators encoding a systematic difference between primes and targets, the inclusion of prime visibility as a moderator significantly explains additional heterogeneity only in interaction with notation, modality with the last visibility measurement and size with the first visibility measurement, $p s<.05$.

We encoded the relation between prime and target in the congruent condition with five levels. Estimating a model with this moderator revealed its contribution to the heterogeneity $\left(p<.0001, \tau^{2}=0.0822\right.$, AIC $\left.=767.72\right)$. Furthermore, the results suggested that cases with a relation through semantic, orthography or phonology do not differ in their effect sizes $(p s>.05)$, while they showed significantly smaller effects than cases with a relation through response similarity or prime and target being identical $(p s<.05)$, both not differing in their effect sizes either $(p>.05)$. We reduced the levels of the moderator to two by combining a semantic, orthographical and phonological relation under the label of an association through meaning and combining cases with primes and targets, being identical in appearance or response association, under the label of a direct relation. This moderator explained the same amount of heterogeneity. Therefore it is, with a reduced number of levels, the better model $(\mathrm{AIC}=756.61)$. A direct prime-target relation generates larger priming 
Table 2.3

Test Statistics for estimated Models with Single Moderators of Priming-Effects and their Interaction with d' of the first visibility measurement

\begin{tabular}{|c|c|c|c|c|c|c|c|c|c|c|c|c|c|}
\hline \multicolumn{2}{|c|}{$\begin{array}{r}\text { Moderator/ } \\
\text { Level }\end{array}$} & \multicolumn{4}{|c|}{$\beta_{1}$} & $95 \% \mathrm{CI}$ & \multicolumn{2}{|c|}{$\beta_{2} \times \mathrm{d}^{\mathrm{\varsigma}}$} & $95 \% \mathrm{CI}$ & $\tau^{2}$ & AIC & $\begin{array}{c}p \\
\text { (ToM) }\end{array}$ & $\begin{array}{c}p \\
\text { (LRT) }\end{array}$ \\
\hline \multirow[t]{2}{*}{ MOD } & 1 & 0.62 & $* * *$ & & & {$[0.58 ; 0.67]$} & 0.00 & & {$[-0.05 ; 0.05]$} & 0.0926 & 795.84 & 0.0497 & 0.5240 \\
\hline & 2 & 0.37 & $* * *$ & -0.26 & $*$ & {$[0.17 ; 0.56]$} & 0.15 & & {$[-0.11 ; 0.42]$} & & & & \\
\hline \multirow[t]{2}{*}{ NOT } & 1 & 0.65 & $* * *$ & & & {$[0.60 ; 0.70]$} & -0.05 & & {$[-0.10 ; 0.01]$} & 0.0886 & 787.95 & 0.0004 & 0.0019 \\
\hline & 2 & 0.44 & $* * *$ & -0.21 & $* * *$ & {$[0.35 ; 0.54]$} & 0.18 & $* *$ & {$[0.07 ; 0.29]$} & & & & \\
\hline \multirow[t]{2}{*}{ SIZ } & 1 & 0.60 & $* * *$ & & & {$[0.54 ; 0.67]$} & 0.11 & $*$ & {$[0.02 ; 0.19]$} & 0.0935 & 796.21 & 0.0151 & 0.0164 \\
\hline & 2 & 0.61 & $* * *$ & 0.01 & & {$[0.55 ; 0.67]$} & -0.05 & & {$[-0.11 ; 0.01]$} & & & & \\
\hline \multirow[t]{2}{*}{ SHA } & 1 & 0.69 & $* * *$ & & & {$[0.59 ; 0.78]$} & 0.23 & $*$ & {$[0.03 ; 0.03]$} & 0.0883 & 785.12 & 0.0002 & 0.0679 \\
\hline & 2 & 0.57 & $* * *$ & -0.12 & $*$ & {$[0.52 ; 0.62]$} & 0.00 & & {$[-0.05 ; 0.05]$} & & & & \\
\hline \multirow[t]{2}{*}{$\mathrm{COL}$} & 1 & 0.63 & $* * *$ & & & {$[0.58 ; 0.68]$} & -0.01 & & {$[-0.06 ; 0.04]$} & 0.0930 & 795.39 & 0.0391 & 0.3198 \\
\hline & 2 & 0.44 & $* * *$ & -0.19 & ** & {$[0.31 ; 0.57]$} & 0.07 & & {$[-0.22 ; 0.36]$} & & & & \\
\hline \multirow[t]{2}{*}{ PER } & 1 & 0.61 & $* * *$ & & & {$[0.57 ; 0.66]$} & 0.00 & & {$[-0.05 ; 0.05]$} & 0.0950 & 800.54 & 0.7718 & 0.7877 \\
\hline & 2 & 0.48 & $* * *$ & -0.13 & & {$[0.23 ; 0.74]$} & 0.20 & & {$[-0.24 ; 0.64]$} & & & & \\
\hline \multirow[t]{5}{*}{ PTR5 } & 1 & 0.79 & $* * *$ & & & {$[0.44 ; 1.15]$} & -0.14 & & {$[-0.91 ; 0.63]$} & 0.0837 & 786.67 & $<0.0001$ & 0.6948 \\
\hline & 2 & 0.71 & $* * *$ & -0.09 & & {$[0.64 ; 0.77]$} & 0.07 & & {$[-0.04 ; 0.18]$} & & & & \\
\hline & 3 & 0.52 & $* * *$ & -0.27 & & {$[0.46 ; 0.58]$} & 0.01 & & {$[-0.05 ; 0.07]$} & & & & \\
\hline & 4 & 0.39 & $* *$ & -0.40 & . & {$[0.15 ; 0.64]$} & 0.22 & & {$[-0.17 ; 0.60]$} & & & & \\
\hline & 4 & 0.40 & $* *$ & -0.39 & . & {$[0.13 ; 0.67]$} & -0.05 & & {$[-0.44 ; 0.34]$} & & & & \\
\hline \multirow[t]{2}{*}{ PTR2 } & 1 & 0.71 & $* * *$ & & & {$[0.64 ; 0.77]$} & 0.07 & & {$[-0.05 ; 0.18]$} & 0.0831 & 768.27 & $<0.0001$ & 0.4741 \\
\hline & 2 & 0.50 & $* * *$ & -0.21 & $* * *$ & {$[0.45 ; 0.56]$} & 0.01 & & {$[-0.04 ; 0.07]$} & & & & \\
\hline \multirow[t]{3}{*}{$\mathrm{PN}$} & 1 & 0.66 & $* * *$ & & & {$[0.61 ; 0.72]$} & 0.05 & & {$[-0.05 ; 0.15]$} & 0.0845 & 771.59 & $<0.0001$ & 0.5667 \\
\hline & 2 & 0.44 & $* * *$ & -0.23 & $* * *$ & {$[0.36 ; 0.51]$} & 0.03 & & {$[-0.03 ; 0.09]$} & & & & \\
\hline & 3 & 0.65 & . & -0.01 & & {$[-0.07 ; 1.38]$} & -1.13 & & {$[-7.36 ; 5.10]$} & & & & \\
\hline \multirow[t]{2}{*}{ RA } & 1 & 0.52 & $* * *$ & & & {$[0.45 ; 0.60]$} & -0.01 & & {$[-0.07 ; 0.05]$} & 0.0823 & 772.32 & $<0.0001$ & 0.0001 \\
\hline & 2 & 0.61 & $* * *$ & 0.08 & . & {$[0.56 ; 0.66]$} & 0.24 & $* * *$ & {$[0.13 ; 0.36]$} & & & & \\
\hline \multirow[t]{3}{*}{$\mathrm{TL}$} & 1 & 0.79 & $* * *$ & & & {$[0.70 ; 0.88]$} & 0.09 & & {$[-0.07 ; 0.25]$} & 0.0832 & 746.45 & $<0.0001$ & 0.3675 \\
\hline & 2 & 0.53 & $* * *$ & -0.26 & $* * *$ & {$[0.46 ; 0.60]$} & 0.00 & & {$[-0.06 ; 0.06]$} & & & & \\
\hline & 3 & 0.54 & $* * *$ & -0.25 & $* * *$ & {$[0.48 ; 0.61]$} & 0.09 & & {$[-0.04 ; 0.22]$} & & & & \\
\hline \multirow[t]{2}{*}{ RTY } & 1 & 0.62 & $* * *$ & & & {$[0.57 ; 0.66]$} & -0.06 & . & {$[-0.11 ; 0.00]$} & 0.0892 & 788.95 & $<0.0001$ & 0.0019 \\
\hline & 2 & 0.61 & $* * *$ & -0.01 & & {$[0.49 ; 0.73]$} & 0.16 & $* *$ & {$[0.06 ; 0.26]$} & & & & \\
\hline PDU & & 0.0000 & & & & {$[-0.0018 ; 0.0019]$} & 0.0003 & & {$[-0.0002 ; 0.0008]$} & 0.0929 & 810.60 & 0.0139 & 0.2544 \\
\hline PMS & & -0.0003 & & & & {$[-0.0013 ; 0.0007]$} & 0.0002 & & {$[-0.0002 ; 0.0006]$} & 0.0951 & 818.33 & 0.2734 & 0.2461 \\
\hline MTS & & -0.0003 & $* *$ & & & {$[-0.0005 ;-0.0001]$} & 0.0000 & & {$[-0.0001 ; 0.0001]$} & 0.0906 & 812.74 & 0.0025 & 0.9560 \\
\hline \multirow[t]{2}{*}{ MAM } & 1 & 0.89 & $* * *$ & & & {$[0.78 ; 1.00]$} & 0.09 & & {$[-0.11 ; 0.30]$} & 0.0805 & 759.82 & $<0.0001$ & 0.5952 \\
\hline & 2 & 0.55 & $* * *$ & -0.34 & $* * *$ & {$[0.51 ; 0.60]$} & 0.01 & & {$[-0.04 ; 0.06]$} & & & & \\
\hline $\mathrm{D}$ & & 0.0021 & & & & {$[-0.05 ; 0.05]$} & / & / & / & 0.0945 & 793.97 & 0.9356 & \\
\hline
\end{tabular}

Note. The different moderators are described in detail in Table 2.1. ToM = Test of Moderators; LRT = Likelihood ratio tests (Comparison of the model with and without visibility as an interacting moderator, estimated with maximum likelihood estimation); ‘***' $p<0.001$; ‘**’ $p<0.01$; ‘*’ $p<0.05$; ‘.' $p<0.1$. 
Table 2.4

Test Statistics for estimated Models with Single Moderators of Priming-Effects and their Interaction with d' of the last visibility measurement

\begin{tabular}{|c|c|c|c|c|c|c|c|c|c|c|c|c|c|}
\hline \multicolumn{2}{|c|}{$\begin{array}{r}\text { Moderator/ } \\
\text { Level }\end{array}$} & \multicolumn{4}{|c|}{$\beta_{1}$} & \multirow{2}{*}{$\frac{95 \% \mathrm{CI}}{[0,55 ; 0,64]}$} & \multicolumn{2}{|c|}{$\beta_{2} \times \mathrm{d}^{\prime}$} & \multirow{2}{*}{$\begin{array}{c}95 \% \mathrm{CI} \\
{[0,02 ; 0,13]}\end{array}$} & \multirow{2}{*}{$\begin{array}{c}\tau^{2} \\
0.0925\end{array}$} & \multirow{2}{*}{$\frac{\text { AIC }}{791.21}$} & \multirow{2}{*}{$\begin{array}{c}\begin{array}{c}p \\
(\mathrm{ToM})\end{array} \\
0.0052\end{array}$} & \multirow{2}{*}{$\begin{array}{c}\begin{array}{c}p \\
\text { (LRT) }\end{array} \\
0.0449\end{array}$} \\
\hline MOD & 1 & 0.59 & $* * *$ & & & & 0.07 & $*$ & & & & & \\
\hline & 2 & 0.43 & $* * *$ & -0.16 & & {$[0,23 ; 0,63]$} & 0.02 & & {$[-0,19 ; 0,23]$} & & & & \\
\hline \multirow[t]{2}{*}{ NOT } & 1 & 0.61 & $* * *$ & & & {$[0,57 ; 0,66]$} & 0.04 & & {$[-0,02 ; 0,11]$} & 0.0930 & 793.52 & 0.0061 & 0.0330 \\
\hline & 2 & 0.46 & $* * *$ & -0.15 & $* *$ & {$[0,36 ; 0,56]$} & 0.12 & * & {$[0,02 ; 0,23]$} & & & & \\
\hline \multirow[t]{2}{*}{ SIZ } & 1 & 0.61 & $* * *$ & & & {$[0,54 ; 0,67]$} & 0.09 & $*$ & {$[0,01 ; 0,18]$} & 0.0947 & 798.48 & 0.0462 & 0.0563 \\
\hline & 2 & 0.57 & $* * *$ & -0.04 & & {$[0,51 ; 0,62]$} & 0.04 & & {$[-0,03 ; 0,11]$} & & & & \\
\hline \multirow[t]{2}{*}{ SHA } & 1 & 0.72 & $* * *$ & & & {$[0,62 ; 0,81]$} & 0.09 & & {$[0,50 ; 0,59]$} & 0.0890 & 785.32 & 0.0001 & 0.0623 \\
\hline & 2 & 0.54 & $* * *$ & -0.17 & $* *$ & {$[0,50 ; 0,59]$} & 0.06 & * & {$[0,00 ; 0,12]$} & & & & \\
\hline \multirow[t]{2}{*}{$\mathrm{COL}$} & 1 & 0.60 & $* * *$ & & & {$[0,56 ; 0,65]$} & 0.05 & . & {$[0,00 ; 0,11]$} & 0.0935 & 792.19 & 0.0087 & 0.1647 \\
\hline & 2 & 0.44 & $* * *$ & -0.16 & $*$ & {$[0,30 ; 0,58]$} & 0.06 & & {$[-0,20 ; 0,32]$} & & & & \\
\hline \multirow[t]{2}{*}{ PER } & 1 & 0.59 & $* * *$ & & & {$[0,54 ; 0,63]$} & 0.06 & $*$ & {$[0,00 ; 0,11]$} & 0.0951 & 796.54 & 0.1707 & 0.0964 \\
\hline & 2 & 0.48 & $* * *$ & -0.10 & & {$[0,23 ; 0,74]$} & 0.20 & & {$[-0,24 ; 0,64]$} & & & & \\
\hline \multirow[t]{5}{*}{ PTR5 } & 1 & 0.78 & $* * *$ & & & {$[0,46 ; 1,10]$} & -0.10 & & {$[-0,80 ; 0,61]$} & 0.0818 & 778.34 & $<0.0001$ & 0.0273 \\
\hline & 2 & 0.72 & $* * *$ & -0.05 & & {$[0,66 ; 0,79]$} & 0.01 & & {$[-0,09 ; 0,11]$} & & & & \\
\hline & 3 & 0.48 & $* * *$ & -0.29 & . & {$[0,42 ; 0,54]$} & 0.11 & $* *$ & {$[0,04 ; 0,18]$} & & & & \\
\hline & 4 & 0.41 & $* *$ & -0.37 & . & {$[0,15 ; 0,66]$} & 0.17 & & {$[-0,20 ; 0,55]$} & & & & \\
\hline & 4 & 0.48 & $* * *$ & -0.30 & & {$[0,23 ; 0,72]$} & -0.13 & & {$[-0,37 ; 0,12]$} & & & & \\
\hline \multirow[t]{2}{*}{ PTR2 } & 1 & 0.73 & $* * *$ & & & {$[0,66 ; 0,79]$} & 0.01 & & {$[-0,09 ; 0,10]$} & 0.0822 & 762.92 & $<0.0001$ & 0.0312 \\
\hline & 2 & 0.47 & $* * *$ & -0.26 & $* * *$ & {$[0,41 ; 0,52]$} & 0.08 & $* *$ & {$[0,02 ; 0,15]$} & & & & \\
\hline \multirow[t]{3}{*}{ PN } & 1 & 0.69 & $* * *$ & & & {$[0,64 ; 0,75]$} & -0.03 & & {$[-0,11 ; 0,05]$} & 0.0810 & 759.95 & $<0.0001$ & 0.0030 \\
\hline & 2 & 0.39 & $* * *$ & -0.30 & $* * *$ & {$[0,32 ; 0,46]$} & 0.13 & $* * *$ & {$[0,06 ; 0,20]$} & & & & \\
\hline & 3 & 0.65 & . & -0.04 & & {$[-0,06 ; 1,37]$} & -1.12 & & {$[-7,27 ; 5,03]$} & & & & \\
\hline \multirow[t]{2}{*}{ RA } & 1 & 0.46 & $* * *$ & & & {$[0,39 ; 0,54]$} & 0.07 & $*$ & {$[0,01 ; 0,14]$} & 0.0823 & 770.83 & $<0.0001$ & $<0.0001$ \\
\hline & 2 & 0.61 & $* * *$ & 0.15 & $* *$ & {$[0,56 ; 0,67]$} & 0.22 & $* * *$ & {$[0,11 ; 0,33]$} & & & & \\
\hline \multirow[t]{3}{*}{$\mathrm{TL}$} & 1 & 0.79 & $* * *$ & & & {$[0,70 ; 0,88]$} & 0.09 & & {$[-0,07 ; 0,24]$} & 0.0816 & 767.90 & $<0.0001$ & 0.0222 \\
\hline & 2 & 0.47 & $* * *$ & -0.32 & $* * *$ & {$[0,40 ; 0,54]$} & 0.09 & $* *$ & {$[0,02 ; 0,16]$} & & & & \\
\hline & 3 & 0.55 & $* * *$ & -0.25 & $* * *$ & {$[0,48 ; 0,61]$} & 0.08 & & {$[-0,04 ; 0,21]$} & & & & \\
\hline \multirow[t]{2}{*}{ RTY } & 1 & 0.59 & $* * *$ & & & {$[0,54 ; 0,64]$} & 0.01 & & {$[-0,05 ; 0,08]$} & 0.0912 & 792.04 & 0.0037 & 0.0099 \\
\hline & 2 & 0.61 & $* * *$ & 0.02 & & {$[0,49 ; 0,73]$} & 0.16 & $* *$ & {$[0,06 ; 0,26]$} & & & & \\
\hline PDU & & -0.0003 & & & & {$[-0,0023 ; 0,0016]$} & 0.0004 & & {$[-0,0001 ;-0,0001]$} & 0.0927 & 809.64 & 0.0089 & 0.1399 \\
\hline PMS & & -0.0006 & & & & {$[-0,0016 ; 0,0004]$} & 0.0003 & . & {$[0,0000 ; 0,0007]$} & 0.0943 & 816.41 & 0.1059 & 0.0713 \\
\hline MTS & & -0.0004 & $* * *$ & & & {$[-0,0006 ;-0,0002]$} & -0.0002 & $*$ & {$[0,0000 ; 0,0004]$} & 0.0898 & 806.97 & 0.0002 & 0.0192 \\
\hline \multirow[t]{2}{*}{ MAM } & 1 & 0.89 & $* * *$ & & & {$[0,78 ; 1,00]$} & 0.09 & & {$[-0,11 ; 0,30]$} & 0.0795 & 752.96 & $<0.0001$ & 0.0202 \\
\hline & 2 & 0.52 & $* * *$ & -0.37 & $* * *$ & {$[0,48 ; 0,57]$} & 0.07 & $* *$ & {$[0,02 ; 0,13]$} & & & & \\
\hline $\mathrm{D}$ & & 0.06 & $*$ & & & {$[0,0035 ; 0,1126]$} & l & l & / & 0.0944 & 789.51 & 0.037 & \\
\hline
\end{tabular}

Note. The different moderators are described in detail in Table 2.1. ToM = Test of Moderators; LRT = Likelihood ratio tests (Comparison of the model with and without visibility as an interacting moderator, estimated with maximum likelihood estimation); '***' $p<0.001$; ‘**' $p<0.01$; '*’ $p<0.05$; '’ $p<0.1$. 
effects $(\beta=0.22, p<.001)$. Including prime visibility as an interacting moderator, revealed a significant impact of $d^{\prime}$ on the effect sizes only with a prime-target relation on a level of meaning and when estimated with the last visibility measurement $\left(\beta_{M 2}=0.08, p<.01\right)$. This model explains significantly more heterogeneity than the model with prime-target relation as a single moderator, $p<.05$. As a single moderator in a model prime novelty has a negative impact $(\beta=-0.23, p<.001)$ on the effect sizes $\left(\tau^{2}=0.0828, \mathrm{AIC}=762.52\right)$. Including prime visibility this pattern remains $\left(\beta_{M 1}=-0.23 ; \beta_{M 2}=-0.30 ; p s<.001\right)$, but only with the last visibility measurement prime visibility has an impact on the priming effects when primes never appear as targets $\left(\beta_{M 2}=-0.23, p<.001\right)$. A smaller AIC of 759.95 and a likelihood ratio test $(p<.005)$ indicate that this is the preferable model.

A clear influence on the effect size had the response association $\left(p<.0001, \tau^{2}=\right.$ 0.0868$, AIC $=777.19)$ with a larger effect size $(\beta=0.15)$, when a congruency of prime and target in the congruency-defining feature confounds with a congruency of the responses, triggered by prime and target. The model improved by adding the interaction of visibility and response association to the model, calculated with both data sets $\left(\tau_{\mathrm{M} 1}^{2}=0.0823, \mathrm{AIC}_{\mathrm{M} 1}=\right.$ 772.32; $\left.\tau_{\mathrm{M} 2}^{2}=0.0823, \mathrm{AIC}_{\mathrm{M} 2}=770.83\right), p s \leq .0001$. Both estimations yielded a significant effect, even when a response association was not present $\left(\beta_{M 1}=0.52 ; \beta_{M 2}=0.46 ; p s<.0001\right)$, and increasing effect sizes in line with increasing $d$ '-values, when a response congruency is present $\left(\beta_{M 1}=0.24 ; \beta_{M 2}=0.22 ; p s \leq .0001\right)$. Task level as a single moderator and in a model with prime visibility as an interacting moderator significantly accounts for heterogeneity $\left(p s<.0001 ; \tau^{2}=0.0821, \mathrm{AIC}=759.71 ; \tau_{\mathrm{M} 1}^{2}=0.0832, \mathrm{AIC}_{\mathrm{M} 1}=774.44 ; \tau_{\mathrm{M} 2}^{2}=0.0832\right.$, $\left.\mathrm{AIC}_{\mathrm{M} 2}=767.90\right)$. A necessary processing on a perceptual level yields larger priming effect than on an abstract level $\left(\beta=-0.29 ; \beta_{M 1}=-0.26 ; \beta_{M 2}=-0.32\right)$ or a semantic level $(\beta=-0.29$; $\beta_{M 1}=-0.26 ; \beta_{M 2}=-0.32$ ), all $p s<.001$. Prime visibility has a positive impact on effect sizes only when the model is estimated with the last visibility measurement and the task demanded 
a processing on an abstract level $\left(\beta_{M 2}=0.09, p<.01\right)$. Estimated with last visibility measurement, the model including prime visibility explains significantly more heterogeneity, $p<.05$

Another possible moderator of the effect sizes is the response type. The corresponding model revealed the response type to be a significant moderator $(p<.05)$ with tasks, demanding a motor decision $(\beta=0.59)$, leading to a smaller effect sizes than tasks, demanding an expression $(\beta=0.71)$. For the sake of completeness, interactions with prime visibility are reported in Table 2.3 and Table 2.4. This moderator is thought to effect the task fulfillment with the target. A contentual meaningful interaction is therefore not reasonable.

Concerning temporal parameters prime duration has a significant positive impact on the effect sizes $\left(\beta=0.001, p<.01, \tau^{2}=0.0925, \mathrm{AIC}=795.19\right)$ and the mask-target SOA a significant negative impact $\left(\beta=-0.0003, p<.001, \tau^{2}=0.0902, \mathrm{AIC}=793.57\right)$ being a single moderator in a model. The prime-mask SOA does not significantly moderate the priming effect neither as a single moderator nor in interaction with prime visibility $(p s>.1)$. Only in interaction with the mask-target SOA (estimated with the last visibility measurement) prime visibility has an impact on the effect sizes $\left(\beta_{M 2}=-0.0002, p<.05\right)$. This model is preferable to one without prime visibility, $p<.05$.

Model estimations with the masking method as a moderator of priming effects revealed pattern masking to be associated with smaller effect sizes $\left(\beta=-0.36 ; \beta_{M 1}=-0.34\right.$; $\left.\beta_{M 2}=-0.37 ; p s<.001\right)$ than metacontrast masking $\left(\tau^{2}=0.0798, \mathrm{AIC}=748.73 ; \tau^{2}{ }_{\mathrm{M} 1}=0.0805\right.$, $\left.\mathrm{AIC}_{\mathrm{M} 1}=759.82 ; \tau_{\mathrm{M} 2}^{2}=0.0795, \mathrm{AIC}_{\mathrm{M} 2}=752.96\right)$. An influence of prime visibility could only be detected within estimation with the last visibility measurement and when pattern masks are applied $\left(\beta_{M 2}=0.001, p<.01\right)$. 


\subsubsection{Meta-analytic Models for the Interplay of Moderators affecting Priming Effects}

Before proceeding with more complex model estimations, we computed Person correlations and variance inflations factors for all potential moderators of priming effects (see Table 2.5 and 2.6). To compute these correlations, we reduced the levels of the moderator prime novelty to two, excluding the category "not available" with five cases. As the moderator task level is ordinal, we assigned the value 1, 2 and 3 to the levels "perceptual", "abstract" and "semantic" to minimize the loss of information. Moderate correlations $(0.4<r<0.6)$ were identified between a systematic difference in modality and notation; systematic difference in shape and the prime-target relation; prime-target relation and task level as well as masking method; prime novelty and prime-target relation; task level and response association as well as response type; prime duration and prime visibility (M1); and between prime-mask SOA and prime visibility. Strong correlations $(r<0.6)$ were identified between prime duration and prime visibility (M2); prime duration and the prime-mask SOA; and between task level and masking method. The variance inflation factor exceeded with 4.45 the limit of four for task level (O'Brien, 2007). Especially the high correlation between task level and masking method (second highest VIF of 3.11) suggest a strong confounding between these moderators. Eliminating one of them, all remaining VIF values decline below the critical threshold (see Table 2.6). It is therefore not indicated to compute a model containing all moderators.

The proposed psychological model consists of four complexes of moderators (see Figure 2.1): (1) Prime processing, (2) Support of the target processing, (3) Response preparation and (4) Target processing. At first, we focused on single complexes (mechanisms) of moderator and the assumed interaction between these moderators. The first three mechanisms rely on prime processing and are therefore thought to be influenced by (interact with) prime visibility. Afterwards the fitted models are pooled in two comprehensive 


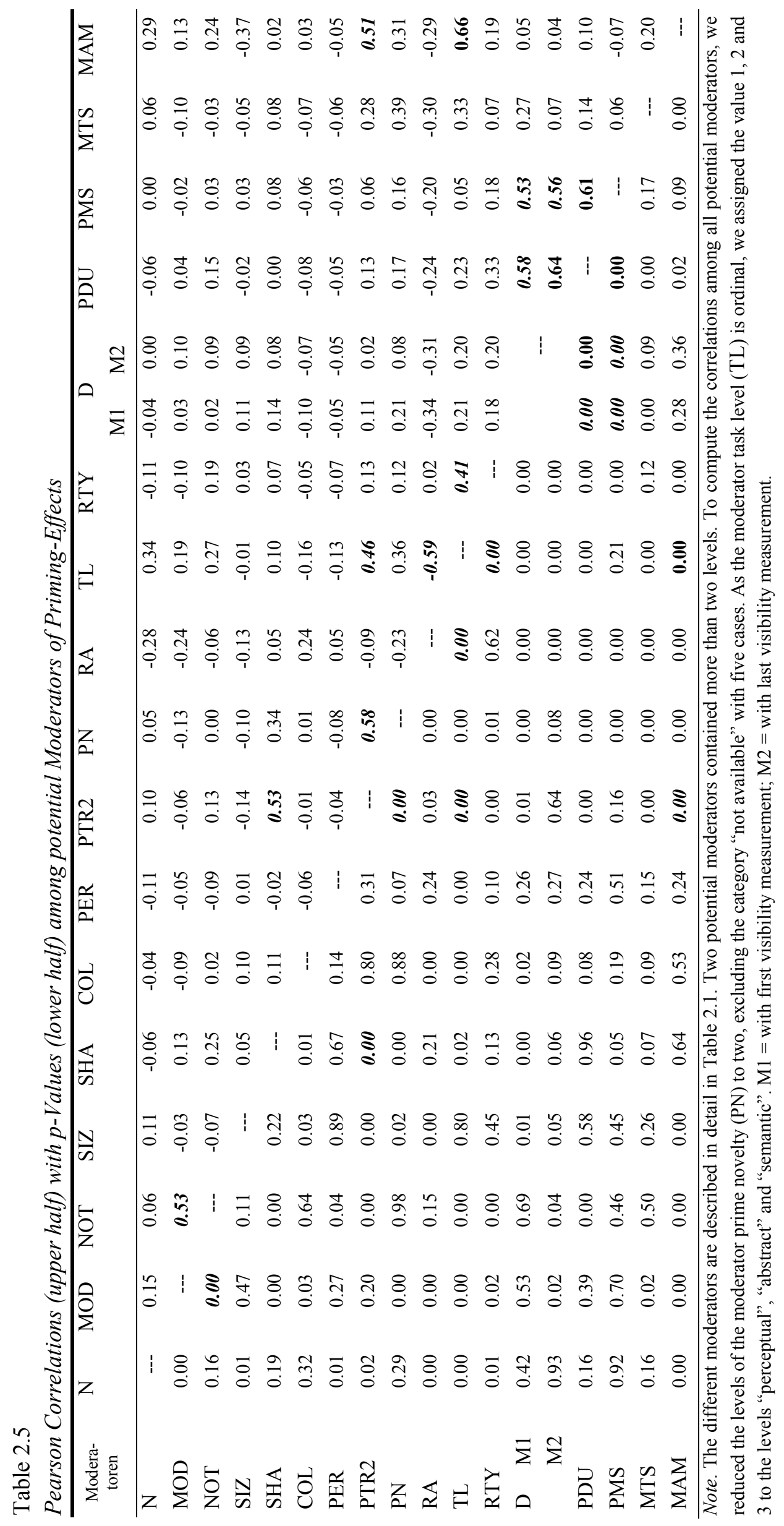


Table 2.6

Variance Inflation Factors (VIF) among potential Moderators of Priming-Effects

\begin{tabular}{lrrrrrr}
\hline \multirow{2}{*}{ Moderators } & \multicolumn{2}{c}{ With all potential Moderators } & \multicolumn{2}{c}{ Without D(P/T)P } & \multicolumn{2}{c}{ Without MAM } \\
\cline { 2 - 6 } M1 & \multicolumn{2}{c}{ M2 } & M1 & M1 & M2 \\
\hline MOD & 1.76 & 1.76 & 1.73 & 1.73 & 1.76 & 1.76 \\
NOT & 1.70 & 1.69 & 1.70 & 1.68 & 1.68 & 1.67 \\
SIZ & 1.48 & 1.45 & 1.41 & 1.40 & 1.14 & 1.13 \\
SHA & 1.98 & 1.95 & 1.98 & 1.95 & 1.79 & 1.78 \\
COL & 1.22 & 1.22 & 1.20 & 1.20 & 1.12 & 1.12 \\
PER & 1.04 & 1.04 & 1.03 & 1.03 & 1.03 & 1.03 \\
PTR2 & 3.02 & 3.03 & 2.90 & 2.90 & 2.59 & 2.61 \\
PN & 1.82 & 1.82 & 1.81 & 1.82 & 1.81 & 1.82 \\
RA & 2.52 & 2.51 & 1.76 & 1.78 & 2.52 & 2.51 \\
TL & 4.45 & 4.45 & --- & 3.46 & 3.48 \\
RTY & 1.82 & 1.82 & 1.33 & 1.33 & 1.81 & 1.81 \\
D & 1.95 & 2.03 & 1.95 & 2.02 & 1.94 & 2.02 \\
PDU & 2.18 & 2.33 & 2.18 & 2.33 & 2.17 & 2.33 \\
PMS & 1.85 & 1.86 & 1.83 & 1.84 & 1.82 & 1.84 \\
MTS & 1.37 & 1.32 & 1.35 & 1.29 & 1.36 & 1.31 \\
MAM & 3.13 & 3.11 & 2.43 & 2.43 & --- & - \\
\hline
\end{tabular}

Note. The different moderators are described in detail in Table 2.1. M1 = with first visibility measurement; M2 = with last visibility measurement.

models - one including task level and one including masking method as a moderator.

Estimated models are written-out in Table 2.7. Characteristic values (e.g. $\tau^{2} \&$ AIC) are summarized for comparison in Table 2.8.

The masking method could influence the processing of primes. As mentioned in the previous section, it appears as a significant moderator of priming effects with pattern masking leading to smaller effect sizes than metacontrast masking (see Table $2.7 \& 2.8$, Model 1). Prime visibility appears as a significant interacting moderator when the model is estimated with the last visibility measurement (Model 1d). In this case, prime visibility has a positive impact on the effect sizes when a pattern mask is applied. This model explains significantly more heterogeneity than the model without prime visibility, $p<.05$. To prevent a loss of information, moderators and interactions were only excluded, when they appeared insignificant in the estimations with the first and the last visibility measurement. 
The second complex of moderators concerns the support of the target processing. Systematic differences between primes and targets form one subgroup of moderators within this complex. While the model including all six single moderators yielded less residual heterogeneity $\left(\tau^{2}=0.0875\right)$ with the test of moderators reaching significance $(p<.0001)$, single moderators were nonsignificant (Model 2.1). The fitted model included only a difference in modality $(\beta=-0.16)$, shape $(\beta=-0.14)$ and color $(\beta=-0.16)$ to significantly reduce the priming effects, $p s<.05$. Including $d^{\prime}$ as a moderator in interaction with the six moderators, the model was to be fitted with two sets of data. The conjunction of the fitted models included a difference in notation $\left(\beta_{M 1}=-0.16 ; \beta_{M 2}=-0.11, p_{M 2}=0.059\right)$, shape $\left(\beta_{M 1}=\right.$ $\left.-0.05, p_{M 1}=0.37 ; \beta_{M 2}=-0.12\right)$ and color $\left(\beta_{M 1}=-0.15 ; \beta_{M 1}=-0.15\right)$ as separate moderators and a difference in modality $\left(\beta_{M 1}=-0.29 ; \beta_{M 1}=-0.26\right)$, notation $\left(\beta_{M 1}=0.33 ; \beta_{M 2}=0.22\right)$, size $\left(\beta_{M 1}=-0.20 ; \beta_{M 2}=-0.13\right)$ and shape $\left(\beta_{M 1}=-0.28 ; \beta_{M 2}=-0.09, p_{M 2}=0.27\right)$ in interaction with $d$ ' (remaining $p s<.05$ ). The fitted models including interactions with prime visibility (Model 2.1d (fit)) explained significantly more heterogeneity than the model without prime visibility, $p s<.005$. Another subgroup of moderators, which might contribute to the support of target processing, captures similarities between primes and targets in the congruent condition. Both moderators in this section (prime-target relation and prime novelty) were significant as single moderators of priming effects. An interaction was not to be estimated as no case applied a direct prime-target relation without reporting the prime novelty. Therefore the two moderators were combined to a single one with five levels. The new model with this combined moderator (Model 2.2) achieves a smaller amount of residual heterogeneity $\left(\tau^{2}=\right.$ 0.0801) and likelihood ratio tests confirm the preferability of this model in comparison to models with prime-target relation and prime novelty as single moderators, $p s<.05$. Including prime visibility leads to a preferable model (Model 2.2d) when estimated with the second visibility measurement, $p_{M 1}>.7$ and $p_{M 2}<.01$. The interaction between prime visibility and 
Table 2.7

List of calculated Model with their components

\begin{tabular}{|c|c|}
\hline Name & Moderators \\
\hline Model (empty) & non \\
\hline \multicolumn{2}{|c|}{$\begin{array}{l}\text { Models with one first-order complex of moderator } \\
\text { Without Visibility (calculated with the whole data set) }\end{array}$} \\
\hline Model 1 & MAM \\
\hline Model 2.1 & $\mathrm{MOD}+\mathrm{NOT}+\mathrm{SIZ}+\mathrm{SHA}+\mathrm{COL}+\mathrm{PER}$ \\
\hline Model 2.1 (fit) & $\mathrm{MOD}+\mathrm{SHA}+\mathrm{COL}$ \\
\hline Model 2.2 & {$[\mathrm{PRT} 2 / \mathrm{PN}]$} \\
\hline Model 3.1 & RA \\
\hline Model 3.2 & TL \\
\hline Model $3.2(3.1=\mathrm{No})$ & $\mathrm{TL}^{1}$ \\
\hline Model $3.2(3.1=$ Yes $)$ & $\mathrm{TL}^{2}$ \\
\hline Model 4.1 & TL \\
\hline Model 4.2 & RTY \\
\hline \multicolumn{2}{|c|}{ With Visibility (calculated with each visibility measurement set) } \\
\hline Model 1d & $\mathrm{MAM}+\mathrm{D} \times \mathrm{MAM}$ \\
\hline Model 2.1d & $\begin{array}{l}\mathrm{D}+\mathrm{MOD}+\mathrm{NOT}+\mathrm{SIZ}+\mathrm{SHA}+\mathrm{COL}+\mathrm{PER}+\mathrm{D} \times \mathrm{MOD}+\mathrm{D} \times \mathrm{NOT}+\mathrm{D} \times \mathrm{SIZ} \\
+\mathrm{D} \times \mathrm{SHA}+\mathrm{D} \times \mathrm{COL}+\mathrm{D} \times \mathrm{PER}\end{array}$ \\
\hline Model 2.1d (fit mit M1) & $\mathrm{D}+\mathrm{NOT}+\mathrm{COL}+\mathrm{D} \times \mathrm{MOD}+\mathrm{D} \times \mathrm{NOT}+\mathrm{D} \times \mathrm{SIZ}+\mathrm{D} \times \mathrm{SHA}^{3}$ \\
\hline Model 2.1d (fit mit M2) & $\mathrm{D}+\mathrm{SHA}+\mathrm{COL}+\mathrm{D} \times \mathrm{MOD}+\mathrm{D} \times \mathrm{NOT}+\mathrm{D} \times \mathrm{SIZ}^{4}$ \\
\hline Model 2.1d (fit) & $\mathrm{D}+\mathrm{NOT}+\mathrm{SHA}+\mathrm{COL}+\mathrm{D} \times \mathrm{MOD}+\mathrm{D} \times \mathrm{NOT}+\mathrm{D} \times \mathrm{SIZ}+\mathrm{D} \times \mathrm{SHA}$ \\
\hline Model 2.2d & {$[\mathrm{PRT} 2 / \mathrm{PN}]+\mathrm{D} \times[\mathrm{PRT} 2 / \mathrm{PN}]$} \\
\hline Model 3.1d & $\mathrm{RA}+\mathrm{D} \times \mathrm{RA}$ \\
\hline Model 3.2d & $\mathrm{TL}+\mathrm{D} \times \mathrm{TL}$ \\
\hline Model 3.2d (3.1=No) & $\mathrm{TL}+\mathrm{D} \times \mathrm{TL}^{1}$ \\
\hline Model 3.2d (3.1=Yes) & $\mathrm{TL}+\mathrm{D} \times \mathrm{TL}^{2}$ \\
\hline
\end{tabular}

Models with one complex of moderators

With Visibility (calculated with each visibility measurement set)

\begin{tabular}{ll}
\hline Model $1 \mathrm{~d}$ & MAM $+\mathrm{D} \times \mathrm{MAM}$ \\
Model 2 & Model $2.1 \mathrm{~d}(\mathrm{fit})+\mathrm{Model} 2.2 \mathrm{~d}$ \\
Model 3 & {$[\mathrm{RA} / \mathrm{TL}]+\mathrm{D} \times[\mathrm{RA} / \mathrm{TL}]$} \\
Model 4 & $\mathrm{TL}+\mathrm{RTY}^{5}$ \\
\hline Comprehensive models $($ calculated with each visibility measurement set $)$ \\
\hline Model 1,2,3,4 (without TL) & $\mathrm{D}+\mathrm{MAM}+\mathrm{D} \times \mathrm{MAM}+\mathrm{NOT}+\mathrm{SHA}+\mathrm{COL}+\mathrm{D} \times \mathrm{MOD}+\mathrm{D} \times \mathrm{NOT}+\mathrm{D} \times \mathrm{SIZ}$ \\
& $+\mathrm{D} \times \mathrm{SHA}+[\mathrm{PRT} / \mathrm{PN}]+\mathrm{D} \times[\mathrm{PRT} / \mathrm{PN}]+\mathrm{RA}+\mathrm{D} \times \mathrm{RA}+\mathrm{RTY}$ \\
Model C1 $(1,2,3,4 ;$ fit without TL) & $\mathrm{D}+\mathrm{MAM}+\mathrm{D} \times \mathrm{MAM}+\mathrm{NOT}+\mathrm{COL}+\mathrm{D} \times \mathrm{NOT}+\mathrm{D} \times \mathrm{SIZ}+\mathrm{D} \times \mathrm{SHA}+$ \\
& {$[\mathrm{PRT} 2 / \mathrm{PN}]+\mathrm{D} \times[\mathrm{PRT} 2 / \mathrm{PN}]+\mathrm{RA}+\mathrm{D} \times \mathrm{RA}$} \\
Model 2,3,4 & Model $2+\mathrm{Model} 3+\mathrm{Model} 4$ \\
Model C2 $(2,3,4 ;$ fit $)$ & $\mathrm{D}+\mathrm{NOT}+\mathrm{COL}+\mathrm{D} \times \mathrm{NOT}+\mathrm{D} \times \mathrm{SIZ}+\mathrm{D} \times \mathrm{SHA}+[\mathrm{PRT} 2 / \mathrm{PN}]+\mathrm{D} \times[\mathrm{PRT} 2 / \mathrm{PN}]$ \\
& $+\mathrm{D} \times[\mathrm{RA} / \mathrm{DPP}]+\mathrm{D}(\mathrm{P} / \mathrm{T}) \mathrm{P}+\mathrm{RTY}$ \\
\hline
\end{tabular}

Note. The different moderators are described in detail in Table 2.1. ${ }^{1}$ estimated with cases without response congruency; ${ }^{2}$ estimated with cases with response congruency; ${ }^{3}$ estimated with the first visibility measurement; ${ }^{4}$ estimated with the last visibility measurement; ${ }^{5}$ estimated with the whole data set. 
Table 2.8

Test Statistics for all estimated Models with their Residual Amount of Heterogeneity $\left(\tau^{2}\right)$ and the Akaike Information Criterions (AIC)

\begin{tabular}{|c|c|c|c|c|c|}
\hline Name & $\tau^{2}$ & AIC & $p(\mathrm{ToM})$ & $p(\mathrm{ToRH})$ & n.s. factors \\
\hline Model (empty) & 0.0940 & 786.53 & --- & $<.0001$ & --- \\
\hline \multicolumn{6}{|c|}{$\begin{array}{l}\text { Models with one first-order complex of moderator } \\
\text { Without Visibility }\end{array}$} \\
\hline Model 1 & 0.0798 & 748.73 & $<.0001$ & $<.0001$ & 0 \\
\hline Model 2.1 & 0.0875 & 793.47 & $<.0001$ & $<.0001$ & 4 \\
\hline Model 2.1_fit & 0.0864 & 778.24 & $<.0001$ & $<.0001$ & 0 \\
\hline Model 2.2 & 0.0801 & 761.35 & $<.0001$ & $<.0001$ & 0 \\
\hline Model 3.1 & 0.0868 & 777.19 & $<.0001$ & $<.0001$ & 0 \\
\hline Model 3.2 & 0.0821 & 759.71 & $<.0001$ & $<.0001$ & 0 \\
\hline Model $3.2(3.1=\mathrm{No})^{1}$ & 0.0921 & 268.11 & 0.1610 & $<.0001$ & 1 \\
\hline Model $3.2(3.1=\mathrm{Yes})^{2}$ & 0.0676 & 494.57 & $<.0001$ & $<.0001$ & 0 \\
\hline Model 4.1 & 0.0821 & 759.70 & $<.0001$ & $<.0001$ & 0 \\
\hline Model 4.2 & 0.0927 & 788.20 & 0.0395 & $<.0001$ & 0 \\
\hline \multicolumn{6}{|c|}{ With first visibility measurement } \\
\hline Model 1d & 0.0805 & 759.82 & $<.0001$ & $<.0001$ & 1 \\
\hline Model 2.1d & 0.0796 & 789.33 & $<.0001$ & $<.0001$ & 7 \\
\hline Model 2.1d (fit mit M1) & 0.0792 & 766.84 & $<.0001$ & $<.0001$ & 0 \\
\hline Model 2.1d (fit) & 0.0791 & 772.01 & $<.0001$ & $<.0001$ & 1 \\
\hline Model $2.2 \mathrm{~d}$ & 0.0824 & 776.59 & $<.0001$ & $<.0001$ & 1 \\
\hline Model 3.1d & 0.0823 & 772.32 & $<.0001$ & $<.0001$ & 1 \\
\hline Model 3.2d & 0.0832 & 774.44 & $<.0001$ & $<.0001$ & 1 \\
\hline Model $3.2 \mathrm{~d}(3.1=\mathrm{No})^{1}$ & 0.0934 & 277.71 & 0.2829 & $<.0001$ & 2 \\
\hline Model $3.2 \mathrm{~d}(3.1=\mathrm{Yes})^{2}$ & 0.0580 & 485.18 & $<.0001$ & $<.0001$ & 0 \\
\hline \multicolumn{6}{|c|}{ With last visibility measurement } \\
\hline Model 1d & 0.0795 & 752.96 & $<.0001$ & $<.0001$ & 0 \\
\hline Model 2.1d & 0.0869 & 811.02 & $<.0001$ & $<.0001$ & 9 \\
\hline Model 2.1d (fit mit M2) & 0.0851 & 784.53 & $<.0001$ & $<.0001$ & 0 \\
\hline Model 2.1d (fit) & 0.0854 & 791.31 & $<.0001$ & $<.0001$ & 2 \\
\hline Model 2.2d & 0.0784 & 763.55 & $<.0001$ & $<.0001$ & 0 \\
\hline Model 3.1d & 0.0823 & 770.83 & $<.0001$ & $<.0001$ & 0 \\
\hline Model 3.2d & 0.0816 & 767.90 & $<.0001$ & $<.0001$ & 0 \\
\hline Model $3.2 \mathrm{~d}(3.1=\mathrm{No})^{1}$ & 0.0875 & 267.99 & 0.0066 & $<.0001$ & 1 \\
\hline Model 3.2d $(3.1=\text { Yes })^{2}$ & 0.0594 & 488.77 & $<.0001$ & $<.0001$ & 0 \\
\hline \multicolumn{6}{|c|}{$\begin{array}{l}\text { Models with one complex of moderators } \\
\text { With first visibility measurement }\end{array}$} \\
\hline Model 1d & 0.0805 & 759.82 & $<.0001$ & $<.0001$ & 1 \\
\hline Model 2 & 0.0714 & 760.20 & $<.0001$ & $<.0001$ & 3 \\
\hline Model 3 & 0.0739 & 763.52 & $<.0001$ & $<.0001$ & 1 \\
\hline \multicolumn{6}{|c|}{ With last visibility measurement } \\
\hline Model 1d & 0.0795 & 752.96 & $<.0001$ & $<.0001$ & 0 \\
\hline Model 2 & 0.0732 & 770.14 & $<.0001$ & $<.0001$ & 3 \\
\hline Model 3 & 0.0722 & 756.48 & $<.0001$ & $<.0001$ & 1 \\
\hline \multicolumn{6}{|l|}{ Without Visibility } \\
\hline Model 4 & 0.0775 & 749.83 & $<.0001$ & $<.0001$ & 0 \\
\hline \multicolumn{6}{|l|}{ Comprehensive models } \\
\hline Model $1,2,3,4$ (without TL) & 0.0596 & 748.43 & $<.0001$ & $<.0001$ & 6 \\
\hline Model C1 & 0.0587 & 735.76 & $<.0001$ & $<.0001$ & 2 \\
\hline Model 2,3,4 & 0.0620 & 766.42 & $<.0001$ & $<.0001$ & 5 \\
\hline Model C2 & 0.0610 & 750.07 & $<.0001$ & $<.0001$ & 1 \\
\hline \multicolumn{6}{|c|}{ With last visibility measurement } \\
\hline Model 1,2,3,4 (without TL) & 0.0595 & 753.791 & $<.0001$ & $<.0001$ & 7 \\
\hline Model C1 & 0.0585 & 739.51 & $<.0001$ & $<.0001$ & 3 \\
\hline Model 2,3,4 & 0.0613 & 770.09 & $<.0001$ & $<.0001$ & 8 \\
\hline Model C2 & 0.0597 & 750.83 & $<.0001$ & $<.0001$ & 3 \\
\hline \multicolumn{6}{|c|}{$\begin{array}{l}\text { Note. The models are itemized in Table } 2.6 \text {. Moderators are likely to account for a part of the heterogeneity, when there is a } \\
\text { reduction of the residual amount of heterogeneity }\left(\tau^{2}\right) \text { and a significant Test of Moderators (ToM). The reference for this is } \\
\text { the empty model. A smaller Akaike information criterion (AIC) is an indication of a preferable model. ToRH }=\text { Test of } \\
\text { Residual Heterogeneity. }{ }^{1} \text { The empty reference model is estimated with a reduced set and has a } \tau^{2} \text { of } 0.0935 \text { and an AIC of } \\
262.55 .{ }^{2} \text { The empty reference model has a } \tau^{2} \text { of } 0.0813 \text { and an AIC of } 516.37 \text { (reduced set). }\end{array}$} \\
\hline
\end{tabular}


the moderator that captures similarities between primes and targets was therefore kept for further modelling. Combining the two subgroups of moderators ("systematic differences between prime and target" and "similarity in the congruent condition") including their interactions with prime visibility yields a model (Model 2) that explains significantly more heterogeneity than the models with only one subgroup, $p s<.0001$.

The third complex of moderators, response preparation, includes the moderators response association and task level. Both moderators explain heterogeneity as single moderators and the models including prime visibility as an interacting moderator are preferable with at least one set of visibility measurements (see Table 2.2, $2.3 \&$ \& 2.4). An interaction of response association and task level was not to be estimated as the cells were not filled sufficiently. Theoretical considerations predicted the moderator task level to be relevant for response preparation with the prime, if a response association is present. In accordance with this thought, we restrained the set once to cases with response congruency and once to cases without response congruency. The results revealed task level to be a significant moderator in cases with response association, $p<.0001$. It cannot be considered as a relevant moderator in cases without response association $(p=0.16)$. Figure 2.3 illustrates this discrepancy and the hypothesis-confirming hierarchical order of moderator levels in cases with response association. The same pattern arose by including visibility as a moderator. This model, estimated with the set with response association, explained $28.66 \%$ of the heterogeneity with the first and $26.94 \%$ with the last visibility measurement. To preserve the informative content of this pattern we combined the two moderators to one and compiled a model with this moderator and its interaction with prime visibility. The resulting model (Model 3) accounts for significantly more heterogeneity than the models with only one moderator and its interaction with prime visibility, irrespectively which set of visibility measurements was used, $p s<.0001$. 

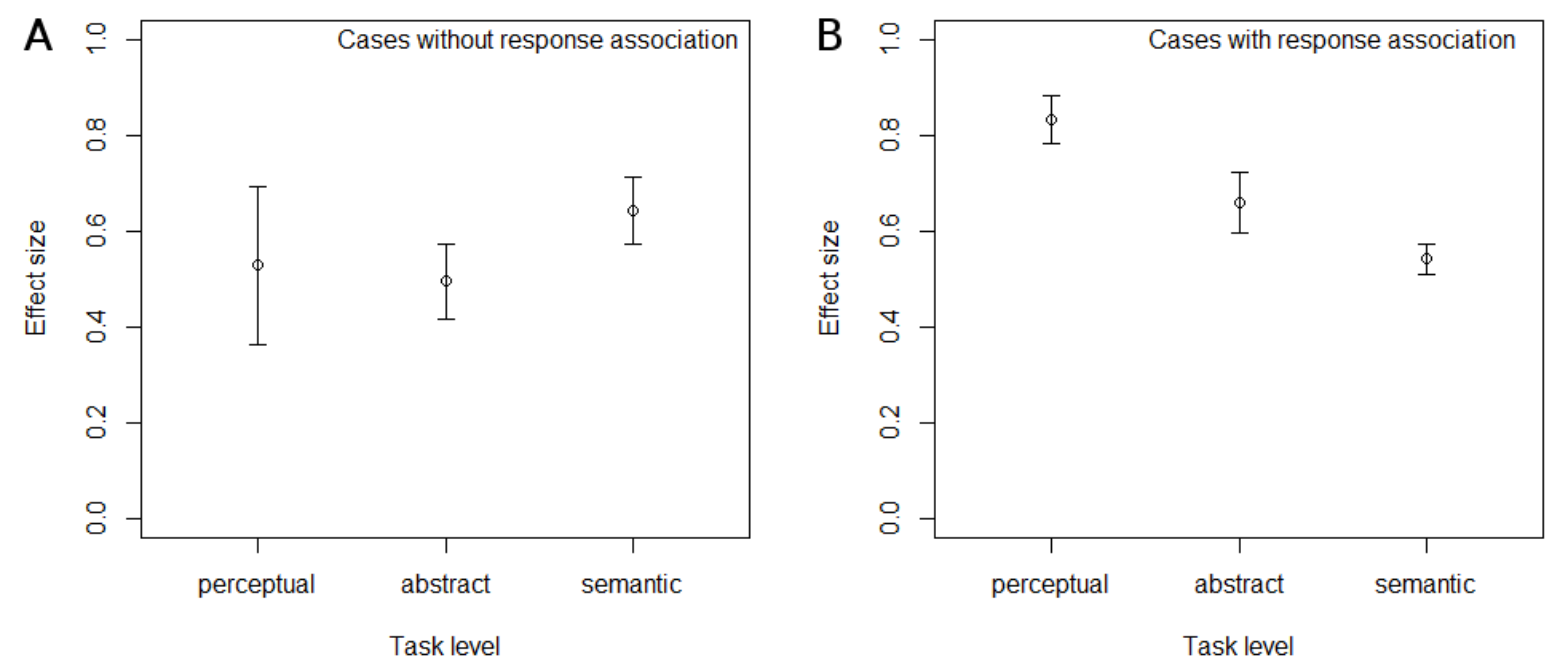

Figure 2.3. Effect sizes for different task levels. (A) Only cases without response association are included. (B) Only cases with response association are included. Response association refers to the prime-target congruency, confounding with the relation between the response, activated by the prime, and the response, activated by the target. Error bars represent one standard error.

The last complex of moderators concerns target processing. The moderators task level and response type are thought to have impacts on this process. Models including only one of these moderators account significantly for heterogeneity (Table 2.2). As contentual considerations suggest no interplay with prime visibility, this interaction was not included. An interaction of response type and task level was not to be estimated as the cells were not filled sufficiently. A moderator combining both moderators did not explain more heterogeneity than a model including the summation of these moderators (Model 4), $p>.9$, which in turn accounted for more heterogeneity than both models with only one moderator, ps $<.0001$.

To achieve comprehensive models we combined the different complexes of moderators as single noninteracting mechanisms. As the data suggest a confounding between the moderators masking method and task level, we compiled and fitted two comprehensive models, (1) one including masking method (Model C1) and (2) one including the moderator 
task level (Model C2). The models are outlined in Table 2.9 and 2.10, respectively, with the regression coefficients $(\beta)$, the corresponding standard errors $(S E)$ and $p$-values for the estimation with the first and the last visibility measurement. As both models share most moderators, we peruse them in parallel. The moderator masking method and its interaction with prime visibility is only present in one comprehensive model (see Table 2.9 and 2.10).

Table 2.9

Fitted third-order Model with estimated Parameters, including the moderator "Masking Method" and without the moderator "Depth of Prime/Target Processing" (Model C1)

\begin{tabular}{|c|c|c|c|c|c|c|c|}
\hline Moderator & Level & \multicolumn{2}{|c|}{$\beta$} & \multicolumn{2}{|c|}{$S E$} & \multicolumn{2}{|c|}{$p$} \\
\hline \multirow[t]{2}{*}{$($ Reference $=$ Level 1$)$} & & \multicolumn{2}{|c|}{ Visibility measurement } & \multicolumn{2}{|c|}{ Visibility measurement } & \multicolumn{2}{|c|}{ Visibility measurement } \\
\hline & & 1 & 2 & 1 & 2 & 1 & 2 \\
\hline Intercept & & 0.8558 & 0.8237 & 0.0684 & 0.0672 & $<.0001$ & $<.0001$ \\
\hline $\mathrm{D}$ & & 0.1770 & 0.0561 & 0.6792 & 0.1524 & 0.2679 & 0.7127 \\
\hline \multicolumn{8}{|c|}{ Complex 1: Prime Processing } \\
\hline MAM & 2 & -0.2369 & -0.2208 & 0.0697 & 0.0695 & 0.0007 & 0.0015 \\
\hline $\mathrm{D} \times \mathrm{MAM}$ & 2 & 0.0527 & 0.0754 & 0.1360 & 0.1331 & 0.6982 & 0.5710 \\
\hline \multicolumn{8}{|c|}{$\begin{array}{l}\text { Complex 2: Support of Target Processing } \\
\text { Complex 2.1: Systematic differences between prime and target }\end{array}$} \\
\hline NOT & 2 & -0.1215 & -0.0840 & 0.0493 & 0.0508 & 0.0137 & 0.0981 \\
\hline $\mathrm{COL}$ & 2 & -0.2099 & -0.2314 & 0.0573 & 0.0571 & 0.0003 & $<.0001$ \\
\hline $\mathrm{D} \times \mathrm{NOT}$ & 2 & 0.2400 & 0.1326 & 0.0638 & 0.0630 & 0.0002 & $\mathbf{0 . 0 3 5 3}$ \\
\hline $\mathrm{D} \times \mathrm{SIZ}$ & 2 & -0.1345 & -0.0522 & 0.0442 & 0.0480 & 0.0023 & 0.2769 \\
\hline $\mathrm{D} \times \mathrm{SHA}$ & 2 & -0.3915 & -0.2740 & 0.0994 & 0.0846 & $<.0001$ & 0.0012 \\
\hline \multicolumn{8}{|c|}{ Complex 2.2: Similarity in the congruent condition } \\
\hline \multirow[t]{5}{*}[\mathrm{PRT}2/\mathrm{PN}]{} & $1 / 2$ & -0.1035 & -0.1183 & 0.1561 & 0.1559 & 0.5071 & 0.4477 \\
\hline & $1 / 3$ & --- & --- & --- & --- & --- & --- \\
\hline & $2 / 1$ & -0.0141 & -0.0262 & 0.0650 & 0.0621 & 0.8282 & 0.6730 \\
\hline & $2 / 2$ & -0.1933 & -0.2459 & 0.0528 & 0.0520 & 0.0003 & $<.0001$ \\
\hline & $2 / 3$ & 0.1374 & 0.1103 & 0.3405 & 0.3406 & 0.6866 & 0.7461 \\
\hline \multirow[t]{5}{*}{$\mathrm{D} \times[\mathrm{PRT} 2 / \mathrm{PN}]$} & $1 / 2$ & 0.0697 & 0.0594 & 0.3516 & 0.3513 & 0.8429 & 0.8658 \\
\hline & $1 / 3$ & --- & --- & --- & --- & --- & --- \\
\hline & $2 / 1$ & -0.0455 & -0.0578 & 0.1267 & 0.0987 & 0.7193 & 0.5582 \\
\hline & $2 / 2$ & 0.2458 & 0.2749 & 0.0888 & 0.0794 & 0.0057 & 0.0005 \\
\hline & $2 / 3$ & -2.1495 & -2.0650 & 2.8855 & 2.8830 & 0.4563 & 0.4738 \\
\hline \multicolumn{8}{|c|}{$\begin{array}{l}\text { Complex 3: Response Preparation } \\
\text { Complex 3.1: Response Association }\end{array}$} \\
\hline RA & 2 & 0.0706 & 0.1054 & 0.0486 & 0.0465 & 0.1465 & 0.0233 \\
\hline $\mathrm{D} \times \mathrm{RA}$ & 2 & 0.3231 & 0.2841 & 0.0936 & 0.0812 & 0.0006 & 0.0005 \\
\hline
\end{tabular}

Note. The different moderators are described in detail in Table 2.1. The model was generated by combining three complexes of moderators. The model estimation was conducted with two different data sets, including the first and the last visibility measurement, respectively. All moderators, that were nonsignificant in both estimations, were excluded. The listed moderators with their levels are explained in Table 2.1. 
Table 2.10

Fitted third-order Model with estimated Parameters, including the moderator "Depth of Prime/Target Processing" and without the moderator "Masking Method" (Model C2)

\begin{tabular}{|c|c|c|c|c|c|c|c|}
\hline Moderator & Level & \multicolumn{2}{|c|}{$\beta$} & \multicolumn{2}{|c|}{$S E$} & \multicolumn{2}{|c|}{$p$} \\
\hline \multirow[t]{2}{*}{$($ Reference $=$ Level 1) } & & \multicolumn{2}{|c|}{ Visibility measurement } & \multicolumn{2}{|c|}{ Visibility measurement } & \multicolumn{2}{|c|}{ Visibility measurement } \\
\hline & & 1 & 2 & 1 & 2 & 1 & 2 \\
\hline Intercept & & 0.8411 & 0.8400 & 0.0453 & 0.0451 & $<.0001$ & $<.0001$ \\
\hline $\mathrm{D}$ & & -0.7084 & -0.8293 & 0.6792 & 0.6753 & 0.2969 & 0.2195 \\
\hline \multicolumn{8}{|c|}{$\begin{array}{l}\text { Complex 2: Support of Target Processing } \\
\text { Complex 2.1: Systematic differences between prime and target }\end{array}$} \\
\hline NOT & 2 & -0.1204 & -0.0885 & 0.0509 & 0.0522 & 0.0181 & 0.0901 \\
\hline $\mathrm{COL}$ & 2 & -0.2244 & -0.2396 & 0.0570 & 0.0568 & $<.0001$ & $<.0001$ \\
\hline $\mathrm{D} \times \mathrm{NOT}$ & 2 & 0.1820 & 0.0911 & 0.0684 & 0.0662 & 0.0078 & 0.1688 \\
\hline $\mathrm{D} \times \mathrm{SIZ}$ & 2 & -0.1229 & -0.0613 & 0.0531 & 0.0535 & 0.0205 & 0.2522 \\
\hline $\mathrm{D} \times \mathrm{SHA}$ & 2 & -0.3412 & -0.2289 & 0.1024 & 0.0881 & 0.0009 & 0.0094 \\
\hline \multicolumn{8}{|c|}{ Complex 2.2: Similarity in the congruent condition } \\
\hline \multirow[t]{5}{*}{ [PRT2/PN] } & $1 / 2$ & -0.1612 & -0.1539 & 0.1611 & 0.1603 & 0.3170 & 0.3369 \\
\hline & $1 / 3$ & --- & --- & --- & --- & --- & --- \\
\hline & $2 / 1$ & -0.0535 & -0.0585 & 0.0680 & 0.0655 & 0.4319 & 0.3715 \\
\hline & $2 / 2$ & -0.1920 & -0.2453 & 0.0579 & 0.0574 & 0.0009 & $<.0001$ \\
\hline & $2 / 3$ & 0.0198 & -0.0025 & 0.3458 & 0.3441 & 0.9544 & 0.9942 \\
\hline \multirow[t]{5}{*}{$\mathrm{D} \times[\mathrm{PRT} 2 / \mathrm{PN}]$} & $1 / 2$ & 0.2010 & 0.1502 & 0.3475 & 0.3461 & 0.5629 & 0.6642 \\
\hline & $1 / 3$ & --- & --- & --- & --- & --- & --- \\
\hline & $2 / 1$ & 0.0027 & -0.0349 & 0.1386 & 0.1041 & 0.9846 & 0.7374 \\
\hline & $2 / 2$ & 0.1694 & 0.2206 & 0.1012 & 0.0886 & 0.0941 & 0.0128 \\
\hline & $2 / 3$ & -1.8236 & -1.8001 & 2.9216 & 2.9064 & 0.5325 & 0.5357 \\
\hline \multicolumn{8}{|c|}{$\begin{array}{c}\text { Complex 3: Response Preparation } \\
\text { Complex } 3.1 \text { \& 3.2: Response Assoc }\end{array}$} \\
\hline \multirow[t]{5}{*}{$\mathrm{D} \times[\mathrm{RA} / \mathrm{TL}]$} & $1 / 2$ & 0.9688 & 0.9917 & 0.6797 & 0.6759 & 0.1541 & 0.1423 \\
\hline & $1 / 3$ & 0.8667 & 0.8364 & 0.6841 & 0.6803 & 0.2052 & 0.2189 \\
\hline & $2 / 1$ & 1.1585 & 1.1610 & 0.6724 & 0.6695 & 0.0849 & 0.0829 \\
\hline & $2 / 2$ & 1.0744 & 1.1352 & 0.6831 & 0.6803 & 0.1157 & 0.0952 \\
\hline & $2 / 3$ & 1.4611 & 1.4446 & 0.6910 & 0.6871 & 0.0345 & 0.0355 \\
\hline \multicolumn{8}{|c|}{$\begin{array}{l}\text { Complex 4: Target Processing (and Complex 3.2) } \\
\text { Complex 4.1: Depth of Target Processing }\end{array}$} \\
\hline \multirow[t]{2}{*}{ TL } & 2 & -0.2522 & -0.2676 & 0.0706 & 0.0709 & 0.0004 & 0.0002 \\
\hline & 3 & -0.1645 & -0.1357 & 0.0698 & 0.0691 & 0.0184 & 0.0496 \\
\hline \multicolumn{8}{|l|}{ Complex 4.2: Response type } \\
\hline RTY & 2 & 0.1951 & 0.1934 & 0.0706 & 0.0669 & 0.0057 & 0.0038 \\
\hline
\end{tabular}

Note. The different moderators are described in detail in Table 2.1. The model was generated by combining three complexes of moderators. The model estimation was conducted with two different data sets, including the first and the last visibility measurement, respectively. All moderators, that were nonsignificant in both estimations, were excluded. The listed moderators with their levels are explained in Table 2.1. 
Previous estimations already indicated that studies applying pattern masks achieve smaller priming effects than studies with metacontrast masks. This was also confirmed by this comprehensive model (Model C1), estimated with the first and the last visibility measurement, $p s<.001$. The interaction between masking method and prime visibility did not reach significance with these estimations. Previous estimations provided evidence for an impact of prime visibility when pattern masks are applied. Interestingly, the moderator prime visibility reached significance when pattern masks were taken as the reference level of the moderator masking method, at least when estimated with the first visibility measurement, $p_{M 1}<.05$ and $p_{M 2}=.1$. Therefore we kept the interaction as the role of prime visibility is the main issue of the current meta-analysis and it seems informatively valuable for this concern. A difference in notation between primes and targets led to smaller priming effects within the first comprehensive model $\left(\beta_{M 1}=-0.12 ; \beta_{M 2}=-0.09\right)$ and the second comprehensive model $\left(\beta_{M 1}=-0.12 ; \beta_{M 2}=-0.09\right)$. In both models, this influence was only significant, when the model was estimated with the first visibility measurement, $p s_{M 1}<.05$ and $p s_{M 2}>.09$. In the second comprehensive model, a positive influence of the prime visibility $\left(\beta_{M 1}=0.18 ; \beta_{M 2}=\right.$ 0.09) with a difference in notation was also only significant with the first visibility measurement, $p_{M 1}<.01$ and $p_{M 2}=.17$. This pattern reached significance with both sets of visibility measurements with the first comprehensive model $\left(\beta_{M 1}=0.24 ; \beta_{M 2}=0.13\right)$, $p s<.05$. A change in color between primes and targets had a negative impact on priming effect irrespectively of prime visibility within the first $\left(\beta_{M 1}=-0.21 ; \beta_{M 2}=-0.23\right)$ and the second comprehensive model $\left(\beta_{M 1}=-0.22 ; \beta_{M 2}=-0.24\right)$, ps $<0.0005$. Within the first comprehensive model, the moderators size $\left(\beta_{M 1}=-0.13, p_{M 1}<0.005 ; \beta_{M 2}=-0.05, p_{M 2}=0.28\right)$ and shape $\left(\beta_{M 1}=-0.39 ; \beta_{M 2}=-0.27 ; p s<0.005\right)$ had a greater negative influence with increasing prime visibility as well as within the second comprehensive model (Size: $\beta_{M 1}=-$ $0.12, p_{M 1}<0.05 ; \beta_{M 1}=-0.06, p_{M 1}=0.25$; Shape: $\left.\beta_{M 1}=-0.34 ; \beta_{M 2}=-0.23 ; p s<0.01\right)$. When 
prime and target were related through meaning and the primes never appeared as targets, the effect sizes were smaller within the first $\left(\beta_{M 1}=-0.19 ; \beta_{M 2}=-0.25\right)$ and the second comprehensive model $\left(\beta_{M 1}=-0.19 ; \beta_{M 2}=-0.25\right)$, ps $<0.001$. Within the second comprehensive model, an enhancement of the effect in these cases with increasing visibility was only significant, when the model was estimated with the second visibility measurement $\left(\beta_{M 1}=0.17, p_{M 1}=0.09 ; \beta_{M 2}=0.22, p_{M 2}<0.05\right)$. This pattern reached significance with both sets of visibility measurements within the first comprehensive model $\left(\beta_{M 1}=0.25 ; \beta_{M 2}=\right.$ 0.27), $p s<0.01$. The first and the second comprehensive model differ with regards to the complex of moderators concerning the response preparation. In the first model, response association is included as a single moderator and in interaction with prime visibility. Priming effects seem to be larger in cases with a response association $\left(\beta_{M 1}=0.07, p_{M 1}=0.15 ; \beta_{M 2}=\right.$ $\left.0.11, p_{M 2}<0.05\right)$ and prime visibility has a positive impact on priming effects in these cases $\left(\beta_{M 1}=0.32 ; \beta_{M 2}=0.28 ; p s<0.001\right)$. Within the second comprehensive model, the moderator response association is combined with the moderator task level. After fitting the model, by excluding insignificant moderators and interactions, the interaction between prime visibility and this combined moderator remained within the model. In cases with primes and targets inducing the same response only and always in the congruent condition, prime visibility had a positive influence on the effect with a semantic depth of prime processing $\left(\beta_{M 1}=1.46 ; \beta_{M 2}=\right.$ 1.44 ; ps $<0.05$ ). Concerning the task level, priming effects are significantly smaller with an abstract task level $\left(\beta_{M 1}=-0.25 ; \beta_{M 2}=-0.27\right)$ and with a semantic task level $\left(\beta_{M 1}=-0.16 ; \beta_{M 2}\right.$ $=-0.14$ ) compared to a perceptual task level, $p s<0.05$. There no difference in effect sizes of studies with an abstract task level compared to a semantic task level, $p=.09$.

After fitting both comprehensive models, the moderator response type remained only in the second model. This model provides evidence that the request of expressions leads to larger priming effects than the demand of motor responses $\left(\beta_{M 1}=0.20 ; \beta_{M 2}=0.19\right)$, ps $<.01$. 
Beside the model estimations in line with the psychological model, we estimated the influence of the prime duration, which was revealed to be a positive one $(\beta=0.001, p<.01)$. Concerning the SOA a differentiation of the prime-mask SOA and the mask-target SOA in interaction with the prime-target relation yielded a model with less residual heterogeneity than the single-moderator models $\left(\tau^{2}=0.0800\right.$, AIC $\left.=806.27\right), p s<.01$. The interactions between the moderator prime-target relation and moderators concerning stimulus onset asynchronies were of interest on the basis of an observation by Van den Bussche and colleagues (2009) that different kinds of priming differ with regards to the influence of the prime-target SOA. Our model revealed a significant positive relation of the prime-mask SOA and the effect size, when there was a direct prime-target relation $(\beta=0.004, p<.0001)$, and a significant negative relation between mask-target SOA and effect size, when the prime-target relation was through associated meanings $(\beta=-0.0002, p<.05)$. Possible influences of the mask-target SOA with a direct prime-target relation and the prime-mask SOA with a primetarget relation through the meanings were not significant $(p=.65 ; p=.36)$. Adding the prime duration to the model led to a further reduction of the heterogeneity $\left(\tau^{2}=0.0783\right.$, AIC $=$ 811.06), $p<.001$. We added the resulting model to the previously fitted comprehensive models, aligned with the psychological deliberations. Estimated with both visibility measurements, no substantial changes of the regression coefficients emerged. Within the second comprehensive model, the residual amounts of heterogeneity were reduced further $\left(\tau_{\mathrm{M} 1}^{2}=0.0589 ; \tau_{\mathrm{M} 2}^{2}=0.0584\right), p s<.01$.

\subsubsection{Influences on Prime Visibility and Visibility Measurements}

The main attempt of the current meta-analysis is to specify the role of prime visibility in masked priming paradigms. Therefore, we analyzed the impact of a series of characteristics of these paradigms in interaction with prime visibility within meta-analytic 
model estimations. In this context the question arises whether there might be any characteristics of the studies that have an impact on prime visibility itself. Besides the impact of moderators on the actual prime visibility, it is interesting whether there are moderators that have an influence on the prime visibility measurements. Of course we cannot distinguish the two aspects statistically, but it is important to do this on a theoretical level. In contrast to moderators that affect actual prime visibility, moderators with an impact on the visibility measurement may cause distortions in conclusions based on the $d^{\prime}$-values (see Figure 2.4). Small differences in model estimations with the first and the last visibility measurement set provide first evidence that those moderators may cause small distortions. We further investigate this presumption in this section. First, we peruse potential moderators of the actual prime visibility. As $d^{\prime}$-values cannot be treated as effect sizes with corresponding sampling variances, we relied on linear regressions. An extended data set, including all visibility measurements, was used for these estimations.

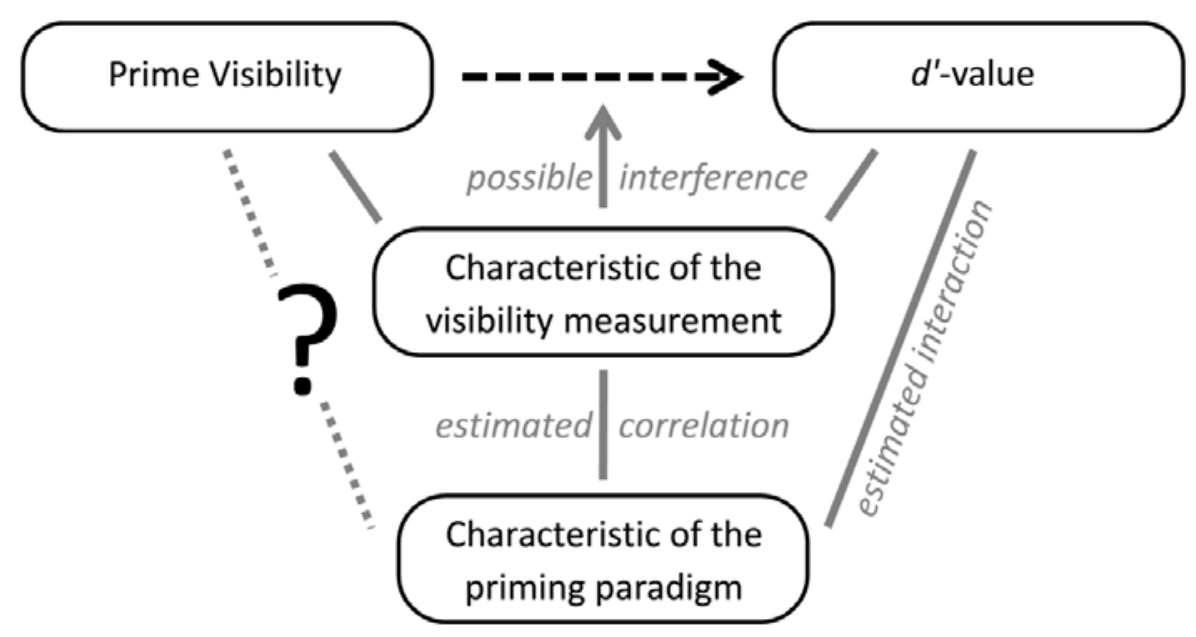

Figure 2.4. Schematic depiction of a possible interference of moderators with an impact on the visibility measurement. An estimated interaction between a moderator of priming effects and $d^{\prime}$ values may be caused by a characteristic of the visibility measurement if there is a confounding between this moderator and the characteristic of the visibility measurement and this characteristic has an influence on the $d^{\prime}$-values. 
Four moderators were examined to have an influence on the actual prime visibility. With increasing prime duration $(\beta=0.007, \mathrm{AIC}=1158.72)$, prime-mask SOA $(\beta=0.005$, $\mathrm{AIC}=1225.18)$ and mask-target SOA $(\beta=0.0007, \mathrm{AIC}=1376.71)$, the $d$ '-value also increased significantly, $p s<.0001$. A model, including all three moderators with their interactions, offered the same picture and additional negative interactions of prime duration with the prime-mask and the mask-target SOA, separately, and a positive interaction of prime-mask and mask-target SOA (AIC $=1083.46), p s<.05$. The fourth moderator, we examined, was the applied type of masking method (see Table 2.1). The kind of masking method did not affect the achieved $d^{\prime}$-values, when only distinguishing metacontrast and pattern masks, $p=.13$. We took a closer look and distinguished seven masking techniques and estimated their influence on the $d$ '-value with a linear regression model. Masking with flanker or using interocular suppression was both applied in less than ten cases. Therefore, interpreting the results, concerning these methods, has to be done with caution. Two groups, in terms of their masking strength, emerged. Masking with only the target and with one additional backward or forward mask obtained a greater $d^{\prime}$-value and therefore a worse masking performance than masking with a forward and a backward mask, with metacontrast masking and with interocular suppression, $p s<.01$. Using flanker to reduce the visibility of the prime obtained a $d$ '-value not significantly different than the ones achieved with other masking techniques ( $p s>.1)$.

Beside the influence of certain moderators on the actual prime visibility, some moderators are possible candidates to have an impact on the visibility measurement and therefore on the gap between the actual value and the measured one (see Figure 2.4). One of these moderators is the type of task, which the subjects have to fulfill in order to specify their detection performance. We distinguished five visibility tasks: the detection task, the discrimination task, the prime-target discrimination, the identification of alternatives and the 
identification. The linear regression identified the visibility task as a significant moderator of the visibility measure $d^{\prime}, p<.0001$. Other interesting candidates for moderating the $d^{\prime}$-values are those proposed by Khalid, König and Ansorge (2011): Prime-target similarity, primeresponse similarity and prime-response mapping. When prime and target were similar, the $d^{\prime}-$ value was significantly smaller, $p<.0001$. A compatibility of the required response and a long-term response to the prime had also a negative influence on the $d^{\prime}$-value, $p<.01$. A fixed response mapping was significantly associated with greater $d$ '-values compared to a variable response mapping, $p<.001$. Furthermore, the appliance of the same targets in the visibility measurement and the priming session (target-target similarity) was accompanied by a significantly smaller $d$ '-value, $p<.0001$.

To reason whether these characteristics may generate the estimated interactions between moderators of priming effects and prime visibility, we calculated correlations between the characteristics of the visibility measurements and the moderators of the comprehensive models (see Table 2.11). Three moderate correlations $(0.4<r<0.6)$ were identified, one between a difference of primes and targets in size and a dissimilarity between primes and targets in the visibility task, one between the task level and the visibility task and one between the response type and the visibility task, $p s<.0001$. The interaction between response type and prime visibility was not included in comprehensive model estimations on the basis of theoretical considerations, although this interaction reached significance in the simple models (see Table 2.3 and 2.4). To learn whether a confounding of response type and visibility task caused this interaction, we estimated the simple models again, only including cases with a discrimination task as a visibility measurement (majority of the cases, see Table 2.1). The Tests of Moderators neither reached significance for response type as a single moderator nor in interaction with $d^{\prime}$-values, $p s>.05$. This validates our decision to exclude the interaction between response type and prime visibility. To examine the impact of a 
Table 2.11

Correlations between characteristics of visibility measurements and the moderators of the comprehensive models (Model C1 und C2) as well as moderators of the actual prime visibility

\begin{tabular}{|c|c|c|c|c|c|c|c|c|c|c|c|c|c|c|c|c|c|c|}
\hline & VIS[1] & & VIS[2] & & VIS[3] & & VIS[4] & & VIS[5] & & PTS & & PRS & & PRM & & TTS & \\
\hline D & 0.00 & & -0.12 & $* *$ & -0.11 & $* *$ & -0.12 & $* *$ & 0.41 & $* * *$ & -0.21 & $* * *$ & -0.09 & $*$ & 0.13 & $* *$ & -0.18 & $* * *$ \\
\hline MAM & 0.14 & $* * *$ & -0.17 & $* * *$ & -0.09 & $*$ & 0.10 & $*$ & 0.14 & $* * *$ & 0.30 & $* * *$ & -0.05 & & 0.06 & & -0.24 & $* * *$ \\
\hline NOT & -0.11 & $*$ & 0.01 & & -0.07 & & -0.02 & & 0.18 & $* * *$ & 0.05 & & 0.04 & & 0.09 & $*$ & -0.07 & \\
\hline SIZ & 0.04 & & -0.17 & $* * *$ & 0.20 & $* * *$ & -0.04 & & 0.06 & & -0.48 & $* * *$ & -0.28 & $* * *$ & -0.23 & $* * *$ & -0.05 & \\
\hline SHA & 0.15 & $* * *$ & -0.09 & $*$ & 0.05 & & -0.20 & $* * *$ & 0.08 & & 0.00 & & -0.14 & $* *$ & 0.02 & & 0.11 & * \\
\hline $\mathrm{COL}$ & -0.04 & & 0.18 & $* * *$ & -0.11 & $*$ & -0.07 & & -0.11 & $*$ & 0.16 & $* * *$ & -0.16 & $* * *$ & 0.12 & $* *$ & 0.08 & \\
\hline PRT2 & 0.30 & $* * *$ & -0.21 & $* * *$ & -0.06 & & -0.12 & ** & 0.17 & $* * *$ & 0.15 & $* * *$ & -0.14 & $* * *$ & 0.09 & $*$ & -0.12 & $* *$ \\
\hline $\mathrm{PN}$ & 0.37 & $* * *$ & -0.19 & $* * *$ & -0.17 & $* * *$ & -0.07 & & 0.14 & $* *$ & 0.00 & & -0.11 & $*$ & 0.19 & $* * *$ & -0.25 & $* * *$ \\
\hline RA & -0.28 & $* * *$ & 0.29 & $* * *$ & 0.18 & $* * *$ & -0.09 & * & -0.31 & $* * *$ & 0.22 & $* * *$ & 0.23 & $* * *$ & -0.18 & $* * *$ & 0.38 & $* * *$ \\
\hline $\mathrm{TL}$ & 0.27 & $* * *$ & -0.42 & $* * *$ & 0.03 & & 0.07 & & 0.33 & $* * *$ & -0.15 & $* * *$ & -0.26 & $* * *$ & -0.02 & & -0.30 & $* * *$ \\
\hline RTY & -0.10 & $*$ & -0.46 & $* * *$ & 0.39 & $* * *$ & 0.02 & & 0.47 & $* * *$ & -0.23 & $* * *$ & -0.09 & $*$ & -0.34 & $* * *$ & -0.15 & $* * *$ \\
\hline PDU & -0.08 & & -0.20 & $* * *$ & -0.03 & & 0.01 & & 0.46 & $* * *$ & -0.07 & & -0.02 & & 0.02 & & -0.26 & $* * *$ \\
\hline PMS & -0.03 & & -0.11 & $*$ & -0.02 & & -0.07 & & 0.28 & $* * *$ & -0.18 & $* * *$ & -0.08 & & 0.04 & & -0.18 & $* * *$ \\
\hline MTS & 0.56 & $* * *$ & -0.40 & $* * *$ & -0.12 & $* *$ & 0.13 & * & 0.09 & $*$ & -0.03 & & -0.09 & $*$ & 0.07 & & -0.46 & $* * *$ \\
\hline
\end{tabular}

Note. The different moderators are described in detail in Table 2.1. Three potential moderators contained more than two levels. To compute the correlations among all potential moderators, we reduced the levels of the moderator "Prime Novelty" (PN) to two, excluding the category "not available" with five cases. As the moderator "Depth of Prime/Target Processing" (DP) is ordinal, we assigned the value 1, 2 and 3 to the levels "visual", "abstract" and "semantic". The nominal moderator "Visibility task" (VIS) split up in five dummy variables. “***' $p<0.001$; ‘**' $p<0.01$; ‘*’ $p<0.05$; '.' $p<0.1$.

confounding between a difference of primes and targets in size and a dissimilarity between primes and targets in the visibility task, we estimated both comprehensive models (see Table 2.9 and 2.10) again, only including cases without prime-target similarity (majority of the cases, see Table 2.1). The interaction of prime visibility and the moderator encoding a change in size between primes and targets reached significance in both comprehensive models, estimated with at least one visibility measurement sets, $p_{C I M 1}<0.005, p_{C 1 M 2}=0.33, p_{C 2 M 1}=$ $0.0001, p_{C 2 M 2}<0.05$. This interaction is therefore not based on a confounding with the primetarget similarity of the visibility measurement. The kind of visibility task also correlated with the task level of the priming task. We estimated the second comprehensive model again (see Table 2.10), only including cases with a discrimination task as a visibility measurement. The impact of prime visibility in studies with a response association and a semantic task level did not reach significance marginally, $p_{C 2 M 1}=0.15, p_{C 2 M 2}=0.15$. An intensification of the 
estimated interaction by a confounding with the visibility task is therefore not inconceivable. Likely however is the assumption that the correlation between $d$ '-values and visibility tasks is caused by correlations between visibility tasks and moderators of the actual prime visibility, like prime duration and mask-target SOA (see Table 2.11 and Figure 2.4).

\subsubsection{Biases}

In experimental studies, counterbalancing conditions and choosing adequate samples are responsibilities of experimenters and are thought to minimize biases. While the search of suitable studies in meta-analytic proceedings is very essential, it is not sufficient to to preclude the occurrence of biases. One prominent bias is the publication bias that is caused by the inaccessibility of some studies (see "Methods - Coding and Meta-analytic Procedures"). By a visual examination of the funnel graph in Figure 2.5, with the sample sizes plotted against the effect sizes, the pyramid of data points can be recognized as tilted to the left. Hence, a negative correlation between the sample size and the effect size can be assumed and is confirmed $(r=-0.30, p<.0001)$ as well as the impact of the sample size as a moderator of the effect size in a random-effects model $(\beta=-0.0097, p<.0001)$. Therefore, the summary effect should be considered as overestimated. To assess whether a distortion of the meta-regression results is likely, we estimated for each relevant moderator a model including the sample size and the interaction of the moderator with the sample size. When each level of a single moderator is biased in the same way, the results, concerning the impact of the moderator on the effect size, should be free of this bias. A nonsignificant interaction between sample size and the examined moderator (with $p>.05$ ) is evidence for this. We found this kind of pattern with the following moderators: a difference between primes and targets concerning modality, notation, size and case, shape, color and perspective as well as response congruency, task level, prime duration and mask-target SOA. The models with the moderator prime novelty and the moderator response type were estimated, revealing a 
nonsignificant interaction between the specific moderator and the sample size and also a nonsignificant main effect of the sample size $(p s>.05)$. A significant interaction between the sample size and the moderator was found in model estimations with the prime-target relation $(\beta=0.007, p<.05)$, the prime-mask SOA $(\beta=-0.00004, p<.01)$ and $d^{\prime}\left(\beta_{M 1}=-0.009\right.$, $\left.p<.001 ; \beta_{M 2}=-0.006, p<.05\right)$. These imbalanced distributions are illustrated in Figure 2.6.

Evidence for another kind of bias is revealed by examining the frequency of the $d^{\prime}$ values (see Figure 2.7). More than half of the cases, namely 305 and 321 of 538 for the first and second visibility measurement, respectively, were within a range of -0.2 and +0.2 with an actual range between -0.24 and 3.83. Reasons and implications of this bias are discussed in the following section.

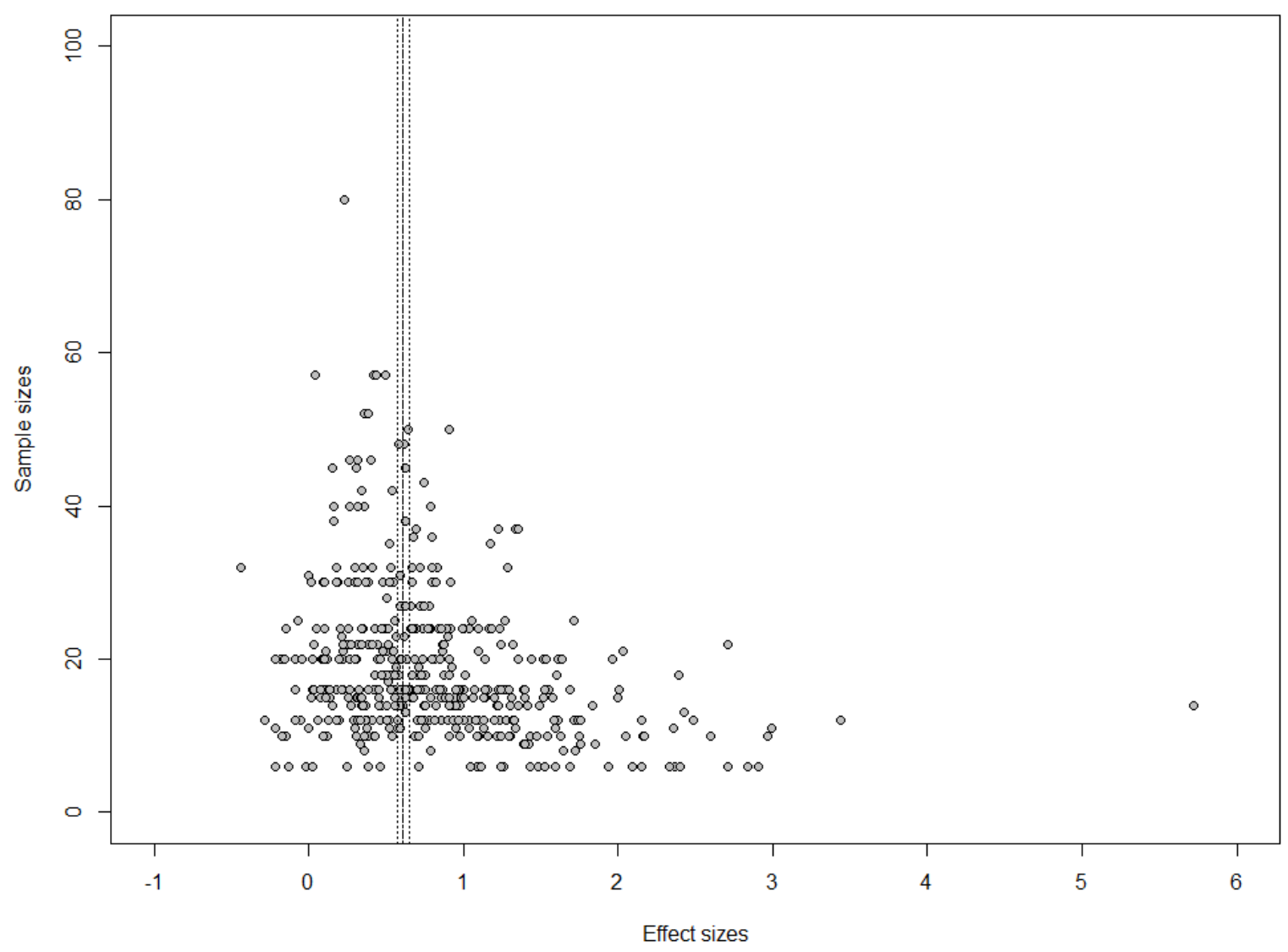

Figure 2.5. Funnel plot with estimated summery effect and corresponding standard error. 

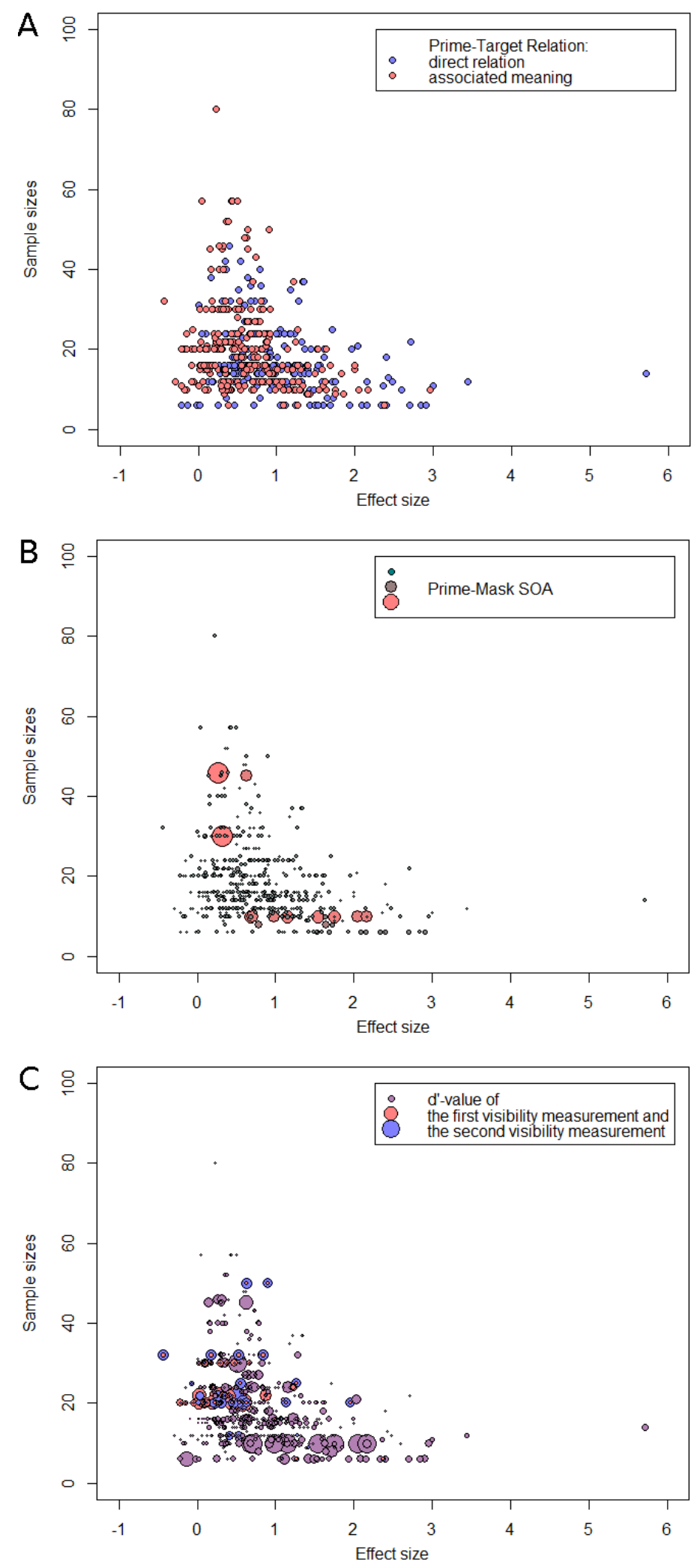

Figure 2.6. Funnel plots with illustrated levels of three moderators. Corresponding random-effects model estimations indicated possible distortions through a publication bias of the moderators (A) prime-target relation, (B) prime-mask SOA and (C) the $d^{\prime}$-value. The continuous variables prime duration and $d^{\prime}$ are visualized through diameters of data points, with higher values being illustrated through larger points. 


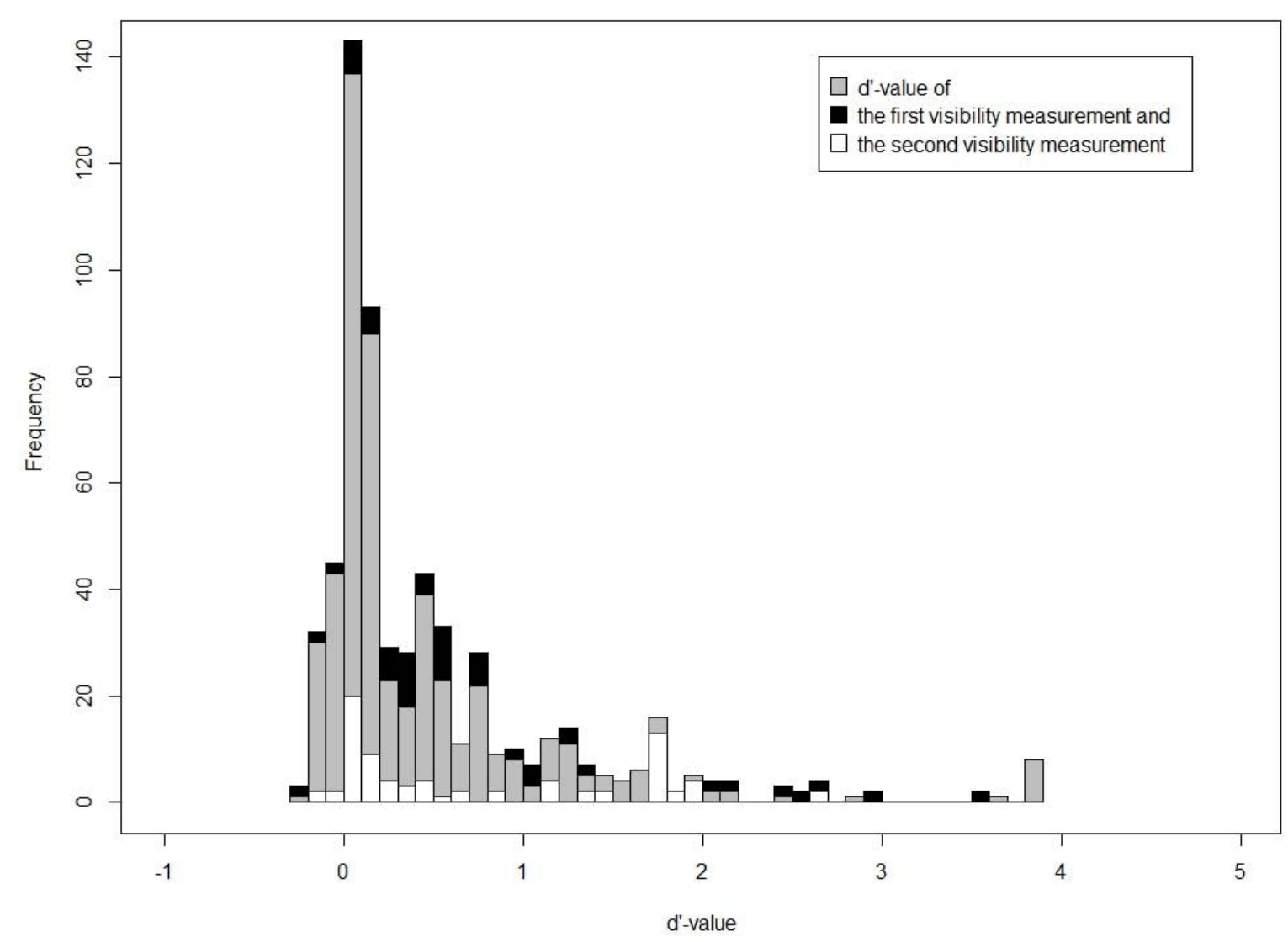

Figure 2.7. Histogram of the frequencies of included $d^{\prime}$-values. Values of the first and the second visibility measurement are visualized separately.

\subsection{Discussion}

The initial empty random-effects model without moderators was estimated with 538 cases that were reported in 84 studies. The highly significant summary effect of 0.61 is likely to be present, even under the assumption of an overestimation through a publication bias. With $56.9 \%$ of the variability due to heterogeneity, the inclusion of moderators was legitimate. In an exploratory approach we distinguished 23 moderators. To handle this large number of moderators, we proposed a psychological model which relates moderators to four mechanism of the model. Due to a confounding of the moderators masking method and task level, we fitted two comprehensive models excluding one or the other moderator. The model including masking method (Model C1) explains $37.5 \%$ and $37.6 \%$ of the heterogeneity, with 
measures of prime visibility in the first or the last test of some studies, respectively. The second comprehensive model (Model C2) accounts for slightly less heterogeneity with 35.1 $\%$ and $36.4 \%$, respectively. In the following we address the effects of moderators that were specifically related to one of the four mechanisms of our psychological model.

\subsubsection{Moderators of Priming Effects}

We propose a psychological model which assumes four mechanisms (see Figure 2.1). According to our model, moderators can influence how primes are processed, whether and how primes support target processing and whether and how primes prepare responses. In addition, the model assumes that a group of moderators influences how target stimuli are processed and an adequate response is generated.

One moderator is thought to influence the prime processing. This moderator describes the masking method and is only included in the first comprehensive model (Model $\mathrm{C} 1$; Table 2.9) due to a confounding. In a model with only this moderator and its interaction with prime visibility, applying pattern masks is associated with smaller priming effects than applying metacontrast masks. This is accompanied by a positive impact of prime visibility in studies with pattern masks. This pattern emerges similarly in the comprehensive model with the linkage between an influence of prime visibility and the usage of pattern masks being weakened. A difference between an influence of prime visibility with pattern masks and with metacontrast masks appears insignificant. However regarded separately, the impact of prime visibility remains significant with pattern masks and insignificant with metacontrast masks. One interpretation of this pattern would be in line the concept of recurrent processing as a necessity for conscious perception (Lamme \& Roelfsema, 2000). Pattern masks might operate on an early level of stimulus processing, while metacontrast masks reduce prime visibility on a higher level (recurrent processing). Prime related activation is passed to higher 
areas in a feedforward sweep. Is the activation reduced by a pattern mask at this stage, priming effects and prime visibility are influenced. When a metacontrast mask disturbs the recurrent processing, priming effects are unimpaired while prime visibility is reduced. Note that this pattern might also be based on the confounding with the moderator task level and its impact on priming effects.

Support of target processing depends on similarities between prime and target. Certain systematic differences between primes and targets were identified to have a negative influence on the primes supporting the target processing. In the model, including only these moderators, a difference in notation, shape and color, and a difference in modality, notation, size and shape in interaction with the visibility showed a significant impact on the priming effects. The main effect of the shape difference and the interaction of prime visibility and a difference in modality were eliminated during fitting processes in both comprehensive models. Generally, systematic differences between primes and targets seem to have a negative impact on priming effects. This notion is in line with our hypothesis (1) and is supported by a negative impact of a difference in notation and color as well as an increasing negative impact of a difference in size and shape with increasing prime visibility. A positive interaction of prime visibility and notation indicates that the negative impact of a difference in notation is reduced with increasing prime visibility. This can be due to a better recognition of the link between prime and target, when the prime is more visible. The support of target processing depends also on the moderators prime-target relation and prime novelty. In both comprehensive models, the priming effects were significantly smaller, when primes and targets are linked through an associated meaning (prime-target relation) and the primes never appear as targets (prime novelty). This pattern of effects accords with our theoretical considerations (hypothesis 2). A positive effect of prime visibility in interaction with a primetarget relation on a higher level of processing was found unconditionally with the first 
comprehensive model (Model C1). Regarding the second comprehensive model (Model C2; Table 2.10) this interaction appeared only significant, when $d$ 'values from the last test were included. An impact of prime visibility on priming effects with a distant prime-target relation (associated meaning) would be in line with previous formulated hypothesis (3). For processes on a higher level more integration of information is necessary. Therefore this pattern provides further evidence for the assumption that integration of information is attached to conscious access (Tononi \& Edelman, 1998; Lamme \& Roelfsema; 2000; Dehaene \& Changeux, 2011; Dehaene \& Naccache, 2001). Note that analyses of a potential influence of a publication bias revealed that the moderator prime-target relation might suffer from this bias. The conduction of a specific experiment to confirm this pattern would be more conclusive.

The first comprehensive model (Model C1) does not include both moderators of the response preparation mechanism due to a confounding. Regarding the second comprehensive model (Model C2), response preparation effects of the prime were clearly modulated by response association and task level. Priming effects were increased when primes and targets were associated with the same responses. Task level was a significant moderator only when a response association was given (hypothesis 4). Corresponding to our hypothesis (6), the visibility of primes increased priming effects only when two conditions were given: prime and target had to be associated to the same responses and semantic stimulus processing is necessary to fulfill the task. In total, 24 studies contributing 137 cases realized this condition (see Appendix I). Note that this pattern might also be induced by a confounding between masking method and task level.

Target Processing was modulated by the task level, which was only included in the second comprehensive model (Model C2). Priming effects are smaller when an abstract or semantic level of processing is necessary to fulfill the task compared to a perceptual level (hypothesis 5). A difference between an abstract and a semantic level of target processing 
was not confirmed. After the fitting process, the response type appeared as a significant moderator only in the second comprehensive model (Model C2). According to our analyses, priming effects are larger when the response consisted in an expression rather than a motor response. This finding might be useful for the construction of experimental designs.

Beyond the moderators which we related to specific mechanisms of our psychological model, we examined effects of modulators that are related to the dynamic of priming effects. First of all, the positive influence of the Prime Duration was confirmed (hypothesis 7). An interesting insight into the general time course of priming effects was gained, by our distinction between prime-mask SOA and mask-target SOA. A differential effect of primetarget SOA on response priming and semantic priming has been reported in the literature (see Van den Bussche, Van den Noortgate \& Reynvoet, 2009) might result from a failure to distinguish prime-mask and mask-target SOA. According to our analyses, an increasing prime-mask SOA leads to increasing priming effects in studies with a direct prime-target relation. An increasing mask-target SOA, in contrast, leads to decreasing priming effects in studies with a prime-target association through a common meaning. We speculate that this pattern of results might be a consequence of the different paradigms used to study response priming and semantic priming. Paradigms differ in respect of stimuli and masking procedures because response priming can be studied with simple stimuli, whereas semantic priming requires complex stimuli. Increasing priming effects with increasing prime-mask SOA and decreasing priming-Effects with increasing mask-target SOA accord with findings from response priming which emphasize the role of the masking stimulus that can produce even inverse priming effects (e.g., Eimer \& Schlaghecken, 2002; Lleras \& Enns, 2004; Mattler, $2005 ; 2006 ; 2007)$. Our findings suggest to examine different priming effects in comparable paradigms. 


\subsubsection{Moderators of Prime Visibility}

We also examined moderators of prime visibility. We distinguished moderators which change true prime visibility in the participants and moderators which change performance in prime visibility tests in other ways. Dividing the two groups of moderators is particularly important, when a possible distortion of visibility measurements affects only subgroups of studies. Timing parameters, like prime duration, prime-mask SOA, and mask-target SOA, as well as the masking method were thought to have an influence on the participants true prime visibility. Corresponding to our hypothesis (7) and in line with previous findings, prime duration and prime-mask SOA had a positive effect on $d$ ' (Enns \& Di Lollo, 2000; Breitmeyer \& Ögmen, 2006). Mask-target SOA also had a positive impact on $d$ '. Analyses revealed that the Masking method is also a moderator of the $d^{\prime}$. Masking with the target or one additional backward or forward mask appeared to be less effective than masking with a forward and a backward mask, with metacontrast masking and with interocular suppression. Masking with flanker was applied in few cases. This might have been the reason for our failure to find a significant difference to other masking procedures. Although our analyses suggest differences between masking procedures modulate masking effects, we note that our results ought to be interpreted with caution. The masking procedures are most likely chosen to be suitable for masking a specific kind of stimulus under defined circumstances. To avoid these confounders, experimental assurance is in demand.

The possibilities to measure conscious perception have been debated for several decades (for a review, see Merikle \& Reingold, 1992) but it still remains unclear how to grasp participants subjective experience in an objective measure. A recent experimental approach to this issue has been reported by Khalid and colleagues (2011). These authors examined the effects of three attributes of visibility tests on $d$ ': Prime-target similarity, Prime-response similarity and Prime-response mapping. We included these variables in our 
meta-analysis on moderators of $d^{\prime}$. Prime-target similarity has been reported to bias participants' answers towards responding to the target (Khalid et al., 2011; Albrecht \& Mattler; 2012a, 2012b). Our analyses could not examine this congruency effect simply because studies typically do not report separate $d^{\prime}$-values for congruent and incongruent trials (for a good reason, as shown by Vorberg et al. 2004). Nevertheless, our analyses confirmed that prime-target similarity declines performance in visibility tests, which is expected when participants respond to the target instead to the prime (hypothesis 8). Prime-response similarity, in contrast, did not increase $d^{\prime}-$ as predicted by Khalid and colleagues. However, Khalid and colleagues (2011) did not find any effect of this variable. Our analyses, in contrast, revealed decreased $d^{\prime}$ in studies with prime-response similarity. Prime-response mapping should increase $d$ ' when the mapping is fixed rather than varied (Khalid et al., 2011). However, the empirical study of Khalid and colleagues did not support this view. Our analysis, in contrast, provided evidence for this effect. Beyond previous studies, we included target-target similarity as a moderator of $d^{\prime}$ in our meta-analysis. In a number of studies, the target used in the priming task differed from the target in the visibility test - and in some studies the target was even absent. This was done to reduce the possibility of a prime-target confusion or to simplify the design and reduce the duration of one trial (e.g., Dell'Acqua \& Grainger, 1999). However, it is possible that the target has an additional masking effect. Therefore, changing or eliminating the target in the visibility test might lead to an overestimation of prime visibility compared to the situation in which priming effects were measured. Our analyses confirm this effect. Although an experimental validation is recommended, our results indicate that this aspect is relevant for the construction of visibility assessments.

The moderator visibility task distinguished five different kinds of tasks that were used to measure prime visibility in an objective manner: detection, discrimination, prime-target 
discrimination, identification of alternatives, and prime identification tasks. Our analyses revealed a significant effect of this modulator on $d^{\prime}$. The literature gives the impression that comparing different kinds of visibility tests is a growing field of research (e.g., Ansorge, Breitmeyer \& Becker, 2007; Ansorge, Becker \& Breitmeyer, 2009; Fisk \& Haase, 2011; Merikle \& Reingold, 1990). However, the relation between the different types of visibility tests is still unclear. For instance, Fisk and Haase (2011) compared performance in a detection task with performance in a two alternative, forced-choice identification task. These authors reported increased performance in a detection task rather than a two alternative, forced-choice identification task. Merikle \& Reingold (1990), in contrast, reported that it is possible to recognize the correct alternative at a level above chance, even when subjects cannot detect the masked stimulus. The results of the present meta-analysis provide a basis for further investigations. The five kinds of visibility tests that we distinguished aim to gather objective data of participants' subjective experience and are frequently used. In addition, recent studies also include subjective methods, in form of self-reports (e.g., Mattler, 2003; Sergent \& Dehaene, 2004b; Overgaard, Rote, Mouridsen \& Ramsøy, 2006) and even phenomenological reports to access participants' subjective experience (e.g., Mattler \& Fendrich, 2010; Albrecht \& Mattler, 2012b). Here we did not include data from subjective visibility tasks because there are too few studies with comparable methods. The combined analyses of different visibility tests and different masking procedures might provide an access to the different facets of consciousness.

\subsubsection{Limitations}

To put the present study in perspective, we have to note the limitations of metaanalyses in general and the present meta-analyses in particular. A potential publication bias is a problem of meta-analyses in general. In the present data studies with large priming effects have used small sample sizes and vice versa. In addition to this, our meta-analyses are 
potentially distorted by a bias towards studies with a visibility of the masked stimuli at chance level because lots of studies attempted to show priming effects of unconscious stimuli. Evidence for this is given by an imbalanced distribution of $d^{\prime}$-values (Figure 2.7). The moderator $d$ ' interacts with several other moderators including sample size. Therefore, a publication bias might have affected the results based on this moderator. However, a decisive distortion is unlikely as a publication bias mainly affects the summary effect with singles studies attempting to confirm this effect. Experiments with small effect sizes often remain unpublished unless they were conducted with large sample sizes.

A high variance inflation factor for the moderator task level and a strong correlation with masking method confirmed a confounding between these moderators. Several additional correlations between moderators were reported. This suggests that certain proceedings are established in the different fields of research, using this kind of paradigm. The effects of single moderators should therefore be validated in experimental studies - especially with regards to the moderators task level and masking method.

Further limitations arise from the general problem of any meta-analysis that necessarily includes studies from a broad field of research. Liberal criteria have to be applied concerning standards of quality and the resulting inhomogeneity between studies' qualities might distort the results. Furthermore, the sources of information were studies, written with a different focus than our meta-analysis. Therefore, the estimations might have some imprecision. A number of studies tested visibility with more than one task. We decided to run our analyses twice, instead of averaging across the different visibility measures. In consequence, some analyses produced two different results which complicates the interpretation. These differences demonstrate again that different visibility tests might produce different results. Therefore, future research should examine the relations between the different types of visibility tests. 


\subsubsection{Conclusion}

Overall, our findings emphasize the necessity of an experimental examination of the impact of prime visibility on priming effects. We proposed four mechanisms that contribute to the emergence of priming effects as a basis for meta-analytic model estimations: Prime processing, support of target processing, response preparation and target processing. Our results attest the viability of the developed model, which can serve as a basis for subsequent experiments. Coping with a confounding between the moderators masking method and task level, we fitted two comprehensive models (Table $2.9 \& 2.10$ ). Both models provide evidence that the impact of certain systematic differences between primes and targets - namely in notation, size and shape - is modulated by prime visibility. Visibility also seems to have an influence on the mechanism of primes supporting target processing, when the link between primes and targets reside at a higher level of processing. This result is in accordance with theories of consciousness that emphasizes the role of information integration in the emergence of consciousness (Dehaene \& Changeux, 2011). In revealing a confounding between masking method and task level, we detected an interesting question for further research. We generated two competing models - one attributing smaller priming effects and an influence of prime visibility to pattern masks and one bringing this in line a semantic task level. Such an effect of the mask would be in line the concept of recurrent processing as a necessity for conscious perception (Lamme \& Roelfsema, 2000), when pattern masks reduce prime processing on an early level whereas metacontrast masks disturb recurrent processing. Higher level processing (e.g. semantic) depending on prime visibility seems to be in line with integration of information as a basis for conscious processing. Which model represents underlying processes more adequately? And is the influence of prime visibility on priming effect moderated by both masking method and task level? Experimental studies are needed to answer this question. 
2 | Approaching Consciousness with Masked Priming 


\section{Prime Visibility in Perceptual and Semantic Priming: Differences based on Masking Method}

\subsection{Abstract}

The impact of conscious perception on the processing of visual stimuli is still indeterminate. Various studies approach this question using masked priming. In accordance with the assumption that consciousness integrates and provides information (e.g. Dehaene \& Changeux, 2011), we followed the hypothesis that early stages of stimulus processing are not impaired by a reduction of the visibility. More complex processes, such as the ones involved in semantic priming, should be influenced by stimulus visibility. Previous research substantiates this pattern (e.g. Van den Bussche et al., 2009; Chapter 2). In the current experiments we compared perceputal and semantic priming applying identical stimulus sequences. We used metacontrast masks, which are often applied in the context of perceptual priming, and pattern masks, which are rather used in studies examining semantic priming. Our results indicate no differences between perceptual und semantic priming, but corresponding differences between the masking methods are revealed. We conclude that previously found differences between motor und semantic priming concerning the role of prime visibility are based on a confounding. 


\subsection{Introduction}

Studies frequently apply masked priming paradigms to address the functional principles and structures of certain information-processing systems. Some studies for example examine the language processing system with semantic (e.g., Dell'Acqua \& Grainger, 1999), phonological (e.g., Rastle \& Brysbaert, 2006) and orthographical priming (e.g., Bonin, Fayol \& Peereman, 1998). Other studies focus mainly on perceptual processing or the motor system (e.g., Vorberg, Mattler, Heinecke, Schmidt \& Schwarzbach, 2003). Differences between different priming effects provide evidence that different mechanisms are operating in a different manner. A legitimate interpretation of these differences relies on certainty that they can be attributed to the attended experimental manipulation. Confounding factors should be virtually excludable.

We conducted a meta-analysis to investigate the role of prime visibility through different priming paradigms (Chapter 2). Visibility is an interesting moderator, primarily because independence of visibility can be interpreted as independence of conscious processing. Therefore, masked priming is also an essential tool to investigate characteristics of conscious and unconscious processing (Dehaene, Changeux, Naccache, Sackur \& Sergent, 2006). The extent to which certain information can be processed without consciousness provides insights into specific information-processing systems as well as characteristics and functions of consciousness. Prime visibility is generally quantified with reference to signal detection theory ( $d$ '-values, Macmillan \& Creelman, 1991). Our meta-analysis actually revealed a relevant confounding between task level and masking method. The variance inflation factor (VIF) for task level exceeded the limit of 4 and masking method achieved the second highest VIF (O'Brien, 2007). Excluding one or the other moderators was essential for further modelling. Consequently, two comprehensive models were estimated - one attributing 
smaller priming effects and an influence of prime visibility to pattern masks and one bringing this in line with a semantic task level. Either metacontrast masking or a perceptual task level determines larger priming effects, which are independent of prime visibility. Which model is more adequate? The current study aims to provide an answer to this this question.

Besides evidence across different studies (Van den Bussche, Van den Noortgate \& Reynvoet, 2009; Chapter 2), indications of perceptual and semantic priming effects being differently modulated through prime visibility can be found on a single study level. Several studies of perceptual and motor priming report an independence of priming effects and prime visibility (e.g. Albrecht, Klapötke \& Matter, 2010; Francken, van Gaal \& de Lange, 2011; Mattler 2003; Vorberg et al., 2003). Some of these studies even report a double dissociation: Priming effects increase with increasing prime-mask SOA (stimulus onset asynchrony) whereas prime visibility decreases (e.g. Vorberg et al., 2003). The influence of prime visibility on semantic priming effects is more debated and results are more ambiguous. Kouider and Dehaene (2007) reviewed that early studies report an association of semantic priming effects and prime visibility (Nolan \& Caramazza, 1982; Purcell, Stewart \& Stanovich, 1983). Van den Bussche and colleagues (2009) examined semantic priming effects and hypothesized that stronger priming effects might be associated with greater prime visibility. This assumption was confirmed across studies with a semantic categorization task. Holcomb, Reder, Misra and Grainger (2005) found a modulation through visibility of an event-related potential component that is strongly associated with semantic processing (N400). Furthermore, positive correlations between semantic priming effects and prime visibility are reported occasionally (Eckstein \& Henson, 2012; Kouider \& Dehaene, 2009; Naccache \& Dehaene, 2001). Negative or no correlations between semantic priming effects and prime visibility are also found (e.g. Adams \& Kiefer, 2012; Kiefer, 2002). The degree to which those correlations are informative is questionable. Many studies try to reach maximal 
reduction of prime visibility without varying prime visibility. A widely approved way of confirming unconscious processing is testing whether prime visibility $\left(d^{\prime}\right)$ differs from zero, assuming that dispersion is normally distributed. Furthermore, interindividual differences may have an impact on those correlations when estimated across subjects.

What kind of conclusions could we draw if perceptual processing is in fact independent of prime visibility and semantic processing not? First of all, it would provide evidence that the chosen designs of perceptual and semantic priming actually address different processes. For instance, semantic response priming is called into question by the action trigger account (Kiesel, Kunde \& Hoffmann, 2007b; Kunde, Kiesel \& Hoffmann, 2003). However, more extensive conclusions might be drawn with respect to theories of conscious processing. An important function of consciousness might be the integration of information, which is achieved by a recurrent connectivity and top-down processing (e.g. Baars, 1988, Dehaene \& Changeux, 2011; Tononi \& Edelman, 1998). In line with this idea, simple and less complex aspects of stimuli might be processed independently of consciousness. This could pertain to features - like color, shape or orientation - that are processed on a perceptual level. Other aspects of stimuli - like affiliation to a semantic category - could be processed on a higher level. Several features of the stimulus have to be integrated to unveil its meaning and interactions of several areas might be necessary for that. The representation of the stimulus might necessarily enter the global workspace and be perceived consciously (Baars, 1988, Dehaene \& Changeux, 2011). Should perceptual priming effects be independent of prime visibility whereas semantic priming effects increase with increasing prime visibility, theories of consciousness would be supported that underline a function of information integration.

Alternatively, priming effects might be independent of prime visibility when metacontrast masks are applied and increase with prime visibility when pattern masks are 
used. For decades, different mechanisms have been thought to operate with pattern masks and metacontrast masks. Pattern masks that appear after the stimulus are thought to interrupt the processing of the stimulus (Spencer \& Shuntich, 1970). The masking effect of metacontrast masks was assumed to originate in interaction of neurons that encode contours of the stimulus and the mask (Breitmeyer, 1984). More recently, the role of recurrent processing for different masking methods was discussed (Enns \& Di Lollo, 2000). Communication between brain areas is not unidirectional (e.g. Zeki, 1993). After an initial transfer of information from one area to another, re-entrant pathways allow information to be passed back. Interference with this process might occur at different times with different masking methods. On the basis of previous studies, one might assume that pattern masks disturb an early level of stimulus processing (e.g. Enns \& Di Lollo; 2000, Kahneman, 1968; Macknik \& Livingstone, 1998; Rieger, Braun, Bülthoff \& Gegenfurtner, 2005). Metacontrast masks might act on a higher level of stimulus processing with a disturbance of prime visibility and no affection of priming effects (e.g., Enns \& Di Lollo, 2000; Breitmeyer \& Ögmen, 2006; Van Aalderen-Smeets, Oostenveld \& Schwarzbach 2006). Recently, Bruno Breitmeyer (2014) proposed a hierarchy of masking methods and suggested that this might be a methodological access to consciousness. In this hierarchy, pattern masking is followed by metacontrast masking, crowding, and object substitution. If our experiments indicate that masking method rather than task level is the critical factor, this approach would be supported.

The current study aims to investigate perceptual and semantic priming with pattern masks and metacontrast masks systematically. Crucially, we wanted to avoid any confoudings. Many studies use different primes, targets and masks even when directly comparing perceptual and semantic priming (e.g. Martens, Ansorge \& Kiefer, 2011). This is reasonable when one intents to apply ideal parameters for each design. However, our metaanalysis (Chapter 2) revealed an impact of even small variations like changes in notation, 
color, size and shape. Therefore, we tried to vary only parameters that are essential to our experimental manipulations. Task level and masking method were varied between subjects. Each subject of the perceptual priming experiments received the exact same stimulations as a subject of the semantic priming experiments. Only the task and therefore the relevant congruency differed. Masking method was varied with this meticulousness between Experiment 3.2 and 3.3. Accordingly, special metacontrast masks and pattern masks were created in a balancing act between comparable masking efficiency and comparable basic stimulus features. Bottom-up energy and color were considered. Expected outcomes are definable for perceptual priming with metacontrast masks and semantic priming with pattern masks. These experiments also serve as a control for an adequate choice of experimental parameters. If we want to provide insights into the confounding between task level and masking method, it is essential to replicate findings of the meta-analysis with these combinations. Perceptual priming effects should be independent of prime visibility when metacontrast masks are used. Semantic priming effects should increase with increasing prime visibility when pattern masks are applied. Either perceptual priming with pattern masks or semantic priming with metacontrast masks should also increase with increasing prime visibility. Task level and masking method are confounded factors, however, an impact of task level does not rule out an impact of masking method. Possibly, both factors modulate the interplay of prime visibility and priming effects. This could also be reflected in our results. Equally, perceptual priming effects with metacontrast masks should be larger than semantic priming effects with pattern masks. Semantic priming effects could be generally smaller than semantic priming effects. This would be in line with semantic information being processed on a higher level than perceptual information. Priming effects could also be generally smaller with pattern masks compared to priming effects achieved with metacontrast masks. This 
would be in line with pattern masks disturbing prime processing at an early stage. Again, both mechanisms might operate separately or in interaction with each other.

\subsection{Experiment 3.1: Priming on a Perceptual and Semantic Level}

The aim of the first experiment was the comparison of perceptual and semantic priming under identical circumstances. In Experiment 3.1A, we applied a perceptual priming task whereas the subjects of Experiment 3.1B had to fulfill a task requiring semantic processing of the stimuli. In an attempt to use the same stimulation in both subexperiments, we used colored letters and numbers as primes and targets, which allow, discrimination on the dimension of color and discrimination regarding the kind of the sign. As prime visibility is a variable of interest, we constructed four types of pattern masks with increasing masking strength. We varied the prime-mask SOA to compare the time courses of the priming effects and draw inferences about underlying mechanisms. On basis of the outlined evidence, we assumed to find an independence of the priming effect and prime visibility in Experiment 3.1A (perceptual priming) and priming effects increasing with increasing prime visibility in Experiment 3.1B (semantic priming). Contrary to former conjectures (e.g. Van den Bussche et al., 2009) and in line with our meta-analysis (Chapter 2), we expected to find an equal behavior (increasing) of perceptual and semantic priming effects with regards to an increasing prime-mask SOA.

\subsubsection{Method}

Participants. Twelve students from the University of Göttingen participated in Experiment 3.1A ( 8 female, 4 male) as well as in Experiment 3.1B (12 female, 0 male) with ages between 19 and $28(M=21.75)$ and 18 and $26(M=21.92)$, respectively. After four sessions of one hour, the participants received 28 Euro or course credit for participation. One 
additional subject abandoned the experiment after three sessions. All subjects were righthanded by self-report. Twelve participants (3.1A: 10; 3.1B: 2) had a normal and twelve corrected-to-normal vision. Ishihara color plates were used to verify a normal color perception.

Stimuli. The stimuli were presented on a CRT-monitor with a refreshing rate of 100 Hz. The background color was white while a fixation point $\left(0.03^{\circ}\right.$ width $\times 0.03^{\circ}$ height of visual angle) and a fixation cross $\left(0.15^{\circ} \times 0.15^{\circ}\right)$ appeared in black color. The letters $A, G, S$ and $U$ and the numbers 2, 3, 7 and 8 in red and green served as primes and targets (Figure 3.1). The stimuli were selected with regards to an equal distribution within the alphabet and the series of numbers. Considering potential subsequent experiments, we chose two vocals, two consonants, two odd numbers and two even numbers. The signs were displayed in Gandhi-Sans and subtended visual angles of about $0.56^{\circ} \times 0.71^{\circ}$. Luminance was constant for all primes and targets $\left(24.6 \mathrm{~cd} / \mathrm{m}^{2}\right)$. Single primes were presented in the center of the screen. Targets were duplicated and presented $2.27^{\circ}$ of visual angle below and above center. Pattern masks were constructed by decomposing the stimuli (quarters) and rearranging them in an area of $1.52^{\circ} \times 1.52^{\circ}$ of visual angle randomly (Figure 3.1). Four different stages of masking strength were achieved by arranging the stimulus pieces in a grid with a mesh width of $0.15^{\circ}$, $0.30^{\circ}, 0.61^{\circ}$ and $1.21^{\circ}$ of visual angle. Using this technique different masks were generated to assure that each individual mask appeared only once during the priming sessions. As a feedback signal a sinusoidal tone of $440 \mathrm{~Hz}$ was presented for $150 \mathrm{~ms}$.

Tasks. Both subexperiments involved two kinds of tasks. In the first three sessions the subjects had to fulfill a forced-choice reaction time task by responding to the target with one of the response buttons (both Crtl-keys) using their index fingers. In Experiment 3.1A, participants responded to green targets with one key and to red targets with the other key. In Experiment 3.1B, subjects had to decide whether the target was a letter or a number. The 
assignments of the keys were counterbalanced between subjects. In the last session, the subjects had to focus on the prime stimulus. After each trial, they first reported the quality of their subjective perception of the stimulus using the perceptual awareness scale (PAS) developed by Ramsøy and Overgaard (2004). With four fingers of their left hand they reported if they saw "nothing", "a weak glimpse (without recognition)", "an almost clear image (with recognition)" or "a clear image". After a response was given, two alternatives appeared on the screen. The subject had to decide which alternative matched with the prime by pressing the left key with index finger or the right key with the middle finger of the right hand. In Experiment 3.1A, alternatives matched in shape and differed in colors whereas they differed in shape and matched in color in Experiment 3.1B.

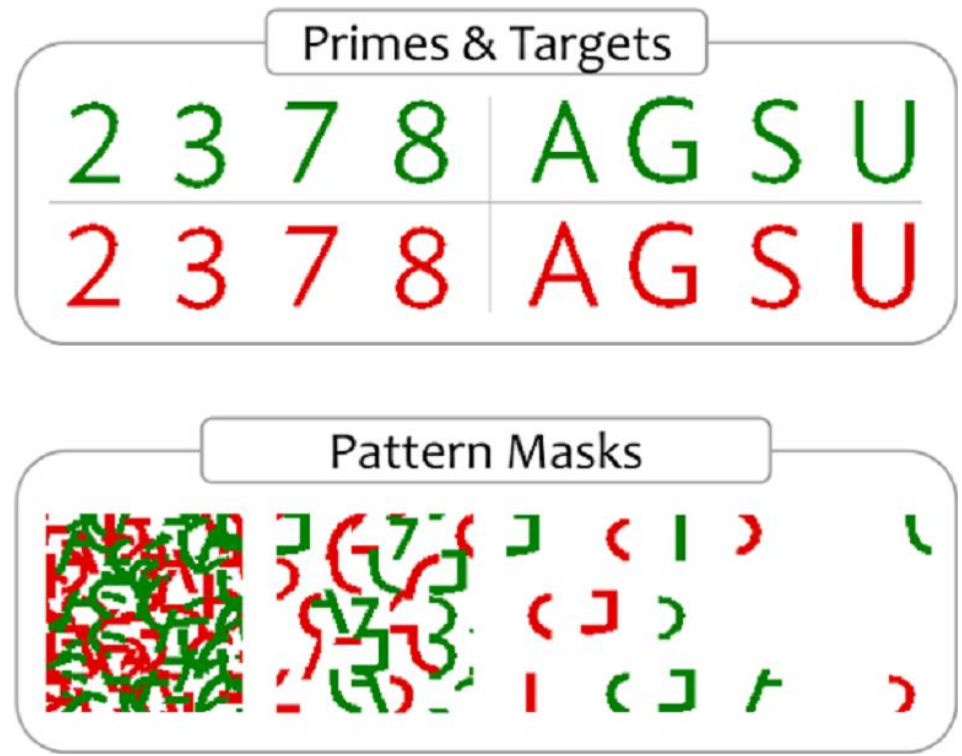

Figure 3.1. Stimuli applied in Experiment 3.1. One prime and one target were paired randomly in each trial, restricted by a balanced occurrence of relevant conditions. Primes and targets never consisted of the same sign. Pattern masks were applied in Experiment 3.1. Parts of prime stimuli were rearranged randomly. With different distances between segments $\left(0.15^{\circ}, 0.30^{\circ}, 0.61^{\circ} \& 1.21^{\circ}\right.$ of visual angle, center to center), different masking strengths were implemented. 
Procedure. The four experimental sessions were held on different days. In the beginning of the first session the proceedings were explained to the subjects, demographic data was collected and they were tested for normal vision and color perception. The participants were instructed to focus on the center of the screen during the whole presentation even though the targets appeared below and above fixation. They were advised to response as fast and accurate as possible. After a short demonstration of 8 trials with no reaction time window and a training block of 48 trials, the subjects received 512 experimental trials divided to 8 runs. The sequence of one trial is depicted in Figure 3.2. The onset of a fixation cross, presented for $500 \mathrm{~ms}$, marked the beginning of each trial. After a fixation point was displayed for $300 \mathrm{~ms}$, a premask appeared on the middle of the screen for $50 \mathrm{~ms}$. The premask-prime interstimulus interval (ISI) of $10 \mathrm{~ms}$ was accompanied by the presentation of a fixation point. The prime was displayed for $20 \mathrm{~ms}$, also in the middle of the screen. Depending on the condition, a prime-mask SOA of $30 \mathrm{~ms}, 60 \mathrm{~ms}, 90 \mathrm{~ms}$ or $120 \mathrm{~ms}$ was realized. The mask was presented in the center of the screen for $100 \mathrm{~ms}$ and shared the on- and offset with the target.

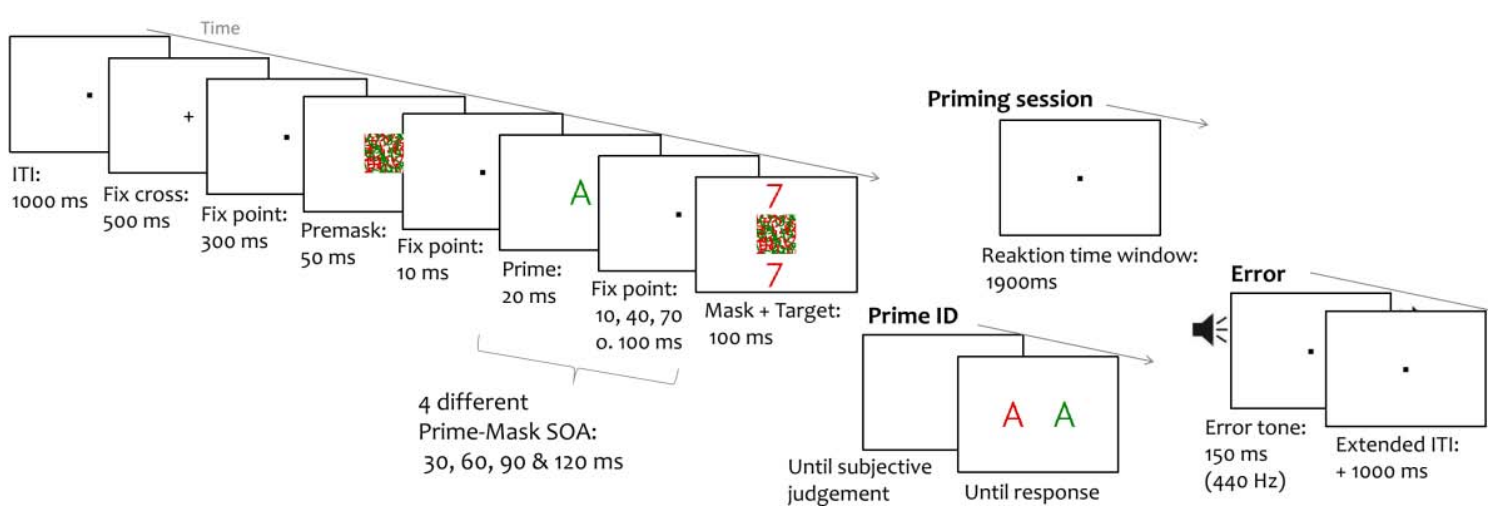

Figure 3.2. The sequence of a trial used in Experiments 3.1 - 3.3. Stimuli are depicted as applied in Experiments 1A and 1B. Different primes and targets were used in Experiments 3.2 and 3.3 (Figure 3.8). In Experiment 3.2, metacontrast masks were used without presenting a pre-mask. Different pattern masks were applied in Experiment 3.3. 
A response window of $2000 \mathrm{~ms}$ started with target onset. The intertrial interval of $1000 \mathrm{~ms}$ started immediately after a correct response. In case of an error, the presentation of an error tone $(440 \mathrm{~Hz} ; 150 \mathrm{~ms})$ and an extension of the intertrial interval for $1000 \mathrm{~ms}$ were interposed. After each run, participants received the opportunity for a self-paced break. The second and the third session also began with 48 training trials. Afterwards the subjects received 768 trials in each session also divided to 8 runs. At the end of the third session, participants were debriefed with regards to detection and recognition of the primes and, at last, informed about their appearance. In the last session, the subjects received 512 trials in 8 runs that were essentially identical to the trials presented before. The trials differed merely after the presentation of the targets. Participants had unlimited time to evaluate the prime perception with the PAS and to choose an alternative afterwards. To get comfortable with the new task, eight trials with an extended prime presentation $(150 \mathrm{~ms})$ were presented at the beginning of the session.

Design and Statistical Analysis. With 4 types of primes ( 2 colors $\times 2$ categories $), 4$ types of targets, 4 prime-mask SOAs $(30,60,90$ and $120 \mathrm{~ms})$ and masks with 4 different strengths, 256 conditions were realized. Each subject received 2048 experimental trials in priming sessions, with specific primes and targets assigned randomly. Primes and targets never consisted of the same sign. Trials were arranged evenly throughout the priming sessions, regarding prime-mask SOA, masking strength and prime-target congruency based on color and semantic category. For each subject of one experiment, trial order was assigned randomly and 512 trials were drawn from the pool of priming trials to serve in a primeidentification session. Each subject of Experiment 3.1B received a trial sequence identical to one subject of Experiment 3.1A. Error and post-error trials as well as the fastest and two slowest reaction times (RT) of each condition were excluded in estimation of mean RTs. Repeated measures ANOVAs were performed with RTs and arc-sin transformed error rates 
(ER) of Experiment 3.1A and 3.1B, respectively. Masking strength, SOA and congruency (based on color and semantic category, respectively) were included as factors. In line with signal detection theory (Macmillan \& Creelman, 1991), $d$ '- values were calculated with the hit rates $(\mathrm{H})$ and false-alarms rates $(\mathrm{F})$ of the two-alternative choices:

$$
d^{\prime}=z(H)-z(F)
$$

with $\mathrm{z}$ as the inverse of the normal distribution function. Using repeated measures ANOVAs, $d$ '-values and subjective visibility judgments were analyzed to check a manipulation of prime visibility through masking strength, simultaneously considering an impact of prime-mask SOA. Mauchly's sphericity test and Greenhouse-Geisser correction for violations of sphericity were applied (Mauchly, 1940; Greenhouse \& Geisser, 1959). The pooled data of Experiment $3.1 \mathrm{~A}$ and $3.1 \mathrm{~B}$ were analyzed with regards to the impact of the task level on priming effects and visibility measurements. Task level was therefore included as a betweensubjects factor.

\subsubsection{Results}

Errors. In Experiment 3.1A, subjects committed errors (ER) in $4.9 \%$ of all trials. Less errors occurred in congruent trials $(3.5 \%)$ compared to incongruent ones $(6.4 \%$; $\left.F(1,11)=21.83, M S_{e}=0.069, p<.001\right)$. Error rates increased with increasing prime-mask $\operatorname{SOA}\left(F(3,33)=5.79, M S_{e}=0.038, p<.005\right)$. Errors were detected in $3.4 \%, 4.6 \%, 5.5 \%$ and $6.4 \%$ of the trials with prime-mask SOAs of $30 \mathrm{~ms}, 60 \mathrm{~ms}, 90 \mathrm{~ms}$ and $120 \mathrm{~ms}$, respectively. With decreasing masking strength, error rates increased $(4.4 \%, 4.5 \%, 5.7 \%$ and $5.3 \%$; $\left.F(3,33)=5.45, M S_{e}=0.019, p<.005\right)$. The interaction between perceptual congruency and masking strength also reached significance $\left(F(3,33)=6.16, M S_{e}=0.018, p<.005\right)$ reflecting larger perceptual priming effects $\left(\mathrm{PE}_{\mathrm{ER}}=\mathrm{ER}_{\text {incongruent }}-\mathrm{ER}_{\text {congruent }}\right)$ with weaker masks namely $1.8 \%, 1.4 \%, 2.9 \%$ and $5.5 \%$ with masks' mesh width of $0.15^{\circ}, 0.30^{\circ}, 0.61^{\circ}$ and 
$1.21^{\circ}$ of visual angle, respectively. Perceptual priming effects also increased with increasing prime-mask SOAs with $30 \mathrm{~ms}, 60 \mathrm{~ms}, 90 \mathrm{~ms}$ and $120 \mathrm{~ms}$ to $0.6 \%, 2 \%, 3.6 \%$ and $5.5 \%$, respectively $\left(F(3,33)=4.19, M S_{e}=0.032, p<.05\right)$. Perceptual priming effect on errors are depicted in Figure 3.3 separately for each masking strength and prime-mask SOA. Mean error rate of Experiment $3.1 B$ was $4.5 \%$. Again, a main effect of semantic congruency was confirmed $\left(F(1,11)=79.01, M S_{e}=0.037, p<.0001\right)$, with fewer errors in congruent $(3.0 \%)$ compared to incongruent trials $(6.0 \%)$. Fewer errors occurred with denser masks $(3.6 \%$ with $0.15^{\circ}, 4.6 \%$ with $0.30^{\circ}, 4.7 \%$ with $0.61^{\circ}$ and $5.2 \%$ with $1.21^{\circ}$ of visual angle; $\left.F(3,33)=3.76, M S_{e}=0.023, p<.05\right)$. Errors increased with increasing prime-mask SOA $\left(3.5 \%, 4.7 \%, 4.6 \%\right.$ and $\left.5.3 \% ; F(3,33)=5.21, M S_{e}=0.021, p<.05\right)$. Semantic priming effects increased with deceasing masking strength $(1.8 \%, 2.2 \%, 3.1 \%$ and $4.9 \%$; interactions of semantic congruency and masking strength $F(3,33)=4.42, M S_{e}=0.019$, $p<.05)$. Longer prime-mask SOAs also enhanced semantic priming effects $(0.6 \%, 2.9 \%$, $3.5 \%$ and $\left.5.0 \% ; F(3,33)=11.48, M S_{e}=0.020, p<.0005\right)$. Semantic priming effects are illustrated in Figure 3.3. Experiment 3.1A and 3.1B differ with regards to the task and the corresponding kind of priming effects. Including task level as a between-subjects factor in an analysis with both data sets revealed neither a main effect of task level nor any significant interaction with this factor $(p s>.05$; Table B1.1 in Appendix II). Main effects and interactions, revealed in the analysis of Experiment 3.1A as well in the analysis of Experiment 3.1B, also reached significance in the conjoint analysis $(p s<.05)$.

Reaction Times. The mean reaction time in Experiment 3.1A was $416 \mathrm{~ms}$. Subjects responded faster in congruent trials (404 ms) compared to incongruent trials (429 ms; $\left.F(1,11)=58.13, M S_{e}=1019, p<.0001\right)$. Reaction times increased with decreasing masking strength $\left(F(3,33)=9.49, M S_{e}=140, p<.0001\right)$. Mask densities of $0.15^{\circ}, 0.30^{\circ}, 0.61^{\circ}$ and $1.21^{\circ}$ of visual angle produced RTs of $413 \mathrm{~ms}, 414 \mathrm{~ms}, 417 \mathrm{~ms}$ and $422 \mathrm{~ms}$, respectively. 

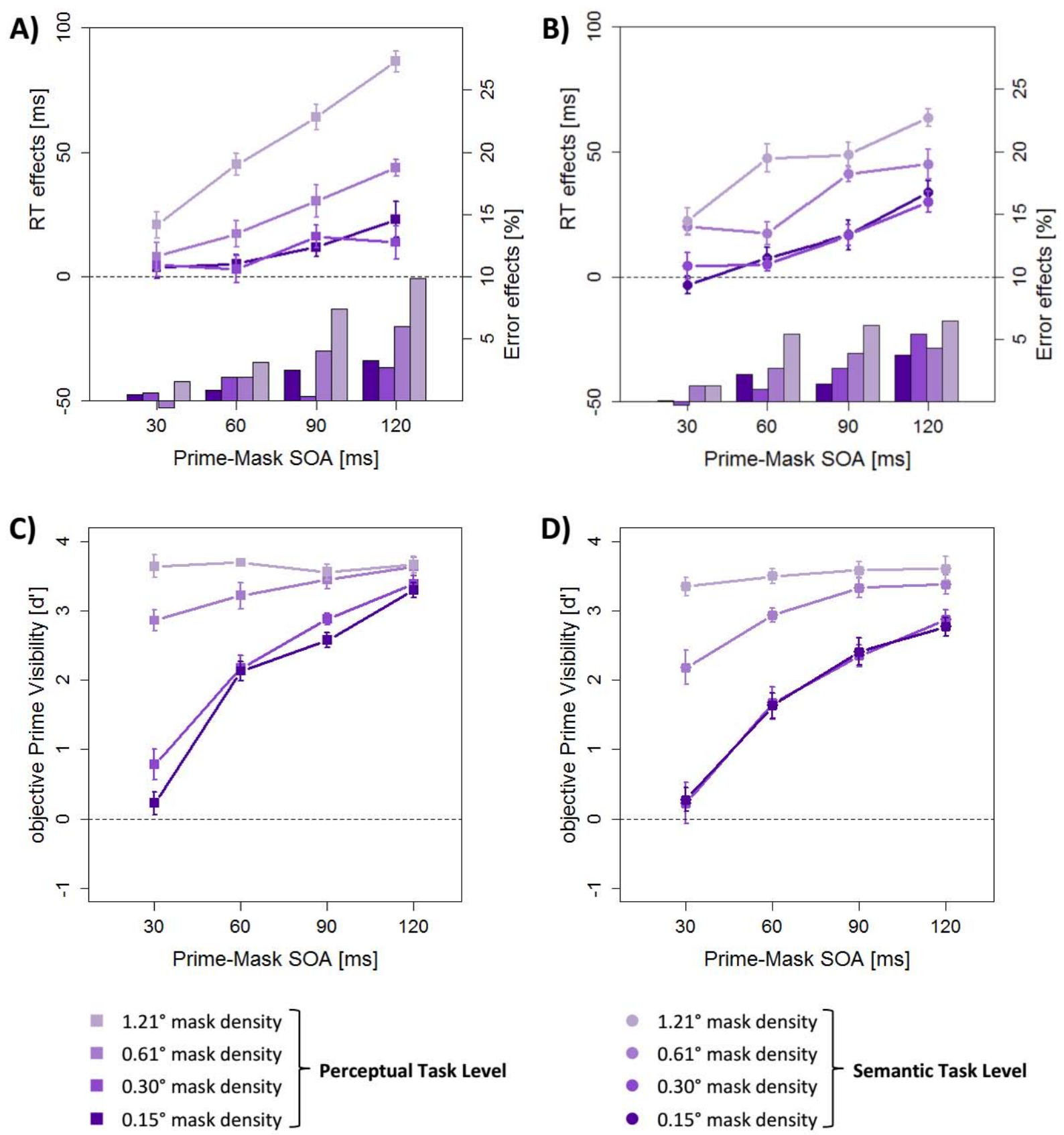

Figure 3.3. Priming effects and $d^{\prime}$-values in Experiment 3.1. Priming effects (A \& B) on reaction times (RT; lines) and errors (ER; bars) and $d$ '-values (C \& D) are displayed for each masking strength and prime-mask SOA. A \& C: Experiment 3.1A with a perceptual task level. B \& D: Experiment 3.1B with a semantic task level. Confidence intervals are in accordance with Loftus and Masson (1994). 
The prime-mask SOA also affected reaction times with smaller SOAs being associated with longer RTs - $422 \mathrm{~ms}, 418 \mathrm{~ms}, 413 \mathrm{~ms}$ and $412 \mathrm{~ms}$ with prime-mask SOAs of $30 \mathrm{~ms}, 60 \mathrm{~ms}$, $90 \mathrm{~ms}$ and $120 \mathrm{~ms}$, respectively $\left(F(3,33)=17.62, M S_{e}=114, p<.0001\right)$. A significant interaction between perceptual congruency and masking strength attested the effect of masking strength on perceptual priming effects $\left(\mathrm{PE}_{\mathrm{RT}}=\mathrm{RT}_{\text {incongruent }}-\mathrm{RT}_{\text {congruent }}\right.$; $\left.F(3,33)=44.68, M S_{e}=231, p<.0001\right)$. Perceptual priming effects of $11 \mathrm{~ms}, 9 \mathrm{~ms}, 25 \mathrm{~ms}$ and $54 \mathrm{~ms}$ occurred with mask densities of $0.15^{\circ}, 0.30^{\circ}, 0.61^{\circ}$ and $1.21^{\circ}$ of visual angle. Primemask SOA also modulated perceptual priming effects with priming effects of $9 \mathrm{~ms}, 18 \mathrm{~ms}$, $31 \mathrm{~ms}$ and $42 \mathrm{~ms}$ after $30 \mathrm{~ms}, 60 \mathrm{~ms}, 90 \mathrm{~ms}$ and $120 \mathrm{~ms}$ prime-mask SOA, respectively $\left(F(3,33)=29.02, M S_{e}=168, p<.0001\right)$. The three-way interaction between perceptual congruency, masking strength and prime-mask SOA also reached significance $\left(F(9,99)=4.50, M S_{e}=145, p<.0001\right)$. Perceptual priming effects and the characteristics of this three-way interaction are depicted in Figure 3.3. Reaction times for congruent and incongruent trials are displayed in Figure 3.4. In Experiment 3.1B, subjects responded after $459 \mathrm{~ms}$ on average. A semantic congruency effect of primes and targets was reflected in faster reactions with $446 \mathrm{~ms}$ in congruent trials compared to $472 \mathrm{~ms}$ in incongruent trials $\left(F(1,11)=106.96, M S_{e}=608, p<.0001\right)$. Reaction times for congruent and incongruent trials are displayed in Figure 3.5. A main effect of masking strength also reached significance $\left(F(3,33)=23.35, M S_{e}=299, p<.0001\right)$. Subjects responded after $452 \mathrm{~ms}, 453 \mathrm{~ms}, 460 \mathrm{~ms}$ and $470 \mathrm{~ms}$, when masks with a mesh width of $0.15^{\circ}, 0.30^{\circ}, 0.61^{\circ}$ and $1.21^{\circ}$ of visual angle were used, respectively. Semantic congruency interacted with masking strength $\left(F(3,33)=29.83, M S_{e}=186, p<.0001\right)$ and prime-mask SOA $\left(F(3,33)=30.97, M S_{e}=150\right.$, $p<.0001)$. Semantic priming effects increased with decreasing masking strength (14 ms, $14 \mathrm{~ms}, 31 \mathrm{~ms}$ and $45 \mathrm{~ms}$ ) and increasing prime-mask SOA (11 ms, $19 \mathrm{~ms}, 31 \mathrm{~ms}$ and $43 \mathrm{~ms})$. Courses of semantic priming effects are itemized for each masking strength and SOA in 
Figure 3.3. The conjoint analysis of both experiment revealed a main effect of task level with smaller reactions times with a perceptual task level compared to a semantic task level $\left(F(1,22)=8.84, M S_{e}=38594, p<.01\right.$; Table B1.2). The interaction between task level and masking strength reached significance as well as the four-way interaction between task level, congruency, masking strength and prime-mask SOA $\left(F(3,66)=5.09, M S_{e}=219, p<.005\right.$ and $\left.F(9,198)=2.38, M S_{e}=137, p<.05\right)$. Main effects and interactions, revealed in the analysis of Experiment 3.1A as well in the analysis of Experiment 3.1B, also reached significance in the conjoint analysis $(p s<.05)$.

\section{Experiment 3.1A}
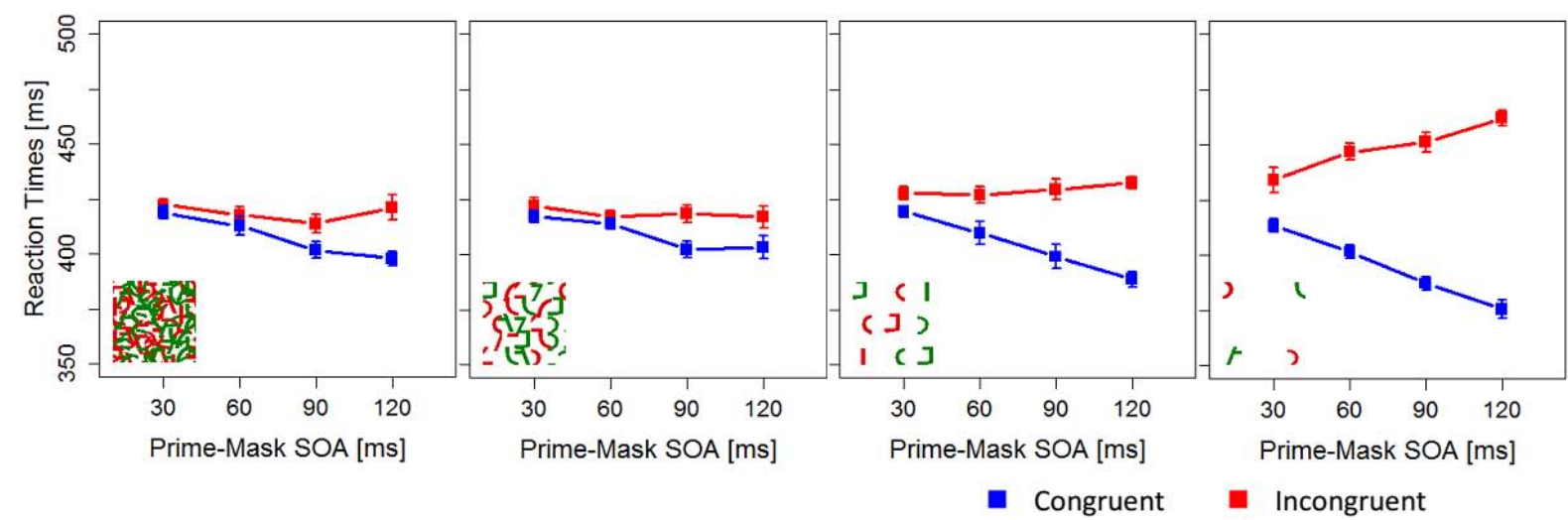

Experiment 3.1B
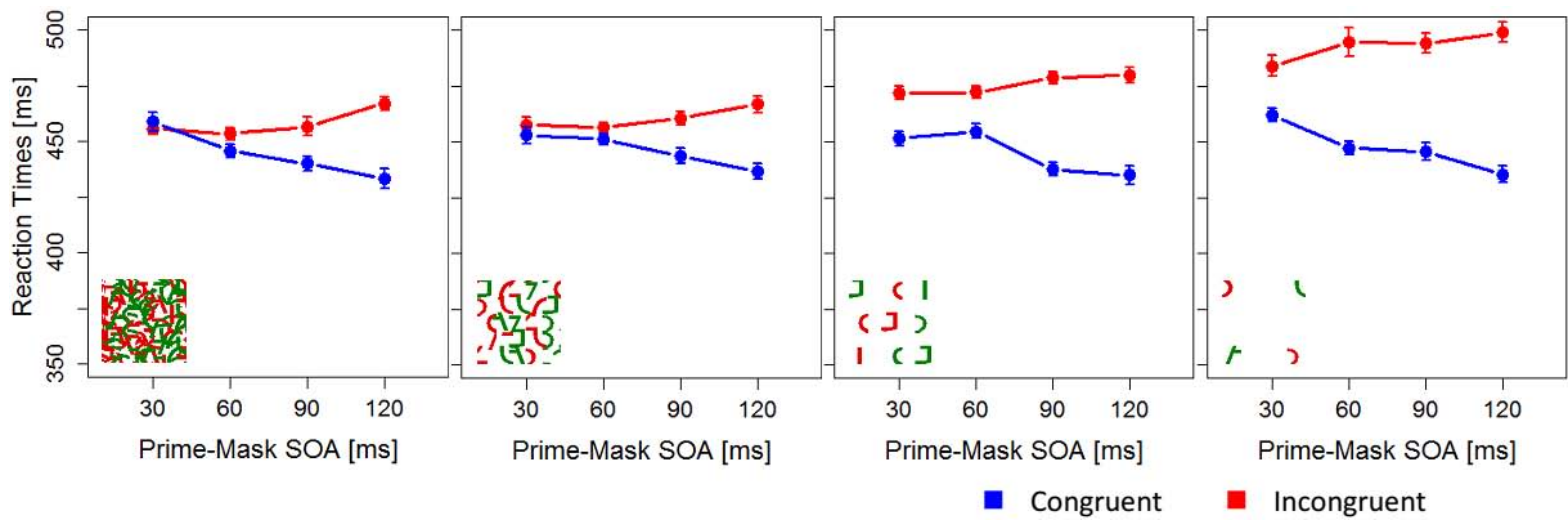

Figure 3.4. Reaction times in Experiment 3.1. Reaction times are displayed for congruent and incongruent trials with each masking strength and prime-mask SOA. Confidence intervals are in accordance with Loftus and Masson (1994). 
Prime visibility. In Experiment 3.1A, subjects chose the correct alternative of primes with a mean $d^{\prime}$-value of 2.82 and rated prime visibility with 3.31 on average (PAS rating; Figure 3.5). Masking strength modulated $d$ '-values with mean values of 2.06, 2.30, 3.29 and 3.64 and subjective ratings with $2.75,3.02,3.51$ and 3.86 in order of descending masking strength $\quad\left(F(3,33)=53.01, \quad M S_{e}=0.528, \quad p<.0001 \quad\right.$ and $\quad F(3,33)=63.13, \quad M S_{e}=0.199$, $p<.0001$, respectively). Prime visibility also increased with increasing prime-mask SOA. In trials with prime-mask SOAs of $30 \mathrm{~ms}, 60 \mathrm{~ms}, 90 \mathrm{~ms}$ and $120 \mathrm{~ms}$ mean $d^{\prime}$-values of 1.88 , $2.81,3.12$ and 3.50 as well as mean subjective ratings of $2.81,3.23,3.54$ and 3.66 were produced, respectively $\left(F(3,33)=123.16, M S_{e}=0.187, p<.0001\right.$ and $F(3,33)=91.74$, $M S_{e}=0.076, p<.0001$, respectively). The interaction between masking strength and primemask SOA also reached significance with $d^{\prime}$-values as well as subjective judgment $\left(F(9,99)=24.83, M S_{e}=0.194, p<.0001\right.$ and $F(9,99)=31.51, M S_{e}=0.049, \quad p<.0001$, respectively). Objective prime visibility $\left(d^{\prime}\right)$ is depicted in Figure 3.3, separately for each masking strength and prime-mask SOA. Debriefing at the end of the last priming session revealed that seven of twelve subjects did not detect primes. One subject was capable of detecting flashes without being able to report their identity. The remaining four subjects identified primes as numbers or letters. At the end of the prime-identification session, eleven subjects reported that they saw the primes better in this session compared to the priming sessions. Only one subject recognized no influence of masking strength on prime visibility. In Experiment $3.1 B, d^{\prime}$-values averaged to 2.51 and subjective ratings to 3.00 . Objective prime visibility $\left(d^{\prime}\right)$ was modulated by masking strength, prime-mask SOA and their interaction (Figure 3.3; $F(3,33)=46.32, \quad M S_{e}=0.790, \quad p<.0001, \quad F(3,33)=106.75, \quad M S_{e}=0.239$, $p<.0001$ and $F(9,99)=9.57, M S_{e}=0.305, p<.0001$, respectively). Objective prime visibility increased with decreasing density of masks $\left(1.77\right.$ with $0.15^{\circ}, 1.78$ with $0.30^{\circ}, 2.96$ with $0.61^{\circ}$ and 3.52 with $1.21^{\circ}$ of visual angle). Increasing prime-mask SOA had an 
increasing influence on objective prime visibility. Trials with prime-mask SOAs of $30 \mathrm{~ms}$, $60 \mathrm{~ms}, 90 \mathrm{~ms}$ and $120 \mathrm{~ms}$ yielded mean $d$ '-values of 1.51, 2.44, 2.92 and 3.16, respectively. Subjective prime visibility was also modulated by masking strength, prime-mask SOA and their interaction (Figure 3.5; $F(3,33)=58.15, M S_{e}=0.309, p<.0001, F(3,33)=136.30$, $M S_{e}=0.037, p<.0001$ and $F(9,99)=12.35, M S_{e}=0.043, p<.0001$, respectively). Subjective prime visibility also increased with decreasing density of masks $\left(2.35\right.$ with $0.15^{\circ}, 2.67$ with $0.30^{\circ}, 3.31$ with $0.61^{\circ}$ and 3.71 with $1.21^{\circ}$ of visual angle). Similar to the impact on objective prime visibility, increasing prime-mask SOA had an increasing influence on subjective prime visibility. Trials with prime-mask SOAs of $30 \mathrm{~ms}, 60 \mathrm{~ms}, 90 \mathrm{~ms}$ and $120 \mathrm{~ms}$ yielded mean PAS ratings of $2.58,2.94,3.19$ and 3.32, respectively. After the last priming session of Experiment 3.1B, three subjects reported no detection and recognition of primes. Only one subject noticed no improvement of prime perception in the prime-identification session. Ten out of twelve subjects perceived a manipulation of prime visibility through masking strength. A conjoint analysis of both experiment revealed no impact of the task level (3.1A: perceptual; 3.1B: semantic) on $d^{\prime}$-values and subjective rating ( $p s>.1$; Table B1.3 and B1.4). Main effects and interactions revealed in the analysis of Experiment 3.1A as well as in the analysis of Experiment 3.2B, also reached significance in the conjoint analysis $(p s<.05)$.

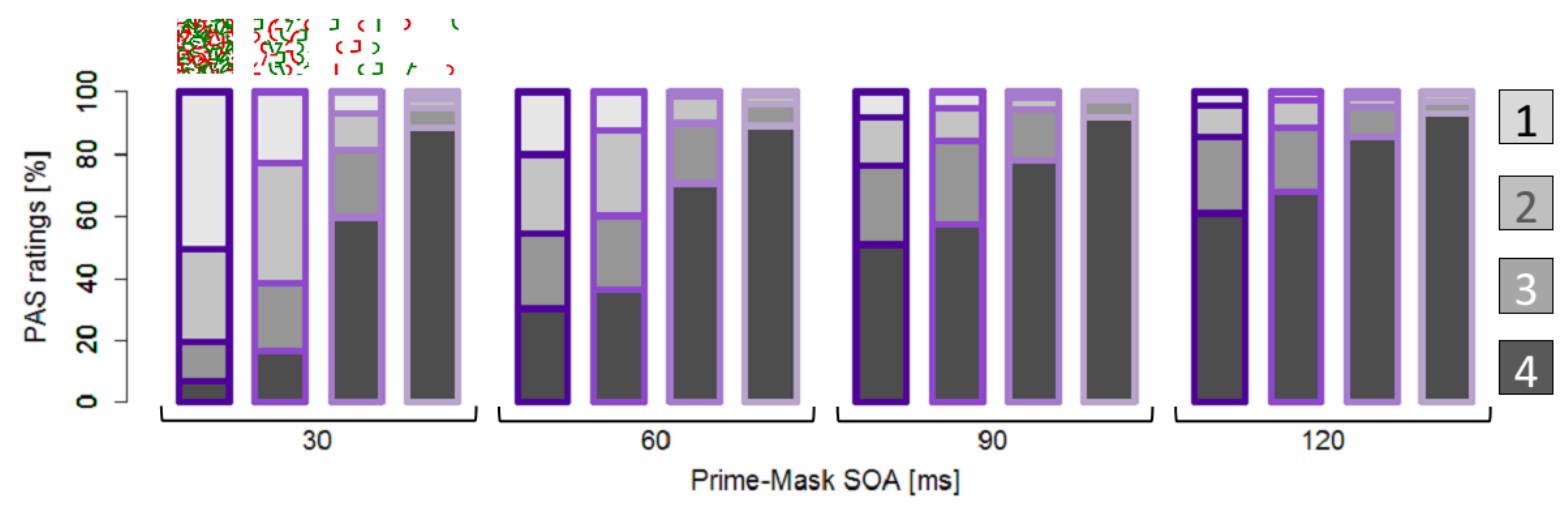

Figure 3.5. Ratings of subjective prime visibility with the PAS in Experiment 3.1A (Ramsøy \& Overgaard, 2004). Percentages of ratings with each masking strength and prime-mask SOA are displayed. Ratings: 1 = 'nothing', 2 = 'weak glimpse', 3 = 'almost clear image' \& $4=$ 'clear image'. 


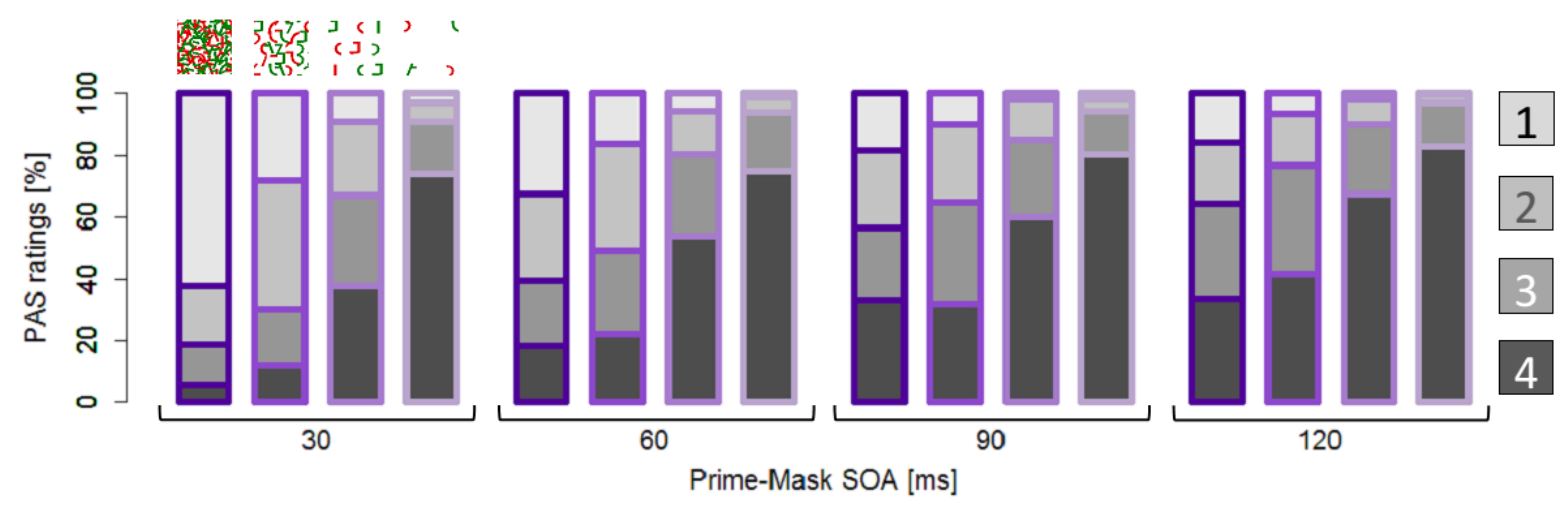

Figure 3.6. Ratings of subjective prime visibility with the PAS in Experiment 3.1B (Ramsøy \& Overgaard, 2004). Percentages of ratings with each masking strength and prime-mask SOA are displayed. Ratings: 1 = 'nothing', 2 = 'weak glimpse', 3 = 'almost clear image' \& 4 = 'clear image'.

\subsubsection{Discussion}

Experiment 3.1 was conducted to compare perceptual and semantic priming with special focus on the modulation of priming effects through prime visibility. Masks with different strengths were applied to realize a controlled variation of prime visibility. Analyses of objective and subjective prime visibility confirmed successful modulation of prime visibility through masking strength. Prime visibility $-d$ '-values as well as PAS-ratings increased with decreasing masking strength. In trials with mask densities of $0.15^{\circ}, 0.30^{\circ}$, $0.61^{\circ}$ and $1.21^{\circ}$ of visual angle, subjects recognized $78 \%, 80 \%, 93 \%$ and $99 \%$ of the alternatives correctly with a chance level of $50 \%$. The manipulation of prime visibility through masking strength was noticed by almost all subjects (21 of 24). Prime visibility was also modulated by prime-mask SOA. Subjects recognized the correct alternative in $75 \%$, $88 \%, 93 \%$ and $96 \%$ of the trials with prime-mask SOAs of $30 \mathrm{~ms}, 60 \mathrm{~ms}, 90 \mathrm{~ms}$ and $120 \mathrm{~ms}$, respectively. However, by choosing a very conservative method of measuring prime visibility, it is likely that we overestimated prime visibility systematically. Almost half of the subjects (10 of 24) did not detect the primes in the priming session and 22 of 24 subjects reported an improvement of prime perception in the prime-identification session. Concerning 
the modulations of prime visibility, no differences between Experiment 3.1A (perceptual task level) and Experiment 3.1B (semantic task level) were assessed. We therefore succeeded in measuring perceptual and semantic priming effects with the same stimulation and under very similar circumstances.

To access the impact of primes, we esteemed error rates as well as reaction times as priming effects might manifest themselves in one or the other measure (Vorberg et al., 2003; Mattler \& Palmer, 2012). Analyses of arc-sin transformed error rated and RTs with repeated measures ANOVAs revealed very similar patterns. This substantiates found effects by eliminating alternative explanations based on different speed-accuracy tradeoffs (Yellott, 1971). Perceptual priming effects (Experiment 3.1A) as well as semantic priming effects (Experiment 3.1B) were found with error rates and RTs. In both subexperiments, error effects increased with increasing prime-mask SOA as well as with decreasing masking strength equatable to increasing prime visibility. Error rates were generally not modulated by the task level. Reaction times were modulated by task level as well as task level in interaction with masking strength and in four-way interaction with congruency, masking method and primemask SOA. Nevertheless, RTs also increased with increasing prime-mask SOA as well as with decreasing masking strength in both subexperiments. Therefore, the data does not support our initial hypothesis that the impact of prime visibility on priming effects depends on the task level. As to be seen in Figure 3.3, the four-way interaction between task level, congruency, masking strength and prime-mask SOA provides indices for differences between perceptual and semantic priming. However, these differences do not concern the basic manipulability of priming effects through prime visibility.

In our meta-analysis (Chapter 2) two competing models were generated on the basis of a confounding between task level and masking method (variance inflations factors of 4.5 and 3.1, respectively). One model (excluding masking method) dedicated an impact of prime 
visibility to a semantic task level and one model (excluding task level) to the use of pattern masks. Experiment 3.1 provides no evidence supporting relevance of tasks level for a modulation of priming effect through prime visibility. In the current experiment, pattern masks were applied and priming effects were successfully modulated by masking strength. Essentially, the results of Experiment 3.1 point to relevance of masking method instead of task level. However, this assumption requires additional experimental validation.

\subsection{Experiment 3.2: Priming with Metacontrast Masking}

To examine a potential influence of the masking method on the modulation of perceptual and semantic priming effects through prime visibility, we implemented metacontrast masks in the current design. An adaptation of the primes and targets was necessary to ensure comparable and sufficient masking of all stimuli. To eliminate the possibility of prime stimulus features generating a response in the experiment with the semantic categorization task, we deployed different stimuli as primes and targets. Priming effects should not be modulated by masking strength, if masking method is the critical factor regarding an impact of prime visibility on priming effects.

\subsubsection{Method}

Participants. Similar to Experiment 3.1, twelve subjects performed four experimental sessions in Experiment 3.2A (8 female, 4 male) as well as in experiment 3.2B ( 9 female, 3 male). In Experiment 3.2A, the ages of the subjects ranged between 22 and $33(M=24.67)$ and six subjects had normal whereas six had corrected-to-normal vision. In Experiment 3.2B, the age of the subjects ranged between 21 and $38(M=23.17)$ with four having normal and eight corrected-to-normal vision. One additional subject received the wrong stimulus sequence and was excluded subsequently. 
Stimuli. The settings and stimuli were identical to those in Experiment 3.1 with following exceptions. The prime and target presentation $\left(0.76^{\circ} \times 0.83^{\circ}\right)$ was adjusted so that all signs could be masked with one metacontrast mask. The depiction of the stimuli was based on a seven-segment display. To ensure an equivalent masking of all prime stimuli, prevent a confusion of certain numbers and letters, and a clustering of visual features in one semantic category, the stimuli were changed (Figure 3.7). The letters A, G, H and $U$ and the numbers 2, 6, 8 and 9 served as primes. The letters $\mathrm{C}, \mathrm{E}, \mathrm{F}$ and $\mathrm{P}$ and the numbers 3, 4, 5 and 7 were used as targets. The metacontrast masks were constructed by placing eight layers in altering color (red and green) around the 8-shaped section surrounding the primes and four layers within the holes of the 8-shaped section (Figure 3.7). Each layer was as thick as the stroke width of the written letters and numbers $\left(0.08^{\circ}\right.$ of visual angle). Therefore, the metacontrast masks subtended visual angles of $1.97^{\circ} \times 2.05^{\circ}$. Six different color patterns were achieved by coloring the outer layers two of four quarters of the mask in green and the other outer layers in red. The masking strength was varied by removing one, two or seven inner layers of the masks, based on a preliminary test.
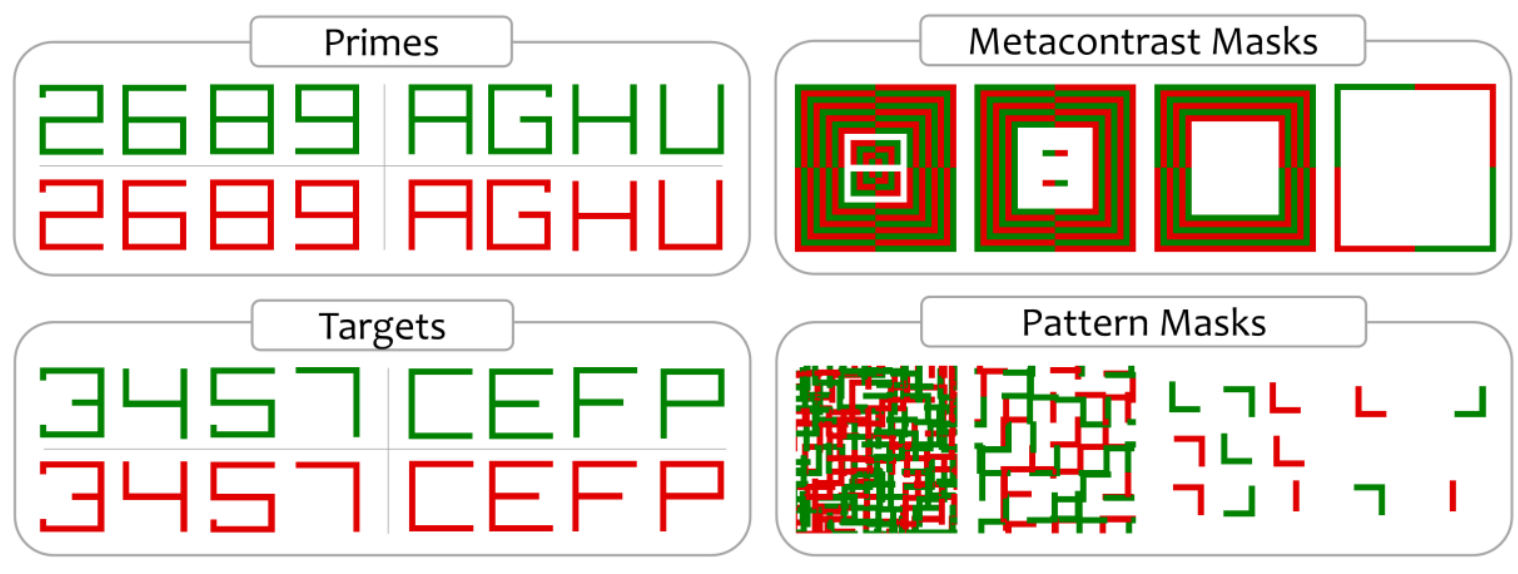

Figure 3.7. Stimuli applied in Experiments 3.2 and 3.3. Primes and targets are based on a sevensegment display to ensure similar masking of all primes by metacontrast masks. Metacontrast masks were applied in Experiment 3.2. To decrease masking strength the amount of inner layers was reduced. Pattern masks were applied in Experiment 3.3. Parts of primes were rearranged randomly and different masking strengths were implemented with different distances between segments. 
Tasks and Procedure. The tasks of Experiments 3.2A (perceptual task level) and 3.2B (semantic task level) were identical to the tasks of Experiments $3.1 \mathrm{~A}$ and 3.1B, respectively. The proceedings in Experiments 3.1 and 3.2 were equal as well.

Design and Statistical Analysis. Design and statistical analysis were applied equivalently to Experiment 3.1 .

\subsubsection{Results}

Errors. In Experiment 3.2A, subjects committed errors in $3.8 \%$ of all trials. Repeated measures ANOVA revealed a main effect of perceptual congruency $(F(1,11)=8.18$, $\left.M S_{e}=0.315, p<.05\right)$, an interaction of perceptual congruency and prime-mask SOA $\left(F(3,33)=7.05, M S_{e}=0.045, p<.01\right)$ and a three-way interaction of perceptual congruency, masking strength and prime-mask $\operatorname{SOA}\left(F(9,99)=2.94, M S_{e}=0.021, p<.05\right)$. Subjects committed more errors in incongruent trials $(5.7 \%)$ compared to congruent trials $(2.0 \%)$. Perceptual priming effects increased with increasing prime-mask SOA. Errors were committed in $0.4 \%, 2.7 \%, 5.3 \%$ and $6.7 \%$ of trials with prime-mask SOAs of $30 \mathrm{~ms}, 60 \mathrm{~ms}$, $90 \mathrm{~ms}$ and $120 \mathrm{~ms}$, respectively. Perceptual priming effects for each condition are depicted in Figure 3.8. In Experiment 3.2B, $5.8 \%$ of trials were error trials. Repeated measures ANOVA yielded a main effect of semantic congruency $\left(F(1,11)=16.67, M S_{e}=0.122, p<.005\right)$, a main effect of masking strength $\left(F(3,33)=6.06, M S_{e}=0.012, p<.005\right)$ and an interaction of semantic congruency and prime-mask SOA $\left(F(3,33)=7.82, M S_{e}=0.045, p<.01\right)$. Less errors occurred in congruent trials $(4.1 \%)$ compared to incongruent trials $(7.2 \%)$. Errors occurred in $5.3 \%, 6.3 \%, 5.8 \%$ and $5.2 \%$ of trials including masks with $8,7,6$ and 1 layer, respectively. Semantic priming effects increased with increasing prime-mask SOA. Semantic priming effects of $0.4 \%, 1.4 \%, 3.8 \%$ and $6.9 \%$ were estimated using trials with primemask SOAs of $30 \mathrm{~ms}, 60 \mathrm{~ms}, 90 \mathrm{~ms}$ and $120 \mathrm{~ms}$, respectively. Semantic priming effects for 
each condition are illustrated in Figure 3.8. In a conjoint analysis of Experiments 3.2A and 3.2B, task level was included as a between-subjects factor. The main effect of task level did not reach significance $(p>.2)$, but task level interacted with masking strength $\left(F(3,66)=5.98, M S_{e}=0.016, p<.005 ;\right.$ Table B2.1). Main effects and interactions revealed in the analysis of Experiment 3.2A as well in the analysis of Experiment 3.2B also reached significance in the conjoint analysis $(p s<.05)$.

Reaction Times. On average, subjects responded $438 \mathrm{~ms}$ after target onset in Experiment 3.2A. Repeated measures ANOVA yielded a main effect of perceptual congruency $\left(F(1,11)=72.33, M S_{e}=2142, p<.0001\right)$ and an interaction of perceptual congruency and prime-mask SOA $\left(F(3,33)=20.77, M S_{e}=703, p<.0005\right)$. Subjects reacted faster in congruent trials $(418 \mathrm{~ms})$ compared to incongruent trials (458 ms; Figure 3.9). Perceptual priming effects increased with increasing prime-mask SOA. In trials with primemask SOAs of $30 \mathrm{~ms}, 60 \mathrm{~ms}, 90 \mathrm{~ms}$ and $120 \mathrm{~ms}$, subjects responded $11 \mathrm{~ms}, 30 \mathrm{~ms}, 50 \mathrm{~ms}$ and 69 ms faster when congruent primes were presented, respectively. In Experiment 3.2B, mean RT was 445 ms. Repeated measures ANOVA revealed a main effect of semantic congruency $\left(F(1,11)=54.65, M S_{e}=1040, p<.0001\right)$ and an interaction of semantic congruency and prime-mask SOA $\left(F(3,33)=19.54, M S_{e}=808, p<.0001\right)$. In congruent trials, subjects responded on average $434 \mathrm{~ms}$ after target onset. In incongruent trials, mean response time was $457 \mathrm{~ms}$. Semantic priming effects increased with increasing prime-mask SOA (see Figure 3.9). Compared to incongruent primes, congruent primes yielded an advantage of $8 \mathrm{~ms}, 16 \mathrm{~ms}, 33 \mathrm{~ms}$ and $40 \mathrm{~ms}$ with prime-mask SOAs of $30 \mathrm{~ms}, 60 \mathrm{~ms}, 90 \mathrm{~ms}$ and $120 \mathrm{~ms}$, respectively. Perceptual priming effects and semantic priming effects are depicted in Figure 3.8. A conjoint analysis of Experiments $3.2 \mathrm{~A}$ and $3.2 \mathrm{~B}$ with task level as an additional between-subject factor revealed an interaction between task level and congruency $\left(F(1,22)=7.57, M S_{e}=1591, p<.05\right.$; Table B2.2). Perceptual priming effects (40 ms) were 
significantly larger than semantic priming effects $(24 \mathrm{~ms})$. Main effects and interactions, revealed in the analysis of Experiment 3.2A as well in the analysis of Experiment 3.2B also reached significance in the conjoint analysis $(p s<.05)$.
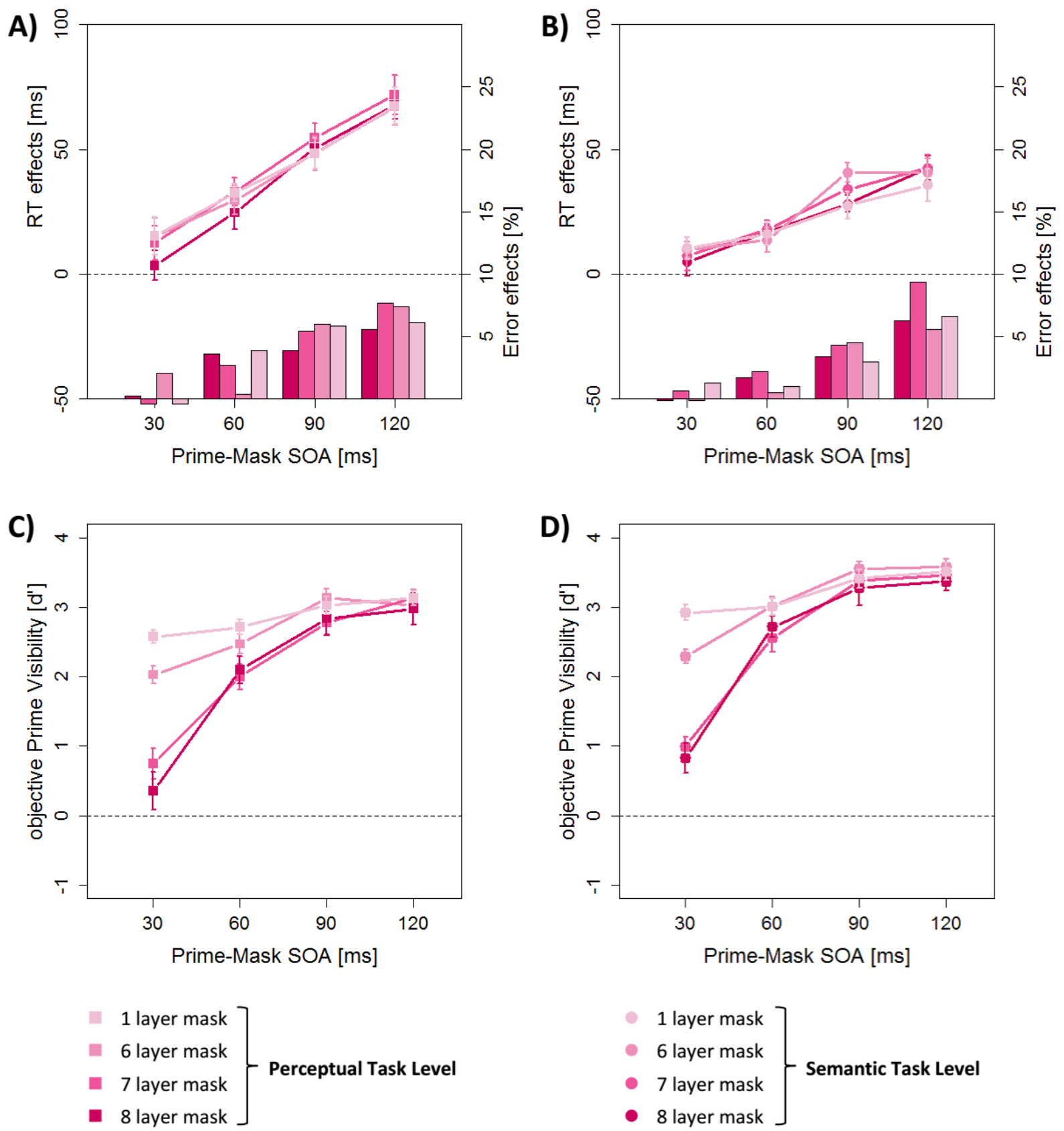

Figure 3.8. Priming effects and $d^{\prime}$-values in Experiment 3.2. Priming effects (A \& B) on reaction times (lines) and errors (bars) and $d$ '-values (C \& D) are displayed for each masking strength and prime-mask SOA. A \& C: Experiment 3.2A with a perceptual task level. B \& D: Experiment 3.2B with a semantic task level. Confidence intervals are in accordance with Loftus and Masson (1994). 


\section{Experiment 3.2A}

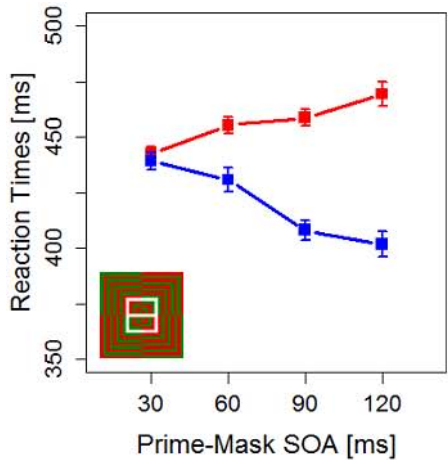

Prime-Mask SOA [ms]

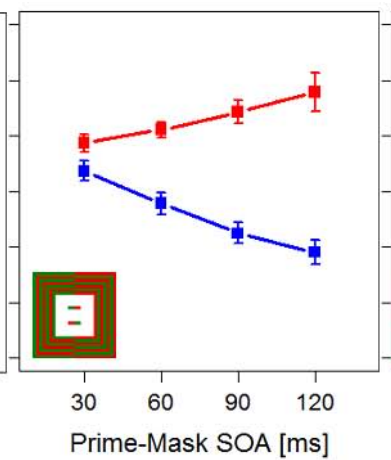

Prime-Mask SOA [ms]

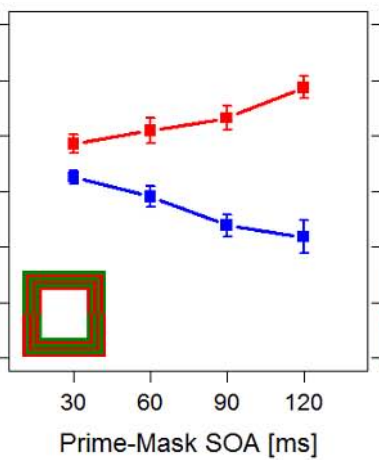

- Congruent

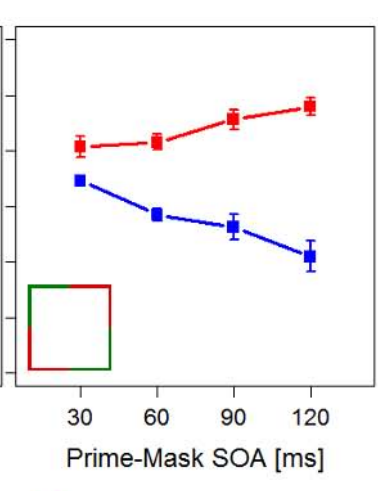

- Incongruent

\section{Experiment 3.2B}
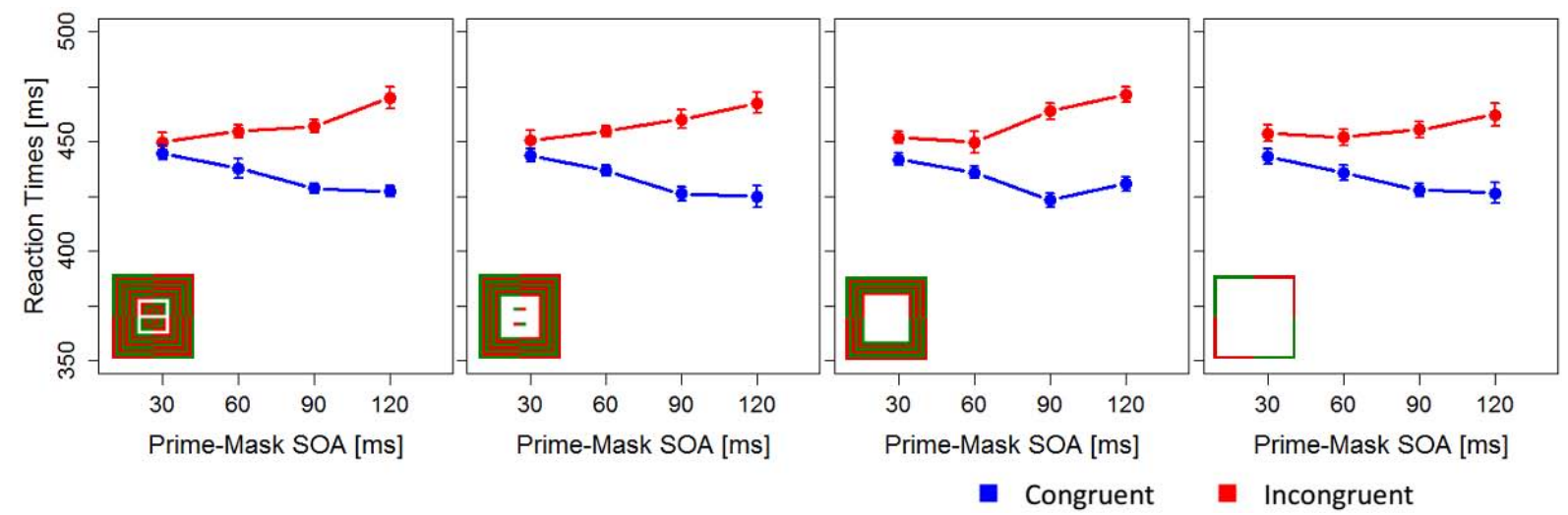

Figure 3.9. Reaction times in Experiment 3.2. Reaction times are displayed for congruent and incongruent trials with each masking strength and prime-mask SOA. Confidence intervals are in accordance with Loftus and Masson (1994).

Prime visibility. In Experiment 3.2A, subjects recognized correct alternatives with a mean $d^{\prime}$ of 2.44 and a mean subjective rating of 3.31. Repeated measures ANOVA on $d$ 'values revealed a main effect of masking strength $\left(F(3,33)=18.38, M S_{e}=0.388, p<.0005\right)$, a main effect of prime-mask SOA $\left(F(3,33)=33.53, M S_{e}=0.809, p<.0001\right)$ and an interaction of masking strength and prime-mask SOA $\left(F(9,99)=13.01, M S_{e}=0.199\right.$, $p<.0001)$. Objective prime visibility $\left(d^{\prime}\right)$ increased with decreasing masking strength. When masks with 8, 7, 6 or 1 layer were applied, mean $d^{\prime}$-values of 2.07, 2.17, 2.67 and 2.86 were achieved, respectively. With increasing prime-mask SOA (30 ms, $60 \mathrm{~ms}, 90 \mathrm{~ms}$ and $120 \mathrm{~ms}$ ), mean $d$ '-values also increased $(1.43,2.32,1.94,3.07)$. Figure 3.8 illustrates objective prime 
visibility and Figure 3.10 displays subjective prime visibility, itemized for each prime-mask SOA and masking strength. Repeated measures ANOVA on subjective ratings (PAS) also revealed a main effect of masking strength $\left(F(3,33)=15.69, M S_{e}=0.087, p<.005\right)$, a main effect of prime-mask SOA $\left(F(3,33)=25.06, M S_{e}=0.242, p<.0005\right)$ and an interaction of masking strength and prime-mask SOA $\left(F(9,99)=12.05, M S_{e}=0.049, p<.005\right)$. Subjective prime visibility increased with decreasing masking strength $(3.14,3.22,3.38,3.52$ for each masking strength, respectively) and increasing prime-mask SOA $(2.83,3.26,3.54,3.61$ for each SOA, respectively). Six of twelve subjects reported to have noticed primes in the priming sessions. Four of them were able to report the identities of the primes at the end of the third session. Ten subjects reported an improvement of prime perception in the prime identification session. Manipulation of prime visibility through masking strength was noticed by ten subjects. In Experiment 3.2B, subjects achieved a mean $d^{\prime}$ of 2.87 and rated prime visibility with 3.36. Repeated measures ANOVA on $d$ '-values yielded a main effect of masking strength $\left(F(3,33)=17.98, M S_{e}=0.315, p<.0001\right)$, a main effect of prime-mask SOA $\left(F(3,33)=57.94, M S_{e}=0.526, p<.0001\right)$ and an interaction of masking strength and prime-mask SOA $\left(F(9,99)=13.04, M S_{e}=0.192, p<.0001\right)$. With decreasing number of layers in applied masks $(8,7,6$ and 1 layers) objective prime visibility increased $(2.55,2.60$, 3.12, 3.22). Increasing prime-mask SOA (30 ms, $60 \mathrm{~ms}, 90 \mathrm{~ms}$ and $120 \mathrm{~ms})$ was accompanied by increasing $d$ '-values $(1.76,2.83,3.41,3.48)$. Objective prime visibility is itemized for each SOA and masking strength in Figure 3.8. A similar pattern was reflected in analysis of subjective prime visibility (see Figure 3.10). Repeated measures ANOVA revealed a main effect of masking strength $\left(F(3,33)=9.68, M S_{e}=0.140, p<.001\right)$, a main effect of prime-mask SOA $\left(F(3,33)=34.87, M S_{e}=0.210, p<.0001\right)$ and an interaction of masking strength and prime-mask SOA $\left(F(9,99)=13.76, M S_{e}=0.045, p<.0001\right)$. Subjective prime visibility increased with decreasing masking strength (3.27 with 8-layer masks, 3.18 
with 7-layer masks, 3.43 with 6-layer masks and 3.56 with 1-layer masks). PAS-ratings also increased with increasing prime-mask SOA $(2.83,3.30,3.61,3.70$ for each SOA, respectively). Nine of twelve subjects reported prime perception in the priming session. Seven of these subjects described the primes accurately. Prime perception of all subjects was improved in the prime-identification session compared to the priming session by their own admission. Only two subjects reported no modulation of prime perception through masking strength. A repeated measures ANOVA with both data sets and task level as an additional between subjects factor revealed no impact of task level on visibility measurements $(d$ 'values and PAS-ratings; $p s>.3$; Table B2.3 and Table B2.4). Main effects and interactions revealed in the analysis of Experiment 3.2A as well in the analysis of Experiment 3.2B also reached significance in the conjoint analysis $(p s<.05)$.

\section{Experiment 3.2A}

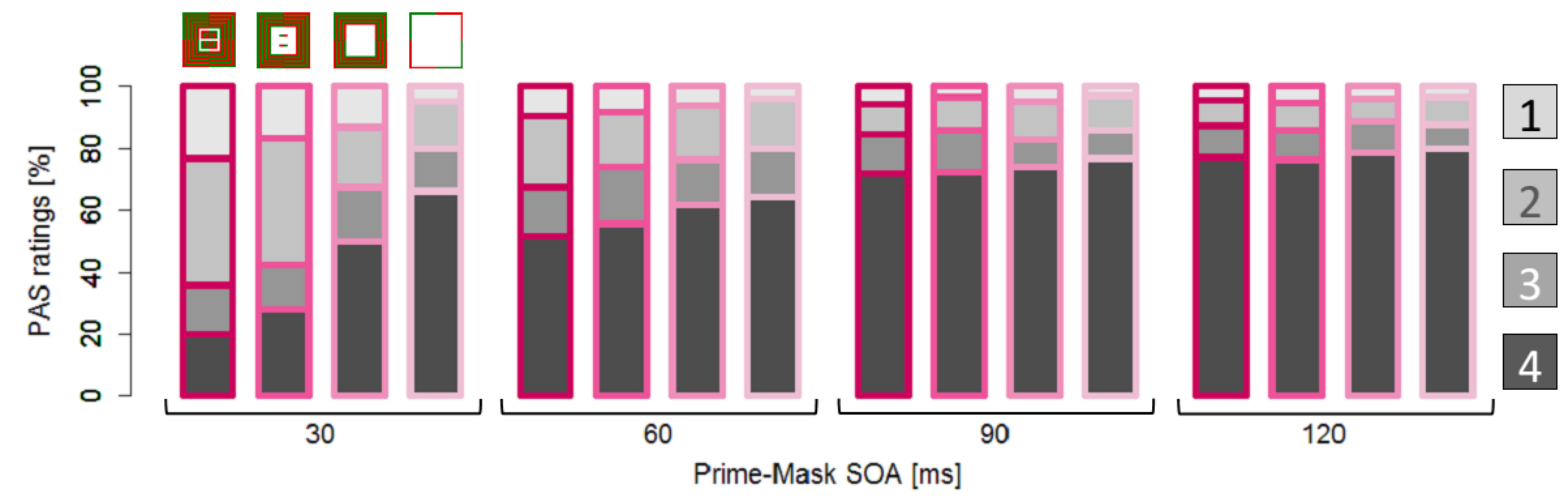

Experiment 3.2B

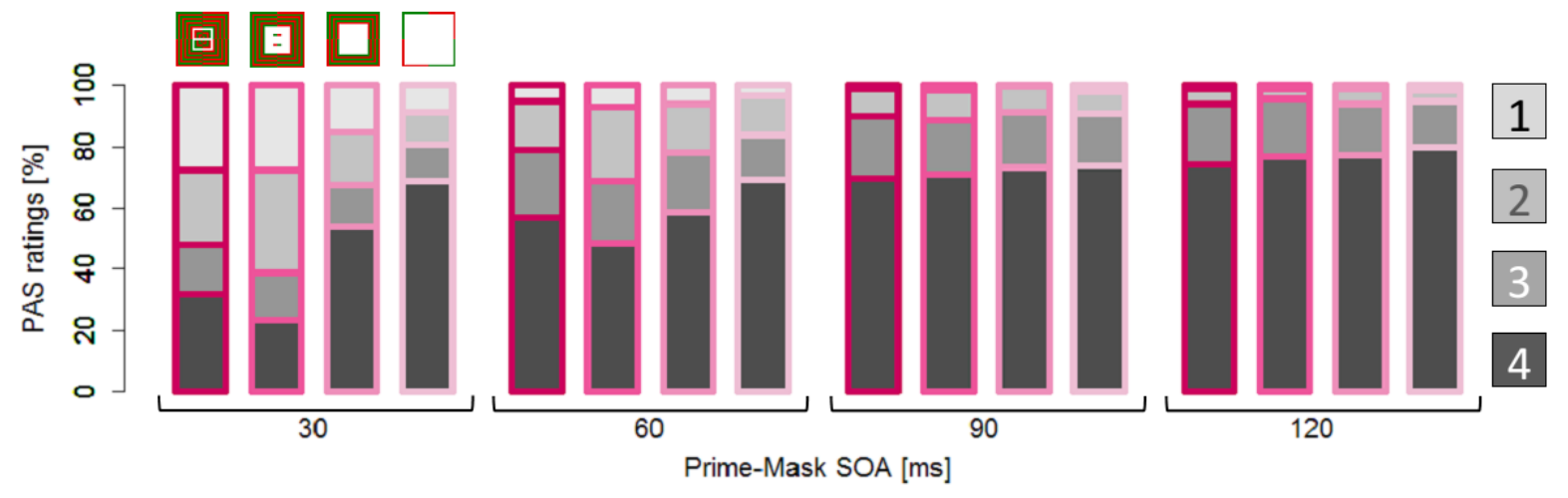

Figure 3.10. Ratings of subjective prime visibility with the PAS in Experiment 3.2 (Ramsøy \& Overgaard, 2004). Percentages of ratings with each masking strength and prime-mask SOA are displayed. Ratings: 1 = 'nothing', 2 = 'weak glimpse', 3 = 'almost clear image' $\& 4$ = 'clear image'. 


\subsubsection{Discussion}

In Experiment 3.2, similar to Experiment 3.1, objective and subjective prime visibility was modulated by masking strength and prime-mask SOA irrespectively of the task level. With masks with 8, 7, 6 and 1 layer subjects recognized the correct alternative in $84 \%, 84 \%$, $91 \%$ and $93 \%$ of the cases, respectively. This manipulation of prime visibility was noticed by 20 of 24 subjects. The correct alternative was chosen in $74 \%, 88 \%, 94 \%$ and $95 \%$ of trials for each SOA, respectively. An overestimation of prime visibility is also likely in Experiment 3.2. Primes were only noticed by 15 of 24 subjects during the priming session and 22 subjects reported an improvement of prime perception in the prime-identification session.

Positive perceptual and semantic priming effects were found in error rates as well as reaction times. An increase of priming effects (in ERs and RTs) with prime-mask SOA was confirmed in Experiments 3.2A (perceptual task level) and 3.2B (semantic task level). The analyses of reaction times consistently revealed only these two effects. Error rates of Experiment $3.2 \mathrm{~A}$ seem to be modulated by an interaction of perceptual congruency, masking strength and prime-mask SOA. However, the underlying pattern seems unsystematic with regards to an impact of increasing prime visibility (see Figure 3.8). Additional effects of a variation of masking strength, besides the manipulation of prime visibility, cannot be ruled out. In Experiment 3.2B, error rates were affected by masking strength. This could be due to different distracting effects of different masking strength $(5.3 \%, 6.3 \%, 5.8 \%$ and $5.2 \%$ with 8, 7, 6 and 1 layer, respectively). When asked about a distracting effect of the masks at the end of each priming session, five subjects said that masks with more layers were more distracting. Only two subjects noted that masks with fewer layers were more distracting. Four subjects stated that it was distracting when numbers or letters appeared in the mask. The intermediate masks are almost as massive as the strongest mask, but are less effective in 
masking the primes. Thus higher error rates with intermediate masks might be due to these two effects in Experiment 3.2B. On the bottom line, prime visibility seems to have no influence on priming effects in Experiment 3.2A (semantic priming) and Experiment 3.2B (perceptual priming).

Another interesting effect of task level was revealed in the conjoint analyses (3.2A and 3.2B) of reaction times. Perceptual priming effects (3.2A) were on average $16 \mathrm{~ms}$ larger than semantic priming effects (3.2B). This is in line with findings of our meta-analysis (Chapter 2) and the notion that semantic processing occurs later and on higher levels of processing (e.g. Martens, Ansorge \& Kiefer, 2011).

\subsection{Experiment 3.3: Priming with Pattern Masking}

To ensure that the revealed differences between Experiment 3.1 and 3.2 are based on the masking procedure only, we replicated Experiment 3.1 with the primes and targets of Experiment 3.2 and adapted pattern masks. In an attempt to keep all variables, except masking procedure and subjects, constant, we applied the same stimulus sequences used in Experiment 3.2. Expected results include a modulation of priming effects through masking strength (i.e. prime visibility).

\subsubsection{Method}

Participants. As before, twelve subjects participated in each experiment with eight female subjects in Experiment 3.3A and nine female subjects in Experiment 3.3B. The ages of the participants spanned from 21 to $34(M=24.5)$ in Experiment $3.3 \mathrm{~A}$ and from 22 to 28 $(M=24)$ in Experiment 3.3B. Normal vision was registered in eleven subjects $(3.3 \mathrm{~A}: 3 ; 3.3 \mathrm{~B}$ : 8). The remaining subjects reported corrected-to-normal vision. 
Stimuli. The settings and stimuli were identical to those in Experiment 3.2 with the following exceptions. Similarly to Experiment 3.1, the pattern masks were created with segments of the primes (Figure 3.7). The sizes of the masks were matched with those used in Experiment 3.2.

Tasks and Procedure. The tasks of Experiments 3.3A (perceptual task level) and 3.3B (semantic task level) were identical to the tasks of Experiments 3.1A/3.2A and 3.1B/3.2B, respectively. The proceedings in Experiments 3.1, 3.2 and 3.3 were equal as well.

Design and Statistical Analysis. Design and statistical analysis are applied equivalently to Experiments 3.1 and 3.2.

\subsubsection{Results}

Errors. In Experiment 3.3A, subjects responded incorrectly in $3.3 \%$ of trials. Repeated measures ANOVA with arc-sin transformed error rates yielded a main effect of perceptual congruency $\left(F(1,11)=30.91, M S_{e}=0.024, p<.0005\right)$ and an interaction of perceptual congruency and prime-mask $\operatorname{SOA}\left(F(3,33)=4.40, M S_{e}=0.022, p<.05\right)$. More errors occurred in incongruent trials $(4.0 \%)$ compared to congruent trials $(2.6 \%)$. With increasing prime-mask SOA (30 ms, $60 \mathrm{~ms}, 90 \mathrm{~ms}$ and $120 \mathrm{~ms}$ ) perceptual priming effects on errors increased as well $(0.1 \%, 1.4 \%, 1.8 \%$ and $2.3 \%)$. For each condition, characterized by masking strength and prime-mask SOA, perceptual priming effects are plotted in Figure 3.11. A different pattern arose in Experiment 3.3B. Errors occurred in $4.4 \%$ of all trials. Only the interaction of semantic congruency and masking strength reached significance $\left(F(3,33)=7.78, M S_{e}=0.020, p<.005\right)$. Semantic priming effects (see Figure 3.11$)$ of $0.7 \%$, $-0.4 \%, 0.4 \%$ and $3.3 \%$ were achieved with mask densities of $0.15^{\circ}, 0.30^{\circ}, 0.61^{\circ}$ and $1.21^{\circ}$ of visual angle, respectively. A repeated measures ANOVA with the conjoint data of both experiments revealed no effects of task level ( $p s>.05$; Table B3.1). Main effects and 

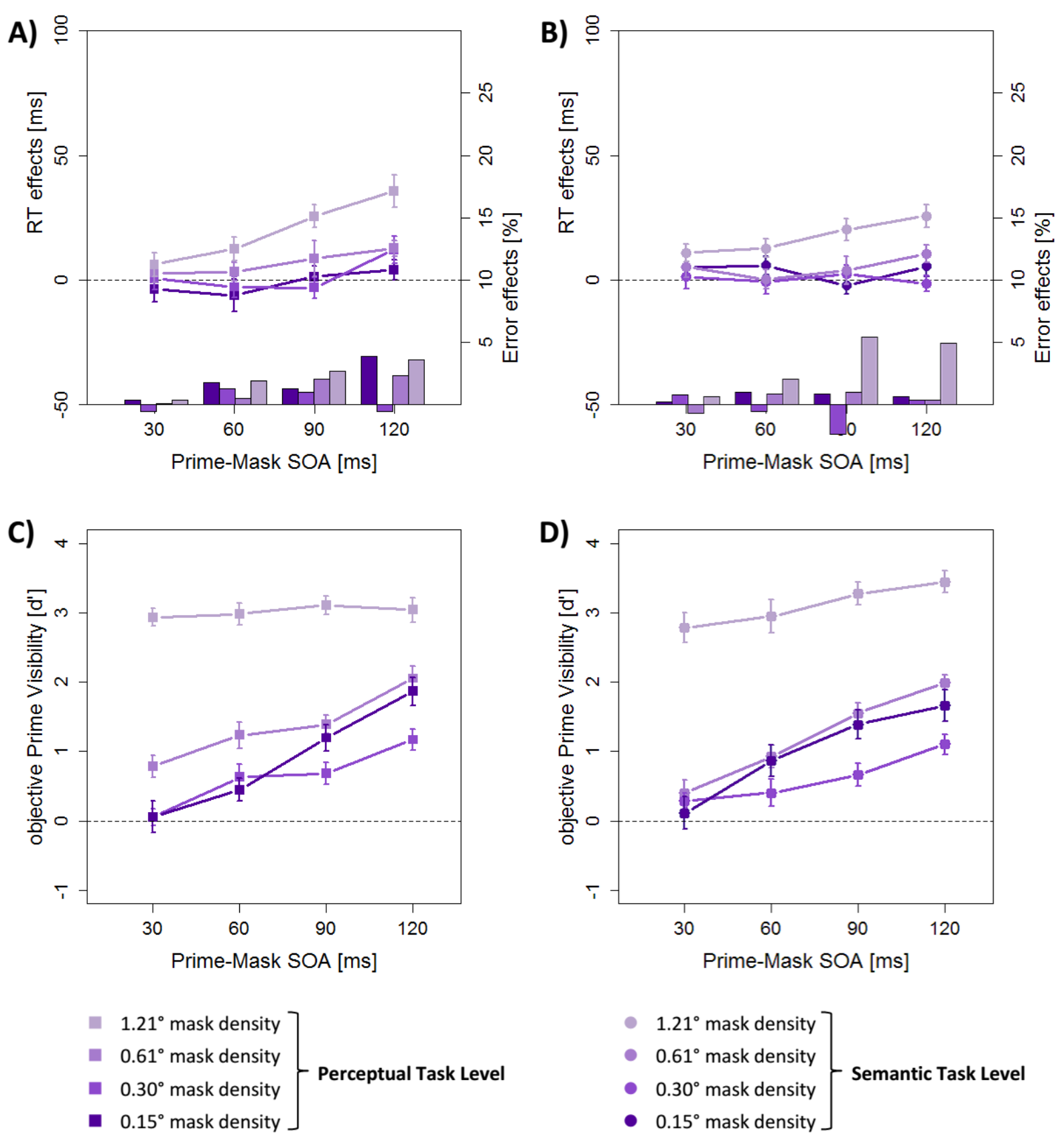

Figure 3.11. Priming effects and $d^{\prime}$-values in Experiment 3.1. Priming effects (A \& B) on reaction times (RT; lines) and errors (ER; bars) and $d$ '-values (C \& D) are displayed for each masking strength and prime-mask SOA. A \& C: Experiment 3.1A with a perceptual task level. B \& D: Experiment 3.1B with a semantic task level. Confidence intervals are in accordance with Loftus and Masson (1994). 
interactions revealed in the analysis of Experiment 3.3A as well in the analysis of Experiment 3.3B also reached significance in the conjoint analysis $(p s<.05)$. Although not prominent in both separate analyses the main effect of congruency $\left(F(1,22)=18.76, M S_{e}=0.037\right.$, $p<.0005)$, the main effect of prime-mask SOA $\left(F(3,66)=5.05, M S_{e}=0.030, p<.01\right)$, the interaction between congruency and masking strength $\left(F(3,66)=7.99, M S_{e}=0.022\right.$, $p<.0005)$ as well as the interaction between congruency and prime-mask SOA $\left(F(3,66)=3.09, M S_{e}=0.023, p<.05\right)$ reached significance.

Reaction Times. In Experiment 3.3A, a mean RT of $422 \mathrm{~ms}$ was calculated. Repeated measures ANOVA yielded a main effect of perceptual congruency $(F(1,11)=23.76$, $\left.M S_{e}=193, p<.0005\right)$, a main effect of masking strength $\left(F(3,33)=13.82, M S_{e}=181\right.$, $p<.0001)$, a main effect of prime-mask $\operatorname{SOA}\left(F(3,33)=18.21, M S_{e}=235, p<.0001\right)$ and an interaction of perceptual congruency and masking strength $\left(F(3,33)=7.36, M S_{e}=219\right.$, $p<.001)$ as well as perceptual congruency and $\operatorname{SOA}\left(F(3,33)=9.59, M S_{e}=155, p<.01\right)$. Mean reaction times were shorter in congruent trials (419 ms) compared to incongruent trials (426 ms; Figure 3.12). Denser masks $\left(0.15^{\circ}, 0.30^{\circ}, 0.61^{\circ}\right.$ and $1.21^{\circ}$ of visual angle) produced shorter RTs (418 ms, $419 \mathrm{~ms}, 423 \mathrm{~ms}$ and $429 \mathrm{~ms}$, respectively). RTs decreased with increasing prime-mask SOA. In trials with SOAs of $30 \mathrm{~ms}, 60 \mathrm{~ms}, 90 \mathrm{~ms}$ and $120 \mathrm{~ms}$, mean RTs of $431 \mathrm{~ms}, 424 \mathrm{~ms}, 420 \mathrm{~ms}$ and $415 \mathrm{~ms}$ were yielded, respectively. With decreasing masking strength, perceptual priming effects increased $(-1 \mathrm{~ms}, 2 \mathrm{~ms}, 7 \mathrm{~ms}$ and $20 \mathrm{~ms}$ with mask densities of $0.15^{\circ}, 0.30^{\circ}, 0.61^{\circ}$ and $1.21^{\circ}$ of visual angle, respectively). Perceptual priming effects also increased with increasing prime-mask SOA $(2 \mathrm{~ms}, 2 \mathrm{~ms}, 8 \mathrm{~ms}$ and $16 \mathrm{~ms}$ with each SOA, respectively). In Experiment 3.3B, subjects responded on average after $434 \mathrm{~ms}$. Repeated measures ANOVA revealed a main effect of semantic congruency $\left(F(1,11)=23.47, M S_{e}=181, p<.001\right)$, a main effect of masking strength $(F(3,33)=16.22$, $\left.M S_{e}=145, p<.0001\right)$, a main effect of prime-mask SOA $\left(F(3,33)=14.20, M S_{e}=126\right.$, 


\section{Experiment 3.3A}

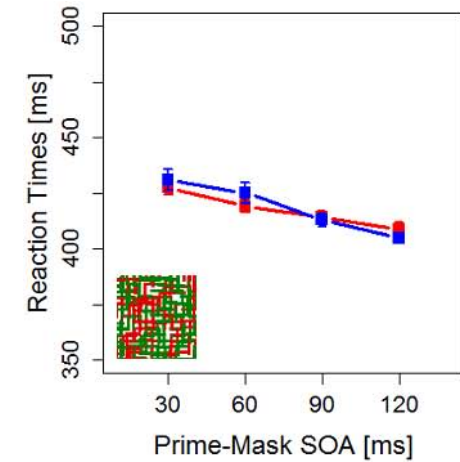

Prime-Mask SOA [ms]

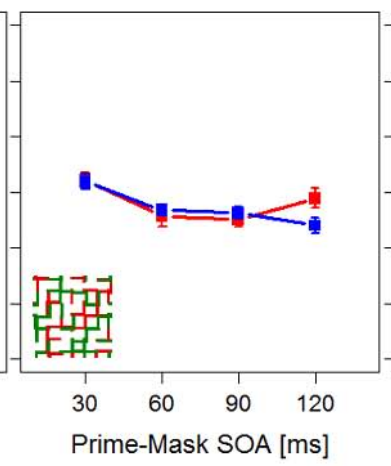

Prime-Mask SOA [ms]

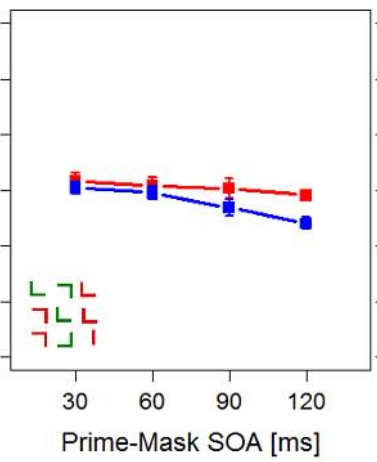

- Congruent

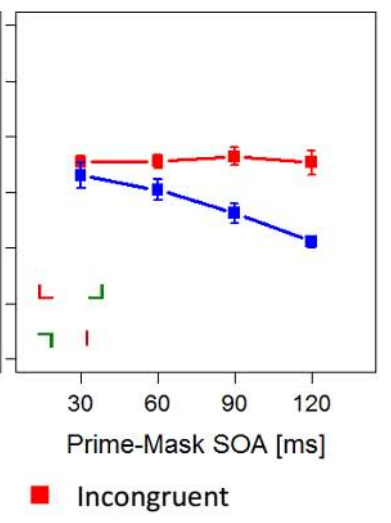

Experiment 3.3B
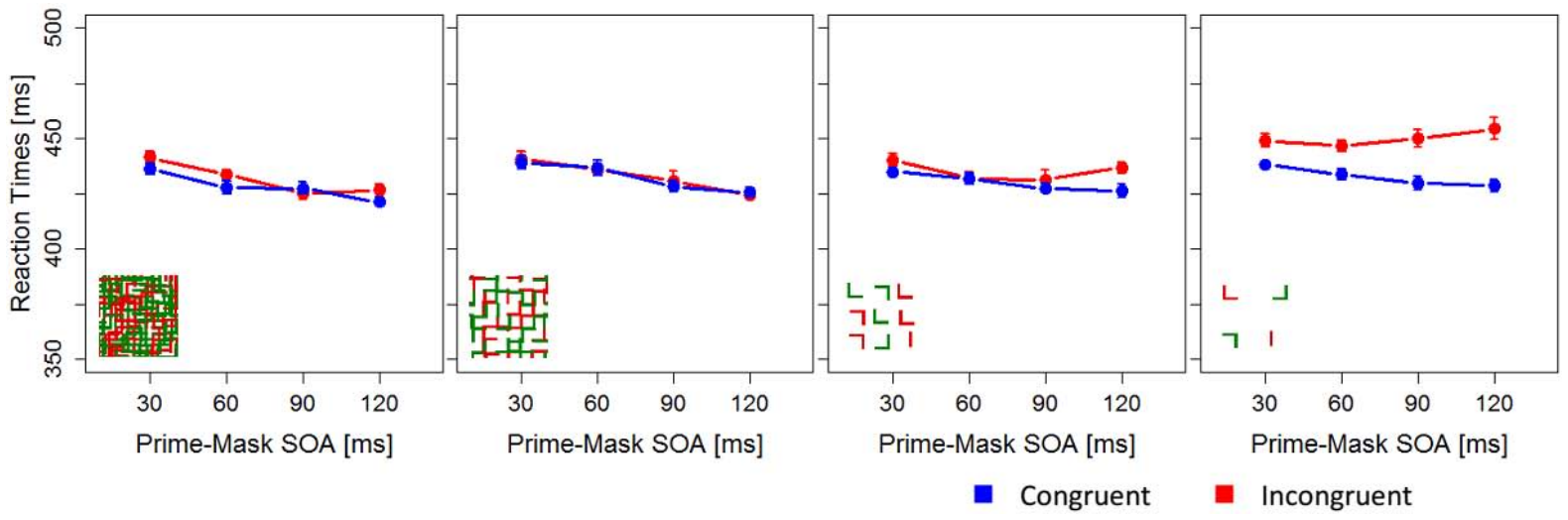

Figure 3.12. Reaction times in Experiment 3.3. Reaction times are displayed for congruent and incongruent trials with each masking strength and prime-mask SOA. Confidence intervals are in accordance with Loftus and Masson (1994).

$p<.0001)$ and an interaction of semantic congruency and masking strength $(F(3,33)=9.19$, $\left.M S_{e}=145, p<.0005\right)$ as well as masking strength and $\operatorname{SOA}\left(F(9,99)=2.78, M S_{e}=81\right.$, $p<.05$ ). With a mean $\mathrm{RT}$ of $431 \mathrm{~ms}$, subjects responded faster in congruent trials than in incongruent trials (mean RT of $438 \mathrm{~ms}$; Figure 3.12). With decreasing masking strength, subjects responded slower $(430 \mathrm{~ms}, 433 \mathrm{~ms}, 433 \mathrm{~ms}$ and $441 \mathrm{~ms}$ with each mask density, respectively). Subjects responded faster with increasing prime-mask SOA (440 ms, $435 \mathrm{ms,}$ $431 \mathrm{~ms}$ and $431 \mathrm{~ms}$ with each SOA, respectively). Semantic priming effects of $4 \mathrm{~ms}, 0 \mathrm{~ms}$, $5 \mathrm{~ms}$ and $17 \mathrm{~ms}$ were achieved with mask densities of $0.15^{\circ}, 0.30^{\circ}, 0.61^{\circ}$ and $1.21^{\circ}$ of visual angle, respectively. Perceptual priming effects and semantic priming effects are plotted in 
Figure 3.11. A conjoint analysis of both experiments with task level (perceptual or semantic) as an additional between-subjects factor revealed a significant interaction of task level, masking strength and prime-mask $\operatorname{SOA}\left(F(9,198)=2.51, M S_{e}=111, p<.05\right.$; Table B3.2). Main effects and interactions revealed in the analysis of Experiment 3.3A as well in the analysis of Experiment 3.3B also reached significance in the conjoint analysis $(p s<.05)$. Although not prominent in both separate analyses, the interaction between congruency and prime-mask SOA $\left(F(3,66)=8.07, M S_{e}=126, p<.005\right)$ as well as the interaction between masking strength and prime-mask SOA $\left(F(9,198)=2.29, M S_{e}=111, p<.05\right)$ reached significance.

Prime visibility. In Experiment 3.3A, a mean $d^{\prime}$ of 1.48 was achieved and subjects rated prime visibility on average with 2.57. Repeated measures ANOVA on objective prime visibility revealed a main effect of masking strength $\left(F(3,33)=74.38, M S_{e}=0.741\right.$, $p<.0001)$, a main effect of prime-mask SOA $\left(F(3,33)=38.58, M S_{e}=0.256, p<.0001\right)$ and an interaction between these factors $\left(F(9,99)=4.74, M S_{e}=0.267, p<.005\right)$. In Figure 3.11, $d$ '-values are depicted for each masking strength and SOA. In trials with a mask density of $0.15^{\circ}, 0.30^{\circ}, 0.61^{\circ}$ and $1.21^{\circ}$ of visual angle a mean $d^{\prime}$ of $0.89,0.63,1.37$ and 3.02 was achieved, respectively. Objective prime visibility increased with increasing prime-mask SOA (0.96, 1.33, 1.59 and 2.03 for each SOA, respectively). Subjective prime visibility (PASrating) was also modulated by masking strength $\left(F(3,33)=31.11, M S_{e}=0.555, p<.0001\right)$, prime-mask SOA $\left(F(3,33)=26.41, M S_{e}=0.081, p<.0001\right)$ and the interaction of masking strength and prime-mask SOA $\left(F(9,99)=8.22, M S_{e}=0.037, p<.0005\right.$; Figure 3.13). Mean subjective ratings increased with masking strength $(2.12,2.19,2.53$ and 3.42 for each mask density, respectively) and prime-mask SOA $(2.30,2.49,2.69$ and 2.78 for each SOA, respectively). At the end of the last priming session, five of twelve subjects reported to have 
noticed an impression of primes. Four of these subjects were able to describe the prime stimuli in more detail (e.g. "numbers", "letters", "signs" etc.). Debriefing subsequently to the prime-identification session revealed that eight subjects were sure of an improvement of prime perception during this session. All subjects noticed a variation of prime visibility through masking strength. In Experiment 3.3B, subjects recognized the correct alternative with a mean $d^{\prime}$ of 1.49 and rated prime visibility on average with 2.72 . Repeated measures ANOVA on $d$ '-values yielded a main effect of masking strength $(F(3,33)=124.95$, $\left.M S_{e}=0.476, p<.0001\right)$ and a main effect of prime-mask SOA $\left(F(3,33)=31.59, M S_{e}=0.387\right.$, $p<.0001)$. Objective prime visibility is itemized for each masking strength and SOA in Figure 3.11 . Mask densities of $0.15^{\circ}, 0.30^{\circ}, 0.61^{\circ}$ and $1.21^{\circ}$ of visual angle produced mean $d$-values of $1.01,0.62,1.22$ and 3.12 , respectively. With increasing prime-mask SOA, objective prime visibility increased as well $(0.90,1.29,1.72$ and 2.05 for each SOA, respectively). Repeated measures ANOVA on mean subjective ratings revealed a main effect of masking strength $\left(F(3,33)=64.06, M S_{e}=0.305, p<.0001\right)$, a main effect of prime-mask SOA $\left(F(3,33)=65.65, M S_{e}=0.071, p<.0001\right)$ and an interaction between masking strength and prime-mask SOA $\left(F(9,99)=6.85, M S_{e}=0.049, p<.01\right.$; Figure 3.13). Subjective prime visibility increased with masking strength $(2.28,2.28,2.68$ and 3.63 for each mask density, respectively) and prime-mask SOA $(2.34,2.62,2.85$ and 3.06 for each SOA, respectively). Seven of twelve subjects reported detections of primes during the priming sessions, as retrieved at the end of the third session. Primes were correctly recognized by five of these subjects. At the end of the prime-identification session, ten subjects reported better prime perception in this session compared to the priming session. A variation of prime visibility through masking strength was noticed by ten subjects. Repeated measures ANOVA with the conjoint data of Experiment 3.3A and 3.3B and task level as an additional between-subject factor revealed no impact of task level on objective prime visibility ( $p s>0.3$; Table B3.3). 
Although not prominent in both separate analyses, the interaction between masking strength and prime-mask SOA significantly moderates objective prime visibility, as revealed by a repeated measures ANOVA with the conjoint data $\left(F(9,198)=4.19, M S_{e}=0.361, p<.005\right)$. Analysis of subjective prime visibility with the conjoint data revealed a significant interaction of task level and prime-mask SOA $\left(F(6,66)=3.40, M S_{e}=0.075, p<.05\right.$; Table B3.4). Main effects and interactions revealed in the analysis of Experiment 3.3A as well in the analysis of Experiment 3.3B also reached significance in the conjoint analysis with objective prime visibility as well as subjective prime visibility $(p s<.05)$.

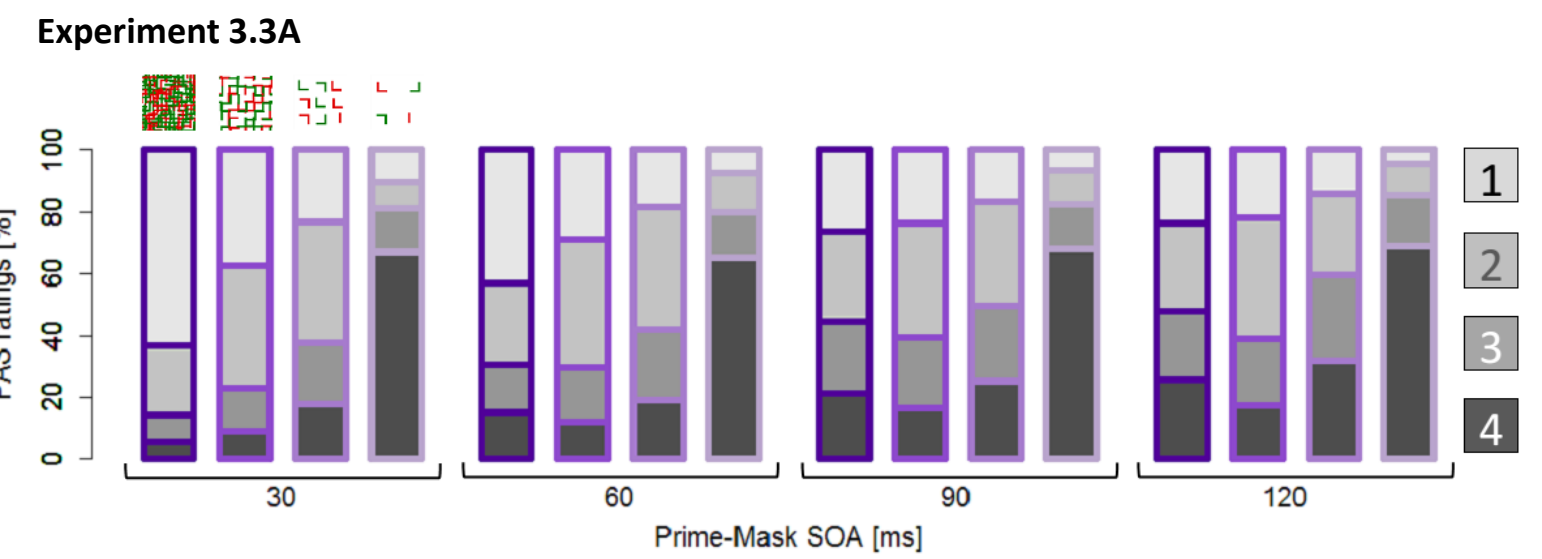

\section{Experiment 3.3B}

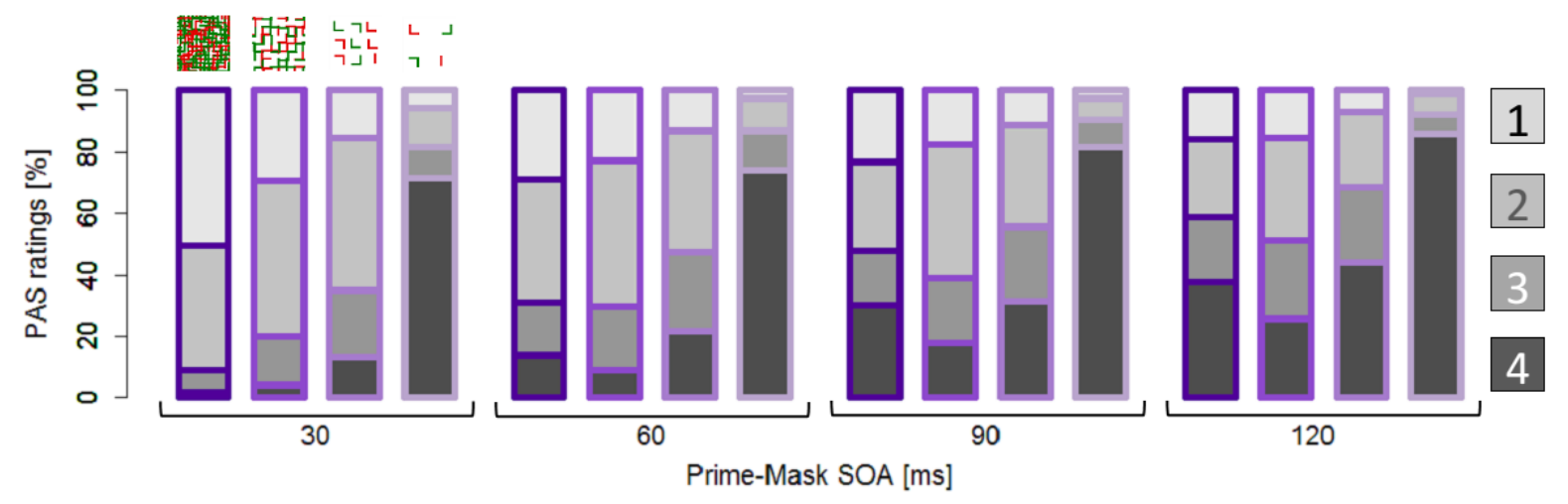

Figure 3.13. Ratings of subjective prime visibility with the PAS in Experiment 3.3 (Ramsøy \& Overgaard, 2004). Percentages of ratings with each masking strength and prime-mask SOA are displayed. Ratings: 1 = 'nothing', 2 = 'weak glimpse', 3 = 'almost clear image' $\& 4$ = 'clear image'. 


\subsubsection{Discussion}

Objective and subjective prime visibility was again successfully modulated through masking strength in Experiment 3.3. This manipulation was noticed by 18 of 24 subjects. Masks with a density of $0.15^{\circ}, 0.30^{\circ}, 0.61^{\circ}$ and $1.21^{\circ}$ of visual angle enabled subjects to recognize $66 \%, 61 \%, 71 \%$ and $93 \%$ of the alternatives correctly, respectively. Masks with the highest density were therefore less effective than masks with a density of $0.30^{\circ}$ of visual angle. Prime visibility was also modulated by prime-mask SOA $-64 \%, 70 \%, 76 \%$ and $81 \%$ of alternatives were correctly identifies for each SOA, respectively. Analyses of prime visibility were very consistent through subexperiments and measurements. A systematic overestimation of prime visibility is likely as half of the subjects did not detect primes during the priming session and 18 of 24 subjects recognized an improvement of prime perception during the prime-identification session.

Concerning the analysis of error rates, the results of Experiment $3.3 \mathrm{~A}$ and $3.3 \mathrm{~B}$ seem quite different, when taken separately. Nevertheless, the conjoint analysis reveals no impact of task level and, most importantly, a significant interaction between congruency and masking strength. Analyses of reaction times were fairly consistent between Experiment 3.3A and Experiment 3.3B. In both experiments, reaction times were modulated by masking strength and prime-mask SOA. Most importantly, priming effects occurred in both experiments and were modulated through masking strength. In contrast to Experiment 3.3A, the interaction between semantic congruency and prime-mask SOA did not reach significance in the analysis of Experiment 3.3B. Even though the three-way interaction of task level, congruency and prime-mask SOA did not reach significance either $(p=.13)$, one might argue that a similar pattern with error rates provides evidence for a difference between perceptual and semantic priming on this account. The relevance of this argument, however, is challenge by the apparent impact of a floor effect concerning effects achieved with strong masks in 
Experiment 3.3B (Figure 3.11). Similar to Experiment 3.1, the results support the assumption that prime visibility modulates priming effects when pattern masks are applied.

\subsection{Analysis across Experiments}

Experiment 3.2 and 3.3 were matched with the intention to conduct a comprehensive analysis examining the impact of prime visibility - varied through masking strength - in interaction with masking method and task level. Therefore, repeated measure ANOVAs were performed on reaction times and arc-sin transformed error rates, combining data of Experiments 3.2 and 3.3. Congruency, prime-mask SOA and masking strength were included as within-subject factors. Masking method and task level were varied between subjects. The aim of applying masks with different strength was the modulation of prime visibility. We therefore reranked the masks with regards to induced objective prime visibility ( $d$ '). Objective prime visibility increased in line with the removal of mask layers in Experiments 3.2A and 3.2B (mean $d^{\prime}$ ' of 2.31, 2.38, 2.89 and 3.04 for each masking strength, respectively). In Experiments 3.3A and 3.3B, masks with the highest density induced better objective prime visibility $(0.95)$ than masks with the second highest density $(0.62)$, followed by masks with the second lowest density (1.29) and masks with the lowest density (3.06).

For reasons of clarity, comprehensibility and conciseness, only interactions with masking method and task level are referred to in the following section. In our attempt to specify the role of prime visibility in perceptual and semantic priming, modulations of priming effects (i.e. interactions with congruency) are of special interest. References to separate analyses of Experiments 3.2 and 3.3 facilitate the insight into the data. Corresponding test statistics are listed in the respective sections in detail and in Appendix II (Table B4.1 - B4.4). 


\subsubsection{Results}

Errors. Repeated measures ANOVA on error rates revealed an interaction of masking method and congruency $\left(F(1,44)=6.72, M S_{e}=0.128, p<.05\right)$, a three-way interaction of masking method, congruency and masking strength $\left(F(3,132)=2.73, M S_{e}=0.021, p<.05\right)$, a three-way interaction of masking method, congruency and prime-mask SOA $\left(F(3,132)=4.76, M S_{e}=0.034, p<.01\right)$ as well as a three-way interaction of masking method, task level and masking strength $\left(F(3,132)=3.16, M S_{e}=0.025, p<.05\right)$. Priming effects on errors were larger with metacontrast masks (Experiment 3.2; $3.5 \%$ ) than priming effects with pattern masks (Experiment 3.3; 1.2\%). Metacontrast masks with 8, 7, 6 and 1 layer induced priming effects of $3.1 \%, 4.0 \%, 3.3 \%$ and $3.4 \%$. These effects did not differ with reference to the separate analysis of Experiment $3.2(p=0.8)$. With pattern masks, priming effects increased with increasing prime visibility as confirmed by the separate analysis of Experiment $3.3(p<.0005)$. Mask densities of $0.30^{\circ}, 0.15^{\circ}, 0.61^{\circ}$ and $1.21^{\circ}$ of visual angle are associated with $d^{\prime}$-values of $0.62,0.95,1.29$ and 3.06 and induced priming effects $0.0 \%$, $1.3 \%, 0.8 \%$ and $2.7 \%$. Priming effects also increased with increasing prime-mask SOA when metacontrast masks were applied $(0.4 \%, 2.0 \%, 4.6 \%$ and $6.8 \%$ for each SOA, respectively) - confirmed by the separate analysis of Experiment $3.2(p<.0001)$. When pattern masks were used, priming effects increased with smaller slope $(0.2 \%, 1.1 \%, 1.5 \%$ and $2.0 \%$ for each SOA, respectively) - confirmed by the separate analysis of Experiment $3.3(p<.05)$.

Reaction Times. Repeated measures ANOVA on RTs yielded an interaction of masking method and congruency $\left(F(1,44)=7.01, M S_{e}=889, p<.0001\right)$, an interaction of masking method and masking strength $\left(F(3,132)=13.86, M S_{e}=149, p<.0001\right)$, an interaction of masking method and prime-mask $\operatorname{SOA}\left(F(3,132)=7.50, M S_{e}=222, p<.0005\right)$, a three-way interaction of masking method, congruency and masking strength 
$\left(F(3,132)=11.35, M S_{e}=160, p<.0001\right)$ and a three-way interaction of masking method, congruency and prime-mask SOA $\left(F(3,132)=19.13, M S_{e}=306, p<.0001\right)$. Furthermore, an interaction of task level and congruency $\left(F(1,44)=6.99, M S_{e}=889, p<.01\right)$, an interaction of task level and prime-mask $\operatorname{SOA}\left(F(3,132)=2.86, M S_{e}=222, p<.05\right)$, a three-way interaction of task level, congruency and prime-mask SOA $\left(F(1,132)=4.23, M S_{e}=306\right.$, $p<.05)$ as well as a three-way interaction of masking method, task level and congruency $\left(F(1,44)=6.56, M S_{e}=889, p<.05\right)$ reached significance. When metacontrast masks were applied, priming effects were larger $(32 \mathrm{~ms})$ and differed broadly between a perceptual task level (40 ms) and a semantic task level $(24 \mathrm{~ms})$ as confirmed in the separate analysis of Experiment $3.2(p<0.05)$. When pattern masks were used, priming effects were smaller (7 ms) and differed only slightly between a perceptual task level $(6.90 \mathrm{~ms})$ and a semantic task level $(6.66 \mathrm{~ms})$. In the separate analysis of Experiment 3, this difference did not reach significance $(p=0.9)$. Priming effects increased with decreasing masking strength, when pattern masks were applied $\left(1 \mathrm{~ms}\right.$ with $0.30^{\circ}, 1 \mathrm{~ms}$ with $0.15^{\circ}, 6 \mathrm{~ms}$ with $0.61^{\circ}$ and $19 \mathrm{~ms}$ with $1.21^{\circ}$ of visual angle) as confirmed by the separate analysis of Experiment 3.3 $(p<.0001)$. Priming effects did not differ between metacontrast masks with different numbers of layers as confirmed by the separate analysis of Experiment $3.2(p>.2)$. Masks with 8, 7, 6 and 1 layer induced mean priming effects of $30 \mathrm{~ms}, 34 \mathrm{~ms}, 33 \mathrm{~ms}$ and $32 \mathrm{~ms}$, respectively. Separate analyses of Experiments 3.2 and 3.3 conveyed that priming effects increased with prime-mask SOA in both experiments $(p s<.005)$. The analysis across experiments revealed that priming effects increased more steeply with metacontrast masks (10 ms, $23 \mathrm{~ms}, 42 \mathrm{~ms}$ and $54 \mathrm{~ms}$ for each SOA, respectively) than with pattern masks (4 ms, $3 \mathrm{~ms}, 7 \mathrm{~ms}$ and $13 \mathrm{~ms}$ for each SOA, respectively). Priming effects also increased more steeply with a perceptual task level $(7 \mathrm{~ms}, 16 \mathrm{~ms}, 29 \mathrm{~ms}$ and $42 \mathrm{~ms}$ for each SOA, respectively) compared to a semantic task level $(7 \mathrm{~ms}, 10 \mathrm{~ms}, 19 \mathrm{~ms}$ and $25 \mathrm{~ms}$ for each 
SOA, respectively). Separate analyses revealed that priming effects increased with primemask SOA in Experiments 3.2A, 3.2B and 3.3A $(p s<.01)$. Prime-mask SOA did not interact significantly with congruency in Experiment 3.3B $(p>.2)$.

Prime visibility. Repeated measures ANOVA on objective prime visibility (d') only revealed significant interactions with masking method $(p s<.0001)$ and none with task level (ps > .2). A main effect of masking method $\left(F(1,44)=34.87, M S_{e}=7.584, p<.0001\right)$, an interaction of masking method and masking strength $\left(F(3,132)=65.16, M S_{e}=0.480\right.$, $p<.0001)$, an interaction of masking method and prime-mask $\operatorname{SOA}(F(3,132)=12.63$, $\left.M S_{e}=0.495, p<.0005\right)$ as well as the three-way interaction of masking method, masking strength and prime-mask SOA $\left(F(9,396)=7.18, \quad M S_{e}=0.278, \quad p<.0001\right)$ reached significance. Objective prime visibility was on average higher with metacontrast masks (2.66) than with pattern masks (1.48). In all experiments, prime visibility was modulated through masking strength as confirmed by analyses of Experiments 3.2 and 3.3 (ps<.0005). The analysis across experiments revealed a different course of $d$ ' with a flatter increase for the manipulation with metacontrast masks $(2.31,2.38,2.89$ and 3.04 with $8,7,6$ and 1 layer, respectively) compared to pattern masks $\left(0.62,0.95,1.29\right.$ and 3.07 with $0.30^{\circ}, 0.15^{\circ}, 0.61^{\circ}$ and $1.21^{\circ}$ of visual angle, respectively). In separate analyses of Experiments 3.2 and 3.3, an increase of $d^{\prime}$ with prime-mask SOA was found in each experiment $(p<.0001)$. Analysis across experiments confirmed a different course of $d$ ' with metacontrast masks $(1.59,2.58$, 3.18 and 3.28 for each SOA, respectively) compared to pattern masks $(0.93,1.31,1.66$ and 2.04 for each SOA, respectively). A comparable pattern became apparent with subjective prime visibility. Repeated measures ANOVA on PAS-ratings revealed a main effect of masking method $\left(F(1,44)=21.66, M S_{e}=4.291, p<.0001\right)$, an interaction of masking method and masking strength $\left(F(3,132)=38.08, M S_{e}=0.272, p<.0001\right)$, an interaction of masking method and prime-mask SOA $\left(F(3,132)=5.28, M S_{e}=0.151, p<.05\right)$ as well as the three- 
way interaction of masking method, masking strength and prime-mask SOA $\left(F(9,396)=5.72, M S_{e}=0.045, p<.005\right)$. Subjective prime visibility was on average higher with metacontrast masks (3.34) than with pattern masks (2.64). In all experiments, prime visibility was modulated through masking strength as confirmed by analyses of Experiments 3.2 and $3.3(p s<.005)$. The analysis across experiments revealed a different course of PASrating with a flatter increase for the manipulation with metacontrast masks $(3.20,3.20,3.40$ and 3.54 with $8,7,6$ and 1 layer, respectively) compared to pattern masks $(2.23,2.20,2.60$ and 3.53 with $0.30^{\circ}, 0.15^{\circ}, 0.61^{\circ}$ and $1.21^{\circ}$ of visual angle, respectively). In separate analyses of Experiments 3.2 and 3.3, an increase of PAS-rating with prime-mask SOA was found in each experiment $(p<.0005)$. Analysis across experiments confirmed a different course of PAS-ratings with metacontrast masks $(2.83,3.28,3.58$ and 3.66 for each SOA, respectively) compared to pattern masks $(2.32,2.56,2.77$ and 2.92 for each SOA, respectively).

\subsubsection{Discussion}

The analyses of error rates as well as reaction times revealed larger priming effects with metacontrast masks than with pattern masks. A three-way interaction between masking method, congruency and masking strength was also supported by both accuracy data and reaction times. With both measures, priming effects increased with masking strength when pattern masks were applied. No modulation of priming effects through masking strength was found with metacontrast masks. An interaction between between masking method, congruency and prime-mask SOA was also apparent with both accuracy data and reaction times. Priming effects on errors and RTs increased with a smaller slope when pattern masks were used. Furthermore, the analysis of reaction times revealed smaller priming effects with a semantic task level compared to perceptual priming effects. With increasing prime-mask SOA, semantic priming effect also increased with a smaller slope than perceptual priming effects. 
As intended, prime visibility was modulated through masking strength in all experiments. Task level had no impact on prime visibility. Therefore, comparisons of semantic and perceptual priming effect can be interpreted without restrictions. Unfortunately, prime visibility was affected by masking method. Prime visibility was higher with metacontrast masks and varied with masking strength in a smaller range. Prime visibility seems to increase in different manners with prime-mask SOA and decreasing masking strength with metacontrast masks compared to pattern masks (see Figure 3.8 and 3.11). This seems reasonable when thinking of different mechanisms that cause a reduction of prime visibility with different masking methods. Nevertheless, on basis of Experiment 3.1 and previous tests of different metacontrast masks, more similar courses of prime visibility were anticipated.

\subsection{Analysis of Visible Priming}

Prime visibility was modulated by different masking strengths in Experiments 3.2 and 3.3. Unfortunately, this modulation differed between masking methods. Nevertheless, data strongly suggest a difference between metacontrast masks and pattern masks in modulating priming effects through masking strength and prime visibility. Besides the different impact of masking strength on priming effects, priming effects generally differed between masking methods. Visual inspection of Figure 3.8 and 3.11 suggest similar and very high objective prime visibility with both metacontrast masks and pattern masks when the weakest masks were applied $-93 \%$ of primes were correctly identified with metacontrast masks as well as pattern masks. Analysis of only this condition might provide stronger evidence for a general modulation of priming effects through masking method - irrespectively of prime visibility. 


\subsubsection{Results}

Errors. A repeated measures ANOVA on arc-sin transformed error rates was conducted with congruency and prime-mask SOA as within-subjects factors and masking method and task level as between-subjects factors. A main effect of congruency $\left(F(1,44)=38.44, \quad M S_{e}=0.054, \quad p<.0001\right), \quad$ a main effect of prime-mask SOA $\left(F(3,132)=3.99, M S_{e}=0.028, p<.05\right)$ and an interaction of congruency and prime-mask SOA $\left(F(3,132)=10.83, M S_{e}=0.023, p<.0001\right)$ reached significance. Masking method and task level did not modulate error rates significantly $(p s>.2)$.

Reaction Times. An equivalent repeated measures ANOVA on RTs revealed a main effect of congruency $\left(F(1,44)=151.65, M S_{e}=403, p<.0001\right)$, a main effect of prime-mask $\operatorname{SOA}\left(F(3,132)=6.43, M S_{e}=147, p<.0005\right)$, an interaction of congruency and prime-mask SOA $\left.F(3,132)=23.74, M S_{e}=180, p<.0001\right)$ as well as an interaction of congruency and masking method $\left.F(1,44)=6.61, M S_{e}=403, p<.005\right)$ and an interaction of congruency and task level $\left.F(1,44)=10.02, M S_{e}=403, p<.05\right)$. Priming effects were larger with metacontrast masks $(32 \mathrm{~ms})$ than with pattern masks $(19 \mathrm{~ms})$. Furthermore, perceptual priming effects (31 ms) were larger than semantic priming effects $(20 \mathrm{~ms})$.

Prime visibility. Repeated measures ANOVA on objective prime visibility (d') yielded a main effect of prime-mask $\operatorname{SOA}\left(F(3,132)=11.09, M S_{e}=0.230, p<.0001\right)$. Masking method and task level did not significantly modulate objective prime visibility $(p>.3)$. A similar pattern was found with subjective prime visibility. A main effect of primemask SOA reached significance $\left(F(3,132)=19.68, M S_{e}=0.030, p<.0001\right)$ while no impact of masking method or task level could be confirmed $(p>.2)$. 


\subsubsection{Discussion}

The analyses of error rates did not reveal an impact of masking method or task level on priming effects. However, priming effects on reactions times were smaller with pattern masks than with metacontrast masks. Perceptual priming effects were also larger than semantic priming effects, when estimated with reaction times. As expected, prime visibility did not differ between task level and masking method, when only the weakest masks were taken into account. The estimated differences, irrespective of prime visibility, are therefore on a solid ground.

\subsection{General Discussion}

The current study was conducted to resolve a confounding between task level and masking method. Previous research indicated that perceptual priming is independent of prime visibility whereas semantic priming increases with increasing prime visibility (Chapter 2). However, different masking methods were systematically applied in perceptual and semantic priming studies. Experiment 3.1 was conducted to examine perceptual (3.1A) and semantic priming (3.1B) with pattern masks. Identical stimuli were presented in Experiments 3.1A and 3.1B - merely the task and the relevant congruency differed. Subsequently, Experiment 3.2 was conducted to examine perceptual (3.2A) and semantic priming (3.2B) with metacontrast masks. Using metacontrast masks required a change of primes and target in size and shape. Our meta-analysis (Chapter 2) indicated that even small changes might have an impact on priming effects. To exclude the possibility of further confoundings Experiment 3.3 was conducted. Again, perceptual and semantic priming with pattern masks was examined, but primes, targets and masks were adapted to those in Experiment 3.2. 
In all three experiments, objective and subjective prime visibility was modulated through masking strength. All masks comprised elements or aspects of primes and targets. Masks of different strength differed with respect to their bottom-up energy. Objective prime visibility was operationalized through a two-alternative forced choice task of prime recognition ( $d$ '-values, Macmillan \& Creelman, 1991). Subjective prime visibility was assessed with the perceptual awareness scale (PAS) developed by Ramsøy and Overgaard (2004). Subjective judgments reflected objective prime visibility in all three experiments. Prime visibility was successfully modulated in all experiments. In Experiments 3.1 and 3.2, prime visibility increased with decreasing masking strength. In Experiment 3, masks with a density of $0.30^{\circ}$ reduced prime visibility more efficiently than masks with a density of $0.15^{\circ}$. This was considered in the conjoint analysis of Experiments 3.2 and 3.3. Prime visibility also increased with increasing prime-mask SOA in each experiment. Prime visibility and its modulation through masking strength and prime-mask SOA did not differ between semantic and perceptual priming experiments. Note that subjects had to focus on the color of primes in perceptual experiments and on the identity of primes in semantic experiments. Unfortunately, prime visibility was different with metacontrast masks (Experiment 3.2) than with pattern masks (Experiment 3). Prime visibility was generally higher with metacontrast masks and varied with masking strength in a smaller range, apparently due to a ceiling effect.

Priming effects on reaction times and errors were analyzed to draw inferences about the processing of primes. Congruency effects were found in each experiment. Generally, priming effects seem to increase with increasing prime-mask SOA. Only in Experiment 3.3A, statistical analysis did not support this modulation of priming effects. However, a floor effect might have caused the absence of a significant interaction between congruency and primemask SOA. Experiments 3.1B, 3.2A and 3.3B can be seen as control experiments. In previous studies, perceptual priming was mostly combined with metacontrast masking (3.2A) and 
semantic priming with pattern masking (3.1B \& 3.3B; Chapter 2). One of our expectations was that perceptual priming with metacontrast masks exceeds semantic priming with pattern masks. This pattern was confirmed by the data. Across experiments, task level and masking method were analyzed to investigate the origin of this effect. The conjoint analysis of Experiments 3.2 and 3.3 revealed that perceptual priming effects were larger than semantic priming effects. Additionally, priming effects were larger with metacontrast masks than with pattern masks. However, prime visibility was higher with metacontrast masks and therefore only limited conclusions can be drawn at this point. Fortunately, prime visibility with very weak masks was not different in experiments with metacontrast masks and experiments with pattern masks. The analysis of visible primes (weak masks) confirmed priming effects being smaller with pattern masks than with metacontrast masks. Another expectation was that perceptual priming effects with metacontrast masks would be independent of prime visibility where semantic priming effects with pattern masks would increase with increasing prime visibility. The data confirmed this pattern as well. The conjoint analysis of Experiments 3.2 and 3.3 revealed an interaction of masking method, congruency and masking strength with reaction times and errors, but no interaction of task level, congruency and masking strength. Priming effects increased clearly with prime visibility, when pattern masks were applied. With metacontrast masks, no modulation of priming effects through prime visibility occurred. Task level had no influence on this modulation.

\subsubsection{Impact of Masking Method}

Masking method seems to be crucial for the modulation of priming effects through prime visibility. Possible conclusions seem to be limited by a lack of variation of prime visibility in experiments with metacontrast masks (3.2A \& 3.2B) compared to experiments with pattern masks (3.3A \& 3.3B). However, priming effects also clearly increased with prime visibility in Experiment 3.1, although modulations of prime visibility were more 
similar to Experiment 3.2 than 3.3. Especially difficult to achieve was variation of prime visibility with metacontrast masks at long prime-mask SOA. However, this seems to be a result of a ceiling effect. With this in mind, a possible overestimation of prime visibility becomes important. Evidence for an overestimation of prime visibility is present. First, prime visibility was always measured subsequently to the priming sessions. Tasks were given in this order to prevent an influence of the prime visibility task on priming effects. However, subjects become probably more familiar with the stimuli during the course of the experiment. Second, objective prime visibility was measured with a delay after stimulus presentation. A subjective rating of prime visibility was interposed to prevent an influence of residual motor activation. However, $d$ '-values are higher when a delay between stimulus presentation and retrieval is applied (Vermeiren \& Cleeremans, 2012). A third line of evidence is provided through report of subjects. In each experiment, only approximately half of subjects noticed primes. Around four fifths of subjects recognized an improvement of prime visibility in the prime identification session. Modulation of prime visibility through masking strength is therefore likely even with metacontrast masks at long prime-mask SOA. On this basis, differences between pattern masking and metacontrast masking are substantial.

In contrast to priming effects with metacontrast masks, priming effects with pattern masks are smaller and increase with prime visibility. In line with previous research, this pattern suggests that pattern masks indeed disturb prime processing on an early level (e.g. Enns \& Di Lollo; 2000, Kahneman, 1968; Macknik \& Livingstone, 1998; Rieger, Braun, Bülthoff \& Gegenfurtner, 2005). In contrast, metacontrast masking seems to reduce prime visibility on a higher level of prime processing (e.g., Enns \& Di Lollo, 2000; Van AalderenSmeets, Oostenveld \& Schwarzbach 2006). Bruno Breitmeyer (2014) distinguished recently several masking techniques and compiled a hierarchy. Backward pattern masking and metacontrast masking were proposed to act on a similar functional level of unconscious 
processing. However, different relations of prime visibility and priming effects with pattern masking and metacontrast masking provide evidence that pattern masking acts on a lower level of processing. The role of the forward pattern mask might be crucial. Prime processing that causes priming effects seems to occur earlier than processing that results in prime visibility. This is line with the model of recurrent processing by Lamme and Roelfsema (2000). Pattern masks seem to disturb prime processing during the feedforward sweep (Figure 3.14). As a result, priming effects and prime visibility are affected. Metacontrast masks seem to impair recurrent processing (Figure 3.14). Response activation is unaffected, whereas prime visibility is reduced.

Collected data also provide evidence concerning another aspect that is relevant for theories of consciousness. Intermediate judgments of prime visibility with the perceptual awareness scale (Ramsøy and Overgaard, 2004) were extensively chosen by subjects. Although this is not unusually in studies that apply the PAS, it is another indication that consciousness is gradual rather than dichotomous (Sandberg, Timmermans, Overgaard \& Cleeremans, 2010).
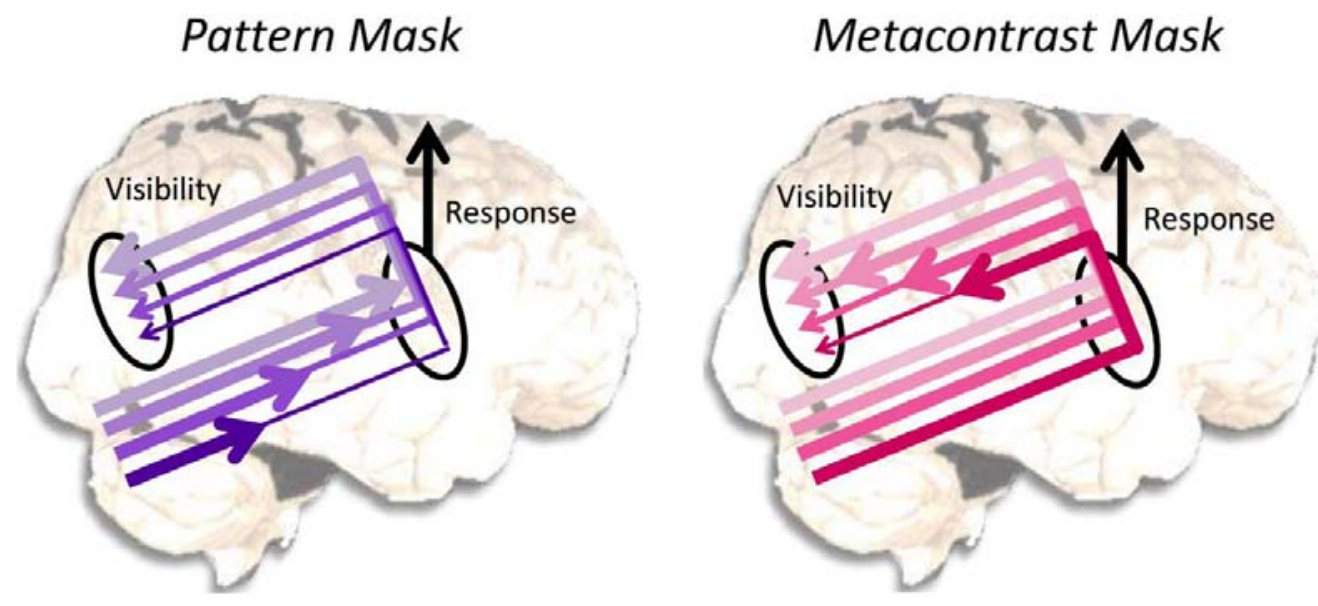

Figure 3.14. Reduction of prime visibility with pattern masks and metacontrast masks. Pattern masks are thought to reduce prime visibility on an early level of stimulus processing (feedforward sweep), whereas metacontrast masks disturb recurrent processing (Lamme \& Roelfsema, 2000). 
In contrast to metacontrast masks which were only presented after the prime, pattern masks were applied as forward and backward masks in our design. This decision was based on two reasons. First, effective masking of colored and complex stimuli is difficult to achieve with only backward pattern masks (see Experiment 4.3 of Chapter 4). Second, forward masks are typically used when pattern masks are applied. Our meta-analysis included 439 cases with pattern masks and only 84 of these cases did not present a forward mask (Chapter 2). Interestingly, the forward mask might be essential to the differences which were found between experiments with pattern masks (1 and 3) and experiments with metacontrast masks (2). According to Turvey (1973), forward masking that concerns the peripheral encoding mechanism is more pronounced than backward masking and depends on the intensity of the mask. Further research might address priming with only backward pattern masks. It is questionable whether courses of priming effect with backward pattern masks are similar to courses of priming effect with forward and backward pattern masks. However, a variation of prime visibility might be difficult with colored letters and numbers as primes. Alternatively, the perceptual task could concern a different perceptual feature. For instance, shape or orientation might be suitable.

Prime visibility was manipulated through mask density when pattern masks were applied (cf. Eimer \& Schlaghecken, 2002). In experiments with metacontrast masks, prime visibility was varied through different intercontour distances (cf. Lefton, 1973; Mattler \& Palmer, 2012). However, it is questionable whether masks with different strength induce additional effects. Many previous attempts to manipulate prime visibility included confounding factors. For instance, prime duration, prime-mask SOA and prime intensity were varied (e.g., Cheesman \& Merikle, 1984; 1986; De Gardelle \& Kouider, 2009; Eckstein, Kubat \& Perrig, 2011; Fisk \& Haase, 2011; Kouider \& Dehaene, 2009; Kunde, 2003; Van Gaal, Ridderinkhof, Scholte \& Lamme 2010; Van Gaal, Scholte, Lamme, Fahrenfort \& 
Ridderinkhof, 2010). However, the duration of primes effectivity increases priming effects as well (e.g. Mattler, 2003; Vorberg et al., 2003). With varying masking strength, we also changed the stimulation. Some evidence indicates that other manipulation occurred besides the manipulation of prime visibility. First, debriefing indicates that distracting effects were different between masks of different strength. Second, the kind of mask seems to have an impact in the field of inverse priming (e.g. Eimer \& Schlaghecken, 2002). Finally and already mentioned, the use of both forward and backward pattern masks reduces priming effects. However, we were interested in solving the confounding between task level and masking method that was revealed in our meta-analysis (Chapter 2). This intent seems to be unaffected by the raised objections: First, masks with different strength were also applied across studies included in the meta-analysis. This factor was not included in the meta-regression because stimuli varied extensively across studies. The modulation of prime visibility through masking strength seems therefore rather suitable. Second, perceptual and semantic priming were measured with identical stimulation. A comparison of perceptual and semantic priming is therefore possible without any limitations. Furthermore, masking strength was varied very similarly with metacontrast masks and pattern masks. Comparisons of both masking methods are therefore also legitimate. Finally, primes are more visibility with a mask density of $0.15^{\circ}$ than with a density of $0.30^{\circ}$ of visual angle in Experiment 3.3. However, priming effects increase with increasing prime visibility rather than with the mask energy. Prime visibility seems to be the crucial factor.

Objective prime visibility was measured differently in perceptual and semantic priming experiments. Subjects had to choose between two alternatives in both types of experiments. However, alternatives differed in color in perceptual priming experiments, whereas they differed in identity in semantic priming experiments. Prime visibility is therefore comparable with regards to the relevant dimension. Differences between visibility 
of perceptual attributes and recognition of primes' identity were expected. Windey, Gevers and Cleeremans (2013) recently compared masked color discrimination with masked number discrimination. A graded relationship between stimulus duration and prime visibility was found with the low-level task (color discrimination). A non-linear relation was found with the high-level task (number discrimination). Results were interpreted as evidence for a gradual awareness of basic stimulus features and a dichotomous recognition of higher-order classifications. In our experiments, visibility of perceptual features did not differ from visibility of stimulus identity. These findings might challenge the theory of Windey, Gevers and Cleeremans. However, there are two possibly relevant differences between their experiment and the current study. First, Windey and colleagues applied the objective visibility task in advance to the subjective judgment. We chose a reversed order to prevent that residual motor activition triggers an answer. The assignment of keys also differed between trials. Furthermore, subjects were instructed to assess global visibility with the PAS. Second, Windey and colleagues varied the task within subjects. In the current study, tasks were varied between subjects. Further research is required to clarify possible differences between visibilities of lower- and higher-order stimulus attributes. However, similar visibilities of color and identity are beneficial to compare perceptual and semantic priming in our study. Differences between perceptual and semantic priming effects are therefore not simply based on different visibilities of the relevant feature.

\subsubsection{Impact of Task Level}

Perceptual and semantic priming effects were equally independent of prime visibility with metacontrast masks and equally modulated through prime visibility with pattern masks. Dehaene and colleagues (1998) showed that priming effects emerge on the motor level in a semantic priming experiment similar to our semantic priming experiments. Perceptual response priming effects should also arise on a motor level as shown by Leuthold and Kopp 
(1998). Similar modulations of these effects seem therefore reasonable as similar mechanisms are involved. However, perceptual priming effects were larger than semantic priming effects in our study.

Several explanations can account for this pattern. First, semantic priming effects might be based on actual semantic processing in advance to motor activations. In contrast to perceptual processing, semantic processing is thought to occur on a higher level. Therefore, activations might be more extended, less intensive and more variable. Second, although equally detectable, perceptual features might be more salient than semantic attributes. Larger priming effects on a perceptual dimension might be based on a difference in saliency. A third explanation is based the action trigger account by Kunde, Kiesel and Hoffmann (2003; Kiesel, Kunde \& Hoffmann, 2007b). The authors argue that activation of pre-specified cognitive action trigger generates semantic response priming effects instead of automatic semantic categorization. Within this framework, priming effects also depend on target set size (Kiesel, Kunde, Pohl \& Hoffmann, 2006). Another aspect might also account for the difference between perceptual and semantic priming effects. Meta-regression estimations revealed a negative impact of a systematic difference between primes and targets in color (Chapter 2). In contrast to perceptual priming effects, primes and targets differed partly in color in semantically congruent as well as semantically incongruent trials.

The current study revealed differences between perceptual and semantic priming effects, but multiple explanations are reasonable. Some of these explanations could be ruled out by the comparison of perceptual and semantic priming without response association. Priming effects without response association can be examined with a lexical decision task (e.g. Meyer \& Schvaneveldt, 1971). Typically, semantic processing is activated by a word versus non-word categorization. Congruency effects of related and unrelated word-word pairs are examined. A similar principle can be used with perceptual features. For Instance, 
Martens, Ansorge and Kiefer (2011) instructed subjects to respond to certain symbols with one hand and to other symbols with the other hand. However, some symbols were more similar in shape with symbols of the other response category. Shape congruency effects were therefore examined independently of response associations. In Chapter 5, perceptual and semantic priming effects are compared with an adaption of this principle. Future research might address whether semantic priming effects without response association vary with prime visibility. Nonetheless, the confounding between task level and masking method is resolved: Masking method seems to be the effective factor in modulating the impact of prime visibility on priming effects.

\subsubsection{Impact of Prime-Mask SOA}

Another confounding was revealed in our meta-analysis (Chapter 2): Priming effects seem to be differently modulated through prime-target SOA (cf. Van den Bussche et al., 2009). However, perceptual priming studies vary mostly prime-mask SOA. In studies with semantic priming, mask-target SOA is varied predominantly. The current study provides first evidence that differences in modulation through prime-target SOA between perceptual and semantic priming are actually based on this confounding. Both perceptual and semantic priming effects increase with increasing prime-mask SOA. This is also in line with the results of the meta-regression. Effects of mask-target SOA variation in perceptual and semantic priming are addressed in the following chapter.

\subsubsection{Conclusion}

The current study provides evidence for an impact of task level and masking method on priming effects. Priming effects are smaller and vary with prime visibility when pattern masks are applied. Priming effects are larger and independent of prime visibility when metacontrast mask are applied. These findings support the assumption that pattern masks 
3 | Approaching Consciousness with Masked Priming

operate on an early level of stimulus processing and disturb the feedforward sweep. Metacontrast masks seem to reduce prime visibility later by disturbing recurrent processing. Furthermore, the data suggest that task level affects the extent of priming effects, but not their modulation trough prime visibility. Perceptual priming effects are larger than semantic priming effects. 


\section{Temporal Parameters in Perceptual and Semantic Priming}

\subsection{Abstract}

Van den Bussche and colleagues (2009) noted in their meta-analysis that response priming increases with increasing stimulus onset asynchrony (SOA) between prime and target and semantic priming decreases at long SOAs. However, our meta-analysis (Chapter 1) confirmed the importance of considering the role of the masks and analyzing the modulations through prime-mask SOA and mask-target SOA separately. Previous experiments already attested that perceptual as well as semantic priming effects increase with increasing primemask SOA (chaper 2). The current study was conducted to examine modulations of perceptual and semantic priming effects through mask-target SOA. The results suggest that simple decay and self-inhibition (Klapp, 2005) affect perceptual as well as semantic priming effects. Additionally, perceptual interactions between prime and mask (e.g. object updating; Lleras \& Enns, 2004) cause an inversion of perceptual priming effects at 100-150 ms masktarget SOA. Overall, both perceptual and semantic priming effects decline with increasing mask-target SOA. 


\subsection{Introduction}

Masked priming is a frequently applied method to address several research questions. For instance, limits of unconscious processing are examined with this paradigm (Dehaene, Changeux, Naccache, Sackur \& Sergent, 2006). Many studies with masked priming also address the functional principles and structures of certain information-processing systems. Some studies examine the language processing system with semantic priming (e.g., Dell'Acqua \& Grainger, 1999), phonological priming (e.g., Rastle \& Brysbaert, 2006) and orthographical priming (e.g., Bonin, Fayol \& Peereman, 1998). Other studies focus on perceptual processing or the motor system (e.g., Vorberg, Mattler, Heinecke, Schmidt \& Schwarzbach, 2003). Comparisons between different priming paradigms are used to identify specific features of certain information-processing mechanisms (e.g. Carr \& Dagenbach, 1990; Martens, Ansorge \& Kiefer, 2011). To draw legitimate conclusions it is crucial that discovered differences cannot be attributed to simple differences in experimental designs.

We conducted a meta-analysis with the intention to pinpoint one specific difference between perceptual and semantic priming (Chapter 2). This difference concerned the modulability of priming effects through prime visibility. Another meta-analysis with a focus on semantic priming reported an association between semantic priming effects and prime visibility (Van den Bussche, Van den Noortgate \& Reynvoet, 2009). In contrast, studies report that perceptual priming and motor priming are independent of prime visibility (e.g. Vorberg et al., 2003). Our meta-analysis revealed a confounding between task level (e.g. perceptual and semantic) and masking method (e.g. metacontrast and pattern masking) in experimental designs. Subsequent experiments attributed the modulability of priming effects through prime visibility to the applied masking method (Chapter 3). 
Several moderators besides task level and masking method were gathered and included in our meta-analytic model (Chapter 2). Following the intention to compile comprehensive model, many moderators were gathered and another confounding was revealed. Van den Bussche and colleagues (2009) noted in their meta-analysis that response priming increases with increasing stimulus onset asynchrony (SOA) between prime and target and semantic priming decreases at long SOAs. Although this hypothesis was not confirmed in their meta-analysis, several studies are in line with this dissociation at first glance (e.g. Mattler, 2003; Vorberg et al., 2003; Greenwald, Draine \& Abrams, 1996; Kiefer \& Spitzer, 2000). However, our meta-analysis confirmed the importance of considering the role of the masks and analyzing the modulations through prime-mask SOA and mask-target SOA separately. It was revealed that the increasing course of perceptual priming effects relies only on modulation by prime-mask SOA whereas the decreasing course of semantic priming effects relies on modulation by mask-target SOA (Table 4.1). The prime-mask SOA and the mask-target SOA had no significant impact on semantic priming effects and perceptual priming effects, respectively. However, prime-mask SOA and mask-target SOA seem to be unequally varied in studies examining perceptual and semantic priming (Figure 4.1). Primemask SOA is frequently varied in single studies that address perceptual and motor priming (e.g. Mattler, 2003; Vorberg et al., 2003). A variation of mask-target SOA is mainly applied in simple priming studies when the specific phenomenon of inverse priming is of interest (Eimer \& Schlaghecken, 1998). Many studies aim to provide evidence for effective prime processing when primes are invisible (e.g. Dehaene et al., 1998; Vorberg et al., 2003). With this intention, a variation of prime-mask SOA seems problematic in semantic priming studies as stimuli are complex and therefore difficult to mask. Therefore, pattern masks are usually applied (Chapter 2) and prime visibility increases notably with increasing prime-mask SOA (Chapter 3). Presumably, prime visibility does not vary with mask-target SOA to the same 
4 | Approaching Consciousness with Masked Priming

Table 4.1

Meta-analytic model including effect type, prime-mask SOA and mask-target SOA as moderators of priming effects

\begin{tabular}{|c|c|c|c|c|c|c|}
\hline Moderators & $\beta$ & $S E$ & $95 \% \mathrm{CI}$ & $z$ & $p$ & \\
\hline Intercept & 0.5308 & 0.0275 & {$[0.4769 ; 0.5847]$} & 19.29 & $<.0001$ & $* * *$ \\
\hline $\begin{array}{l}\text { Perceptual priming } \\
\quad \times \text { prime-mask SOA }\end{array}$ & 0.0038 & 0.0006 & {$[0.0025 ; 0.0050]$} & 5.80 & $<.0001$ & $* * *$ \\
\hline $\begin{array}{l}\text { Semantic priming } \\
\quad \times \text { prime-mask SOA }\end{array}$ & 0.0002 & 0.0002 & {$[-0.0002 ; 0.0006]$} & 0.91 & 0.36 & \\
\hline $\begin{array}{l}\text { Perceptual priming } \\
\quad \times \text { mask-target SOA }\end{array}$ & 0.0001 & 0.0002 & {$[-0.0003 ; 0.0005]$} & 0.45 & 0.65 & \\
\hline $\begin{array}{l}\text { Semantic priming } \\
\quad \times \text { mask-target SOA }\end{array}$ & -0.0002 & 0.0001 & {$[-0.0004 ;-0.0000]$} & -1.99 & $<.05$ & $*$ \\
\hline
\end{tabular}

Note. A mixed-effects model was estimated with restricted maximum likelihood (REML) approach. $\square \square^{2}$ (residual heterogeneity) $=0.0800$; Test for Residual Heterogeneity: $Q E(533)=1177.13, p<.0001$; Test of Moderators: $Q M(4)=$ $49.94, p<.0001$
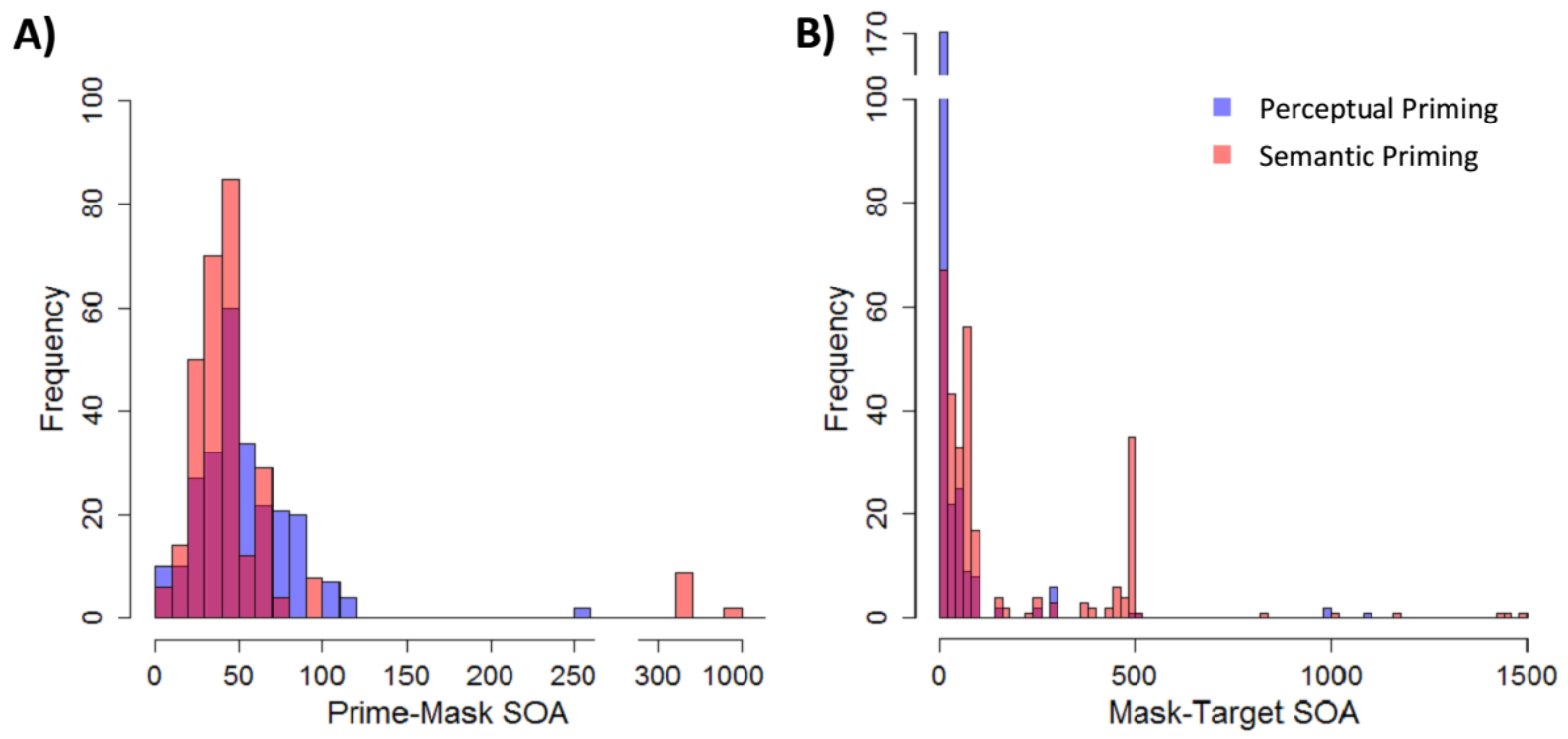

Figure 4.1. Distribution of applied prime-mask SOA (A) and mask-target SOA (B) in perceptual and semantic priming studies (reviewed in chapter 2). 
degree. Perceptual priming studies frequently use metacontrast masks (Chapter 2). With metacontrast masks, prime visibility can be dissociated from prime-mask SOA with certain experimental parameters (Breitmeyer \& Öğmen, 2006) and on the basis of interindividual differences (Albrecht, Klapötke \& Matter, 2010; Albrecht \& Mattler 2012b). As yet there is no systematic variation of prime-mask SOA and mask-target SOA in perceptual and semantic priming with otherwise identical designs (including all stimulus features). We intended to fill this gap with the current study.

Previous experiments already attested that perceptual as well as semantic priming effects increase with increasing prime-mask SOA (Chapter 3). In six experiments with different samples, this increase was found irrespectively of the masking method. These results are in line with the conception that the duration of prime effectiveness is prolonged until the presentation of the mask (Mattler, 2003). In the current study, prime-mask SOA was varied again to replicate the findings and consider possible interactions between prime-mask and mask-target SOA. However, the main focus was laid on a possible modulation of perceptual and semantic priming effects through mask-target SOA. Studies suggest that semantic priming effects decrease with increasing mask-target SOA (Van den Bussche et al. 2009; Chapter 2 and Table 4.1). A slightly positive impact of mask-target SOA on perceptual priming effects was calculated with the data of our meta-analysis (Table 4.1). However this modulation was insignificant $(p=0.65)$. Other studies suggest a decrease of perceptual priming effects with increasing mask-target SOA up to an inversion (e.g. Schlaghecken \& Eimer, 2000). Some mechanisms that supposedly support this inversion are thought to depend on low prime visibility (e.g. mechanisms of inhibition, Klapp, 2005). Therefore, masks with two different masking strengths were included in our design. We expected to find selfinhibition only in conditions with low prime visibility. Four different mask-target SOA were applied. A mask-target SOA of $0 \mathrm{~ms}$ was chosen to allow comparisons with previous 
experiments (Chapter 3). Mask-target SOAs of $100 \mathrm{~ms}$ and $150 \mathrm{~ms}$ were applied as literature concerning inverse priming effects suggests an inversion of effects around this time window (Eimer \& Schlaghecker, 2003; Schlaghecker \& Eimer, 2000; Sumner, 2007). Another long mask-target SOA (250 ms) was included because long mask-target SOAs are typically used semantic priming studies (Figure 4.1). Thereby, we wanted to capture a decline of semantic priming effects, even if this decline might be delayed or weaker. On the basis of the described state of research, we expected an overall decline of perceptual and semantic priming effects with increasing mask-target SOA. Furthermore, an inversion of perceptual priming effects around 100 ms prime-mask SOA was expected. A main focus was set on possible similarities and differences between perceptual and semantic priming to gather insights into stimulus processing on different cognitive levels.

\subsection{Experiment 4.1: SOA-Variation with Metacontrast Masking}

As previous research underlined the impact of the masking procedure on the manifestation of priming effects (Chapter 3), we chose to pursue further comparative research of perceptual and semantic priming effects, applying both metacontrast and pattern masks. In the first experiment, metacontrast masks, typically used in studies examining perceptual priming, were applied. In two groups of subjects, perceptual priming (4.1A) and semantic priming (4.1B) were examined under the same terms. The main focus of the experiment was on the courses of perceptual and semantic priming effects modulated by SOA variation. Therefore, the prime-mask SOA was varied in two steps (60 ms and $90 \mathrm{~ms})$ and the masktarget SOA was varied in four steps $(0 \mathrm{~ms}, 100 \mathrm{~ms}, 150 \mathrm{~ms}$ and $250 \mathrm{~ms})$. Our previous research suggests an increase of perceptual and semantic priming effects with increasing prime-mask SOA (Chapter 3), which we hoped to replicate. The meta-analysis we conducted previously indicated an impact of an increasing mask-target SOA on the decrease of semantic 
priming effects with increasing prime-target SOA (Chapter 2). Literature on the negative compatibility effect (or inverse priming) suggests a decrease of perceptual priming effects with increasing mask-target SOA as well (e.g. Schlaghecker \& Eimer, 2000). Therefore, a decrease of the priming effect with increasing mask-target SOA was expected in the condition with perceptual priming and in the subexperiment with semantic priming. The occurrence of an inverse priming effect is suspected after $100 \mathrm{~ms}$ in Experiment 4.1A (cf. Schlaghecker \& Eimer, 2000).

\subsubsection{Method}

Participants. For each experiment, twelve students from the University of Göttingen were invited to participate. The age of the subjects was between 21 and $32(M=24.33)$ in Experiment 4.1A ( 7 female, 5 male) and between 20 and $33(M=25)$ in Experiment 4.1B ( 7 female, 5 male). The participants attended four experimental sessions of one hour and received 28 Euro or course credits for participation. Ishihara color plates were used to verify a normal color perception. Twelve subjects were with normal vision (4.1A: 6; 4.1B: 6). The remaining twelve subjects had corrected-to-normal vision.

Stimuli. Stimuli, tasks and procedure were substantially derived from the approach by Wernicke and Mattler (Chapter 3, Experiment 3.2). A CRT-monitor with a refreshing rate of $100 \mathrm{~Hz}$ was used for stimulus presentation on a white background. A fixation point $\left(0.03^{\circ}\right.$ width $\times 0.03^{\circ}$ height of visual angle) in black was presented at all times and a fixation cross $\left(0.15^{\circ} \times 0.15^{\circ}\right)$ indicated the beginning of a trial. The letters $A, G, H$ and $\mathrm{U}$ and the numbers 2, 6, 8 and 9 appeared as primes. The letters $\mathrm{C}, \mathrm{E}, \mathrm{F}$ and $\mathrm{P}$ and the numbers 3, 4, 5 and 7 served as targets. Primes and targets $\left(0.76^{\circ} \times 0.83^{\circ}\right)$ were presented with constant luminance $\left(24.6 \mathrm{~cd} / \mathrm{m}^{2}\right)$ in a seven-segment display format (Figure 4.2). Primes were displayed in the middle of the screen. Targets appeared $2.27^{\circ}$ of visual angle below and above center. 


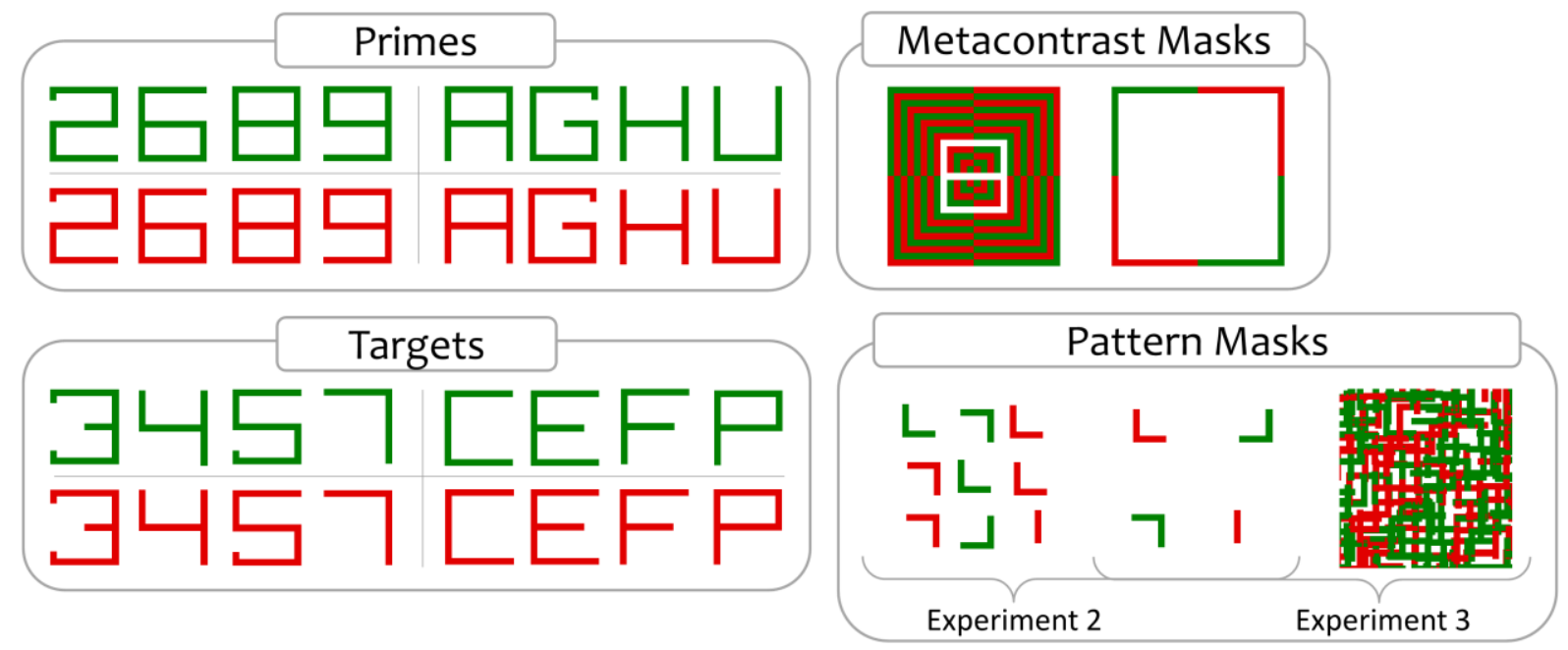

Figure 4.2. Stimuli used in Experiments 4.1 - 4.3. Primes and targets were based on a seven-segment display to ensure similar masking of all primes by metacontrast masks. One strong and weak metacontrast mask were applied in Experiment 4.1. Pattern masks were applied in Experiment 4.2. Parts of primes were rearranged randomly and two different masking strengths were implemented with different distances between segments $\left(0.61^{\circ} \& 1.21^{\circ}\right.$ of visual angle).

An 8-shaped section (all seven segments) formed a hole within a metacontast mask $\left(1.97^{\circ} \times\right.$ $2.05^{\circ}$ ), which was constructed by placing eight layers in altering color (red and green) around the section and four layers within the holes of the section (Figure 4.2). In two of four quarters of the mask one color surrounded the prime directly. By removing seven layers another mask with less masking strength was created.

Tasks. In the first three sessions a forced-choice reaction time task was given. The subjects had to react to a target stimulus by pressing one of the two Crtl-keys with their left or right index finger. Subjects assigned to Experiment 4.1A responded to green stimuli with one finger and to red stimuli with the other. In Experiment 4.1B, the subjects had to press one button in reaction to a letter and the other button in reaction to a number. The assignments of the key were counterbalanced between subjects. The last session was dedicated to prime identification. Using the perceptual awareness scale (PAS) developed by Ramsøy and Overgaard (2004), the subjects had to assess clarity of their prime perception directly after 
each trial by pressing one of four buttons with their left hand ( $1=$ "no perception", 2 = "a weak glimpse without recognition", 3 = "an almost clear image with recognition", $4=$ "a clear image"). Following this decision, two alternatives appeared on the screen with one of them matching the prime stimulus. The alternatives differed in color and matched in shape in Experiment 4.1A, whereas they matched in color and differed in shape in Experiment 4.1B. The subjects had to decide which alternative matched the prime by pressing a left or a right key with the index or middle finger of the right hand.

Procedure. Following Wernicke and Mattler (Chapter 3) in many aspects, subjects participated in three priming sessions and one prime identification session. Every appointment was scheduled for a different day. Demographic data were collected and vision tests regarding visual acuity and color perception were applied in advance of the actual experiment. At the beginning of the first and fourth session, the respective tasks were explained to the subjects. They were always advised to focus on the center of the screen, marked with a fixation cross. In the first session, subjects received 8 trials of demonstration, 48 training trials and 512 experimental trials in 8 runs (Figure 4.3). A trial started with the presentation of a fixation cross for $500 \mathrm{~ms}$, followed by a fixation point for $360 \mathrm{~ms}$. Subsequently, the prime was displayed for $20 \mathrm{~ms}$. Realizing two prime-mask SOAs, the following fixation point was presented for $40 \mathrm{~ms}$ or $70 \mathrm{~ms}$. In one fourth of the trials, a central mask and two equal targets, below and above fixation, were presented simultaneously, afterwards. A mask-target SOA of $100 \mathrm{~ms}$ was realized by presenting a mask for $100 \mathrm{~ms}$, directly followed by the pair of targets, also for $100 \mathrm{~ms}$ (1/4 of the trials). In the last two fourths of the trials, a fixation point was interposed between mask and targets for $50 \mathrm{~ms}$ or $100 \mathrm{~ms}$. A response window was set for $2000 \mathrm{~ms}$. The trial was aborted after a response was given. In case of an error, a feedback tone $(440 \mathrm{~Hz} ; 150 \mathrm{~ms})$ was given and the general inter trial interval of $1000 \mathrm{~ms}$ was extended to $2000 \mathrm{~ms}$. Each run ended with a break. The duration 
was set by the subjects. The second and third priming session contained 768 experimental trials in 8 runs, respectively. A training run of 48 trials preceded the experimental runs. At the end of the last priming session, subjects were questioned and informed about the appearance of primes. The final session was dedicated to prime identification. Subjects received 8 demonstration trials with a prolonged prime presentation $(150 \mathrm{~ms})$. Afterwards, 512 experimental trials were processed. Trials were essentially equal in structure to those presented in the priming sessions. Subjects were instructed to focus on the prime. At the end of each trial, they were expected to evaluate the prime perception with the PAS. For this, the response window was unlimited. Triggered by this response, two alternatives appeared on the screen, one the left and one on the right side. One of these alternatives matched the prime and subjects were instructed to identify it by pressing the assigned key. An error was again indicated by a feedback tone.

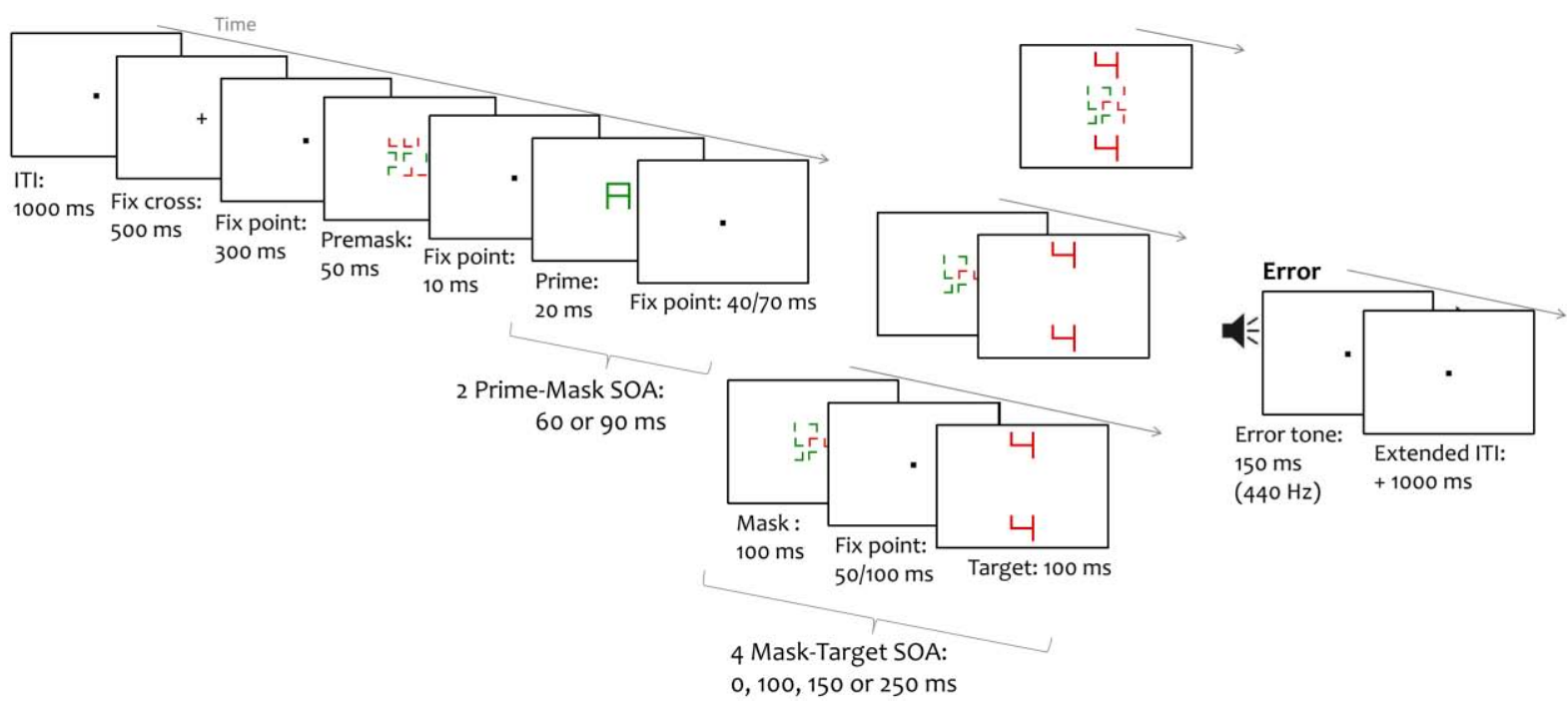

Figure 4.3. Sequence of a trial used in Experiments 4.1 - 4.3. In Experiment 4.1 metacontrast masks were used without presenting a pre-mask. In Experiment 4.2, pattern masks were applied as forward and backward masks. 
Design and Statistical Analysis. Four types of primes ( 2 colors $\times 2$ categories), four types of targets, two prime-mask SOAs (60 \& $90 \mathrm{~ms})$, four mask-target SOAs $(0,100,150 \&$ $250 \mathrm{~ms}$ ) and masks with two different strengths yield 256 conditions. In the priming experiment, participants received 2048 experimental trials. 512 of these trials were used for prime identification in a subsequent session. Specific signs (4 for each type of primes and targets) were assigned randomly for each subject. The occurrence of trials was spread evenly throughout the priming sessions with regards to prime-mask SOA, mask-target SOA, masking strength and prime-target congruency based on color and semantic category. Trial order was assigned randomly. Error and post-error trials as well as the fastest and two slowest reaction times (RT) were excluded in estimation of mean RTs for each condition. Mean RTs and arc-sin transformed error rates (ER) were subjected to repeated measures ANOVAs. Prime-mask SOA, mask-target SOA, masking strength and congruency - based on color and semantic category for Experiment 4.1A and 4.1B, respectively - were included as factors. Mauchly's sphericity test was used to examine whether a violation of the sphericity assumption occurred (Mauchly, 1940). In such cases, Greenhouse-Geisser correction was applied (Greenhouse \& Geisser, 1959). In line with signal detection theory (Macmillan \& Creelman, 1991) $d$ '- values were calculated with the hit rates $(\mathrm{H})$ and false-alarms rates $(\mathrm{F})$ of the two-alternative choices:

$$
d^{\prime}=z(H)-z(F)
$$

with $\mathrm{z}$ as the inverse of the normal distribution function. Subjective ratings (PAS) and $d$ '-values were analyzed with repeated measures ANOVAs to determine the impact of primemask SOA, mask-target SOA and masking strength. 


\subsubsection{Results}

Errors. In Experiment 4.1A, errors occurred in $3.2 \%$ of all trials. Repeated measures ANOVA revealed an interaction of perceptual congruency and mask-target $\operatorname{SOA}(F(3,33)=$ 7.27, $\left.M S_{e}=0.026, p<.001\right)$ and a three-way interaction of perceptual congruency, masking strength and mask-target $\operatorname{SOA}\left(F(3,33)=3.14, M S_{e}=0.028, p<.05\right)$. When weak masks were applied, error effects $\left(\mathrm{PE}_{\mathrm{ER}}=\mathrm{ER}_{\text {incongruent }}-\mathrm{ER}_{\text {congruent }}\right)$ decreased with increasing masktarget SOA $(1.6 \%, 0.4 \%, 0.2 \%$ and $-0.1 \%$ with mask-target SOAs of $0 \mathrm{~ms}, 100 \mathrm{~ms}, 150 \mathrm{~ms}$ and $250 \mathrm{~ms}$, respectively). Initially, error effects also decreased when strong masks were used, but with a mask-target SOA of $250 \mathrm{~ms}$ error effects increased again $(2.4 \%,-0.8 \%$, $1.1 \%$ and $3.1 \%$ with mask-target SOAs of $0 \mathrm{~ms}, 100 \mathrm{~ms}, 150 \mathrm{~ms}$ and $250 \mathrm{~ms}$, respectively). Neither the perceptual priming effect at 100 ms mask-target SOA $(t(11)=-0.72, p>.2)$ nor the perceptual priming effect at $150 \mathrm{~ms}$ mask-target SOA $(t(11)=-1.28, p>.2)$ was significantly negative. In Experiment $4.1 \mathrm{~B}$, subjects committed errors in $4.2 \%$ of trials. Repeated measures ANOVA revealed a main effect of semantic congruency $(F(1,11)=6.04$, $\left.M S_{e}=0.022, p<.05\right)$ and a main effect mask-target SOA $\left(F(3,33)=3.07, M S_{e}=0.025\right.$, $p<.05)$. More errors occurred in incongruent trials $(4.5 \%)$ compared to congruent trials (3.9\%). Errors increased with increasing mask-target SOA $(3.7 \%, 4.0 \%, 4.3 \%$ and $4.7 \%$ with mask-target SOAs of $0 \mathrm{~ms}, 100 \mathrm{~ms}, 150 \mathrm{~ms}$ and $250 \mathrm{~ms}$, respectively). The conjoint analysis of Experiments 4.1A and 4.1B (Table C1.1 in Appendix III) revealed a main effect of congruency $\left(F(1,22)=10.54, M S_{e}=0.025, p<.005\right)$, an interaction of congruency and mask-target SOA $\left(F(3,66)=6.62, M S_{e}=0.024, p<.001\right)$ and a three-way interaction of task level, congruency and mask-target $\operatorname{SOA}\left(F(3,66)=3.21, M S_{e}=0.024, p<.05\right)$. Perceptual and semantic priming effects on error rates are displayed in Figure 4.4. 

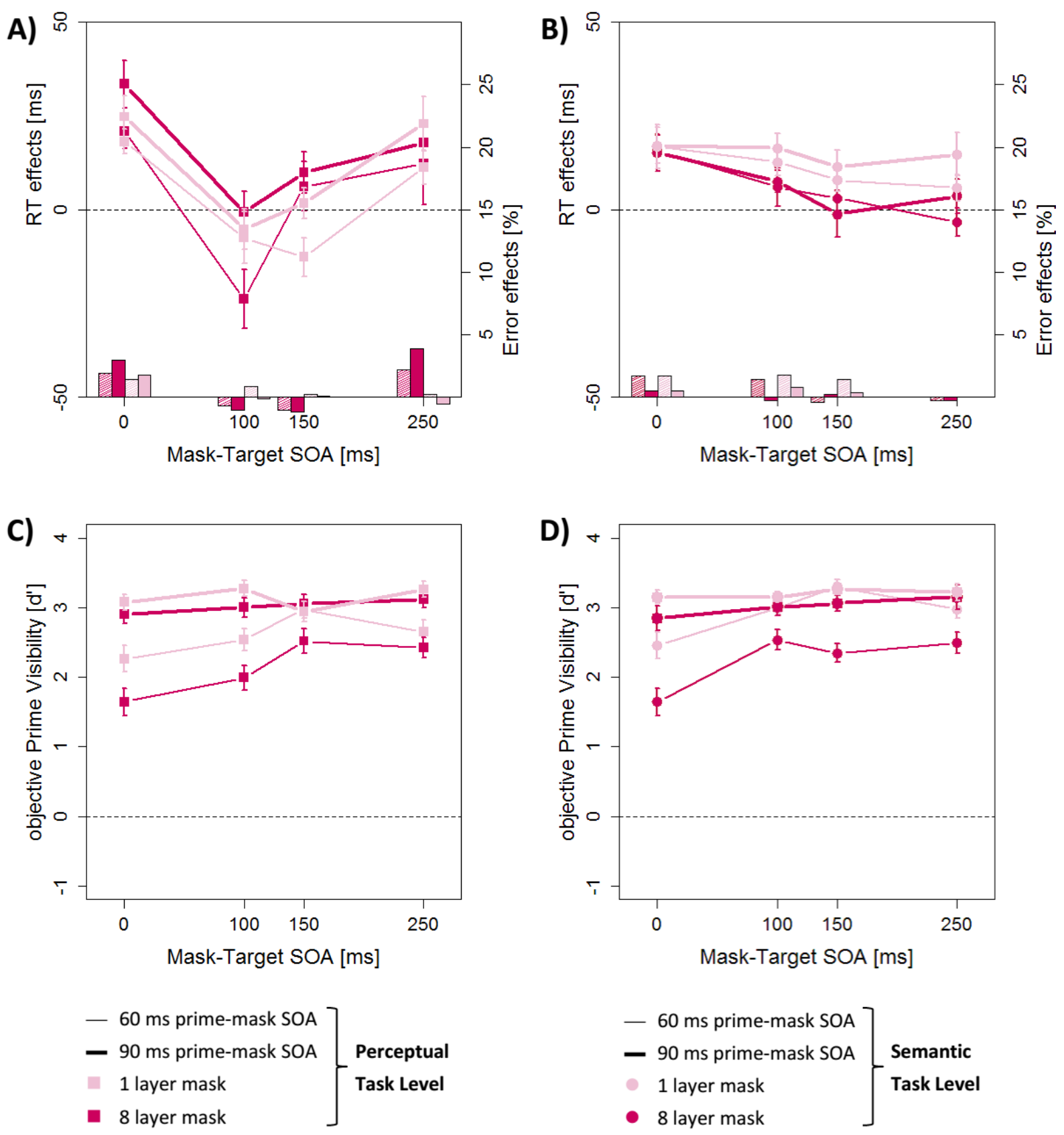

Figure 4.4. Priming effects and $d$ '-values in Experiment 4.1. Priming effects (A \& B) on reaction times (RT; lines) and errors (ER; bars) and $d$ '-values (C \& D) are displayed for each masking strength, prime-mask SOA and mask-target SOA. A \& C: Experiment 4.1A with a perceptual task level. B \& D: Experiment 4.1B with a semantic task level. Confidence intervals are in accordance with Loftus and Masson (1994). 
Reaction Times. In Experiment 4.1A, subjects responded on average $424 \mathrm{~ms}$ after target onset. Repeated measures ANOVA revealed an main effect of perceptual congruency $\left(F(1,11)=14.20, M S_{e}=448, p<.005\right)$, masking strength $\left(F(1,11)=10.41, M S_{e}=135\right.$, $p<.01)$, prime-mask SOA $\left(F(1,11)=14.24, M S_{e}=70, p<.005\right)$ and mask-target SOA $\left(F(3,33)=63.10, M S_{e}=456, p<.0001\right)$, an interaction of masking strength and mask-target SOA $\left(F(3,33)=4.32, M S_{e}=139, p<.05\right)$, an interaction of perceptual congruency and prime-mask SOA $\left(F(1,11)=5.26, M S_{e}=453, p<.05\right)$, an interaction of perceptual congruency and mask-target SOA $\left(F(3,33)=13.88, M S_{e}=390, p<.0001\right)$ and a four-way interaction of perceptual congruency, masking strength, prime-mask SOA and mask-target SOA $\left(F(3,33)=3.00, M S_{e}=99, p<.05\right.$; see Figure 4.4). Subjects responded faster in congruent trials $(420 \mathrm{~ms})$ than in incongruent trials $(428 \mathrm{~ms}$; Figure 4.5$)$. Subjects also responded slightly faster when weak masks were applied (422 ms) compared to trials with strong masks (426 ms). Responses were slower after $60 \mathrm{~ms}$ prime-mask SOA (426 ms) than after $90 \mathrm{~ms}$ prime-mask SOA (423 ms). Response times decreased with increasing masktarget SOA when strong masks were used (449 ms, $422 \mathrm{~ms}, 414 \mathrm{~ms}$ and $403 \mathrm{~ms}$ with masktarget SOAs of $0 \mathrm{~ms}, 100 \mathrm{~ms}, 150 \mathrm{~ms}$ and $250 \mathrm{~ms}$, respectively). Response times also decreased with weak masks, but with a less negative slope (445 ms, $426 \mathrm{~ms}, 421 \mathrm{~ms}$ and $409 \mathrm{~ms}$ with mask-target SOAs of $0 \mathrm{~ms}, 100 \mathrm{~ms}, 150 \mathrm{~ms}$ and $250 \mathrm{~ms}$, respectively). Perceptual priming effects $\left(\mathrm{PE}_{\mathrm{RT}}=\mathrm{RT}_{\text {incongruent }}-\mathrm{RT}_{\text {congruent }}\right)$ were smaller with a prime-mask SOA of $60 \mathrm{~ms}(3 \mathrm{~ms})$ compared to perceptual priming effects achieved with a prime-mask SOA of $90 \mathrm{~ms}(13 \mathrm{~ms})$. Perceptual priming effects were also modulated through mask-target SOA. An initially positive perceptual priming effect of $24 \mathrm{~ms}$ decreased after $100 \mathrm{~ms}$ masktarget SOA to a negative value of $-9 \mathrm{~ms}$. Afterward, perceptual priming effects increased again to $1 \mathrm{~ms}$ after $150 \mathrm{~ms}$ prime-mask SOA and $16 \mathrm{~ms}$ after $250 \mathrm{~ms}$ prime-mask SOA. Perceptual priming effect were significantly negative $(-16 \mathrm{~ms})$ with a $60 \mathrm{~ms}$ prime-mask SOA 
and a $100 \mathrm{~ms}$ mask-target SOA $(t(11)=-3.35, p<.01)$. In Experiment $4.1 B$, participants responded on average after $457 \mathrm{~ms}$. Repeated measures ANOVA revealed a main effect of semantic congruency $\left(F(1,11)=20.71, M S_{e}=407, p<.001\right)$, a main effect of mask-target $\operatorname{SOA}\left(F(3,33)=56.21, M S_{e}=735, p<.0001\right)$, an interaction of masking strength and masktarget SOA $\left(F(3,33)=3.30, M S_{e}=117, p<.05\right)$, an interaction of semantic congruency and masking strength $\left(F(1,11)=5.95, M S_{e}=200, p<.05\right)$ and an interaction of semantic congruency and mask-target $\operatorname{SOA}\left(F(3,33)=5.06, M S_{e}=128, p<.01\right.$; see Figure 4.4).

\section{Experiment 4.1A}
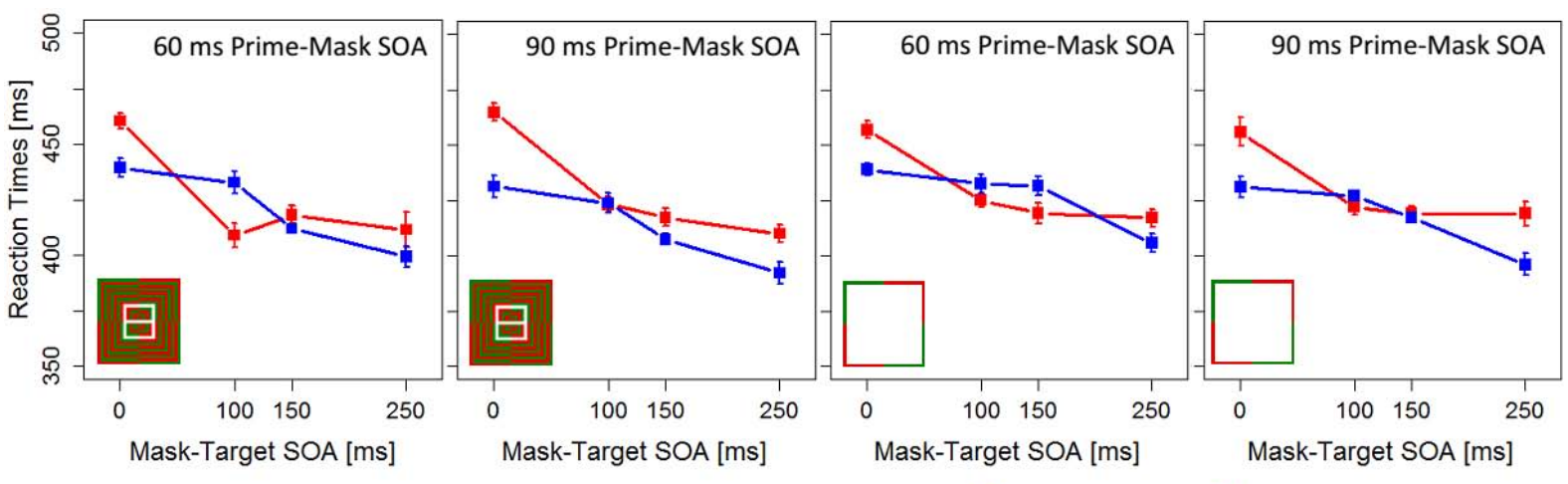

Experiment 4.1B
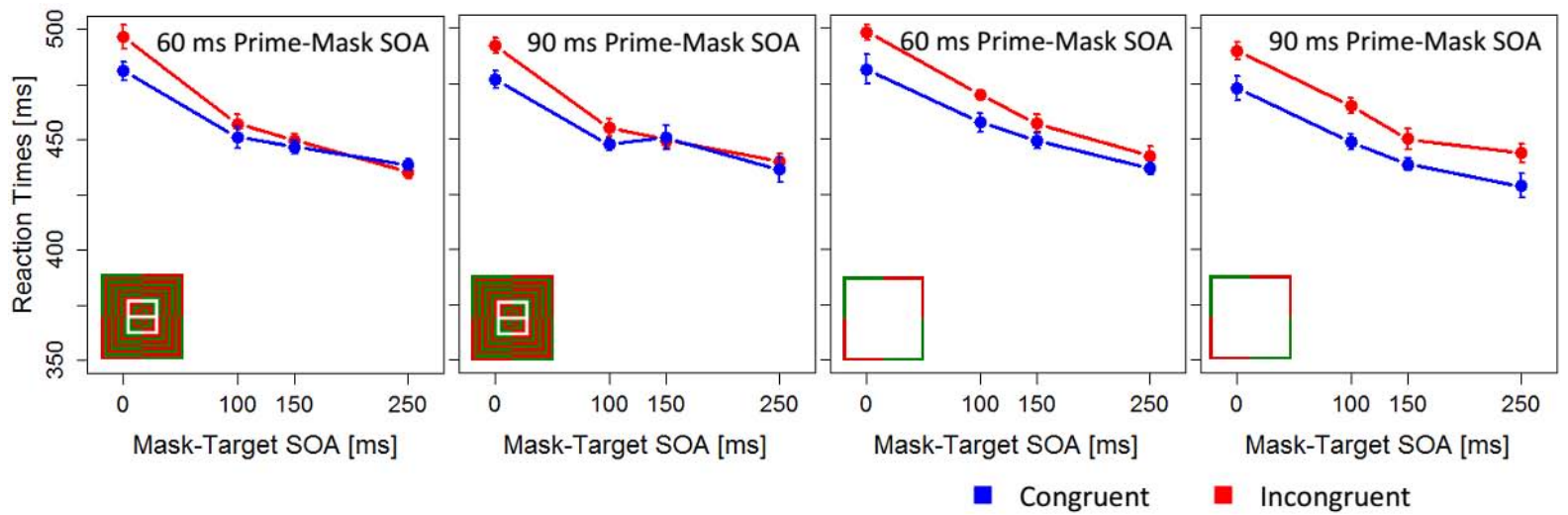

Figure 4.5. Reaction times in Experiment 4.1. Reaction times are displayed for congruent and incongruent trials with each masking strength, prime-mask SOA and mask-target SOA. Confidence intervals are in accordance with Loftus and Masson (1994). 
Subjects responded faster in congruent trials $(453 \mathrm{~ms})$ compared to incongruent trials (462 ms; Figure 4.5). Similar to Experiment 4.1A, response times decreased with increasing mask-target SOA, when strong masks were used $(487 \mathrm{~ms}, 453 \mathrm{~ms}, 449 \mathrm{~ms}$ and $438 \mathrm{~ms}$ with mask-target SOAs of $0 \mathrm{~ms}, 100 \mathrm{~ms}, 150 \mathrm{~ms}$ and $250 \mathrm{~ms}$, respectively) and decreased with a slightly different curve when weak masks were applied (485 ms, $460 \mathrm{~ms}, 449 \mathrm{~ms}$ and $438 \mathrm{~ms}$, respectively). Semantic priming effects differed between masking strengths. Smaller semantic priming effects were found with strong masks $(6 \mathrm{~ms})$ than with weak masks (13 ms). Semantic priming effects were also modulated through prime-mask SOA. They decreased with increasing prime-mask SOA $(16 \mathrm{~ms}, 11 \mathrm{~ms}, 5 \mathrm{~ms}$ and $5 \mathrm{~ms}$ with mask-target SOAs of $0 \mathrm{~ms}, 100 \mathrm{~ms}, 150 \mathrm{~ms}$ and $250 \mathrm{~ms}$, respectively). The conjoint analysis of Experiments 4.1A and 4.1B (Table $\mathrm{C} 1.2$ ) revealed a main effect of congruency $(F(1,22)=$ $\left.34.42, M S_{e}=428, p<.0001\right)$, masking strength $\left(F(1,22)=7.47, M S_{e}=186, p<.05\right)$, primemask SOA $\left(F(1,22)=11.72, M S_{e}=220, p<.005\right)$ and mask-target $\operatorname{SOA}(F(3,66)=116.57$, $\left.M S_{e}=595, p<.0001\right)$, an interaction of masking strength and prime-mask $\operatorname{SOA}(F(1,22)=$ $\left.6.92, M S_{e}=142, p<.05\right)$, an interaction of masking strength and mask-target $\operatorname{SOA}(F(3,66)=$ $\left.4.58, M S_{e}=128, p<.01\right)$, an interaction of congruency and prime-mask SOA SOA $(F(1,22)=$ $\left.7.00, M S_{e}=268, p<.05\right)$, an interaction congruency and mask-target SOA $(F(3,66)=14.24$, $\left.M S_{e}=259, p<.0001\right)$, a three-way interaction of task level masking strength and mask-target SOA $\left(F(3,66)=3.13, M S_{e}=128, p<.05\right)$ and a three-way interaction of task level, congruency and mask-target $\operatorname{SOA}\left(F(3,66)=9.15, M S_{e}=259, p<.0001\right)$.

Prime visibility. In Experiment 4.1A, subjects chose correct alternatives with a mean $d$ ' of 2.73. Repeated measures ANOVA revealed a main effect of prime-mask SOA $\left(F(1,11)=70.92, M S_{e}=0.336, p<.0001\right)$, a main effect of mask-target $\operatorname{SOA}(F(3,33)=8.25$, $\left.M S_{e}=0.205, p<.0005\right)$, an interaction on masking strength and prime-mask SOA $(F(1,11)=$ $\left.5.01, M S_{e}=0.285, p<.05\right)$ and an interaction of prime-mask SOA and mask-target SOA 
$\left(F(3,33)=5.79, M S_{e}=0.241, p<.05\right)$. Objective prime visibility increased with prime-mask SOA (Figure 4.4). This increase was stronger with strong masks (2.15 to 3.03) compared to a weak masks (2.61 to 3.14$)$. Objective prime visibility also increased with increasing masktarget SOA. This increase was stronger with prime-mask SOA of $60 \mathrm{~ms}(1.96,2.27,2.75$ and 2.54 with mask-target SOAs of $0 \mathrm{~ms}, 100 \mathrm{~ms}, 150 \mathrm{~ms}$ and $250 \mathrm{~ms}$, respectively) compared to a prime-mask SOA of $90 \mathrm{~ms}(3.00,3.14,3.00$ and 3.20, respectively). Subjective prime visibility was rated with 3.26 on average. Repeated measures ANOVA revealed a main effect of masking strength $\left(F(1,11)=5.54, M S_{e}=0.122, p<.05\right)$, prime-mask SOA $(F(1,11)=$ 16.57, $\left.M S_{e}=0.197, p<.005\right)$ and mask-target $\operatorname{SOA}\left(F(3,33)=9.45, M S_{e}=0.018, p<.0005\right)$. Subjective prime visibility was smaller with strong masks (3.21) than with weak masks (3.32; Figure 4.6). It was also smaller with a prime-mask SOA of $60 \mathrm{~ms}$ (3.13) compared to a prime-mask SOA of $90 \mathrm{~ms}$ (3.40). Subjective prime visibility also increased with increasing mask-target SOA $(3.18,3.26,3.31$ and 3.31 with mask-target SOAs of $0 \mathrm{~ms}, 100 \mathrm{~ms}, 150 \mathrm{~ms}$ and $250 \mathrm{~ms}$, respectively). Debriefing at the end of the last priming session revealed that only five of twelve subjects detected the primes and four were able to describe them. Eleven subjects reported an improvement of prime perception during the prime identification session. Eight participants noted a manipulation of prime visibility through masking strength. In Experiment 4.1B, subjects achieved a mean $d$ ' of 2.85. Repeated measures ANOVA revealed a main effect of masking strength $\left(F(1,11)=12.02, M S_{e}=0.738, p<.01\right)$, prime-mask SOA $\left(F(1,11)=37.32, M S_{e}=0.343, p<.0001\right)$ and mask-target SOA $\left(F(3,33)=10.34, M S_{e}=\right.$ $0.223, p<.0001)$, an interaction on masking strength and prime-mask SOA $(F(1,11)=27.24$, $\left.M S_{e}=0.108, p<.0005\right)$ and an interaction of prime-mask SOA and mask-target SOA $\left(F(3,33)=4.40, M S_{e}=0.240, p<.05\right)$. Strong masks reduced prime visibility more effectively (2.64) than weak masks (3.01). Objective prime visibility increased with primemask SOA (Figure 4.4). This increase was stronger with strong masks (from 2.26 to 3.02) 
compared to a weak masks (from 2.94 to 3.20 ). Objective prime visibility also increased with increasing mask-target SOA. This increase was stronger with a prime-mask SOA of $60 \mathrm{~ms}$ $(2.05,2.77,2.82$ and 2.74 with mask-target SOAs of $0 \mathrm{~ms}, 100 \mathrm{~ms}, 150 \mathrm{~ms}$ and $250 \mathrm{~ms}$, respectively) compared to a prime-mask SOA of $90 \mathrm{~ms}(3.00,3.08,3.17$ and 3.19, respectively). Subjective prime visibility was rated with 3.37 on average. Repeated measures ANOVA revealed a main effect of masking strength $\left(F(1,11)=15.42, M S_{e}=0.061, p<.005\right)$, prime-mask SOA $\left(F(1,11)=21.54, M S_{e}=0.080, p<.001\right)$, a main effect of mask-target SOA $\left(F(3,33)=14.70, M S_{e}=0.043, p<.005\right)$ and an interaction on masking strength and primemask SOA $\left(F(1,11)=22.26, M S_{e}=0.021, p<.001\right)$. Participants rated prime visibility lower when strong masks were applied (3.30) compared to trials with weak masks (3.44). Subjective prime visibility increased with prime-mask SOA (Figure 4.6). This increase was stronger with strong masks (3.15 to 3.44 ) compared to a weak masks (3.39 to 3.48). Subjective prime visibility also increased with increasing mask-target SOA $(3.19,3.43,3.43$ and 3.42 with mask-target SOAs of $0 \mathrm{~ms}, 100 \mathrm{~ms}, 150 \mathrm{~ms}$ and $250 \mathrm{~ms}$, respectively). Debriefing revealed that five subjects detected and four subjects recognized the primes during the priming sessions. Eleven subjects noticed an improvement of prime perception during the prime identification session and seven subjects noticed a manipulation of prime visibility through masking strength. The conjoint analysis (Experiments 4.1A and 4.1B; Table C1.3) of objective prime visibility revealed a main effect of masking strength $\left(F(1,22)=14.95, M S_{e}=\right.$ $0.831, p<.001)$, prime-mask SOA $\left(F(1,22)=105.38, M S_{e}=0.339, p<.0001\right)$ and masktarget SOA $\left(F(3,66)=18.11, M S_{e}=0.214, p<.0001\right)$, an interaction on masking strength and prime-mask SOA $\left(F(1,22)=21.56, M S_{e}=0.196, p<.0005\right)$ and an interaction of prime-mask SOA and mask-target SOA $\left(F(3,66)=8.31, M S_{e}=0.240, p<.005\right)$. The conjoint analysis (Experiments 4.1A and 4.1B; Table C1.4) of subjective prime visibility revealed a main effect of masking strength $\left(F(1,22)=17.54, M S_{e}=0.091, p<.0005\right)$, prime-mask SOA 
$\left(F(1,22)=35.14, M S_{e}=0.139, p<.0001\right)$ and mask-target SOA $\left(F(3,66)=22.90, M S_{e}=\right.$ $0.030, p<.0001)$, an interaction on masking strength and prime-mask $\operatorname{SOA}(F(1,22)=22.41$, $\left.M S_{e}=0.018, p<.0005\right)$, an interaction of prime-mask SOA and mask-target $\operatorname{SOA}(F(3,66)=$ 4.86, $\left.M S_{e}=0.019, p<.05\right)$ and a three-way interaction of task level, masking strength and prime-mask SOA $\left(F(1,22)=6.27, M S_{e}=0.018, p<.05\right)$.

\section{Experiment 4.1A}

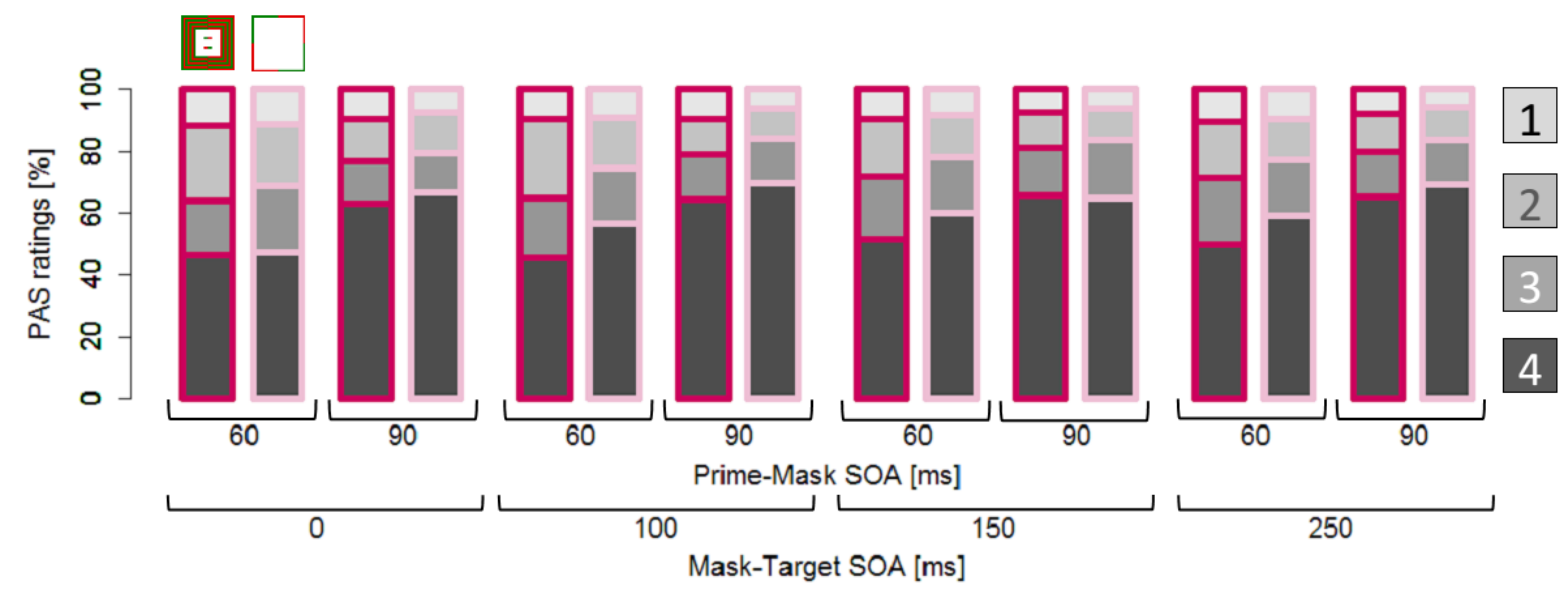

Experiment 4.1B

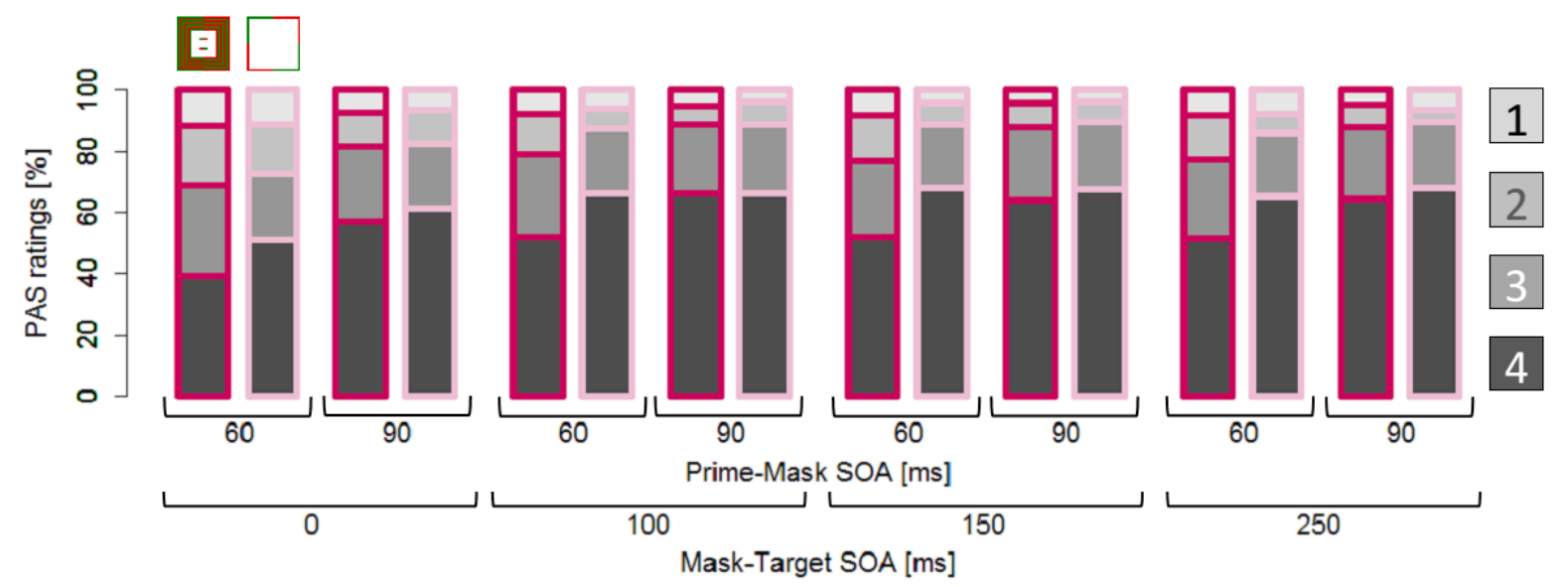

Figure 4.6. Ratings of subjective prime visibility with the PAS in Experiment 4.1 (Ramsøy \& Overgaard, 2004). Percentages of ratings with each masking strength, prime-mask SOA and masktarget SOA are displayed. Ratings: 1 = 'nothing', 2 = 'weak glimpse', 3 = 'almost clear image' \& 4 $=$ 'clear image'. 


\subsubsection{Discussion}

Experiment 4.1 was conducted to compare perceptual and semantic priming with regards to a possibly different modulation through mask-target SOA. Additionally, the primemask SOA was varied to replicate a positive impact of prime-mask SOA on priming effects (Chapter 3). Error effects were not modulated through prime-mask SOA in both subexperiments. This might be due to the small difference between the short prime-mask SOA (60 ms) and the long one $(90 \mathrm{~ms})$. Furthermore, accuracy data is less sensitive than reaction times. Analyses of reaction times revealed an interaction of perceptual congruency and prime-mask SOA (Experiment 4.1A), but no interaction of semantic congruency and prime-mask SOA (Experiment 4.1B). The lack of significant modulation of semantic priming effects through prime-mask SOA could be due to a floor effect with strong masks (Figure 4.4). Semantic priming effects were smaller with $60 \mathrm{~ms}$ prime-mask SOA compared to $90 \mathrm{~ms}$ prime-mask SOA when weak masks were used.

Four different mask-target SOAs were applied to examine a modulation of priming effects through mask-target SOA. A condition with no onset asynchrony between mask and target was included to relate the results to previous experiments (Chapter 3). Mask-target SOAs of $100 \mathrm{~ms}$ and $150 \mathrm{~ms}$ were chosen to capture possible inversions of priming effects (Eimer \& Schlaghecker, 2003; Schlaghecker \& Eimer, 2000; Sumner, 2007). In line with other studies (Figure 4.1), another long (250 ms) mask-target SOA was included to capture a decay of semantic priming effects. As expected, perceptual as well as semantic RT priming effects were modulated through mask-target SOA. Additionally, a similar modulation of error effects through mask-target SOA was found in Experiment 4.1A. In both subexperiments, maximal RT priming effects occurred with $0 \mathrm{~ms}$ mask-target SOA. Perceptual RT priming effects (Experiment 4.1A) decreased rapidly at first. After $100 \mathrm{~ms}$ mask-target SOA a less steep increase of perceptual RT priming effects began. This pattern was reflected through 
similar modulations of error effects with strong masks. Perceptual error effects with weak masks decreased continuously with increasing mask-target SOA. Semantic RT priming effects also decreased continuously with increasing mask-target SOA. This pattern was also reflected through similar modulations of semantic error effects. The mean perceptual priming effect was significantly negative with $60 \mathrm{~ms}$ prime-mask SOA and $100 \mathrm{~ms}$ mask-target SOA. Semantic priming effect did not drop below zero in the period examined. The described pattern suggests that, at least partially, different mechanisms are involved in Experiment 4.1A and Experiment 4.1B. One possible mechanism might be a simple decay of priming effects due to decline of activity over time, but this mechanism cannot account for the occurrence of negative priming effects. Several possible mechanisms are discussed in the literature concerning the occurrence of inverse priming effects (Sumner, 2007). Some of these mechanisms rely on low prime visibility (e.g. self-inhibition; Klapp, 2005) whereas other mechanisms seem to be independent of prime visibility (e.g. object updating; Lleras \& Enns, 2004). Especially, inverse priming effects with irrelevant masks might rely on low prime visibility (Eimer \& Schlaghecken, 2002; Klapp, 2005; Klapp \& Hinkley, 2002). A perceptual interaction of prime and mask is a possible cause of inverse priming effects with relevant masks and might be independent of prime visibility. Lleras and Enns (2004) describe this mechanism within the framework of object updating. Verleger and colleagues (2004) postulate the active-mask hypothesis. Previous experiments (Chapter 3) also indicated that prime visibility might interact with temporal parameters in modulating priming effects. Therefore, masks with two different strengths were used to vary prime visibility.

Subjective prime visibility was modulated through masking strength in both subexperiments. Stronger masks reduced subjective prime visibility more effectively than weaker masks. Objective prime visibility was also modulated through masking strength in Experiment 4.1B. Subjects recognized the correct alternative more often with weak masks 
than with strong masks. No main effect of masking strength modulating objective prime visibility was found in Experiment 4.1A, but a significant main effect of prime-mask SOA and an interaction of masking strength and prime-mask SOA. In conditions with a primemask SOA of $90 \mathrm{~ms}$, participants recognized correct alternatives in $93 \%$ of trials with strong masks and in $94 \%$ of trials with weak masks. This suggests the presence of a ceiling effect. Furthermore, objective visibility might be overestimated through a strong attentional focus on the primes and a delayed retrieval (Vermeiren \& Cleeremans, 2012) - before subjects responded to the objective visibility task, a subjective visibility assessment was demanded. Subsequent debriefing supports this assumption. Taking this and the modulation of subjective prime visibility through masking strength into account, prime visibility seems to be successfully modulated through masking strength in both subexperiments. Additionally, no main effect of task level or interaction with task level in modulating objective prime visibility was found. The identity of primes and color of primes seems to be equally reportable under these circumstances. Interestingly, objective as well subjective visibility data provide evidence for an impact of mask-target SOA on prime visibility. This indicates a masking effect of the target stimuli.

The successful modulation of prime visibility through masking strength legitimates a straightforward interpretation of priming effects modulated by masking strength. Perceptual priming effects (Experiment 4.1A) on errors were modulated through masking strength in interaction with mask-target SOA. Perceptual RT priming effects were modulated through masking strength in interaction with prime-mask SOA, mask-target SOA. Seemingly, prime visibility - varied through masking strength - has an impact on perceptual priming effects, but this impact is of a complex nature. Furthermore, the rapid decay of perceptual priming effects until $100 \mathrm{~ms}$ prime mask SOA seems unaffected by simple prime visibility (Figure 4.4). Maximal perceptual priming effects on reaction times occurred with $0 \mathrm{~ms}$ mask- 
target SOA. An impact of simple decay on perceptual priming effect is therefore plausible. However, indications of an inversion of perceptual priming effects cannot be caused by simple decay. Mechanisms of inhibition can lead to inverse priming effects, but depend on low prime visibility (Eimer \& Schlaghecken, 2002; Klapp, 2005; Klapp \& Hinkley, 2002; Sumner, 2007). In our experiments, prime visibility moderates perceptual priming effects, but especially the inversion does not depend on low prime visibility. Presumably, mechanisms of inhibition are effective in modulating perceptual priming effects, but further mechanisms are involved. The u-shaped course of perceptual priming effects with increasing mask-target SOA and the inversion around $100 \mathrm{~ms} / 150 \mathrm{~ms}$ mask-target SOA is typical for conditions with relevant masks (e.g. Schlaghecker \& Eimer, 2000). A perceptual interaction of prime and mask in the sense of object updating is feasible (Lleras \& Enns, 2004). The priming task of Experiment 4.1A concerns the discrimination of red and green. Masks always contain red and green sections. Following a prime of one color, the other color might be more salient within the mask, due to its novelty. Alternatively, an afterimage in complementary color might account for inverse priming effects.

Only semantic priming effects (Experiment 4.1B) on reaction times were significantly modulated through masking strength. Priming effects were smaller with strong masks than with weak masks. The three-way interaction of congruency, masking strength and masktarget SOA did not reach significance. Nevertheless, semantic priming effects did not differ between masks in conditions with $0 \mathrm{~ms}$ mask-target SOA. Semantic priming effects seem to decay faster with increasing mask-target SOA when strong mask are applied (Figure 4.4). Speculatively, larger initial priming effects and a larger difference in masking efficiency between strong and weak masks would enable the three-way interaction of congruency, masking strength and mask-target SOA. Unfortunately, both parameters could only be regulated further through prime-mask SOA variation - diametrically. A simple decay seems 
to contribute to the decline of semantic priming effects as they also decrease slightly with increasing mask-target SOA when weak masks are applied. The impact of prime visibility in facilitating a decline of semantic priming effects suggests a contribution of inhibitory mechanisms to this decline (Eimer \& Schlaghecken, 2002; Klapp, 2005; Klapp \& Hinkley, 2002; Sumner, 2007). However, the mechanism of object updating (Lleras \& Enns, 2004) cannot be not effective in Experiment 4.1B. Novel and therefore more salient aspects of masks do not provide an advantage for incongruent targets with this kind of task.

\subsection{Experiment 4.2: SOA-Variation with Pattern Masking}

In the second experiment, pattern masks, typically used in studies examining semantic priming, were applied. As the mask plays a crucial part in some mechanisms thought to induce inverse priming, it is an interesting question if those mechanisms are supported by pattern masks, too. Different courses of perceptual and semantic priming effects with increasing mask-target SOA would provide evidence for this.

\subsubsection{Method}

Participants. Twelve subjects participated in Experiment 4.2A (10 female, 2 male) as well as in Experiment 4.2B ( 7 female, 5 male). The subjects were from 20 to 33 years of age $(M=23.75)$ in Experiment $4.2 \mathrm{~A}$ and from 19 to 29 years of age $(M=24.08)$ in Experiment 4.2B. Three participants in Experiment 4.2A and five participants in Experiment 4.2B had normal vision. The remaining subjects had corrected-to-normal vision. The proceedings were equivalent to Experiment 4.1.

Stimuli. The same stimuli used in Experiment 4.1 were applied in Experiment 4.2 except for the masks. Instead of metacontrast masks, pattern masks were constructed with segments of the primes $(25 \mathrm{px} \times 25 \mathrm{px})$ in the same size. The segments were rearranged 
randomly in a grid with a mesh width of $0.61^{\circ}$ and $1.21^{\circ}$ of visual angle to achieved masks with different masking strengths. For each trial, two masks (one forward mask and a backward mask) were generated this way.

Tasks. The tasks of Experiments $4.2 \mathrm{~A}$ and $4.2 \mathrm{~B}$ were identical to the tasks of Experiments 4.1A and 4.1B, respectively.

Procedure. Proceedings in Experiment 4.2 matched proceedings in Experiment 4.1. The only difference concerned the appliance of masks. Instead of metacontrast masks, pattern masks were used. To achieve a reduction of prime visibility and maintain comparability to previous experiments (e.g. Chapter 3, for a review see Chapter 4), a forward mask was applied. Thus, each trial began with a fixation cross for $500 \mathrm{~ms}$, a fixation point for $300 \mathrm{~ms}$, a pre-mask for $50 \mathrm{~ms}$ and an inter stimulus interval of $10 \mathrm{~ms}$. Afterwards, prime and subsequent stimulus sequence were displayed analogously to Experiment 4.1.

Design and Statistical Analysis. Design and statistical analysis were equivalent to Experiment 4.1 .

\subsubsection{Results}

Errors. In Experiment $4.2 \mathrm{~A}$, subjects committed errors in $2.4 \%$ of trials. Repeated measures ANOVA revealed a main effect of mask-target $\operatorname{SOA}\left(F(3,33)=8.60, M S_{e}=0.020\right.$, $p<.0005$; see Figure 4.7). Error rates increased with increasing mask-target SOA $(1.6 \%$, $2.7 \%, 2.4 \%$ and $2.8 \%$ with mask-target SOAs of $0 \mathrm{~ms}, 100 \mathrm{~ms}, 150 \mathrm{~ms}$ and $250 \mathrm{~ms}$, respectively). In Experiment $4.2 B$, errors occurred in $2.4 \%$ of trials. Repeated measures ANOVA revealed an interaction of masking strength and prime-mask $\operatorname{SOA}(F(1,11)=12.43$, $\left.M S_{e}=0.011, p<.005\right)$ and a four-way interaction of semantic congruency, masking strength, prime-mask SOA and mask-target $\operatorname{SOA}\left(F(3,33)=3.09, M S_{e}=0.014, p<.05\right.$; Figure 4.7). 

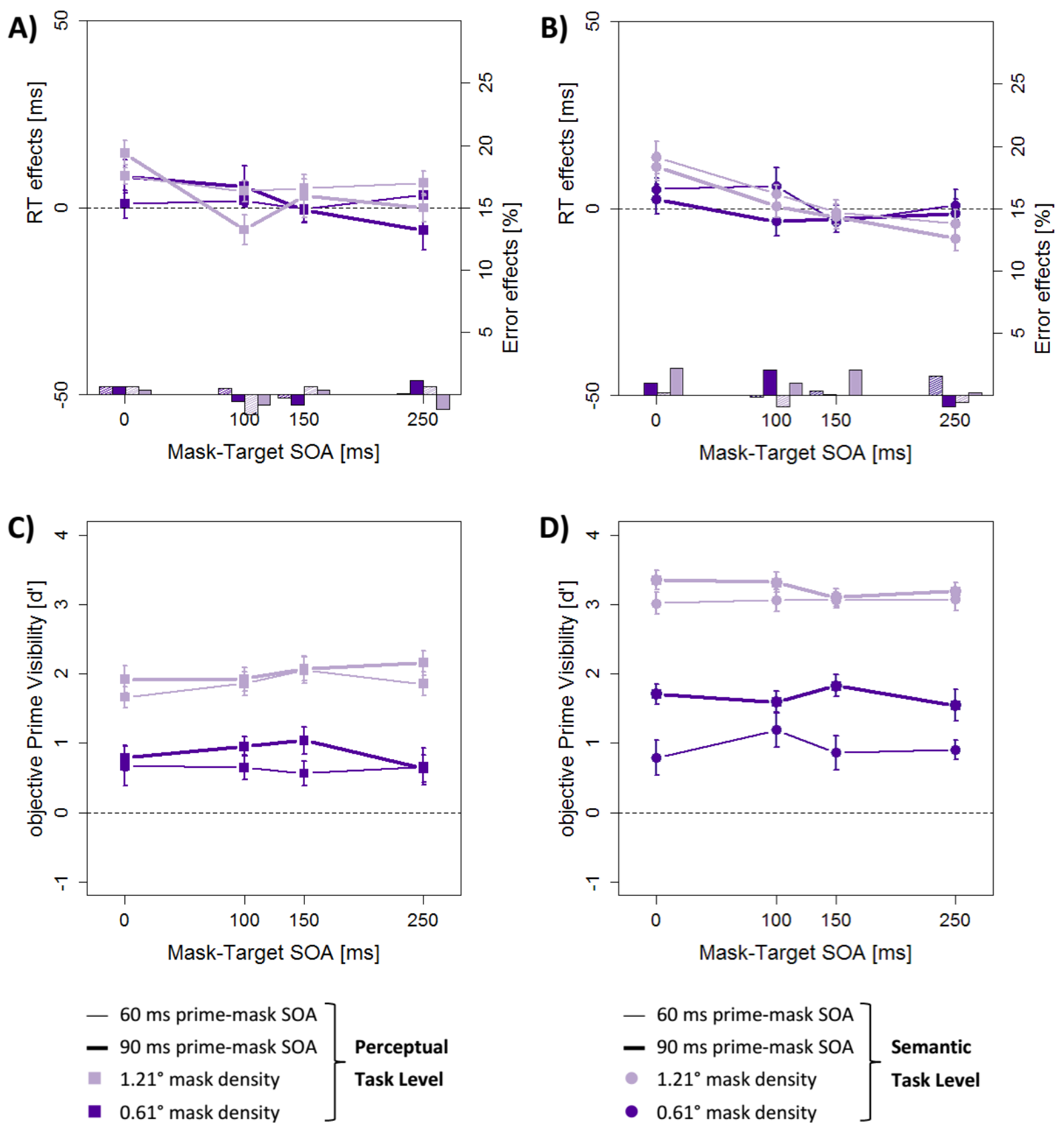

Figure 4.7. Priming effects and $d$ '-values in Experiment 4.2. Priming effects (A \& B) on reaction times (RT; lines) and errors (ER; bars) and $d$ '-values (C \& D) are displayed for each masking strength, prime-mask SOA and mask-target SOA. A \& C: Experiment 4.2A with a perceptual task level. B \& D: Experiment 4.2B with a semantic task level. Confidence intervals are in accordance with Loftus and Masson (1994). 
Error rate increased with prime-mask SOA when strong masks were applied (from $2.6 \%$ to $2.93 \%$ ). Errors decreased with prime-mask SOA when weak masks were applied (from $3.3 \%$ to $2.6 \%$ ). The conjoint analysis of Experiments 4.2A and 4.2B (Table C2.1) revealed a main effect of mask-target SOA $\left(F(3,66)=2.96, M S_{e}=0.030, p<.05\right)$, an interaction of masking strength and prime-mask SOA $\left(F(1,22)=9.08, M S_{e}=0.013, p<.01\right)$, an interaction of task level and prime-mask SOA $\left(F(1,22)=5.80, M S_{e}=0.027, p<.05\right)$, an interaction of task level and mask-target $\operatorname{SOA}\left(F(3,66)=3.91, M S_{e}=0.030, p<.05\right)$ and a five-way interaction of task level, congruency, masking strength, prime-mask SOA and mask-target $\operatorname{SOA}\left(F(3,66)=4.16, M S_{e}=0.018, p<.01 ;\right.$ Figure 4.7).

Reaction Times. Subjects responded on average after $416 \mathrm{~ms}$ in Experiment 4.2A. Repeated measures ANOVA revealed main effects of perceptual congruency $(F(1,11)=$ $\left.14.75, M S_{e}=65, p<.005\right)$ and mask-target $\operatorname{SOA}\left(F(3,33)=55.84, M S_{e}=412, p<.0001\right)$ and an interaction of perceptual congruency and mask-target $\operatorname{SOA}\left(F(3,33)=3.26, M S_{e}=83\right.$, $p<.05$; Figure 4.7). Subjects responded faster in congruent trials (414 ms) compared to incongruent trials (418 ms; Figure 4.8). Response times decreased with increasing masktarget SOA (433 ms, $424 \mathrm{~ms}, 412 \mathrm{~ms}$ and $396 \mathrm{~ms}$ with mask-target SOAs of $0 \mathrm{~ms}, 100 \mathrm{~ms}$, $150 \mathrm{~ms}$ and $250 \mathrm{~ms}$, respectively). Perceptual priming effects also decreased with increasing mask-target SOA $(8 \mathrm{~ms}, 2 \mathrm{~ms}, 2 \mathrm{~ms}$ and $1 \mathrm{~ms}$ with mask-target SOAs of $0 \mathrm{~ms}, 100 \mathrm{~ms}$, $150 \mathrm{~ms}$ and $250 \mathrm{~ms}$, respectively). In Experiment 4.2B, correct responses occurred on average after $441 \mathrm{~ms}$. Repeated measures ANOVA revealed main effects of masking strength $\left(F(1,11)=14.70, M S_{e}=102, p<.005\right)$ and mask-target $\operatorname{SOA}\left(F(3,33)=100.86, M S_{e}=155\right.$, $p<.0001)$ and an interaction of semantic congruency and mask-target $\operatorname{SOA}(F(3,33)=5.91$, $M S_{e}=108, p<.005 ;$ Figure 4.7). Responses were faster in trials with strong masks (439 ms) compared to trials with weak masks (443 ms; Figure 4.8). Similar to Experiment 4.2A, response times decreased with increasing mask-target SOA $(456 \mathrm{~ms}, 445 \mathrm{~ms}, 438 \mathrm{~ms}$ and 
$426 \mathrm{~ms}$ with mask-target SOAs of $0 \mathrm{~ms}, 100 \mathrm{~ms}, 150 \mathrm{~ms}$ and $250 \mathrm{~ms}$, respectively). Semantic priming effects also decreased with increasing mask-target SOA ( $8 \mathrm{~ms}, 2 \mathrm{~ms},-2 \mathrm{~ms}$ and $-3 \mathrm{~ms}$ with mask-target SOAs of $0 \mathrm{~ms}, 100 \mathrm{~ms}, 150 \mathrm{~ms}$ and $250 \mathrm{~ms}$, respectively). The conjoint analysis of Experiments 4.2A and 4.2B revealed main effects of congruency $(F(1,22)=$ $\left.15.49, M S_{e}=57, p<.001\right)$, masking strength $\left(F(1,22)=10.91, M S_{e}=196, p<.005\right)$, prime$\operatorname{mask} \operatorname{SOA}\left(F(1,22)=7.27, M S_{e}=99, p<.05\right)$ and mask-target $\operatorname{SOA}\left(F(3,66)=134.59, M S_{e}\right.$ $=283, p<.0001)$, an interaction of masking strength and prime-mask $\operatorname{SOA}(F(1,22)=5.83$, $\left.M S_{e}=56, p<.05\right)$, an interaction of congruency and mask-target $\operatorname{SOA}\left(F(3,66)=8.74, M S_{e}=\right.$ $287, p<.0001)$ and a four-way interaction of task level, masking strength, prime-mask SOA and mask-target SOA $\left(F(3,66)=3.81, M S_{e}=56, p<.05\right.$; Figure 4.7 and Table C2.2).

\section{Experiment 4.2A}

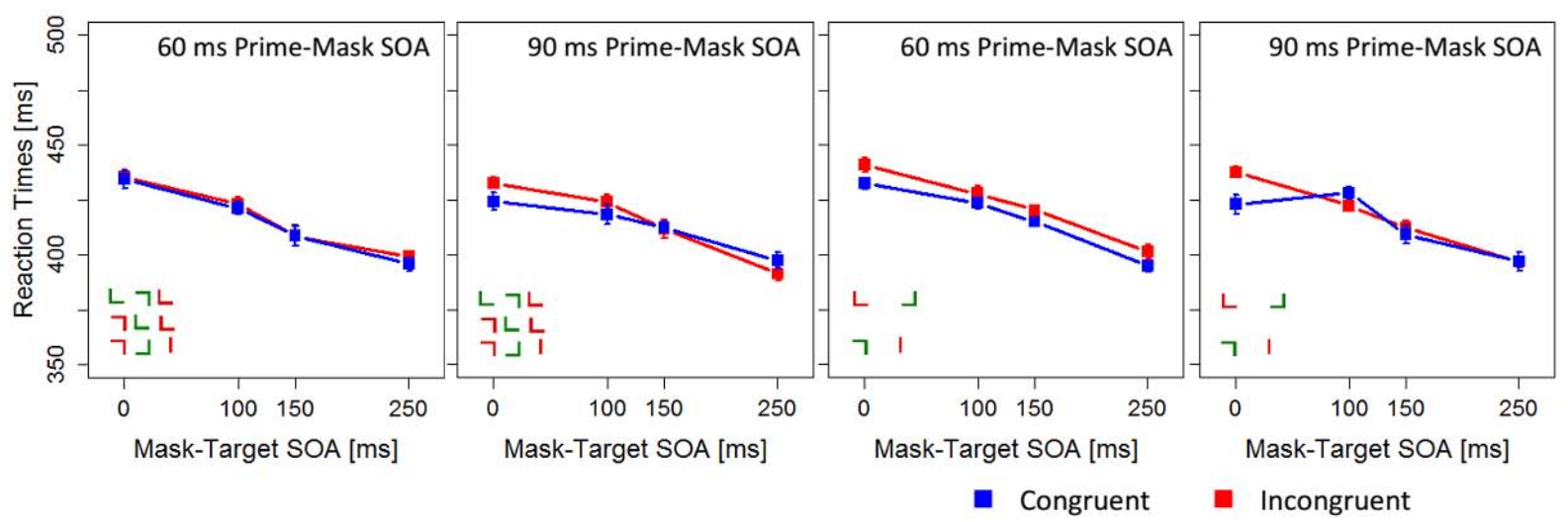

\section{Experiment 4.2B}

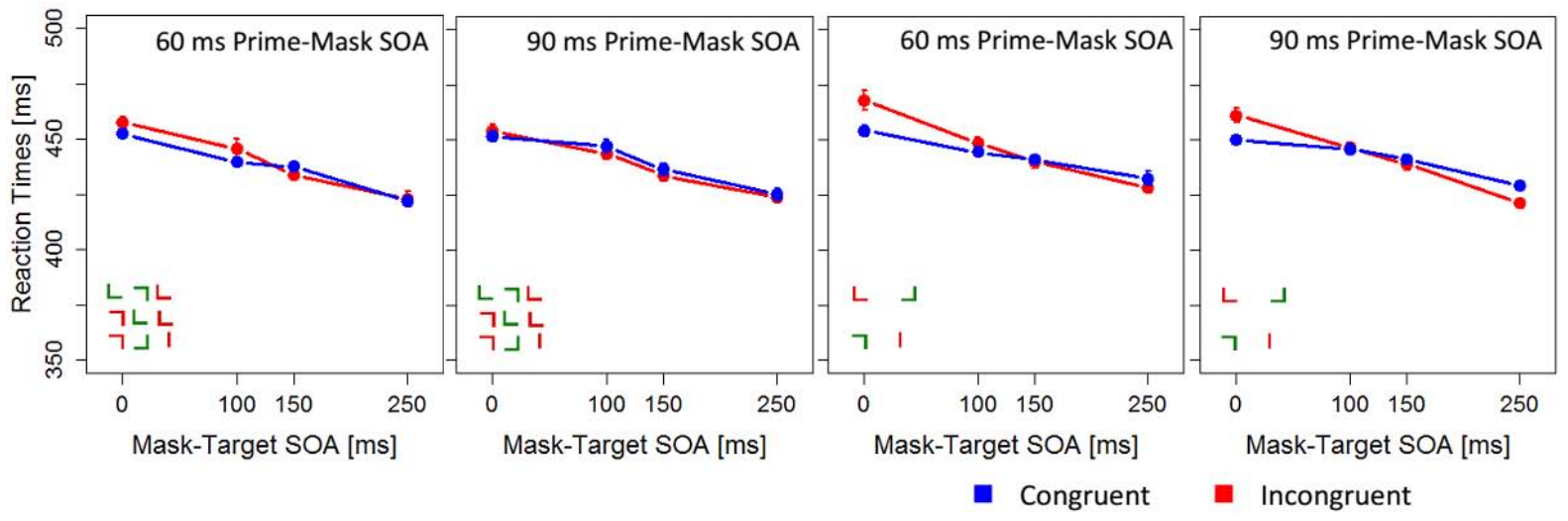

Figure 4.8. Reaction times in Experiment 4.2. Reaction times are displayed for congruent and incongruent trials with each masking strength, prime-mask SOA and mask-target SOA. Confidence intervals are in accordance with Loftus and Masson (1994). 
Prime visibility. In Experiment 4.2A, subjects achieved a mean $d^{\prime}$ of 1.34 . Repeated measures ANOVA revealed a main effect of masking strength $\left(F(1,11)=16.76, M S_{e}=4.085\right.$, $p<.005)$ and a main effect of prime-mask SOA $\left(F(1,11)=6.40, M S_{e}=0.272, p<.05\right.$; Figure 4.7). More effective reduction of objective prime visibility was achieved with strong masks (0.75) compared to weak masks (1.94). Subjects also recognized the correct alternative more often in trials with a prime-mask SOA of $90 \mathrm{~ms}$ (1.44) compared to trials with a prime-mask SOA of $60 \mathrm{~ms}$ (1.25). Subjective prime visibility was rated with 2.56 on average. Repeated measures ANOVA revealed a main effect of masking strength $\left(F(1,11)=11.94, M S_{e}=1.198\right.$, $p<.01)$, a main effect of prime-mask SOA $\left(F(1,11)=7.44, M S_{e}=0.055, p<.05\right)$ and an interaction of masking strength and mask-target $\operatorname{SOA}\left(F(3,33)=4.56, M S_{e}=0.015, p<.01\right.$; Figure 4.9). Prime visibility was rated lower with strong masks (2.29) than with weak masks (2.84). Prime visibility was also rated lower with the short prime-mask SOA (2.52) than with the long prime-mask SOA (2.61). Subjective prime visibility decreased slightly with increasing mask-target SOA when strong masks were applied $(2.35,2.27,2.27$ and 2.27 with mask-target SOAs of $0 \mathrm{~ms}, 100 \mathrm{~ms}, 150 \mathrm{~ms}$ and $250 \mathrm{~ms}$, respectively). It increased slightly with increasing mask-target SOA when weak masks were used (2.80, 2.80, 2.89 and 2.86 with mask-target SOAs of $0 \mathrm{~ms}, 100 \mathrm{~ms}, 150 \mathrm{~ms}$ and $250 \mathrm{~ms}$, respectively). Five subjects reported that they detected the primes during the priming sessions. However, only two subjects were able to describe the primes. Eleven participants reported an improvement of prime perception during the prime identifications session. Five subjects noticed a manipulation of prime visibility through masking strength. In Experiment $4.2 B$, subjects recognized correct alternatives with a mean $d$ ' of 2.23. Repeated measures ANOVA revealed a main effect of masking strength $\left(F(1,11)=69.25, M S_{e}=2.363, p<.0001\right)$, a main effect of prime-mask SOA $\left(F(1,11)=16.22, M S_{e}=0.631, p<.005\right)$ and an interaction of masking strength and prime-mask $\operatorname{SOA}\left(F(1,11)=11.97, M S_{e}=0.294, p<.01\right.$; Figure 4.7). More 
effective reduction of objective prime visibility was achieved with strong masks (1.30) compared to weak masks (3.15). Objective prime visibility increased with prime-mask SOA when strong masks were used (0.94 and 1.67 with prime-mask SOAs of $60 \mathrm{~ms}$ and $90 \mathrm{~ms}$, respectively). Objective prime visibility also increased with prime-mask SOA when weak masks were applied, but with a smaller slope (3.56 and 3.25 with prime-mask SOAs of $60 \mathrm{~ms}$ and $90 \mathrm{~ms}$, respectively). Subjective prime visibility was rated with 2.82 on average. Repeated measures ANOVA revealed a main effect of masking strength $(F(1,11)=47.84$, $\left.M S_{e}=1.181, p<.0001\right)$, a main effect of prime-mask SOA $\left(F(1,11)=37.05, M S_{e}=0.055\right.$, $p<.0001)$ and an interaction of masking strength and prime-mask $\operatorname{SOA}\left(F(1,11)=7.49, M S_{e}\right.$ $=0.060, p<.05$; Figure 4.9). Subjective prime visibility was rated lower with strong masks (2.28) compared to weak masks (3.36). Visibility ratings increased with prime-mask SOA in trials with strong masks (2.13 and 2.43 with prime-mask SOAs of $60 \mathrm{~ms}$ and $90 \mathrm{~ms}$, respectively). Visibility ratings also increased with prime-mask SOA in trials with weak masks, but with a smaller slope (3.31 and 3.42 with prime-mask SOAs of $60 \mathrm{~ms}$ and $90 \mathrm{~ms}$, respectively). Again, five subjects detected the primes and four subjects recognized them during the priming sessions. Eleven subjects reported that prime perception improved during the prime identification session. Eight participants noticed a manipulation of prime visibility through masking strength. The conjoint analysis (Experiments 4.2A and 4.2B; Table C2.3) of objective prime visibility revealed main effects of task level $\left(F(1,22)=5.99, M S_{e}=12.568\right.$, $p<.05)$, masking strength $\left(F(1,22)=68.82, M S_{e}=3.224, p<.0001\right)$ and prime-mask SOA $\left(F(1,22)=22.62, M S_{e}=0.452, p<.0001\right)$, an interaction of masking strength and prime-mask SOA $\left(F(1,22)=7.91, M S_{e}=0.267, p<.05\right)$ and a three-way interaction of task level, masking strength and prime-mask SOA $\left(F(1,22)=5.40, M S_{e}=0.267, p<.05\right)$. The conjoint analysis (Experiments 4.2A and 4.2B; Table C2.4) of subjective prime visibility revealed a main effect of masking strength $\left(F(1,22)=53.57, M S_{e}=1.190, p<.0001\right)$, a main effect of 
prime-mask SOA $\left(F(1,22)=38.76, M S_{e}=0.055, p<.0001\right)$, an interaction of masking strength and prime-mask SOA $\left(F(1,22)=10.80, M S_{e}=0.042, p<.005\right)$, an interaction of masking strength and mask-target $\operatorname{SOA}\left(F(3,66)=3.73, M S_{e}=0.013, p<.05\right)$, an interaction of task level and masking strength $\left(F(1,22)=5.86, M S_{e}=1.190, p<.05\right)$ and an interaction of task level and prime-mask $\operatorname{SOA}\left(F(1,22)=5.55, M S_{e}=0.055, p<.05\right)$.

\section{Experiment 4.2A}

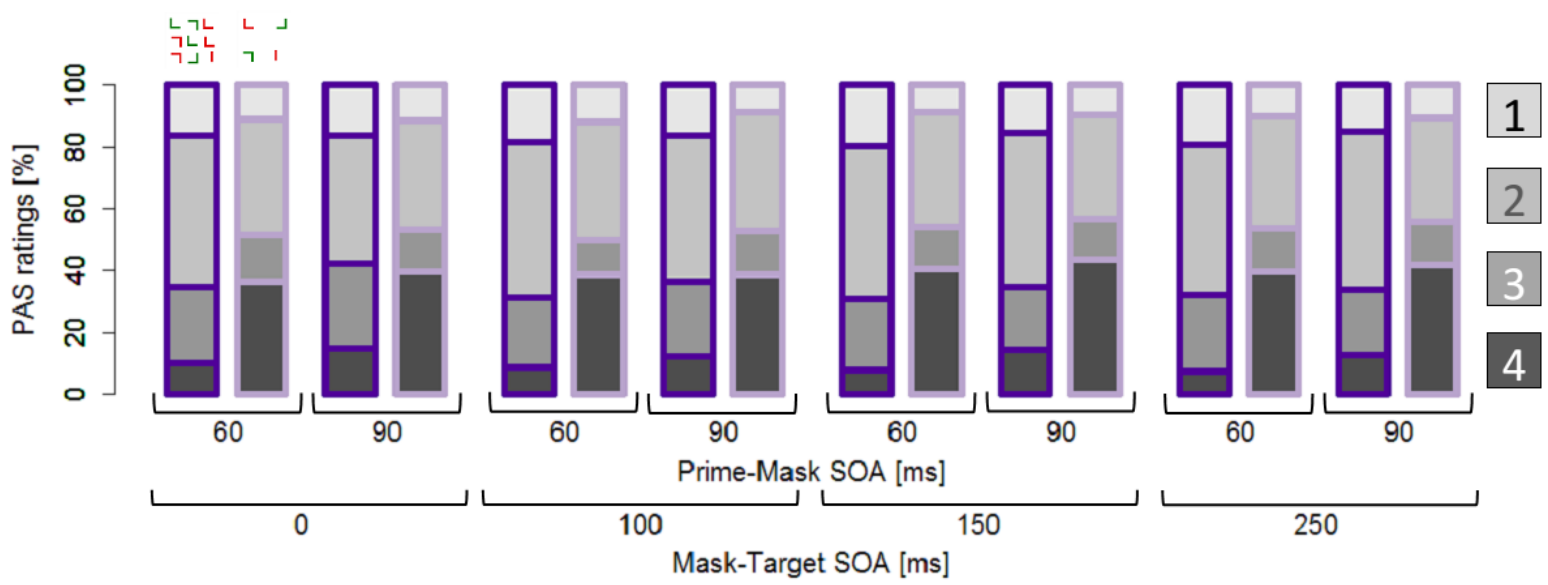

\section{Experiment 4.2B}

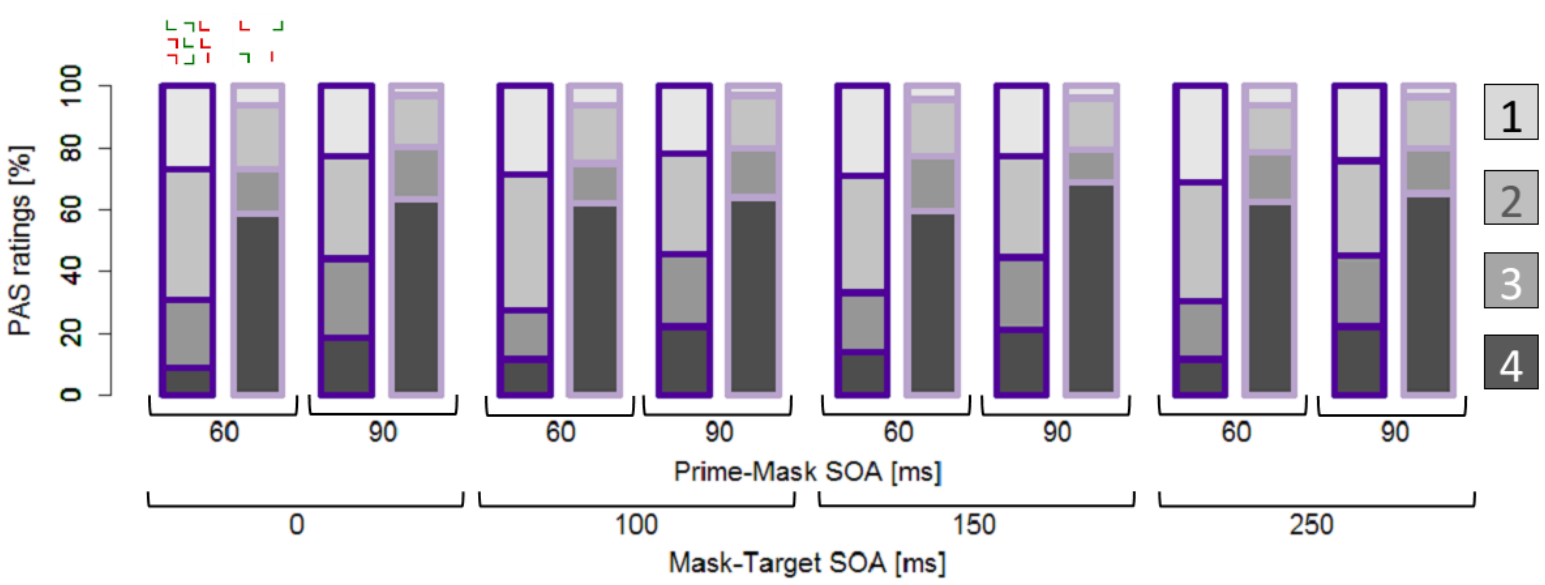

Figure 4.9. Ratings of subjective prime visibility with the PAS in Experiment 4.2 (Ramsøy \& Overgaard, 2004). Percentages of ratings with each masking strength, prime-mask SOA and masktarget SOA are displayed. Ratings: 1 = 'nothing', 2 = 'weak glimpse', 3 = 'almost clear image' \& 4 $=$ 'clear image'. 


\subsubsection{Discussion}

Experiment 4.2 was conducted to examine the impact of mask-target SOA on perceptual and semantic priming with pattern masks. Prime-mask SOA was varied again to replicate a positive impact of prime-mask SOA on priming effects (Chapter 3). Perceptual priming effects on error rates were not modulated through prime-mask SOA. The analysis of semantic ER priming effects revealed a four-way interaction of semantic congruency, masking strength, prime-mask SOA and mask-target SOA. Neither semantic nor perceptual RT priming effects were modulated significantly through prime-mask SOA. A floor effect might be responsible for the lack of evidence that would support a modulation of priming effects through prime-mask SOA. Small priming effects in all condition and the small difference between $60 \mathrm{~ms}$ and $90 \mathrm{~ms}$ prime-mask SOA support this explanation.

Perceptual and semantic priming effects on reaction times were modulated equally through mask-target SOA. Priming effects decreased with increasing mask-target SOA up to $250 \mathrm{~ms}$ in both subexperiments. No evidence for inverse priming effects or a late recovery was found. Subjective and objective prime visibility was modulated through masking strength in Experiments 4.2A and 4.2B. Strong masks reduced prime visibility more efficiently than weak masks in both subexperiments. However, masking strength had no impact on priming effects. Therefore, courses of semantic and perceptual priming effects with increasing masktarget SOA can be explained by simple decay.

\subsection{Analysis across Experiments 4.1 and 4.2}

We intended to examine the impact of masking method and task level on priming effects. For this purpose, repeated measure ANOVAs were performed on reaction times and arc-sin transformed error rates with combined data of Experiments 4.1 and 4.2 (Tables C4). 


\subsubsection{Results}

Errors. Repeated measures ANOVA revealed a main effect of congruency $(F(1,44)=$ $\left.8.50, M S_{e}=0.023, p<.01\right)$, a main effect of mask-target SOA $\left(F(3,132)=2.97, M S_{e}=0.033\right.$, $p<.05)$, an interaction of congruency and mask-target SOA $\left(F(3,132)=6.83, M S_{e}=0.021\right.$, $p<.0005)$, an interaction of task level and prime-mask SOA $\left(F(1,44)=4.36, M S_{e}=0.027\right.$, $p<.05)$, an interaction of task level and mask-target SOA $\left(F(3,132)=2.81, M S_{e}=0.033\right.$, $p<.05$ ), a three-way interaction masking method, masking strength and prime-mask SOA $\left(F(1,44)=8.91, M S_{e}=0.014, p<.005\right)$, a three-way interaction of masking method, task level and mask-target SOA $\left(F(3,132)=2.73, M S_{e}=0.033, p<.05\right)$, a three-way interaction of task level, congruency and mask-target $\operatorname{SOA}\left(F(3,132)=3.36, M S_{e}=0.021, p<.05\right)$, a fourway interaction of masking method, congruency, masking strength and mask-target SOA $\left(F(3,132)=3.00, M S_{e}=0.026, p<.05\right)$ and a five-way interaction of task level, congruency, masking strength, prime-mask SOA and mask-target SOA $\left(F(3,132)=3.08, M S_{e}=0.019\right.$, $p<.05)$.

Reaction Times. Repeated measures ANOVA revealed a main effect of congruency $\left(F(1,44)=47.07, M S_{e}=242, p<.0001\right)$, masking strength $\left(F(1,44)=18.27, M S_{e}=191\right.$, $p<.0005)$, prime-mask SOA $\left(F(1,44)=18.88, M S_{e}=160, p<.0001\right)$ and mask-target SOA $\left(F(3,132)=233.15, M S_{e}=439, p<.0001\right)$, an interaction of masking strength and primemask SOA $\left(F(1,44)=12.33, M S_{e}=99, p<.005\right)$, an interaction of masking strength and mask-target $\operatorname{SOA}\left(F(3,132)=2.95, M S_{e}=108, p<.05\right)$, an interaction of congruency and mask-target SOA $\left(F(3,132)=19.95, M S_{e}=177, p<.0001\right)$, an interaction of masking method and mask-target SOA $\left(F(3,132)=11.61, M S_{e}=439, p<.0001\right)$, an interaction of masking method and congruency $\left(F(1,44)=17.33, M S_{e}=242, p<.0005\right)$, a three-way interaction of masking method, masking strength and mask-target $\operatorname{SOA}\left(F(3,132)=2.71, M S_{e}=108\right.$, $p<.05$ ), a three-way interaction of masking method, congruency and prime-mask SOA 
$\left(F(1,44)=10.64, M S_{e}=164, p<.005\right)$, a three-way interaction of task level, congruency and mask-target SOA $\left(F(3,132)=7.84, M S_{e}=177, p<.0001\right)$, a three-way interaction of masking method, congruency and mask-target $\operatorname{SOA}\left(F(3,132)=5.56, M S_{e}=177, p<.005\right)$, a fourway interaction of masking method, congruency, masking strength and mask-target SOA $\left(F(3,132)=3.74, M S_{e}=132, p<.05\right)$, a four-way interaction of masking method, task level, congruency and masking strength $\left(F(1,44)=4.39, M S_{e}=179, p<.05\right)$ and a four-way interaction of masking method, task level, congruency and mask-target SOA $(F(3,132)=$ 5.95, $\left.M S_{e}=177, p<.001\right)$.

Prime visibility. Repeated measures ANOVA on objective prime visibility $\left(d^{\prime}\right)$ revealed a main effect of masking strength $\left(F(1,44)=83.68, M S_{e}=2.028, p<.0001\right)$, primemask SOA $\left(F(1,44)=106.45, M S_{e}=0.395, p<.0001\right)$, mask-target $\operatorname{SOA}(F(3,132)=11.65$, $\left.M S_{e}=0.218, p<.0001\right)$, task level $\left(F(1,44)=5.11, M S_{e}=9.547, p<.05\right)$ and masking method $\left(F(1,44)=20.44, M S_{e}=9.547, p<.0001\right)$, an interaction of masking strength and prime-mask SOA $\left(F(1,44)=26.61, M S_{e}=0.232, p<.0001\right)$, an interaction of prime-mask SOA and mask-target SOA $\left(F(3,132)=5.42, M S_{e}=0.227, p<.005\right)$, an interaction of masking method and masking strength $\left(F(1,44)=31.87, M S_{e}=2.028, p<.0001\right)$, an interaction of masking method and prime-mask SOA $\left(F(1,44)=9.80, M S_{e}=0.395, p<.005\right)$, an interaction of masking method and mask-target SOA $\left(F(3,132)=7.02, M S_{e}=0.218\right.$, $p<.0005$ ), a three-way interaction of masking strength, prime-mask SOA and mask-target SOA $\left(F(3,132)=3.03, M S_{e}=0.199, p<.05\right)$, a three-way interaction of task level, masking strength and prime-mask SOA $\left(F(1,44)=5.32, M S_{e} 0.232, p<.05\right)$, a three-way interaction of masking method, prime-mask SOA and mask- target SOA $\left(F(3,132)=4.03, M S_{e}=0.227\right.$, $p<.05)$ and a three-way interaction of masking method, task level and prime-mask SOA $\left(F(1,44)=6.38, M S_{e}=0.395, p<.05\right)$. Repeated measures ANOVA on subjective prime visibility (PAS-ratings) revealed a main effect of masking strength $\left(F(1,44)=66.87, M S_{e}=\right.$ 
$0.640, p<.0001)$, prime-mask SOA $\left(F(1,44)=69.44, M S_{e}=0.097, p<.0001\right)$, mask-target $\operatorname{SOA}\left(F(3,132)=19.64, M S_{e}=0.021, p<.0001\right)$ and masking method $\left(F(1,44)=10.77, M S_{e}\right.$ $=6.916, p<.005)$, an interaction of masking strength and prime-mask $\operatorname{SOA}(F(1,44)=28.58$, $\left.M S_{e}=0.030, p<.0001\right)$, an interaction of masking method and mask-target SOA $(F(3,132)=$ 3.70, $\left.M S_{e}=0.014, p<.05\right)$, an interaction of prime-mask SOA and mask-target SOA $\left(F(3,132)=3.60, M S_{e}=0.014, p<.05\right)$, an interaction of task level and masking strength $\left(F(1,44)=5.88, M S_{e}=0.640, p<.05\right)$, an interaction of masking method and masking strength $\left(F(1,44)=35.32, M S_{e}=0.640, p<.0001\right)$, an interaction of task level and masktarget SOA $\left(F(3,132)=4.49, M S_{e}=0.021, p<.05\right)$, an interaction of masking method and mask-target SOA $\left(F(3,132)=14.74, M S_{e}=0.021, p<.0001\right)$, a three-way interaction of task level, masking strength and prime-mask $\operatorname{SOA}\left(F(1,44)=6.37, M S_{e}=0.030, p<.05\right)$, a threeway interaction of masking method, prime-mask SOA and mask-target $\operatorname{SOA}(F(3,132)=$ 3.18, $\left.M S_{e}=0.014, p<.05\right)$, a three-way interaction of masking method, task level and masking strength $\left(F(1,44)=5.02, M S_{e}=0.640, p<.05\right)$ and a a three-way interaction of masking method, task level and prime-mask $\operatorname{SOA}\left(F(1,44)=4.20, M S_{e}=0.097, p<.05\right)$.

\subsubsection{Discussion}

Analyses across Experiments 4.1 and 4.2 were performed to examine the impact of masking method and task level on priming effects and prime visibility. Several interactions between congruency and masking method or task level were confirmed. Priming effects on reaction times and errors were modulated through masking method. Priming effects were generally larger with metacontrast mask than with pattern masks. Analysis of reaction times revealed a three-way interaction of masking method, congruency and prime-mask SOA. Priming effects were only modulated through prime-mask SOA when metacontrast masks were applied. Previously (Chapter 3), an impact of prime-mask SOA on priming effects was also found with pattern masks. In this respect, generally small priming effects with pattern 
masks might have caused a floor effect that prevented a detectable impact of prime-mask SOA on priming effects. RT priming effects were also modulated through masking method and mask-target SOA. A steeper decline of priming effects with increasing mask-target SOA was found with metacontrast masks. This might be due to larger priming effects with metacontrast masks than pattern masks at $0 \mathrm{~ms}$ mask-target SOA. A four-way interaction of masking method, congruency, masking strength and mask-target SOA reached significance in analysis of reaction times and errors. Prime visibility modulated the decline of priming effects with increasing mask-target SOA only when metacontrast masks were applied. Evidence for active inhibition was only provided in Experiment 4.1. Task level modulated the impact of mask-target SOA on RT priming effects and ER priming effects. The mechanism of object updating (Lleras \& Enns, 2004) can only operate with a perceptual task level and seems to be the cause of this difference between perceptual and semantic priming. Furthermore, error rates were modulated through task level in interaction with congruency, masking method, prime-mask SOA and mask-target SOA. This provides further evidence for similar mechanisms operating on both perceptual priming experiments and both semantic priming experiments, respectively. This is particularly perceivable with error effects in Experiments $4.1 \mathrm{~A}$ and $4.2 \mathrm{~A}$ (Figure 4.4 and 4.7). Reaction times were also modulated through a four-way interaction of masking method, task level, congruency and masking strength and a four-way interaction of masking method, task level, congruency and masktarget SOA. The four-way interaction with masking strength reflects different priming effects with strong and weak masks only in the semantic priming experiment with metacontrast masks. The four-way interaction with mask-target SOA attests different courses of perceptual and semantic priming effects with increasing prime-mask SOA when metacontrast masks were applied. These courses of priming effects also differ from courses of priming effects achieved with pattern masks. 
Besides the modulation of priming effects, prime visibility was affected through masking method and task level. Objective and subjective prime visibility was higher in experiments with metacontrast masks compared to experiments with pattern masks. An interaction between masking method and masking strength indicates a smaller variation of objective and subjective prime visibility through masking strength with metacontrast masks than with pattern masks. An interaction between masking method and mask-target SOA indicates an increase of objective and subjective prime visibility with increasing mask-target SOA only with metacontrast masks. A three-way interaction of masking method, prime-mask SOA and mask-target SOA was revealed in the analyses of objective and subjective prime visibility. It reflects a ceiling effect that caused a modulation of prime visibility through prime-mask SOA and mask-target SOA with metacontrast masking. Objective prime visibility was also modulated through task level. Primes were more visible in semantic priming experiments compared to perceptual priming experiments. An interaction between task level and masking strength reached significance with subjective prime visibility as well as an interaction between task level and mask-target SOA. This confirms less variation of prime visibility in experiments with a perceptual task level and a more dominant increase of prime visibility with mask-target SOA in perceptual priming experiments. A three-way interaction of task level, masking strength and prime-mask SOA indicates that objective and subjective prime visibility was less influenced by prime-mask SOA with weak masks than strong masks in semantic priming experiments (Figure 4.4 and 4.7). The impact of primemask SOA on prime visibility was not strongly modulated through masking strength in perceptual priming experiments. An interaction of masking method, task level and primemask SOA indicates different impacts of prime-mask SOA on objective and subjective prime visibility in each subexperiment. 
Although mechanisms of inhibition and object updating were not established with pattern masks, slight evidence was found that indicates an impact of similar mechanisms with each task level. According to previous studies, mechanisms of inhibition also operate with pattern masks (e.g. Klapp \& Hinkley, 2002). Furthermore, pattern masks applied in Experiment 4.2 should also operate as relevant mask with a perceptual task level (Lleras \& Enns, 2004). One problem might be the very small priming effects with pattern masks in Experiment 4.2. Another prominent difference between Experiment 4.1 and 4.2 is the application of premasks in Experiment 4.2.

\subsection{Experiment 4.3: Pattern Masking without Premasks}

We conducted another pair of experiments to examine the contribution of premasks to the specific courses of priming effects with pattern masks. We expected the masking to be less effective without premasks and enhanced the density of the strong mask.

\subsubsection{Method}

Participants. Twelve subjects participated in Experiment 4.3A (7 female, 5 male) as well as in Experiment 4.3B ( 9 female, 3 male). The subjects were from 20 to 27 years of age $(M=23.67)$ in Experiment 4.3A and from 19 to 34 years of age $(M=25.33)$ in Experiment 4.3B. Seven participants in Experiment 4.3A and five participants in Experiment 4.3B had normal vision. The remaining subjects had corrected-to-normal vision. The proceeding was equivalent to Experiments 4.1 and 4.2 .

Stimuli. The same stimuli used in Experiment 4.2 were applied in Experiment 4.3 except for the strong pattern masks. Instead of masks with a density of $0.61^{\circ}$ we used masks with a density of $0.15^{\circ}$ (Figure 4.2). This change was applied to compensate for the omission of presmasks. 
Tasks. The tasks of Experiments $4.3 \mathrm{~A}$ and $4.3 \mathrm{~B}$ were identical to the tasks of Experiments 4.1A and 4.1B, respectively.

Procedure. Proceeding in Experiment 4.3 matched proceeding in Experiment 4.1. The only difference concerned the appliance of masks. Instead of metacontrast masks, pattern masks were used.

Design and Statistical Analysis. Design and statistical analysis are equivalent to Experiments 4.1 and 4.2 .

\subsubsection{Results}

Errors. Errors occurred in $4.3 \%$ of trials in Experiment $4.3 \mathrm{~A}$. Repeated measures ANOVA revealed a main effect of masking strength $\left(F(1,11)=6.59, M S_{e}=0.003, p<.05\right.$; Figure 4.10). Subjects committed more errors with weak masks (4.8\%) compared to strong masks (3.8 \%). In Experiment $4.3 B$, errors occurred in $4.4 \%$ of trials. Repeated measures ANOVA revealed a main effect of semantic congruency $\left(F(1,11)=8.13, M S_{e}=0.035\right.$, $p<.05$; Figure 4.10). More errors were committed in incongruent trials (4.8\%) compared to congruent trials $(4.0 \%)$. The conjoint analysis of Experiments $4.3 \mathrm{~A}$ and $4.3 \mathrm{~B}$ (Table C3.1) revealed a main effect of congruency $\left(F(1,22)=4.77, M S_{e}=0.030, p<.05\right)$, a main effect of masking strength $\left(F(1,22)=4.72, M S_{e}=0.032, p<.05\right)$, an interaction of congruency and mask-target $\operatorname{SOA}\left(F(3,66)=2.77, M S_{e}=0.031, p<.05\right)$, an interaction of task level and congruency $\left(F(1,22)=4.57, M S_{e}=0.030, p<.05\right)$, an interaction of task level and masking strength $\left(F(1,22)=5.33, M S_{e}=0.032, p<.05\right)$ and a four way interaction of task level, congruency, prime-mask SOA and mask-target SOA $\left(F(3,66)=2.90, M S_{e}=0.021, p<.05\right)$. Priming effects were negative with a perceptual task level (Experiment 4.3A) at $100 \mathrm{~ms}$ mask-target SOA (-0.3\%) and 150 ms mask-target SOA (-0.8\%). Both negative effects did not reach significance $(t(11)=-0.25, p=0.80$ and $t(11)=-1.22, p=0.24$, respectively), but 

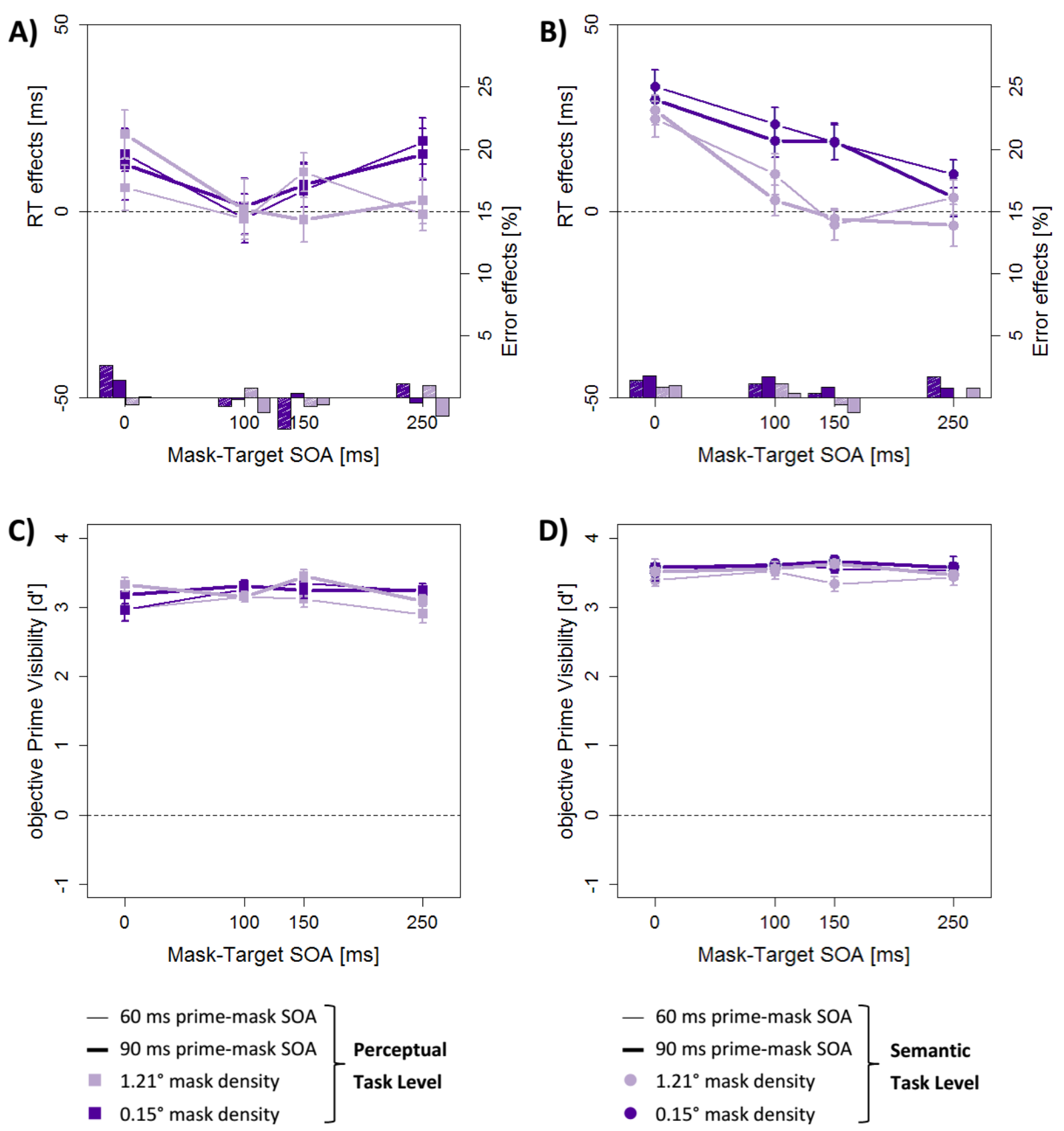

Figure 4.10. Priming effects and $d^{\prime}$-values in Experiment 4.3. Priming effects (A \& B) on reaction times (RT; lines) and errors (ER; bars) and $d$ '-values (C \& D) are displayed for each masking strength, prime-mask SOA and mask-target SOA. A \& C: Experiment 4.3A with a perceptual task level. B \& D: Experiment 4.3B with a semantic task level. Confidence intervals are in accordance with Loftus and Masson (1994). 
this inversion corresponded to a similar modulation of reaction times. Furthermore, we found an equally insignificant negative semantic priming effect (Experiment 4.3B) at $150 \mathrm{~ms}$ masktarget $\operatorname{SOA}(-0.8 \% ; t(11)=-0.89, p=0.39)$ that also matches with reaction times.

Reaction Times. In Experiment 4.3A, subjects responded on average after $433 \mathrm{~ms}$. Repeated measures ANOVA revealed a main effect of perceptual congruency $(F(1,11)=8.5$, $\left.M S_{e}=533, p<.05\right)$, masking strength $\left(F(1,11)=41.25, M S_{e}=341, p<.0001\right)$, prime-mask $\operatorname{SOA}\left(F(1,11)=12.17, M S_{e}=113, p<.01\right)$ and mask-target SOA $\left(F(3,33)=57.31, M S_{e}=\right.$ $615, p<.0001)$ and an interaction of masking strength and prime-mask SOA $(F(1,11)=7.82$, $M S_{e}=51, p<.05$; Figure 4.10). Subjects responded faster in congruent trials (430 ms) than in incongruent trials (437 ms; Figure 4.11). Reaction times decreased with increasing primemask SOA, when strong masks were applied (from $430 \mathrm{~ms}$ to $424 \mathrm{~ms}$ ). When weak masks were used, reactions times were generally slower and decreased with a smaller slope (from $440 \mathrm{~ms}$ to $438 \mathrm{~ms}$ ). Response times also decreased with increasing mask-target SOA (459 ms, $433 \mathrm{~ms}, 428 \mathrm{~ms}$ and $413 \mathrm{~ms}$ with mask-target SOAs of $0 \mathrm{~ms}, 100 \mathrm{~ms}, 150 \mathrm{~ms}$ and $250 \mathrm{~ms}$, respectively). In Experiment $4.3 \mathrm{~B}$, participants responded on average $462 \mathrm{~ms}$ after target onset. Repeated measures ANOVA revealed main effects of semantic congruency $(F(1,11)=$ $\left.17.47, M S_{e}=1001, p<.005\right)$, masking strength $\left(F(1,11)=38.71, M S_{e}=130, p<.0001\right)$ and mask-target $\operatorname{SOA}\left(F(3,33)=82.01, M S_{e}=380, p<.0001\right)$, an interaction of masking strength and mask-target SOA $\left(F(3,33)=4.41, M S_{e}=93, p<.05\right)$, an interaction of semantic congruency and masking strength $\left(F(1,11)=20.00, M S_{e}=178, p<.001\right)$, an interaction of semantic congruency and mask-target $\operatorname{SOA}\left(F(3,33)=18.02, M S_{e}=163, p<.0001\right)$ and a three-way interaction of semantic congruency, masking strength and mask-target SOA $\left(F(3,33)=4.32, M S_{e}=73, p<.05 ;\right.$ Figure 4.10). Response times decreased with increasing mask-target SOA when strong masks were used $(483 \mathrm{~ms}, 458 \mathrm{~ms}, 447 \mathrm{~ms}$ and $443 \mathrm{~ms}$ with mask-target SOAs of $0 \mathrm{~ms}, 100 \mathrm{~ms}, 150 \mathrm{~ms}$ and $250 \mathrm{~ms}$, respectively). Response times were 


\section{Experiment 4.3A}

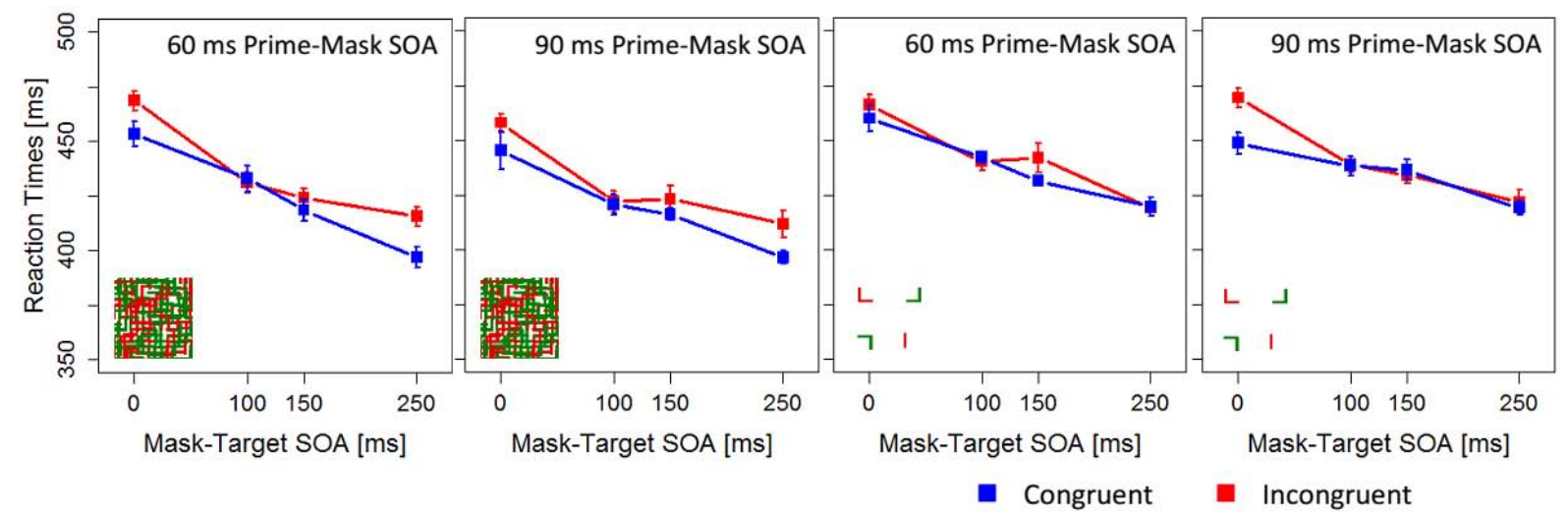

\section{Experiment 4.3B}
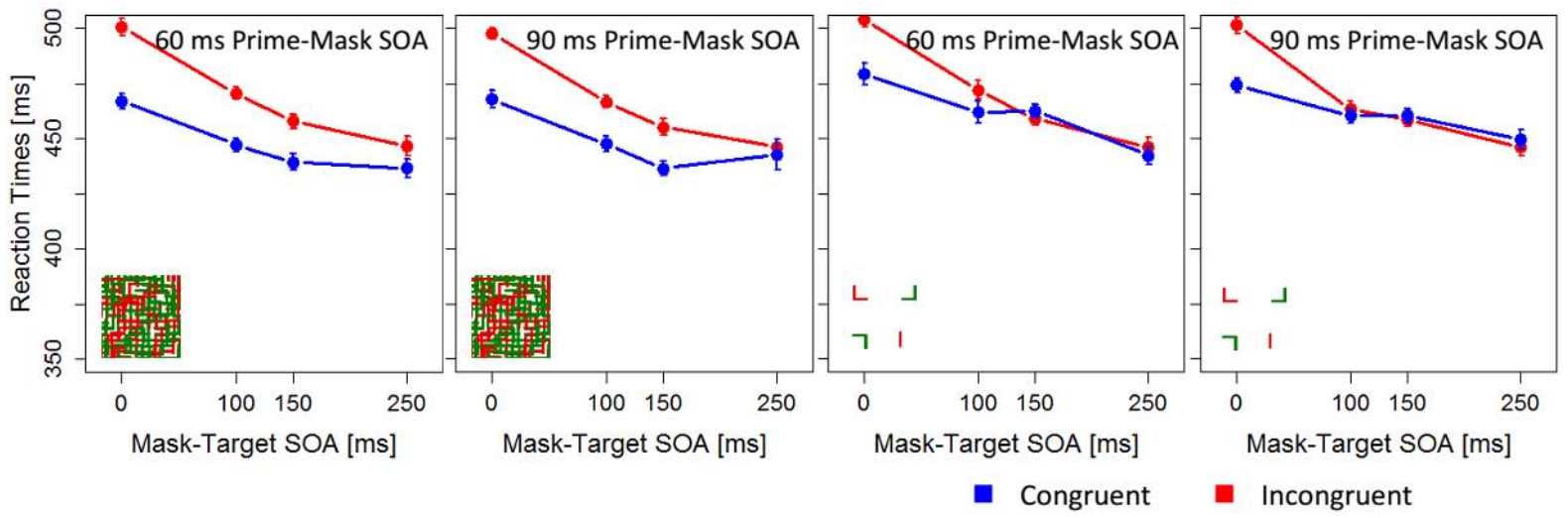

Figure 4.11. Reaction times in Experiment 4.3. Reaction times are displayed for congruent and incongruent trials with each masking strength, prime-mask SOA and mask-target SOA. Confidence intervals are in accordance with Loftus and Masson (1994).

generally slower and also decreased with weak masks, but with a different course (490 ms, $464 \mathrm{~ms}, 460 \mathrm{~ms}$ and $446 \mathrm{~ms}$ with mask-target SOAs of $0 \mathrm{~ms}, 100 \mathrm{~ms}, 150 \mathrm{~ms}$ and $250 \mathrm{~ms}$, respectively). Semantic priming effects were positive on average (13ms; Figure 4.11). When strong masks were applied, semantic priming effects decreased with increasing mask-target SOA $(32 \mathrm{~ms}, 21 \mathrm{~ms}, 19 \mathrm{~ms}$ and $7 \mathrm{~ms}$ with mask-target SOAs of $0 \mathrm{~ms}, 100 \mathrm{~ms}, 150 \mathrm{~ms}$ and $250 \mathrm{~ms}$, respectively). Semantic priming effects also decreased with weak masks, but with a more negative slope $(26 \mathrm{~ms}, 6 \mathrm{~ms},-3 \mathrm{~ms}$ and $-0.003 \mathrm{~ms}$ with mask-target SOAs of $0 \mathrm{~ms}$, $100 \mathrm{~ms}, 150 \mathrm{~ms}$ and $250 \mathrm{~ms}$, respectively). The negative semantic priming effect with weak masks and a mask-target SOA of $150 \mathrm{~ms}$ did not reach significance $(t(11)=-0.71, p=0.50)$, 
but matches the error effect. The conjoint analysis of Experiments 4.3A and 4.3B (Table C3.2) revealed a main effect of congruency $\left(F(1,22)=25.97, M S_{e}=767, p<.0001\right)$, masking strength $\left(F(1,22)=76.28, M S_{e}=235, p<.0001\right)$, prime-mask SOA $\left(F(1,22)=9.96, M S_{e}=\right.$ $121, p<.005)$ and mask-target SOA $\left(F(3,66)=132.77, M S_{e}=498, p<.0001\right)$, an interaction masking strength and mask-target $\operatorname{SOA}\left(F(3,66)=3.25, M S_{e}=181, p<.05\right)$, an interaction of congruency and mask-target SOA $\left(F(3,66)=8.31, M S_{e}=316, p<.001\right)$, an interaction of task level and masking strength $\left(F(1,22)=4.82, M S_{e}=235, p<.05\right)$, a three-way interaction of task level, masking strength and prime-mask $\operatorname{SOA}\left(F(1,22)=4.33, M S_{e}=72, p<.05\right)$, a three-way interaction of task level, congruency and mask-target SOA $\left(F(3,66)=3.76, M S_{e}=\right.$ $316, p<.05)$ and a four-way interaction of task level, congruency, masking strength and mask-target $\operatorname{SOA}\left(F(3,66)=2.87, M S_{e}=160, p<.05\right)$.

Prime visibility. In Experiment 4.3A, subjects chose correct alternatives with a mean $d^{\prime}$ of 3.19. Repeated measures ANOVA revealed a main effect of prime-mask SOA $(F(1,11)$ $=6.16, M S_{e}=0.135, p<.05 ;$ Figure 4.10). Subjects achieved a smaller mean $d$ '-value (3.12) with a prime-mask SOA of $60 \mathrm{~ms}$ than with a prime-mask of $90 \mathrm{~ms}$ (3.25). Participants rated subjective prime visibility with 3.60 on average. Repeated measures ANOVA revealed main effects of masking strength $\left(F(1,11)=8.30, M S_{e}=0.023, p<.05\right)$ and prime-mask SOA $\left(F(1,11)=7.54 M S_{e}=0.015, p<.05\right)$ and an interaction of prime-mask SOA and mask-target SOA $\left(F(1,11)=3.44, M S_{e}=0.006, p<.05\right.$; Figure 4.12). Prime visibility was rated higher with strong masks (3.63) than weak masks (3.56). Subjects also assessed prime visibility higher with a prime-mask SOA of $90 \mathrm{~ms}$ (3.62) than with a prime-mask SOA of $60 \mathrm{~ms}$ (3.63). Subjective prime visibility was also modulated through mask-target SOA with a prime-mask SOA of $60 \mathrm{~ms}(3.55,3.60,3.58$ and 3.56 with mask-target SOAs of $0 \mathrm{~ms}, 100 \mathrm{~ms}, 150 \mathrm{~ms}$ and $250 \mathrm{~ms}$, respectively) and a prime-mask SOA of $90 \mathrm{~ms}(3.60,3.64,3.63$ and 3.62 with mask-target SOAs of $0 \mathrm{~ms}, 100 \mathrm{~ms}, 150 \mathrm{~ms}$ and $250 \mathrm{~ms}$, respectively). Six participants 
detected and recognized the primes during the priming sessions. Eleven subjects noted an improvement of prime perception during the prime identification session. Only three subjects thought that prime visibility was manipulated through masking strength. In Experiment 4.3B, subjects achieved a mean $d^{\prime}$ of 3.54. Repeated measures ANOVA revealed main effects of masking strength $\left(F(1,11)=5.24, M S_{e}=0.090, p<.05\right)$ and prime-mask SOA $(F(1,11)=$ 7.53, $M S_{e}=0.053, p<.05$; Figure 4.10). Objective prime visibility was higher with strong masks (3.59) than with weak masks (3.49). It was also higher with a prime-mask SOA of $90 \mathrm{~ms}$ (3.58) than with a prime-mask SOA of $60 \mathrm{~ms}$ (3.49). Subjective prime visibility was rated with 3.88 on average. Repeated measures ANOVA revealed main effects of masking strength $\left(F(1,11)=9.67, M S_{e}=0.011, p<.01\right)$, prime-mask SOA $\left(F(1,11)=7.42, M S_{e}=\right.$ $0.008, p<.05)$ and mask-target $\operatorname{SOA}\left(F(3,33)=3.00, M S_{e}=0.004, p<.05 ;\right.$ Figure 4.12). Prime visibility was rated higher with strong mask (3.90) than with weak masks (3.85). Participants also rated prime visibility higher in trials with a prime-mask SOA of $90 \mathrm{~ms}$ (3.89) compared to trials with a prime-mask SOA of $60 \mathrm{~ms}$ (3.85). Subjective prime visibility also increased with increasing mask-target SOA $(3.85,3.87,3.89$ and 3.89 with mask-target SOAs of $0 \mathrm{~ms}, 100 \mathrm{~ms}, 150 \mathrm{~ms}$ and $250 \mathrm{~ms}$, respectively). The conjoint analysis (4.3A \& 4.3B; Table C3.3) of objective prime visibility revealed main effects of masking strength $\left(F(1,22)=9.14, M S_{e}=0.084, p<.01\right)$ and prime-mask SOA $\left(F(1,22)=12.68, M S_{e}=0.094\right.$, $p<.005)$. All subjects detected the primes during the priming sessions and only one subject was not able to describe them. Eleven subjects reported an improvement of prime perception during the prime identification session. Only one subject noted a manipulation of prime visibility through masking strength. The conjoint analysis (4.3A \& 4.3B; Table C3.4) of subjective prime visibility revealed main effects of masking strength $\left(F(1,22)=17.11, M S_{e}=\right.$ $0.017, p<.0005)$ and prime-mask SOA $\left(F(1,22)=14.68, M S_{e}=0.012, p<.001\right)$ and an interaction of masking strength and mask-target $\operatorname{SOA}\left(F(3,66)=5.18, M S_{e}=0.006, p<.005\right)$. 


\section{Experiment 4.3A}

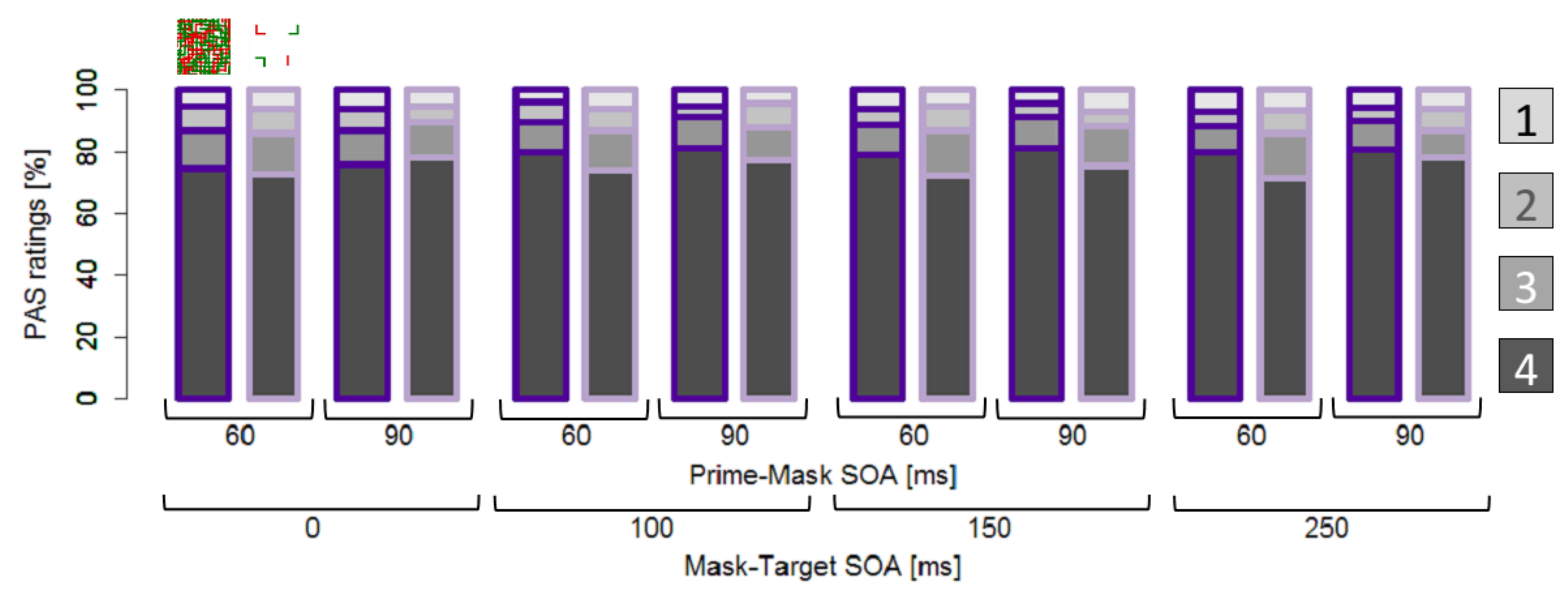

Experiment 4.3B

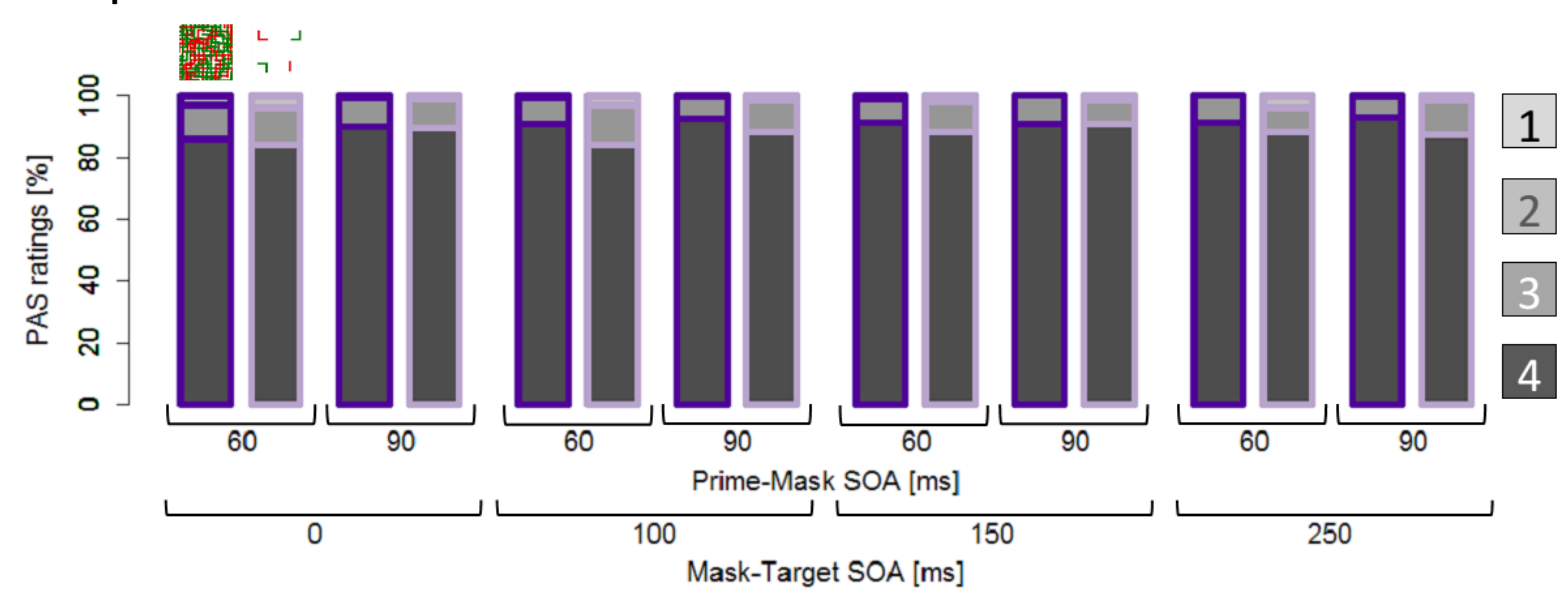

Figure 4.12. Ratings of subjective prime visibility with the PAS in Experiment 4.3 (Ramsøy \& Overgaard, 2004). Percentages of ratings with each masking strength, prime-mask SOA and masktarget SOA are displayed. Ratings: 1 = 'nothing', 2 = 'weak glimpse', 3 = 'almost clear image' \& 4 $=$ 'clear image'.

\subsubsection{Discussion}

Experiment 4.3 was conducted to examine the contribution of premasks to the specific courses of priming effects with pattern masks. Different courses of priming effects should be found in Experiments 4.1 and 4.3, if there are fundamental differences between metacontrast masks and pattern masks in modulating a decline of priming effects with increasing primemask SOA. If premasks are crucial for the emergence of differences between Experiment 4.1 
and 2, courses of priming effects in Experiment 4.3 should align with courses of priming effects in Experiment 4.2. Prime-mask SOA was varied again to replicate a positive impact of prime-mask SOA on priming effects (Chapter 3). Neither ER priming effects nor RT priming effects were significantly modulated through prime-mask SOA. Given the results of previous studies (Chapter 3) that report a modulation of priming effect through prime-mask SOA, the time lag between $60 \mathrm{~ms}$ and $90 \mathrm{~ms}$ prime-mask SOA seems to be too small to detect the expected modulation.

Only the analysis of semantic priming effects on reaction times revealed a significant modulation through mask-target SOA. Semantic priming effects declined continuously with increasing mask-target SOA. Prime visibility is again an interesting moderator of this modulation. Prime visibility was only slightly varied through masking strength. Objective prime visibility in Experiment 4.3A was not modulated by masking strength. However, subjective visibility data of Experiment $4.3 \mathrm{~A}$ as well as objective and subjective visibility data in Experiment 4.3B indicates that prime visibility was significantly lower with weak masks than with strong masks. The difference in masking efficiency of strong and weak masks is very small. Subjects recognized the correct alternative in $99 \%$ of trials with strong masks and in $98 \%$ of trials with weak masks. Although an overestimation of prime visibility is again likely, a direct comparison between Experiment 4.1, 4.2 and 4.3 is therefore problematic. However, this interesting inversion is especially relevant for interpretation of priming effects (Figure 4.10 and 4.12).

Only semantic priming effects on reaction times seem to be modulated through masking strength in Experiment 4.3. Besides a simple interaction of semantic congruency and masking strength, masking strength also modulated the interaction between semantic congruency and mask-target SOA. Semantic priming effects were smaller and declined faster with increasing mask-target SOA when weak masks were applied. At first glance, this seems 
counterintuitive and in conflict with results of Experiment 4.1. However, prime visibility was modulated reversely through masking strength in Experiments 4.1 compared to 4.3 . Therefore, strong evidence is provided that priming effects are actually modulated through prime visibility and not through a third variable like bottom-up energy of masks. Interestingly, such a small variation in prime visibility already has an impact on priming effects. Furthermore, semantic RT and ER priming effects with weak masks (low prime visibility) are numerically negative with a mask-target SOA of $150 \mathrm{~ms}$. Both effects are not significant, but similar RT and ER effects indicate that they might be meaningful. This provides evidence for mechanisms of inhibition operating after the presentation of a pattern mask (Klapp, 2005). However, mechanisms of inhibition might be modulated by prime visibility, but seem to operate even when primes are detectable.

Courses of priming effects achieved with backward pattern masks (Experiment 4.3) matched courses of priming effects achieved with metacontrast mask (Experiment 4.1) by visual inspection (Figure 4.10 and Figure 4.4). Even though perceptual priming effects were not significantly modulated through mask-target SOA, the specific u-shaped course indicates an operating mechanism of object updating (Lleras \& Enns, 2004). The data also suggests an inversion of perceptual priming effects with mask-target SOAs of 100 and $150 \mathrm{~ms}$, when reaction times and errors are taken into account. Therefore, evidence is provided that similar mechanisms are causing a decline of priming effects after the presentation of metacontrast masks and pattern masks. Data of Experiment 4.3B suggest more clearly that mechanisms of inhibition are operating than data of Experiment 4.1B. This implicitly supports the assumption that mechanisms of inhibition are also operating in Experiment 4.1B. 


\subsection{General Discussion}

The current study was conducted to resolve a confounding revealed in our metaanalysis (Chapter 2). Van den Bussche and colleagues (2009) already observed that response priming increases with increasing stimulus onset interval (SOA) between prime and target and semantic priming decreases at long SOAs. This pattern was confirmed with regards to a prime-target SOA variation in our meta-analysis. However, it was also revealed that primemask SOA is often varied in perceptual priming studies whereas mask-target SOA is more varied in the context of semantic priming (Figure 4.1). Previously (Chapter 3), evidence was provided that both perceptual and semantic priming increase with increasing prime-mask SOA. In the current study, we focused on a variation of mask-target SOA. A comparison between perceptual and semantic priming was intended. In line with previous experiments that emphasize the importance of the masking method, we applied metacontrast masks and pattern masks (e.g. Chapter 3; Lleras \& Enns, 2004). Two different prime-mask SOAs were implemented to replicate the modulation of priming effects through prime-mask SOA. Two different masking strengths were included to differentiate between strong and weak reductions of prime visibility.

We chose to apply prime-mask SOAs of $60 \mathrm{~ms}$ and $90 \mathrm{~ms}$. This decision was based on two factors. First, strong priming effects were expected to manifest after $60 \mathrm{~ms}$ prime-mask SOA. Second, prime visibility was expected to reach its maximum even with strong metacontrast masks after $90 \mathrm{~ms}$ prime-mask SOA. Unfortunately, this left little room for variation and the modulation of priming effects was hardly replicated in the current study. Only priming effects on reaction times in Experiment 4.1A were significantly modulated through prime-mask SOA. Besides the small time lag between both prime-mask SOA, priming effects were generally small floor effects might have had an impact. Strong 
indications for this assumption were found in Experiment 4.1B. In this experiment, modulation through prime-mask SOA increases with weak masks (i.e. larger priming effects). Despite the lack of additional evidence for a modulation of perceptual and semantic priming effects through prime-mask SOA, previous research strongly indicates that prime-mask SOA has an impact on priming effects (Chapter 3).

Modulation of prime visibility through masking strength was partly successful. In Experiment 4.1, subjective prime visibility was generally lower with strong masks than with weak masks. Objective prime visibility was also modulated through masking strength in Experiment 4.1B. In Experiment 4.1A, the main effect of masking strength did not reach significance. However, an interaction between masking strength and prime-mask SOA with generally high objective prime visibility indicated the impact of a ceiling effect. In line with a possible overestimation of objective prime visibility (Vermeiren \& Cleeremans, 2012), a successful modulation of prime visibility is likely even in Experiment 4.1A. Subjective and objective prime visibility was clearly modulated through masking strength in Experiment 4.2. In Experiment 4.3, only backward pattern masks were applied and prime visibility was hardly modulated through masking strength as expected. Objective prime visibility of Experiment 4.3A was not modulated by masking strength. However, subjective visibility data of Experiment $4.3 \mathrm{~A}$ as well as objective and subjective visibility data of Experiment 4.3B indicates that prime visibility was significantly lower with weak masks than with strong masks. The reversed modulation of prime visibility through masking strength in Experiment 4.3 should be kept in mind with regards to a modulation of priming effects through prime visibility. Turvey (1973) already showed that mask intensity modulates peripheral forward masking, but not peripheral backward masking. The term "peripheral mechanisms" refers to encoding mechanisms. In this context, the reversal seems plausible. Furthermore, prime visibility was modulated through mask-target SOA in Experiment 4.1. This is important 
evidence that the peripheral targets also reduce the visibility of priming. A potential masking effect of targets was also emphasized in our meta-analysis (Chapter 2). Studies that applied the same targets in the priming task and the prime identification task reported significantly smaller $d$ '-values (target-target similarity). Obviously, it is important to keep stimuli presentation very similar between priming sessions and prime identification session.

The main focus of the current study was set on a possible modulation of priming effects through mask-target SOA. Prime visibility might interfere with this modulation and was varied for this reason. Except for Experiment 4.3A, priming effects were modulated through mask-target SOA in all experiments. However, the numerical variation of priming effects on reaction times and errors with mask-target SOA was very characteristic in Experiment 4.3A and also very similar to Experiment 4.1A. Unfortunately, error effects and reaction time effects cannot be totaled up in a simple way.

Generally, priming effects declined with increasing mask-target SOA. However, very characteristic courses of priming effects emerged in different experiments. In Experiment 4.2, forward and backward pattern masks were applied. Priming effects were generally small and declined irrespectively of variations in prime-mask SOA or masking strength. No evidence for active processes was found as inverse priming effects were not identified. A different pattern occurred in Experiments 4.1 and 4.3. Courses of priming effects with increasing mask-target SOA were very similar between 4.1A and 4.3A (perceptual priming) as well as 4.1B and 4.3B (semantic priming). Initially, perceptual priming effects decreased very fast. Especially in Experiment 4.1A, indications for an inversion of priming effects after $100 \mathrm{~ms}$ mask-target SOA were provided. Afterwards, priming effects revived, but not to their full extent. At least in Experiment 4.1A, prime visibility had an impact on perceptual priming effects in interaction with mask-target SOA. However, the rapid decline of priming effects 
occurred irrespectively of masking strength. The decline of semantic priming effects (4.1B and 4.3B) was more continuous and seems to rely on poor prime visibility to a high degree.

Different patterns of priming effects in perceptual and semantic paradigms are in line with the assumption of different mechanisms that contribute to the decline of priming effects with increasing mask-target SOA. First, a simple decay of priming effect might occur in all experiments. Second, an active process of inhibition can lead to a decline of priming effects and can also cause an inversion of priming effects (Eimer \& Schlaghecken, 2002; Klapp, 2005; Klapp \& Hinkley, 2002; Sumner, 2007). This mechanism can also operate in all experiments, but relies on low prime visibility. Maybe the initial priming effect ( 0 ms masktarget SOA) has to be pronounced to trigger the mechanism and its impact is therefore less obvious in Experiment 4.2. Third, a perceptual interaction of prime and mask in the sense of object updating might cause the rapid decline of perceptual priming effects in Experiments 4.1A and 4.3A (Lleras \& Enns, 2004). The u-shaped course of perceptual priming effects with increasing mask-target SOA and the inversion around 100/150 ms mask-target SOA is typical for experiments with relevant masks (e.g. Schlaghecker \& Eimer, 2000; Lleras \& Enns, 2004). A mask is relevant when both prime alternatives are included in the mask. After the presentation of the prime, parts of the mask are less salient when they are similar to the prime. After the presentation of a green prime, green parts are less salient in the mask than red parts and red targets benefit. This mechanism cannot occur in our semantic paradigm, because not all primes are included as a whole in the mask. Therefore, this mechanism can only modify perceptual priming effects. Although priming effects are very small and evidence is not strong, reaction time data and error data might also indicate a small impact of this mechanism in Experiment 4.2A (Figure 4.7).

Nevertheless, the forward pattern masks in Experiment 4.2 seem to intensively impair priming effects. Previously (Chapter 3), we proposed that pattern masks disturb the 
feedforward sweep, whereas metacontrast masks reduce prime visibility by interfering with recurrent processing. Feedforward sweep and recurrent processing are distinguished within the framework of a theory of consciousness proposed by Lamme and Roelfsema (2000). The authors propose that consciousness relies on recurrent processing. Unconscious priming effects are therefore based on processing within the feedforward sweep. In line with Breitmeyer (2014), we emphasized the fertility of examining different masking methods as an access to conscious processing (Chapter 3). Differences between pattern masks and metacontrast masks seemed to be crucial. However, the current study narrows it down further. Forward pattern masks seem to disturb the feedforward sweep in contrast to backward masks. This conclusion would be fostered further by replicating Experiment 3.3 without forward pattern masks. However, prime visibility was very high without forward pattern masks in Experiment 4.3 and variations of prime visibility with masking strength were minimal. Nevertheless, these variations still affected priming effects. It should therefore be considered whether more extensive changes should be implemented. Masking of colored stimuli with pattern masks seems to be a difficult aspect. Experiment 3.3 could be replicated with a different perceptual task. A better reduction of prime visibility would be possible, but comparability between experiments would be limited.

In contrast to previous experiments (Chapter 3), differences between perceptual and semantic priming were found in the current study. Different courses of priming effects in increasing mask-target SOA were revealed. However, these differences can be explained by the mechanism of object updating that only facilitates incongruent target processing when relevant masks are applied (Lleras \& Enns, 2004). In our design, masks are only relevant with perceptual priming. Therefore, the differences between perceptual and semantic priming are probably not based on different levels of processing, but can be attributed to differences between the specific tasks in interplay with our masks. Whether perceptual and semantic 
priming with these stimuli actually differ in level of processing, is still a question open for further research. This point of view is for instance challenged by the action trigger account by Kunde, Kiesel and Hoffmann (2003; Kiesel, Kunde \& Hoffmann 2007b). In the next chapter, we address this question in an experiment with electroencephalography (EEG).

\subsubsection{Conclusion}

The current study provides evidence that mostly similar mechanisms modulate perceptual and semantic priming effects with variations of temporal parameters. An increase of priming effects with prime-mask SOA was not replicated. However, previous research strongly indicates such a modulation (Chapter 3). The absence of significant interactions between congruency and prime-mask SOA in the current study can be attributed to generally small priming effects and a small variation of prime-mask SOA in interplay with floor effects. Priming effects generally declined with increasing mask-target SOA, but courses of priming effects differed between perceptual and semantic priming. Different mechanisms are thought to contribute differently to the modulation of perceptual and semantic priming effects with increasing mask-target SOA in our experiments. Three mechanisms are thought to modify priming effects with increasing prime-mask SOA: 1) a simple decay, 2) active processes of inhibition with low prime visibility (e.g. Klapp, 2005) and 3) active processes of object updating with a perceptual task level (Lleras \& Enns, 2004). 
4 | Approaching Consciousness with Masked Priming 


\section{Perceptual and Semantic Priming without Response Association: Electrophysiological Differences}

\subsection{Abstract}

The current study compares perceptual and semantic priming without response association. Crucially, identical stimulation was applied in the perceptual and the semantic priming experiment to avoid confounding factors (cf. Chapter 2). The designs of the experiments allowed examinations of behavioral priming effects (reaction times, errors) and electrophysiological priming effects (target-locked event-related potentials) without response execution. Both, perceptual and semantic priming effects on reaction times were found, but differed in magnitude. Smaller semantic priming effects can be attributed to higher level processing with activations being more extended, less intensive and more variable. Perceptual congruency modulated P2 amplitude and P3 amplitude. These components are associated with repetition suppression, transdimensional feature detection (P2) and memory processes (P3) involved in updating of representations (e.g. Brang et al., 2008; Freunberger et al., 2007; Hsu, Hämäläinen \& Waszak, 2014; Luck \& Hillyard, 1994; Polich, 2007). Semantic congruency was reflected in a N400-like effect with a delayed latency. Perceptual ERP effects were observed earlier and with different distributions than semantic ERP effects. This pattern suggests different mechanisms being involved in the emergence of perceptual and semantic priming effects. 


\subsection{Introduction}

Masked priming is a method frequently applied to investigate unconscious processing and functional principles and structures of certain information-processing systems (e.g. Bonin, Fayol \& Peereman, 1998; Dehaene, Changeux, Naccache, Sackur \& Sergent, 2006; Dell'Acqua \& Grainger, 1999; Rastle \& Brysbaert, 2006; Vorberg, Mattler, Heinecke, Schmidt \& Schwarzbach, 2003). Different kinds of masked priming paradigms are distinguished based on a long tradition. Semantic priming is characterized though a semantic relation between prime and target. Semantic processing in general and the possibility of semantic processing without consciousness has been an issue of research for a long time (e.g. Marcel \& Forrin, 1974). Phonological priming (e.g., Rastle \& Brysbaert, 2006) and orthographical priming (e.g., Bonin, Fayol \& Peereman, 1998) are very closely related to semantic priming (Chapter 2). As indicated by the labels, primes and targets are related through similar pronunciation in phonological priming paradigms and related trough similar spelling in orthographical priming paradigms. Semantic, phonological and orthographical priming were already intensively examined with electrophysiological methods (EEG; e.g. Kutas, 1997). Several event-related potentials (ERP) were associated with language processing. Especially, the N400 is thought to reflect semantic processing and was discovered early (Kutas \& Hillyard, 1980). It is characterized through a negative deflection associated with a semantic violation or mismatch.

Semantic priming is distinguished from response priming (e.g. Klotz \& Wolff, 1995; Vorberg et al., 2003), repetition priming (e.g. Dehaene et al., 2001; Forster \& Davis, 1984; Logan, 1990) and perceptual priming (Wiggs \& Martin, 1998) in a long tradition. In contrast to semantic priming effects, response priming effects as well as repetition priming effects and perceptual priming effects are thought to emerge on lower levels of processing and it is 
largely accepted that prime awareness is not necessary for these processes (Kouider \& Dehaene, 2007). Semantic priming effects are thought to occur on higher levels of processing and it is still being debated whether semantic priming can occur without and independently of prime visibility (e.g. Eriksen, 1960; Dixon, 1971; Holender, 1986; Greenwald, 1992, Kouider \& Dehaene, 2007). Differences between perceptual and semantic priming effects became also apparent in a meta-analytic examination of 84 studies (Chapter 2). In accordance with the assumption that perceptual priming effects emerge on a lower level of processing than semantic priming effects, perceptual priming effects were generally larger than semantic priming effects. Furthermore, meta-analytic modelling suggested that semantic priming effects might increase with increasing prime-mask SOA (stimulus onset asynchrony), whereas perceptual priming seemed independent of prime visibility. However, a confounding of task level (perceptual, abstract and semantic) and masking method (pattern masking and metacontrast masking) called this finding into question. Subsequent experiments revealed that priming effects were indeed independent of prime visibility with metacontrast masks and increased with increasing prime visibility when pattern masks were applied (Chapter 3). Task level did not moderate this relation. However, perceptual priming effects were again larger than semantic priming effects. Four possible origins of this pattern were proposed. First, this might be an indication of perceptual and semantic priming being located on lower and higher levels of processing, respectively. Second, there might be a difference in saliency between perceptual and semantic attributes. Third, semantic response priming might be based on activations of action triggers (Kiesel, Kunde \& Hoffmann, 2007; Kunde, Kiesel \& Hoffmann, 2003). Fourth, a systematic difference between primes and targets in color might induce smaller semantic priming effects (Chapter 2). Differences between perceptual and semantic priming were also found with regards to a modulation trough mask-target SOA (Chapter 4). Priming effects generally deceased with increasing mask-target SOA. However, perceptual 
priming effects deceased rapidly at first and recovered after $100 \mathrm{~ms} / 150 \mathrm{~ms}$. Semantic priming effects decreased continuously with increasing mask-target SOA. Yet, these differences seem to be based on the specific stimuli that were used. At least, three mechanisms were thought to modulate priming effects with increasing mask-target SOA: 1) a simple decay, 2) an active mechanism of inhibition with low prime visibility (Klapp, 2005) and 3) an active mechanism of object updating with relevant masks (Lleras and Enns, 2004). Masks were only relevant with a perceptual task level in this study and therefore a difference between perceptual and semantic task level emerged. Consequently, one might wonder whether there are substantial differences between perceptual and semantic priming. Several studies already suggested that different mechanisms operate with perceptual and semantic priming, respectively (e.g. Carr \& Dagenbach, 1990; Martens, Ansorge \& Kiefer, 2011). However, our current design allows for a comparison of perceptual and semantic priming with the exact same stimulation. This is very beneficial considering that disregarded confounding factors might be reasons for differences (Chapter 2 - 4).

In the current study, we attempted to compare perceptual and semantic priming with electroencephalography (EEG). Previously, we focused on perceptual and semantic response priming (Chapter $3 \& 4$ ). After respective prime processing, a preactivation of a motor response seems to contribute mainly to these priming effects (Dehaene et al., 1998). Perceptual and semantic response priming seem to share this mechanism. As we were interested in differences between perceptual and semantic priming, we wanted to focus on a possible support of target processing through the prime (i.e. priming without response association; Chapter 2). If primes are differently processed in perceptual and semantic priming, this could be reflected in a different time course and different areas being involved. For instance, perceptual priming effects may be based on early processing in the visual 
cortex. Semantic priming effects may be based on later processing with involvement of Wernicke's area (Kutas, 1997).

Examination of priming without response association requires disentangling of task and congruency and this has to be done with caution. In our previous studies, task and congruency were closely matched (Chapter $3 \& 4$ ). In some experiments, subjects had to respond to green and red targets with one or another button. Trials with green primes and green targets as well as red primes and red targets were defined as congruent trials. In other experiments, subjects had to respond to number targets and letter targets with one or another button. Trials with number primes and number targets as well as letter primes and letter targets were defined as congruent trials. However, in all experiments primes and targets were always numbers and letters in green and red. Therefore, besides the relevant congruency (i.e. effect type matches task level) an irrelevant congruency effect could be examined. The reanalysis of the six experiments revealed no perceptual priming effects on reaction times with a semantic task level and no semantic priming effects on reaction times with a perceptual task level (Appendix IV). A possible explanation of this pattern is provided by the attentional sensitization model by Kiefer and Martens (2010). The authors propose that a sensitization of task-congruent processing pathways is necessary for unconscious processing. This sensitization is provided through top-down attention.

The current design was developed to examine priming without response association and nevertheless guarantee attentional sensitization of relevant pathways. We chose to apply a go/no-go task. All trials of main interest were no-go trials as the EEG response was to be measured without interference of response activation. Two experiments were conducted. In one experiment, subjects performed a perceptual categorization. In the other experiment, participants fulfilled a semantic categorization. The exact same no-go trials with green and red numbers and letters were presented to the subjects of both experiments. However, go 
trials differed between experiments. In one experiment, cyan and magenta targets were used as go targets. In the other experiment, subjects were asked to respond to small numbers and letters late in the alphabet that were applied in red and green. We chose to combine small numbers with letter late in the alphabet to minimize the possible impact of a SNARC effect (Spatial-Numerical Association of Response Codes effect; Dehaene, Bossini \& Giraux, 1993; Gevers, Reynvoet, \& Fias, 2003).

The chosen design has several advantages. First, we can compare perceptual and semantic priming effects on event-related potentials without response execution with the exact same stimulation (no-go trials). Second, we can compare relevant and irrelevant priming effects with the exact same stimulation across experiments and therefore assess the impact of attentional sensitization. Third, perceptual and semantic priming effects on reactions times (RT) and errors (ER) can also be compared as well as relevant and irrelevant priming effects on RTs and ERs (go trials). Although go targets and no-go targets differ, they are quite similar (see "Stimuli"). Therefore, ERP effects (no-go trials) and behavioral priming effects (go trials) are comparable.

The main aim of the current study is to reveal possibly differed mechanisms which contribute to the occurrence of perceptual and semantic priming effects. Previously, perceptual priming effects appeared to be larger than semantic priming effects in a behavioral study (Chapter 3). One benefit of the current design is that not only ERP effects but also behavioral priming effects can be assessed. In the current study, we hoped to replicate that relevant perceptual priming effects on RTs and ERs are larger than relevant semantic priming effects on RTs and ERs. Finding this pattern would forge a bridge to previous research. Furthermore, two different prime-mask SOAs were applied (60 ms and $120 \mathrm{~ms}$ ). Previously, priming effects increased with increasing prime-mask SOA (Chapter 3). A replication of the modulation of relevant priming effects through prime-mask SOA is expected. Besides these 
relevant priming effects, irrelevant priming effects can be examined. However, irrelevant priming effects are not expected to occur on the basis of previous research and the attentional sensitization model (Appendix IV; Kiefer and Martens, 2010).

Concerning electrophysiological data, two alternative patterns could respectively attest similar or different mechanisms at work with perceptual and semantic priming. Semantic priming effects could occur on the same ERP components with the same topographical distribution as perceptual priming effects. The difference in magnitude between perceptual and semantic priming effects on RTs might be reflected by a similar difference in magnitude between ERP amplitude effects. Alternatively, effects on different components and different topographical distributions would be indications of a different time course and different areas being involved in emergence of perceptual and semantic priming effects. Several hypotheses can be formulated with regards to specific components and distribution. Generally, perceptual processing is thought to be low level processing whereas semantic processing occurs on a higher level (Kouider \& Dehaene, 2007). Therefore, perceptual priming effects might occur on early components. A modulation of the P2 component through perceptual congruency would be in line with studies interpreting P2 as a correlation of repetition suppression (e.g. Freunberger, Klimesch, Doppelmayr \& Höller, 2007). It is thought to reflect perceptual processing (Brang, Edwards, Ramachandran \& Coulson, 2008; Kranczioch, Debener \& Engel, 2003), feature detection and recognition processes (Dunn, Dunn, Languis \& Andrews, 1988, Evans \& Federmeier, 2007; Luck \& Hillyard, 1994) and distractor feature suppression (Phillips \& Takeda, 2009). The P2 component is expected to be larger in incongruent trials than in congruent trials (e.g. Brang et al., 2008; Freunberger et al., 2007). Another component that might be modulated through perceptual congruency is the P3. The P3 component consists of two subcomponents which are associated with different, but linked, processes (reviewed in Polich, 2007). P3a reflects 
stimulus-driven frontal attention that is engaged to support context updating (P3b) through inhibition of on-going activity. Task relevant stimulus information is passed on to temporalparietal areas. P3b originates from these areas and reflects context updating which is associated with memory processes. Kok (2001) proposes that the P3 reflects activation in an event-categorization network and is associated with response decisions. It is expected that the P3 amplitude is larger in congruent trials than in incongruent trials (Hillyard \& Münte, 1984; Martens et al., 2011; Zovko \& Kiefer, 2013). The N400 component is thought to reflect semantic processing (reviewed in Kutas, 1997). Therefore it might be modulated through semantic congruency. N400 is a negative deflection associated with a semantic violation or mismatch. It is maximal at posterior sites of the right hemisphere (Holcomb, 1993; Luck, 2005; Neville, Mills \& Lawson, 1992; Van Petten, 1993; Van Petten \& Luka, 2005). The N400 should be more negative in incongruent trials than in congruent trials. This pattern would confirm semantic processing of prime and target.

\subsection{Method}

Participants. Twenty students from the University of Göttingen participated in Experiment 5A (10 female, 10 male) as well as in Experiment 5B (15 female, 5 male) with ages between 18 and $32(M=23.6)$ and 19 and $29(M=22.6)$, respectively. After two sessions of overall 3.5 hours, the participants received 25 Euro or course credits for participation. All subjects were right-handed by self-report. Twenty participants (A: 10; B: 10) had a normal and Twenty corrected-to-normal vision. Ishihara color plates were used to verify a normal color perception.

Stimuli. The stimuli were presented on a CRT-monitor with a refreshing rate of 100 Hz. The background color was white. A fixation point $\left(0.03^{\circ}\right.$ width $\times 0.03^{\circ}$ height of visual angle) and a fixation cross $\left(0.15^{\circ} \times 0.15^{\circ}\right)$ appeared in black color. Numbers and letters in 
different colors were used as prime and target stimuli $\left(0.76^{\circ} \times 0.83^{\circ}\right.$; see Figure 5.1$)$. The depiction of prime and target stimuli was based on the one achieved by a seven-segment display in order to assure equivalent masking with one metacontrast mask. The letters A, G, P and $\mathrm{U}$ and the numbers 2, 4, 8 and 9 served as primes in Experiments $5 \mathrm{~A}$ and 5B. The letters $\mathrm{C}, \mathrm{E}$ and $\mathrm{F}$ and the numbers 5, 6 and 7 were used as no-go targets in Experiments 5A and 5B. Primes and no-go targets were presented in red and green. Different go targets were applied in Experiment 5A and Experiment 5B. In Experiment 5A, go and no-go targets differed in color. No-go targets were presented in red and green. Go targets were displayed in magenta and cyan. In Experiment 5B, the letters $\mathrm{H}, \mathrm{J}$, and $\mathrm{L}$ and the numbers 0,1 and 3 were used as go targets and all stimuli were presented in red and green. Luminance was constant for all primes and targets $\left(24.6 \mathrm{~cd} / \mathrm{m}^{2}\right)$. Single primes were presented in the center of the screen. Targets were duplicated and presented at a distance of $2.27^{\circ}$ of visual angle left and right from center. The metacontrast masks were constructed by placing eight layers in altering color (red and green) around the the 8-shaped section surrounding the primes and four layers within the holes of the 8-shaped section (Figure 5.1). Each layer was as thick as the stroke width of the written letters and numbers $\left(0.08^{\circ}\right.$ of visual angle $)$. Therefore the metacontrast masks subtended visual angles of $1.97^{\circ} \times 2.05^{\circ}$. Six different color patterns were achieved by coloring the outer layers two of four quarters of the mask in green and the other outer layers in red. As an error signal, a sinusoidal tone of $440 \mathrm{~Hz}$ was presented for $150 \mathrm{~ms}$. A grey line drawing of an eye was presented between trials to indicate appropriate moments for blinking (Figure 5.2).

Tasks. Both experiments involved two kinds of tasks. In the first session subjects had to fulfill a go/no-go reaction time task by responding to go targets with one of their index fingers (balanced across subjects) on a response button box. In Experiment 5A, participants responded to magenta and cyan targets. In Experiment 5B, subjects responded to the letters 
$\mathrm{H}, \mathrm{J}$, and $\mathrm{L}$ (late in the alphabet) and the numbers 0,1 and 3 (small numbers). These go targets were chosen to compensate a possible influence of the SNARC effect (Dehaene, et al., 1993). Small numbers are associated with the left side and letters late in the alphabet are associated with the right side (Dehaene et al., 1993; Gevers et al., 2003). In the last session, subjects had to focus on prime stimuli. After each trial, they first reported the quality of their subjective perception of the stimulus using the perception awareness scale (PAS) developed by Ramsøy and Overgaard (2004). With four fingers of their left hand they reported if they saw "nothing", “a weak glimpse (without recognition)", "an almost clear image (with recognition)" or "a clear image". After a response was given, two alternatives appeared on the screen. The subjects had to decide which alternative matched with the prime by pressing the left key with index finger or the right key with the middle finger of the right hand. In Experiment 5A, alternatives matched in shape and differed in colors whereas they differed in shape and matched in color in Experiment 5B.
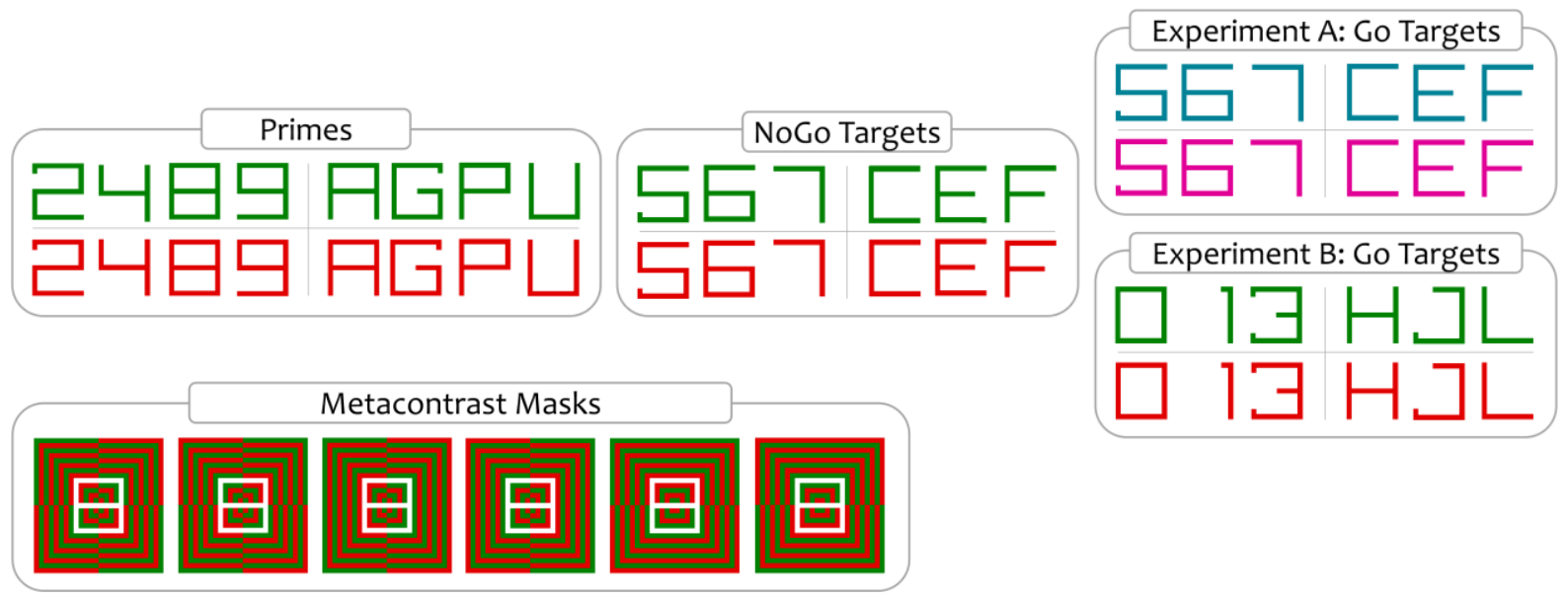

Figure 5.1. Stimuli applied in Experiments 5A and 5B. Primes and targets are based on a sevensegment display to ensure similar masking of all primes by metacontrast masks. Identical primes and no-go targets were used in both experiments. Go targets differed between experiments. In Experiment 5A, go and no-go targets differed in color (warm vs. cold). In Experiment 5B, they differed semantically (early vs. late in the alphabet/large vs. small numbers). Metacontrast masks were applied in Experiment 5A and Experiment 5B 
Procedure. Two experimental sessions were held on different days. In the first session, EEG, reaction times and errors were recorded. In the second session, PAS ratings and responses to the forced-choice task were collected. In the beginning of the first session, the proceedings were explained to the subjects, demographic data was collected and they were tested for normal vision and color perception. The participants were instructed to focus on the center of the screen during the whole presentation, even though the targets appeared beside fixation. They were advised to response as fast and accurate as possible and refrain from blinking, except in between trials and after responding. After a short demonstration of 8 trials with no reaction time window and a training block of 80 trials, the subjects received 960 experimental trials divided into 12 runs. The sequence of one trial is depicted in Figure 5.2. A fixation cross was presented for $650 \mathrm{~ms}$ and marked the beginning of each trial. Subsequently, the prime was displayed for $20 \mathrm{~ms}$ in the center of the screen. Afterwards, a fixation point was presented for $40 \mathrm{~ms}$ or $100 \mathrm{~ms}$. In one half of the trials, a prime-mask SOA of $60 \mathrm{~ms}$ was realized and in the other half of the trials a prime-mask SOA of $120 \mathrm{~ms}$. The mask was presented in the center of the screen for $100 \mathrm{~ms}$ and shared the on- and offset with the targets. A response window of $1000 \mathrm{~ms}$ started with target onset. The intertrial interval started immediately after a correct response and lasted between 800 and $1200 \mathrm{~ms}$. In case of an error, the presentation of an error tone $(440 \mathrm{~Hz} ; 150 \mathrm{~ms})$ and an extension of the intertrial interval for $1000 \mathrm{~ms}$ were interposed. After each run, participants received the opportunity for a self-paced break. In the last session, subjects received the same 960 trials in different order, again divided into 12 runs. The trials differed merely after the presentation of the targets. Participants had unlimited time to evaluate their prime perception with the PAS and to choose an alternative afterwards. To get comfortable with the new task, eight trials with an extended prime presentation $(150 \mathrm{~ms})$ were presented at the beginning of the session. 


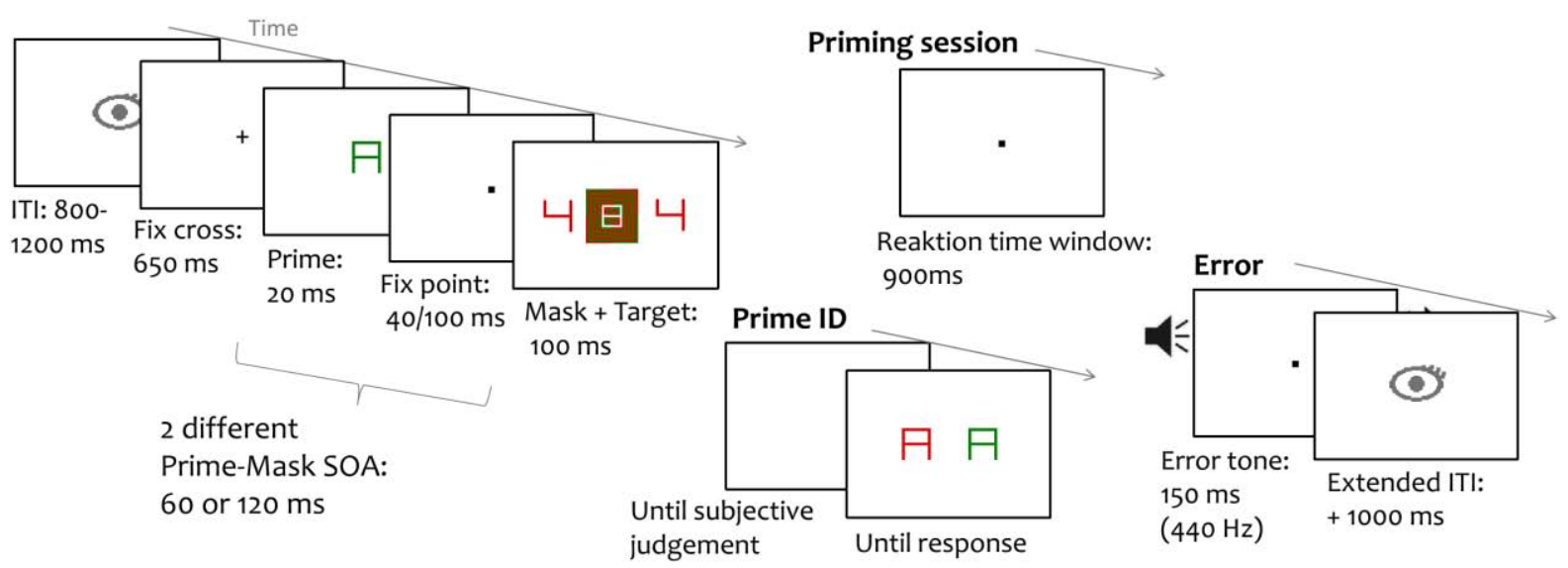

Figure 5.2. The sequence of a trial used in Experiments 5A and 5B.

Design and Behavioral Analysis. With 4 types of primes ( 2 colors $\times 2$ categories $), 8$ types of targets (4 go, 4 no-go) and 2 prime-mask SOA (60 and $120 \mathrm{~ms}$ ), 64 conditions were realized. Each subject received 768 experimental trials in each session, with specific primes and targets assigned randomly. Additionally, 192 trials were presented without target to prune target ERPs of overlapping activity induced by prime and mask. Each subject of Experiment 5B received a trial sequence identical to one subject of Experiment 5A. Error and post-error trials as well as the fastest and two slowest reaction times of each condition were excluded in estimation of mean RTs. Repeated measures ANOVAs were performed with RTs and arc-sin transformed error rates. SOA and congruency were included as factors. Note that primes and go targets were never of the exact same color. Trials were treated as perceptually congruent when a red prime was followed by a magenta target as well as when a green prime was followed by a cyan target. Four data sets were examined: 1) perceptual task level (Experiment 5A) and perceptual congruency (based on color), 2) perceptual task level (Experiment 5A) and semantic congruency (based on category), 3) semantic task level (Experiment 5B) and perceptual congruency and 4) semantic task level (Experiment 5B) and semantic congruency. Prime visibility was analyzed in line with signal detection theory 
(Macmillan \& Creelman, 1991). D'- values were calculated with the hit rates (H) and falsealarms rates $(\mathrm{F})$ of the two-alternative choices:

$$
d^{\prime}=z(H)-z(F)
$$

with $\mathrm{z}$ as the inverse of the normal distribution function. Using repeated measures ANOVAs, $d$ '-values and subjective visibility judgments were analyzed with regards to possible manipulations through prime-mask SOA and task level. Mauchly's sphericity test and Greenhouse-Geisser correction for violations of sphericity were applied (Mauchly, 1940; Greenhouse \& Geisser, 1959).

EEG Recording and Statistical Analysis. A BioSemi ActiveTwo recording system with 64 electrodes was used to record EEG (sampling rate $=512 \mathrm{~Hz}$; BioSemi Inc., The Netherlands). In line with the International 10-20 system, Electrodes were arranged with BioSemi head caps. Electrodes were applied to the external canthi and below the left eye to record horizontal and vertical EOG (electro-oculographem). EEG data was analyzed with EEGLAB (Delorme \& Makeig, 2004) and ERPLAB (Lopez-Calderon \& Luck, 2010). The data was referenced to the mastoid electrodes. A band-pass filter was applied $(0.53 \mathrm{~Hz}-30$ Hz). Trials with errors and blinks during stimuli presentation were excluded. Ocular artifacts were corrected with independent component analysis. A simple voltage threshold was set to \pm $100 \mu \mathrm{V}$. A sample-to-sample voltage threshold was set to $50 \mu \mathrm{V}$. The data was segmented in reference to the target $(-320 \mathrm{~ms}$ to $800 \mathrm{~ms})$ and corrected with a baseline of $200 \mathrm{~ms}(-320 \mathrm{~ms}$ to $-120 \mathrm{~ms})$. ANOVAs were performed with data pruned of prime and mask activation. Perceptual priming effects were analyzed with a focus on the P2 and P3 component at occipital and centro-parietal electrodes (Figure 5.3; Brang et al. 2008; Jaśkowski, Skalska \& Verleger, 2003; Martens et al., 2011; Zovko \& Kiefer, 2013): O1/O2, PO3/PO4, PO7/PO8, P5/P6, P7/P8, P9/P10 and TP7/TP8 (occipital cluster) as well as P1/P2, P3/P4, CP1/CP2, 
$\mathrm{CP} 3 / \mathrm{CP} 4, \mathrm{C} 1 / \mathrm{C} 2$ and $\mathrm{C} 3 / \mathrm{C} 4$ (centro-parietal cluster). Semantic priming effects were expected to be located on a higher level of processing. In line with Kiefer and collegues (Kiefer, 2002; Kiefer \& Brendel, 2006; Martens et al., 2011), an N400-like component was analyzed and the peak was expected at about $600 \mathrm{~ms}$ after target onset. Major effects were expected at temporal/centro-posterior electrodes (capturing Wernicke's area; Figure 5.3) with larger modulations over the right hemisphere (Holcomb, 1993; Kutas \& Hillyard, 1982; Luck, 2005; Neville, Mills \& Lawson, 1992; Van Petten, 1993; Van Petten \& Luka, 2005): (PO7)/PO8, (P5)/P6, (P7)/P8, (P9)/P10, (CP5)/CP6, (TP7)/TP8, (C5)/C6 and (T7)/T8 (temporal cluster). Possible modulations of congruency effects through prime-mask SOA, area and hemisphere were of interest. Furthermore, we intended to examine effect specificities (effect type) of ERP modulations and the role of task relevance (effect type $\times$ task level).

\section{Occipital Cluster}

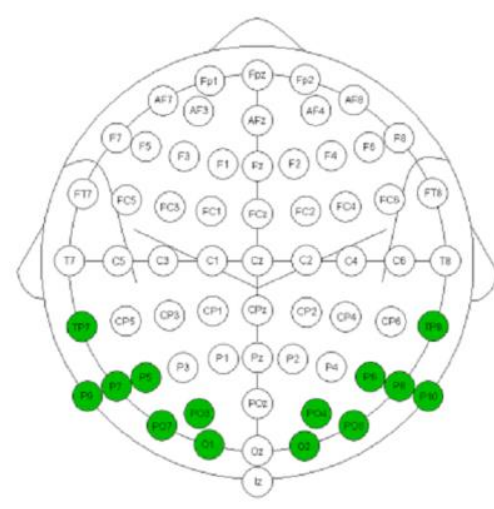

Centro-Parietal Cluster

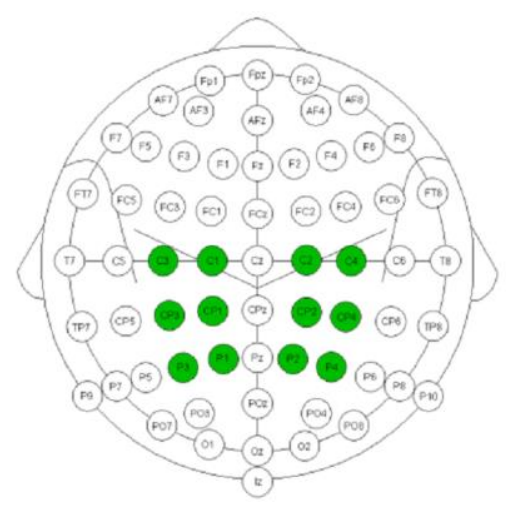

Temporal Cluster

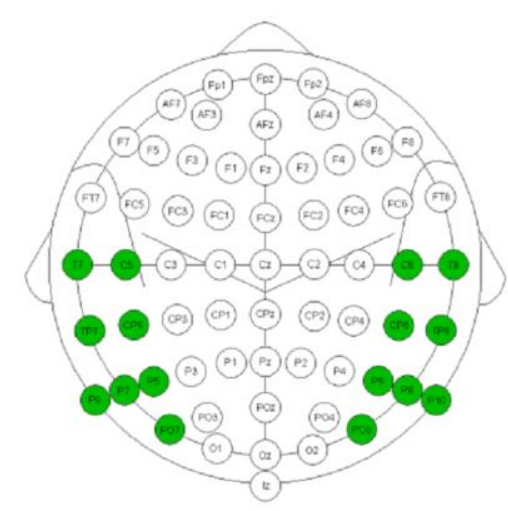

Figure 5.3. Occipital, centro-parietal and temporal electrode clusters used for analysis of perceptual (occipital and centro-parietal) and semantic priming effects (temporal). Note that each cluster is applied for the analysis of a different time window. 


\subsection{Results}

\subsubsection{Behavioral}

Errors. Across experiments, errors occurred in $4.6 \%$ of trials. Participants of Experiment 5A (perceptual task level) committed errors in $7.6 \%$ of trials. Subjects of Experiment 5B (semantic task level) responded more accurately with an error rate of $1.6 \%$. Planned analyses of perceptual and semantic priming effects in Experiment 5A as well as B revealed only a significant main effect of perceptual congruency with a semantic task level $\left(F(1,19)=5.51, M S_{e}=0.011, p<.05\right)$. An irrelevant perceptual priming effect $\left(\mathrm{PE}_{\mathrm{ER}}=\right.$ $\left.\mathrm{ER}_{\text {incongruent }}-\mathrm{ER}_{\text {congruent }}\right)$ of $-0.8 \%$ was found.

Reaction Times. Subjects responded on average $469 \mathrm{~ms}$ after target onset. Reaction times of each condition are displayed in Figure 5.4. In analyses of perceptual and semantic priming effects in Experiment $5 \mathrm{~A}$ as well as $\mathrm{B}$ only relevant congruency effects reached significance (perceptual: $F(1,19)=26.79, M S_{e}=85, p<.0001$; semantic: $F(1,19)=4.56$, $\left.M S_{e}=117, p<.05\right)$. In Experiment 5A, relevant perceptual priming $\left(\mathrm{PE}_{\mathrm{RT}}=\mathrm{RT}_{\text {incongruent }}-\right.$ $\mathrm{RT}_{\text {congruent }}$ ) effects of $11 \mathrm{~ms}$ were achieved. In Experiment $5 \mathrm{~B}$, relevant semantic priming effects of $5 \mathrm{~ms}$ were found. Furthermore, an interaction between prime-mask SOA and perceptual congruency in Experiment 5A reached significance $\left(F(1,19)=13.91, M S_{e}=135\right.$, $p<.005)$. Relevant perceptual priming effects were smaller with a prime-mask SOA of 60 $\mathrm{ms}(1 \mathrm{~ms})$ than with a prime-mask SOA of $120 \mathrm{~ms}(20 \mathrm{~ms})$.

Prime visibility. Objective as well as subjective prime visibility differed significantly between experiments (i.e. task levels) and prime-mask SOA (see Figure 5.5). Subjects reported the color of the prime less accurately (Experiment 5A: $d^{\prime}$ of 2.79) than the identity of the prime (Experiment 5B: $d^{\prime}$ of $3.65 ; F(1,38)=11.33, M S_{e}=1.292, p<.005$ ). Subjective ratings reflected this pattern (Experiment 5A: PAS-rating of 3.19; Experiment 5B: PAS- 
rating of 3.53; $\left.\left(F(1,38)=11.33, M S_{e}=1.292, p<.005\right)\right)$. Furthermore, objective prime visibility increased with prime-mask SOA (60 ms: $d$ ' of $2.19 ; 120 \mathrm{~ms}: d^{\prime}$ of $4.25 ; F(1,38)=$ $525.41, M S_{e}=0.162, p<.0001$ ) as well as subjective prime visibility (60 ms: PAS-rating of 3.03; 120 ms: PAS-rating of 3.68; $\left.F(1,38)=119.42, M S_{e}=0.071, p<.0001\right)$.

Perceptual effect

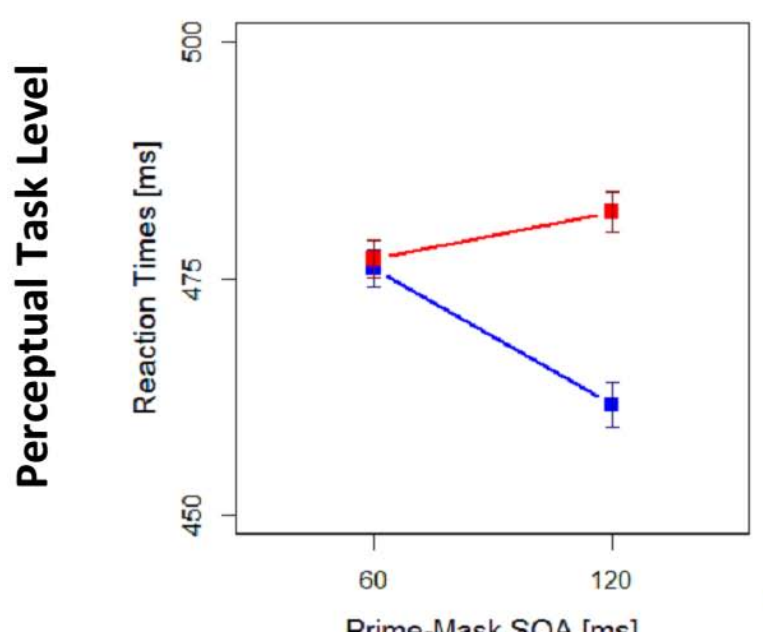

Prime-Mask SOA [ms]

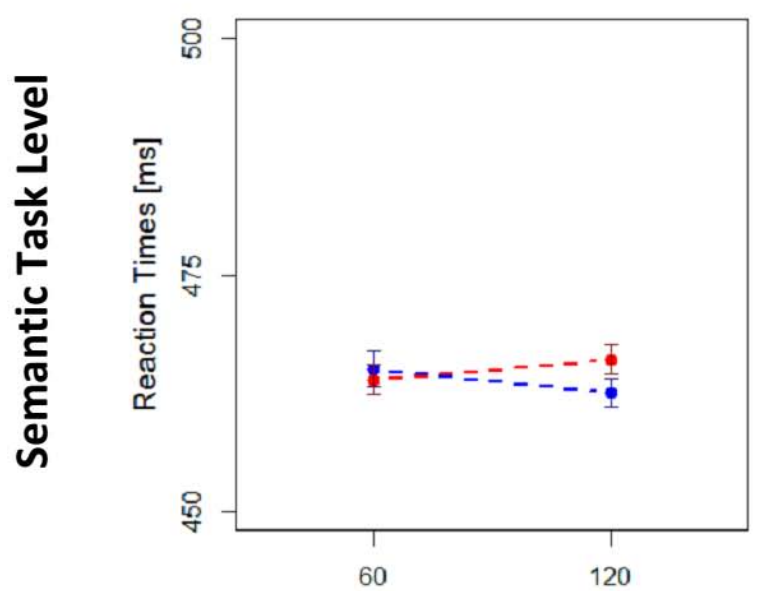

Prime-Mask SOA [ms]
Semantic effect
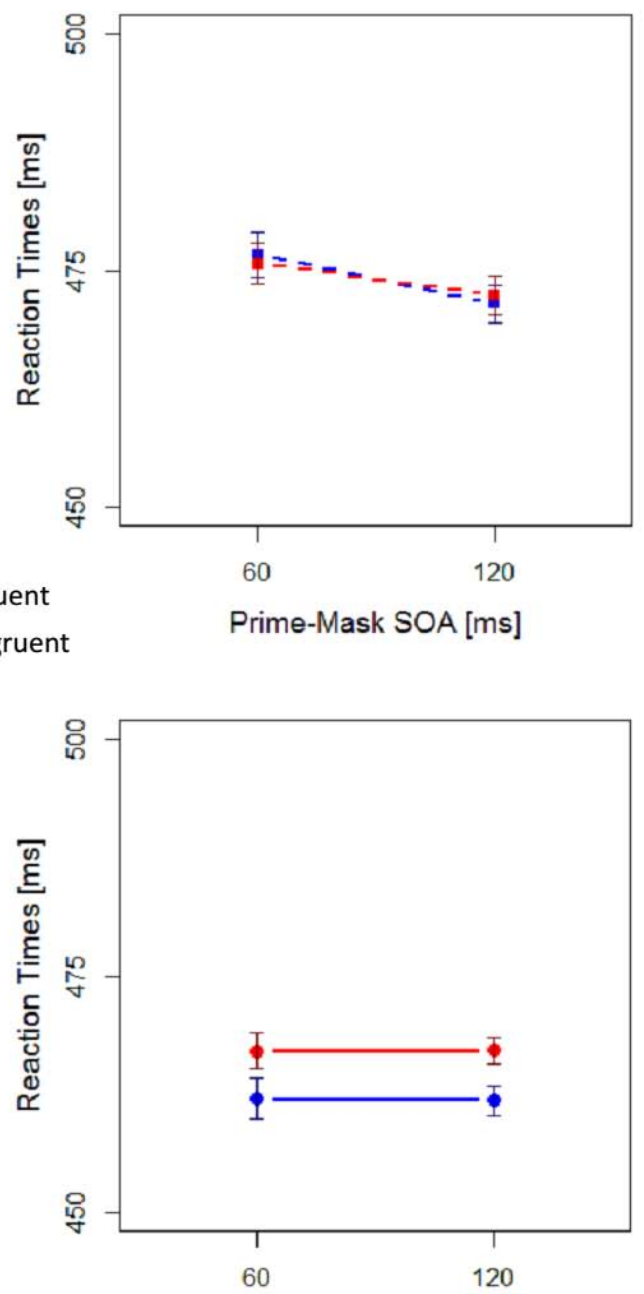

Prime-Mask SOA [ms]

Figure 5.4. Priming effects on reaction times measured in Experiments 5A (upper section) and 5B (lower section). Relevant priming effects are represented by solid lines and irrelevant effects by dashed lines. Confidence intervals are in accordance with Loftus and Masson (1994). 

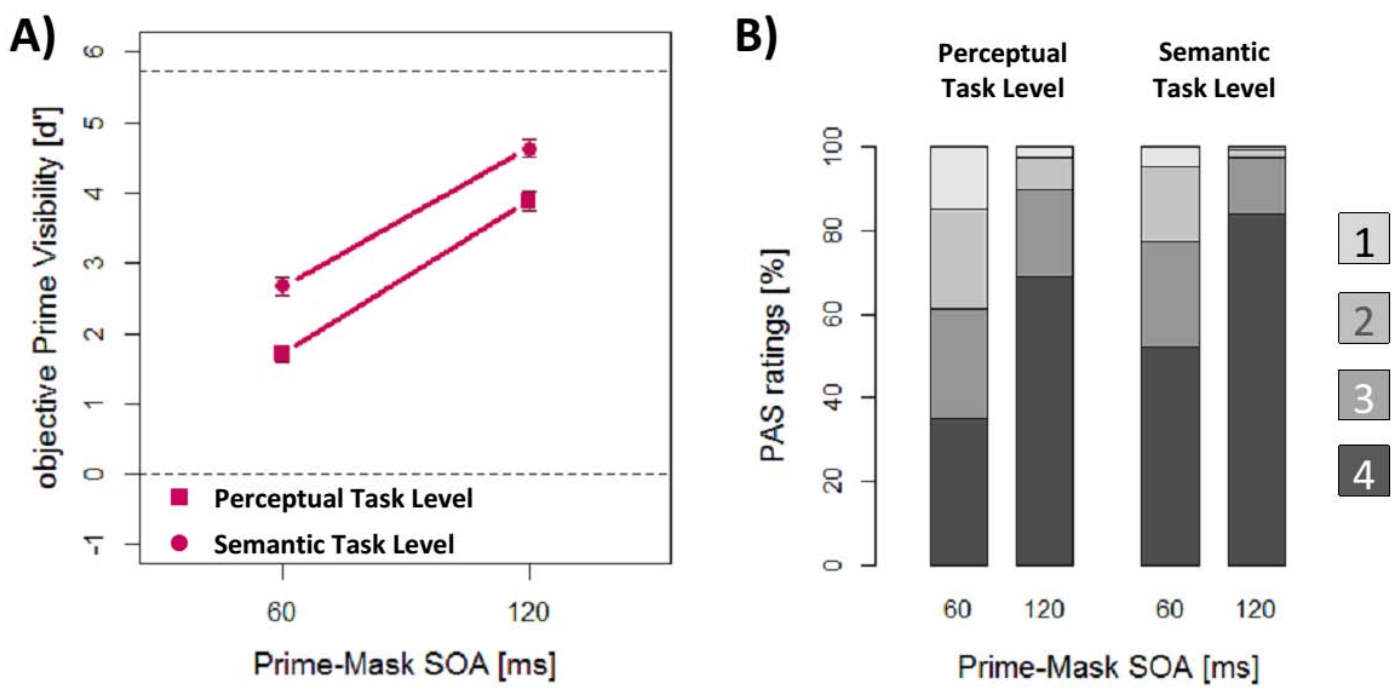

Figure 5.5. Objective and subjective prime visibility in Experiment 5. A: Objective prime visibility. B: Subjective prime visibility was rated with the perceptual awareness scale $(1=$ "nothing", 2 = "a weak glimpse", 3 = "an almost clear image", and 4 = "a clear image"; Ramsøy \& Overgaard, 2004).

\subsubsection{Event-related Potentials}

Perceptual priming: early effect. Mean amplitude (Amp) measures between $175 \mathrm{~ms}$ and $275 \mathrm{~ms}$ after target onset were analyzed to capture the P2 component in Experiment 5A (perceptual task level). The primary focus was set on an expected modulation through perceptual congruency at occipital and centro-parietal electrodes. Repeated measures ANOVA revealed a main effect of hemisphere $\left(F(1,19)=14.24, M S_{e}=2.50, p<.005\right)$ and an interaction of congruency and prime-mask SOA $\left(F(1,19)=4.53, M S_{e}=2.02, p<.05\right)$. Planned analyses of each SOA and area revealed a significant effect of perceptual congruency only at occipital electrodes with a prime-mask SOA of $120 \mathrm{~ms}(F(1,19)=7.98$, $M S_{e}=0.48, p<.05$; Figure 5.6 and 5.7). A planned analysis of perceptual congruency effects in Experiment 5B (semantic task level) revealed no main effect of the irrelevant congruency or interaction with the irrelevant congruency ( $p s>0.1$; Figure 5.6). A planned analysis of semantic congruency effects in Experiment 5B (semantic task level) revealed no main effect of semantic congruency or interaction with semantic congruency within this time window and at occipital and centro-parietal electrodes ( $p s>0.5$; Figure 5.8). 


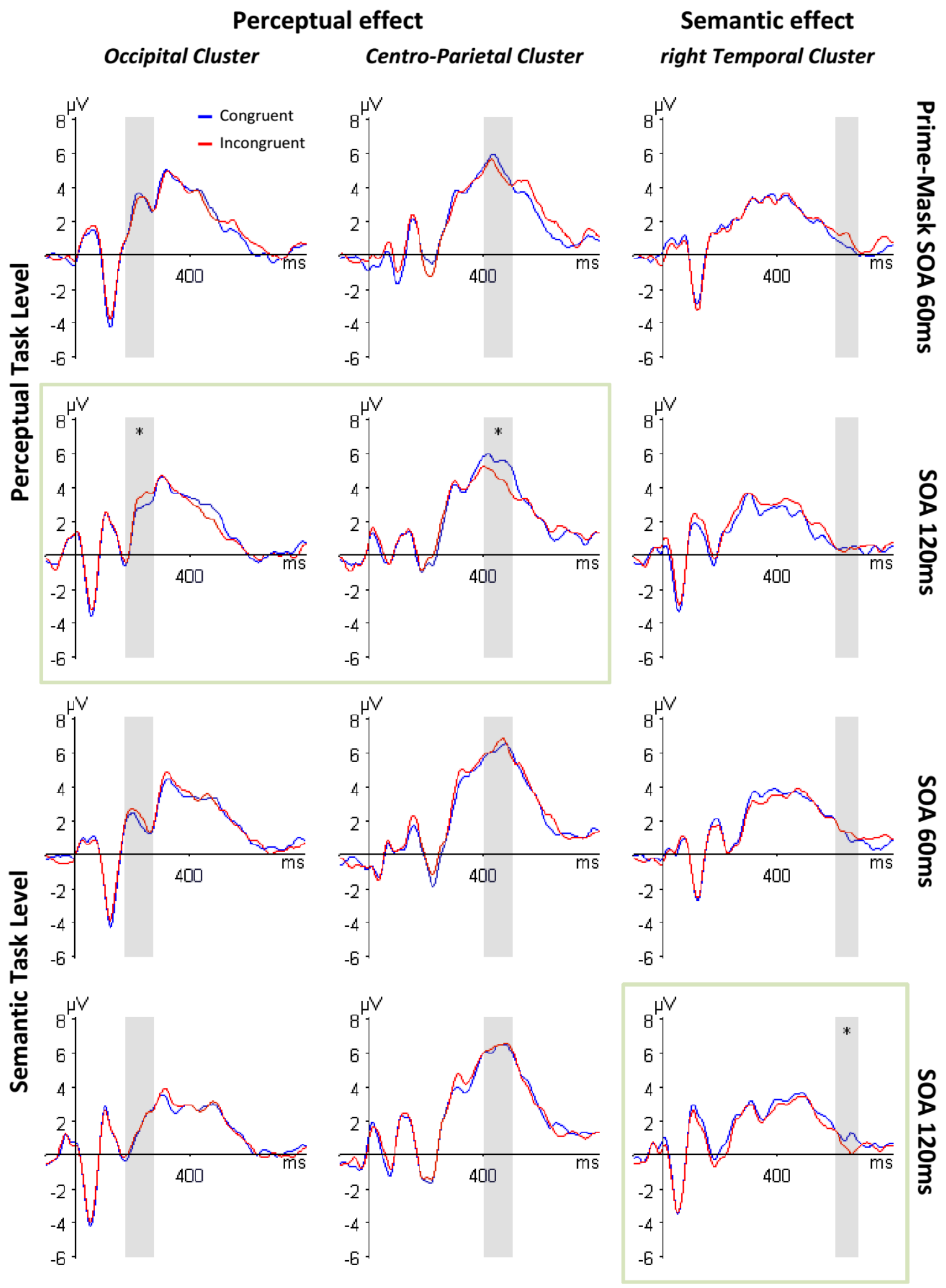

Figure 5.6. Modulations of grand average event-related potentials (target-locked ERPs) through task relevance and prime-mask SOA. Waveforms are depicted for congruent (blue) and incongruent (red) trials from occipital, centro-parietal (perceptual effect) and right temporal electrodes (semantic effect). ERPs are plotted separately for task level (perceptual \& semantic), effect type (perceptual \& semantic) and prime-mask SOA (60 ms \& $90 \mathrm{~ms})$. 

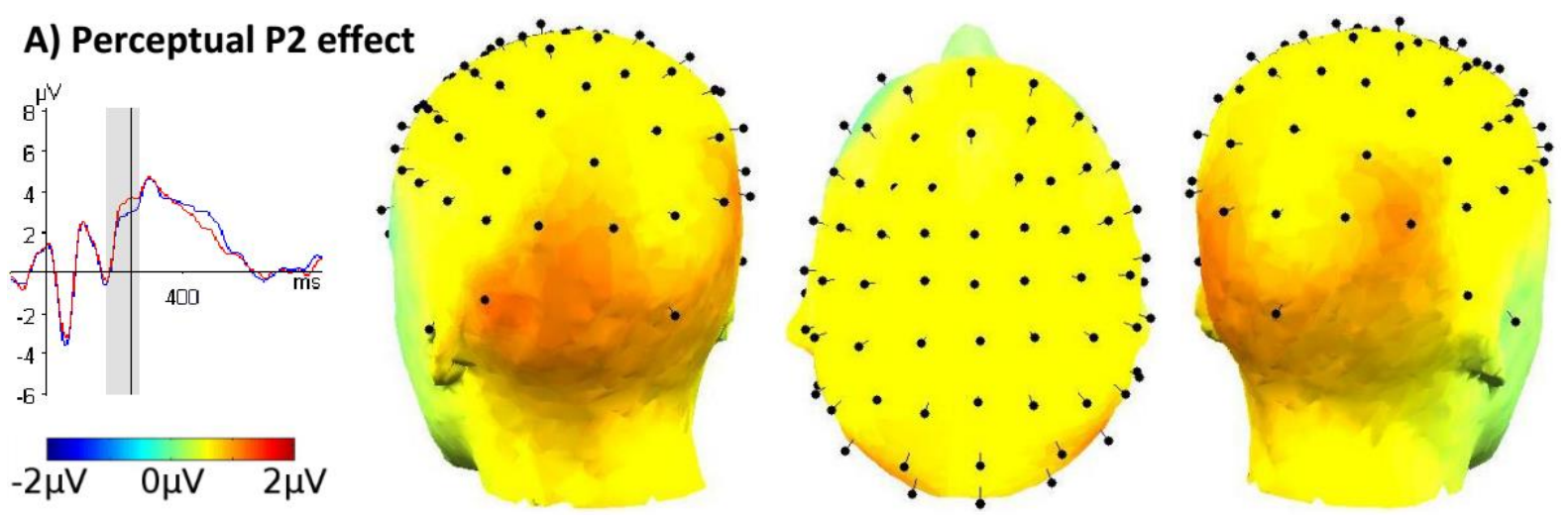

\section{B) Perceptual P3 effect}
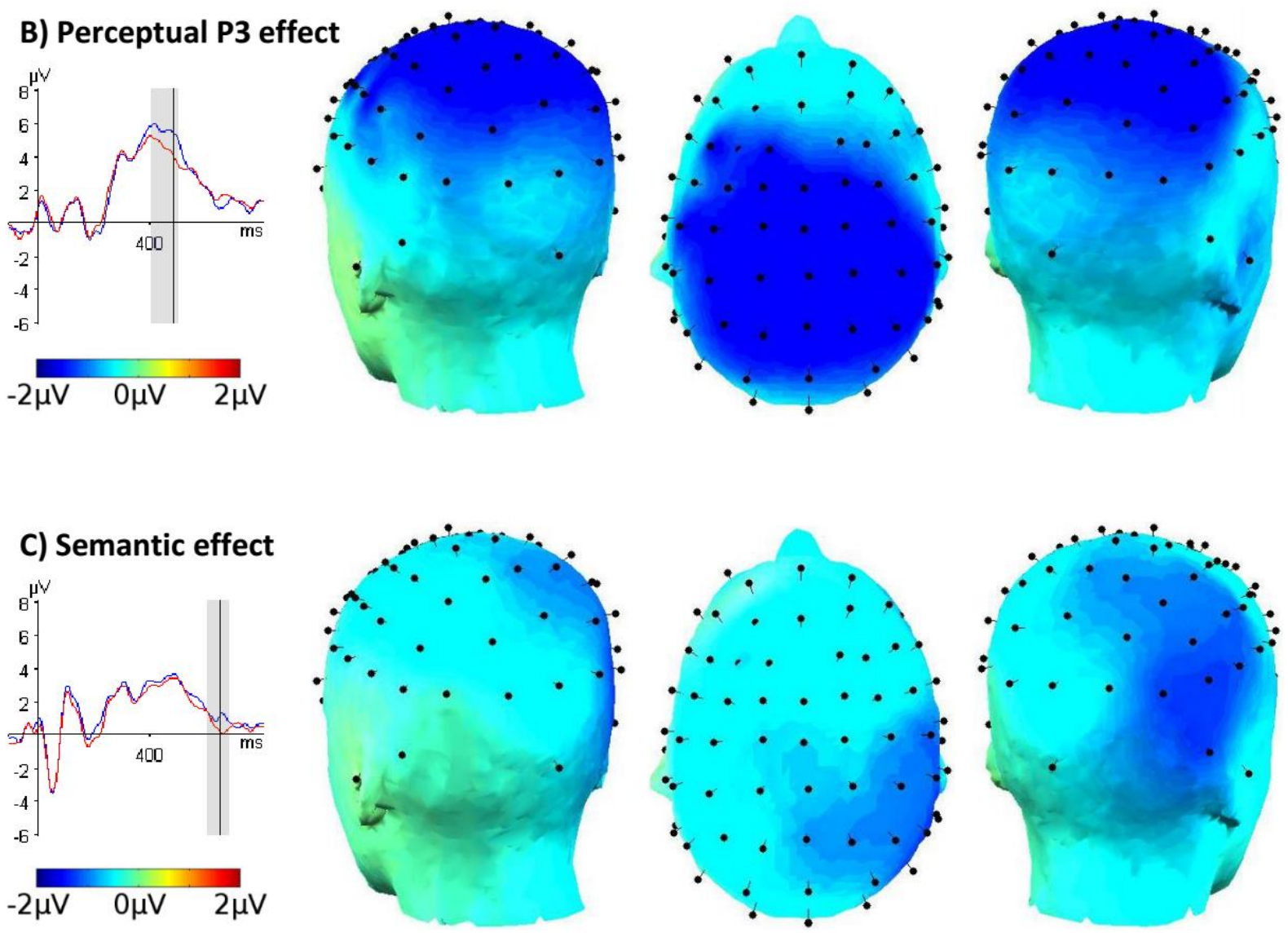

Figure 5.7. Voltage maps with topographical distributions for priming effects $\left(\mathrm{PE}_{\mathrm{Amp}}=\mathrm{Amp}_{\text {incongruent }}-\right.$

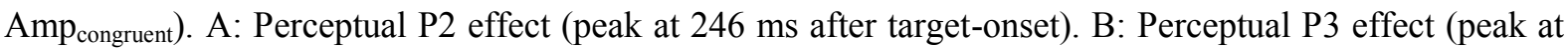
$492 \mathrm{~ms}$ ). C: Semantic effect (peak at $652 \mathrm{~ms}$ ). 


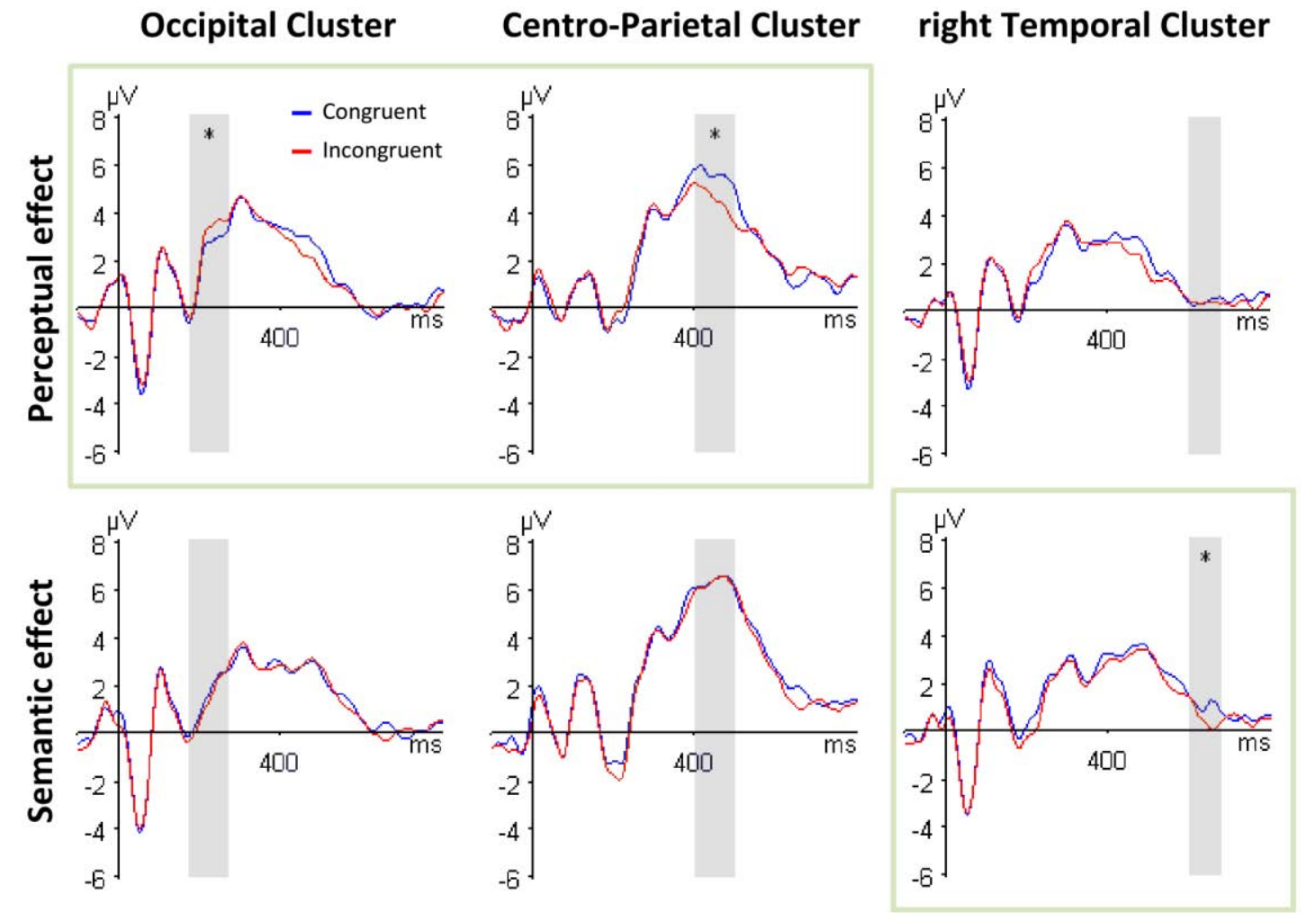

Figure 5.8. Modulations of grand average event-related potentials (target-locked ERPs) through effect types. The effect specificities of perceptual and semantic ERP effects are illustrated. Waveforms are depicted for congruent (blue) and incongruent (red) trials from occipital, centroparietal and right temporal electrodes. Relevant ERP priming effects are plotted separately for each effect type (perceptual and semantic) and electrode cluster at $120 \mathrm{~ms}$ prime-mask SOA.

Perceptual priming: late effect. Mean amplitude measures between $400 \mathrm{~ms}$ and $500 \mathrm{~ms}$ after target onset were analyzed to capture the P3 component in Experiment $5 \mathrm{~A}$ (perceptual task level). The primary focus was set on an expected modulation through perceptual congruency at occipital and centro-parietal electrodes. Repeated measures ANOVA revealed a main effect of area $\left(F(1,19)=6.88, M S_{e}=6.07, p<.05\right)$ and a three-way interaction of congruency, prime-mask SOA and area $\left(F(1,19)=4.59, M S_{e}=0.12, p<.05\right)$. Planned analyses of each SOA and area revealed a significant effect of perceptual congruency only from centro-parietal electrodes with a prime-mask SOA of $120 \mathrm{~ms}(F(1,19)$ $=5.12, M S_{e}=4.22, p<.05$; Figure 5.6 and Figure 5.7). A planned analysis of perceptual congruency effects in Experiment 5B (semantic task level) revealed no main effect of the 
irrelevant congruency or interaction with the irrelevant congruency ( $p s>0.1$; Figure 5.6). A planned analysis of semantic congruency effects in Experiment 5B (semantic task level) revealed no main effect of semantic congruency or interaction with semantic congruency within this time window and at occipital and centro-parietal electrodes ( $p s>0.5$; Figure 5.8).

Semantic priming effects. Mean amplitude measures between $600 \mathrm{~ms}$ and $675 \mathrm{~ms}$ after target onset (cf. Martens et al., 2011) were analyzed to capture an N400-like component in Experiment $5 B$ (semantic task level). The primary focus was set on an expected modulation through semantic congruency at temporal electrodes. Repeated measures ANOVA revealed a main effect of hemisphere $\left(F(1,19)=5.68, M S_{e}=2.52, p<.05\right)$ and a three-way interaction of congruency, prime-mask SOA and hemisphere $\left(F(1,19)=8.79, M S_{e}\right.$ $=0.26, p<.01)$. Planned analyses of each SOA and hemisphere revealed a significant effect of semantic congruency only from right temporal electrodes with a prime-mask SOA of $120 \mathrm{~ms}\left(F(1,19)=4.53, M S_{e}=0.79, p<.05\right.$; Figure 5.6 and Figure 5.7). A planned analysis of semantic congruency effects in Experiment 5A (perceptual task level) revealed no main effect of the irrelevant congruency $(p>0.5)$, but an interaction of the irrelevant congruency, prime-mask SOA and hemisphere $\left(F(1,19)=5.59, M S_{e}=0.10, p<.05\right)$. No significant effect of the irrelevant congruency was identified in a single SOA or hemisphere ( $p s>0.1$; Figure 5.6). A planned analysis of perceptual congruency effects in Experiment 5A (perceptual task level) revealed no main effect of perceptual congruency or interaction with perceptual congruency within this time window and at temporal electrodes ( $p s>0.5$; Figure 5.8).

\subsubsection{Behavior and Electrophysiology}

We computed correlations to detect possible direct links between behavioral priming effects and prime related amplitude differences. Perceptual priming effects (Experiment 5A and Experiment 5B included) on reaction times and mean amplitude differences between incongruent and congruent trials measured between $175 \mathrm{~ms}$ and $275 \mathrm{~ms}$ (P2) did not correlate 
significantly $(r=-0.02, p>.5$; Figure 5.9A). However, perceptual priming effects on reaction times and mean amplitude differences between incongruent and congruent trials measured between $400 \mathrm{~ms}$ and $500 \mathrm{~ms}$ (P3) correlated significantly in the predicted direction $(r=-0.24, p<.05$; Figure 5.9B). A correlation test between semantic priming effects on reaction times and mean amplitude differences measured between $600 \mathrm{~ms}$ and $675 \mathrm{~ms}$ did not reveal a significant correlation $(r=-0.07, p>.2$; Figure 5.9C).

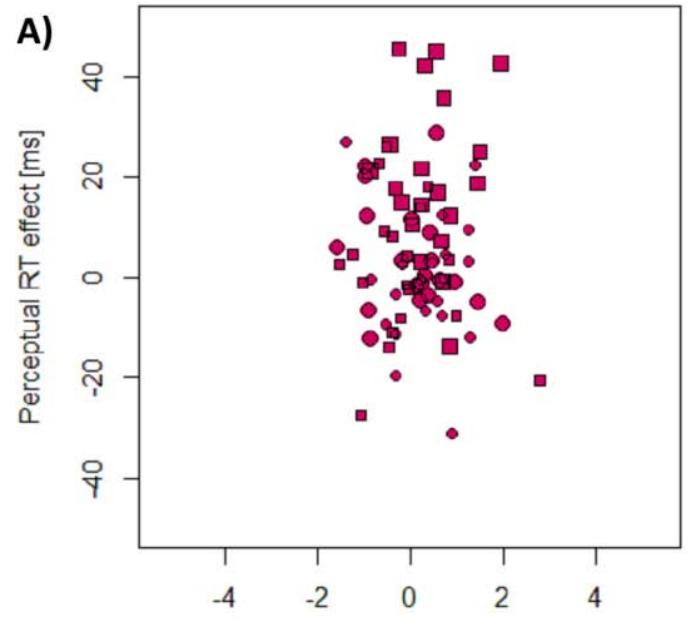

P2 amplitude difference $[\mu \mathrm{V}]$

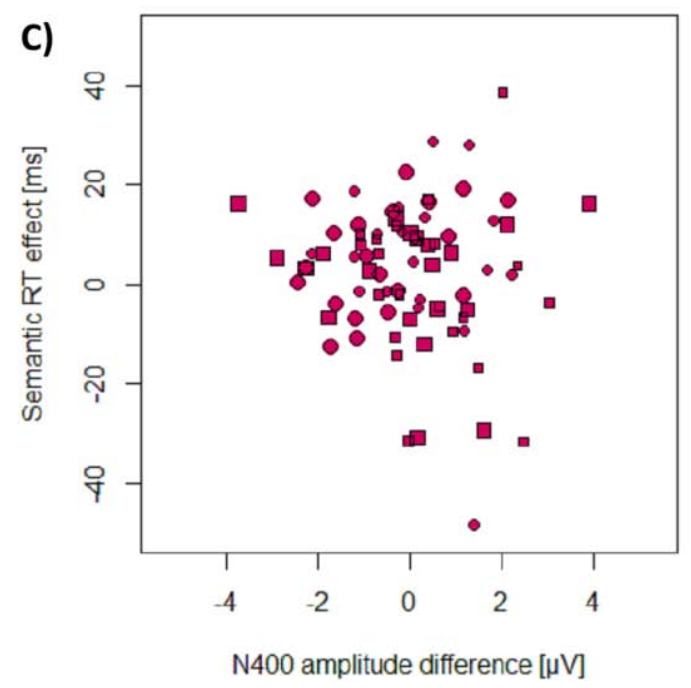

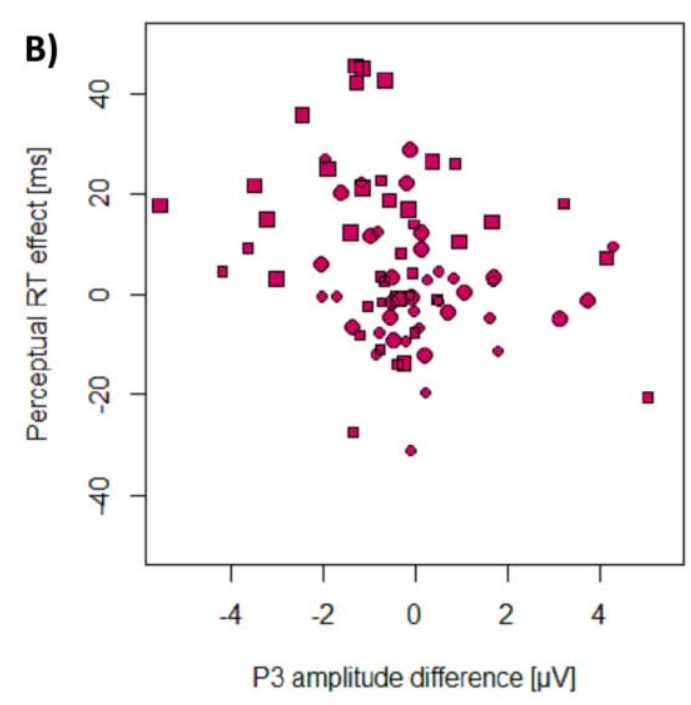

Perceptual Task Level
- $60 \mathrm{~ms}$ prime-mask SOA
- 120 ms prime-mask SOA

Semantic Task Level

- 60 ms prime-mask SOA

- 120 ms prime-mask SOA

Figure 5.10. Correlations between behavioral priming effects (reaction times) and ERP priming effects. A: Perceptual priming effects and P2 amplitude differences at occipital electrodes $(r=-0.02$, $p>.5)$. B: Perceptual priming effects and P3 amplitude differences at centro-parietal electrodes $(r=$ $-0.24, p<.05)$. C: Semantic priming effects and N400 amplitude differences at right temporal electrodes $(r=-0.07, p>.2)$. 


\subsection{Discussion}

The current study was conducted to examine possible differences between perceptual and semantic priming. Furthermore, we wanted to assess the impact of task relevance. Primarily, we were interested in modulations of ERPs through perceptual and semantic congruency. However, our design also enabled us to compute behavioral priming effects. With this opportunity, behavioral priming effects and ERP priming effects without response execution can be linked. Furthermore, this enhances the comparability between our results and previous research (Chapter $3 \& 4)$.

In the current study, metacontrast masks were applied. This decision was based on previous experiments that indicated an unimpeded feedforward processing with metacontrast masks (Chapter 3; Lamme \& Roelfsema, 2000). Priming effects were not modulated through prime visibility when metacontrast mask were applied. However, prime visibility was assessed in the current study to place possible impacts of prime visibility beyond question. Objective as well as subjective prime visibility was modulated through task level and primemask SOA. Prime visibility was higher with a semantic task level than with a perceptual task level. Prime visibility also increased with increasing prime-mask SOA. This relation was also observed in previous studies (e.g. Chapter $3 \& 4$ ).

Priming effects on error rates were hardly found in the current study. An interaction between task level and congruency reached significance. The interaction reflects a negative perceptual congruency effect in the experiment with the semantic task level. Other modulations of error rates through congruency did not reach significance. The negative perceptual congruency effect might reflect the necessity of inhibiting interferences of color processing when the semantic of a stimulus is relevant. However, this effect should be interpreted with caution as the reaction time effect shows a different polarity. Reaction times 
were modulated through congruency more clearly. Subjects reacted generally faster in congruent trials compared to incongruent trials and these priming effects increased with increasing prime-mask SOA. This observation is in line with results of previous studies (e.g. Chapter 3 \& 4; Mattler, 2003; Vorberg et al., 2003). The increase of perceptual priming effects was larger than the increase of semantic priming effects. Perceptual priming effects were larger than semantic priming effects and relevant priming effects larger than irrelevant priming effects. Indeed, neither irrelevant perceptual nor irrelevant semantic priming effects reached significance in separate analyses.

First of all, perceptual and semantic priming effects without response association were attested in the current study. Semantic priming effects without response association are examined in a long tradition with the lexical decision task (Meyer \& Schvaneveldt, 1971; reviewed in Van den Bussche, 2009). Typically, subjects categorize words and non-words and subsequently respond with one of two buttons. Within the framework of the attentional sensitization model, one would argue that relevant pathways are sensitized by attention to the meaning of the stimuli (Kiefer \& Martens, 2010). Congruency effects of related and unrelated word-word pairs are examined. With this approach, priming effects are pruned of effects on a motor level. Perceptual priming effects without response association can be examined in a similar way. However, this approach was only applied in a few studies. For instance, the impact of shape congruency was examined (Martens et al., 2011; Zovko \& Kiefer, 2013). The current study extended the findings concerning perceptual congruency effects without response association to the attribute of color. Furthermore, we attested perceptual and semantic priming effects without response association in very similar conditions. Differences between these effect types and task levels can be interpreted without restrictions.

Priming effects increased with prime-mask SOA and were only reliable with $120 \mathrm{~ms}$ prime-mask SOA. In this condition, perceptual priming effects were clearly larger than 
semantic priming effects. This pattern is in line with previous research (Chapter 3). It provides first evidence that perceptual and semantic priming effects might rely on different mechanisms. This finding cannot be attributed to differences in prime visibility as prime visibility is higher in Experiment 5B (semantic task level) and prime visibility is positively associated with priming effects, if at all (Chapter 3). Furthermore, similar overall reaction times with small error rates in both experiments (A and B) indicate similar task difficulty. Priming effects were also only reliable when effect type matched task level (i.e. relevant priming effects). This pattern is in line with the attentional sensitization model by Kiefer \& Martens (2010). The authors assume that attention to attributes is necessary for unconscious processing of these attributes. Two mechanisms are thought to be involved: 1) enhancement of target activation (Posner, Snyder \& Davidson, 1980) and 2) relative reduction of distractor activation (Desimone \& Duncan, 1995). However, there is an insignificant tendency that perceptual priming effects might evolve with increasing prime-mask SOA even when the task level is semantic. There was no hint for this tendency in previous experiments (Appendix IV). This might be due to an enhanced task complexity in the current study. Subjects were asked to focus on more than a one attribute in the current experiments and this could have impeded attentional focusing. However, the current study does not provide sufficient evidence that perceptual priming can occur without attention. Nevertheless, it provides reason for further investigations. Future studies could vary the width of attentional focus as well as the complexity of the prime-target relation to address this question.

Besides the results of the current study, the finding of priming effects supports the viability of the current design. Future studies might use this design to examine the impact of prime visibility on perceptual and semantic priming effects without response association when pattern masks and metacontrast mask are applied (cf. Chapter 3). If behavioral data is of interest one should use the no-go targets of current study as go targets and vice versa. 
Analyses of behavioral data appeared to be insightful. However, the primary focus of the current study was set on the analyses of ERP priming effects. Interestingly, the design justifies the examination of correlations between behavioral priming effects and ERP priming effects. These correlations forge a bridge to previous research (Chapter $3 \& 4$ ) and are discussed in the following sections.

\subsubsection{ERP effects of Perceptual Congruency}

Although the P2 component generally overlaps with the N2, P3a an MMN, P2 was well detectable in the current study (Crowley \& Colrain, 2004; Luck, 2005). As expected, the P2 amplitude was larger in incongruent trials than in congruent trials and this is in line with the assumption that $\mathrm{P} 2$ reflects repetitions suppression (e.g. Brang et al., 2008; Freunberger et al., 2007; Hsu, Hämäläinen \& Waszak, 2014). A correlation between behavioral perceptual priming effects and P2 priming effects did not reach significance. However, similar to behavioral priming effects, $\mathrm{P} 2$ priming effects were only reliable with the long prime-mask SOA (120 ms).

The P2 component reflects early stimulus processing as it is associated with perceptual processing (Brang et al., 2008; Kranczioch, Debener \& Engel, 2003), feature detection and recognition processes (Dunn et al., 1988, Evans \& Federmeier, 2007; Luck \& Hillyard, 1994) and distractor feature suppression (Phillips \& Takeda, 2009). The relation of P2 and distractor suppression is supported by the increase of this amplitude with age. GarciaLarrea and colleagues (1992) propose that this is due to a progressive deficit in the ability to withdraw attentional resources. P2 might reflect aspects of a stimulus classification process as P2 amplitude is enhanced with nontargets in oddball paradigms (Crowley \& Colrain, 2004; Garcia-Larrea et al., 1992; Novak, Ritter \& Vaughan, 1992). It is thought to reflect a comparison between the stimulus and mental representations and is therefore also associated 
with memory (Costa-Faidella, Baldeweg, Grimm \& Escera, 2011; Evans \& Federmeier, 2007; Rugg \& Doyle, 1994). P2 amplitude is increased for correctly remembered items compared to items correctly identified as new. It seems to be generally enhanced for repeated stimuli compared to novel stimuli (Curran \& Dien, 2003; Misra \& Holcombe, 2003). However, it is improbable that this effect modulates priming effects in the current design as all stimuli are repeated several times. Primes and targets appear balanced between congruent and incongruent trials and the sequence is generated randomly.

Interestingly, P2 amplitude is larger for improbable targets (Luck \& Hillyard, 1994). Maybe this contributed to the priming effect as the visual system might adjust to primes and incongruent targets are perceived as improbable. In an auditory cuing paradigm, P2 amplitude was also smaller in trials with a valid cue compared to trials with an invalid cue (Golob, Pratt \& Starr, 2002). P2 could also reflect cognitive processing demands as dissonance occurs when the prime activates a context that does not match the target (Freunberger et al., 2007). Contrary to this proposal, Phillips and Takeda (2009) report that P2 peaks are higher for efficient search conditions compared to inefficient conditions.

In the current study, the P2 component was found between $175 \mathrm{~ms}$ and $275 \mathrm{~ms}$ after target onset. This time window is in line with previous research (Brang et al., 2008; Crowley \& Colrain, 2004; Evans \& Federmeier, 2007; Freunberger et al., 2007; Luck \& Hillyard, 1994). Similar to findings of comparable studies, the perceptual P2 effect was mainly found over occipital electrodes (Jaśkowski et al., 2003; Martens et al., 2011; Zovko \& Kiefer, 2013). The generator of the $\mathrm{P} 2$ is assumed to be in parieto-occipital regions (Freunberger et al., 2007).

P2 priming effects were only found with a perceptual effect type and not with a semantic effect type. This is in line with the notion of Luck (2005) that P2 is responsive when 
the target is defined by simple stimulus features. Furthermore, P2 seems to reflect analysis of stimuli rather than postperceptual processing (Luck \& Hillyard, 1994; Näätänen \& Picton, 1986; Ritter, Simson \& Vaughan, 1983). In a visual search paradigm, P2 was larger for popout targets (Luck \& Hillyard, 1994). This effect was more pronounced for color pop-outs compared to pop-outs based on orientation or shape. However, orientation pop-outs and size pop-outs also modulated P2 and it is therefore thought to reflect a transdimensional feature detection process. Nevertheless, enhanced P2 amplitudes were mainly observed when subjects attended to color (Hillyard \& Münte, 1984; Kenemans, Kok \& Smulders, 1993; Wijers, Mulder, Okita, Mulder \& Scheffers, 1989). Transferred to the results of the current study, the color of the target might have popped out more intensively when preceded by a prime of different color. The P2 priming effect might have been less pronounced if we had chosen another perceptual attribute not related to color. Besides the effect of color processing on P2 amplitude, selections based on brightness were also associated with P2 changes (Wastell \& Kleinman, 1980). However, luminance was adjusted equally for all stimuli in the current study.

Furthermore, Luck and Hillyard (1994) only found P2 effects for target pop-outs and not for nontarget pop-outs. They inferred that top-down information about stimulus classification affected pop-out processing. The impact of attention, top-down activation and context matching is also assumed by other authors (e.g. Federmeier, Mai \& Kutas, 2005; Freunberger et al., 2007; Hsu et al., 2014; Kranczioch et al., 2003; Luck, Vogel, \& Shapiro 1996). The current results support this notion as P2 amplitude was not affected by irrelevant congruency (e.g. perceptual congruency with a semantic task level). A bridge can be forged between an impact of attention on P2 modulation and the attentional sensitization model by Kiefer \& Martens (2010). However, P2 effects seem to be most reliable with moderate 
attention (Hsu et al., 2014) and it is also argued that P2 is at least partially exogenous (Crowley \& Colrain, 2004)

Evidence against effect specificity of the P2 amplitude modulation is provided by Freunberger and colleagues (2007). The authors found P2 effects with a classical semantic task - a categorization of living and non-living things. However, pictures were used and it is likely that pictures of houses or cars also differ perceptually from pictures of plants or animals. The modulation of P2 through congruency seems similar to the modulation that was found in the current study. The authors found a significant P2 effect only in left hemisphere. The perceptual P2 effect in the current study had a broader distribution over the left hemisphere (Figure 5.7). The asymmetry between hemispheres was also reported in other studies (Federmeier \& Kutas, 2002; Kanske \& Kotz, 2007). For instance, Federmeier and Kutas (2002) found a modulation of P2 only when stimuli were presented to the right hemifield. Kanske and Kotz (2007) reported that P2 was generally larger for words presented in the right hemifield. The left hemisphere seems to be more sensitive with regards to these processes.

Besides a modulation of $\mathrm{P} 2$, the $P 3$ component was modulated through perceptual congruency. As expected, the P3 amplitude was larger in congruent trials than in incongruent trials (Hillyard \& Münte, 1984; Martens et al., 2011; Zovko \& Kiefer, 2013). Perceptual congruency had a reversed impact on P2 and P3. A separation of these generally overlapping components was therefore distinct (Crowley \& Colrain, 2004; Luck, 2005). However, P3 consists of two subcomponents and it is difficult to distinguish P3a and P3b (reviewed in Polich, 2007). Many studies only refer to the historically first reported P300 which corresponds to P3b (Sutton, Zubin \& John, 1965). Yet, P3a and P3b are thought to reflect processes that are linked, but still different. 
Visual inspection of ERPs suggests that both subcomponents might be modulated through perceptual congruency (Figure 5.6). However, there are some indications that congruent and incongruent trials might differ mainly in processes associated with P3b. First of all, the P3 component was identified between $400 \mathrm{~ms}$ and $500 \mathrm{~ms}$ after target onset. This is in line with previous research (e.g. Hillyard \& Münte, 1984). Polich (2007) noted that P3 can be found between $250 \mathrm{~ms}$ and $500 \mathrm{~ms}$, but the range is quite variable. Nevertheless, it can be assumed that we mainly found a pronounced P3b in this time window. Furthermore, we found the P3 mainly over centro-parietal electrodes (Figure 5.7). P3a and P3b differ with regards to their topographical distributions (Polich, 2007). For instance, Squires and colleagues (1975) reported a frontally maximal P3a and a parietally maximal P3b (Luck, 2005). P3a seems to be associated with frontal (dopaminergic) pathways whereas P3b reflects operations of parietal (norepinephrine) pathways (Polich, 2007). The generator of P3b seems to be in the medial temporal lobe (Halgren et al., 1980; McCarthy, Wood, Williamson \& Spencer, 1989). Another aspect indicates that we mainly observed a P3b. P3 is larger with improbable targets (Duncan-Johnson \& Donchin, 1977; Luck \& Hillyard, 1994). Verleger, Jaśkowski and Wauschkuhn (1994) found a P3b when stimuli were presented rarely, but subjects still expected them (Luck, 2005). P3a was pronounced with novel stimuli. In the current study, 24 target stimuli were presented in 768 experimental trials. Each target was therefore not predictable, but hardly novel.

P3b modulations were reported in many different studies and many studies referring to P3 or P300 report findings which concern P3b (Luck, 2005). The polarity of the perceptual P3 priming effect might be due to a smaller P3 amplitude when subjects are uncertain whether a specific target was presented or not (Luck, 2005). It is reasonable to assume that certainty is reduced in incongruent trials and P3 amplitude is attenuated. P3 amplitude is also lower with inefficient search conditions compared to efficient search conditions (Phillips \& 
Takeda, 2009). Smaller P3 amplitudes are also reported in association with increased memory load (Kok, 2001; Wijers, Mulder, Okita \& Mulder 1989). It was also proposed that smaller P3 amplitudes are induced in tasks with a high demand of attention (Kok, 2001; Polich, 1987, 2007). Incongruent trials might be more difficult and require more attentional resources. Furthermore, Hillyard and Münte (1984) found a larger P400-500 (P3b) that was induced by targets compared to nontargets with the same color and at the same location. Targets were defined through the height of the stimulus. Therefore, this effect seems to occur with different perceptual features. In the current study, P3 amplitude was not modulated through semantic congruency. Counterintuitively, P3 amplitude seems to increase with increasing task demand and one might assume that task demand is higher in incongruent trials (Johnson and Donchin, 1985; Nittono et al., 1999; Ruchkin et al., 1980; Smith and Guster, 1993). However, the decisive factor seems to be the effort that was put into fulfilling the task (Isreal et al., 1980). P3 amplitude declines when performance is negatively affected (Johnson \& Donchin, 1985; Nittono, Nageishi, Nakajima \& Ullsperger, 1999; Ruchkin, Johnson, Canoune \& Ritter, 1980; Smith \& Guster, 1993). Performance is indeed affected through perceptual congruency in the current study as behavioral priming effects on reactions times were found.

In contrast to $\mathrm{P} 2$ priming effects, $\mathrm{P} 3$ priming effects correlate with behavioral priming effects. This seems reasonable as P3 occurs subsequently to an initial classification (P2) and is more related to decision masking (Hillyard \& Münte, 1984; Kok, 2001; Luck, 2005). It was proposed that P2 might reflect identification of items which are subsequently matched with targets represented in the working memory (Kok, 2001; Phillips \& Takeda, 2009). The P3b component was frequently associated with memory and correlates with recall and recognition of items (e.g. Evans \& Federmeier, 2007; Lefebvre, Marchand, Eskes \& Connolly, 2005; reviewed in Polich, 2007). More generally, it was proposed that P3 reflects the updating of consciousness (Dehaene et al., 2001; Donchin \& Coles, 1988; Picton, 1992). 
Only perceptual P3 priming effects with a perceptual task level were observed in the current study. This is in line with previous research indicating that P3b is affected through task relevancy and attention (McCarthy \& Nobre, 1993; Nobre, 2001; Squires, Squire \& Hillyard, 1975). Again, a bridge can be forged between impact of attention on P3 modulation and the attentional sensitization model by Kiefer \& Martens (2010).

P3a seems to be involved in capturing stimulus-driven attention (reviewed in Polich, 2007). Polich (2007) proposes that P3a indicates stimulus-driven attentional mechanisms that are especially pronounced with novel stimuli. Inhibition of irrelevant brain activation allows a rapid transmission of stimulus information to temporal-parietal areas where the $\mathrm{P} 3 \mathrm{~b}$ is generated. $\mathrm{P} 3 \mathrm{~b}$ is furthermore associated with memory processes generating an update of the representation.

\subsubsection{ERP effects of Semantic Congruency}

ERP modulations through semantic congruency were expected later than modulations though perceptual congruency. Especially the N400 component was thought to reflect differences in semantic processing of targets in congruent and incongruent trials (reviewed in Kutas, 1997). In accordance with Martens and colleagues (2011), we found a negative deflection between $600 \mathrm{~ms}$ and $675 \mathrm{~ms}$ after target onset in semantically incongruent trials compared to semantically congruent trials. The semantic ERP priming effect was distributed over temporal/centro-posterior electrodes on the right hemisphere. Similar distributions of N400 over Wernicke's homolog were found frequently with content words (Holcomb, 1993; Kutas \& Hillyard, 1982; Luck, 2005; Neville et al., 1992; Van Petten, 1993; Van Petten \& Luka, 2005).

However, the time window is not typical for usually observed N400 effects. The N400 was reported by Kutas and Hillyard for the first time in 1980 and its peak was found at about 
$400 \mathrm{~ms}$ after stimulus onset. In this study, N400 effects were found after semantic violations. Sentences were presented and subjects were instructed to read them and told that questions would be asked at the end of the experiment. Each word was presented for $100 \mathrm{~ms}$ and stimulus onset asynchronies were $1000 \mathrm{~ms}$. The focus was set on the processing of the last word in a sentence. These words were either semantically appropriate, semantic violations or physically different. A negative deflection was only found with semantic violations and this difference in ERP of semantically appropriate and inappropriate words was named N400 effect. The design of Kutas and Hillyard (1980) seems rather different compared to studies applying semantic priming or masked semantic priming. Nevertheless, several semantic priming experiments found comparable modulations through prime-target congruency, subsequently (e.g. Bentin, McCarthy \& Wood, 1985; Holcomb, 1988; Rugg, 1985; reviewed in Osterhout \& Holcomb, 1995). However, these studies also reported a late ERP positivity that was also partially affected by semantic congruency (e.g. 550 to $650 \mathrm{~ms}$; Bentin et al. 1985).The relatively late ERP effect that was found in the current study might be linked to this. Alternatively, specific parameters of the current design might have caused a shift of the N400 latency. Although very few factors have an impact on N400 latency, increasing presentation rates seems to have an effect (Kutas \& Federmeier, 2009). The peak of N400 is delayed with increasing presentation rates (Kutas, 1993). Stimulus onset asynchronies between primes and targets of $60 \mathrm{~ms}$ and $120 \mathrm{~ms}$ were applied in the current study - one tenth of the original SOA. This effect might have also caused a shift in N400 latency in the experiment of Martens and colleagues (2011). The authors used a prime-target SOA of $67 \mathrm{~ms}$.

Crucially, we found a semantic ERP effect with a topographical distribution that is in line with previously found distributions of N400 effects with a larger amplitude effects over the right hemisphere (Holcomb, 1993; Kutas \& Hillyard, 1982; Luck, 2005; Neville et al., 
1992; Van Petten, 1993; Van Petten \& Luka, 2005). Subjects were asked whether they directed their attention to one side as targets were presented on the left as well as the right side. Eleven subjects attended to both sides, four subjects preferred the right side whereas five attended mainly to the left target in Experiment 5B. Therefore, the distribution is balanced and it is unlikely that one-sided attention allocation evoked the hemispheric asymmetry. Furthermore, the polarity of the semantic ERP effect is equivalent to previously found N400 effects (reviewed in Kutas, 1997; Kutas \& Federmeier 2009; Osterhout \& Holcomb, 1995). Therefore, similar generators can be assumed. The semantic neural system is complex and widespread (Binder, Desai, Gravers \& Conant, 2009). In their meta-analytic review, Binder and colleagues (2009) identified 3 categories of areas: 1) the posterior heteromodal association cortex, 2) subregions of heteromodal prefrontal cortex and 3) medial paralimbic regions with connections to the hippocampal formation. The N400 is thought to be mainly generated in the posterior heteromodal association cortex, including temporal areas (reviewed in Kutas \& Federmeier 2009). Various methods besides EEG were applied to identify the sources of the N400, including magnetoencephalography, event-related optical signals and intracranial recordings. These studies suggest involvement of temporal areas (e.g. superior and middle temporal gyrus, temporo-parietal junction and medial temporal lobe), but also prefrontal regions (Helenius, Salmelin, Service \& Connolly, 1998; Halgren et al., 2002; Tse et al., 2007). In line with these findings, patients with Wernicke's aphasia have comprehension deficits induced by lesions in the superior temporal lobe, middle temporal gyrus or temporal-parietal-occipital junction (Hickok \& Poeppel, 2000). The critical involvement of the posterior heteromodal association cortex suggests that semantic processing of stimuli exceeds basal perceptual processing. In line with this inference, the semantic ERP effect was not observed with perceptual congruency (Figure 5.8). Furthermore, the N400 effect is only fully developed with attention to the meaning of the stimulus (e.g. 
Holcomb, 1988; reviewed in Kutas \& Federmeier 2009). This is in line with the attentional sensitization model by Kiefer and Martens (2010). The current study provided further evidence for this modulation as only relevant ERP priming effects were found (Figure 5.6).

Behavioral as well as electrophysiological semantic priming effects were found in the current study. However, the correlation between these effects did not reach significance. Several factors might account for this discrepancy. Semantic priming effects on reaction times were very small and not particularly stable between or within subjects. Furthermore, behavioral and electrophysiological data was collected on different trials. It is notable that reactions occurred to a considerable extent prior to the latency of the semantic ERP effect. This might be due to the relatively small number of different targets. Maybe the task was therefore quite simple and it was possible to solve it without semantic processing later in the experiment. However, the pure semantic priming effects can only be attributed to semantic processing as the specific categorization into numbers and letters was irrelevant to the task. Nevertheless, it would be interesting to replicate the experiment with a more difficult task and an extended reaction time window.

\subsubsection{Conclusion}

In the current study, perceptual and semantic priming effects without response association were found under identical conditions. Considering the impact of stimulus parameters was proved to be increasingly important as several parameters can have an impact on priming effects (Chapter 2). Therefore, the same masking method was applied in the perceptual and semantic priming experiment. Previous research indicated that masking method has a crucial impact on the development of priming effects (Chapter 3). Perceptual priming effects on reaction times appeared to be larger than semantic priming effects on reaction times. This could indicate that semantic processing occurs on a higher level with 
activations being more extended, less intensive and more variable. This would be in line with observed ERP priming effects. P2 amplitude and P3 amplitude were modulated through perceptual congruency. P2 is associated with repetition suppression (e.g. Brang et al., 2008; Freunberger et al., 2007; Hsu, Hämäläinen \& Waszak, 2014) and seems to reflect a transdimensional feature detection process (Luck \& Hillyard, 1994). P3(b) is associated with memory processes which generate an update of the stimulus representation (Polich, 2007). The modulation of P3 amplitude through perceptual congruency correlated with perceptual priming effects on reactions times. Event-related potentials were also modulated through semantic congruency. An N400-like priming effect occurred with a delayed latency. Perceptual ERP effects were observed earlier and with different distributions than semantic ERP effects. Evidence was provided that different mechanisms contribute to perceptual and semantic priming effects with identical stimulation. 


\section{Overall Discussion}

The current work addresses masked priming as a tool for investigating unconscious processing. Limits of unconscious processing shed light on the structure of consciousness. Initially, 84 studies were examined to get an overview of the current state of research. These studies provided 538 cases (i.e. effect sizes) for meta-analytic examination. Heterogeneity between studies was expectably high. In an exploratory approach, 23 different moderators were identified (Table 2.1). A psychological model (Figure 2.1) was developed to provide a framework for meta-regression estimations. The impact of prime visibility on priming effects was of particular interest as low prime visibility indicates effective masking and establishes unconscious processing.

Two comprehensive models were estimated due to confounding factors which should not be included in the same model. These confounding factors concerned the masking method and the task level. Both models match to a vast extent and differ predominantly with regards to the confounding factors. Six systematic differences between primes and targets were identified. These were differences in modality, notation, size and case, shape, color and perspective. Only differences in notation, size/case, shape and color had significant impacts on effect sizes in comprehensive model estimations. A negative impact of differences in size and shape became more pronounced with increasing prime visibility. The negative impact of different prime and target notations was reduced with increasing prime visibility. Systematic differences between primes and targets seem to emphasize the distinctness of primes and targets. The system might inhibit activations induced by primes as a response to recognizing the distinctness. This process is promoted by increasing prime visibility. However, the system also recognizes higher level similarities with increasing prime visibility when primes and targets differ in notation. Priming effects were generally smaller when congruency was based 
on the meaning of the stimuli compared to effect sizes achieved in studies with a direct relation of primes and targets. Prime novelty in semantic priming paradigms was especially important for this difference. However, pure semantic priming effects increased with increasing prime visibility. Response type remained in only one comprehensive model after fitting. On the basis of this model, priming effects seem to be larger when subjects are asked to express themselves verbally or by writing compared to giving a motor response.

The impact of masking method or task level cannot be interpreted without restrictions as a confounding of these factors was identified. Two groups of masking methods were distinguished: pattern masking and metacontrast masking. One comprehensive model estimation revealed larger priming effects with metacontrast masks than with pattern masks. This model also suggested that priming effects are independent of prime visibility when metacontrast mask are applied. Prime effects seem to increase with increasing prime visibility when pattern masks are used. However, a similar pattern was found with the confounding factor task level in another comprehensive model estimation. Semantic response priming effects were generally smaller than perceptual response priming effects and increased with increasing prime visibility. Perceptual response priming effects seemed to be independent of prime visibility. It is unclear whether this pattern is due to differences between masking methods or differences between task levels. Contributions of both factors are also conceivable. Subsequent experiments were conducted to resolve the confounding by examining perceptual and semantic priming effects with metacontrast masks and pattern masks (Chapter 3). Manipulation of prime visibility was essential to determine whether it contributes only to semantic priming effects or generally to priming effects when pattern masks are applied.

Another confounding was revealed through the distinction of prime-mask SOA and mask-target SOA. It was previously proposed that response priming effects increase with 
increasing prime-target SOA and semantic priming effects decrease with long prime-target SOA (Van den Bussche et al., 2009). The current meta-analysis revealed the same pattern when prime-mask SOA and mask-target SOA were aggregated. However, direct priming effects seem to increase with increasing prime-mask SOA whereas semantic priming effects decrease with increasing mask-target SOA. Prime-mask SOA and mask-target SOA were varied to a different extent in studies applying different priming paradigms (Figure 4.1). Six experiments were conducted to resolve this confounding and compare the time courses of different priming paradigms (Chapter 4). These experiments did not focus predominately on prime visibility. However, some mechanisms that might operate between the presentations of mask and target are thought to rely on low prime visibility (Klapp, 2005). Therefore, effective manipulation of prime visibility was necessary again.

\subsection{Manipulation and Measurement of Prime Visibility}

The meta-analysis (Chapter 2) revealed several moderators of prime visibility as well as moderators that have an impact on the measurement of visibility. Khalid and colleagues (2011) proposed three potential factors that might have an impact on the estimation of prime visibility: prime-target similarity, prime-response similarity and prime-response mapping. Prime-target similarity is thought to lead to confusions of prime and target. In accordance with this assumption, prime visibility was significantly larger in studies without prime-target similarity. Furthermore, prime visibility was reduced in studies with primes that were naturally associated with the response. Studies with a fixed prime-response mapping reported higher $d^{\prime}$-values than studies with a varied mapping. In addition to moderators of Khalid and colleagues (2011), target-target similarity was introduced as a potential moderator of $d$ '. Target-target similarity encodes whether the same target was applied in priming and prime identification sessions or not. Meta-analytic estimations suggested that changing or 
eliminating the target caused an overestimation of prime visibility. This might attest an additional masking effect of targets. Furthermore, five different visibility tasks were distinguished: detection, discrimination, prime-target discrimination, identification of alternatives and prime identifications tasks. The moderator visibility tasks had a significant impact on the measurement of prime visibility as well. The design of subsequent experiments was adapted with regards to these factors. It was aimed to achieve estimations of prime visibility as precise as possible and ensure comparability between experiments. In every experiment, except Experiment 3.1, primes never appeared as targets. Impacts of possible response associations (e.g. SNARC effects; Dehaene, Bossini \& Giraux, 1993; Gevers, Reynvoet, \& Fias, 2003) were avoided through a delay between presentation and response as well as indicating the response mapping after stimulus presentation. Targets were equally applied in priming sessions and prime identification sessions. The identification of alternatives was chosen as visibility task for all experiments. Furthermore, subjects were asked to assess prime visibility with the perception awareness scale (PAS) developed by Ramsøy and Overgaard (2004).

Besides moderators of the prime visibility measurement, several moderators of actual prime visibility were identified. These moderators are of particular interest as it was aimed to manipulate prime visibility. Prime duration and prime-mask SOA had a positive impact on prime visibility as expected (Enns \& Di Lollo, 2000; Breitmeyer \& Ögmen, 2006). Masktarget SOA also had a positive impact on prime visibility. This attests an additional masking effect of targets. However, it was aimed to manipulate prime visibility without changing the duration of prime effectivity as well. It is known that priming effects increase with increasing duration of prime effectivity (e.g. Mattler, 2003; Vorberg et al., 2003). The meta-analysis also revealed that prime visibility differed between different masking methods. However, masking methods are most likely chosen to be suitable for masking a specific kind of 
stimulus. Furthermore, masking method is also a possible moderator of priming effects. However, pattern masks as well as metacontrast masks can be constructed with different masking strength.

In twelve experiments, prime visibility was varied through masking strength. Two kinds of masks were applied in different experiments. Parts of primes were arranged in a grid to construct pattern masks. Different masking strengths were realized through four different mesh widths: $0.15^{\circ}, 0.30^{\circ}, 0.61^{\circ}$ and $1.21^{\circ}$ of visual angle. The strongest metacontrast masks consisted of eight layers in altering colors (red and green) encompassing the primes. The layers were as thick as the stroke width of the primes. Four different masking strengths were realized through removing zero, one, two or seven layers (cf. Lefton, 1973; Mattler \& Palmer, 2012). In Chapter 3, four different masking strengths were applied with metacontrast masks and pattern masks. Furthermore, four different prime-mask SOAs were applied: $30 \mathrm{ms,}$ $60 \mathrm{~ms}, 90 \mathrm{~ms}$ and $120 \mathrm{~ms}$. Subjective judgments generally reflected objective prime visibility. As expected, prime visibility increased with increasing prime-mask SOA. Prime visibility also increased with decreasing masking strength in Experiments 3.1 and 3.2. In Experiment 3.3 , prime visibility was reduced more efficiently with a mask density of $0.30^{\circ}$ than with a density of $0.15^{\circ}$. This was considered in subsequent analyses and deductions. Prime visibility was less effectively reduced with metacontrast masks and varied with masking strength in a smaller range. An impact of a ceiling effect was suspected. Only two different prime-mask SOAs (60 ms \& $90 \mathrm{~ms}$ ) and two masking strengths were applied in experiments of Chapter 4. As prime visibility increases with increasing prime-mask SOA, ceiling effects were expected to be more pronounced. Consequently, modulations of prime visibility were less evident. Metacontrast masks were applied in Experiment 4.1 and subjective prime visibility was generally modulated through masking strength. However, objective prime visibility was only significantly modulated through masking strength in Experiment 4.1B. In Experiment 4.2, 
pattern masks were applied and subjective as well as objective prime visibility was clearly modulated through masking strength. However, prime visibility was hardly modulated in Experiment 4.3 with only backward pattern masks. Nevertheless, the slight modulation of subjective prime visibility reached significance in both subexperiments as well as the modulation of objective prime visibility in Experiment 4.3B. Interestingly, masks with less bottom-up energy $\left(1.21^{\circ}\right.$ mesh width) reduced prime visibility more effectively.

Generally, prime visibility was successfully modulated through masking strength. This modulation was especially pronounced and important in experiments of Chapter 3. However, prime visibility was quite high in certain conditions and ceiling effects became a problem. For instance, variation of prime visibility was small with metacontrast masks at long SOAs. In this context, the possibility of an overestimation of prime visibility is relevant and there is evidence that suggests such an overestimation. First, prime visibility was always assessed after the priming session. Second, objective prime visibility was assessed with a delay between presentation and retrieval (Vermeiren \& Cleeremans, 2012). Third, several subjects did not detect the prime during the priming sessions and many subjects noticed an improvement of prime perception during the prime identification session. Nevertheless, primes were visible in Experiment 4.3. This emphasizes the importance of forward masks for effective pattern masking and is in line with the observation that pattern masking is usually applied with forward and backward masks (Chapter 2).

Prime visibility was manipulated through mask density with pattern masks (cf. Eimer \& Schlaghecken, 2002) and through intercontour distances with metacontrast masks (cf. Lefton, 1973; Mattler \& Palmer, 2012). Consequently, stimulation differed between trials of high and low prime visibility and additional effects of masks are conceivable. Evidence for such an effect is provided through debriefing. Subjects reported that masks of different strength were differently distracting. Furthermore, several mechanisms are triggered by 
masks (Chapter 4; e.g. Klapp, 2005; Lleras and Enns, 2004). These mechanisms modulate priming effects with increasing mask-target SOA. Moreover, experiments of Chapter 3 revealed that priming effects increase with prime visibility when pattern masks are used and are independent of prime visibility with metacontrast masks. Nevertheless, modulation of prime visibility through masking strength seems to be appropriate. Initially, we were interested in resolving confoundings that were revealed in the meta-analysis. Masks with different strengths were also applied across studies. Furthermore, prime visibility was not always reduced more effectively by masks with higher bottom-up energy (Experiment 3.3 and 4.3). In these cases, priming effects were rather modulated through prime visibility than bottom-up energy of masks. This provides further evidence that effects of prime visibility were observed.

\subsection{Perceptual and Semantic Priming - Similarities and Differences}

Perceptual and semantic effects were compared in three studies focusing on different aspects. Chapter 3 concerns the relation of prime visibility and priming effects. More precisely, six experiments were conducted to determine whether perceptual and semantic priming effects differed with regards to a modulation through prime visibility. A metaanalytic review of previous research (Chapter 2) indicated that perceptual priming effects might be independent of prime visibility whereas semantic priming effects increase with increasing prime visibility. However, these differences might have also emerged on the basis of different masking methods. Experiments of Chapter 4 were conducted to examine and compare the time courses of perceptual and semantic priming with increasing prime-mask and mask-target SOA. It was previously observed that perceptual priming effects increase with prime-target SOA, whereas semantic priming effect decrease at long SOAs (Van den Bussche et al., 2009). However, our meta-analysis suggested that different SOA were 
predominantly varied in perceptual and semantic priming studies. Finally, perceptual and semantic priming effects without response association were examined with electroencephalography (EEG) to reveal similarities and differences on a neuronal level.

The first study revealed that perceptual as well as semantic priming effects increase with prime visibility when pattern masks are applied. Priming effects are independent of prime visibility when metacontrast masks are used. This supposed difference between perceptual and semantic priming was based on a confounding. However, perceptual priming effects appeared to be larger than semantic priming effects. Four explanations were proposed which might account for this difference. First, primes might facilitate actual semantic processing with the applied semantic task. Semantic processing is thought to occur on a higher level than perceptual processing. Therefore, primes might induce activations that are more extended, less intensive and more variable. Second, the color of stimuli might be more salient than the form which is the basis for semantic categorization. However, color and identity were equally reportable in prime identification sessions. Contrary, prime identity was recognized more correctly than color in the EEG experiment. Third, semantic priming effects might be smaller due to a larger set size in the current design (Kiesel et al., 2006). Kunde and colleagues (2003) proposed the action trigger account and argued that semantic response priming is based on activation of pre-specified cognitive action triggers (Kiesel, Kunde \& Hoffmann, 2007). Fourth, a systematic difference between primes and targets in color occurs only with semantic priming effects in the current design and might reduce these effects (Chapter 2).

Further differences between perceptual and semantic priming effects were assumed with regards to time courses with increasing prime-mask and mask-target SOA. Four different prime-mask SOAs were applied in experiments of Chapter 3. Perceptual as well as semantic priming effects increased with increasing prime-mask SOA. In experiments of 
Chapter 4, a replication of these modulations was aimed for with two intermediate primemask SOAs. However, evidence was less compelling. Nevertheless, evidence suggests that priming effects increase with increasing prime-mask SOA as the lack of additional evidence can be attributed to the small difference between $60 \mathrm{~ms}$ and $90 \mathrm{~ms}$ prime-mask SOA. Perceptual and semantic priming effects are modulated equally through prime-mask SOA. However, differences were found with increasing mask-target SOA (Chapter 4). Perceptual and semantic priming effects were equally reduced at long mask-target SOA, but the courses differed characteristically. Perceptual priming effects declined rapidly at first. At $100 \mathrm{~ms}$ mask-target SOA, priming effects became inverse, i.e. reactions were faster or more accurate in incongruent trials compared to congruent trials. Subsequently, perceptual priming effects became increasingly positive again. In contrast, semantic priming effects decreased continuously. However, this decrease was more pronounced with less prime visibility and indications for an inversion of priming effects were found. Three mechanisms are thought to contribute to the courses of priming effects with increasing mask-target SOA. First, a simple decay of activation can occur with perceptual and semantic priming. Masks seem to interrupt prime processing and any residual activation can decay over time. Second, masks might trigger an active process of inhibition (e.g., Klapp, 2005). However, this process is thought to rely on low prime visibility. Perceptual and semantic priming can be equally affected by this process. A third mechanism might be based on perceptual interaction of primes and masks. This mechanism of object updating was introduced by Lleras and Enns (2004) and acts when primes and masks share features. As masks are presented subsequently to primes, all aspects that differed between a specific prime and the mask are more salient. Consequently, processing of perceptually incongruent trials is facilitated when the impact of the relevant masks had time to evolve. The impact of this mechanism is maximal with a mask-target SOA of approximately $100 \mathrm{~ms}$ to $150 \mathrm{~ms}$. Crucially, this mechanism only affects perceptual 
priming effects. The three mechanisms were developed and intensively discussed since the phenomenon of inverse priming effects was introduced (Eimer \& Schlaghecken, 1998). The current work provides further evidence for these mechanisms. It was possible, to detect and distinguish both active processes in experiments with the exact same stimulation. Therefore, the mechanism of object updating can be directly attributed to the relevance of masks. Furthermore, active processes of inhibition were more pronounced with low prime visibility. This pattern was found in Experiments 4.1 and 4.3. Interestingly, masks with more bottom-up energy reduced prime visibility more effectively in Experiment 4.1 and less effectively in Experiment 4.3. However, priming effects were rather modulated through prime visibility than mask energy. This emphasizes the role of actual prime visibility for active processes of inhibition. Generally, perceptual and semantic priming effects were modulated through prima-mask and mask-target SOA very similarly. Priming effects increase with increasing prime-mask SOA and decrease with increasing mask-target SOA. This pattern was expected on the basis of our meta-analysis (Chapter 2). Differences between perceptual and semantic priming occurred with regards to the specific courses of effects with increasing mask-target SOA. However, these differences can be attributed to the specific tasks and masks rather than to different levels of processing.

The current work revealed that perceptual and semantic priming effects are very similar with regards to possible modulations through prime visibility and temporal parameters. Nevertheless, perceptual priming effects appeared to be larger than semantic priming effects. Characteristics of the current design might account for this pattern. However, it might also be an indication that semantic processing occurs on a higher level than perceptual processing. The induced activations might be more extended, less intensive and more variable at this level. An experiment was designed to examine this possibility more precisely with electroencephalography (Chapter 5). The design allowed a comparison of 
behavioral perceptual and semantic priming effects without response association. Furthermore, ERP priming effects were observed without response execution. We were mainly interested in relevant priming effects, i.e. effect type and task level match. However, irrelevant priming effects (e.g. perceptual congruency effects with a semantic task level) were also computed. Neither behavioral nor ERP congruency effects were found on a dimension that is irrelevant for the task. This is in line with the attentional sensitization model (Kiefer \& Martens, 2010). Relevant perceptual congruency effects were again larger than relevant semantic congruency effects on a behavioral level. Examination of target-locked ERPs revealed distinctive modulations through perceptual and semantic congruency. The P2 component was modulated through perceptual congruency with an occipital distribution. Modulations of the P3 component through perceptual congruency were found with a more centro-parietal distribution. These components are thought to be involved in transdimensional feature detection processes (P2; Luck \& Hillyard, 1994) as well as updating of the stimulus representation (P3(b); Polich, 2007). P2 was previously associated with repetition suppression (e.g. Brang et al., 2008; Freunberger et al., 2007; Hsu, Hämäläinen \& Waszak, 2014). P3 reflects processes that closer to response decisions (Hillyard \& Münte, 1984; Kok, 2001; Luck, 2005). In line with this, P3 priming effects correlated with behavioral priming effects on reaction times. Event-related potentials were also modulated through semantic congruency. A N400-like priming effect occurred with a delayed latency. This delay might be due to the presentation of primes and targets in quick succession (Kutas, 1993) and is in line with a study using similar temporal parameters (Martens et al., 2011). Nevertheless, the distribution of the N400-like priming effect is over temporal/centro-posterior electrodes on the right hemisphere and is line with studies that found N400 over Wernicke's homolog (Holcomb, 1993; Kutas \& Hillyard, 1982; Luck, 2005; Neville et al., 1992; Van Petten, 1993; Van Petten \& Luka, 2005). N400 is thought to reflect activations in the posterior heteromodal 
association cortex and prefrontal areas (reviewed in Kutas \& Federmeier 2009; Binder et al., 2009). This suggests that semantic priming effects are based on semantic processing of stimuli that exceeds basal perceptual processing in the current study.

In current work, perceptual and semantic effects were compared with an intense focus on three characteristics. Our meta-analysis (Chapter 2) emphasized the importance of avoiding confoudings with regards to the applied stimuli. Initially, both effect types were examined with regards to a possible modifiability through prime visibility. However, the impact of prime visibility differed between masking methods rather than effect types. Furthermore, development of perceptual and semantic priming effects with different temporal parameters was of interest. Priming effects increased generally with increasing prime-mask SOA and declined with increasing mask-target SOA. Differences with regards to the specific courses of perceptual and semantic priming effects can be attributed to the specific tasks and masks rather than to different levels of processing. Nevertheless, an electrophysiological study suggested that perceptual priming and semantic priming differ substantially even when semantic congruency is based on a categorization of letters and numbers. Semantic priming is thought to occur on a higher level of processing than perceptual priming.

\subsection{Masking Method - a Key to the Structure of Consciousness}

The current work was mainly focused on masked priming as a method for examining unconscious processing of stimuli. This is a common approach for getting insights into consciousness (Kouider \& Dehaene, 2007). Prime visibility is an important factor as no prime visibility indicates unconscious processing of stimuli. Identifying processes that rely on consciousness allows inferences to be drawn concerning the function and structure of consciousness. A meta-analysis (Chapter 2) was therefore conducted with a main focus on the impact of prime visibility. Meta-regression estimations revealed several interactions with 
prime visibility. An observed interaction between prime visibility and task level was of great interest as it indicates that higher level processing might rely on consciousness. However, a confounding between task level and masking method was also revealed. Subsequent experiments (Chapter 3) showed that the impact of priming visibility is independent of task level. Instead, priming effects were independent of prime visibility with metacontrast masks and increased with increasing prime visibility when pattern masks were applied.

In experiments of Chapter 4, perceptual and semantic priming effects were examined with regards to an impact of temporal parameters. Again, perceptual and semantic priming effects were modulated quite similarly. Differences occurred with regards to the specific courses of priming effects with increasing mask-target SOA. However, these differences are rather based on the relevancy of masks.

The masking method seems to determine which processes can occur. Therefore, examining priming with different masking methods might be a key to the structure and function of consciousness. This approach was also recently proposed by Breitmeyer (2014). The author compiled a hierarchy of psychophysical blinding techniques relative to the functional level of unconscious processing. Binocular-rivalry suppression is thought to act on a very low level of processing that is partly precortical. Continuous flash suppression operates on a higher level of processing, followed by backward pattern masking and metacontrast masking. Crowding and blinding through attentional blink seem to act on an even higher level. These techniques are followed by object-substitution masking. Breitmeyer emphasized that the hierarchy is tentative and open for additions and revisions. Based on the current work, we like to propose that pattern masking acts on a lower level of processing than metacontrast masking (Chapter 3). However, the application of a forward pattern mask might be crucial (Chapter 4). The current work supports the idea that such a hierarchy combined with the use of brain imaging might reveal important insights into consciousness. 


\subsection{Implications for Theories of Consciousness}

Revealing characteristics of consciousness was the overarching goal of the current work. Masked priming was the method of choice (Kouider \& Dehaene, 2007). A metaanalysis was conducted with the main intention to reinterpret accumulated evidence for a distinction of processes that rely on consciousness and processes that are independent of consciousness. At first glance, it was very interesting that meta-regression revealed an interaction of task level and prime visibility. It seemed that perceptual priming effects are independent of prime visibility whereas semantic priming effects increase with increasing prime visibility. This pattern would have been in line with the Global Neuronal Workspace Theory (GNW; Dehaene et al., 1998). The theory received considerable support (reviewed in Blackmore, 2003) and proclaims a distinction of a unique global workspace and modular perceptual, motor, memory, evaluative, and attentional processors. These modular processors are functionally specialized. The global neuronal workspace consists of a distributed set of cortical neurons. These (pyramidal) neurons have long-range axons and interact with each other across cortical areas. Especially in effortful tasks, neurons of the global workspace contribute to processing. Transferred to the current differentiation of task levels, simple perceptual processing might occur without activations of global workspace neurons whereas more complex semantic processing might rely on cortico-cortical interactions. However, subsequent experiments revealed that the interaction of task level and prime visibility across studies was based on a confounding (Chapter 3). Nevertheless, this also cannot be considered as dissenting evidence. Within the framework of GNW Theory, semantic processing might also be accomplished by perceptual circuits (Dehaene et al., 1998).

In Chapter 3, experiments revealed that metacontrast masking reduces prime visibility without affecting priming effects. Pattern masking reduced prime visibility as well as priming 
effects. The examination of different masking methods seems be to a promising approach to grasp consciousness (cf. Breitmeyer, 2014). The found pattern suggests that processes leading to priming effects occur prior to processes of consciousness. Metacontrast masking seems to disturb processing after the emergence of activations that are relevant for priming effects. Pattern masking seems to reduce prime visibility on a low level and priming effects are compromised. This is in accordance with the model of recurrent processing (Lamme \& Roelfsema, 2000). Lamme and Roelfsema propose a distinction of feedforward and recurrent processing. In multiple parallel feedforward sweeps, information is passed rapidly from lower to higher visual areas. This initial processing is characterized as unidirectional. This is reflected through increasing response latencies of areas that are hierarchically higher and higher. The speeds of these streams of processing differ due to different contributions of magnocellular and parvocellular pathways and depend on stimulus attributes. Many aspects of stimuli can be processed within the feedforward sweep. Additionally, the authors propose that recurrent connections allow feedback from higher areas to lower areas. This is reflected by a late change in tuning of neurons and a late responsiveness to information outside of the receptive field. Furthermore, Lamme and Roelfsema suggest that recurrent processing is the essence of consciousness. It seems that metacontrast masking disturbs the recurrent processing between higher and lower visual areas as proposed by the authors. Pattern masking, in contrast, interferes with the feedforward sweep. The results of the current work support the model of recurrent processing.

A different aspect of the complied data provides information regarding another possible characteristic of consciousness. Whether consciousness is gradual or dichotomous is controversial (e.g. Nieuwenhuis \& Kleijn, 2011; Overgaard, Rote, Mouridsen \& Ramsøy, 2006; Sandberg, Timmermans, Overgaard \& Cleeremans, 2010; Sergent \& Dehaene, 2004a). Several imaging studies show correlations between cortical activity and subjective reports of 
stimulus perception in a gradual fashion (e.g. Bar et al., 2001; Grill-Spector, Kushnir, Hendler, \& Malach, 2000; Moutoussis \& Zeki, 2002; reviewed in Sergent \& Dehaene, 2004a). However, theories of consciousness that emphasize the importance of recurrent interaction between cortices proclaim a dichotomous character of consciousness, including Global Neuronal Workspace Theory (Dehaene et al., 1998) and the model of recurrent processing (Lamme \& Roelfsema, 2000). Once activation exceeds a threshold, reverberation and self-amplification within the global workspace leads to conscious perception. Sergent and Dehaene (2004a) provided evidence for the all-or-none character of consciousness. The authors investigated subjective perception in a study using the attentional blink paradigm. Subjects rated stimulus perception with a continuous scale, but used predominantly the extreme values. This pattern was interpreted as an indication of the dichotomous character of consciousness. However, this study was not received without criticisms (e.g. Nieuwenhuis \& Kleijn, 2011; Overgaard et al., 2006). For instance, only the extreme values were labeled on the scale and this might entice subjects to neglect intermediate responses. It was also noted that it is important to emphasize the request of introspective reports rather than reports of stimulus features (Overgaard et al., 2006; Ramsøy \& Overgaard, 2004). Furthermore, subjects were asked to rate the visibility of words and the cognitive system is often able to infer the whole word from perception of single letters (McClelland \& Rumelhart, 1981; Nieuwenhuis \& Kleijn, 2011). Subsequently, evidence was provided that suggests a gradual characteristic of consciousness (Nieuwenhuis \& Kleijn, 2011; Overgaard et al., 2006; Sandberg et al., 2010). Sandberg and colleagues (2010) compared different methods of assessing conscious perception: the Perceptual Awareness Scale by Ramsøy and Overgaard (2004), confidence ratings (e.g. Cheesman \& Merikle, 1986) and post-decision wagering introduced by Persaud, McLeod, and Cowey (2007). The authors revealed that the Perceptual Awareness Scale (PAS) is especially sensitive to intermediate levels of awareness. The PAS 
was also applied in 14 experiments of the current work. In each experiment, intermediate ratings were used extensively. This indicated that consciousness has indeed a gradual characteristic. Does this implicate a rejection of Global Neuronal Workspace Theory (Dehaene et al., 1998) and the model of recurrent processing (Lamme \& Roelfsema, 2000)? Our data strongly support the model of recurrent processing and both theories suggest a dichotomous character of consciousness rather than rely on the assumption of a dichotomous character.

Another proposed model can be evaluated on the basis of compiled data: the attentional sensitization model by Kiefer \& Martens (2010). The authors do not provide a theory of conscious in a strict sense. However, the model proposes that an attention based sensitization of relevant pathways is necessary for unconscious processing. Unconscious processing is therefore sensitive to top-down control as well as conscious processing. The authors concede that top-down control is more flexible with conscious processing and refer to the distinction of preemptive and reactive control (cf. Ansorge \& Horstmann, 2007; Kiefer, 2007). They propose that unconscious processing is only modulated through preemptive topdown control. In the current experiments, subjects were asked to either concentrate on the color of stimuli or focus on the semantic. However, the design always allowed a computation of perceptual and semantic priming effects. In each study, only relevant priming effects reached significance, i.e. when task level and effect type matched (Chapter 5). Neither the analysis of behavioral data (reaction times and error rates) nor event-related potentials indicated a modulation through the irrelevant congruency. Therefore, the current work supports the attentional sensitization model in 14 experiments.

In summary, the current work provided data that is in line with the model of recurrent processing by Lamme \& Roelfsema (2000). However, subjective ratings of prime perception with the Perceptual Awareness Scale by Ramsøy and Overgaard (2004) also indicated a 
gradual character of consciousness. This is not in accordance with the original theory. Furthermore, our data emphasized the impact of attentional top-down control on unconscious processing. This was predicted by the attentional sensitization model by Kiefer \& Martens (2010).

\subsection{Pending Questions}

In our attempt to include various studies in our meta-analysis (Chapter 2), several differences between these studies were identified and included as moderators. The impact of prime visibility on effects sizes and interactions of prime visibility and specific moderators (e.g. task level and masking method) were of special interest. However, meta-regression model estimations suggested that several additional moderators also have an impact on effect sizes. Other moderators were excluded during model fitting. Although meta-analytic model estimations provide a promising approach to exploit previous research, it is important to mind that most studies were not designed to investigate the impact of several moderators. Consequently, these moderators were not varied systematically. Variance inflations factors (VIF) might indicate whether there are severe confoundings (O'Brien, 2007), but a low VIF does not substitute for proper experimental variation. One cannot rule out possible impacts of moderators that were not gathered. Identified coherences between moderators and effect sizes can be regarded as profound hypotheses. The current work addressed some of these moderators: prime visibility, masking method, task level, effect type, response association, prime-mask SOA and mask-target SOA. However, a psychological model was proposed that concerned the composition of priming effects (Chapter 2) and a validation of this model includes examination of further moderators. Beyond model validation, investigations of further interactions with prime visibility might provide additional insights into conscious and unconscious processing. 
One complex of moderators concerned systematic differences between primes and targets. Generally, these moderators had a negative impact on priming effects. After model fitting, differences in notation, color, size and shape seemed to have a significant impact on effect sizes, whereas differences in modality and perceptive seemed to have no significant impact. Interestingly, differences in size and shape only had an impact in interaction with prime visibility. A difference in color reduced priming effects independently of prime visibility. This pattern indicates that some stimulus aspects might induce sustained activations independently of conscious processing. The impact of attention on these processes is also still obscure (cf. attentional sensitization model; Kiefer \& Martens, 2010).

Another set of moderators seems to have an impact on prime visibility. Especially, the visibility task might be a factor of interest. Only few studies compared different visibility tasks previously. For instance, Fisk and Haase $(2005,2011)$ compared a detection task with a discrimination of alternatives and an identification task. However, the current meta-analysis identified five types of visibility tasks that are frequently applied: detection, discrimination, prime-target discrimination, identification of alternatives and prime identifications tasks. Comparable to a hierarchy of blinding techniques (Breitmeyer, 2014), it might be fruitful to experimentally consolidate a hierarchy of visibility tasks. The application of several tasks might allow a more precise assessment of stimulus visibility. It is conceivable that some stimulus aspects are processed unconsciously whereas other aspects reach consciousness. This approach might be a way of getting closer to the subjective perception. It would also be interesting to establish how these visibility tasks relate to subjective reports of visibility and to which extent performance is sensitive to feedback.

Some pending questions can be derived directly from experiments of the current work. In experiments of Chapter 3, the impact of prime visibility was assessed in the context of different task levels and masking methods. Within the meta-analysis (Chapter 2), two 
competing models were compiled. One model included the moderator masking method and attributed smaller priming effects and an influence of prime visibility to pattern masks. The other model included the moderator task level and brought this in line with a semantic task level. Experiments of Chapter 3 revealed that different impacts of prime visibility only occur with different masking methods. Perceptual and semantic priming effects with response association are equally affected by variations of prime visibility. However, another moderator that encodes the relation between primes and targets was included in both model estimations. This moderator reflects priming effects without response association. Both models indicated that a direct prime-target relation leads to larger priming effects than an association through meaning. Furthermore, priming effects seem to increase with increasing prime visibility when primes and targets are associated through meaning. The moderator prime-target relation was included in both models on a basis of a VIF below 4 (O'Brien, 2007). However, it is to be noted that the moderator prime-target relation had the third highest VIF. The estimated impact of prime-target relation on effect sizes requires experimental validation. A combination of the designs in Chapter 3 and 5 would be suitable to address this question.

Another pool of research questions concerns the impact of attention and top-down control on unconscious processing. The current data consistently supports the attentional sensitization model by Kiefer and Martens (2010). However, the results of the electrophysiological experiments (Chapter 5) revealed a slight insignificant tendency that perceptual priming effects on reaction times might increase with increasing prime-mask SOA (Figure 5.4). This raises the interesting question whether there are basal stimulus attributes that can produce effects without attentional amplification. I hope the current work stimulates further thoughts and new ideas. 


\subsection{Concluding Remarks}

The current works provides a meta-analytic review of masked priming as an approach to consciousness. Several moderators of priming effects were identified and a psychological model that concerns the composition of priming effects was proposed (Chapter 2). Model estimations revealed interesting interactions with prime visibility. Identifying processes that can occur without consciousness and processes that rely on consciousness provides insights into functions and the structure consciousness. Subsequent experiments (Chapter 3) confirmed that priming effects increase with prime visibility when pattern masks are applied. Priming effects are independent of prime visibility with metacontrast masks. Within the framework of the model of recurrent processing (Lamme \& Roelfsema, 2000), pattern masks are thought to reduce prime visibility on an early level and interfere with the feedforward sweep whereas metacontrast masks disturb the recurrent processing. Perceptual and semantic priming effects were affected equally by variations in prime visibility. Both effect types were also modulated through prime-mask SOA and mask-target SOA quite similarly (Chapter 4). Priming effects increase with increasing prime-mask SOA and decline with increasing masktarget SOA. This decline seems to be based on a simple decay and active mechanisms of inhibition (e.g. Klapp, 2005). Courses of perceptual and semantic priming effects with increasing mask-target SOA differed due to an additional mechanism of object updating with perceptual priming (Lleras \& Enns, 2004). However, semantic priming effects were generally smaller than perceptual priming effects. In an electrophysiological study (Chapter 5), perceptual and semantic priming effects were compared with identical stimulation. This study suggested that perceptual and semantic priming effects differ substantially. The current work proposes several approaches to the investigation consciousness and emphasizes the importance of considering the impact of several moderators on priming effects. 
6 | Approaching Consciousness with Masked Priming 


\section{References}

References marked with an asterisk indicate studies included in the meta-analysis (Chapter 2).

Adams, S. C., \& Kiefer, M. (2012). Testing the attentional boundary conditions of subliminal semantic priming: the influence of semantic and phonological task sets. Frontiers in Human Neuroscience, 6, 241. doi:10.3389/fnhum.2012.00241

Albrecht, T., Klapötke, S., \& Mattler, U. (2010). Individual differences in metacontrast masking are enhanced by perceptual learning. Consciousness and Cognition, 19(2), 656-666. doi:10.1016/j.concog.2009.12.002

Albrecht, T., \& Mattler, U. (2012a). Individual differences in metacontrast masking regarding sensitivity and response bias. Consciousness and Cognition, 21(3), 1222-1231. doi:10.1016/j.concog.2012.04.006

Albrecht, T., \& Mattler, U. (2012b). Individual differences in subjective experience and objective performance in metacontrast masking. Journal of Vision, 12(5), 5. doi:10.1167/12.5.5

* Ansorge, U. (2003). Asymmetric influences of temporally vs. nasally presented masked visual information: Evidence for collicular contributions to nonconscious priming effects. Brain and Cognition, 51(3), 317-325. doi:10.1016/S0278-2626(03)00034-4

Ansorge, U., Becker, S. I., \& Breitmeyer, B. (2009). Revisiting the metacontrast dissociation: comparing sensitivity across different measures and tasks. Quarterly Journal of Experimental Psychology (2006), 62(2), 286-309. doi:10.1080/17470210801908492 
Ansorge, U., Breitmeyer, B. G., \& Becker, S. I. (2007). Comparing sensitivity across different processing measures under metacontrast masking conditions. Vision Research, 47(27), 3335-3349. doi:10.1016/j.visres.2007.09.009

Ansorge, U., \& Horstmann, G. (2007). Preemptive control of attentional capture by colour: evidence from trial-by-trial analyses and orderings of onsets of capture effects in reaction time distributions. Quarterly Journal of Experimental Psychology (2006), 60(7), 952-975. doi:10.1080/17470210600822795

Baars, B. J. (1989). A Cognitive Theory of Consciousness. Cambridge England ; New York: Cambridge University Press.

Bar, M., Tootell, R. B. H., Schacter, D. L., Greve, D. N., Fischl, B., Mendola, J. D., ... Dale, A. M. (2001). Cortical Mechanisms Specific to Explicit Visual Object Recognition. Neuron, 29(2), 529-535. doi:10.1016/S0896-6273(01)00224-0

* Barbot, A., \& Kouider, S. (2012). Longer is not better: nonconscious overstimulation reverses priming influences under interocular suppression. Attention, Perception, \& Psychophysics, 74(1), 174-184. doi:10.3758/s13414-011-0226-3

Bentin, S., McCarthy, G., \& Wood, C. C. (1985). Event-related potentials, lexical decision and semantic priming. Electroencephalography and Clinical Neurophysiology, 60(4), 343-355. doi:10.1016/0013-4694(85)90008-2

Binder, J. R., Desai, R. H., Graves, W. W., \& Conant, L. L. (2009). Where Is the Semantic System? A Critical Review and Meta-Analysis of 120 Functional Neuroimaging Studies. Cerebral Cortex, 19(12), 2767-2796. doi:10.1093/cercor/bhp055

Blackmore, S. (2003). Consciousness: An Introduction. Oxford; New York: Oxford University Press. 
* Bodner, G., \& Dypvik, A. (2005). Masked priming of number judgments depends on prime validity and task. Memory \& Cognition, 33(1), 29-47. doi:10.3758/BF03195295

* Bodner, G., \& Masson, M. (2003). Beyond spreading activation: An influence of relatedness proportion on masked semantic priming. Psychonomic Bulletin \& Review, 10(3), 645-652. doi:10.3758/BF03196527

* Bodner, G., \& Mulji, R. (2010). Prime Proportion Affects Masked Priming of Fixed and Free-Choice Responses. Experimental Psychology (formerly Zeitschrift für Experimentelle Psychologie), 57(5), 360-366. doi:10.1027/1618-3169/a000043

* Bonin, P., Fayol, M., \& Peereman, R. (1998). Masked form priming in writing words from pictures: Evidence for direct retrieval of orthographic codes. Acta Psychologica, 99(3), 311-328. doi:10.1016/S0001-6918(98)00017-1

Borenstein, M., Hedges, L. V., Higgins, J. P. T., \& Rothstein, H. R. (2009). Introduction to Meta-Analysis (1. Aufl.). Wiley.

* Boy, F., \& Sumner, P. (2010). Tight coupling between positive and reversed priming in the masked prime paradigm. Journal of experimental psychology. Human perception and performance, 36(4), 892-905. doi:10.1037/a0017173

Brang, D., Edwards, L., Ramachandran, V. S., \& Coulson, S. (2008). Is the sky 2? Contextual priming in grapheme-color synaesthesia. Psychological Science, 19(5), 421-428. doi:10.1111/j.1467-9280.2008.02103.x

Breitmeyer, B. G. (1984). Visual Masking: An Integrative Approach. Clarendon Press.

Breitmeyer, B. G. (2014). The Visual (Un)Conscious and Its (Dis)Contents: A microtemporal approach. New York, NY: Oxford University Press. 
Breitmeyer, B., \& Öğmen, H. (2006). Visual Masking: Time Slices Through Conscious and Unconscious Vision. Oxford University Press.

* Breitmeyer, B. G., Ögmen, H., \& Chen, J. (2004). Unconscious priming by color and form: Different processes and levels. Consciousness and Cognition, 13(1), 138-157. doi:10.1016/j.concog.2003.07.004

Breitmeyer, B. G., Ro, T., \& Singhal, N. S. (2004). Unconscious Color Priming Occurs at Stimulus- Not Percept-Dependent Levels of Processing. Psychological Science, 15(3), 198-202. doi:10.1111/j.0956-7976.2004.01503009.x

* Breitmeyer, B., Ro, T., Öĝmen, H., \& Todd, S. (2007). Unconscious, stimulus-dependent priming and conscious, percept-dependent priming with chromatic stimuli. Attention, Perception, \& Psychophysics, 69(4), 550-557. doi:10.3758/BF03193912

* Carr, T. H., \& Dagenbach, D. (1990). Semantic priming and repetition priming from masked words: Evidence for a center-surround attentional mechanism in perceptual recognition. Journal of Experimental Psychology: Learning, Memory, and Cognition, 16(2), 341-350. doi:10.1037/0278-7393.16.2.341

* Carr, T. H., McCauley, C., Sperber, R. D., \& Parmelee, M. C. (1982). Words, pictures, and priming: On semantic activation, conscious identification, and the automaticity of information processing. Journal of Experimental Psychology: Human Perception and Performance, 8(6), 757-777. doi:10.1037/0096-1523.8.6.757

* Cheesman, J., \& Merikle, P. (1984). Priming with and without awareness. Attention, Perception, \& Psychophysics, 36(4), 387-395. doi:10.3758/BF03202793

Cheesman, J., \& Merikle, P. M. (1986). Distinguishing conscious from unconscious perceptual processes. Canadian Journal of Psychology, 40(4), 343-367. 
Clifford, C. W. G., \& Rhodes, G. (2005). Fitting the Mind to the World: Adaptation and After-Effects in High-Level Vision. Oxford University Press.

Costa-Faidella, J., Baldeweg, T., Grimm, S., \& Escera, C. (2011). Interactions between "What" and "When" in the Auditory System: Temporal Predictability Enhances Repetition Suppression. The Journal of Neuroscience, 31(50), 18590-18597. doi:10.1523/JNEUROSCI.2599-11.2011

Crowley, K. E., \& Colrain, I. M. (2004). A review of the evidence for P2 being an independent component process: age, sleep and modality. Clinical Neurophysiology: Official Journal of the International Federation of Clinical Neurophysiology, 115(4), 732-744. doi:10.1016/j.clinph.2003.11.021

Curran, T., \& Dien, J. (2003). Differentiating amodal familiarity from modality-specific memory processes: An ERP study. Psychophysiology, 40(6), 979-988. doi:10.1111/1469-8986.00116

* De Gardelle, V., Charles, L., \& Kouider, S. (2011). Perceptual awareness and categorical representation of faces: Evidence from masked priming. Consciousness and Cognition, 20(4), 1272-1281. doi:10.1016/j.concog.2011.02.001

* De Pisapia, N., Turatto, M., Lin, P., Jovicich, J., \& Caramazza, A. (2012). Unconscious Priming Instructions Modulate Activity in Default and Executive Networks of the Human Brain. Cerebral Cortex, 22(3), 639-649. doi:10.1093/cercor/bhr146

Dehaene, S., Bossini, S., \& Giraux, P. (1993). The mental representation of parity and number magnitude. Journal of Experimental Psychology: General, 122(3), 371-396. doi:10.1037/0096-3445.122.3.371 
Dehaene, S., \& Changeux, J.-P. (2011). Experimental and Theoretical Approaches to Conscious Processing. Neuron, 70(2), 200-227. doi:10.1016/j.neuron.2011.03.018

Dehaene, S., Changeux, J.-P., Naccache, L., Sackur, J., \& Sergent, C. (2006). Conscious, preconscious, and subliminal processing: a testable taxonomy. Trends in Cognitive Sciences, 10(5), 204-211. doi:10.1016/j.tics.2006.03.007

Dehaene, S., Kerszberg, M., \& Changeux, J.-P. (1998). A neuronal model of a global workspace in effortful cognitive tasks. Proceedings of the National Academy of Sciences, 95(24), 14529-14534. doi:10.1073/pnas.95.24.14529

Dehaene, S., \& Naccache, L. (2001). Towards a cognitive neuroscience of consciousness: basic evidence and a workspace framework. Cognition, 79(1-2), 1-37.

* Dehaene, S., Naccache, L., Clec'H, G. L., Koechlin, E., Mueller, M., Dehaene-Lambertz, G., Moortele, P.-F. van de, Bihan, D. L. (1998). Imaging unconscious semantic priming. Nature, 395(6702), 597-600. doi:10.1038/26967

Dehaene, S., Naccache, L., Cohen, L., Bihan, D. L., Mangin, J.-F., Poline, J.-B., \& Rivière, D. (2001). Cerebral mechanisms of word masking and unconscious repetition priming. Nature Neuroscience, 4(7), 752-758. doi:10.1038/89551

* Dell'Acqua, R., \& Grainger, J. (1999). Unconscious semantic priming from pictures. Cognition, 73(1), B1-B15. doi:10.1016/S0010-0277(99)00049-9

Delorme, A., \& Makeig, S. (2004). EEGLAB: an open source toolbox for analysis of singletrial EEG dynamics including independent component analysis. Journal of Neuroscience Methods, 134(1), 9-21. doi:10.1016/j.jneumeth.2003.10.009

Dennett, D. C. (1991). Consciousness Explained. Little, Brown and Company. 
Desimone, R., \& Duncan, J. (1995). Neural Mechanisms of Selective Visual Attention. Annual Review of Neuroscience, 18(1), 193-222. doi:10.1146/annurev.ne.18.030195.001205

* Di Luca, S., \& Pesenti, M. (2008). Masked priming effect with canonical finger numeral configurations. Experimental Brain Research, 185(1), 27-39. doi:10.1007/s00221$007-1132-8$

Dixon, N. F. (1971). Subliminal Perception: The Nature of a Controversy. McGraw-Hill.

Donchin, E., \& Coles, M. G. H. (1988). Is the P300 component a manifestation of context updating? Behavioral and Brain Sciences, 11(03), 357-374. doi:10.1017/S0140525X00058027

Duncan-Johnson, C. C., \& Donchin, E. (1977). On Quantifying Surprise: The Variation of Event-Related Potentials With Subjective Probability. Psychophysiology, 14(5), 456467. doi:10.1111/j.1469-8986.1977.tb01312.x

Dunn, B. R., Dunn, D. A., Languis, M., \& Andrews, D. (1998). The Relation of ERP Components to Complex Memory Processing. Brain and Cognition, 36(3), 355-376. doi:10.1006/brcg.1998.0998

* Eckstein, D., \& Henson, R. N. (2012). Stimulus/response learning in masked congruency priming of faces: Evidence for covert mental classifications? The Quarterly Journal of Experimental Psychology, 65(1), 92-120. doi:10.1080/17470218.2011.590595

* Eckstein, D., Kubat, M., \& Perrig, W. J. (2011). Visible homonyms are ambiguous, subliminal homonyms are not: A close look at priming. Consciousness and Cognition, 20(4), 1327-1343. doi:10.1016/j.concog.2011.05.010 
Eimer, M., \& Schlaghecken, F. (1998). Effects of masked stimuli on motor activation: Behavioral and electrophysiological evidence. Journal of Experimental Psychology: Human Perception and Performance, 24(6), 1737-1747. doi:10.1037/00961523.24.6.1737

Eimer, M., \& Schlaghecken, F. (2002). Links between conscious awareness and response inhibition: Evidence from masked priming. Psychonomic Bulletin \& Review, 9(3), 514-520. doi:10.3758/BF03196307

Eimer, M., \& Schlaghecken, F. (2003). Response facilitation and inhibition in subliminal priming. Biological Psychology, 64(1-2), 7-26. doi:10.1016/S0301-0511(03)00100-5

* Elsner, K., Kunde, W., \& Kiesel, A. (2008). Limited transfer of subliminal response priming to novel stimulus orientations and identities. Consciousness and Cognition, 17(3), 657-671. doi:10.1016/j.concog.2007.06.007

Enns, J. T., \& Di Lollo, V. (2000). What's new in visual masking? Trends in Cognitive Sciences, 4(9), 345-352. doi:10.1016/S1364-6613(00)01520-5

* Enns, J. T., \& Oriet, C. (2008). Visual similarity in masking and priming: The critical role of task relevance. Advances in Cognitive Psychology, 3(1-2), 211-226. doi:10.2478/v10053-008-0026-Z

Eriksen, C. W. (1960). Discrimination and learning without awareness: A methodological survey and evaluation. Psychological Review, 67(5), 279-300. doi:10.1037/h0041622

Evans, K. M., \& Federmeier, K. D. (2007). The memory that's right and the memory that's left: Event-related potentials reveal hemispheric asymmetries in the encoding and retention of verbal information. Neuropsychologia, 45(8), 1777-1790. doi:10.1016/j.neuropsychologia.2006.12.014 
* Fabre, L., Lemaire, P., \& Grainger, J. (2007). Attentional modulation of masked repetition and categorical priming in young and older adults. Cognition, 105(3), 513-532. doi:10.1016/j.cognition.2006.10.011

* Faivre, N., \& Kouider, S. (2011). Increased sensory evidence reverses nonconscious priming during crowding. Journal of Vision, 11(13). doi:10.1167/11.13.16

Federmeier, K. D., \& Kutas, M. (2002). Picture the difference: electrophysiological investigations of picture processing in the two cerebral hemispheres. Neuropsychologia, 40(7), 730-747. doi:10.1016/S0028-3932(01)00193-2

Federmeier, K. D., Mai, H., \& Kutas, M. (2005). Both sides get the point: Hemispheric sensitivities to sentential constraint. Memory \& Cognition, 33(5), 871-886. doi:10.3758/BF03193082

* Ferrand, L., Grainger, J., \& Segui, J. (1994). A study of masked form priming in picture and word naming. Memory \& Cognition, 22(4), 431-441. doi:10.3758/BF03200868

* Finkbeiner, M. (2011). Subliminal priming with nearly perfect performance in the primeclassification task. Attention, Perception, \& Psychophysics, 73(4), 1255-1265. doi:10.3758/s13414-011-0088-8

* Finkbeiner, M., \& Caramazza, A. (2008). Modulating the masked congruence priming effect with the hands and the mouth. Journal of Experimental Psychology: Human Perception and Performance, 34(4), 894-918. doi:10.1037/0096-1523.34.4.894

* Fischer, R., Kiesel, A., Kunde, W., \& Schubert, T. (2011). Selective impairment of masked priming in dual-task performance. The Quarterly Journal of Experimental Psychology, 64(3), 572-595. doi:10.1080/17470218.2010.505984 
* Fischer, R., Schubert, T., \& Liepelt, R. (2007). Accessory stimuli modulate effects of nonconscious priming. Attention, Perception, \& Psychophysics, 69(1), 9-22. doi:10.3758/BF03194449

Fisk, G. D., \& Haase, S. J. (2005). Unconscious Perception or Not? An Evaluation of Detection and Discrimination as Indicators of Awareness. The American Journal of Psychology, 118(2), 183-212.

* Fisk, G. D., \& Haase, S. J. (2011). The relationship between the objective identification threshold and priming effects does not provide a definitive boundary between conscious and unconscious perceptual processes. Consciousness and cognition, 20(4), 1221-1231. doi:10.1016/j.concog.2011.03.016

Forster, K. I., \& Davis, C. (1984). Repetition priming and frequency attenuation in lexical access. Journal of Experimental Psychology: Learning, Memory, and Cognition, 10(4), 680-698. doi:10.1037/0278-7393.10.4.680

* Francken, J. C., Gaal, S. van, \& de Lange, F. P. (2011). Immediate and long-term priming effects are independent of prime awareness. Consciousness and Cognition, 20(4), 1793-1800. doi:10.1016/j.concog.2011.04.005

Freunberger, R., Klimesch, W., Doppelmayr, M., \& Höller, Y. (2007). Visual P2 component is related to theta phase-locking. Neuroscience Letters, 426(3), 181-186. doi:10.1016/j.neulet.2007.08.062

Freyman, R. L., Balakrishnan, U., \& Helfer, K. S. (2004). Effect of number of masking talkers and auditory priming on informational masking in speech recognition. The Journal of the Acoustical Society of America, 115(5 Pt 1), 2246-2256. 
Gaal, S. van, Ridderinkhof, K. R., Scholte, H. S., \& Lamme, V. A. F. (2010). Unconscious Activation of the Prefrontal No-Go Network. The Journal of Neuroscience, 30(11), 4143-4150. doi:10.1523/JNEUROSCI.2992-09.2010

García-Larrea, L., Lukaszewicz, A.-C., \& Mauguiére, F. (1992). Revisiting the oddball paradigm. Non-target vs neutral stimuli and the evaluation of ERP attentional effects. Neuropsychologia, 30(8), 723-741. doi:10.1016/0028-3932(92)90042-K

* García-Orza, J., Damas-López, J., Matas, A., \& Rod ríguez, J. (2009). “2 x 3” primes naming "6": Evidence from masked priming. Attention, Perception, \& Psychophysics, 71(3), 471-480. doi:10.3758/APP.71.3.471

Gevers, W., Reynvoet, B., \& Fias, W. (2003). The mental representation of ordinal sequences is spatially organized. Cognition, 87(3), B87-B95. doi:10.1016/S00100277(02)00234-2

Gibbons, R. D., Hedeker, D. R., \& Davis, J. M. (1993). Estimation of Effect Size From a Series of Experiments Involving Paired Comparisons. Journal of Educational and Behavioral Statistics, 18(3), 271-279. doi:10.3102/10769986018003271

Golob, E. J., Pratt, H., \& Starr, A. (2002). Preparatory slow potentials and event-related potentials in an auditory cued attention task. Clinical Neurophysiology, 113(10), 1544-1557. doi:10.1016/S1388-2457(02)00220-1

* Grainger, J., Diependaele, K., Spinelli, E., Ferrand, L., \& Farioli, F. (2003). Masked Repetition and Phonological Priming Within and Across Modalities. Journal of Experimental Psychology: Learning, Memory, and Cognition, 29(6), 1256-1269. doi:10.1037/0278-7393.29.6.1256 
Green, D., \& Dai, H. (1991). Probability of being correct with 1 of Morthogonal signals. Attention, Perception, \& Psychophysics, 49(1), 100-101. doi:10.3758/BF03211621

Greenhouse, S. W., \& Geisser, S. (1959). On methods in the analysis of profile data. Psychometrika, 24(2), 95-112. doi:10.1007/BF02289823

Greenwald, A. G. (1992). New Look 3: Unconscious cognition reclaimed. American Psychologist, 47(6), 766-779. doi:10.1037/0003-066X.47.6.766

Greenwald, A. G., Draine, S. C., \& Abrams, R. L. (1996). Three Cognitive Markers of Unconscious Semantic Activation. Science, 273, 1699-1702.

Grill-Spector, K., Kushnir, T., Hendler, T., \& Malach, R. (2000). The dynamics of objectselective activation correlate with recognition performance in humans. Nature Neuroscience, 3(8), 837-843. doi:10.1038/77754

* Grossi, G. (2006). Relatedness proportion effects on masked associative priming: An ERP study. Psychophysiology, 43(1), 21-30. doi:10.1111/j.1469-8986.2006.00383.x

Halgren, E., Dhond, R. P., Christensen, N., Van Petten, C., Marinkovic, K., Lewine, J. D., \& Dale, A. M. (2002). N400-like magnetoencephalography responses modulated by semantic context, word frequency, and lexical class in sentences. NeuroImage, 17(3), $1101-1116$.

Halgren, E., Squires, N. K., Wilson, C. L., Rohrbaugh, J. W., Babb, T. L., \& Crandall, P. H. (1980). Endogenous potentials generated in the human hippocampal formation and amygdala by infrequent events. Science (New York, N.Y.), 210(4471), 803-805.

Hedges, L. V. (1982). Estimation of effect size from a series of independent experiments. Psychological Bulletin, 92(2), 490-499. doi:10.1037/0033-2909.92.2.490 
* Heinemann, A., Kiesel, A., Pohl, C., \& Kunde, W. (2010). Masked response priming in expert typists. Consciousness and Cognition, 19(1), 399-407. doi:10.1016/j.concog.2009.09.003

* Heinemann, A., Kunde, W., \& Kiesel, A. (2009). Context-specific prime-congruency effects: On the role of conscious stimulus representations for cognitive control. Consciousness and Cognition, 18(4), 966-976. doi:10.1016/j.concog.2009.08.009

Helenius, P., Salmelin, R., Service, E., \& Connolly, J. F. (1998). Distinct time courses of word and context comprehension in the left temporal cortex. Brain: A Journal of Neurology, 121 ( Pt 6), 1133-1142.

* Henson, R. N., Mouchlianitis, E., Matthews, W. J., \& Kouider, S. (2008). Electrophysiological correlates of masked face priming. NeuroImage, 40(2), 884-895. doi:10.1016/j.neuroimage.2007.12.003

* Hermens, F., Sumner, P., \& Walker, R. (2010). Inhibition of masked primes as revealed by saccade curvature. Vision Research, 50(1), 46-56. doi:10.1016/j.visres.2009.10.008

Hickok, null, \& Poeppel, null. (2000). Towards a functional neuroanatomy of speech perception. Trends in Cognitive Sciences, 4(4), 131-138.

Hillyard, S. A., \& Münte, T. F. (1984). Selective attention to color and location: An analysis with event-related brain potentials. Perception \& Psychophysics, 36(2), 185-198. doi:10.3758/BF03202679

Holcomb, P. J. (1988). Automatic and attentional processing: an event-related brain potential analysis of semantic priming. Brain and Language, 35(1), 66-85. 
Holcomb, P. J. (1993). Semantic priming and stimulus degradation: implications for the role of the N400 in language processing. Psychophysiology, 30(1), 47-61.

Holender, D. (1986). Semantic Activation Without Conscious Identification in Dichotic Listening, Parafoveal Vision, and Visual Masking: A Survey and Appraisal. Behavioral and Brain Sciences, 9(1), 1-23.

Hsu, Y.-F., Hämäläinen, J. A., \& Waszak, F. (2014). Repetition suppression comprises both attention-independent and attention-dependent processes. NeuroImage, 98, 168-175. doi:10.1016/j.neuroimage.2014.04.084

* Huang, Y., Zhou, T., \& Chen, L. (2011). The precedence of topological change over topdown attention in masked priming. Journal of Vision, 11(12). doi:10.1167/11.12.9

* Hughes, G., Velmans, M., \& De Fockert, J. (2009). Unconscious priming of a no-go response. Psychophysiology, 46(6), 1258-1269. doi:10.1111/j.14698986.2009.00873.x

* Jaśkowski, P., \& Przekoracka-Krawczyk, A. (2005). On the role of mask structure in subliminal priming. Acta neurobiologiae experimentalis, 65(4), 409-417.

Jaśkowski, P., Skalska, B., \& Verleger, R. (2003). How the Self Controls Its "Automatic Pilot" when Processing Subliminal Information. Journal of Cognitive Neuroscience, 15(6), 911-920. doi:10.1162/089892903322370825

Johnson, R., \& Donchin, E. (1985). Second thoughts: multiple P300s elicited by a single stimulus. Psychophysiology, 22(2), 182-194.

Kahneman, D. (1968). Method, findings, and theory in studies of visual masking. Psychological Bulletin, 70(6, Pt.1), 404-425. doi:10.1037/h0026731 
Kanske, P., \& Kotz, S. A. (2007). Concreteness in emotional words: ERP evidence from a hemifield study. Brain Research, 1148, 138-148. doi:10.1016/j.brainres.2007.02.044

Kenemans, J. L., Kok, A., \& Smulders, F. T. (1993). Event-related potentials to conjunctions of spatial frequency and orientation as a function of stimulus parameters and response requirements. Electroencephalography and Clinical Neurophysiology, 88(1), 51-63.

* Kemp-Wheeler, S. M., \& Hill, A. B. (1988). Semantic priming without awareness: Some methodological considerations and replications. The Quarterly Journal of Experimental Psychology Section A, 40(4), 671-692. doi:10.1080/14640748808402293

Khalid, S., König, P., \& Ansorge, U. (2011). Sensitivity of different measures of the visibility of masked primes: Influences of prime-response and prime-target relations. Consciousness and Cognition, 20(4), 1473-1488. doi:10.1016/j.concog.2011.06.014

* Kiefer, M. (2002). The N400 is modulated by unconsciously perceived masked words: further evidence for an automatic spreading activation account of N400 priming effects. Cognitive Brain Research, 13(1), 27-39. doi:10.1016/S0926-6410(01)00085-4

Kiefer, M. (2008). Top-down modulation of unconscious ,automatic“ processes: A gating framework. Advances in Cognitive Psychology, 3(1-2), 289-306. doi:10.2478/v10053-008-0032-2

* Kiefer, M., \& Brendel, D. (2006). Attentional Modulation of Unconscious "Automatic" Processes: Evidence from Event-related Potentials in a Masked Priming Paradigm. Journal of Cognitive Neuroscience, 18(2), 184-198. doi:10.1162/jocn.2006.18.2.184 
* Kiefer, M., \& Martens, U. (2010). Attentional sensitization of unconscious cognition: Task sets modulate subsequent masked semantic priming. Journal of Experimental Psychology: General, 139(3), 464-489. doi:10.1037/a0019561

Kiefer, M., \& Spitzer, M. (2000). Time Course of Conscious and Unconscious Semantic Brain Activation. Neuroreport, 11(11), 2401-2407.

* Kiesel, A., Kunde, W., \& Hoffmann, J. (2007a). Unconscious priming according to multiple S-R rules. Cognition, 104(1), 89-105. doi:10.1016/j.cognition.2006.05.008

Kiesel, A., Kunde, W., \& Hoffmann, J. (2007b). Mechanisms of subliminal response priming. Advances in Cognitive Psychology, 3(1-2), 307-315. doi:10.2478/v10053-008-0032-1

Kiesel, A., Kunde, W., Pohl, C., \& Hoffmann, J. (2006). Priming from novel masked stimuli depends on target set size. Advances in Cognitive Psychology, 2(1), 37-45. doi:10.2478/v10053-008-0043-y

* Kiyonaga, K., Grainger, J., Midgley, K., \& Holcomb, P. J. (2007). Masked Cross-Modal Repetition Priming: An Event-Related Potential Investigation. Language and cognitive processes, 22(3), 337-376. doi:10.1080/01690960600652471

Klapp, S. T. (2005). Two versions of the negative compatibility effect: Comment on Lleras and Enns (2004). Journal of Experimental Psychology: General, 134(3), 431-435. doi:10.1037/0096-3445.134.3.431

Klapp, S. T., \& Hinkley, L. B. (2002). The negative compatibility effect: Unconscious inhibition influences reaction time and response selection. Journal of Experimental Psychology: General, 131(2), 255-269. doi:10.1037/0096-3445.131.2.255 
Klotz, W., \& Wolff, P. (1995). The effect of a masked stimulus on the response to the masking stimulus. Psychological Research, 58(2), 92-101. doi:10.1007/BF00571098

Knoblauch, K. (2012). psyphy: Functions for analyzing psychophysical data in R. R package version 0.1-7. http://CRAN.R-project.org/package=psyphy

Kok, A. (2001). On the utility of P3 amplitude as a measure of processing capacity. Psychophysiology, 38(3), 557-577.

Kouider, S., \& Dehaene, S. (2007). Levels of processing during non-conscious perception: a critical review of visual masking. Philosophical Transactions of the Royal Society B: Biological Sciences, 362(1481), 857-875. doi:10.1098/rstb.2007.2093

* Kouider, S., \& Dehaene, S. (2009). Subliminal Number Priming Within and Across the Visual and Auditory Modalities. Experimental Psychology (formerly Zeitschrift für Experimentelle Psychologie), 56(6), 418-433. doi:10.1027/1618-3169.56.6.418

* Kouider, S., Dehaene, S., Jobert, A., \& Bihan, D. L. (2007). Cerebral Bases of Subliminal and Supraliminal Priming during Reading. Cerebral Cortex, 17(9), 2019-2029. doi:10.1093/cercor/bhl110

* Kouider, S., \& Dupoux, E. (2001). A functional disconnection between spoken and visual word recognition: evidence from unconscious priming. Cognition, 82(1), B35-B49. doi:10.1016/S0010-0277(01)00152-4

* Kouider, S., \& Dupoux, E. (2004). Partial Awareness Creates the "Illusion" of Subliminal Semantic Priming. Psychological Science, 15(2), 75-81. doi:10.1111/j.09637214.2004.01502001.x 
Kouider, S., \& Dupoux, E. (2005). Subliminal Speech Priming. Psychological Science, 16(8), 617-625. doi:10.1111/j.1467-9280.2005.01584.x

* Kouider, S., Eger, E., Dolan, R., \& Henson, R. N. (2009). Activity in Face-Responsive Brain Regions is Modulated by Invisible, Attended Faces: Evidence from Masked Priming. Cerebral Cortex (New York, NY), 19(1), 13-23. doi:10.1093/cercor/bhn048

Kranczioch, C., Debener, S., \& Engel, A. K. (2003). Event-related potential correlates of the attentional blink phenomenon. Cognitive Brain Research, 17(1), 177-187. doi:10.1016/S0926-6410(03)00092-2

Krüger, D., \& Mattler, U. (2012). Inverse cue priming is not limited to masks with relevant features. Consciousness and Cognition, 21(3), 1207-1221. doi:10.1016/j.concog.2012.04.003

Kunde, W. (2003). Sequential modulations of stimulus-response correspondence effects depend on awareness of response conflict. Psychonomic Bulletin \& Review, 10(1), 198-205. doi:10.3758/BF03196485

* Kunde, W. (2004). Response priming by supraliminal and subliminal action effects. Psychological Research, 68(2), 91-96. doi:10.1007/s00426-003-0147-4

Kunde, W., Kiesel, A., \& Hoffmann, J. (2003). Conscious control over the content of unconscious cognition. Cognition, 88(2), 223-242. doi:10.1016/S00100277(03)00023-4

Kutas, M. (1993). In the company of other words: Electrophysiological evidence for singleword and sentence context effects. Language and Cognitive Processes, 8(4), 533-572. doi:10.1080/01690969308407587 
Kutas, M. (1997). Views on how the electrical activity that the brain generates reflects the functions of different language structures. Psychophysiology, 34(4), 383-398.

Kutas, M., \& Federmeier, K. (2009). N400. Scholarpedia, 4(10), 7790. doi:10.4249/scholarpedia.7790

Kutas, M., \& Hillyard, S. (1980). Reading senseless sentences: brain potentials reflect semantic incongruity. Science, 207(4427), 203-205. doi:10.1126/science.7350657

Kutas, M., \& Hillyard, S. A. (1982). The lateral distribution of event-related potentials during sentence processing. Neuropsychologia, 20(5), 579-590.

Lamme, V. A. F., \& Roelfsema, P. R. (2000). The distinct modes of vision offered by feedforward and recurrent processing. Trends in Neurosciences, 23(11), 571-579. doi:10.1016/S0166-2236(00)01657-X

Lefebvre, C. D., Marchand, Y., Eskes, G. A., \& Connolly, J. F. (2005). Assessment of working memory abilities using an event-related brain potential (ERP)-compatible digit span backward task. Clinical Neurophysiology: Official Journal of the International Federation of Clinical Neurophysiology, 116(7), 1665-1680. doi:10.1016/j.clinph.2005.03.015

Lefton, L. A. (1973a). Metacontrast: A review. Perception \& Psychophysics, 13(1-B), 161171.

Lefton, L. A. (1973b). Spatial factors in metacontrast. Perception \& Psychophysics, 14(3), 497-500. doi:10.3758/BF03211189 
Leuthold, H., \& Kopp, B. (1998). Mechanisms of Priming by Masked Stimuli: Inferences From Event-Related Brain Potentials. Psychological Science, 9(4), 263-269. doi:10.1111/1467-9280.00053

Lleras, A., \& Enns, J. T. (2004). Negative Compatibility or Object Updating? A Cautionary Tale of Mask-Dependent Priming. Journal of Experimental Psychology: General, 133(4), 475-493. doi:10.1037/0096-3445.133.4.475

Loftus, G. R., \& Masson, M. E. J. (1994). Using confidence intervals in within-subject designs. Psychonomic Bulletin \& Review, 1(4), 476-490. doi:10.3758/BF03210951

Logan, G. D. (1990). Repetition priming and automaticity: Common underlying mechanisms? Cognitive Psychology, 22(1), 1-35. doi:10.1016/0010-0285(90)90002-L

Lopez-Calderon, J., \& Luck, S. J. (2014). ERPLAB: an open-source toolbox for the analysis of event-related potentials. Frontiers in Human Neuroscience, 8, 213. doi:10.3389/fnhum.2014.00213

Luck, S. J. (2005). An Introduction to the Event-Related Potential Technique. Cambridge, Mass: The Mit Press.

Luck, S. J., \& Hillyard, S. A. (1994). Electrophysiological correlates of feature analysis during visual search. Psychophysiology, 31(3), 291-308. doi:10.1111/j.14698986.1994.tb02218.x

Luck, S. J., Vogel, E. K., \& Shapiro, K. L. (1996). Word meanings can be accessed but not reported during the attentional blink. Nature, 383, 616-618. doi:10.1038/383616a0

Macknik, S. L., \& Livingstone, M. S. (1998). Neuronal correlates of visibility and invisibility in the primate visual system. Nature Neuroscience, 1(2), 144-149. doi:10.1038/393 
Macmillan, N. A., \& Creelman, C. D.(1991). Detection theory: A user's guide. New York: Cambridge University Press.

Marcel, T., \& Forrin, B. (1974). Naming latency and the repetition of stimulus categories. Journal of Experimental Psychology, 103(3), 450-460. doi:10.1037/h0037173

Martens, U., Ansorge, U., \& Kiefer, M. (2011). Controlling the unconscious: attentional task sets modulate subliminal semantic and visuomotor processes differentially. Psychological Science, 22(2), 282-291. doi:10.1177/0956797610397056

* Marzouki, Y., \& Grainger, J. (2008). Effects of prime and target eccentricity on masked repetition priming. Psychonomic Bulletin \& Review, 15(1), 141-148. doi:10.3758/PBR.15.1.141

* Marzouki, Y., Grainger, J., \& Theeuwes, J. (2007). Exogenous spatial cueing modulates subliminal masked priming. Acta Psychologica, 126(1), 34-45. doi:10.1016/j.actpsy.2006.11.002

* Marzouki, Y., Grainger, J., \& Theeuwes, J. (2008). Inhibition of return in subliminal letter priming. Acta Psychologica, 129(1), 112-120. doi:10.1016/j.actpsy.2008.05.002

* Matsumoto, A., Iidaka, T., Nomura, M., \& Ohira, H. (2005). Dissociation of conscious and unconscious repetition priming effect on event-related potentials. Neuropsychologia, 43(8), 1168-1176. doi:10.1016/j.neuropsychologia.2004.11.020

* Mattler, U. (2003). Priming of mental operations by masked stimuli. Attention, Perception, \& Psychophysics, 65(2), 167-187. doi:10.3758/BF03194793

Mattler, U. (2005). Inhibition and decay of motor and nonmotor priming. Attention, Perception, \& Psychophysics, 67(2), 285-300. doi:10.3758/BF03206492 
Mattler, U. (2006). On the locus of priming and inverse priming effects. Perception \& Psychophysics, 68(6), 975-991.

* Mattler, U. (2007). Inverse target- and cue-priming effects of masked stimuli. Journal of Experimental Psychology: Human Perception and Performance, 33(1), 83-102. doi:10.1037/0096-1523.33.1.83

Mattler, U., \& Fendrich, R. (2010). Consciousness mediated by neural transition states: how invisibly rapid motions can become visible. Consciousness and Cognition, 19(1), 172-185. doi:10.1016/j.concog.2009.12.015

Mattler, U., \& Palmer, S. (2012). Time course of free-choice priming effects explained by a simple accumulator model. Cognition, 123(3), 347-360. doi:10.1016/j.cognition.2012.03.002

Mauchly, J. W. (1940). Significance Test for Sphericity of a Normal n-Variate Distribution. The Annals of Mathematical Statistics, 11(2), 204-209.

McCarthy, G., \& Nobre, A. C. (1993). Modulation of semantic processing by spatial selective attention. Electroencephalography and Clinical Neurophysiology, 88(3), 210-219.

McCarthy, G., Wood, C. C., Williamson, P. D., \& Spencer, D. D. (1989). Task-dependent field potentials in human hippocampal formation. The Journal of Neuroscience, 9(12), $4253-4268$.

McClelland, J. L., \& Rumelhart, D. E. (1981). An interactive activation model of context effects in letter perception: I. An account of basic findings. Psychological Review, 88(5), 375-407. doi:10.1037/0033-295X.88.5.375 
Merikle, P. M., \& Reingold, E. M. (1990). Recognition and lexical decision without detection: Unconscious perception? Journal of Experimental Psychology: Human Perception and Performance, 16(3), 574-583. doi:10.1037/0096-1523.16.3.574

Merikle, P. M., \& Reingold, E. M. (1992). Measuring unconscious perceptual processes. In R. F. Bornstein \& T. S. Pittman (Hrsg.), Perception Without Awareness (S. 55-80). New York: Guilford Press.

Meyer, D. E., \& Schvaneveldt, R. W. (1971). Facilitation in recognizing pairs of words: Evidence of a dependence between retrieval operations. Journal of Experimental Psychology, 90(2), 227-234. doi:10.1037/h0031564

Misra, M., \& Holcomb, P. J. (2003). Event-related potential indices of masked repetition priming. Psychophysiology, 40(1), 115-130. doi:10.1111/1469-8986.00012

Morris, S. B., \& DeShon, R. P. (2002). Combining effect size estimates in meta-analysis with repeated measures and independent-groups designs. Psychological methods, 7(1), $105-125$.

Moutoussis, K., \& Zeki, S. (2002). The relationship between cortical activation and perception investigated with invisible stimuli. Proceedings of the National Academy of Sciences of the United States of America, 99(14), 9527-9532. doi:10.1073/pnas.142305699

Näätänen, R., \& Picton, T. W. (1986). N2 and automatic versus controlled processes. Electroencephalography and Clinical Neurophysiology. Supplement, 38, 169-186.

* Naccache, L., Blandin, E., \& Dehaene, S. (2002). Unconscious Masked Priming Depends on Temporal Attention. Psychological Science, 13(5), 416-424. doi:10.1111/14679280.00474 
* Naccache, L., \& Dehaene, S. (2001). Unconscious semantic priming extends to novel unseen stimuli. Cognition, 80(3), 215-229. doi:10.1016/S0010-0277(00)00139-6

* Nakamura, K., Dehaene, S., Jobert, A., Bihan, D. L., \& Kouider, S. (2007). Task-specific change of unconscious neural priming in the cerebral language network. Proceedings of the National Academy of Sciences, 104(49), 19643-19648. doi:10.1073/pnas.0704487104

Neville, H. J., Mills, D. L., \& Lawson, D. S. (1992). Fractionating Language: Different Neural Subsystems with Different Sensitive Periods. Cerebral Cortex, 2(3), 244-258. doi:10.1093/cercor/2.3.244

Nieuwenhuis, S., \& de Kleijn, R. (2011). Consciousness of targets during the attentional blink: a gradual or all-or-none dimension? Attention, Perception, \& Psychophysics, 73(2), 364-373. doi:10.3758/s13414-010-0026-1

Nittono, H., Nageishi, Y., Nakajima, Y., \& Ullsperger, P. (1999). Event-related potential correlates of individual differences in working memory capacity. Psychophysiology, $36(6), 745-754$.

Nobre, A. C. (2001). Orienting attention to instants in time. Neuropsychologia, 39(12), 13171328.

Nolan, K. A., \& Caramazza, A. (1982). Unconscious perception of meaning: A failure to replicate. Bulletin of the Psychonomic Society, 20(1), 23-26. doi:10.3758/BF03334791

Novak, G., Ritter, W., \& Vaughan, H. G. (1992). Mismatch Detection and the Latency of Temporal Judgments. Psychophysiology, 29(4), 398-411. doi:10.1111/j.14698986.1992.tb01713.x 
O’Brien, R. M. (2007). A Caution Regarding Rules of Thumb for Variance Inflation Factors. Quality \& Quantity, 41(5), 673-690. doi:10.1007/s11135-006-9018-6

* O’Connor, P. A., \& Neill, W. T. (2011). Does subliminal priming of free response choices depend on task set or automatic response activation? Consciousness and Cognition, 20(2), 280-287. doi:10.1016/j.concog.2010.08.007

* Ortells, J. J., Frings, C., \& Plaza-Ayllon, V. (2012). Influence of spatial attention on conscious and unconscious word priming. Consciousness and Cognition, 21(1), 117138. doi:10.1016/j.concog.2011.10.012

Osterhout, L., \& Holcomb, P. J. (1995). Event related potentials and language comprehension. In M. D. Rugg \& M. G. H. Coles (Hrsg.), Electrophysiology of mind: Event-related brain potentials and cognition (S. 171-215). New York, NY, US: Oxford University Press.

Overgaard, M., Rote, J., Mouridsen, K., \& Ramsøy, T. Z. (2006). Is conscious perception gradual or dichotomous? A comparison of report methodologies during a visual task. Consciousness and Cognition, 15(4), 700-708. doi:10.1016/j.concog.2006.04.002

Persaud, N., McLeod, P., \& Cowey, A. (2007). Post-decision wagering objectively measures awareness. Nature Neuroscience, 10(2), 257-261. doi:10.1038/nn1840

* Persuh, M., \& Ro, T. (2012). Context-dependent brightness priming occurs without visual awareness. Consciousness and Cognition, 21(1), 177-185. doi:10.1016/j.concog.2011.11.002

Picton, T. W. (1992). The P300 wave of the human event-related potential. Journal of Clinical Neurophysiology: Official Publication of the American Electroencephalographic Society, 9(4), 456-479. 
Phillips, S. \& Takeda, Y. (2009). An EEG/ERP study of efficient versus inefficient visual search. In N. A. Taatgen \& H. van Rijin (Eds.), Proceedings of the 31st Annual Conference of the Cognitive Science Society, pp. 383-388. Amsterdam.

Polich, J. (1987). Task difficulty, probability, and inter-stimulus interval as determinants of P300 from auditory stimuli. Electroencephalography and Clinical Neurophysiology, 68(4), 311-320.

Polich, J. (2007). Updating P300: an integrative theory of P3a and P3b. Clinical Neurophysiology: Official Journal of the International Federation of Clinical Neurophysiology, 118(10), 2128-2148. doi:10.1016/j.clinph.2007.04.019

* Pollatsek, A., Perea, M., \& Carreiras, M. (2005). Does conal prime CANAL more than cinal? Masked phonological priming effects in Spanish with the lexical decision task. Memory \& cognition, 33(3), 557-565.

Posner, M. I., Snyder, C. R., \& Davidson, B. J. (1980). Attention and the detection of signals. Journal of Experimental Psychology, 109(2), 160-174.

* Pratte, M., \& Rouder, J. (2009). A task-difficulty artifact in subliminal priming. Attention, Perception, \& Psychophysics, 71(6), 1276-1283. doi:10.3758/APP.71.6.1276

Purcell, D. G., Stewart, A. L., \& Stanovich, K. E. (1983). Another look at semantic priming without awareness. Perception \& Psychophysics, 34(1), 65-71. doi:10.3758/BF03205897

Ramsøy, T. Z., \& Overgaard, M. (2004). Introspection and subliminal perception. Phenomenology and the Cognitive Sciences, 3(1), 1-23. doi:10.1023/B:PHEN.0000041900.30172.e8 
* Rastle, K., \& Brysbaert, M. (2006). Masked phonological priming effects in English: Are they real? Do they matter? Cognitive Psychology, 53(2), 97-145. doi:10.1016/j.cogpsych.2006.01.002

* Ratinckx, E., Brysbaert, M., \& Fias, W. (2005). Naming two-digit Arabic numerals: Evidence from masked priming studies. Journal of Experimental Psychology: Human Perception and Performance, 31(5), 1150-1163. doi:10.1037/0096-1523.31.5.1150

* Reynvoet, B., Gevers, W., \& Caessens, B. (2005). Unconscious Primes Activate Motor Codes Through Semantics. Journal of Experimental Psychology: Learning, Memory, and Cognition, 31(5), 991-1000. doi:10.1037/0278-7393.31.5.991

* Reynvoet, B., \& Ratinckx, E. (2004). Hemispheric differences between left and right number representations: effects of conscious and unconscious priming. Neuropsychologia, 42(6), 713-726. doi:10.1016/j.neuropsychologia.2003.11.013

Rieger, J. W., Braun, C., Bülthoff, H. H., \& Gegenfurtner, K. R. (2005). The dynamics of visual pattern masking in natural scene processing: A magnetoencephalography study. Journal of Vision, 5(3), 10. doi:10.1167/5.3.10

Ritter, W., Simson, R., \& Vaughan, H. G. (1983). Event-Related Potential Correlates of Two Stages of Information Processing in Physical and Semantic Discrimination Tasks. Psychophysiology, 20(2), 168-179. doi:10.1111/j.1469-8986.1983.tb03283.x

Ruchkin, D. S., Johnson, R., Canoune, H., \& Ritter, W. (1990). Short-term memory storage and retention: an event-related brain potential study. Electroencephalography and Clinical Neurophysiology, 76(5), 419-439. 
Rugg, M. D. (1985). The Effects of Semantic Priming and Word Repetition on Event-Related Potentials. Psychophysiology, 22(6), 642-647. doi:10.1111/j.14698986.1985.tb01661.x

Rugg, M. D., \& Doyle, M. C. (1994). Event-Related Potentials and Stimulus Repetition in Direct and Indirect Tests of Memory. In H.-J. Heinze, T. F. Münte, \& G. R. Mangun (Hrsg.), Cognitive Electrophysiology (S. 124-148). Birkhäuser Boston. Abgerufen von http://link.springer.com/chapter/10.1007/978-1-4612-0283-7_5

Rosenthal, R. (1991). Meta-Analytic Procedures for Social Research. SAGE.

* Rouder, J. N., Morey, R. D., Speckman, P. L., \& Pratte, M. S. (2007). Detecting chance: a solution to the null sensitivity problem in subliminal priming. Psychonomic bulletin \& review, 14(4), 597-605.

* Ruz, M., Madrid, E., Lupiáñez, J., \& Tudela, P. (2003). High density ERP indices of conscious and unconscious semantic priming. Cognitive Brain Research, 17(3), 719731. doi:10.1016/S0926-6410(03)00197-6

Sandberg, K., Timmermans, B., Overgaard, M., \& Cleeremans, A. (2010). Measuring consciousness: Is one measure better than the other? Consciousness and Cognition, 19(4), 1069-1078. doi:10.1016/j.concog.2009.12.013

Schlaghecken, F., \& Eimer, M. (2000). A central-peripheral asymmetry in masked priming. Perception \& Psychophysics, 62(7), 1367-1382. doi:10.3758/BF03212139

* Schlaghecken, F., \& Eimer, M. (2004). Masked prime stimuli can bias "free" choices between response alternatives. Psychonomic Bulletin \& Review, 11(3), 463-468. doi:10.3758/BF03196596 
Schmidt, T., \& Vorberg, D. (2006). Criteria for unconscious cognition: three types of dissociation. Perception \& Psychophysics, 68(3), 489-504.

* Seiss, E., \& Praamstra, P. (2004). The basal ganglia and inhibitory mechanisms in response selection: evidence from subliminal priming of motor responses in Parkinson's disease. Brain, 127(2), 330-339. doi:10.1093/brain/awh043

* Seiss, E., \& Praamstra, P. (2006). Time-course of masked response priming and inhibition in Parkinson's disease. Neuropsychologia, 44(6), 869-875. doi:10.1016/j.neuropsychologia.2005.09.001

Sergent, C., \& Dehaene, S. (2004a). Is consciousness a gradual phenomenon? Evidence for an all-or-none bifurcation during the attentional blink. Psychological Science, 15(11), 720-728. doi:10.1111/j.0956-7976.2004.00748.x

Sergent, C., \& Dehaene, S. (2004b). Neural processes underlying conscious perception: Experimental findings and a global neuronal workspace framework. Journal of Physiology-Paris, 98(4-6), 374-384. doi:10.1016/j.jphysparis.2005.09.006

Smith, M. E., \& Guster, K. (1993). Decomposition of recognition memory event-related potentials yields target, repetition, and retrieval effects. Electroencephalography and Clinical Neurophysiology, 86(5), 335-343.

Spencer, T. J., \& Shuntich, R. (1970). Evidence for an interruption theory of backward masking. Journal of Experimental Psychology, 85(2), 198-203. doi:10.1037/h0029510

Squires, N. K., Squires, K. C., \& Hillyard, S. A. (1975). Two varieties of long-latency positive waves evoked by unpredictable auditory stimuli in man. Electroencephalography and Clinical Neurophysiology, 38(4), 387-401. 
Stanislaw, H., \& Todorov, N. (1999). Calculation of signal detection theory measures. Behavior research methods, instruments, \& computers: a journal of the Psychonomic Society, Inc, 31(1), 137-149.

* Stone, A. (2012). Centre-surround inhibition is a general aspect of famous-person recognition: Evidence from negative semantic priming from clearly visible primes. Memory \& Cognition, 40(4), 652-662. doi:10.3758/s13421-011-0176-y

Sumner, P. (2007). Negative and positive masked-priming - implications for motor inhibition. Advances in Cognitive Psychology, 3(1-2), 317-326. doi:10.2478/v10053-008-0033-0

Sutton, S., Braren, M., Zubin, J., \& John, E. R. (1965). Evoked-potential correlates of stimulus uncertainty. Science (New York, N.Y.), 150(3700), 1187-1188.

* Tapia, E., Breitmeyer, B. G., \& Shooner, C. R. (2010). Role of task-directed attention in nonconscious and conscious response priming by form and color. Journal of Experimental Psychology: Human Perception and Performance, 36(1), 74-87. doi:10.1037/a0017166

Tononi, G., \& Edelman, G. M. (1998). Consciousness and Complexity. Science, 282(5395), 1846-1851. doi:10.1126/science.282.5395.1846

Tse, C.-Y., Lee, C.-L., Sullivan, J., Garnsey, S. M., Dell, G. S., Fabiani, M., \& Gratton, G. (2007). Imaging cortical dynamics of language processing with the event-related optical signal. Proceedings of the National Academy of Sciences, 104(43), 1715717162. doi:10.1073/pnas.0707901104

Turvey, M. T. (1973). On peripheral and central processes in vision: inferences from an information-processing analysis of masking with patterned stimuli. Psychological Review, 80(1), 1-52. 
Van Aalderen-Smeets, S. I., Oostenveld, R., \& Schwarzbach, J. (2006). Investigating neurophysiological correlates of metacontrast masking with magnetoencephalography. Advances in Cognitive Psychology, 2(1), 21-35. doi:10.2478/v10053-008-0042-z

* Van den Bussche, E., Hughes, G., Humbeeck, N. V., \& Reynvoet, B. (2010). The relation between consciousness and attention: An empirical study using the priming paradigm. Consciousness and Cognition, 19(1), 86-97. doi:10.1016/j.concog.2009.12.019

* Van den Bussche, E., Notebaert, K., \& Reynvoet, B. (2009). Masked Primes Can Be Genuinely Semantically Processed. Experimental Psychology (formerly Zeitschrift für Experimentelle Psychologie), 56(5), 295-300. doi:10.1027/1618-3169.56.5.295

* Van den Bussche, E., \& Reynvoet, B. (2007). Masked Priming Effects in Semantic Categorization Are Independent of Category Size. Experimental Psychology (formerly „Zeitschrift für Experimentelle Psychologie“), 54(3), 225-235. doi:10.1027/16183169.54.3.225

* Van den Bussche, E., Segers, G., \& Reynvoet, B. (2008). Conscious and unconscious proportion effects in masked priming. Consciousness and Cognition, 17(4), 13451358. doi:10.1016/j.concog.2007.08.005

Van den Bussche, E., Van den Noortgate, W., \& Reynvoet, B. (2009). Mechanisms of masked priming: A meta-analysis. Psychological Bulletin, 135(3), 452-477. doi:10.1037/a0015329 
Van Gaal, S., Scholte, H. S., Lamme, V. A. F., Fahrenfort, J. J., \& Ridderinkhof, K. R. (2010). Pre-SMA Gray-matter Density Predicts Individual Differences in Action Selection in the Face of Conscious and Unconscious Response Conflict. Journal of Cognitive Neuroscience, 23(2), 382-390. doi:10.1162/jocn.2010.21444

Van Petten, C., \& Luka, B. J. (2006). Neural localization of semantic context effects in electromagnetic and hemodynamic studies. Brain and Language, 97(3), 279-293. doi:10.1016/j.bandl.2005.11.003

Van Petten, C. V. (1993). A comparison of lexical and sentence-level context effects in event-related potentials. Language and Cognitive Processes, 8(4), 485-531. doi:10.1080/01690969308407586

* Verleger, R., Jaśkowski, P., Aydemir, A., van der Lubbe, R. H. J., \& Groen, M. (2004). Qualitative Differences Between Conscious and Nonconscious Processing? On Inverse Priming Induced by Masked Arrows. Journal of Experimental Psychology: General, 133(4), 494-515. doi:10.1037/0096-3445.133.4.494

Verleger, R., Jaskowski, P., \& Wauschkuhn, B. (1994). Suspense and surprise: on the relationship between expectancies and P3. Psychophysiology, 31(4), 359-369.

Vermeiren, A., \& Cleeremans, A. (2012). The Validity of d' Measures. PLoS ONE, 7(2), e31595. doi:10.1371/journal.pone.0031595

Viechtbauer, W. (2005). Bias and Efficiency of Meta-Analytic Variance Estimators in the Random-Effects Model. Journal of Educational and Behavioral Statistics, 30(3), 261-293.

Viechtbauer, W. (2010). Conducting meta-analyses in R with the metafor package. Journal of Statistical Software, 36(3), 1-48. 
Vorberg, D., Mattler, U., Heinecke, A., Schmidt, T., \& Schwarzbach, J. (2003). Different time courses for visual perception and action priming. Proceedings of the National Academy of Sciences of the United States of America, 100(10), 6275-6280. doi:10.1073/pnas.0931489100

Vorberg, D., Mattler, U., Heinecke, A., Schmidt, T., \& Schwarzbach, J. (2004). Invariant time course of priming with and without awareness. In C. Kaernbach, E. Schröger, \& H. Müller (Eds.), Psychophysics beyond sensation. Laws and invariants of human cognition (pp. 271-288). Mahwah, NJ: Lawrence Erlbaum Associates, Publishers.

Wastell, D. G., \& Kleinman, D. (1980). Evoked potential correlates of visual selective attention. Acta Psychologica, 46(2), 129-140. doi:10.1016/0001-6918(80)90005-0

* Wenke, D., Fleming, S. M., \& Haggard, P. (2010). Subliminal priming of actions influences sense of control over effects of action. Cognition, 115(1), 26-38. doi:10.1016/j.cognition.2009.10.016

Wiggs, C. L., \& Martin, A. (1998). Properties and mechanisms of perceptual priming. Current Opinion in Neurobiology, 8(2), 227-233. doi:10.1016/S0959-4388(98)80144$\mathrm{X}$

Wijers, A. A., Mulder, G., Okita, T., \& Mulder, L. J. (1989). Event-related potentials during memory search and selective attention to letter size and conjunctions of letter size and color. Psychophysiology, 26(5), 529-547.

Wijers, A. A., Mulder, G., Okita, T., Mulder, L. J. M., \& Scheffers, M. K. (1989). Attention to Color: An Analysis of Selection, Controlled Search, and Motor Activation, Using Event-Related Potentials. Psychophysiology, 26(1), 89-109. doi:10.1111/j.14698986.1989.tb03137.x 
7 | Approaching Consciousness with Masked Priming

Windey, B., Gevers, W., \& Cleeremans, A. (2013). Subjective Visibility Depends on Level of Processing. Cognition, 129(2), 404-409.

Yellott Jr., J. I. (1971). Correction for fast guessing and the speed-accuracy tradeoff in choice reaction time. Journal of Mathematical Psychology, 8(2), 159-199. doi:10.1016/00222496(71)90011-3

Zeki, S. (1993). A vision of the brain (Bd. xi). Cambridge, MA, US: Blackwell Scientific Publications.

* Zhou, F., \& Davis, G. (2012). Unconscious priming of task sets: The role of spatial attention. Attention, Perception, \& Psychophysics, 74(1), 105-114. doi:10.3758/s13414-011-0221-8

Zovko, M., \& Kiefer, M. (2013). Do different perceptual task sets modulate electrophysiological correlates of masked visuomotor priming? Attention to shape and color put to the test. Psychophysiology, 50(2), 149-157. doi:10.1111/j.14698986.2012.01492.x 


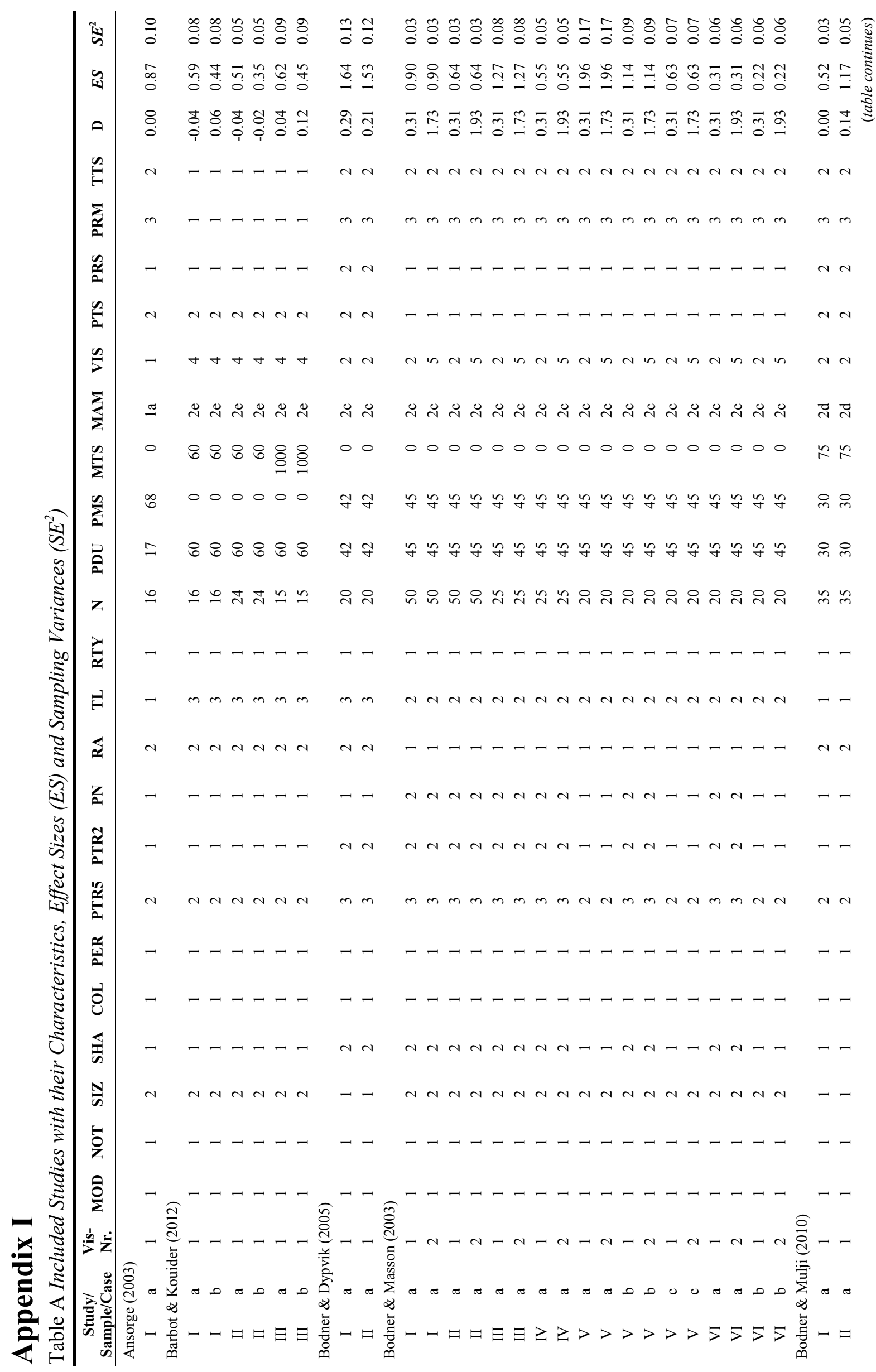




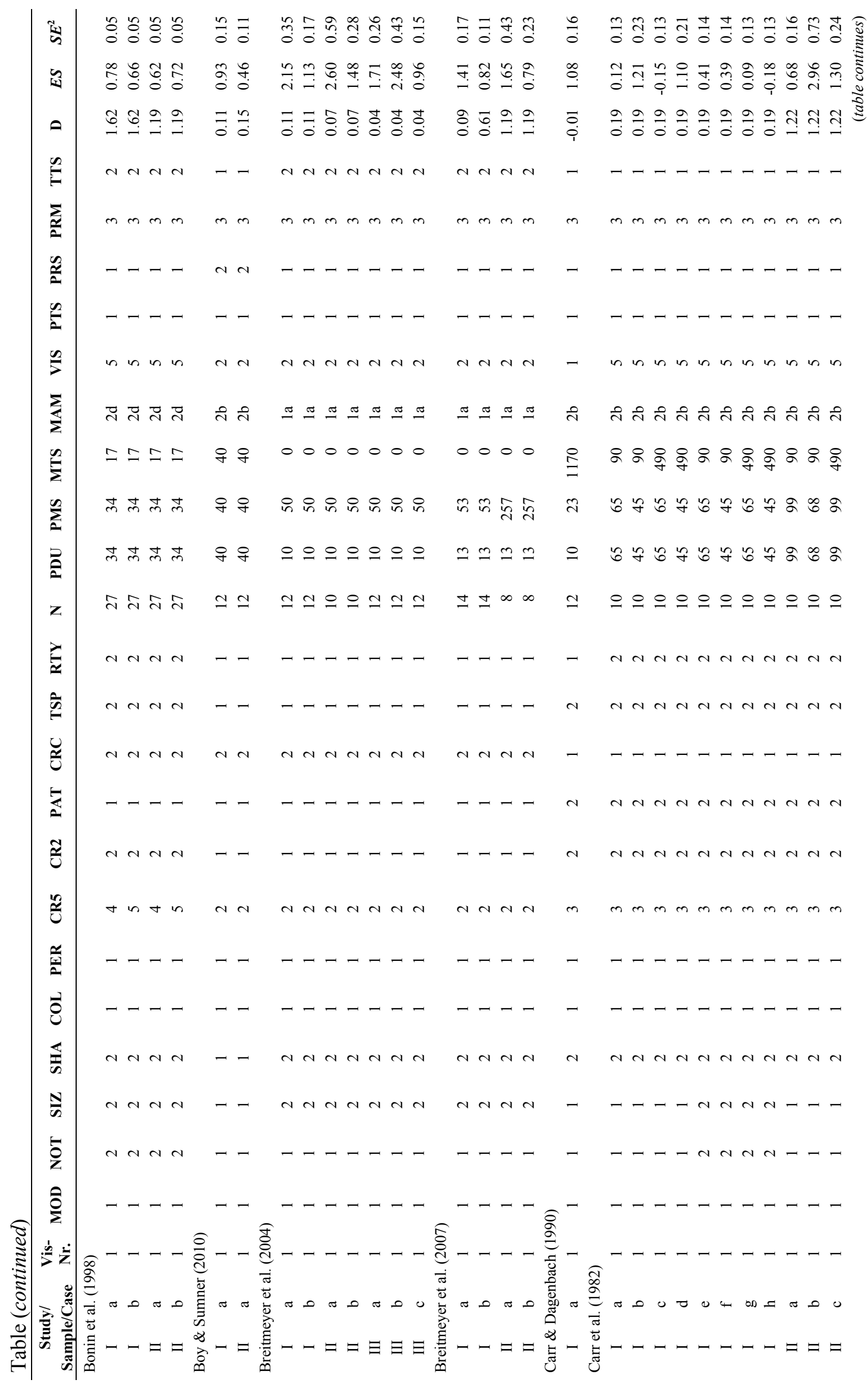




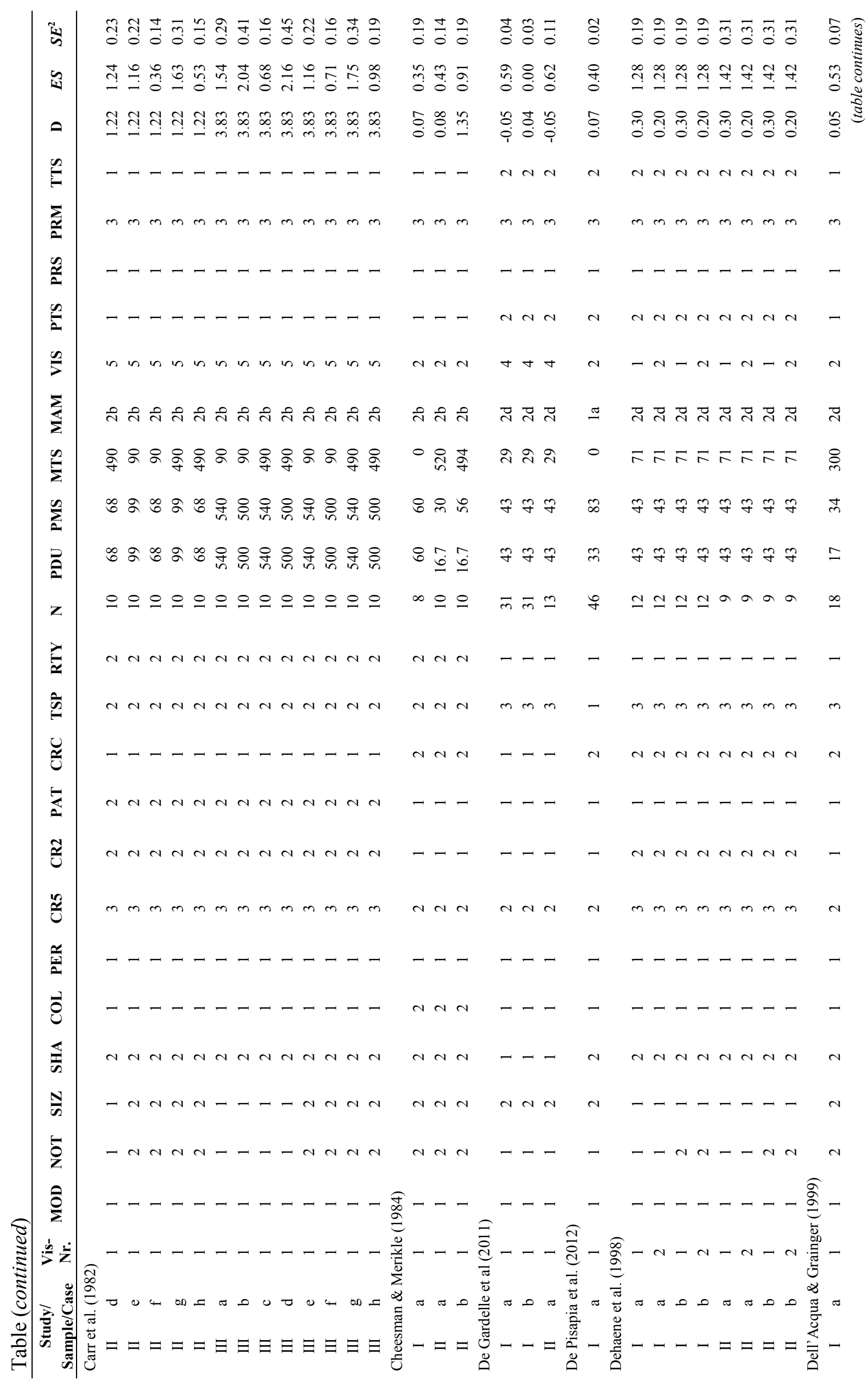




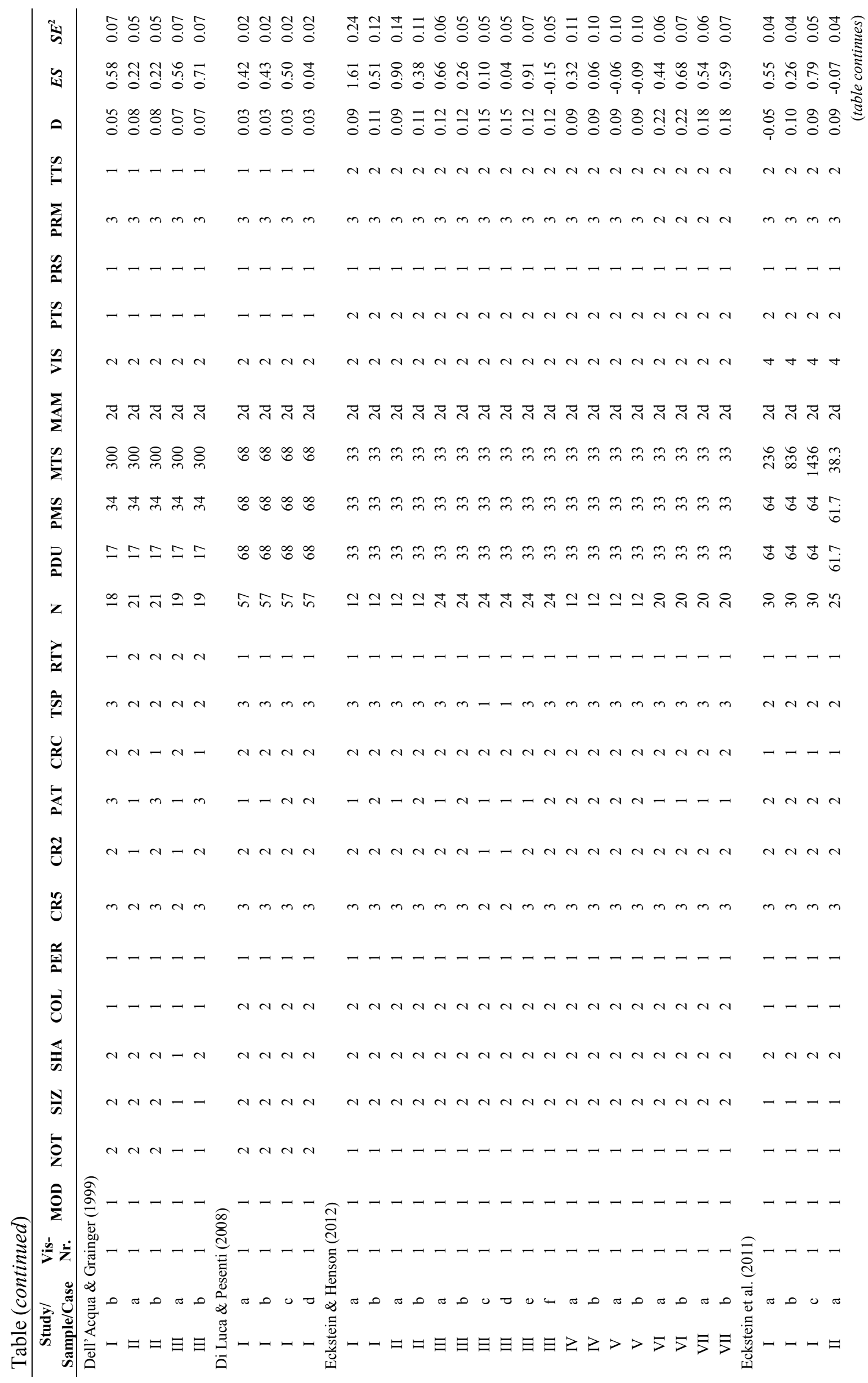




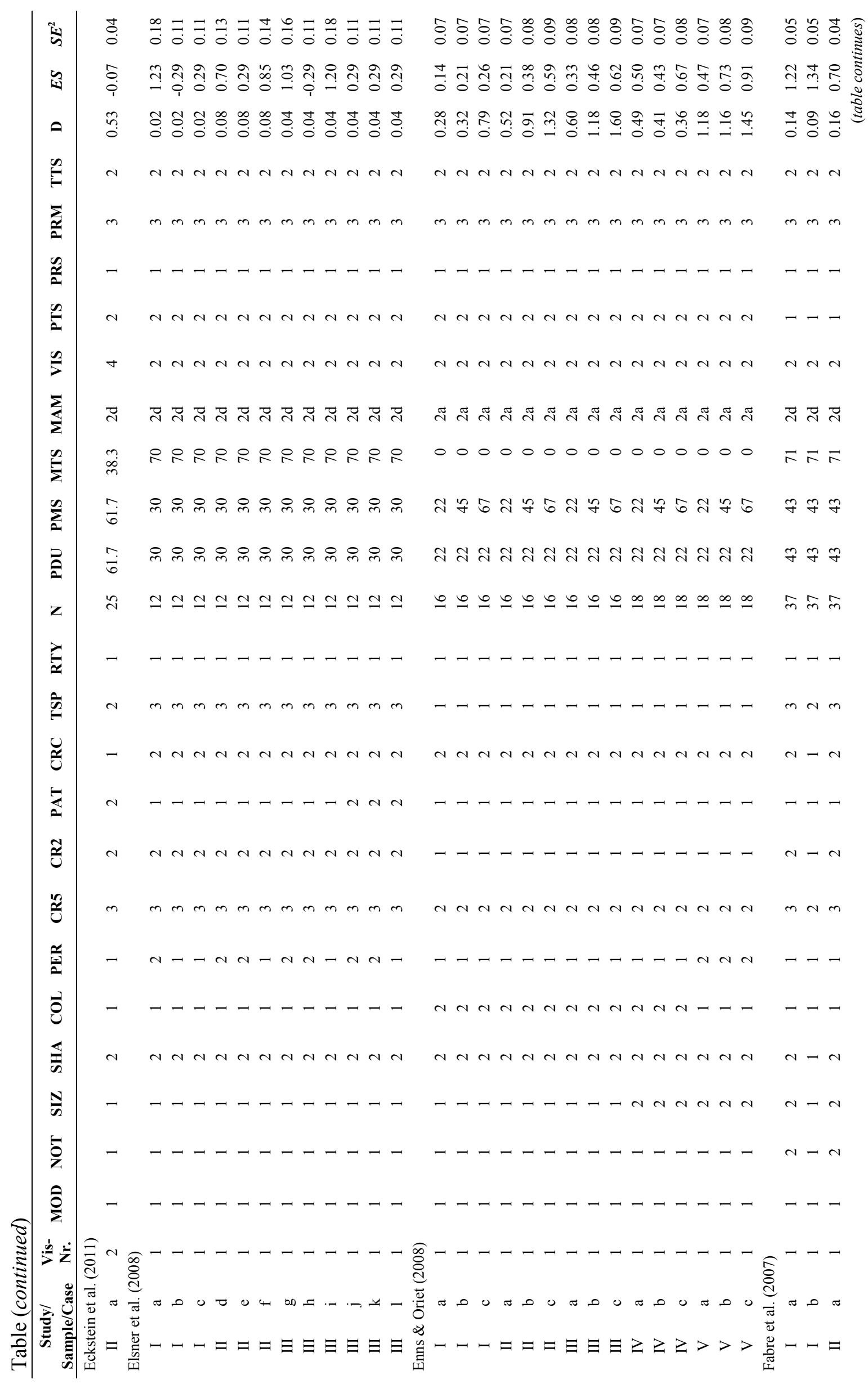




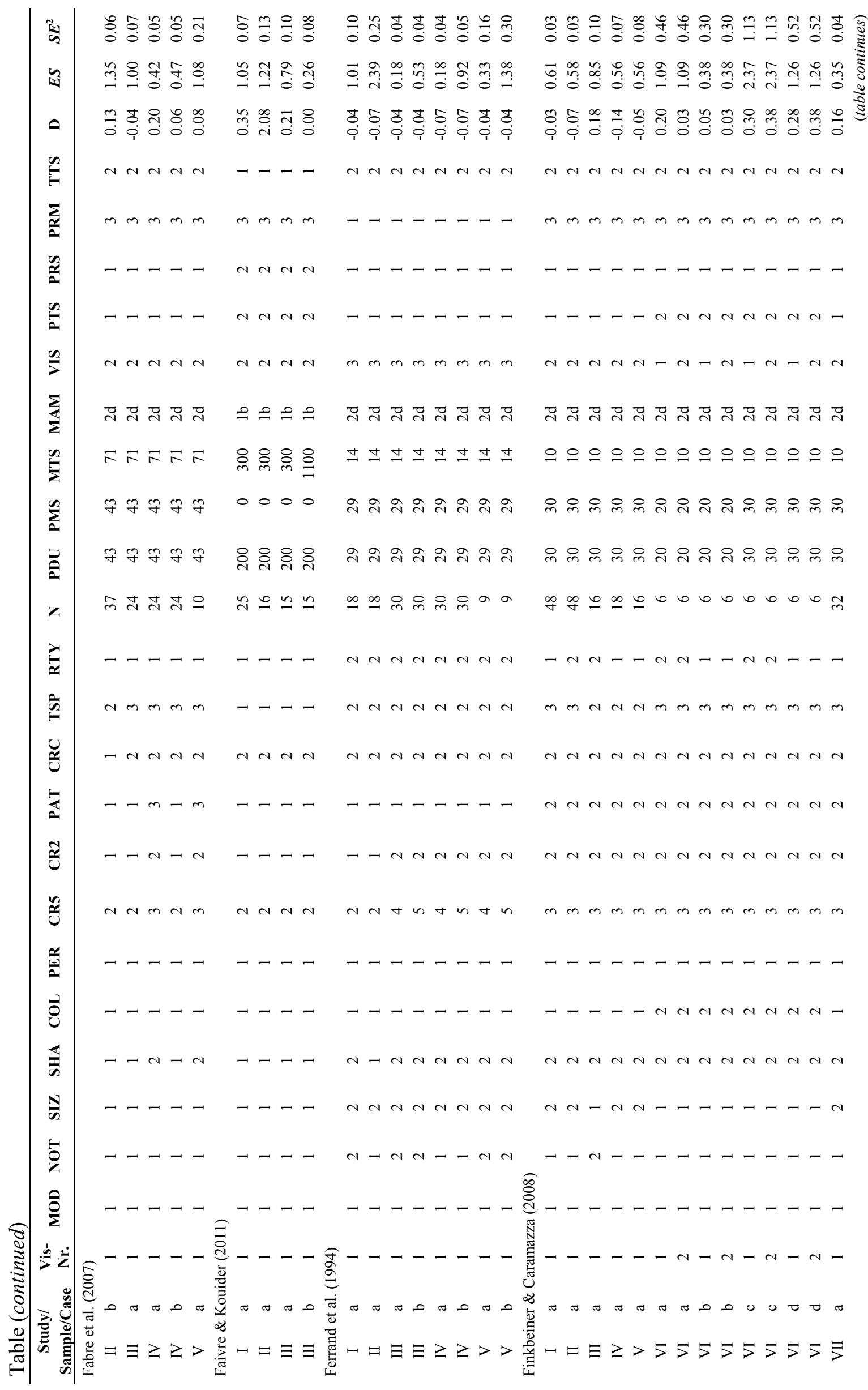




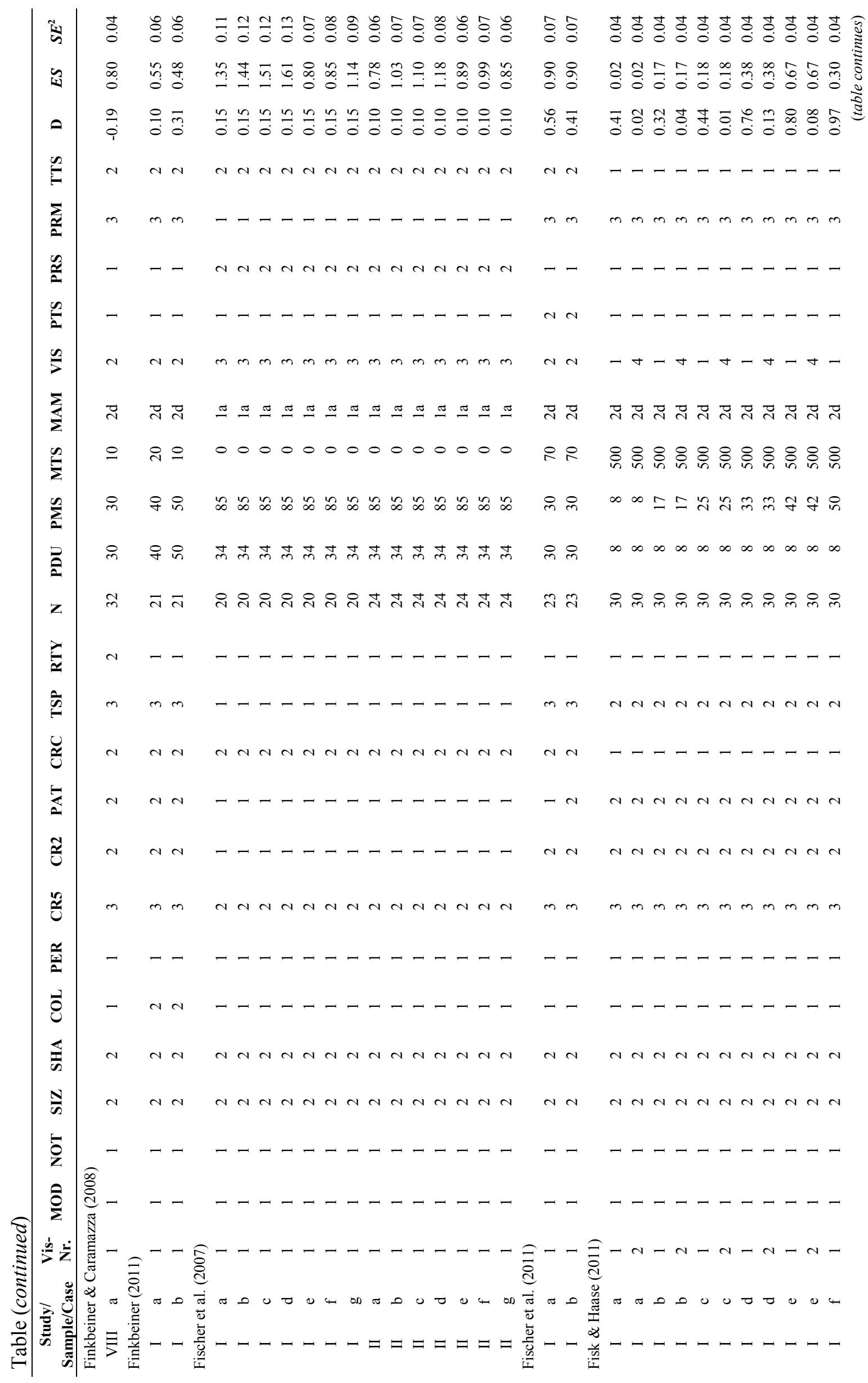




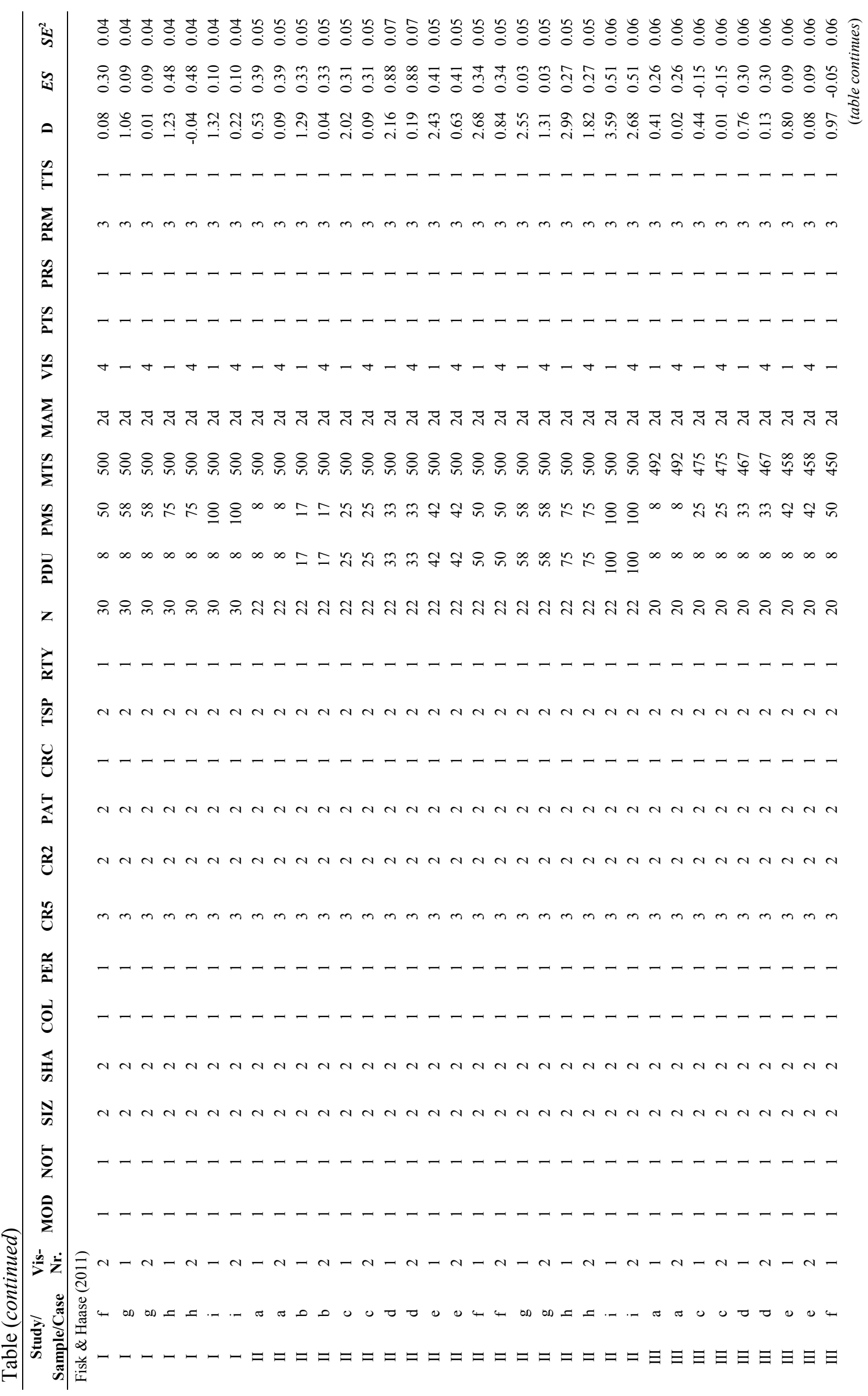




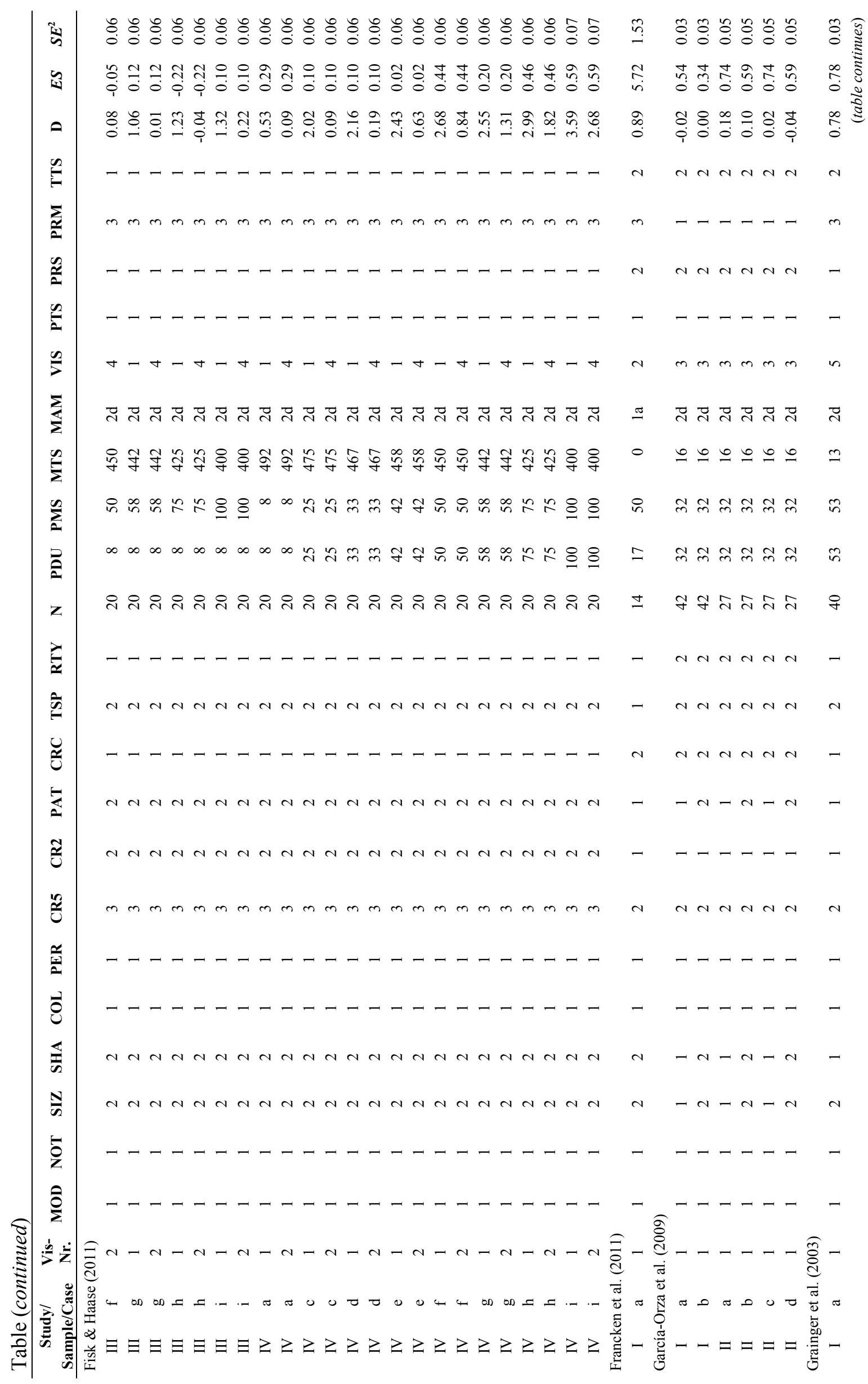




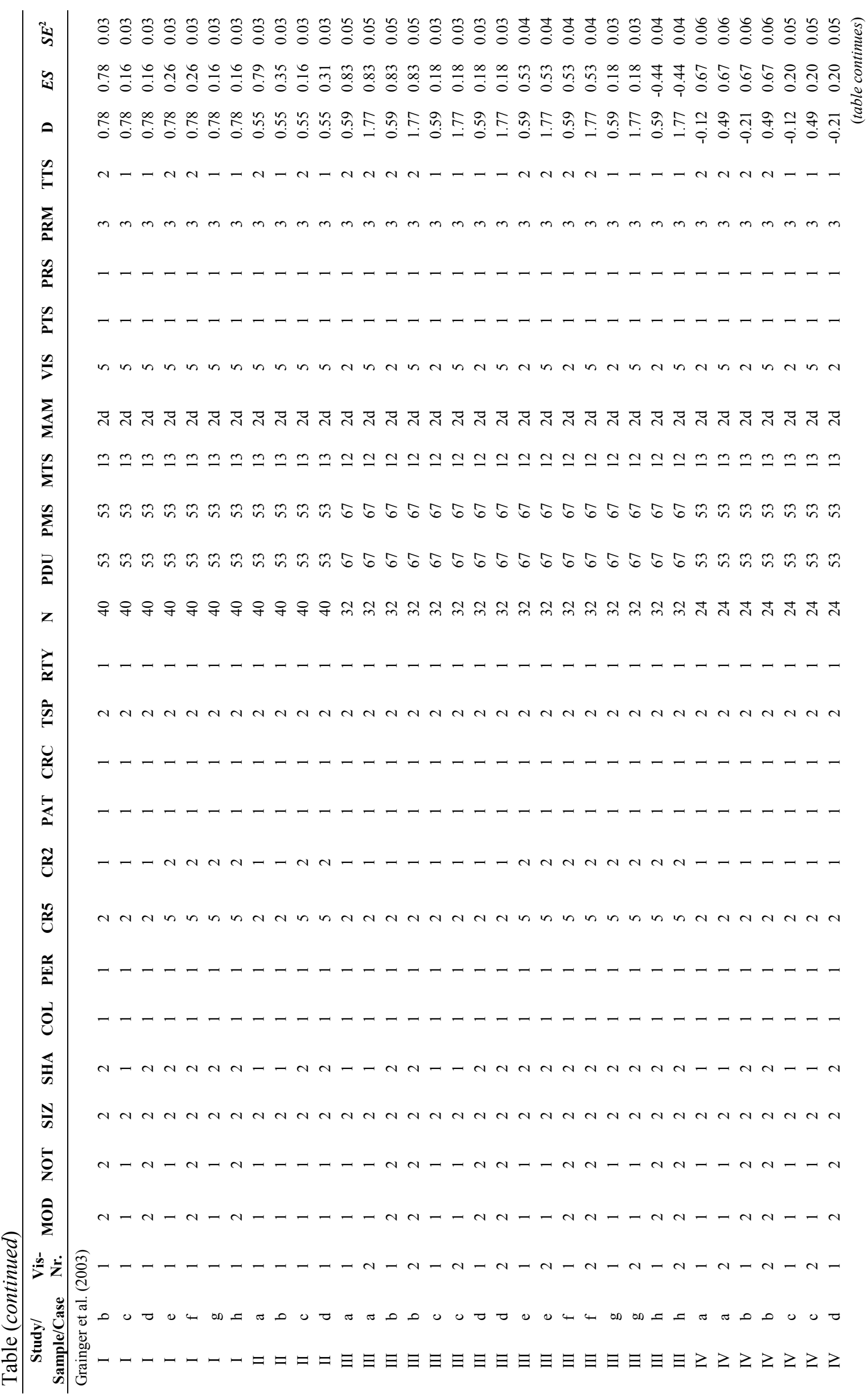




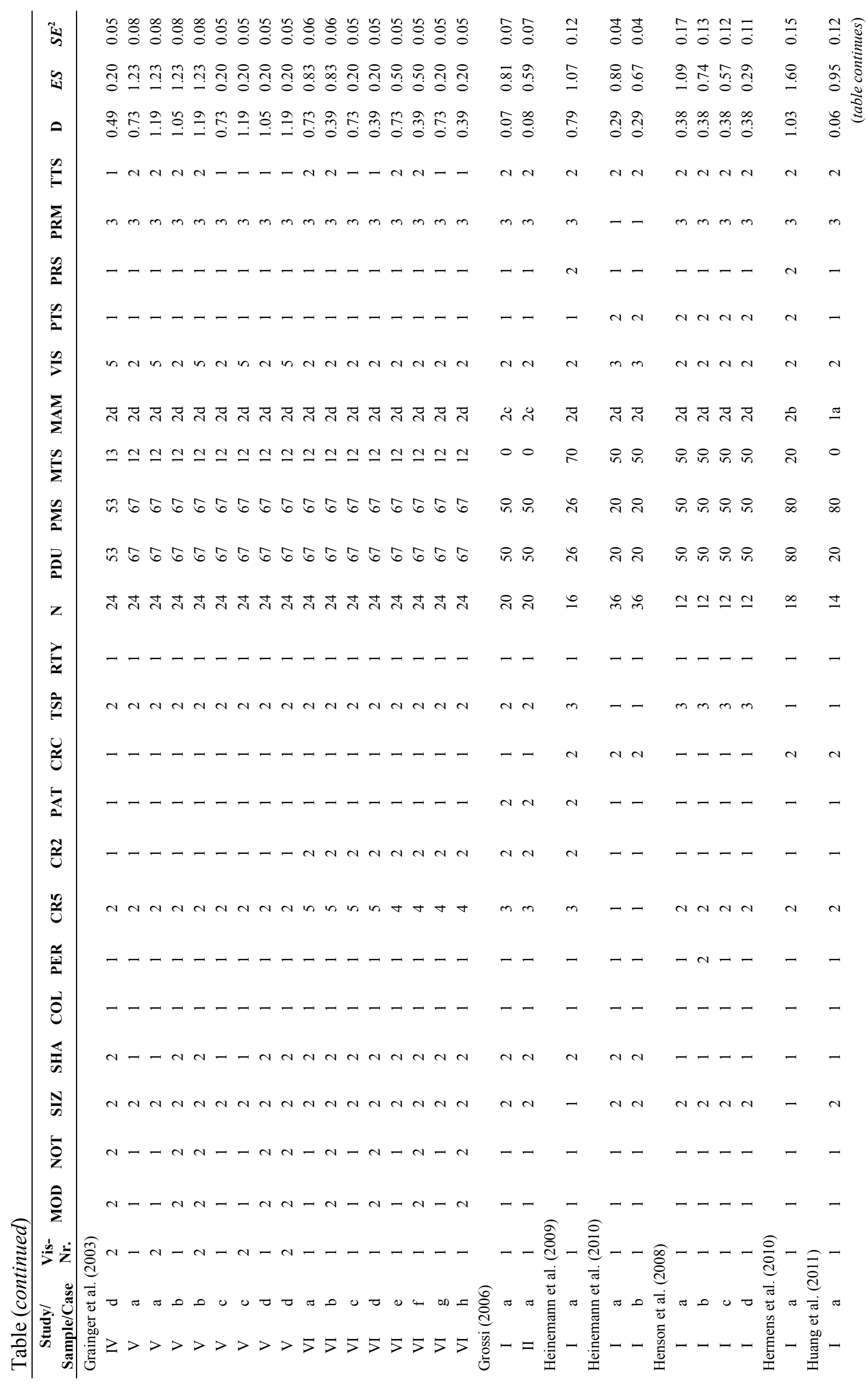




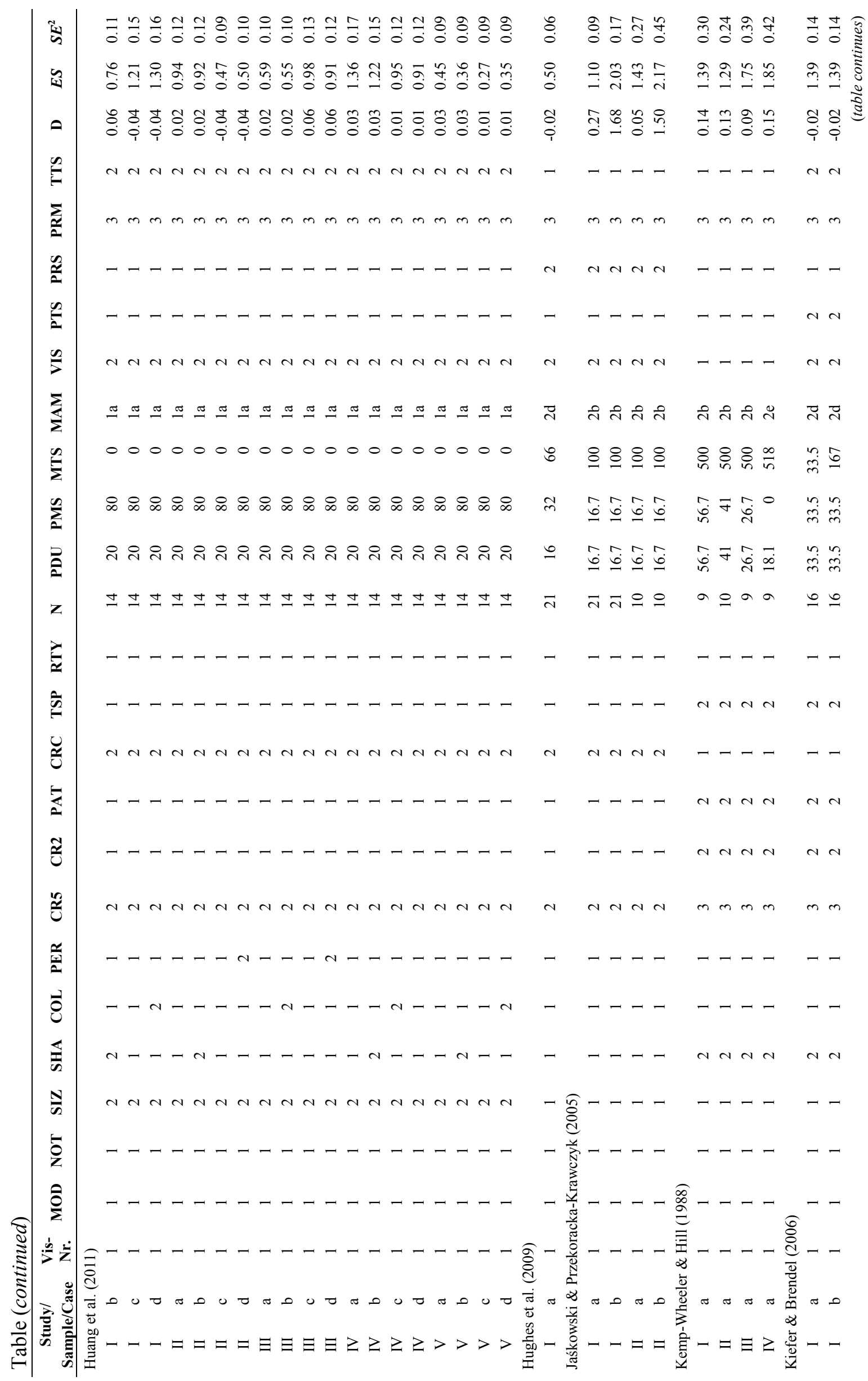




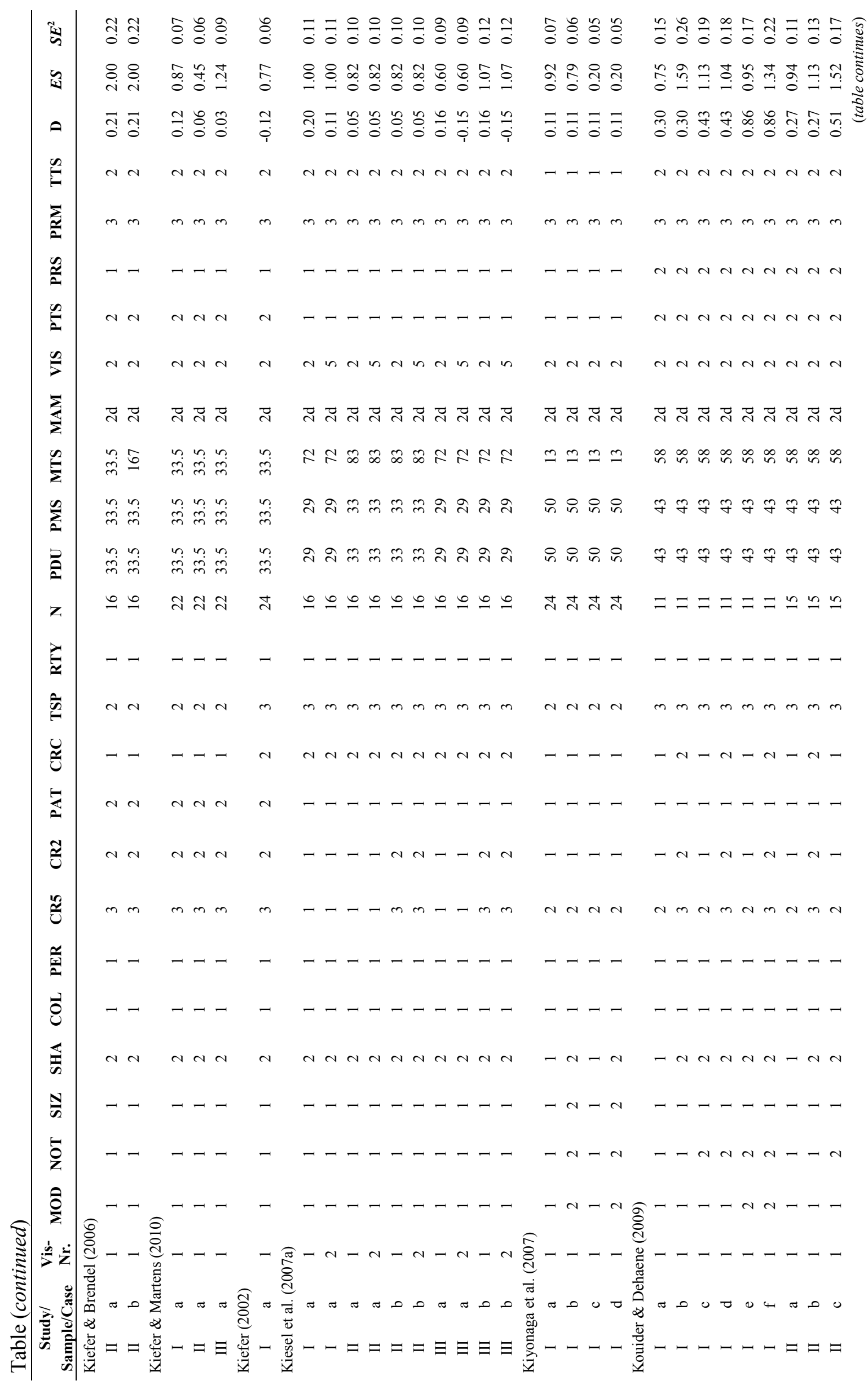




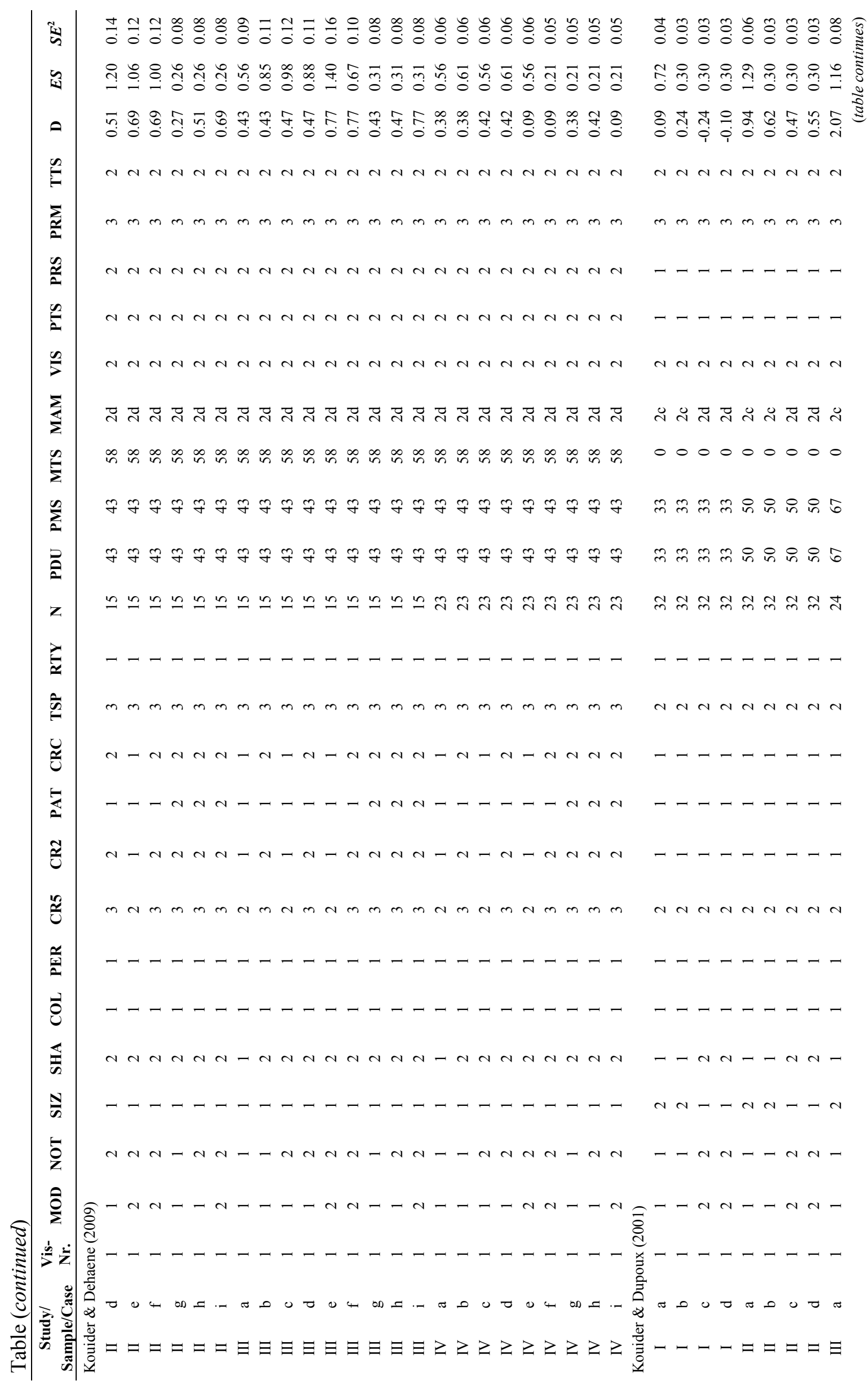




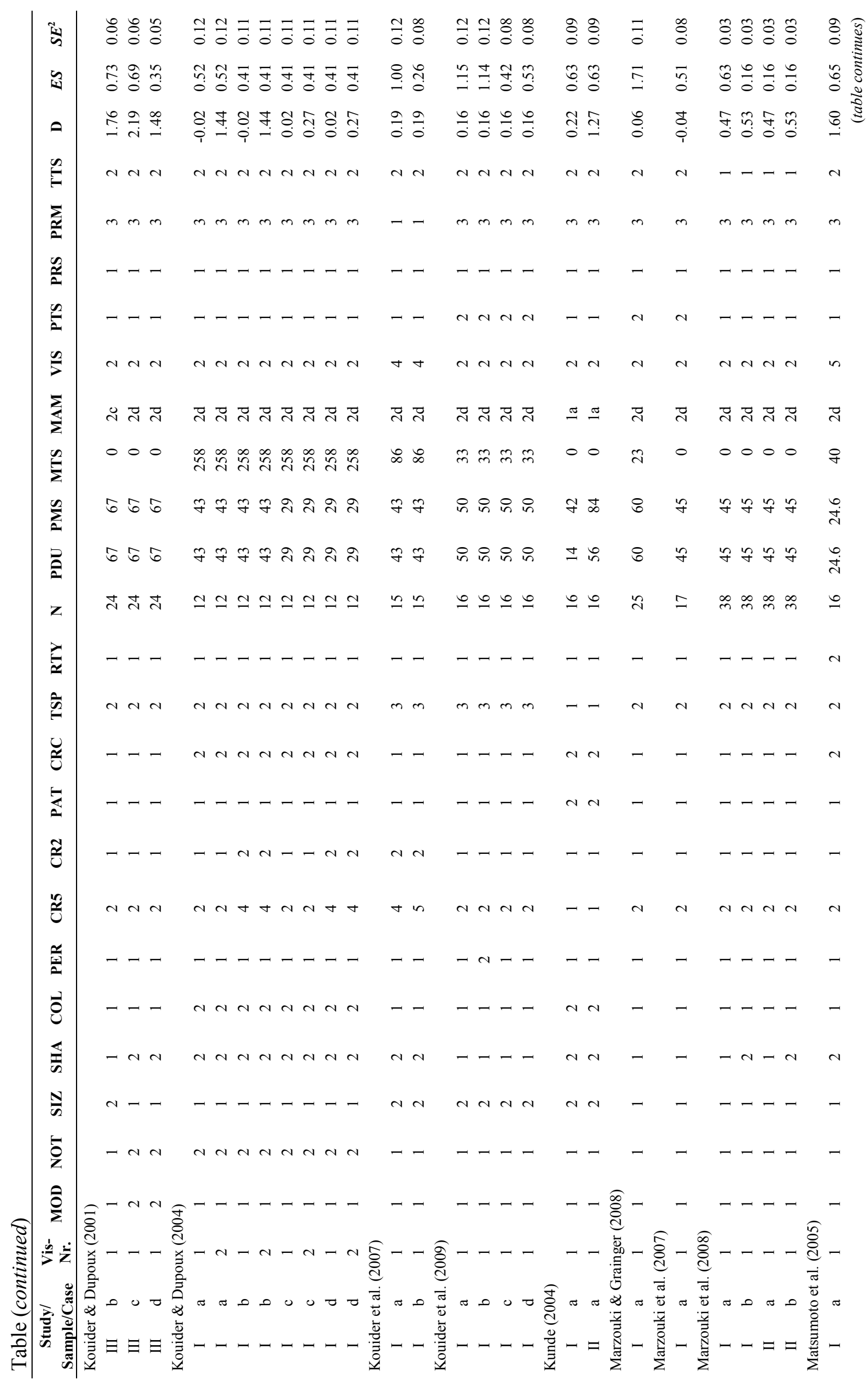




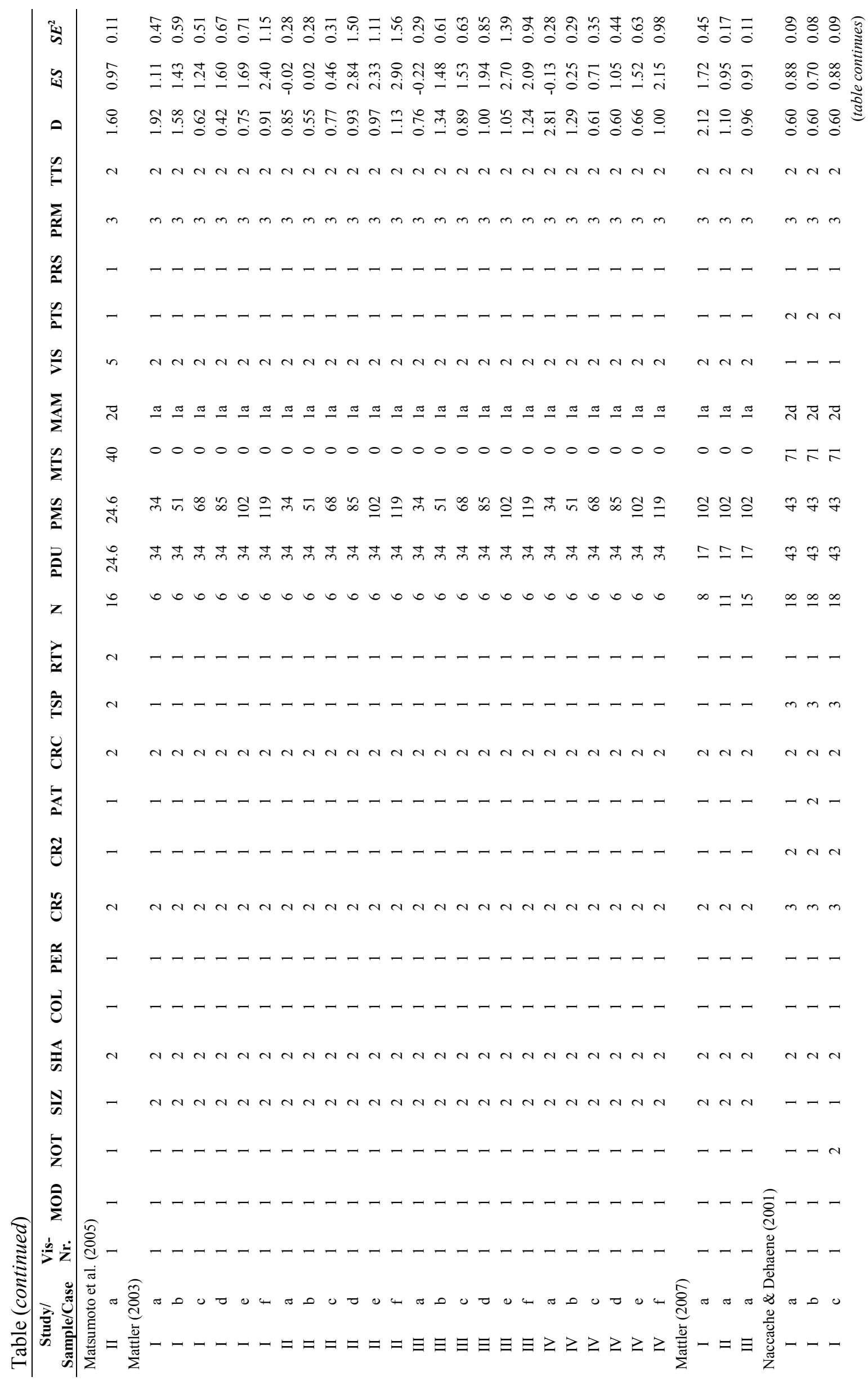




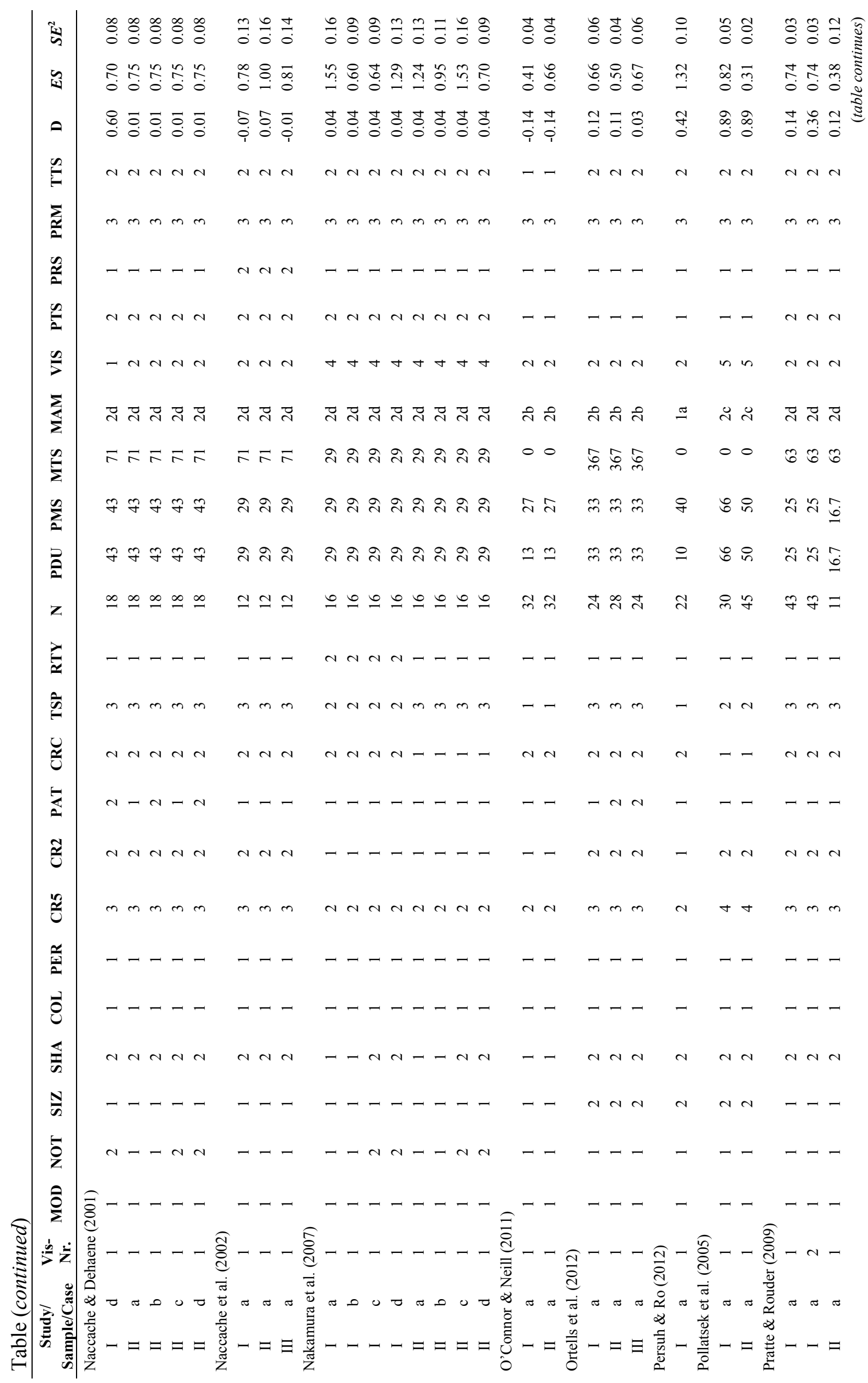




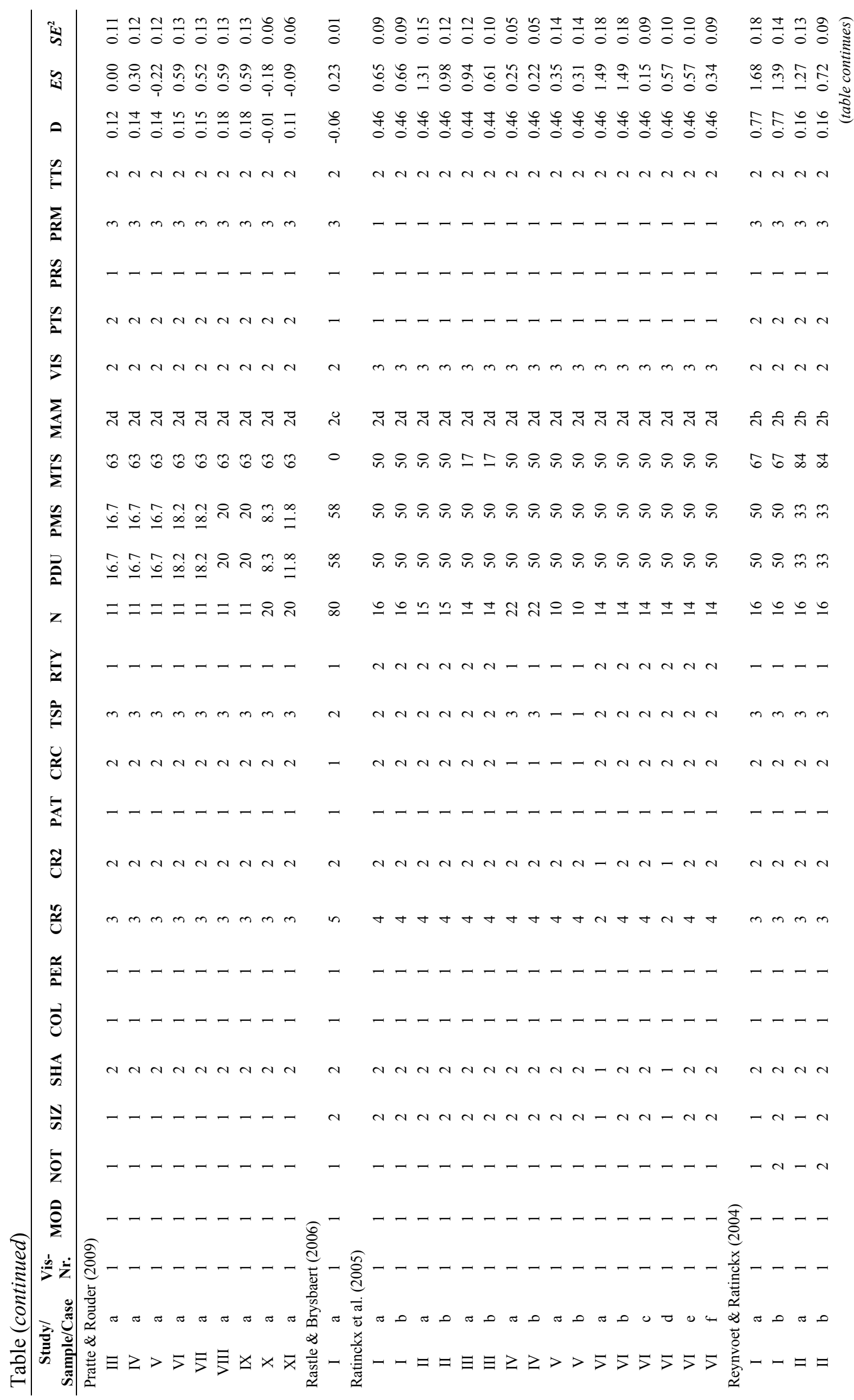




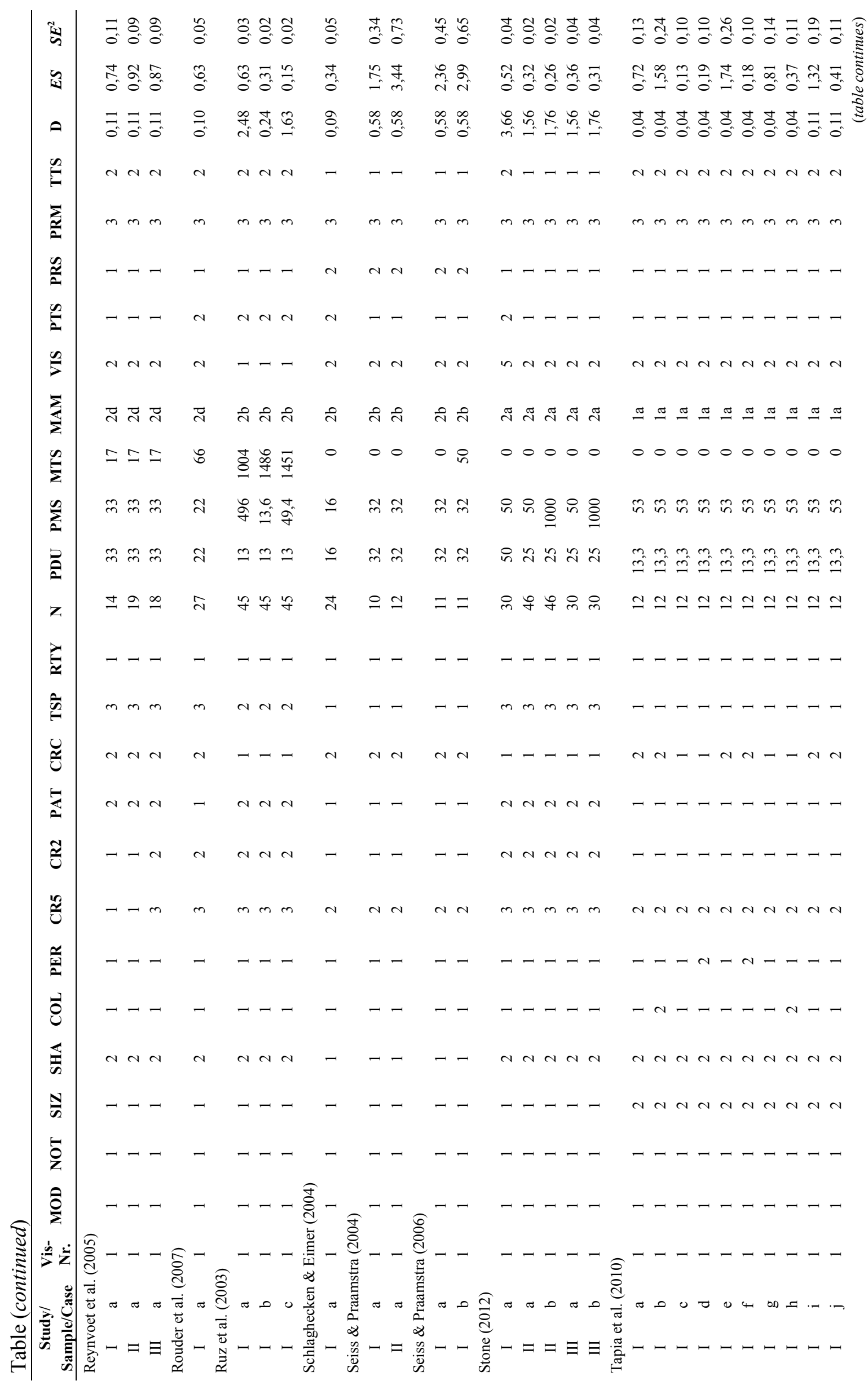




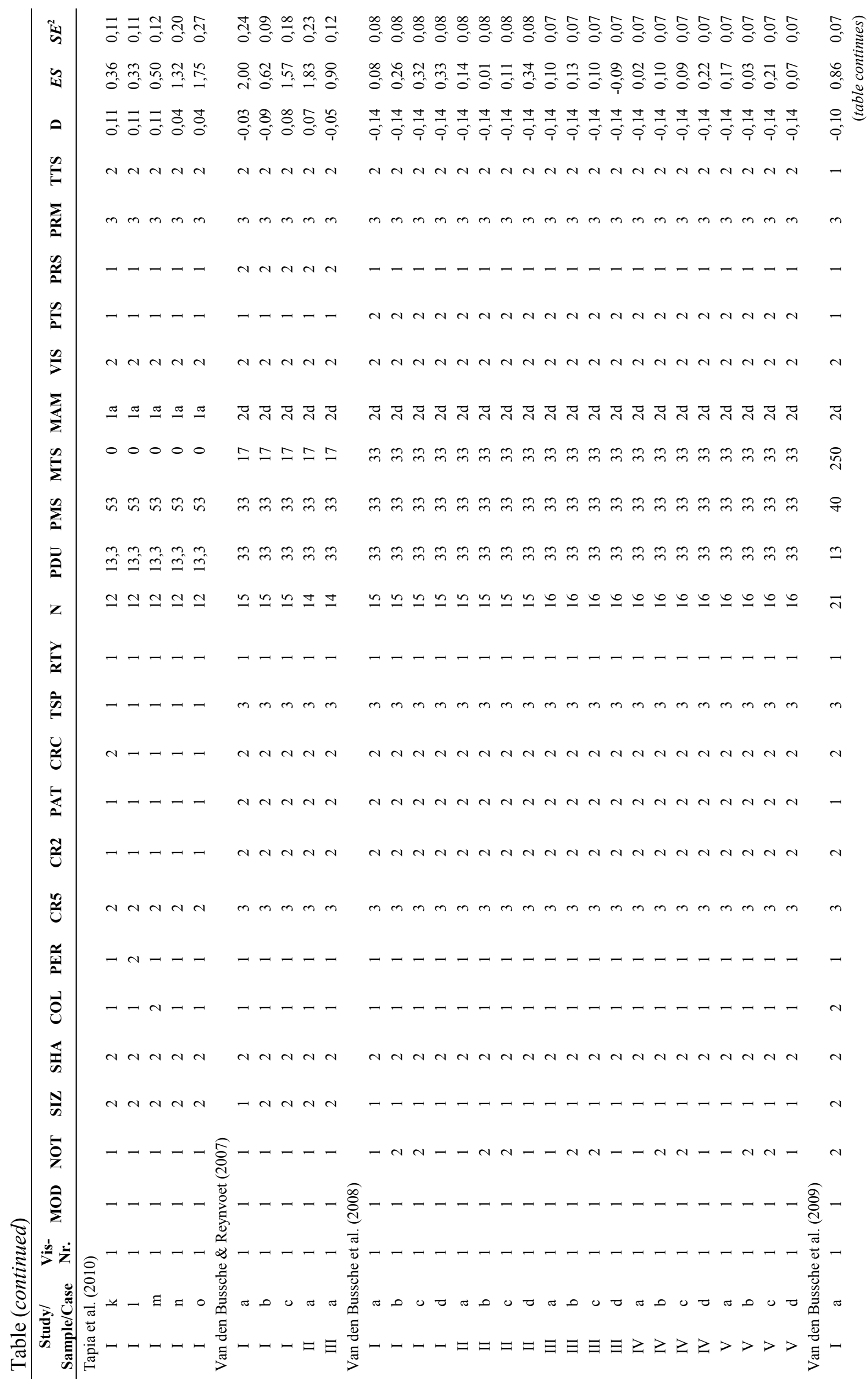




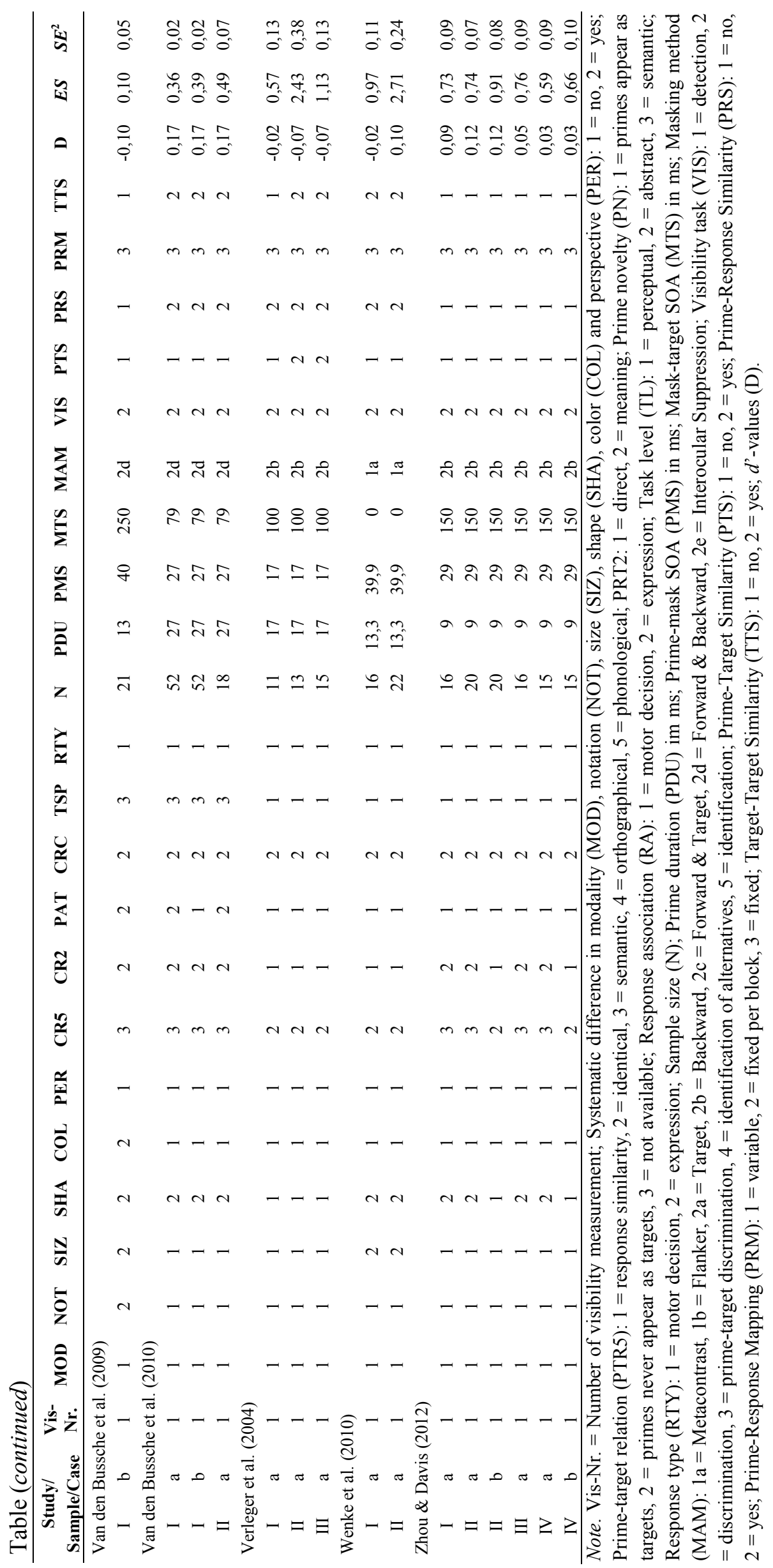


I | Approaching Consciousness with Masked Priming 


\section{Appendix II}

Table B1.1

ANOVA on Errors of Experiments $3.1 \mathrm{~A}$ and $3.1 \mathrm{~B}$ with the relevant congruency as a factor

\begin{tabular}{lccccc}
\hline Effect & $\boldsymbol{F}$ & $\boldsymbol{d f}$ & $\boldsymbol{M S}_{\boldsymbol{e}}$ & $\boldsymbol{p}$ (p.r.n. GG) \\
\hline (Intercept) & 158.48 & 1,22 & 0.696 & $<.0001$ & $* * *$ \\
Congruency & 81.01 & 1,22 & 0.053 & $<.0001$ & $* * *$ \\
Masking strength & 6.56 & 3,66 & 0.021 & $<.001$ & $* * *$ \\
Prime-mask SOA & 10.23 & 3,66 & 0.030 & $<.0001$ & $* * *$ \\
Congruency $\times$ Masking strength & 10.05 & 3,66 & 0.019 & $<.0001$ & $* * *$ \\
Congruency $\times$ Prime-mask SOA & 13.41 & 3,66 & 0.026 & $<.0001$ & $* * *$ \\
Masking strength $\times$ Prime-mask SOA & 0.54 & 9,198 & 0.025 & 0.85 & \\
Congruency $\times$ Masking strength $\times$ Prime-mask SOA & 0.77 & 9,198 & 0.022 & 0.64 & \\
& & & & & 0.62 \\
Task level & 0.25 & 1,22 & 0.696 & 0.17 \\
Task level $\times$ Congruency & 2.06 & 1,22 & 0.053 & 0.021 & 0.68 \\
Task level $\times$ Masking strength & 2.49 & 3,66 & 0.032 \\
Task level $\times$ Prime-mask SOA & 0.94 & 3,66 & 0.030 & 0.42 \\
Task level $\times$ Congruency $\times$ Masking strength & 0.52 & 3,66 & 0.019 & 0.67 \\
Task level $\times$ Congruency $\times$ Prime-mask SOA & 0.50 & 3,66 & 0.026 & 0.68 \\
Task level $\times$ Masking strength $\times$ Prime-mask SOA & 0.86 & 9,198 & 0.025 & 0.56 \\
Task level $\times$ Congruency $\times$ Masking strength & 1.10 & 9,198 & 0.022 & 0.36 \\
$\quad \times$ Prime-mask SOA & & & &
\end{tabular}

Note. GG $=$ Greenhouse-Geisser correction was applied, if Mauchly's sphericity test indicated a violation of the sphericity assumption. '***' $p<0.001$; '**' $p<0.01$; '*' $p<0.05$; '.' $p<0.1$.

Table B1.2

ANOVA on RTs of Experiments $3.1 \mathrm{~A}$ and $3.1 \mathrm{~B}$ with the relevant congruency as a factor

\begin{tabular}{|c|c|c|c|c|c|}
\hline Effect & $F$ & $d f$ & $M S_{e}$ & \multicolumn{2}{|c|}{$\boldsymbol{p}$ (p.r.n. GG) } \\
\hline (Intercept) & 3811.79 & 1,22 & 38594 & $<.0001$ & $* * *$ \\
\hline Congruency & 152.67 & 1,22 & 814 & $<.0001$ & $* * *$ \\
\hline Masking strength & 32.80 & 3,66 & 219 & $<.0001$ & $* * *$ \\
\hline Prime-mask SOA & 16.71 & 3,66 & 138 & $<.0001$ & $* * *$ \\
\hline Congruency $\times$ Masking strength & 73.51 & 3,66 & 208 & $<.0001$ & $* * *$ \\
\hline Congruency $\times$ Prime-mask SOA & 59.84 & 3,66 & 159 & $<.0001$ & $* * *$ \\
\hline Masking strength $\times$ Prime-mask SOA & 0.70 & 9,198 & 143 & 0.64 & \\
\hline Congruency $\times$ Masking strength $\times$ Prime-mask SOA & 4.37 & 9,198 & 127 & $<.05$ & $*$ \\
\hline Task level & 8.94 & 1,22 & 38594 & $<.01$ & $* *$ \\
\hline Task level $\times$ Congruency & 0.08 & 1,22 & 814 & 0.78 & \\
\hline Task level $\times$ Masking strength & 5.09 & 3,66 & 219 & $<.01$ & $* *$ \\
\hline Task level $\times$ Prime-mask SOA & 1.75 & 3,66 & 138 & 0.17 & \\
\hline Task level $\times$ Congruency $\times$ Masking strength & 2.59 & 3,66 & 208 & 0.06 & . \\
\hline Task level $\times$ Congruency $\times$ Prime-mask SOA & 0.04 & 3,66 & 159 & 0.10 & . \\
\hline Task level $\times$ Masking strength $\times$ Prime-mask SOA & 0.44 & 9,198 & 143 & 0.85 & \\
\hline $\begin{array}{l}\text { Task level } \times \text { Congruency } \times \text { Masking strength } \\
\times \text { Prime-mask SOA }\end{array}$ & 2.39 & 9,198 & 127 & $<.05$ & $*$ \\
\hline
\end{tabular}

Note. GG = Greenhouse-Geisser correction was applied, if Mauchly's sphericity test indicated a violation of the sphericity assumption. '***' $p<0.001$; ‘**’ $p<0.01$; '*’’ $p<0.05$; '’ $p<0.1$. 
II | Approaching Consciousness with Masked Priming

Table B1.3

ANOVA on objective prime visibility of Experiments $3.1 \mathrm{~A}$ and $3.1 \mathrm{~B}$

\begin{tabular}{lccccc}
\hline Effect & $\boldsymbol{F}$ & $\boldsymbol{d f}$ & $\boldsymbol{M S}_{\boldsymbol{e}}$ & \multicolumn{2}{c}{$\boldsymbol{p}$ (p.r.n. GG) } \\
\hline (Intercept) & 687.36 & 1,22 & 3.973 & $<.0001$ & $* * *$ \\
Masking strength & 97.02 & 3,66 & 0.659 & $<.0001$ & $* * *$ \\
Prime-mask SOA & 227.12 & 3,66 & 0.213 & $<.0001 *^{* * *}$ \\
Masking strength $\times$ Prime-mask SOA & 29.97 & 9,198 & 0.249 & $<.0001 * * *$ \\
Task level & & & & & 0.13 \\
Task level $\times$ Masking strength & 2.44 & 1,22 & 3.973 & 0.659 & 0.37 \\
Task level $\times$ Prime-mask SOA & 0.98 & 3,66 & 0.213 & 0.52 \\
Task level $\times$ Masking strength $\times$ Prime-mask SOA & 1.04 & 9,198 & 0.249 & 0.39
\end{tabular}

Note. GG = Greenhouse-Geisser correction was applied, if Mauchly's sphericity test indicated a violation of the sphericity assumption. '***' $p<0.001$; ‘**' $p<0.01$; '*’ $p<0.05$; '.’ $p<0.1$.

Table B1.4

ANOVA on subjective prime visibility of Experiments $3.2 \mathrm{~A}$ and $3.2 \mathrm{~B}$

\begin{tabular}{lccccc}
\hline Effect & $\boldsymbol{F}$ & $\boldsymbol{d f}$ & $\boldsymbol{M \boldsymbol { S } _ { \boldsymbol { e } }}$ & \multicolumn{1}{c}{$\boldsymbol{p}$ (p.r.n. GG) } \\
\hline (Intercept) & 1470.00 & 1,22 & 2.607 & $<.0001$ & $* * *$ \\
Masking strength & 119.13 & 3,66 & 0.254 & $<.0001$ & $* * *$ \\
Prime-mask SOA & 211.29 & 3,66 & 0.057 & $<.0001$ & $* * *$ \\
Masking strength $\times$ Prime-mask SOA & 40.68 & 9,198 & 0.041 & $<.0001$ & $* * *$ \\
Task level & 3.33 & 1,22 & 2.607 & 0.08 &. \\
Task level $\times$ Masking strength & 1.07 & 3,66 & 0.254 & 0.34 \\
Task level $\times$ Prime-mask SOA & 1.44 & 3,66 & 0.057 & 0.25 &
\end{tabular}

Note. GG = Greenhouse-Geisser correction was applied, if Mauchly's sphericity test indicated a violation of the sphericity assumption. '***' $p<0.001$; '**' $p<0.01$; ‘*’ $p<0.05$; '. $p<0.1$. 
Table B2.1

ANOVA on Errors of Experiments $3.2 A$ and $3.2 B$ with the relevant congruency as a factor

\begin{tabular}{|c|c|c|c|c|c|}
\hline Effect & $F$ & $\overline{d f}$ & $M S_{e}$ & $p$ (p.r.n & JG) \\
\hline (Intercept) & 61.45 & 1,22 & 1.466 & $<.0001$ & $* * *$ \\
\hline Congruency & 21.05 & 1,22 & 0.218 & $<.0005$ & $* * *$ \\
\hline Masking strength & 0.58 & 3,66 & 0.016 & 0.58 & \\
\hline Prime-mask SOA & 2.60 & 3,66 & 0.044 & 0.06 & 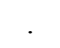 \\
\hline Congruency $\times$ Masking strength & 0.38 & 3,66 & 0.019 & 0.77 & \\
\hline Congruency $\times$ Prime-mask SOA & 14.82 & 3,66 & 0.045 & $<.0001$ & $* * *$ \\
\hline Masking strength $\times$ Prime-mask SOA & 1.36 & 9, 198 & 0.020 & 0.21 & \\
\hline Congruency $\times$ Masking strength $\times$ Prime-mask SOA & 2.20 & 9, 198 & 0.020 & 0.02 & $*$ \\
\hline Task level & 1.35 & 1,22 & 1.466 & 0.26 & \\
\hline Task level $\times$ Congruency & 0.07 & 1,22 & 0.218 & 0.79 & \\
\hline Task level $\times$ Masking strength & 5.98 & 3,66 & 0.016 & $<.005$ & $* * *$ \\
\hline Task level $\times$ Prime-mask SOA & 0.05 & 3,66 & 0.044 & 0.99 & \\
\hline Task level $\times$ Congruency $\times$ Masking strength & 0.11 & 3,66 & 0.019 & 0.96 & \\
\hline Task level $\times$ Congruency $\times$ Prime-mask SOA & 0.05 & 3,66 & 0.045 & 0.94 & \\
\hline Task level $\times$ Masking strength $\times$ Prime-mask SOA & 0.72 & 9,198 & 0.020 & 0.69 & \\
\hline $\begin{array}{l}\text { Task level } \times \text { Congruency } \times \text { Masking strength } \\
\times \text { Prime-mask SOA }\end{array}$ & 1.45 & 9,198 & 0.020 & 0.17 & \\
\hline
\end{tabular}

Table B2.2

ANOVA on RTs of Experiments $3.2 A$ and $3.2 B$ with the relevant congruency as a factor

\begin{tabular}{|c|c|c|c|c|c|}
\hline Effect & $F$ & $d f$ & $M S_{e}$ & $\boldsymbol{p}$ (p.r.n & GG) \\
\hline (Intercept) & 1494.30 & 1,22 & 100289 & $<.0001$ & $* * *$ \\
\hline Congruency & 125.53 & 1,22 & 1591 & $<.0001$ & $* * *$ \\
\hline Masking strength & 0.64 & 3,66 & 135 & 0.54 & \\
\hline Prime-mask SOA & 3.45 & 3,66 & 263 & $<.05$ & $*$ \\
\hline Congruency $\times$ Masking strength & 1.27 & 3,66 & 138 & 0.29 & \\
\hline Congruency $\times$ Prime-mask SOA & 38.32 & 3,66 & 486 & $<.0001$ & $* * *$ \\
\hline Masking strength $\times$ Prime-mask SOA & 1.16 & 9,198 & 149 & 0.33 & \\
\hline Congruency $\times$ Masking strength $\times$ Prime-mask SOA & 0.72 & 9,198 & 132 & 0.62 & \\
\hline Task level & 0.12 & 1,22 & 100289 & 0.73 & \\
\hline Task level $\times$ Congruency & 7.57 & 1,22 & 1591 & $<.05$ & $*$ \\
\hline Task level $\times$ Masking strength & 1.45 & 3,66 & 135 & 0.25 & \\
\hline Task level $\times$ Prime-mask SOA & 1.23 & 3,66 & 263 & 0.30 & \\
\hline Task level $\times$ Congruency $\times$ Masking strength & 0.59 & 3,66 & 138 & 0.62 & \\
\hline Task level $\times$ Congruency $\times$ Prime-mask SOA & 2.54 & 3,66 & 486 & 0.10 & \\
\hline Task level $\times$ Masking strength $\times$ Prime-mask SOA & 0.40 & 9,198 & 149 & 0.86 & \\
\hline $\begin{array}{l}\text { Task level } \times \text { Congruency } \times \text { Masking strength } \\
\times \text { Prime-mask SOA }\end{array}$ & 0.43 & 9,198 & 132 & 0.84 & \\
\hline
\end{tabular}


II | Approaching Consciousness with Masked Priming

Table B2.3

ANOVA on objective prime visibility of Experiments $3.2 A$ and $3.2 B$

\begin{tabular}{lccccc}
\hline Effect & $\boldsymbol{F}$ & $\boldsymbol{d f}$ & $\boldsymbol{M S}_{\boldsymbol{e}}$ & \multicolumn{2}{c}{$\boldsymbol{p}$ (p.r.n. GG) } \\
\hline (Intercept) & 290.07 & 1,22 & 9.349 & $<.0001$ & $* * *$ \\
Masking strength & 36.20 & 3,66 & 0.351 & $<.0001$ & $* * *$ \\
Prime-mask SOA & 86.09 & 3,66 & 0.668 & $<.0001$ & $* * *$ \\
Masking strength $\times$ Prime-mask SOA & 25.68 & 9,198 & 0.196 & $<.0001 * * *$ \\
Task level & 1.91 & 1,22 & 9.349 & 0.18 \\
Task level $\times$ Masking strength & 0.20 & 3,66 & 0.351 & 0.77 \\
Task level $\times$ Prime-mask SOA & 0.19 & 3,66 & 0.668 & 0.82 \\
Task level $\times$ Masking strength $\times$ Prime-mask SOA & 0.37 & 9,198 & 0.196 & 0.87
\end{tabular}

Note. $\mathrm{GG}=$ Greenhouse-Geisser correction was applied, if Mauchly's sphericity test indicated a violation of the sphericity assumption. '***’ $p<0.001$; '**' $p<0.01$; ‘*’ $p<0.05 ;$; ' $p<0.1$.

Table B2.4

ANOVA on subjective prime visibility of Experiments $3.2 \mathrm{~A}$ and $3.2 \mathrm{~B}$

\begin{tabular}{lccccc}
\hline Effect & $\boldsymbol{F}$ & $\boldsymbol{d f}$ & $\boldsymbol{M \boldsymbol { S } _ { \boldsymbol { e } }}$ & \multicolumn{1}{c}{$\boldsymbol{p}$ (p.r.n. GG) } \\
\hline (Intercept) & 940.59 & 1,22 & 4.546 & $<.0001$ & $* * *$ \\
Masking strength & 22.98 & 3,66 & 0.114 & $<.0001$ & $* * *$ \\
Prime-mask SOA & 59.09 & 3,66 & 0.226 & $<.0001$ & $* * *$ \\
Masking strength $\times$ Prime-mask SOA & 24.51 & 9,198 & 0.047 & $<.0001 *^{* * *}$ \\
Task level & 0.05 & 1,22 & 4.556 & 0.83 \\
Task level $\times$ Masking strength & 1.01 & 3,66 & 0.114 & 0.36 \\
Task level $\times$ Prime-mask SOA & 0.13 & 3,66 & 0.226 & 0.80 \\
Task level $\times$ Masking strength $\times$ Prime-mask SOA & 1.21 & 9,198 & 0.047 & 0.31
\end{tabular}

Note. GG = Greenhouse-Geisser correction was applied, if Mauchly's sphericity test indicated a violation of the sphericity assumption. '***' $p<0.001$; '**' $p<0.01$; ‘*’ $p<0.05$; '. $p<0.1$. 
Table B3.1

ANOVA on Errors of Experiments $3.3 \mathrm{~A}$ and $3.3 \mathrm{~B}$ with the relevant congruency as a factor

\begin{tabular}{|c|c|c|c|c|c|}
\hline Effect & $F$ & $\overline{d f}$ & $M S_{e}$ & $p$ (p.r.n & JG) \\
\hline (Intercept) & 133.62 & 1,22 & 0.653 & $<.0001$ & $* * *$ \\
\hline Congruency & 18.76 & 1,22 & 0.037 & $<.0005$ & $* * *$ \\
\hline Masking strength & 0.71 & 3,66 & 0.035 & 0.55 & \\
\hline Prime-mask SOA & 5.05 & 3,66 & 0.030 & $<.01$ & $* *$ \\
\hline Congruency $\times$ Masking strength & 7.99 & 3,66 & 0.022 & $<.0005$ & $* * *$ \\
\hline Congruency $\times$ Prime-mask SOA & 3.08 & 3,66 & 0.023 & $<.05$ & $*$ \\
\hline Masking strength $\times$ Prime-mask SOA & 0.43 & 9,198 & 0.021 & 0.92 & \\
\hline Congruency $\times$ Masking strength $\times$ Prime-mask SOA & 1.18 & 9,198 & 0.021 & 0.31 & \\
\hline Task level & 0.34 & 1,22 & 0.653 & 0.56 & \\
\hline Task level $\times$ Congruency & 3.88 & 1,22 & 0.037 & 0.06 & 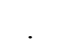 \\
\hline Task level $\times$ Masking strength & 0.31 & 3,66 & 0.035 & 0.82 & \\
\hline Task level $\times$ Prime-mask SOA & 0.38 & 3,66 & 0.030 & 0.72 & \\
\hline Task level $\times$ Congruency $\times$ Masking strength & 1.67 & 3,66 & 0.022 & 0.18 & \\
\hline Task level $\times$ Congruency $\times$ Prime-mask SOA & 1.50 & 3,66 & 0.023 & 0.22 & \\
\hline Task level $\times$ Masking strength $\times$ Prime-mask SOA & 1.06 & 9,198 & 0.021 & 0.40 & \\
\hline $\begin{array}{l}\text { Task level } \times \text { Congruency } \times \text { Masking strength } \\
\times \text { Prime-mask SOA }\end{array}$ & 1.43 & 9,198 & 0.021 & 0.18 & \\
\hline
\end{tabular}

Table B3.2

ANOVA on RTs of Experiments $3.3 A$ and $3.3 B$ with the relevant congruency as a factor

\begin{tabular}{|c|c|c|c|c|c|}
\hline Effect & $F$ & $d f$ & $M S_{e}$ & $p$ (p.r.n & GG) \\
\hline (Intercept) & 1649.38 & 1,22 & 85418 & $<.0001$ & $* * *$ \\
\hline Congruency & 47.24 & 1,22 & 187 & $<.0001$ & $* * *$ \\
\hline Masking strength & 29.02 & 3,66 & 163 & $<.0001$ & $* * *$ \\
\hline Prime-mask SOA & 31.82 & 3,66 & 180 & $<.0001$ & $* * *$ \\
\hline Congruency $\times$ Masking strength & 18.12 & 3,66 & 182 & $<.0001$ & $* * *$ \\
\hline Congruency $\times$ Prime-mask SOA & 8.07 & 3,66 & 126 & $<.005$ & $* *$ \\
\hline Masking strength $\times$ Prime-mask SOA & 2.29 & 9,198 & 111 & $<.05$ & $*$ \\
\hline Congruency $\times$ Masking strength $\times$ Prime-mask SOA & 1.65 & 9,198 & 117 & 0.10 & \\
\hline Task level & 0.32 & 1,22 & 85418 & 0.58 & \\
\hline Task level $\times$ Congruency & 0.02 & 1,22 & 187 & 0.90 & \\
\hline Task level $\times$ Masking strength & 0.75 & 3,66 & 163 & 0.49 & \\
\hline Task level $\times$ Prime-mask SOA & 1.79 & 3,66 & 180 & 0.16 & \\
\hline Task level $\times$ Congruency $\times$ Masking strength & 0.75 & 3,66 & 182 & 0.53 & \\
\hline Task level $\times$ Congruency $\times$ Prime-mask SOA & 2.11 & 3,66 & 126 & 0.13 & \\
\hline Task level $\times$ Masking strength $\times$ Prime-mask SOA & 2.51 & 9,198 & 111 & $<.01$ & $* *$ \\
\hline $\begin{array}{l}\text { Task level } \times \text { Congruency } \times \text { Masking strength } \\
\times \text { Prime-mask SOA }\end{array}$ & 0.70 & 9,198 & 117 & 0.71 & \\
\hline
\end{tabular}


II | Approaching Consciousness with Masked Priming

Table B3.3

ANOVA on objective prime visibility of Experiments $3.3 \mathrm{~A}$ and $3.3 \mathrm{~B}$

\begin{tabular}{lccccc}
\hline Effect & $\boldsymbol{F}$ & $\boldsymbol{d f}$ & $\boldsymbol{M S}_{\boldsymbol{e}}$ & \multicolumn{1}{c}{$\boldsymbol{p}$ (p.r.n. GG) } \\
\hline (Intercept) & 145.28 & 1,22 & 5.819 & $<.0001$ & $* * *$ \\
Masking strength & 187.73 & 3,66 & 0.609 & $<.0001$ & $* * *$ \\
Prime-mask SOA & 68.21 & 3,66 & 0.322 & $<.0001$ & $* * *$ \\
Masking strength $\times$ Prime-mask SOA & 4.19 & 9,198 & 0.361 & $<.005$ & $* *$ \\
Task level & & & & & \\
Task level $\times$ Masking strength & 0.002 & 1,22 & 5.819 & 0.96 \\
Task level $\times$ Prime-mask SOA & 0.59 & 3,66 & 0.609 & 0.59 \\
Task level $\times$ Masking strength $\times$ Prime-mask SOA & 0.53 & 3,66 & 0.322 & 0.60
\end{tabular}

Note. $\mathrm{GG}=$ Greenhouse-Geisser correction was applied, if Mauchly's sphericity test indicated a violation of the sphericity assumption. '***’ $p<0.001$; “**' $p<0.01$; ‘*’ $p<0.05 ;$; ' $p<0.1$.

Table B3.4

ANOVA on subjective prime visibility of Experiments $3.3 \mathrm{~A}$ and $3.3 \mathrm{~B}$

\begin{tabular}{lccccc}
\hline Effect & $\boldsymbol{F}$ & $\boldsymbol{d f}$ & $\boldsymbol{M \boldsymbol { S } _ { \boldsymbol { e } }}$ & $\boldsymbol{p}$ (p.r.n. GG) \\
\hline (Intercept) & 663.71 & 1,22 & 4.036 & $<.0001$ & $* * *$ \\
Masking strength & 85.47 & 3,66 & 0.430 & $<.0001$ & $* * *$ \\
Prime-mask SOA & 86.02 & 3,66 & 0.076 & $<.0001 * * * *$ \\
Masking strength $\times$ Prime-mask SOA & 14.67 & 9,198 & 0.043 & $<.0001 * * *$ \\
Task level & & & & 0.47 \\
Task level $\times$ Masking strength & 0.55 & 1,22 & 4.036 & 0.430 & 0.83 \\
Task level $\times$ Prime-mask SOA & 0.12 & 3,66 & 0.076 & $<.05$ & $*$ \\
Task level $\times$ Masking strength $\times$ Prime-mask SOA & 3.40 & 3,66 & 0.043 & 0.84
\end{tabular}

Note. GG = Greenhouse-Geisser correction was applied, if Mauchly's sphericity test indicated a violation of the sphericity assumption. '***' $p<0.001$; '**' $p<0.01$; ‘*’ $p<0.05$; '. $p<0.1$. 
Table B4.1

ANOVA on Errors of Experiments 3.2A, 3.2B, 3.3A and 3.3B with the relevant congruency as a factor

\begin{tabular}{|c|c|c|c|c|c|}
\hline Effect & $\boldsymbol{F}$ & $d f$ & $M S_{e}$ & \multicolumn{2}{|c|}{$\boldsymbol{p}$ (p.r.n. GG) } \\
\hline (Intercept) & 167.36 & 1,44 & 1.060 & $<.0001$ & $* * *$ \\
\hline Congruency & 34.71 & 1,44 & 0.128 & $<.0001$ & $* * *$ \\
\hline Masking strength & 0.96 & 3,132 & 0.025 & 0.41 & \\
\hline Prime-mask SOA & 7.10 & 3,132 & 0.037 & $<.0005$ & $* * *$ \\
\hline Congruency $\times$ Masking strength & 6.07 & 3,132 & 0.021 & $<.001$ & $* * *$ \\
\hline Congruency $\times$ Prime-mask SOA & 17.05 & 3,132 & 0.034 & $<.0001$ & $* * *$ \\
\hline Masking strength $\times$ Prime-mask SOA & 1.12 & 9,396 & 0.021 & 0.35 & \\
\hline Congruency $\times$ Masking strength $\times$ Prime-mask SOA & 2.47 & 9,396 & 0.020 & $<.01$ & $* *$ \\
\hline Task level & 1.67 & 1,44 & 1.060 & 0.20 & \\
\hline Task level $\times$ Congruency & 1.00 & 1,44 & 0.128 & 0.32 & \\
\hline Task level $\times$ Masking strength & 1.02 & 3,132 & 0.025 & 0.39 & \\
\hline Task level $\times$ Prime-mask SOA & 0.10 & 3,132 & 0.037 & 0.96 & \\
\hline Task level $\times$ Congruency $\times$ Masking strength & 1.01 & 3,132 & 0.021 & 0.39 & \\
\hline Task level $\times$ Congruency $\times$ Prime-mask SOA & 0.54 & 3,132 & 0.034 & 0.61 & \\
\hline Task level $\times$ Masking strength $\times$ Prime-mask SOA & 1.28 & 9,396 & 0.021 & 0.25 & \\
\hline $\begin{array}{l}\text { Task level } \times \text { Congruency } \times \text { Masking strength } \\
\times \text { Prime-mask SOA }\end{array}$ & 1.56 & 9,396 & 0.020 & 0.12 & \\
\hline Masking method & 0.01 & 1,44 & 1.060 & 0.92 & \\
\hline Masking method $\times$ Congruency & 6.72 & 1,44 & 0.128 & $<.05$ & $*$ \\
\hline Masking method $\times$ Masking strength & 0.38 & 3,132 & 0.025 & 0.77 & \\
\hline Masking method $\times$ Prime-mask SOA & 0.07 & 3,132 & 0.037 & 0.98 & \\
\hline Masking method $\times$ Congruency $\times$ Masking strength & 2.73 & 3,132 & 0.021 & $<.05$ & $*$ \\
\hline Masking method $\times$ Congruency $\times$ Prime-mask SOA & 4.76 & 3,132 & 0.034 & $<.01$ & $* *$ \\
\hline Masking method $\times$ Masking strength $\times$ Prime-mask SOA & 0.66 & 9,396 & 0.021 & 0.75 & \\
\hline $\begin{array}{l}\text { Masking method } \times \text { Congruency } \times \text { Masking strength } \\
\times \text { Prime-mask SOA }\end{array}$ & 0.88 & 9,396 & 0.020 & 0.54 & \\
\hline Masking method $\times$ Task level & 0.41 & 1,44 & 1.060 & 0.52 & \\
\hline Masking method $\times$ Task level $\times$ Congruency & 0.25 & 1,44 & 0.128 & 0.62 & \\
\hline Masking method $\times$ Task level $\times$ Masking strength & 3.16 & 3,132 & 0.025 & $<.05$ & $*$ \\
\hline Masking method $\times$ Task level $\times$ Prime-mask SOA & 0.26 & 3,132 & 0.037 & 0.86 & \\
\hline $\begin{array}{l}\text { Masking method } \times \text { Task level } \times \text { Congruency } \\
\times \text { Masking strength }\end{array}$ & 0.86 & 3,132 & 0.021 & 0.46 & \\
\hline $\begin{array}{l}\text { Masking method } \times \text { Task level } \times \text { Congruency } \\
\times \text { Prime-mask SOA }\end{array}$ & 0.53 & 3,132 & 0.034 & 0.62 & \\
\hline $\begin{array}{l}\text { Masking method } \times \text { Task level } \times \text { Masking strength } \\
\times \text { Prime-mask SOA }\end{array}$ & 0.50 & 9,396 & 0.021 & 0.87 & \\
\hline $\begin{array}{l}\text { Masking method } \times \text { Task level } \times \text { Congruency } \\
\times \text { Masking strength } \times \text { Prime-mask SOA }\end{array}$ & 1.32 & 9,396 & 0.020 & 0.22 & \\
\hline
\end{tabular}

Note. GG $=$ Greenhouse-Geisser correction was applied, if Mauchly's sphericity test indicated a violation of the sphericity assumption. '***' $p<0.001$; '**’ $p<0.01$; '*’ $p<0.05$; ' ' $p<0.1$. 
Table B4.2

ANOVA on RTs of Experiments 3.2A, 3.2B, 3.3A and 3.3B with the relevant congruency as a factor

\begin{tabular}{|c|c|c|c|c|c|}
\hline Effect & $\boldsymbol{F}$ & $d f$ & $M S_{e}$ & \multicolumn{2}{|c|}{$\boldsymbol{p}$ (p.r.n. GG) } \\
\hline (Intercept) & 3130.52 & 1,44 & 92853 & $<.0001$ & $* * *$ \\
\hline Congruency & 164.53 & 1,44 & 889 & $<.0001$ & $* * *$ \\
\hline Masking strength & 18.44 & 3,132 & 149 & $<.0001$ & $* * *$ \\
\hline Prime-mask SOA & 22.48 & 3,132 & 222 & $<.0001$ & $* * *$ \\
\hline Congruency $\times$ Masking strength & 10.38 & 3,132 & 160 & $<.0001$ & $* * *$ \\
\hline Congruency $\times$ Prime-mask SOA & 45.07 & 3,132 & 306 & $<.0001$ & $* * *$ \\
\hline Masking strength $\times$ Prime-mask SOA & 1.85 & 9,396 & 130 & 0.06 & . \\
\hline Congruency $\times$ Masking strength $\times$ Prime-mask SOA & 0.35 & 9,396 & 125 & 0.92 & \\
\hline Task level & 0.41 & 1,44 & 92853 & 0.53 & \\
\hline Task level $\times$ Congruency & 6.99 & 1,44 & 889 & $<.05$ & $*$ \\
\hline Task level $\times$ Masking strength & 0.82 & 3,132 & 149 & 0.46 & \\
\hline Task level $\times$ Prime-mask SOA & 2.86 & 3,132 & 222 & $<.05$ & $*$ \\
\hline Task level $\times$ Congruency $\times$ Masking strength & 0.46 & 3,132 & 160 & 0.71 & \\
\hline Task level $\times$ Congruency $\times$ Prime-mask SOA & 4.23 & 3,132 & 306 & $<.05$ & $*$ \\
\hline Task level $\times$ Masking strength $\times$ Prime-mask SOA & 0.82 & 9,396 & 130 & 0.60 & \\
\hline $\begin{array}{l}\text { Task level } \times \text { Congruency } \times \text { Masking strength } \\
\times \text { Prime-mask SOA }\end{array}$ & 0.44 & 9,396 & 125 & 0.87 & \\
\hline Masking method & 0.75 & 1,44 & 92853 & 0.39 & \\
\hline Masking method $\times$ Congruency & 70.08 & 1,44 & 889 & $<.0001$ & $* * *$ \\
\hline Masking method $\times$ Masking strength & 13.85 & 3,132 & 149 & $<.0001$ & $* * *$ \\
\hline Masking method $\times$ Prime-mask SOA & 7.50 & 3,132 & 222 & $<.0005$ & $* * *$ \\
\hline Masking method $\times$ Congruency $\times$ Masking strength & 11.35 & 3,132 & 160 & $<.0001$ & $* * *$ \\
\hline Masking method $\times$ Congruency $\times$ Prime-mask SOA & 19.13 & 3,132 & 306 & $<.0001$ & $* * *$ \\
\hline Masking method $\times$ Masking strength $\times$ Prime-mask SOA & 1.43 & 9,396 & 130 & 0.17 & \\
\hline $\begin{array}{l}\text { Masking method } \times \text { Congruency } \times \text { Masking strength } \\
\times \text { Prime-mask SOA }\end{array}$ & 1.96 & 9,396 & 125 & 0.06 & . \\
\hline Masking method $\times$ Task level & 0.02 & 1,44 & 92853 & 0.90 & \\
\hline Masking method $\times$ Task level $\times$ Congruency & 6.56 & 1,44 & 889 & $<.05$ & $*$ \\
\hline Masking method $\times$ Task level $\times$ Masking strength & 1.32 & 3,132 & 149 & 0.27 & \\
\hline Masking method $\times$ Task level $\times$ Prime-mask SOA & 0.06 & 3,132 & 222 & 0.97 & \\
\hline $\begin{array}{l}\text { Masking method } \times \text { Task level } \times \text { Congruency } \\
\times \text { Masking strength }\end{array}$ & 0.89 & 3,132 & 160 & $<.0001$ & $* * *$ \\
\hline $\begin{array}{l}\text { Masking method } \times \text { Task level } \times \text { Congruency } \\
\times \text { Prime-mask SOA }\end{array}$ & 0.68 & 3,132 & 306 & $<.0001$ & $* * *$ \\
\hline $\begin{array}{l}\text { Masking method } \times \text { Task level } \times \text { Masking strength } \\
\times \text { Prime-mask SOA }\end{array}$ & 1.79 & 9,396 & 130 & 0.06 & . \\
\hline $\begin{array}{l}\text { Masking method } \times \text { Task level } \times \text { Congruency } \\
\times \text { Masking strength } \times \text { Prime-mask SOA }\end{array}$ & 0.67 & 9,396 & 125 & 0.69 & \\
\hline
\end{tabular}

Note. GG $=$ Greenhouse-Geisser correction was applied, if Mauchly's sphericity test indicated a violation of the sphericity assumption. '***' $p<0.001$; '**’ $p<0.01$; '*' $p<0.05$; '.' $p<0.1$. 
Table B4.3

ANOVA on objective prime visibility of Experiments $3.2 \mathrm{~A}, 3.2 \mathrm{~B}, 3.3 \mathrm{~A}$ and $3.3 \mathrm{~B}$

\begin{tabular}{|c|c|c|c|c|c|}
\hline Effect & $F$ & $d f$ & $M S_{e}$ & $p$ (p.r.n & GG) \\
\hline (Intercept) & 434.16 & 1,44 & 7.584 & $<.0001$ & $* * *$ \\
\hline Masking strength & 199.43 & 3,132 & 0.480 & $<.0001$ & $* * *$ \\
\hline Prime-mask SOA & 147.93 & 3,132 & 0.494 & $<.0001$ & $* * *$ \\
\hline Masking strength $\times$ Prime-mask SOA & 16.30 & 9,396 & 0.278 & $<.0001$ & $* * *$ \\
\hline Task level & 1.24 & 1,44 & 7.584 & 0.27 & \\
\hline Task level $\times$ Masking strength & 0.28 & 3,132 & 0.480 & 0.80 & \\
\hline Task level $\times$ Prime-mask SOA & 0.42 & 3,132 & 0.494 & 0.67 & \\
\hline Task level $\times$ Masking strength $\times$ Prime-mask SOA & 1.07 & 9,396 & 0.278 & 0.38 & \\
\hline Masking method & 34.87 & 1,44 & 7.584 & $<.0001$ & $* * *$ \\
\hline Masking method $\times$ Masking strength & 65.16 & 3,132 & 0.480 & $<.0001$ & $* * *$ \\
\hline Masking method $\times$ Prime-mask SOA & 12.63 & 3,132 & 0.494 & $<.0001$ & $* * *$ \\
\hline Masking method $\times$ Masking strength $\times$ Prime-mask SOA & 7.18 & 9,396 & 0.278 & $<.0001$ & $* * *$ \\
\hline Masking method $\times$ Task level & 1.11 & 1,44 & 7.584 & 0.30 & \\
\hline Masking method $\times$ Task level $\times$ Masking strength & 0.61 & 3,132 & 0.480 & 0.57 & \\
\hline Masking method $\times$ Task level $\times$ Prime-mask SOA & 0.19 & 3,132 & 0.494 & 0.84 & \\
\hline $\begin{array}{l}\text { Masking method } \times \text { Task level } \times \text { Masking strength } \\
\times \text { Prime-mask SOA }\end{array}$ & 0.63 & 9,396 & 0.278 & 0.71 & \\
\hline
\end{tabular}

Table B4.4

ANOVA on subjective prime visibility of Experiments $3.2 \mathrm{~A}, 3.2 \mathrm{~B}, 3.3 \mathrm{~A}$ and $3.3 \mathrm{~B}$

\begin{tabular}{|c|c|c|c|c|c|}
\hline \multirow{2}{*}{$\begin{array}{l}\text { Effect } \\
\text { (Intercept) }\end{array}$} & \multirow{2}{*}{$\begin{array}{c}\boldsymbol{F} \\
1599.10\end{array}$} & \multirow{2}{*}{$\frac{d \boldsymbol{f}}{1,44}$} & \multirow{2}{*}{$\begin{array}{l}\boldsymbol{M} \boldsymbol{S}_{\boldsymbol{e}} \\
4.291\end{array}$} & \multicolumn{2}{|c|}{$\boldsymbol{p}$ (p.r.n. GG) } \\
\hline & & & & $<.0001$ & $* * *$ \\
\hline Masking strength & 106.70 & 3,132 & 0.272 & $<.0001$ & $* * *$ \\
\hline Prime-mask SOA & 126.44 & 3,132 & 0.151 & $<.0001$ & $* * *$ \\
\hline Masking strength $\times$ Prime-mask SOA & 33.86 & 9,396 & 0.045 & $<.0001$ & $* * *$ \\
\hline Task level & 0.45 & 1,44 & 4.291 & 0.51 & \\
\hline Task level $\times$ Masking strength & 0.15 & 3,132 & 0.272 & 0.84 & \\
\hline Task level $\times$ Prime-mask SOA & 1.53 & 3,132 & 0.151 & 0.23 & \\
\hline Task level $\times$ Masking strength $\times$ Prime-mask SOA & 0.71 & 9,396 & 0.045 & 0.54 & \\
\hline Masking method & 21.66 & 1,44 & 4.291 & $<.0001$ & $* * *$ \\
\hline Masking method $\times$ Masking strength & 38.08 & 3,132 & 0.272 & $<.0001$ & $* * *$ \\
\hline Masking method $\times$ Prime-mask SOA & 5.28 & 3,132 & 0.151 & $<.05$ & $*$ \\
\hline Masking method $\times$ Masking strength $\times$ Prime-mask SOA & 5.72 & 9,396 & 0.045 & $<.005$ & $* *$ \\
\hline Masking method $\times$ Task level & 0.12 & 1,44 & 4.291 & 0.73 & \\
\hline Masking method $\times$ Task level $\times$ Masking strength & 0.46 & 3,132 & 0.272 & 0.61 & \\
\hline Masking method $\times$ Task level $\times$ Prime-mask SOA & 0.38 & 3,132 & 0.151 & 0.62 & \\
\hline $\begin{array}{l}\text { Masking method } \times \text { Task level } \times \text { Masking strength } \\
\times \text { Prime-mask SOA }\end{array}$ & 0.76 & 9,396 & 0.045 & 0.51 & \\
\hline
\end{tabular}

Note. $\mathrm{GG}=$ Greenhouse-Geisser correction was applied, if Mauchly's sphericity test indicated a violation of the sphericity assumption. '***' $p<0.001$; ‘**' $p<0.01$; '*’ $p<0.05$; ' '’ $p<0.1$. 
II | Approaching Consciousness with Masked Priming 


\section{Appendix III}

Table C1.1

ANOVA on Errors of Experiments $4.1 \mathrm{~A}$ and $4.1 \mathrm{~B}$ with the relevant congruency as a factor

\begin{tabular}{|c|c|c|c|c|c|}
\hline Effect & $F$ & $d f$ & $M S_{e}$ & \multicolumn{2}{|c|}{$\boldsymbol{p}$ (p.r.n. GG) } \\
\hline (Intercept) & 97.25 & 1,22 & 0.819 & $<.0001$ & $* * *$ \\
\hline Congruency & 10.54 & 1,22 & 0.025 & $<.005$ & $* *$ \\
\hline Masking strength & 0.08 & 1,22 & 0.034 & 0.78 & \\
\hline Prime-mask SOA & 1.56 & 1,22 & 0.028 & 0.22 & \\
\hline Mask-target SOA & 0.77 & 3,66 & 0.037 & 0.51 & \\
\hline Congruency $\times$ Masking strength & 0.002 & 1,22 & 0.028 & 0.97 & \\
\hline Congruency $\times$ Prime-mask SOA & 0.30 & 1,22 & 0.018 & 0.59 & \\
\hline Masking strength $\times$ Prime-mask SOA & 1.56 & 1,22 & 0.015 & 0.23 & \\
\hline Congruency $\times$ Mask-target SOA & 6.62 & 3,66 & 0.024 & $<.001$ & $* * *$ \\
\hline Masking strength $\times$ Mask-target SOA & 1.85 & 3,66 & 0.018 & 0.15 & \\
\hline Prime-mask SOA $\times$ Mask-target SOA & 0.81 & 3,66 & 0.019 & 0.50 & \\
\hline Congruency $\times$ Masking strength $\times$ Prime-mask SOA & 0.15 & 1,22 & 0.017 & 0.70 & \\
\hline Congruency $\times$ Masking strength $\times$ Mask-target SOA & 2.08 & 3,66 & 0.024 & 0.11 & \\
\hline Congruency $\times$ Prime-mask SOA $\times$ Mask-target SOA & 1.23 & 3,66 & 0.020 & 0.31 & \\
\hline Masking strength $\times$ Prime-mask SOA $\times$ Mask-target SOA & 1.48 & 3,66 & 0.021 & 0.23 & \\
\hline $\begin{array}{l}\text { Congruency } \times \text { Masking strength } \times \text { Prime-mask SOA } \times \\
\text { Mask-target SOA }\end{array}$ & 0.62 & 3,66 & 0.020 & 0.60 & \\
\hline Task level & 0.74 & 1,22 & 0.819 & 0.40 & \\
\hline Task level $\times$ Congruency & 0.0003 & 1,22 & 0.025 & 0.99 & \\
\hline Task level $\times$ Masking strength & 0.05 & 1,22 & 0.034 & 0.83 & \\
\hline Task level $\times$ Prime-mask SOA & 0.31 & 1,22 & 0.028 & 0.59 & \\
\hline Task level $\times$ Mask-target SOA & 1.84 & 3,66 & 0.037 & 0.15 & \\
\hline Task level $\times$ Congruency $\times$ Masking strength & 2.53 & 1,22 & 0.028 & 0.13 & \\
\hline Task level $\times$ Congruency $\times$ Prime-mask SOA & 0.17 & 1,22 & 0.018 & 0.68 & \\
\hline Task level $\times$ Masking strength $\times$ Prime-mask SOA & 0.74 & 1,22 & 0.015 & 0.40 & \\
\hline Task level $\times$ Congruency $\times$ Mask-target SOA & 3.21 & 3,66 & 0.024 & $<.05$ & $*$ \\
\hline Task level $\times$ Masking strength $\times$ Mask-target SOA & 1.82 & 3,66 & 0.018 & 0.15 & \\
\hline Task level $\times$ Prime-mask SOA $\times$ Mask-target SOA & 0.70 & 3,66 & 0.019 & 0.56 & \\
\hline $\begin{array}{l}\text { Task level } \times \text { Congruency } \times \text { Masking strength } \\
\times \text { Prime-mask SOA }\end{array}$ & 0.83 & 1,22 & 0.017 & 0.37 & \\
\hline $\begin{array}{l}\text { Task level } \times \text { Congruency } \times \text { Masking strength } \\
\times \text { Mask-target SOA }\end{array}$ & 1.70 & 3,66 & 0.024 & 0.18 & \\
\hline $\begin{array}{l}\text { Task level } \times \text { Congruency } \times \text { Prime-mask SOA } \\
\times \text { Mask-target SOA }\end{array}$ & 0.44 & 3,66 & 0.020 & 0.72 & \\
\hline $\begin{array}{l}\text { Task level } \times \text { Masking strength } \times \text { Prime-mask SOA } \\
\times \text { Mask-target SOA }\end{array}$ & 0.25 & 3,66 & 0.021 & 0.86 & \\
\hline $\begin{array}{l}\text { Task level } \times \text { Congruency } \times \text { Masking strength } \\
\times \text { Prime-mask SOA } \times \text { Mask-target SOA }\end{array}$ & 1.02 & 3,66 & 0.020 & 0.39 & \\
\hline
\end{tabular}

Note. GG $=$ Greenhouse-Geisser correction was applied, if Mauchly's sphericity test indicated a violation of the sphericity assumption. '***' $p<0.001$; '**' $p<0.01$; '*’ $p<0.05$; '’ $p<0.1$. 
Table C1.2

ANOVA on RTs of Experiments $4.1 \mathrm{~A}$ and $4.1 \mathrm{~B}$ with the relevant congruency as a factor

\begin{tabular}{|c|c|c|c|c|c|}
\hline Effect & $F$ & $d f$ & $\overline{M S_{e}}$ & \multicolumn{2}{|c|}{$p$ (p.r.n. GG) } \\
\hline (Intercept) & 1029.45 & 1,22 & 144896 & $<.0001$ & $* * *$ \\
\hline Congruency & 34.42 & 1,22 & 428 & $<.0001$ & $* * *$ \\
\hline Masking strength & 7.47 & 1,22 & 186 & $<.05$ & $*$ \\
\hline Prime-mask SOA & 11.72 & 1,22 & 220 & $<.005$ & $* *$ \\
\hline Mask-target SOA & 116.57 & 3,66 & 595 & $<.0001$ & $* * *$ \\
\hline Congruency $\times$ Masking strength & 0.77 & 1,22 & 282 & 0.39 & \\
\hline Congruency $\times$ Prime-mask SOA & 7.00 & 1,22 & 268 & $<.05$ & $*$ \\
\hline Masking strength $\times$ Prime-mask SOA & 6.92 & 1,22 & 142 & $<.05$ & $*$ \\
\hline Congruency $\times$ Mask-target SOA & 14.24 & 3,66 & 259 & $<.0001$ & $* * *$ \\
\hline Masking strength $\times$ Mask-target SOA & 4.58 & 3,66 & 128 & $<.01$ & $* *$ \\
\hline Prime-mask SOA $\times$ Mask-target SOA & 0.36 & 3,66 & 152 & 0.78 & \\
\hline Congruency $\times$ Masking strength $\times$ Prime-mask SOA & 0.01 & 1,22 & 151 & 0.92 & \\
\hline Congruency $\times$ Masking strength $\times$ Mask-target SOA & 1.87 & 3,66 & 163 & 0.17 & \\
\hline Congruency $\times$ Prime-mask SOA $\times$ Mask-target SOA & 0.25 & 3,66 & 187 & 0.86 & \\
\hline Masking strength $\times$ Prime-mask SOA $\times$ Mask-target SOA & 0.54 & 3,66 & 132 & 0.65 & \\
\hline $\begin{array}{l}\text { Congruency } \times \text { Masking strength } \times \text { Prime-mask SOA } \times \\
\text { Mask-target SOA }\end{array}$ & 1.80 & 3,66 & 107 & 0.15 & \\
\hline Task level & 1.47 & 1,22 & 144896 & 0.24 & \\
\hline Task level $\times$ Congruency & 0.17 & 1,22 & 428 & 0.68 & \\
\hline Task level $\times$ Masking strength & 1.32 & 1,22 & 186 & 0.26 & \\
\hline Task level $\times$ Prime-mask SOA & 0.16 & 1,22 & 220 & 0.69 & \\
\hline Task level $\times$ Mask-target SOA & 1.09 & 3,66 & 595 & 0.34 & \\
\hline Task level $\times$ Congruency $\times$ Masking strength & 4.13 & 1,22 & 282 & 0.05 & \\
\hline Task level $\times$ Congruency $\times$ Prime-mask SOA & 2.47 & 1,22 & 268 & 0.13 & \\
\hline Task level $\times$ Masking strength $\times$ Prime-mask SOA & 0.47 & 1,22 & 142 & 0.50 & \\
\hline Task level $\times$ Congruency $\times$ Mask-target SOA & 9.15 & 3,66 & 259 & $<.0001$ & $* * *$ \\
\hline Task level $\times$ Masking strength $\times$ Mask-target SOA & 3.13 & 3,66 & 128 & $<.05$ & $*$ \\
\hline Task level $\times$ Prime-mask SOA $\times$ Mask-target SOA & 0.90 & 3,66 & 152 & 0.45 & \\
\hline $\begin{array}{l}\text { Task level } \times \text { Congruency } \times \text { Masking strength } \\
\times \text { Prime-mask SOA }\end{array}$ & 0.67 & 1,22 & 151 & 0.42 & \\
\hline $\begin{array}{l}\text { Task level } \times \text { Congruency } \times \text { Masking strength } \\
\times \text { Mask-target SOA }\end{array}$ & 1.38 & 3,66 & 163 & 0.26 & \\
\hline $\begin{array}{l}\text { Task level } \times \text { Congruency } \times \text { Prime-mask SOA } \\
\times \text { Mask-target SOA }\end{array}$ & 0.33 & 3,66 & 187 & 0.80 & \\
\hline $\begin{array}{l}\text { Task level } \times \text { Masking strength } \times \text { Prime-mask SOA } \\
\times \text { Mask-target SOA }\end{array}$ & 0.37 & 3,66 & 132 & 0.78 & \\
\hline $\begin{array}{l}\text { Task level } \times \text { Congruency } \times \text { Masking strength } \\
\times \text { Prime-mask SOA } \times \text { Mask-target SOA }\end{array}$ & 1.10 & 3,66 & 107 & 0.36 & \\
\hline
\end{tabular}

Note. GG $=$ Greenhouse-Geisser correction was applied, if Mauchly's sphericity test indicated a violation of the sphericity assumption. '***' $p<0.001$; ‘**’ $p<0.01$; '*’ $p<0.05$; '’ $p<0.1$. 
Table C1.3

ANOVA on objective prime visibility of Experiments $4.1 \mathrm{~A}$ and $4.1 \mathrm{~B}$

\begin{tabular}{lccccc}
\hline Effect & $\boldsymbol{F}$ & $\boldsymbol{d f}$ & $\boldsymbol{M} \boldsymbol{S}_{\boldsymbol{e}}$ & $\boldsymbol{p}$ (p.r.n. GG) \\
\hline Intercept) & 459.19 & 1,22 & 6.526 & $<.0001$ & $* * *$ \\
Masking strength & 14.95 & 1,22 & 0.832 & $<.001$ & $* * *$ \\
Prime-mask SOA & 105.38 & 1,22 & 0.339 & $<.0001$ & $* * *$ \\
Mask-target SOA & 18.11 & 3,66 & 0.214 & $<.0001$ & $* * *$ \\
Masking strength $\times$ Prime-mask SOA & 21.56 & 1,22 & 0.196 & $<.0005$ & $* * *$ \\
Masking strength $\times$ Mask-target SOA & 1.21 & 3,66 & 0.205 & 0.31 & $* *$ \\
Prime-mask SOA $\times$ Mask-target SOA & 8.31 & 3,66 & 0.240 & $<.005$ & $* *$ \\
Masking strength $\times$ Prime-mask SOA $\times$ Mask-target SOA & 1.12 & 3,66 & 0.193 & 0.35 & \\
& & & & & 0.64 \\
Task level & 0.22 & 1,22 & 6.526 & 0.46 \\
Task level $\times$ Masking strength & 0.57 & 1,22 & 0.832 & 0.13 \\
Task level $\times$ Prime-mask SOA & 2.49 & 1,22 & 0.339 & 0.214 & 0.64 \\
Task level $\times$ Mask-target SOA & 0.57 & 3,66 & 0.214 \\
Task level $\times$ Masking strength $\times$ Prime-mask SOA & 0.69 & 1,22 & 0.196 & 0.41 \\
Task level $\times$ Masking strength $\times$ Mask-target SOA & 1.37 & 3,66 & 0.205 & 0.26 \\
Task level $\times$ Prime-mask SOA $\times$ Mask-target SOA & 1.88 & 3,66 & 0.240 & 0.17 \\
Task level $\times$ Masking strength $\times$ Prime-mask SOA & 0.15 & 3,66 & 0.193 & 0.93 \\
$\quad \times$ Mask-target SOA & & & &
\end{tabular}

Note. GG = Greenhouse-Geisser correction was applied, if Mauchly's sphericity test indicated a violation of the sphericity assumption. '***' $p<0.001$; ‘**' $p<0.01$; '*’ $p<0.05$; ' ' $p<0.1$.

Table C1.4

ANOVA on subjective prime visibility of Experiments $4.1 \mathrm{~A}$ and $4.1 \mathrm{~B}$

\begin{tabular}{|c|c|c|c|c|c|}
\hline Effect & $F$ & $d f$ & $M S_{e}$ & $\boldsymbol{p}$ (p.r.n & GG) \\
\hline (Intercept) & 537.57 & 1,22 & 7.851 & $<.0001$ & $* * *$ \\
\hline Masking strength & 17.54 & 1,22 & 0.091 & $<.0005$ & $* * *$ \\
\hline Prime-mask SOA & 35.14 & 1,22 & 0.139 & $<.0001$ & $* * *$ \\
\hline Mask-target SOA & 22.90 & 3,66 & 0.031 & $<.0001$ & $* * *$ \\
\hline Masking strength $\times$ Prime-mask SOA & 22.41 & 1,22 & 0.018 & $<.0005$ & $* * *$ \\
\hline Masking strength $\times$ Mask-target SOA & 1.13 & 3,66 & 0.015 & 0.34 & \\
\hline Prime-mask SOA $\times$ Mask-target SOA & 4.86 & 3,66 & 0.019 & 0.01 & \\
\hline Masking strength $\times$ Prime-mask SOA $\times$ Mask-target SOA & 1.98 & 3,66 & 0.012 & 0.13 & \\
\hline Task level & 0.12 & 1,22 & 7.851 & 0.73 & \\
\hline Task level $\times$ Masking strength & 0.12 & 1,22 & 0.091 & 0.73 & \\
\hline Task level $\times$ Prime-mask SOA & 0.88 & 1,22 & 0.139 & 0.36 & \\
\hline Task level $\times$ Mask-target SOA & 3.32 & 3,66 & 0.031 & 0.06 & \\
\hline Task level $\times$ Masking strength $\times$ Prime-mask SOA & 6.27 & 1,22 & 0.018 & 0.02 & \\
\hline Task level $\times$ Masking strength $\times$ Mask-target SOA & 1.51 & 3,66 & 0.015 & 0.22 & \\
\hline Task level $\times$ Prime-mask SOA $\times$ Mask-target SOA & 0.70 & 3,66 & 0.019 & 0.49 & \\
\hline $\begin{array}{l}\text { Task level } \times \text { Masking strength } \times \text { Prime-mask SOA } \\
\times \text { Mask-target SOA }\end{array}$ & 0.02 & 3,66 & 0.012 & 0.99 & \\
\hline
\end{tabular}


Table C2.1

ANOVA on Errors of Experiments $4.2 A$ and $4.2 B$ with the relevant congruency as a factor

\begin{tabular}{|c|c|c|c|c|c|}
\hline Effect & $\boldsymbol{F}$ & $d f$ & $M \boldsymbol{S}_{e}$ & \multicolumn{2}{|c|}{$\boldsymbol{p}$ (p.r.n. GG) } \\
\hline (Intercept) & 163.45 & 1,22 & 0.345 & $<.0001$ & $* * *$ \\
\hline Congruency & 0.57 & 1,22 & 0.020 & 0.46 & $* *$ \\
\hline Masking strength & 1.10 & 1,22 & 0.027 & 0.31 & \\
\hline Prime-mask SOA & 0.00 & 1,22 & 0.027 & 0.99 & \\
\hline Mask-target SOA & 2.96 & 3,66 & 0.030 & $<.05$ & $*$ \\
\hline Congruency $\times$ Masking strength & 0.65 & 1,22 & 0.029 & 0.43 & \\
\hline Congruency $\times$ Prime-mask SOA & 0.11 & 1,22 & 0.026 & 0.74 & \\
\hline Masking strength $\times$ Prime-mask SOA & 9.08 & 1,22 & 0.013 & $<.01$ & $* *$ \\
\hline Congruency $\times$ Mask-target SOA & 1.20 & 3,66 & 0.019 & 0.32 & \\
\hline Masking strength $\times$ Mask-target SOA & 0.19 & 3,66 & 0.031 & 0.91 & \\
\hline Prime-mask SOA $\times$ Mask-target SOA & 1.22 & 3,66 & 0.023 & 0.31 & \\
\hline Congruency $\times$ Masking strength $\times$ Prime-mask SOA & 0.31 & 1,22 & 0.015 & 0.59 & \\
\hline Congruency $\times$ Masking strength $\times$ Mask-target SOA & 2.11 & 3,66 & 0.028 & 0.11 & \\
\hline Congruency $\times$ Prime-mask SOA $\times$ Mask-target SOA & 1.34 & 3,66 & 0.014 & 0.27 & \\
\hline Masking strength $\times$ Prime-mask SOA $\times$ Mask-target SOA & 0.70 & 3,66 & 0.023 & 0.56 & \\
\hline $\begin{array}{l}\text { Congruency } \times \text { Masking strength } \times \text { Prime-mask SOA } \times \\
\text { Mask-target SOA }\end{array}$ & 0.12 & 3,66 & 0.018 & 0.95 & \\
\hline Task level & 1.16 & 1,22 & 0.345 & 0.29 & \\
\hline Task level $\times$ Congruency & 1.34 & 1,22 & 0.020 & 0.26 & \\
\hline Task level $\times$ Masking strength & 0.11 & 1,22 & 0.027 & 0.75 & \\
\hline Task level $\times$ Prime-mask SOA & 5.80 & 1,22 & 0.027 & $<.05$ & $*$ \\
\hline Task level $\times$ Mask-target SOA & 3.91 & 3,66 & 0.030 & $<.05$ & $*$ \\
\hline Task level $\times$ Congruency $\times$ Masking strength & 0.56 & 1,22 & 0.029 & 0.46 & \\
\hline Task level $\times$ Congruency $\times$ Prime-mask SOA & 2.17 & 1,22 & 0.026 & 0.15 & \\
\hline Task level $\times$ Masking strength $\times$ Prime-mask SOA & 2.22 & 1,22 & 0.013 & 0.15 & \\
\hline Task level $\times$ Congruency $\times$ Mask-target SOA & 0.58 & 3,66 & 0.019 & 0.63 & \\
\hline Task level $\times$ Masking strength $\times$ Mask-target SOA & 1.85 & 3,66 & 0.031 & 0.15 & \\
\hline Task level $\times$ Prime-mask SOA $\times$ Mask-target SOA & 0.42 & 3,66 & 0.023 & 0.74 & \\
\hline $\begin{array}{l}\text { Task level } \times \text { Congruency } \times \text { Masking strength } \\
\times \text { Prime-mask SOA }\end{array}$ & 2.72 & 1,22 & 0.015 & 0.11 & \\
\hline $\begin{array}{l}\text { Task level } \times \text { Congruency } \times \text { Masking strength } \\
\times \text { Mask-target SOA }\end{array}$ & 0.44 & 3,66 & 0.028 & 0.73 & \\
\hline $\begin{array}{l}\text { Task level } \times \text { Congruency } \times \text { Prime-mask SOA } \\
\times \text { Mask-target SOA }\end{array}$ & 1.49 & 3,66 & 0.014 & 0.23 & \\
\hline $\begin{array}{l}\text { Task level } \times \text { Masking strength } \times \text { Prime-mask SOA } \\
\times \text { Mask-target SOA }\end{array}$ & 1.41 & 3,66 & 0.023 & 0.25 & \\
\hline $\begin{array}{l}\text { Task level } \times \text { Congruency } \times \text { Masking strength } \\
\times \text { Prime-mask SOA } \times \text { Mask-target SOA }\end{array}$ & 4.16 & 3,66 & 0.018 & $<.01$ & $* *$ \\
\hline
\end{tabular}

Note. GG $=$ Greenhouse-Geisser correction was applied, if Mauchly's sphericity test indicated a violation of the sphericity assumption. '***' $p<0.001$; ‘**’ $p<0.01$; '*’ $p<0.05$; '’ $p<0.1$. 
Table C2.2

ANOVA on RTs of Experiments $4.2 A$ and $4.2 B$ with the relevant congruency as a factor

\begin{tabular}{|c|c|c|c|c|c|}
\hline Effect & $F$ & $d f$ & $M S_{e}$ & \multicolumn{2}{|c|}{$\boldsymbol{p}$ (p.r.n. GG) } \\
\hline (Intercept) & 1501.89 & 1,22 & 94048 & $<.0001$ & $* * *$ \\
\hline Congruency & 15.49 & 1,22 & 57 & $<.001$ & $* * *$ \\
\hline Masking strength & 10.91 & 1,22 & 196 & $<.005$ & $* *$ \\
\hline Prime-mask SOA & 7.27 & 1,22 & 99 & $<.05$ & $*$ \\
\hline Mask-target SOA & 134.59 & 3,66 & 283 & $<.0001$ & $* * *$ \\
\hline Congruency $\times$ Masking strength & 2.84 & 1,22 & 76 & 0.11 & \\
\hline Congruency $\times$ Prime-mask SOA & 4.16 & 1,22 & 59 & 0.05 & \\
\hline Masking strength $\times$ Prime-mask SOA & 5.83 & 1,22 & 56 & $<.05$ & $*$ \\
\hline Congruency $\times$ Mask-target SOA & 8.74 & 3,66 & 96 & $<.0001$ & $* * *$ \\
\hline Masking strength $\times$ Mask-target SOA & 0.27 & 3,66 & 87 & 0.85 & \\
\hline Prime-mask SOA $\times$ Mask-target SOA & 2.18 & 3,66 & 112 & 0.10 & \\
\hline Congruency $\times$ Masking strength $\times$ Prime-mask SOA & 0.29 & 1,22 & 94 & 0.60 & \\
\hline Congruency $\times$ Masking strength $\times$ Mask-target SOA & 2.19 & 3,66 & 101 & 0.10 & \\
\hline Congruency $\times$ Prime-mask SOA $\times$ Mask-target SOA & 1.74 & 3,66 & 89 & 0.17 & \\
\hline Masking strength $\times$ Prime-mask SOA $\times$ Mask-target SOA & 0.63 & 3,66 & 56 & 0.60 & \\
\hline $\begin{array}{l}\text { Congruency } \times \text { Masking strength } \times \text { Prime-mask SOA } \times \\
\text { Mask-target SOA }\end{array}$ & 0.14 & 3,66 & 85 & 0.93 & \\
\hline Task level & 1.27 & 1,22 & 94048 & 0.27 & \\
\hline Task level $\times$ Congruency & 3.45 & 1,22 & 57 & 0.08 & \\
\hline Task level $\times$ Masking strength & 0.36 & 1,22 & 196 & 0.55 & \\
\hline Task level $\times$ Prime-mask SOA & 1.33 & 1,22 & 99 & 0.26 & \\
\hline Task level $\times$ Mask-target SOA & 1.71 & 3,66 & 283 & 0.17 & \\
\hline Task level $\times$ Congruency $\times$ Masking strength & 0.40 & 1,22 & 76 & 0.54 & \\
\hline Task level $\times$ Congruency $\times$ Prime-mask SOA & 0.54 & 1,22 & 59 & 0.47 & \\
\hline Task level $\times$ Masking strength $\times$ Prime-mask SOA & 0.49 & 1,22 & 56 & 0.49 & \\
\hline Task level $\times$ Congruency $\times$ Mask-target SOA & 0.77 & 3,66 & 96 & 0.51 & \\
\hline Task level $\times$ Masking strength $\times$ Mask-target SOA & 0.55 & 3,66 & 87 & 0.65 & \\
\hline Task level $\times$ Prime-mask SOA $\times$ Mask-target SOA & 0.06 & 3,66 & 112 & 0.98 & \\
\hline $\begin{array}{l}\text { Task level } \times \text { Congruency } \times \text { Masking strength } \\
\times \text { Prime-mask SOA }\end{array}$ & 0.56 & 1,22 & 94 & 0.46 & \\
\hline $\begin{array}{l}\text { Task level } \times \text { Congruency } \times \text { Masking strength } \\
\times \text { Mask-target SOA }\end{array}$ & 1.40 & 3,66 & 101 & 0.25 & \\
\hline $\begin{array}{l}\text { Task level } \times \text { Congruency } \times \text { Prime-mask SOA } \\
\times \text { Mask-target SOA }\end{array}$ & 1.28 & 3,66 & 89 & 0.29 & \\
\hline $\begin{array}{l}\text { Task level } \times \text { Masking strength } \times \text { Prime-mask SOA } \\
\times \text { Mask-target SOA }\end{array}$ & 3.81 & 3,66 & 56 & $<.05$ & $*$ \\
\hline $\begin{array}{l}\text { Task level } \times \text { Congruency } \times \text { Masking strength } \\
\times \text { Prime-mask SOA } \times \text { Mask-target SOA }\end{array}$ & 1.06 & 3,66 & 85 & 0.37 & \\
\hline
\end{tabular}

Note. GG = Greenhouse-Geisser correction was applied, if Mauchly's sphericity test indicated a violation of the sphericity assumption. '***' $p<0.001$; ‘**’ $p<0.01$; '*’ $p<0.05$; '.' $p<0.1$. 
Table C2.3

ANOVA on objective prime visibility of Experiments $4.2 \mathrm{~A}$ and $4.2 \mathrm{~B}$

\begin{tabular}{|c|c|c|c|c|c|}
\hline Effect & $F$ & $\overline{d f}$ & $M S_{e}$ & \multicolumn{2}{|c|}{$\boldsymbol{p}$ (p.r.n. GG) } \\
\hline (Intercept) & 97.39 & 1,22 & 12.568 & $<.0001$ & $* * *$ \\
\hline Masking strength & 68.82 & 1,22 & 3.224 & $<.0001$ & $* * *$ \\
\hline Prime-mask SOA & 22.62 & 1,22 & 0.452 & $<.0001$ & $* * *$ \\
\hline Mask-target SOA & 0.83 & 3,66 & 0.221 & 0.48 & \\
\hline Masking strength $\times$ Prime-mask SOA & 7.91 & 1,22 & 0.267 & $<.05$ & $*$ \\
\hline Masking strength $\times$ Mask-target SOA & 0.73 & 3,66 & 0.207 & 0.53 & \\
\hline Prime-mask SOA $\times$ Mask-target SOA & 0.68 & 3,66 & 0.213 & 0.57 & \\
\hline Masking strength $\times$ Prime-mask SOA $\times$ Mask-target SOA & 2.08 & 3,66 & 0.204 & 0.11 & \\
\hline Task level & 5.99 & 1,22 & 12.568 & $<.05$ & $*$ \\
\hline Task level $\times$ Masking strength & 3.16 & 1,22 & 3.224 & 0.09 & \\
\hline Task level $\times$ Prime-mask SOA & 3.91 & 1,22 & 0.452 & 0.06 & \\
\hline Task level $\times$ Mask-target SOA & 0.74 & 3,66 & 0.221 & 0.53 & \\
\hline Task level $\times$ Masking strength $\times$ Prime-mask SOA & 5.40 & 1,22 & 0.267 & $<.05$ & $*$ \\
\hline Task level $\times$ Masking strength $\times$ Mask-target SOA & 0.94 & 3,66 & 0.207 & 0.43 & \\
\hline Task level $\times$ Prime-mask SOA $\times$ Mask-target SOA & 0.43 & 3,66 & 0.213 & 0.73 & \\
\hline $\begin{array}{l}\text { Task level } \times \text { Masking strength } \times \text { Prime-mask SOA } \\
\times \text { Mask-target SOA }\end{array}$ & 1.29 & 3,66 & 0.204 & 0.28 & \\
\hline
\end{tabular}

Note. GG $=$ Greenhouse-Geisser correction was applied, if Mauchly's sphericity test indicated a violation of the sphericity assumption. '***' $p<0.001$; '**' $p<0.01$; '*’ $p<0.05$; '’ $p<0.1$.

Table C2.4

ANOVA on subjective prime visibility of Experiments $4.2 A$ and $4.2 B$

\begin{tabular}{|c|c|c|c|c|c|}
\hline Effect & $F$ & $d f$ & $M S_{e}$ & $\boldsymbol{p}$ (p.r.n & GG) \\
\hline (Intercept) & 465.41 & 1,22 & 5.981 & $<.0001$ & $* * *$ \\
\hline Masking strength & 53.67 & 1,22 & 1.190 & $<.0001$ & $* * *$ \\
\hline Prime-mask SOA & 38.76 & 1,22 & 0.055 & $<.0001$ & $* * *$ \\
\hline Mask-target SOA & 1.70 & 3,66 & 0.011 & 0.18 & \\
\hline Masking strength $\times$ Prime-mask SOA & 10.80 & 1,22 & 0.042 & $<.005$ & $* *$ \\
\hline Masking strength $\times$ Mask-target SOA & 3.73 & 3,66 & 0.013 & $<.05$ & $*$ \\
\hline Prime-mask SOA $\times$ Mask-target SOA & 0.06 & 3,66 & 0.009 & 0.98 & \\
\hline Masking strength $\times$ Prime-mask SOA $\times$ Mask-target SOA & 0.67 & 3,66 & 0.010 & 0.58 & \\
\hline Task level & 1.08 & 1,22 & 5.981 & 0.31 & \\
\hline Task level $\times$ Masking strength & 5.86 & 1,22 & 1.190 & $<.05$ & $*$ \\
\hline Task level $\times$ Prime-mask SOA & 5.55 & 1,22 & 0.055 & $<.05$ & $*$ \\
\hline Task level $\times$ Mask-target SOA & 1.17 & 3,66 & 0.011 & 0.33 & \\
\hline Task level $\times$ Masking strength $\times$ Prime-mask SOA & 1.89 & 1,22 & 0.042 & 0.18 & \\
\hline Task level $\times$ Masking strength $\times$ Mask-target SOA & 2.09 & 3,66 & 0.013 & 0.11 & \\
\hline Task level $\times$ Prime-mask SOA $\times$ Mask-target SOA & 0.31 & 3,66 & 0.009 & 0.82 & \\
\hline $\begin{array}{l}\text { Task level } \times \text { Masking strength } \times \text { Prime-mask SOA } \\
\times \text { Mask-target SOA }\end{array}$ & 1.38 & 3,66 & 0.010 & 0.26 & \\
\hline
\end{tabular}


Table C3.1

ANOVA on Errors of Experiments $4.3 \mathrm{~A}$ and $4.3 \mathrm{~B}$ with the relevant congruency as a factor

\begin{tabular}{|c|c|c|c|c|c|}
\hline Effect & $F$ & $d f$ & $M S_{e}$ & $p$ (p.r.n & $\mathrm{GG})$ \\
\hline (Intercept) & 92.04 & 1,22 & 1.049 & $<.0001$ & $* * *$ \\
\hline Congruency & 4.77 & 1,22 & 0.030 & $<.05$ & $*$ \\
\hline Masking strength & 4.72 & 1,22 & 0.032 & $<.05$ & $*$ \\
\hline Prime-mask SOA & 3.22 & 1,22 & 0.019 & 0.09 & \\
\hline Mask-target SOA & 0.38 & 3,66 & 0.029 & 0.77 & \\
\hline Congruency $\times$ Masking strength & 2.96 & 1,22 & 0.021 & 0.10 & \\
\hline Congruency $\times$ Prime-mask SOA & 0.07 & 1,22 & 0.015 & 0.80 & \\
\hline Masking strength $\times$ Prime-mask SOA & 1.29 & 1,22 & 0.022 & 0.27 & \\
\hline Congruency $\times$ Mask-target SOA & 2.77 & 3,66 & 0.031 & $<.05$ & $*$ \\
\hline Masking strength $\times$ Mask-target SOA & 0.16 & 3,66 & 0.020 & 0.92 & \\
\hline Prime-mask SOA $\times$ Mask-target SOA & 1.03 & 3,66 & 0.023 & 0.38 & \\
\hline Congruency $\times$ Masking strength $\times$ Prime-mask SOA & 0.06 & 1,22 & 0.032 & 0.82 & \\
\hline Congruency $\times$ Masking strength $\times$ Mask-target SOA & 0.03 & 3,66 & 0.024 & 0.99 & \\
\hline Congruency $\times$ Prime-mask SOA $\times$ Mask-target SOA & 1.09 & 3,66 & 0.021 & 0.36 & \\
\hline Masking strength $\times$ Prime-mask SOA $\times$ Mask-target SOA & 0.47 & 3,66 & 0.020 & 0.71 & \\
\hline $\begin{array}{l}\text { Congruency } \times \text { Masking strength } \times \text { Prime-mask SOA } \times \\
\text { Mask-target SOA }\end{array}$ & 1.48 & 3,66 & 0.020 & 0.24 & \\
\hline Task level & 0.01 & 1,22 & 1.049 & 0.92 & \\
\hline Task level $\times$ Congruency & 4.57 & 1,22 & 0.030 & $<.05$ & $*$ \\
\hline Task level $\times$ Masking strength & 5.33 & 1,22 & 0.032 & $<.05$ & $*$ \\
\hline Task level $\times$ Prime-mask SOA & 0.00 & 1,22 & 0.019 & 0.96 & \\
\hline Task level $\times$ Mask-target SOA & 2.09 & 3,66 & 0.029 & 0.11 & \\
\hline Task level $\times$ Congruency $\times$ Masking strength & 1.00 & 1,22 & 0.021 & 0.33 & \\
\hline Task level $\times$ Congruency $\times$ Prime-mask SOA & 0.18 & 1,22 & 0.015 & 0.67 & \\
\hline Task level $\times$ Masking strength $\times$ Prime-mask SOA & 1.76 & 1,22 & 0.022 & 0.20 & \\
\hline Task level $\times$ Congruency $\times$ Mask-target SOA & 0.38 & 3,66 & 0.031 & 0.77 & \\
\hline Task level $\times$ Masking strength $\times$ Mask-target SOA & 1.66 & 3,66 & 0.020 & 0.18 & \\
\hline Task level $\times$ Prime-mask SOA $\times$ Mask-target SOA & 0.89 & 3,66 & 0.023 & 0.45 & \\
\hline $\begin{array}{l}\text { Task level } \times \text { Congruency } \times \text { Masking strength } \\
\times \text { Prime-mask SOA }\end{array}$ & 0.41 & 1,22 & 0.032 & 0.53 & \\
\hline $\begin{array}{l}\text { Task level } \times \text { Congruency } \times \text { Masking strength } \\
\times \text { Mask-target SOA }\end{array}$ & 0.46 & 3,66 & 0.024 & 0.71 & \\
\hline $\begin{array}{l}\text { Task level } \times \text { Congruency } \times \text { Prime-mask SOA } \\
\times \text { Mask-target SOA }\end{array}$ & 2.90 & 3,66 & 0.021 & $<.05$ & $*$ \\
\hline $\begin{array}{l}\text { Task level } \times \text { Masking strength } \times \text { Prime-mask SOA } \\
\times \text { Mask-target SOA }\end{array}$ & 1.90 & 3,66 & 0.020 & 0.14 & \\
\hline $\begin{array}{l}\text { Task level } \times \text { Congruency } \times \text { Masking strength } \\
\times \text { Prime-mask SOA } \times \text { Mask-target SOA }\end{array}$ & 0.54 & 3,66 & 0.020 & 0.61 & \\
\hline
\end{tabular}

Note. GG = Greenhouse-Geisser correction was applied, if Mauchly's sphericity test indicated a violation of the sphericity assumption. '***' $p<0.001$; ‘**’ $p<0.01$; '*’ $p<0.05$; '.’ $p<0.1$. 
Table C3.2

ANOVA on RTs of Experiments $4.3 A$ and $4.3 B$ with the relevant congruency as a factor

\begin{tabular}{|c|c|c|c|c|c|}
\hline Effect & $\boldsymbol{F}$ & $d f$ & $M S_{e}$ & \multicolumn{2}{|c|}{$p$ (p.r.n. GG) } \\
\hline (Intercept) & 2852.74 & 1,22 & 53894 & $<.0001$ & $* * *$ \\
\hline Congruency & 25.97 & 1,22 & 767 & $<.0001$ & $* * *$ \\
\hline Masking strength & 76.28 & 1,22 & 235 & $<.0001$ & $* * *$ \\
\hline Prime-mask SOA & 9.96 & 1,22 & 121 & $<.005$ & $* *$ \\
\hline Mask-target SOA & 132.77 & 3,66 & 498 & $<.0001$ & $* * *$ \\
\hline Congruency $\times$ Masking strength & 19.47 & 1,22 & 177 & $<.0005$ & $* * *$ \\
\hline Congruency $\times$ Prime-mask SOA & 0.49 & 1,22 & 126 & 0.49 & \\
\hline Masking strength $\times$ Prime-mask SOA & 1.57 & 1,22 & 72 & 0.22 & \\
\hline Congruency $\times$ Mask-target SOA & 8.31 & 3,66 & 316 & $<.001$ & $* * *$ \\
\hline Masking strength $\times$ Mask-target SOA & 3.25 & 3,66 & 181 & $<.05$ & $*$ \\
\hline Prime-mask SOA $\times$ Mask-target SOA & 2.60 & 3,66 & 165 & 0.06 & \\
\hline Congruency $\times$ Masking strength $\times$ Prime-mask SOA & 0.17 & 1,22 & 191 & 0.69 & \\
\hline Congruency $\times$ Masking strength $\times$ Mask-target SOA & 1.21 & 3,66 & 160 & 0.31 & \\
\hline Congruency $\times$ Prime-mask SOA $\times$ Mask-target SOA & 0.45 & 3,66 & 183 & 0.72 & \\
\hline Masking strength $\times$ Prime-mask SOA $\times$ Mask-target SOA & 0.04 & 3,66 & 175 & 0.99 & \\
\hline $\begin{array}{l}\text { Congruency } \times \text { Masking strength } \times \text { Prime-mask SOA } \times \\
\text { Mask-target SOA }\end{array}$ & 1.11 & 3,66 & 161 & 0.35 & \\
\hline Task level & 2.85 & 1,22 & 53894 & 0.11 & \\
\hline Task level $\times$ Congruency & 2.75 & 1,22 & 767 & 0.11 & \\
\hline Task level $\times$ Masking strength & 4.82 & 1,22 & 235 & $<.05$ & $*$ \\
\hline Task level $\times$ Prime-mask SOA & 2.60 & 1,22 & 121 & 0.12 & \\
\hline Task level $\times$ Mask-target SOA & 0.72 & 3,66 & 498 & 0.48 & \\
\hline Task level $\times$ Congruency $\times$ Masking strength & 3.69 & 1,22 & 177 & 0.07 & \\
\hline Task level $\times$ Congruency $\times$ Prime-mask SOA & 1.42 & 1,22 & 126 & 0.25 & \\
\hline Task level $\times$ Masking strength $\times$ Prime-mask SOA & 4.33 & 1,22 & 72 & $<.05$ & $*$ \\
\hline Task level $\times$ Congruency $\times$ Mask-target SOA & 3.76 & 3,66 & 316 & $<.05$ & $*$ \\
\hline Task level $\times$ Masking strength $\times$ Mask-target SOA & 2.20 & 3,66 & 181 & 0.10 & \\
\hline Task level $\times$ Prime-mask SOA $\times$ Mask-target SOA & 0.34 & 3,66 & 165 & 0.80 & \\
\hline $\begin{array}{l}\text { Task level } \times \text { Congruency } \times \text { Masking strength } \\
\times \text { Prime-mask SOA }\end{array}$ & 0.03 & 1,22 & 191 & 0.86 & \\
\hline $\begin{array}{l}\text { Task level } \times \text { Congruency } \times \text { Masking strength } \\
\times \text { Mask-target SOA }\end{array}$ & 2.87 & 3,66 & 160 & $<.05$ & $*$ \\
\hline $\begin{array}{l}\text { Task level } \times \text { Congruency } \times \text { Prime-mask SOA } \\
\times \text { Mask-target SOA }\end{array}$ & 0.80 & 3,66 & 183 & 0.50 & \\
\hline $\begin{array}{l}\text { Task level } \times \text { Masking strength } \times \text { Prime-mask SOA } \\
\times \text { Mask-target SOA }\end{array}$ & 0.56 & 3,66 & 175 & 0.64 & \\
\hline $\begin{array}{l}\text { Task level } \times \text { Congruency } \times \text { Masking strength } \\
\times \text { Prime-mask SOA } \times \text { Mask-target SOA }\end{array}$ & 0.66 & 3,66 & 161 & 0.58 & \\
\hline
\end{tabular}

Note. GG $=$ Greenhouse-Geisser correction was applied, if Mauchly's sphericity test indicated a violation of the sphericity assumption. '***' $p<0.001$; ‘**’ $p<0.01$; '*’ $p<0.05$; '’ $p<0.1$. 
Table C3.3

ANOVA on objective prime visibility of Experiments $4.3 A$ and $4.3 B$

\begin{tabular}{lccccc}
\hline Effect & $\boldsymbol{F}$ & $\boldsymbol{d f}$ & $\boldsymbol{M S}_{\boldsymbol{e}}$ & $\boldsymbol{p}$ (p.r.n. GG) \\
\hline (Intercept) & 1187.74 & 1,22 & 3.656 & $<.0001$ & $* * *$ \\
Masking strength & 9.14 & 1,22 & 0.084 & $<.01$ & $* *$ \\
Prime-mask SOA & 12.68 & 1,22 & 0.094 & $<.005$ & $* *$ \\
Mask-target SOA & 2.39 & 3,66 & 0.147 & 0.08 \\
Masking strength $\times$ Prime-mask SOA & 2.84 & 1,22 & 0.127 & 0.11 \\
Masking strength $\times$ Mask-target SOA & 1.16 & 3,66 & 0.107 & 0.33 \\
Prime-mask SOA $\times$ Mask-target SOA & 2.43 & 3,66 & 0.068 & 0.07 \\
Masking strength $\times$ Prime-mask SOA $\times$ Mask-target SOA & 0.91 & 3,66 & 0.118 & 0.44 \\
& & & & \\
Task level & 3.18 & 1,22 & 3.656 & 0.09 \\
Task level $\times$ Masking strength & 0.10 & 1,22 & 0.084 & 0.75 \\
Task level $\times$ Prime-mask SOA & 0.41 & 1,22 & 0.094 & 0.53 \\
Task level $\times$ Mask-target SOA & 0.62 & 3,66 & 0.147 & 0.60 \\
Task level $\times$ Masking strength $\times$ Prime-mask SOA & 0.55 & 1,22 & 0.127 & 0.46 \\
Task level $\times$ Masking strength $\times$ Mask-target SOA & 1.20 & 3,66 & 0.107 & 0.32 \\
Task level $\times$ Prime-mask SOA $\times$ Mask-target SOA & 1.10 & 3,66 & 0.068 & 0.35 \\
Task level $\times$ Masking strength $\times$ Prime-mask SOA & 0.34 & 3,66 & 0.118 & 0.80 \\
$\quad \times$ Mask-target SOA & & & &
\end{tabular}

Note. GG = Greenhouse-Geisser correction was applied, if Mauchly's sphericity test indicated a violation of the sphericity assumption. '***' $p<0.001$; '**' $p<0.01$; '*’ $p<0.05$; '.' $p<0.1$.

Table C3.4

ANOVA on subjective prime visibility of Experiments $4.3 A$ and $4.3 B$

\begin{tabular}{|c|c|c|c|c|c|}
\hline Effect & $F$ & $d f$ & $M S_{e}$ & $\boldsymbol{p}$ (p.r.n & GG) \\
\hline (Intercept) & 1681.70 & 1,22 & 3.187 & $<.0001$ & $* * *$ \\
\hline Masking strength & 17.11 & 1,22 & 0.017 & $<.0005$ & $* * *$ \\
\hline Prime-mask SOA & 14.68 & 1,22 & 0.012 & $<.001$ & $* * *$ \\
\hline Mask-target SOA & 2.97 & 3,66 & 0.009 & 0.06 & \\
\hline Masking strength $\times$ Prime-mask SOA & 3.23 & 1,22 & 0.005 & 0.09 & \\
\hline Masking strength $\times$ Mask-target SOA & 5.18 & 3,66 & 0.006 & $<.005$ & $* *$ \\
\hline Prime-mask SOA $\times$ Mask-target SOA & 0.59 & 3,66 & 0.007 & 0.55 & \\
\hline Masking strength $\times$ Prime-mask SOA $\times$ Mask-target SOA & 0.52 & 3,66 & 0.007 & 0.59 & \\
\hline Task level & 2.36 & 1,22 & 3.187 & 0.14 & \\
\hline Task level $\times$ Masking strength & 0.37 & 1,22 & 0.017 & 0.55 & \\
\hline Task level $\times$ Prime-mask SOA & 0.31 & 1,22 & 0.012 & 0.58 & \\
\hline Task level $\times$ Mask-target SOA & 0.99 & 3,66 & 0.009 & 0.39 & \\
\hline Task level $\times$ Masking strength $\times$ Prime-mask SOA & 0.12 & 1,22 & 0.005 & 0.73 & \\
\hline Task level $\times$ Masking strength $\times$ Mask-target SOA & 1.42 & 3,66 & 0.006 & 0.24 & \\
\hline Task level $\times$ Prime-mask SOA $\times$ Mask-target SOA & 0.72 & 3,66 & 0.007 & 0.48 & \\
\hline $\begin{array}{l}\text { Task level } \times \text { Masking strength } \times \text { Prime-mask SOA } \\
\times \text { Mask-target SOA }\end{array}$ & 0.62 & 3,66 & 0.007 & 0.54 & \\
\hline
\end{tabular}


Table C4.1

ANOVA on Errors of Experiments 4.1A, 4.1B, 4.2A and 4.2B with the relevant congruency as a factor

\begin{tabular}{|c|c|c|c|c|c|}
\hline Effect & $F$ & $\overline{d f}$ & $M S_{e}$ & $p$ (p.r.n & GG) \\
\hline (Intercept) & 231.99 & 1,44 & 0.582 & $<.0001$ & $* * *$ \\
\hline Congruency & 8.50 & 1,44 & 0.023 & $<.01$ & $* *$ \\
\hline Masking strength & 0.23 & 1,44 & 0.031 & 0.63 & \\
\hline Prime-mask SOA & 0.77 & 1,44 & 0.027 & 0.39 & \\
\hline Mask-target SOA & 2.97 & 3,132 & 0.033 & $<.05$ & $*$ \\
\hline Congruency $\times$ Masking strength & 0.30 & 1,44 & 0.028 & 0.59 & \\
\hline Congruency $\times$ Prime-mask SOA & 0.01 & 1,44 & 0.022 & 0.93 & \\
\hline Masking strength $\times$ Prime-mask SOA & 1.39 & 1,44 & 0.014 & 0.24 & \\
\hline Congruency $\times$ Mask-target SOA & 6.83 & 3,132 & 0.021 & $<.0005$ & $* * *$ \\
\hline Masking strength $\times$ Mask-target SOA & 0.57 & 3,132 & 0.024 & 0.64 & \\
\hline Prime-mask SOA $\times$ Mask-target SOA & 1.56 & 3,132 & 0.021 & 0.20 & \\
\hline Congruency $\times$ Masking strength $\times$ Prime-mask SOA & 0.01 & 1,44 & 0.016 & 0.92 & \\
\hline Congruency $\times$ Masking strength $\times$ Mask-target SOA & 1.19 & 3,132 & 0.026 & 0.31 & \\
\hline Congruency $\times$ Prime-mask SOA $\times$ Mask-target SOA & 1.59 & 3,132 & 0.017 & 0.20 & \\
\hline Masking strength $\times$ Prime-mask SOA $\times$ Mask-target SOA & 0.13 & 3,132 & 0.022 & 0.94 & \\
\hline $\begin{array}{l}\text { Congruency } \times \text { Masking strength } \times \text { Prime-mask SOA } \times \\
\text { Mask-target SOA }\end{array}$ & 0.41 & 3,132 & 0.019 & 0.74 & \\
\hline Task level & 1.72 & 1,44 & 0.582 & 0.20 & \\
\hline Task level $\times$ Congruency & 0.58 & 1,44 & 0.023 & 0.45 & \\
\hline Task level $\times$ Masking strength & 0.00 & 1,44 & 0.031 & 0.96 & \\
\hline Task level $\times$ Prime-mask SOA & 4.36 & 1,44 & 0.027 & $<.05$ & $*$ \\
\hline Task level $\times$ Mask-target SOA & 2.81 & 3,132 & 0.033 & $<.05$ & $*$ \\
\hline Task level $\times$ Congruency $\times$ Masking strength & 2.72 & 1,44 & 0.028 & 0.11 & \\
\hline Task level $\times$ Congruency $\times$ Prime-mask SOA & 0.75 & 1,44 & 0.022 & 0.39 & \\
\hline Task level $\times$ Masking strength $\times$ Prime-mask SOA & 0.17 & 1,44 & 0.014 & 0.69 & \\
\hline Task level $\times$ Congruency $\times$ Mask-target SOA & 3.36 & 3,132 & 0.021 & $<.05$ & $*$ \\
\hline Task level $\times$ Masking strength $\times$ Mask-target SOA & 3.36 & 3,132 & 0.024 & $<.05$ & $*$ \\
\hline Task level $\times$ Prime-mask SOA $\times$ Mask-target SOA & 0.12 & 3,132 & 0.021 & 0.95 & \\
\hline $\begin{array}{l}\text { Task level } \times \text { Congruency } \times \text { Masking strength } \\
\times \text { Prime-mask SOA }\end{array}$ & 3.25 & 1,44 & 0.016 & 0.08 & \\
\hline $\begin{array}{l}\text { Task level } \times \text { Congruency } \times \text { Masking strength } \\
\times \text { Mask-target SOA }\end{array}$ & 1.48 & 3,132 & 0.026 & 0.22 & \\
\hline $\begin{array}{l}\text { Task level } \times \text { Congruency } \times \text { Prime-mask SOA } \\
\times \text { Mask-target SOA }\end{array}$ & 0.78 & 3,132 & 0.017 & 0.50 & \\
\hline $\begin{array}{l}\text { Task level } \times \text { Masking strength } \times \text { Prime-mask SOA } \\
\times \text { Mask-target SOA }\end{array}$ & 1.18 & 3,132 & 0.022 & 0.32 & \\
\hline $\begin{array}{l}\text { Task level } \times \text { Congruency } \times \text { Masking strength } \\
\times \text { Prime-mask SOA } \times \text { Mask-target SOA }\end{array}$ & 3.08 & 3,132 & 0.019 & $<.05$ & $*$ \\
\hline Masking method & 1.73 & 1,44 & 0.582 & 0.20 & \\
\hline Masking method $\times$ Congruency & 3.65 & 1,44 & 0.023 & 0.06 & \\
\hline Masking method $\times$ Masking strength & 0.83 & 1,44 & 0.031 & 0.37 & \\
\hline Masking method $\times$ Prime-mask SOA & 0.81 & 1,44 & 0.027 & 0.37 & \\
\hline
\end{tabular}




\begin{tabular}{|c|c|c|c|c|c|}
\hline Effect & $\boldsymbol{F}$ & $d f$ & $M S_{e}$ & \multicolumn{2}{|c|}{$\boldsymbol{p}$ (p.r.n. GG) } \\
\hline Masking method $\times$ Mask-target SOA & 0.54 & 3,132 & 0.033 & 0.65 & \\
\hline Masking method $\times$ Congruency $\times$ Masking strength & 0.36 & 1,44 & 0.028 & 0.55 & \\
\hline Masking method $\times$ Congruency $\times$ Prime-mask SOA & 0.37 & 1,44 & 0.022 & 0.55 & \\
\hline Masking method $\times$ Masking strength $\times$ Prime-mask SOA & 8.91 & 1,44 & 0.014 & $<.005$ & $* *$ \\
\hline Masking method $\times$ Congruency $\times$ Mask-target SOA & 1.59 & 3,132 & 0.021 & 0.20 & \\
\hline Masking method $\times$ Masking strength $\times$ Mask-target SOA & 1.03 & 3,132 & 0.024 & 0.38 & \\
\hline Masking method $\times$ Prime-mask SOA $\times$ Mask-target SOA & 0.51 & 3,132 & 0.021 & 0.68 & \\
\hline $\begin{array}{l}\text { Masking method } \times \text { Congruency } \times \text { Masking strength } \\
\times \text { Prime-mask SOA }\end{array}$ & 0.44 & 1,44 & 0.016 & 0.51 & \\
\hline $\begin{array}{l}\text { Masking method } \times \text { Congruency } \times \text { Masking strength } \\
\times \text { Mask-target SOA }\end{array}$ & 3.00 & 3,132 & 0.026 & $<.05$ & $*$ \\
\hline $\begin{array}{l}\text { Masking method } \times \text { Congruency } \times \text { Prime-mask SOA } \\
\times \text { Mask-target SOA }\end{array}$ & 0.96 & 3,132 & 0.017 & 0.41 & \\
\hline $\begin{array}{l}\text { Masking method } \times \text { Masking strength } \times \text { Prime-mask SOA } \\
\times \text { Mask-target SOA }\end{array}$ & 2.00 & 3,132 & 0.022 & 0.12 & \\
\hline $\begin{array}{l}\text { Masking method } \times \text { Congruency } \times \text { Masking strength } \\
\times \text { Prime-mask SOA } \times \text { Mask-target SOA }\end{array}$ & 0.36 & 3,132 & 0.019 & 0.78 & \\
\hline Masking method $\times$ Task level & 0.02 & 1,44 & 0.582 & 0.89 & \\
\hline Masking method $\times$ Task level $\times$ Congruency & 0.62 & 1,44 & 0.023 & 0.44 & \\
\hline Masking method $\times$ Task level $\times$ Masking strength & 0.15 & 1,44 & 0.031 & 0.70 & \\
\hline Masking method $\times$ Task level $\times$ Prime-mask SOA & 1.69 & 1,44 & 0.027 & 0.20 & \\
\hline Masking method $\times$ Task level $\times$ Mask-target SOA & 2.73 & 3,132 & 0.033 & $<.05$ & $*$ \\
\hline $\begin{array}{l}\text { Masking method } \times \text { Task level } \times \text { Congruency } \\
\times \text { Masking strength }\end{array}$ & 0.34 & 1,44 & 0.028 & 0.56 & \\
\hline $\begin{array}{l}\text { Masking method } \times \text { Task level } \times \text { Congruency } \\
\times \text { Prime-mask SOA }\end{array}$ & 1.96 & 1,44 & 0.022 & 0.17 & \\
\hline $\begin{array}{l}\text { Masking method } \times \text { Task level } \times \text { Masking strength } \\
\times \text { Prime-mask SOA }\end{array}$ & 2.73 & 1,44 & 0.014 & 0.11 & \\
\hline $\begin{array}{l}\text { Masking method } \times \text { Task level } \times \text { Congruency } \\
\times \text { Mask-target SOA }\end{array}$ & 0.72 & 3,132 & 0.021 & 0.54 & \\
\hline $\begin{array}{l}\text { Masking method } \times \text { Task level } \times \text { Masking strength } \\
\times \text { Mask-target SOA }\end{array}$ & 0.31 & 3,132 & 0.024 & 0.81 & \\
\hline $\begin{array}{l}\text { Masking method } \times \text { Task level } \times \text { Prime-mask SOA } \\
\times \text { Mask-target SOA }\end{array}$ & 0.97 & 3,132 & 0.021 & 0.41 & \\
\hline $\begin{array}{l}\text { Masking method } \times \text { Task level } \times \text { Congruency } \\
\times \text { Masking strength } \times \text { Prime-mask SOA }\end{array}$ & 0.24 & 1,44 & 0.016 & 0.62 & \\
\hline $\begin{array}{l}\text { Masking method } \times \text { Task level } \times \text { Congruency } \\
\times \text { Masking strength } \times \text { Mask-target SOA }\end{array}$ & 0.58 & 3,132 & 0.026 & 0.63 & \\
\hline $\begin{array}{l}\text { Masking method } \times \text { Task level } \times \text { Congruency } \\
\times \text { Prime-mask SOA } \times \text { Mask-target SOA }\end{array}$ & 0.95 & 3,132 & 0.017 & 0.42 & \\
\hline $\begin{array}{l}\text { Masking method } \times \text { Task level } \times \text { Masking strength } \\
\times \text { Prime-mask SOA } \times \text { Mask-target SOA }\end{array}$ & 0.54 & 3,132 & 0.022 & 0.65 & \\
\hline $\begin{array}{l}\text { Masking method } \times \text { Task level } \times \text { Congruency } \\
\times \text { Masking strength } \times \text { Prime-mask SOA } \\
\times \text { Mask-target SOA }\end{array}$ & 1.91 & 3,132 & 0.019 & 0.13 & \\
\hline
\end{tabular}

Note. GG = Greenhouse-Geisser correction was applied, if Mauchly's sphericity test indicated a violation of the sphericity assumption. '***' $p<0.001$; '**' $p<0.01$; '*’ $p<0.05$; '’ $p<0.1$. 
Table C4.2

ANOVA on RTs of Experiments $4.1 \mathrm{~A}, 4.1 \mathrm{~B}, 4.2 \mathrm{~A}$ and $4.2 \mathrm{~B}$ with the relevant congruency as a factor

\begin{tabular}{|c|c|c|c|c|c|}
\hline Effect & $F$ & $d f$ & $M S_{e}$ & $\boldsymbol{p}$ (p.r.n & GG) \\
\hline (Intercept) & 2430.35 & 1,44 & 119472 & $<.0001$ & $* * *$ \\
\hline Congruency & 47.07 & 1,44 & 242 & $<.0001$ & $* * *$ \\
\hline Masking strength & 18.27 & 1,44 & 191 & $<.0005$ & $* * *$ \\
\hline Prime-mask SOA & 18.88 & 1,44 & 160 & $<.0001$ & $* * *$ \\
\hline Mask-target SOA & 233.15 & 3,132 & 439 & $<.0001$ & $* * *$ \\
\hline Congruency $\times$ Masking strength & 2.42 & 1,44 & 179 & 0.13 & \\
\hline Congruency $\times$ Prime-mask SOA & 2.34 & 1,44 & 164 & 0.13 & \\
\hline Masking strength $\times$ Prime-mask SOA & 12.33 & 1,44 & 99 & $<.005$ & $* *$ \\
\hline Congruency $\times$ Mask-target SOA & 19.95 & 3,132 & 177 & $<.0001$ & $* * *$ \\
\hline Masking strength $\times$ Mask-target SOA & 2.95 & 3,132 & 108 & $<.05$ & $*$ \\
\hline Prime-mask SOA $\times$ Mask-target SOA & 1.71 & 3,132 & 132 & 0.17 & \\
\hline Congruency $\times$ Masking strength $\times$ Prime-mask SOA & 0.07 & 1,44 & 123 & 0.80 & \\
\hline Congruency $\times$ Masking strength $\times$ Mask-target SOA & 0.25 & 3,132 & 132 & 0.83 & \\
\hline Congruency $\times$ Prime-mask SOA $\times$ Mask-target SOA & 0.16 & 3,132 & 138 & 0.92 & \\
\hline Masking strength $\times$ Prime-mask SOA $\times$ Mask-target SOA & 0.90 & 3,132 & 94 & 0.44 & \\
\hline $\begin{array}{l}\text { Congruency } \times \text { Masking strength } \times \text { Prime-mask SOA } \times \\
\text { Mask-target SOA }\end{array}$ & 1.34 & 3,132 & 96 & 0.26 & \\
\hline Task level & 2.73 & 1,44 & 119472 & 0.11 & \\
\hline Task level $\times$ Congruency & 0.06 & 1,44 & 242 & 0.80 & \\
\hline Task level $\times$ Masking strength & 0.14 & 1,44 & 191 & 0.71 & \\
\hline Task level $\times$ Prime-mask SOA & 0.10 & 1,44 & 160 & 0.76 & \\
\hline Task level $\times$ Mask-target SOA & 0.81 & 3,132 & 439 & 0.45 & \\
\hline Task level $\times$ Congruency $\times$ Masking strength & 2.30 & 1,44 & 179 & 0.14 & \\
\hline Task level $\times$ Congruency $\times$ Prime-mask SOA & 3.00 & 1,44 & 164 & 0.09 & \\
\hline Task level $\times$ Masking strength $\times$ Prime-mask SOA & 0.92 & 1,44 & 99 & 0.34 & \\
\hline Task level $\times$ Congruency $\times$ Mask-target SOA & 7.84 & 3,132 & 177 & $<.0001$ & $* * *$ \\
\hline Task level $\times$ Masking strength $\times$ Mask-target SOA & 1.62 & 3,132 & 108 & 0.19 & \\
\hline Task level $\times$ Prime-mask SOA $\times$ Mask-target SOA & 0.33 & 3,132 & 132 & 0.80 & \\
\hline $\begin{array}{l}\text { Task level } \times \text { Congruency } \times \text { Masking strength } \\
\times \text { Prime-mask SOA }\end{array}$ & 1.22 & 1,44 & 123 & 0.28 & \\
\hline $\begin{array}{l}\text { Task level } \times \text { Congruency } \times \text { Masking strength } \\
\times \text { Mask-target SOA }\end{array}$ & 0.88 & 3,132 & 132 & 0.44 & \\
\hline $\begin{array}{l}\text { Task level } \times \text { Congruency } \times \text { Prime-mask SOA } \\
\times \text { Mask-target SOA }\end{array}$ & 1.08 & 3,132 & 138 & 0.36 & \\
\hline $\begin{array}{l}\text { Task level } \times \text { Masking strength } \times \text { Prime-mask SOA } \\
\times \text { Mask-target SOA }\end{array}$ & 0.96 & 3,132 & 94 & 0.41 & \\
\hline $\begin{array}{l}\text { Task level } \times \text { Congruency } \times \text { Masking strength } \\
\times \text { Prime-mask SOA } \times \text { Mask-target SOA }\end{array}$ & 2.11 & 3,132 & 96 & 0.10 & \\
\hline Masking method & 0.45 & 1,44 & 119472 & 0.51 & \\
\hline Masking method $\times$ Congruency & 17.33 & 1,44 & 242 & $<.0005$ & $* * *$ \\
\hline Masking method $\times$ Masking strength & 0.21 & 1,44 & 191 & 0.65 & \\
\hline Masking method $\times$ Prime-mask SOA & 1.80 & 1,44 & 160 & 0.19 & \\
\hline
\end{tabular}




\begin{tabular}{|c|c|c|c|c|c|}
\hline Effect & $F$ & $d f$ & $M S_{e}$ & $p$ (p.r.n & JG) \\
\hline Masking method $\times$ Mask-target SOA & 11.61 & 3,132 & 439 & $<.0001$ & $* * *$ \\
\hline Masking method $\times$ Congruency $\times$ Masking strength & 0.00 & 1,44 & 179 & 1.00 & \\
\hline Masking method $\times$ Congruency $\times$ Prime-mask SOA & 10.64 & 1,44 & 164 & $<.005$ & $* *$ \\
\hline Masking method $\times$ Masking strength $\times$ Prime-mask SOA & 0.88 & 1,44 & 99 & 0.35 & \\
\hline Masking method $\times$ Congruency $\times$ Mask-target SOA & 5.56 & 3,132 & 177 & $<.005$ & $* *$ \\
\hline Masking method $\times$ Masking strength $\times$ Mask-target SOA & 2.71 & 3,132 & 108 & $<.05$ & $*$ \\
\hline Masking method $\times$ Prime-mask SOA $\times$ Mask-target SOA & 0.55 & 3,132 & 132 & 0.65 & \\
\hline $\begin{array}{l}\text { Masking method } \times \text { Congruency } \times \text { Masking strength } \\
\times \text { Prime-mask SOA }\end{array}$ & 0.17 & 1,44 & 123 & 0.69 & \\
\hline $\begin{array}{l}\text { Masking method } \times \text { Congruency } \times \text { Masking strength } \\
\times \text { Mask-target SOA }\end{array}$ & 3.74 & 3,132 & 132 & $<.05$ & $*$ \\
\hline $\begin{array}{l}\text { Masking method } \times \text { Congruency } \times \text { Prime-mask SOA } \\
\times \text { Mask-target SOA }\end{array}$ & 1.30 & 3,132 & 138 & 0.28 & \\
\hline $\begin{array}{l}\text { Masking method } \times \text { Masking strength } \times \text { Prime-mask SOA } \\
\times \text { Mask-target SOA }\end{array}$ & 0.24 & 3,132 & 94 & 0.87 & \\
\hline $\begin{array}{l}\text { Masking method } \times \text { Congruency } \times \text { Masking strength } \\
\times \text { Prime-mask SOA } \times \text { Mask-target SOA }\end{array}$ & 0.81 & 3,132 & 96 & 0.49 & \\
\hline Masking method $\times$ Task level & 0.06 & 1,44 & 119472 & 0.81 & \\
\hline Masking method $\times$ Task level $\times$ Congruency & 1.05 & 1,44 & 242 & 0.31 & \\
\hline Masking method $\times$ Task level $\times$ Masking strength & 1.52 & 1,44 & 191 & 0.22 & \\
\hline Masking method $\times$ Task level $\times$ Prime-mask SOA & 0.95 & 1,44 & 160 & 0.33 & \\
\hline Masking method $\times$ Task level $\times$ Mask-target SOA & 1.77 & 3,132 & 439 & 0.18 & \\
\hline $\begin{array}{l}\text { Masking method } \times \text { Task level } \times \text { Congruency } \\
\times \text { Masking strength }\end{array}$ & 4.39 & 1,44 & 179 & $<.05$ & $*$ \\
\hline $\begin{array}{l}\text { Masking method } \times \text { Task level } \times \text { Congruency } \\
\times \text { Prime-mask SOA }\end{array}$ & 1.23 & 1,44 & 164 & 0.27 & \\
\hline $\begin{array}{l}\text { Masking method } \times \text { Task level } \times \text { Masking strength } \\
\times \text { Prime-mask SOA }\end{array}$ & 0.04 & 1,44 & 99 & 0.84 & \\
\hline $\begin{array}{l}\text { Masking method } \times \text { Task level } \times \text { Congruency } \\
\times \text { Mask-target SOA }\end{array}$ & 5.95 & 3,132 & 177 & $<.001$ & $* * *$ \\
\hline $\begin{array}{l}\text { Masking method } \times \text { Task level } \times \text { Masking strength } \\
\times \text { Mask-target SOA }\end{array}$ & 2.54 & 3,132 & 108 & 0.06 & \\
\hline $\begin{array}{l}\text { Masking method } \times \text { Task level } \times \text { Prime-mask SOA } \\
\times \text { Mask-target SOA }\end{array}$ & 0.76 & 3,132 & 132 & 0.52 & \\
\hline $\begin{array}{l}\text { Masking method } \times \text { Task level } \times \text { Congruency } \\
\times \text { Masking strength } \times \text { Prime-mask SOA }\end{array}$ & 0.03 & 1,44 & 123 & 0.86 & \\
\hline $\begin{array}{l}\text { Masking method } \times \text { Task level } \times \text { Congruency } \\
\times \text { Masking strength } \times \text { Mask-target SOA }\end{array}$ & 1.90 & 3,132 & 132 & 0.14 & \\
\hline $\begin{array}{l}\text { Masking method } \times \text { Task level } \times \text { Congruency } \\
\times \text { Prime-mask SOA } \times \text { Mask-target SOA }\end{array}$ & 0.20 & 3,132 & 138 & 0.90 & \\
\hline $\begin{array}{l}\text { Masking method } \times \text { Task level } \times \text { Masking strength } \\
\times \text { Prime-mask SOA } \times \text { Mask-target SOA }\end{array}$ & 1.82 & 3,132 & 94 & 0.15 & \\
\hline $\begin{array}{l}\text { Masking method } \times \text { Task level } \times \text { Congruency } \\
\times \text { Masking strength } \times \text { Prime-mask SOA } \\
\times \text { Mask-target SOA }\end{array}$ & 0.05 & 3,132 & 96 & 0.99 & \\
\hline
\end{tabular}


Table C4.3

ANOVA on objective prime visibility of Experiments $4.1 \mathrm{~A}, 4.1 \mathrm{~B}, 4.2 \mathrm{~A}$ and $4.2 \mathrm{~B}$

\begin{tabular}{|c|c|c|c|c|c|}
\hline Effect & $\boldsymbol{F}$ & $d f$ & $M S_{e}$ & $p$ (p.r.n. & JG) \\
\hline (Intercept) & 421.66 & 1,44 & 9.547 & $<.0001$ & $* * *$ \\
\hline Masking strength & 83.68 & 1,44 & 2.028 & $<.0001$ & $* * *$ \\
\hline Prime-mask SOA & 106.45 & 1,44 & 0.395 & $<.0001$ & $* * *$ \\
\hline Mask-target SOA & 11.65 & 3,132 & 0.218 & $<.0001$ & $* * *$ \\
\hline Masking strength $\times$ Prime-mask SOA & 26.61 & 1,44 & 0.232 & $<.0001$ & $* * *$ \\
\hline Masking strength $\times$ Mask-target SOA & 0.31 & 3,132 & 0.206 & 0.82 & \\
\hline Prime-mask SOA $\times$ Mask-target SOA & 5.42 & 3,132 & 0.227 & $<.005$ & $* *$ \\
\hline Masking strength $\times$ Prime-mask SOA $\times$ Mask-target SOA & 3.03 & 3,132 & 0.199 & $<.05$ & $*$ \\
\hline Task level & 5.11 & 1,44 & 9.547 & $<.05$ & $*$ \\
\hline Task level $\times$ Masking strength & 3.72 & 1,44 & 2.028 & 0.06 & \\
\hline Task level $\times$ Prime-mask SOA & 0.21 & 1,44 & 0.395 & 0.65 & \\
\hline Task level $\times$ Mask-target SOA & 0.72 & 3,132 & 0.218 & 0.54 & \\
\hline Task level $\times$ Masking strength $\times$ Prime-mask SOA & 5.32 & 1,44 & 0.232 & $<.05$ & $*$ \\
\hline Task level $\times$ Masking strength $\times$ Mask-target SOA & 0.64 & 3,132 & 0.206 & 0.59 & \\
\hline Task level $\times$ Prime-mask SOA $\times$ Mask-target SOA & 1.71 & 3,132 & 0.227 & 0.18 & \\
\hline $\begin{array}{l}\text { Task level } \times \text { Masking strength } \times \text { Prime-mask SOA } \\
\times \text { Mask-target SOA }\end{array}$ & 1.01 & 3,132 & 0.199 & 0.39 & \\
\hline Masking method & 20.44 & 1,44 & 9.547 & $<.0001$ & $* * *$ \\
\hline Masking method $\times$ Masking strength & 31.87 & 1,44 & 2.028 & $<.0001$ & $* * *$ \\
\hline Masking method $\times$ Prime-mask SOA & 9.80 & 1,44 & 0.395 & $<.005$ & $* *$ \\
\hline Masking method $\times$ Mask-target SOA & 7.02 & 3,132 & 0.218 & $<.0005$ & $* * *$ \\
\hline Masking method $\times$ Masking strength $\times$ Prime-mask SOA & 0.79 & 1,44 & 0.232 & 0.38 & \\
\hline Masking method $\times$ Masking strength $\times$ Mask-target SOA & 1.63 & 3,132 & 0.206 & 0.18 & \\
\hline Masking method $\times$ Prime-mask SOA $\times$ Mask-target SOA & 4.03 & 3,132 & 0.227 & $<.05$ & $*$ \\
\hline $\begin{array}{l}\text { Masking method } \times \text { Masking strength } \times \text { Prime-mask SOA } \\
\times \text { Mask-target SOA }\end{array}$ & 0.20 & 3,132 & 0.199 & 0.89 & \\
\hline Masking method $\times$ Task level & 2.93 & 1,44 & 9.547 & 0.09 & \\
\hline Masking method $\times$ Task level $\times$ Masking strength & 1.55 & 1,44 & 2.028 & 0.22 & \\
\hline Masking method $\times$ Task level $\times$ Prime-mask SOA & 6.38 & 1,44 & 0.395 & $<.05$ & $*$ \\
\hline Masking method $\times$ Task level $\times$ Mask-target SOA & 0.59 & 3,132 & 0.218 & 0.63 & \\
\hline $\begin{array}{l}\text { Masking method } \times \text { Task level } \times \text { Masking strength } \\
\times \text { Prime-mask SOA }\end{array}$ & 1.49 & 1,44 & 0.232 & 0.23 & \\
\hline $\begin{array}{l}\text { Masking method } \times \text { Task level } \times \text { Masking strength } \\
\times \text { Mask-target SOA }\end{array}$ & 1.66 & 3,132 & 0.206 & 0.18 & \\
\hline $\begin{array}{l}\text { Masking method } \times \text { Task level } \times \text { Prime-mask SOA } \\
\times \text { Mask-target SOA }\end{array}$ & 0.69 & 3,132 & 0.227 & 0.53 & \\
\hline $\begin{array}{l}\text { Masking method } \times \text { Task level } \times \text { Masking strength } \\
\times \text { Prime-mask SOA } \times \text { Mask-target SOA }\end{array}$ & 0.47 & 3,132 & 0.199 & 0.71 & \\
\hline
\end{tabular}


Table C4.4

ANOVA on subjective prime visibility of Experiments $4.1 \mathrm{~A}, 4.1 \mathrm{~B}, 4.2 \mathrm{~A}$ and $4.2 \mathrm{~B}$

\begin{tabular}{|c|c|c|c|c|c|}
\hline Effect & $F$ & $d f$ & $M S_{e}$ & $p$ (p.r.n & GG) \\
\hline (Intercept) & 1001.97 & 1,44 & 6.916 & $<.0001$ & $* * *$ \\
\hline Masking strength & 66.87 & 1,44 & 0.640 & $<.0001$ & $* * *$ \\
\hline Prime-mask SOA & 69.44 & 1,44 & 0.097 & $<.0001$ & $* * *$ \\
\hline Mask-target SOA & 19.64 & 3,132 & 0.021 & $<.0001$ & $* * *$ \\
\hline Masking strength $\times$ Prime-mask SOA & 28.58 & 1,44 & 0.030 & $<.0001$ & $* * *$ \\
\hline Masking strength $\times$ Mask-target SOA & 3.70 & 3,132 & 0.014 & $<.05$ & $*$ \\
\hline Prime-mask SOA $\times$ Mask-target SOA & 3.60 & 3,132 & 0.014 & $<.05$ & $*$ \\
\hline Masking strength $\times$ Prime-mask SOA $\times$ Mask-target SOA & 1.73 & 3,132 & 0.011 & 0.16 & \\
\hline Task level & 0.90 & 1,44 & 6.916 & 0.35 & \\
\hline Task level $\times$ Masking strength & 5.88 & 1,44 & 0.640 & $<.05$ & $*$ \\
\hline Task level $\times$ Prime-mask SOA & 0.21 & 1,44 & 0.097 & 0.65 & \\
\hline Task level $\times$ Mask-target SOA & 4.49 & 3,132 & 0.021 & $<.05$ & $*$ \\
\hline Task level $\times$ Masking strength $\times$ Prime-mask SOA & 6.37 & 1,44 & 0.030 & $<.05$ & $*$ \\
\hline Task level $\times$ Masking strength $\times$ Mask-target SOA & 1.14 & 3,132 & 0.014 & 0.34 & \\
\hline Task level $\times$ Prime-mask SOA $\times$ Mask-target SOA & 0.30 & 3,132 & 0.014 & 0.78 & \\
\hline $\begin{array}{l}\text { Task level } \times \text { Masking strength } \times \text { Prime-mask SOA } \\
\times \text { Mask-target SOA }\end{array}$ & 0.76 & 3,132 & 0.011 & 0.52 & \\
\hline Masking method & 10.77 & 1,44 & 6.916 & $<.005$ & $* *$ \\
\hline Masking method $\times$ Masking strength & 35.32 & 1,44 & 0.640 & $<.0001$ & $* * *$ \\
\hline Masking method $\times$ Prime-mask SOA & 2.89 & 1,44 & 0.097 & 0.10 & \\
\hline Masking method $\times$ Mask-target SOA & 14.74 & 3,132 & 0.021 & $<.0001$ & $* * *$ \\
\hline Masking method $\times$ Masking strength $\times$ Prime-mask SOA & 0.02 & 1,44 & 0.030 & 0.88 & \\
\hline Masking method $\times$ Masking strength $\times$ Mask-target SOA & 0.93 & 3,132 & 0.014 & 0.43 & \\
\hline Masking method $\times$ Prime-mask SOA $\times$ Mask-target SOA & 3.18 & 3,132 & 0.014 & $<.05$ & $*$ \\
\hline $\begin{array}{l}\text { Masking method } \times \text { Masking strength } \times \text { Prime-mask SOA } \\
\times \text { Mask-target SOA }\end{array}$ & 1.03 & 3,132 & 0.011 & 0.38 & \\
\hline Masking method $\times$ Task level & 0.18 & 1,44 & 6.916 & 0.68 & \\
\hline Masking method $\times$ Task level $\times$ Masking strength & 5.02 & 1,44 & 0.640 & $<.05$ & $*$ \\
\hline Masking method $\times$ Task level $\times$ Prime-mask SOA & 4.20 & 1,44 & 0.097 & $<.05$ & $*$ \\
\hline Masking method $\times$ Task level $\times$ Mask-target SOA & 1.00 & 3,132 & 0.021 & 0.37 & \\
\hline $\begin{array}{l}\text { Masking method } \times \text { Task level } \times \text { Masking strength } \\
\times \text { Prime-mask SOA }\end{array}$ & 0.05 & 1,44 & 0.030 & 0.82 & \\
\hline $\begin{array}{l}\text { Masking method } \times \text { Task level } \times \text { Masking strength } \\
\times \text { Mask-target SOA }\end{array}$ & 2.41 & 3,132 & 0.014 & 0.07 & \\
\hline $\begin{array}{l}\text { Masking method } \times \text { Task level } \times \text { Prime-mask SOA } \\
\times \text { Mask-target SOA }\end{array}$ & 0.86 & 3,132 & 0.014 & 0.44 & \\
\hline $\begin{array}{l}\text { Masking method } \times \text { Task level } \times \text { Masking strength } \\
\times \text { Prime-mask SOA } \times \text { Mask-target SOA }\end{array}$ & 0.53 & 3,132 & 0.011 & 0.67 & \\
\hline
\end{tabular}


III | Approaching Consciousness with Masked Priming 


\section{Appendix IV}

Table D1.1

ANOVA on Errors of Experiment 3.1A (perceptual task level) with semantic congruency as a factor

\begin{tabular}{lccccc}
\hline Effect & $\boldsymbol{F}$ & $\boldsymbol{d f}$ & $\boldsymbol{M} \boldsymbol{S}_{\boldsymbol{e}}$ & \multicolumn{1}{c}{$\boldsymbol{p}$ (p.r.n. GG) } \\
\hline (Intercept) & 95.07 & 1,11 & 0.646 & $<.0001$ & $* * *$ \\
Semantic congruency & 0.45 & 1,11 & 0.023 & 0.52 & $* .01$ \\
Masking strength & 5.28 & 3,33 & 0.017 & $<.0$. & $* *$ \\
Prime-mask SOA & 6.09 & 3,33 & 0.048 & $<.005$ & 0.49 \\
Semantic congruency $\times$ Masking strength & 0.83 & 3,33 & 0.020 & 0.027 & 0.25 \\
Semantic congruency $\times$ Prime-mask SOA & 1.43 & 3,33 & 0.023 & 0.35 \\
Masking strength $\times$ Prime-mask SOA & 1.12 & 9,99 & 0.020 & 0.56
\end{tabular}

Note. GG $=$ Greenhouse-Geisser correction was applied, if Mauchly's sphericity test indicated a violation of the sphericity assumption. '***' $p<0.001$; '**’ $p<0.01$; '*’ $p<0.05$; '’ $p<0.1$.

Table D1.2

ANOVA on RTs of Experiment 3.1 A (perceptual task level) with semantic congruency as a factor

\begin{tabular}{lccccc}
\hline Effect & $\boldsymbol{F}$ & $\boldsymbol{d f}$ & $\boldsymbol{M} \boldsymbol{S}_{\boldsymbol{e}}$ & \multicolumn{1}{c}{$\boldsymbol{p}$ (p.r.n. GG) } \\
\hline (Intercept) & 1490.91 & 1,11 & 44524 & $<.0001$ & $* * *$ \\
Semantic congruency & 0.30 & 1,11 & 168 & 0.60 & \\
Masking strength & 7.85 & 3,33 & 127 & $<.0005$ & $* * *$ \\
Prime-mask SOA & 19.82 & 3,33 & 126 & $<.0001$ & $* * *$ \\
Semantic congruency $\times$ Masking strength & 2.55 & 3,33 & 75 & 0.07 &. \\
Semantic congruency $\times$ Prime-mask SOA & 0.49 & 3,33 & 67 & 0.69 \\
Masking strength $\times$ Prime-mask SOA & 0.38 & 9,99 & 178 & 0.94 \\
Semantic congruency $\times$ Masking strength $\times$ & 0.45 & 9,99 & 139 & 0.90
\end{tabular}

Note. GG = Greenhouse-Geisser correction was applied, if Mauchly's sphericity test indicated a violation of the sphericity assumption. '***’ $p<0.001$; ‘**’ $p<0.01$; '*’ $p<0.05$; '’ $p<0.1$.

Table D2.1

ANOVA on Errors of Experiment 3.1B (semantic task level) with perceptual congruency as a factor

\begin{tabular}{lccccc}
\hline Effect & $\boldsymbol{F}$ & $\boldsymbol{d f}$ & $\boldsymbol{M} \boldsymbol{S}_{\boldsymbol{e}}$ & \multicolumn{1}{c}{$\boldsymbol{p}$ (p.r.n. GG) } \\
\hline (Intercept) & 71.05 & 1,11 & 0.760 & $<.0001$ & $* * *$ \\
Perceptual congruency & 0.68 & 1,11 & 0.033 & 0.43 & $* .005$ \\
Masking strength & 6.42 & 3,33 & 0.022 & $<$ \\
Prime-mask SOA & 6.80 & 3,33 & 0.025 & $<.005$ & $* *$ \\
Perceptual congruency $\times$ Masking strength & 4.14 & 3,33 & 0.012 & $<.05$ & $*$ \\
Perceptual congruency $\times$ Prime-mask SOA & 0.46 & 3,33 & 0.031 & 0.72 & 0.94 \\
Masking strength $\times$ Prime-mask SOA & 0.38 & 9,99 & 0.025 & & 0.92
\end{tabular}

Note. GG $=$ Greenhouse-Geisser correction was applied, if Mauchly's sphericity test indicated a violation of the sphericity assumption. '***’ $p<0.001$; “**' $p<0.01$; '*’ $p<0.05$; '.' $p<0.1$. 
Table D2.2

ANOVA on RTs of Experiment $3.1 B$ (semantic task level) with perceptual congruency as a factor

\begin{tabular}{|c|c|c|c|c|c|}
\hline Effect & $\boldsymbol{F}$ & $d f$ & $M S_{e}$ & $p$ (p.r.n & GG) \\
\hline (Intercept) & 2429.79 & 1,11 & 33245 & $<.0001$ & $* * *$ \\
\hline Perceptual congruency & 0.23 & 1,11 & 143 & 0.64 & \\
\hline Masking strength & 22.37 & 3,33 & 289 & $<.0001$ & $* * *$ \\
\hline Prime-mask SOA & 3.53 & 3,33 & 157 & 0.06 & \\
\hline Perceptual congruency $\times$ Masking strength & 1.51 & 3,33 & 120 & 0.23 & \\
\hline Perceptual congruency $\times$ Prime-mask SOA & 0.60 & 3,33 & 118 & 0.62 & \\
\hline Masking strength $\times$ Prime-mask SOA & 0.89 & 9,99 & 109 & 0.54 & \\
\hline $\begin{array}{l}\text { Perceptual congruency } \times \text { Masking strength } \\
\times \text { Prime-mask SOA }\end{array}$ & 0.35 & 9,99 & 93 & 0.95 & \\
\hline
\end{tabular}

Table D3.1

ANOVA on Errors of Experiment 3.2A (perceptual task level) with semantic congruency as a factor

\begin{tabular}{lcccc}
\hline Effect & $\boldsymbol{F}$ & $\boldsymbol{d f}$ & $\boldsymbol{M S}_{\boldsymbol{e}}$ & $\boldsymbol{p}$ (p.r.n. GG) \\
\hline (Intercept) & 25.41 & 1,11 & 1.421 & $<.0005$ \\
Semantic congruency & 0.10 & 1,11 & 0.008 & 0.76 \\
Masking strength & 1.42 & 3,33 & 0.020 & 0.25 \\
Prime-mask SOA & 3.31 & 3,33 & 0.051 & 0.07 \\
Semantic congruency $\times$ Masking strength & 1.27 & 3,33 & 0.021 & 0.30 \\
Semantic congruency $\times$ Prime-mask SOA & 0.30 & 3,33 & 0.035 & 0.82 \\
Masking strength $\times$ Prime-mask SOA & 1.91 & 9,99 & 0.021 & 0.06 \\
Semantic congruency $\times$ Masking strength $\times$ & 1.20 & 9,99 & 0.019 & 0.30
\end{tabular}

Note. $\mathrm{GG}=$ Greenhouse-Geisser correction was applied, if Mauchly's sphericity test indicated a violation of the sphericity assumption. '***' $p<0.001$; '**’ $p<0.01$; '*’ $p<0.05$; '’ $p<0.1$.

Table D3.2

ANOVA on RTs of Experiment $3.2 A$ (perceptual task level) with semantic congruency as a factor

\begin{tabular}{lcccc}
\hline Effect & $\boldsymbol{F}$ & $\boldsymbol{d f}$ & $\boldsymbol{M} \boldsymbol{S}_{\boldsymbol{e}}$ & $\boldsymbol{p}$ (p.r.n. GG) \\
\hline (Intercept) & 524.01 & 1,11 & 139984 & $<.0001$ \\
Semantic congruency & 3.11 & 1,11 & 29 & 0.11 \\
Masking strength & 2.01 & 3,33 & 419 & 0.16 \\
Prime-mask SOA & 2.42 & 3,33 & 426 & 0.12 \\
Semantic congruency $\times$ Masking strength & 0.63 & 3,33 & 170 & 0.60 \\
Semantic congruency $\times$ Prime-mask SOA & 1.29 & 3,33 & 214 & 0.30 \\
Masking strength $\times$ Prime-mask SOA & 0.70 & 9,99 & 184 & 0.58 \\
Semantic congruency $\times$ Masking strength $\times$ & 0.51 & 9,99 & 162 & 0.70 \\
$\quad$ Prime-mask SOA & & & &
\end{tabular}

Note. GG $=$ Greenhouse-Geisser correction was applied, if Mauchly's sphericity test indicated a violation of the sphericity assumption. ‘***’ $p<0.001$; ‘**’ $p<0.01$; ‘*’ $p<0.05$; ‘’ $p<0.1$. 
Table D4.1

ANOVA on Errors of Experiment 3.2B (semantic task level) with perceptual congruency as a factor

\begin{tabular}{lccccc}
\hline Effect & $\boldsymbol{F}$ & $\boldsymbol{d f}$ & $\boldsymbol{M S}_{\boldsymbol{e}}$ & \multicolumn{1}{c}{$\boldsymbol{p}$ (p.r.n. GG) } \\
\hline (Intercept) & 35.25 & 1,11 & 1.780 & $<.0001$ & $* * *$ \\
Perceptual congruency & 2.11 & 1,11 & 0.011 & 0.17 & $*$ \\
Masking strength & 4.29 & 3,33 & 0.013 & $<.05$ & $* .08$ \\
Prime-mask SOA & 2.42 & 3,33 & 0.058 & 0.44 \\
Perceptual congruency $\times$ Masking strength & 0.92 & 3,33 & 0.036 & 0.44 \\
Perceptual congruency $\times$ Prime-mask SOA & 0.82 & 3,33 & 0.016 & 0.49 \\
Masking strength $\times$ Prime-mask SOA & 0.63 & 9,99 & 0.017 & 0.77 \\
Perceptual congruency $\times$ Masking strength & 0.67 & 9,99 & 0.015 & 0.73
\end{tabular}

Note. GG = Greenhouse-Geisser correction was applied, if Mauchly's sphericity test indicated a violation of the sphericity assumption. '***' $p<0.001$; '**' $p<0.01$; '*’' $p<0.05$; '’ $p<0.1$.

Table D4.2

ANOVA on RTs of Experiment 3.2B (semantic task level) with perceptual congruency as a factor

\begin{tabular}{|c|c|c|c|c|c|}
\hline Effect & $\bar{F}$ & $\overline{d f}$ & $M S_{e}$ & \multicolumn{2}{|c|}{$\boldsymbol{p}$ (p.r.n. GG) } \\
\hline (Intercept) & 1116.49 & 1,11 & 68174 & $<.0001$ & $* * *$ \\
\hline Perceptual congruency & 0.65 & 1,11 & 206 & 0.44 & \\
\hline Masking strength & 0.29 & 3,33 & 142 & 0.83 & \\
\hline Prime-mask SOA & 1.93 & 3,33 & 225 & 0.18 & \\
\hline Perceptual congruency $\times$ Masking strength & 0.90 & 3,33 & 108 & 0.45 & \\
\hline Perceptual congruency $\times$ Prime-mask SOA & 0.16 & 3,33 & 122 & 0.92 & \\
\hline Masking strength $\times$ Prime-mask SOA & 0.88 & 9,99 & 101 & 0.54 & \\
\hline $\begin{array}{l}\text { Perceptual congruency } \times \text { Masking strength } \\
\times \text { Prime-mask SOA }\end{array}$ & 0.86 & 9,99 & 00 & 0.57 & \\
\hline
\end{tabular}

Note. $\mathrm{GG}=$ Greenhouse-Geisser correction was applied, if Mauchly's sphericity test indicated a violation of the sphericity assumption. '***' $p<0.001$; '**’ $p<0.01$; '*’ $p<0.05$; ‘’ $p<0.1$.

Table D5.1

ANOVA on Errors of Experiment 3.3A (perceptual task level) with semantic congruency as a factor

\begin{tabular}{lccccc}
\hline Effect & $\boldsymbol{F}$ & $\boldsymbol{d f}$ & $\boldsymbol{M S}_{\boldsymbol{e}}$ & \multicolumn{1}{c}{$\boldsymbol{p}$ (p.r.n. GG) } \\
\hline (Intercept) & 207.90 & 1,11 & 0.190 & $<.0001$ & $* * *$ \\
Semantic congruency & 1.18 & 1,11 & 0.017 & 0.30 \\
Masking strength & 0.17 & 3,33 & 0.037 & 0.92 \\
Prime-mask SOA & 3.43 & 3,33 & 0.036 & $<.05$ & $*$ \\
Semantic congruency $\times$ Masking strength & 3.73 & 3,33 & 0.021 & $<.05$ & $*$ \\
Semantic congruency $\times$ Prime-mask SOA & 0.20 & 3,33 & 0.029 & 0.90 \\
Masking strength $\times$ Prime-mask SOA & 0.83 & 9,99 & 0.022 & 0.59 \\
Semantic congruency $\times$ Masking strength $\times$ & 0.51 & 9,99 & 0.028 & 0.87
\end{tabular}

Note. $\mathrm{GG}=$ Greenhouse-Geisser correction was applied, if Mauchly's sphericity test indicated a violation of the sphericity assumption. '***' $p<0.001$; ‘**’ $p<0.01$; '*’’ $p<0.05$; '’ $p<0.1$. 
Table D5.2

ANOVA on RTs of Experiment 3.3A (perceptual task level) with semantic congruency as a factor

\begin{tabular}{|c|c|c|c|c|c|}
\hline Effect & $F$ & $d f$ & $M S_{e}$ & \multicolumn{2}{|c|}{$\boldsymbol{p}$ (p.r.n. GG) } \\
\hline (Intercept) & 789.67 & 1,11 & 86726 & $<.0001$ & $* * *$ \\
\hline Semantic congruency & 0.24 & 1,11 & 156 & 0.63 & \\
\hline Masking strength & 12.77 & 3,33 & 194 & $<.0001$ & $* * *$ \\
\hline Prime-mask SOA & 17.99 & 3,33 & 237 & $<.0001$ & $* * *$ \\
\hline Semantic congruency $\times$ Masking strength & 1.36 & 3,33 & 130 & 0.27 & \\
\hline Semantic congruency $\times$ Prime-mask SOA & 2.44 & 3,33 & 115 & 0.08 & . \\
\hline Masking strength $\times$ Prime-mask SOA & 2.17 & 9,99 & 138 & $<.05$ & $*$ \\
\hline $\begin{array}{l}\text { Semantic congruency } \times \text { Masking strength } \times \\
\text { Prime-mask SOA }\end{array}$ & 0.51 & 9,99 & 143 & 0.68 & \\
\hline
\end{tabular}

Note. GG $=$ Greenhouse-Geisser correction was applied, if Mauchly's sphericity test indicated a violation of the sphericity assumption. '***' $p<0.001$; '**' $p<0.01$; '*’' $p<0.05$; '’ $p<0.1$.

Table D6.1

ANOVA on Errors of Experiment 3.3B (semantic task level) with perceptual congruency as a factor

\begin{tabular}{lcccc}
\hline Effect & $\boldsymbol{F}$ & $\boldsymbol{d f}$ & $\boldsymbol{M \boldsymbol { S } _ { \boldsymbol { e } }}$ & \multicolumn{1}{c}{$\boldsymbol{p}$ (p.r.n. GG) } \\
\hline (Intercept) & 43.50 & 1,11 & 1.120 & $<.0001$ \\
Perceptual congruency & 0.51 & 1,11 & 0.015 & 0.49 \\
Masking strength & 1.04 & 3,33 & 0.032 & 0.39 \\
Prime-mask SOA & 2.75 & 3,33 & 0.022 & 0.10 \\
Perceptual congruency $\times$ Masking strength & 1.61 & 3,33 & 0.015 & 0.21 \\
Perceptual congruency $\times$ Prime-mask SOA & 1.13 & 3,33 & 0.025 & 0.35 \\
Masking strength $\times$ Prime-mask SOA & 0.52 & 9,99 & 0.021 & 0.85 \\
Perceptual congruency $\times$ Masking strength & 1.43 & 9,99 & 0.023 & 0.19
\end{tabular}

Note. $\mathrm{GG}=$ Greenhouse-Geisser correction was applied, if Mauchly's sphericity test indicated a violation of the sphericity assumption. '***' $p<0.001$; '**’ $p<0.01$; '*’ $p<0.05$; '’ $p<0.1$.

Table D6.2

ANOVA on RTs of Experiment 3.3B (semantic task level) with perceptual congruency as a factor

\begin{tabular}{|c|c|c|c|c|c|}
\hline Effect & $F$ & $d f$ & $M S_{e}$ & $p$ (p.r.n & jG) \\
\hline (Intercept) & 867.87 & 1,11 & 83397 & $<.0001$ & $* * *$ \\
\hline Perceptual congruency & 0.58 & 1,11 & 112 & 0.46 & \\
\hline Masking strength & 15.07 & 3,33 & 147 & $<.0001$ & $* * *$ \\
\hline Prime-mask SOA & 15.60 & 3,33 & 125 & $<.0001$ & $* * *$ \\
\hline Perceptual congruency $\times$ Masking strength & 0.34 & 3,33 & 67 & 0.79 & \\
\hline Perceptual congruency $\times$ Prime-mask SOA & 0.69 & 3,33 & 150 & 0.57 & \\
\hline Masking strength $\times$ Prime-mask SOA & 2.90 & 9,99 & 78 & $<.005$ & $* *$ \\
\hline $\begin{array}{l}\text { Perceptual congruency } \times \text { Masking strength } \\
\times \text { Prime-mask SOA }\end{array}$ & 1.15 & 9,99 & 86 & 0.33 & \\
\hline
\end{tabular}


Table D7.1

ANOVA on Errors of Experiment 4.1 A (perceptual task level) with semantic congruency as a factor

\begin{tabular}{|c|c|c|c|c|c|}
\hline Effect & $F$ & $d f$ & $M S_{e}$ & \multicolumn{2}{|c|}{$\boldsymbol{p}$ (p.r.n. GG) } \\
\hline (Intercept) & 53.01 & 1,11 & 0.638 & $<.0001$ & $* * *$ \\
\hline Semantic congruency & 0.69 & 1,11 & 0.027 & 0.42 & \\
\hline Masking strength & 0.01 & 1,11 & 0.052 & 0.91 & \\
\hline Prime-mask SOA & 0.09 & 1,11 & 0.036 & 0.77 & \\
\hline Mask-target SOA & 0.21 & 3,33 & 0.048 & 0.89 & \\
\hline Semantic congruency $\times$ Masking strength & 0.04 & 1,11 & 0.029 & 0.85 & \\
\hline Semantic congruency $\times$ Prime-mask SOA & 0.42 & 1,11 & 0.023 & 0.53 & \\
\hline Masking strength $\times$ Prime-mask SOA & 0.02 & 1,11 & 0.013 & 0.89 & \\
\hline Semantic congruency $\times$ Mask-target SOA & 1.74 & 3,33 & 0.017 & 0.18 & \\
\hline Masking strength $\times$ Mask-target SOA & 3.18 & 3,33 & 0.015 & $<.05$ & $*$ \\
\hline Prime-mask SOA $\times$ Mask-target SOA & 0.61 & 3,33 & 0.028 & 0.50 & \\
\hline $\begin{array}{l}\text { Semantic congruency } \times \text { Masking strength } \times \\
\text { Prime-mask SOA }\end{array}$ & 1.74 & 1,11 & 0.027 & 0.21 & \\
\hline $\begin{array}{l}\text { Semantic congruency } \times \text { Masking strength } \times \\
\text { Mask-target SOA }\end{array}$ & 1.48 & 3,33 & 0.020 & 0.24 & \\
\hline $\begin{array}{l}\text { Semantic congruency } \times \text { Prime-mask SOA } \\
\times \text { Mask-target SOA }\end{array}$ & 1.77 & 3,33 & 0.021 & 0.17 & \\
\hline $\begin{array}{l}\text { Masking strength } \times \text { Prime-mask SOA } \times \\
\text { Mask-target SOA }\end{array}$ & 0.31 & 3,33 & 0.030 & 0.82 & \\
\hline $\begin{array}{l}\text { Semantic congruency } \times \text { Masking strength } \times \\
\text { Prime-mask SOA } \times \text { Mask-target SOA }\end{array}$ & 0.83 & 3,33 & 0.026 & 0.49 & \\
\hline
\end{tabular}

Note. GG = Greenhouse-Geisser correction was applied, if Mauchly's sphericity test indicated a violation of the sphericity assumption. '***’ $p<0.001$; ‘**’ $p<0.01$; '*’ $p<0.05$; ‘’ $p<0.1$.

Table D7.2

ANOVA on RTs of Experiment 4.1A (perceptual task level) with semantic congruency as a factor

\begin{tabular}{|c|c|c|c|c|c|}
\hline \multirow{2}{*}{$\begin{array}{l}\text { Effect } \\
\text { (Intercept) }\end{array}$} & \multirow{2}{*}{$\begin{array}{c}\boldsymbol{F} \\
759.56\end{array}$} & \multirow{2}{*}{$\begin{array}{c}\boldsymbol{d f} \\
1,11\end{array}$} & \multirow{2}{*}{$\begin{array}{c}M S_{e} \\
90865\end{array}$} & \multicolumn{2}{|c|}{$\boldsymbol{p}$ (p.r.n. GG) } \\
\hline & & & & $<.0001$ & $* * *$ \\
\hline Semantic congruency & 0.02 & 1,11 & 83 & 0.89 & \\
\hline Masking strength & 8.73 & 1,11 & 163 & $<.05$ & $*$ \\
\hline Prime-mask SOA & 10.50 & 1,11 & 93 & $<.01$ & $* *$ \\
\hline Mask-target SOA & 65.49 & 3,33 & 441 & $<.0001$ & $* * *$ \\
\hline Semantic congruency $\times$ Masking strength & 0.03 & 1,11 & 48 & 0.88 & \\
\hline Semantic congruency $\times$ Prime-mask SOA & 3.39 & 1,11 & 100 & 0.09 & . \\
\hline Masking strength $\times$ Prime-mask SOA & 2.09 & 1,11 & 83 & 0.18 & \\
\hline Semantic congruency $\times$ Mask-target SOA & 1.73 & 3,33 & 250 & 0.21 & \\
\hline Masking strength $\times$ Mask-target SOA & 4.31 & 3,33 & 149 & $<.05$ & $*$ \\
\hline Prime-mask SOA $\times$ Mask-target SOA & 0.54 & 3,33 & 246 & 0.66 & \\
\hline $\begin{array}{l}\text { Semantic congruency } \times \text { Masking strength } \times \\
\text { Prime-mask SOA }\end{array}$ & 0.01 & 1,11 & 133 & 0.93 & \\
\hline $\begin{array}{l}\text { Semantic congruency } \times \text { Masking strength } \times \\
\text { Mask-target SOA }\end{array}$ & 1.46 & 3,33 & 123 & 0.25 & \\
\hline $\begin{array}{l}\text { Semantic congruency } \times \text { Prime-mask SOA } \\
\times \text { Mask-target SOA }\end{array}$ & 1.23 & 3,33 & 218 & 0.31 & \\
\hline $\begin{array}{l}\text { Masking strength } \times \text { Prime-mask SOA } \times \\
\text { Mask-target SOA }\end{array}$ & 0.40 & 3,33 & 118 & 0.76 & \\
\hline $\begin{array}{l}\text { Semantic congruency } \times \text { Masking strength } \times \\
\text { Prime-mask SOA } \times \text { Mask-target SOA }\end{array}$ & 0.75 & 3,33 & 113 & 0.53 & \\
\hline
\end{tabular}

Note. $\mathrm{GG}=$ Greenhouse-Geisser correction was applied, if Mauchly's sphericity test indicated a violation of the sphericity assumption. '***' $p<0.001$; ‘**' $p<0.01$; '*’ $p<0.05$; '.' $p<0.1$. 
Table D8.1

ANOVA on Errors of Experiment $4.1 B$ (semantic task level) with perceptual congruency as a factor

\begin{tabular}{|c|c|c|c|c|c|}
\hline Effect & $\boldsymbol{F}$ & $d f$ & $M S_{e}$ & \multicolumn{2}{|c|}{$\boldsymbol{p}$ (p.r.n. GG) } \\
\hline (Intercept) & 46.87 & 1,11 & 1.001 & $<.0001$ & $* * *$ \\
\hline Perceptual congruency & 0.22 & 1,11 & 0.013 & 0.65 & \\
\hline Masking strength & 0.16 & 1,11 & 0.021 & 0.70 & \\
\hline Prime-mask SOA & 0.81 & 1,11 & 0.021 & 0.39 & \\
\hline Mask-target SOA & 3.20 & 3,33 & 0.024 & $<.05$ & $*$ \\
\hline Perceptual congruency $\times$ Masking strength & 1.84 & 1,11 & 0.013 & 0.20 & \\
\hline Perceptual congruency $\times$ Prime-mask SOA & 8.20 & 1,11 & 0.015 & $<.05$ & $*$ \\
\hline Masking strength $\times$ Prime-mask SOA & 1.20 & 1,11 & 0.019 & 0.30 & \\
\hline Perceptual congruency $\times$ Mask-target SOA & 0.61 & 3,33 & 0.026 & 0.61 & \\
\hline Masking strength $\times$ Mask-target SOA & 1.69 & 3,33 & 0.022 & 0.19 & \\
\hline Prime-mask SOA $\times$ Mask-target SOA & 0.15 & 3,33 & 0.019 & 0.91 & \\
\hline $\begin{array}{l}\text { Perceptual congruency } \times \text { Masking strength } \\
\times \text { Prime-mask SOA }\end{array}$ & 0.28 & 1,11 & 0.015 & 0.60 & \\
\hline $\begin{array}{l}\text { Perceptual congruency } \times \text { Masking strength } \\
\times \text { Mask-target SOA }\end{array}$ & 0.90 & 3,33 & 0.027 & 0.45 & \\
\hline $\begin{array}{l}\text { Perceptual congruency } \times \text { Prime-mask SOA } \\
\times \text { Mask-target SOA }\end{array}$ & 1.51 & 3,33 & 0.013 & 0.23 & \\
\hline $\begin{array}{l}\text { Masking strength } \times \text { Prime-mask SOA } \times \\
\text { Mask-target SOA }\end{array}$ & 1.50 & 3,33 & 0.014 & 0.23 & \\
\hline $\begin{array}{l}\text { Perceptual congruency } \times \text { Masking strength } \\
\times \text { Prime-mask SOA } \times \text { Mask-target SOA }\end{array}$ & 0.52 & 3,33 & 0.023 & 0.67 & \\
\hline
\end{tabular}

Note. $\mathrm{GG}=$ Greenhouse-Geisser correction was applied, if Mauchly's sphericity test indicated a violation of the sphericity assumption. '***’ $p<0.001$; ‘**’ $p<0.01$; '*’ $p<0.05$; ‘’ $p<0.1$.

Table D8.2

ANOVA on RTs of Experiment $4.1 B$ (semantic task level) with perceptual congruency as a factor

\begin{tabular}{|c|c|c|c|c|c|}
\hline Effect & $F$ & $d f$ & $M S_{e}$ & $p$ (p.r.n & JG) \\
\hline (Intercept) & 401.06 & 1,11 & 200318 & $<.0001$ & $* * *$ \\
\hline Perceptual congruency & 0.95 & 1,11 & 92 & 0.35 & \\
\hline Masking strength & 0.96 & 1,11 & 225 & 0.35 & \\
\hline Prime-mask SOA & 4.41 & 1,11 & 334 & 0.06 & . \\
\hline Mask-target SOA & 54.93 & 3,33 & 745 & $<.0001$ & $* * *$ \\
\hline Perceptual congruency $\times$ Masking strength & 0.66 & 1,11 & 95 & 0.43 & \\
\hline Perceptual congruency $\times$ Prime-mask SOA & 0.83 & 1,11 & 285 & 0.38 & \\
\hline Masking strength $\times$ Prime-mask SOA & 4.18 & 1,11 & 209 & 0.07 & . \\
\hline Perceptual congruency $\times$ Mask-target SOA & 0.48 & 3,33 & 115 & 0.70 & \\
\hline Masking strength $\times$ Mask-target SOA & 3.36 & 3,33 & 122 & $<.05$ & $*$ \\
\hline Prime-mask SOA $\times$ Mask-target SOA & 1.65 & 3,33 & 95 & 0.20 & \\
\hline $\begin{array}{l}\text { Perceptual congruency } \times \text { Masking strength } \\
\times \text { Prime-mask SOA }\end{array}$ & 1.08 & 1,11 & 138 & 0.32 & \\
\hline $\begin{array}{l}\text { Perceptual congruency } \times \text { Masking strength } \\
\times \text { Mask-target SOA }\end{array}$ & 0.61 & 3,33 & 141 & 0.61 & \\
\hline $\begin{array}{l}\text { Perceptual congruency } \times \text { Prime-mask SOA } \\
\times \text { Mask-target SOA }\end{array}$ & 0.30 & 3,33 & 92 & 0.82 & \\
\hline $\begin{array}{l}\text { Masking strength } \times \text { Prime-mask SOA } \times \\
\text { Mask-target SOA }\end{array}$ & 0.43 & 3,33 & 140 & 0.62 & \\
\hline $\begin{array}{l}\text { Perceptual congruency } \times \text { Masking strength } \\
\times \text { Prime-mask SOA } \times \text { Mask-target SOA }\end{array}$ & 0.60 & 3,33 & 147 & 0.54 & \\
\hline
\end{tabular}


Table D9.1

ANOVA on Errors of Experiment 4.2 A (perceptual task level) with semantic congruency as a factor

\begin{tabular}{lcccc}
\hline Effect & $\boldsymbol{F}$ & $\boldsymbol{d f}$ & $\boldsymbol{M} \boldsymbol{S}_{\boldsymbol{e}}$ & $\boldsymbol{p}$ (p.r.n. GG) \\
\hline Intercept) & 56.46 & 1,11 & 0.419 & $<.0001$ \\
Semantic congruency & 0.05 & 1,11 & 0.015 & 0.83 \\
Masking strength & 0.69 & 1,11 & 0.032 & 0.42 \\
Prime-mask SOA & 3.21 & 1,11 & 0.026 & 0.10 \\
Mask-target SOA & 7.90 & 3,33 & 0.019 & $<.0005$ \\
Semantic congruency $\times$ Masking strength & 0.83 & 1,11 & 0.030 & 0.38 \\
Semantic congruency $\times$ Prime-mask SOA & 0.39 & 1,11 & 0.031 & 0.55 \\
Masking strength $\times$ Prime-mask SOA & 0.35 & 1,11 & 0.015 & 0.57 \\
Semantic congruency $\times$ Mask-target SOA & 0.21 & 3,33 & 0.023 & 0.89 \\
Masking strength $\times$ Mask-target SOA & 0.84 & 3,33 & 0.035 & 0.48 \\
Prime-mask SOA $\times$ Mask-target SOA & 0.07 & 3,33 & 0.023 & 0.98 \\
Semantic congruency $\times$ Masking strength $\times$ & 1.34 & 1,11 & 0.036 & 0.27 \\
$\quad$ Prime-mask SOA & & & & \\
Semantic congruency $\times$ Masking strength $\times$ & 0.20 & 3,33 & 0.020 & 0.90 \\
$\quad$ Mask-target SOA & & & & 0.41 \\
Semantic congruency $\times$ Prime-mask SOA & 0.98 & 3,33 & 0.026 & \\
$\quad \times$ Mask-target SOA & & & & \\
Masking strength $\times$ Prime-mask SOA $\times$ & 1.24 & 3,33 & 0.024 & 0.31 \\
$\quad$ Mask-target SOA & & 3,33 & 0.016 & 0.11 \\
Semantic congruency $\times$ Masking strength $\times$ & 2.19 & & &
\end{tabular}

Note. GG = Greenhouse-Geisser correction was applied, if Mauchly's sphericity test indicated a violation of the sphericity assumption. '***’ $p<0.001$; ‘**’ $p<0.01$; '*’ $p<0.05$; ‘’ $p<0.1$.

Table D9.2

ANOVA on RTs of Experiment $4.2 A$ (perceptual task level) with semantic congruency as a factor

\begin{tabular}{|c|c|c|c|c|c|}
\hline Effect & $F$ & $d f$ & $M S_{e}$ & \multicolumn{2}{|c|}{$\boldsymbol{p}$ (p.r.n. GG) } \\
\hline (Intercept) & 463.17 & 1,11 & 143703 & $<.0001$ & $* * *$ \\
\hline Semantic congruency & 0.38 & 1,11 & 75 & 0.55 & \\
\hline Masking strength & 2.53 & 1,11 & 293 & 0.14 & \\
\hline Prime-mask SOA & 5.51 & 1,11 & 150 & $<.05$ & $*$ \\
\hline Mask-target SOA & 57.28 & 3,33 & 398 & $<.0001$ & $* * *$ \\
\hline Semantic congruency $\times$ Masking strength & 0.15 & 1,11 & 60 & 0.71 & \\
\hline Semantic congruency $\times$ Prime-mask SOA & 0.40 & 1,11 & 84 & 0.54 & \\
\hline Masking strength $\times$ Prime-mask SOA & 1.28 & 1,11 & 44 & 0.28 & \\
\hline Semantic congruency $\times$ Mask-target SOA & 0.74 & 3,33 & 185 & 0.53 & \\
\hline Masking strength $\times$ Mask-target SOA & 0.39 & 3,33 & 115 & 0.76 & \\
\hline Prime-mask SOA $\times$ Mask-target SOA & 1.35 & 3,33 & 119 & 0.28 & \\
\hline $\begin{array}{l}\text { Semantic congruency } \times \text { Masking strength } \times \\
\text { Prime-mask SOA }\end{array}$ & 0.02 & 1,11 & 105 & 0.90 & \\
\hline $\begin{array}{l}\text { Semantic congruency } \times \text { Masking strength } \times \\
\text { Mask-target SOA }\end{array}$ & 0.98 & 3,33 & 97 & 0.41 & \\
\hline $\begin{array}{l}\text { Semantic congruency } \times \text { Prime-mask SOA } \\
\times \text { Mask-target SOA }\end{array}$ & 0.37 & 3,33 & 122 & 0.70 & \\
\hline $\begin{array}{l}\text { Masking strength } \times \text { Prime-mask SOA } \times \\
\text { Mask-target SOA }\end{array}$ & 2.71 & 3,33 & 67 & 0.06 & . \\
\hline $\begin{array}{l}\text { Semantic congruency } \times \text { Masking strength } \times \\
\text { Prime-mask SOA } \times \text { Mask-target SOA }\end{array}$ & 0.40 & 3,33 & 91 & 0.64 & \\
\hline
\end{tabular}

Note. $\mathrm{GG}=$ Greenhouse-Geisser correction was applied, if Mauchly's sphericity test indicated a violation of the sphericity assumption. '***' $p<0.001$; ‘**' $p<0.01$; '*’ $p<0.05$; '.' $p<0.1$. 
Table D10.1

ANOVA on Errors of Experiment 4.2B (semantic task level) with perceptual congruency as a factor

\begin{tabular}{|c|c|c|c|c|c|}
\hline \multirow{2}{*}{$\begin{array}{l}\text { Effect } \\
\text { (Intercept) }\end{array}$} & \multirow{2}{*}{$\begin{array}{c}\boldsymbol{F} \\
114.52\end{array}$} & \multirow{2}{*}{$\frac{d \boldsymbol{d}}{1,11}$} & \multirow{2}{*}{$\begin{array}{l}\boldsymbol{M} \boldsymbol{S}_{e} \\
0.285\end{array}$} & \multicolumn{2}{|c|}{$\boldsymbol{p}$ (p.r.n. GG) } \\
\hline & & & & $<.0001$ & $* * *$ \\
\hline Perceptual congruency & 0.47 & 1,11 & 0.012 & 0.51 & \\
\hline Masking strength & 1.73 & 1,11 & 0.020 & 0.21 & \\
\hline Prime-mask SOA & 1.18 & 1,11 & 0.039 & 0.30 & \\
\hline Mask-target SOA & 0.65 & 3,33 & 0.036 & 0.59 & \\
\hline Perceptual congruency $\times$ Masking strength & 0.46 & 1,11 & 0.023 & 0.51 & \\
\hline Perceptual congruency $\times$ Prime-mask SOA & 0.0002 & 1,11 & 0.024 & 0.99 & \\
\hline Masking strength $\times$ Prime-mask SOA & 8.42 & 1,11 & 0.014 & $<.05$ & $*$ \\
\hline Perceptual congruency $\times$ Mask-target SOA & 3.30 & 3,33 & 0.023 & $<.05$ & $*$ \\
\hline Masking strength $\times$ Mask-target SOA & 1.33 & 3,33 & 0.026 & 0.28 & \\
\hline Prime-mask SOA $\times$ Mask-target SOA & 1.32 & 3,33 & 0.030 & 0.28 & \\
\hline $\begin{array}{l}\text { Perceptual congruency } \times \text { Masking strength } \\
\times \text { Prime-mask SOA }\end{array}$ & 0.14 & 1,11 & 0.026 & 0.72 & \\
\hline $\begin{array}{l}\text { Perceptual congruency } \times \text { Masking strength } \\
\times \text { Mask-target SOA }\end{array}$ & 1.49 & 3,33 & 0.019 & 0.23 & \\
\hline $\begin{array}{l}\text { Perceptual congruency } \times \text { Prime-mask SOA } \\
\times \text { Mask-target SOA }\end{array}$ & 1.59 & 3,33 & 0.016 & 0.21 & \\
\hline $\begin{array}{l}\text { Masking strength } \times \text { Prime-mask SOA } \times \\
\text { Mask-target SOA }\end{array}$ & 1.11 & 3,33 & 0.020 & 0.36 & \\
\hline $\begin{array}{l}\text { Perceptual congruency } \times \text { Masking strength } \\
\times \text { Prime-mask SOA } \times \text { Mask-target SOA }\end{array}$ & 0.27 & 3,33 & 0.020 & 0.85 & \\
\hline
\end{tabular}

Note. $\mathrm{GG}=$ Greenhouse-Geisser correction was applied, if Mauchly's sphericity test indicated a violation of the sphericity assumption. '***’ $p<0.001$; ‘**’ $p<0.01$; '*’ $p<0.05$; ‘’ $p<0.1$.

Table D10.2

ANOVA on RTs of Experiment 4.2B (semantic task level) with perceptual congruency as a factor

\begin{tabular}{|c|c|c|c|c|c|}
\hline Effect & $F$ & $d f$ & $M S_{e}$ & $\boldsymbol{p}$ (p.r.n & JG) \\
\hline (Intercept) & 1684.68 & 1,11 & 44386 & $<.0001$ & $* * *$ \\
\hline Perceptual congruency & 0.00003 & 1,11 & 86 & 0.995 & \\
\hline Masking strength & 13.44 & 1,11 & 107 & $<.005$ & $* *$ \\
\hline Prime-mask SOA & 2.23 & 1,11 & 37 & 0.17 & \\
\hline Mask-target SOA & 105.90 & 3,33 & 147 & $<.0001$ & $* * *$ \\
\hline Perceptual congruency $\times$ Masking strength & 0.61 & 1,11 & 54 & 0.45 & \\
\hline Perceptual congruency $\times$ Prime-mask SOA & 0.004 & 1,11 & 89 & 0.95 & \\
\hline Masking strength $\times$ Prime-mask SOA & 4.02 & 1,11 & 72 & 0.07 & . \\
\hline Perceptual congruency $\times$ Mask-target SOA & 0.34 & 3,33 & 73 & 0.79 & \\
\hline Masking strength $\times$ Mask-target SOA & 0.58 & 3,33 & 68 & 0.63 & \\
\hline Prime-mask SOA $\times$ Mask-target SOA & 1.25 & 3,33 & 97 & 0.31 & \\
\hline $\begin{array}{l}\text { Perceptual congruency } \times \text { Masking strength } \\
\times \text { Prime-mask SOA }\end{array}$ & 0.01 & 1,11 & 72 & 0.91 & \\
\hline $\begin{array}{l}\text { Perceptual congruency } \times \text { Masking strength } \\
\times \text { Mask-target SOA }\end{array}$ & 2.12 & 3,33 & 55 & 0.12 & \\
\hline $\begin{array}{l}\text { Perceptual congruency } \times \text { Prime-mask SOA } \\
\times \text { Mask-target SOA }\end{array}$ & 0.24 & 3,33 & 88 & 0.87 & \\
\hline $\begin{array}{l}\text { Masking strength } \times \text { Prime-mask SOA } \times \\
\text { Mask-target SOA }\end{array}$ & 1.15 & 3,33 & 40 & 0.35 & \\
\hline $\begin{array}{l}\text { Perceptual congruency } \times \text { Masking strength } \\
\times \text { Prime-mask SOA } \times \text { Mask-target SOA }\end{array}$ & 0.57 & 3,33 & 85 & 0.64 & \\
\hline
\end{tabular}


Table D11.1

ANOVA on Errors of Experiment 4.3A (perceptual task level) with semantic congruency as a factor

\begin{tabular}{|c|c|c|c|c|c|}
\hline Effect & $F$ & $d f$ & $M S_{e}$ & $\boldsymbol{p}$ (p.r.n & GG) \\
\hline (Intercept) & 63.86 & 1,11 & 0.781 & $<.0001$ & $* * *$ \\
\hline Semantic congruency & 0.02 & 1,11 & 0.026 & 0.90 & \\
\hline Masking strength & 7.40 & 1,11 & 0.051 & $<.05$ & $*$ \\
\hline Prime-mask SOA & 1.16 & 1,11 & 0.031 & 0.30 & \\
\hline Mask-target SOA & 1.14 & 3,33 & 0.031 & 0.35 & \\
\hline Semantic congruency $\times$ Masking strength & 0.86 & 1,11 & 0.025 & 0.37 & \\
\hline Semantic congruency $\times$ Prime-mask SOA & 0.09 & 1,11 & 0.030 & 0.77 & \\
\hline Masking strength $\times$ Prime-mask SOA & 3.80 & 1,11 & 0.017 & 0.08 & . \\
\hline Semantic congruency $\times$ Mask-target SOA & 0.63 & 3,33 & 0.014 & 0.60 & \\
\hline Masking strength $\times$ Mask-target SOA & 0.34 & 3,33 & 0.022 & 0.69 & \\
\hline Prime-mask SOA $\times$ Mask-target SOA & 2.57 & 3,33 & 0.022 & 0.07 & . \\
\hline $\begin{array}{l}\text { Semantic congruency } \times \text { Masking strength } \times \\
\text { Prime-mask SOA }\end{array}$ & 0.01 & 1,11 & 0.014 & 0.92 & \\
\hline $\begin{array}{l}\text { Semantic congruency } \times \text { Masking strength } \times \\
\text { Mask-target SOA }\end{array}$ & 0.24 & 3,33 & 0.021 & 0.87 & \\
\hline $\begin{array}{l}\text { Semantic congruency } \times \text { Prime-mask SOA } \\
\times \text { Mask-target SOA }\end{array}$ & 1.42 & 3,33 & 0.021 & 0.25 & \\
\hline $\begin{array}{l}\text { Masking strength } \times \text { Prime-mask SOA } \times \\
\text { Mask-target SOA }\end{array}$ & 2.65 & 3,33 & 0.015 & 0.07 & . \\
\hline $\begin{array}{l}\text { Semantic congruency } \times \text { Masking strength } \times \\
\text { Prime-mask SOA } \times \text { Mask-target SOA }\end{array}$ & 0.89 & 3,33 & 0.029 & 0.46 & \\
\hline
\end{tabular}

Table D11.2

ANOVA on RTs of Experiment 4.3 A (perceptual task level) with semantic congruency as a factor

\begin{tabular}{|c|c|c|c|c|c|}
\hline \multirow{2}{*}{$\begin{array}{l}\text { Effect } \\
\text { (Intercept) }\end{array}$} & \multirow{2}{*}{$\begin{array}{c}\boldsymbol{F} \\
810.84\end{array}$} & \multirow{2}{*}{$\begin{array}{c}\boldsymbol{d f} \\
1,11\end{array}$} & \multirow{2}{*}{$\begin{array}{c}\boldsymbol{M S}_{\boldsymbol{e}} \\
88896\end{array}$} & \multicolumn{2}{|c|}{$\boldsymbol{p}$ (p.r.n. GG) } \\
\hline & & & & $<.0001$ & $* * *$ \\
\hline Semantic congruency & 0.86 & 1,11 & 194 & 0.37 & \\
\hline Masking strength & 43.05 & 1,11 & 319 & $<.0001$ & $* * *$ \\
\hline Prime-mask SOA & 11.99 & 1,11 & 138 & $<.01$ & $* *$ \\
\hline Mask-target SOA & 57.68 & 3,33 & 606 & $<.0001$ & $* * *$ \\
\hline Semantic congruency $\times$ Masking strength & 0.01 & 1,11 & 190 & 0.92 & \\
\hline Semantic congruency $\times$ Prime-mask SOA & 0.29 & 1,11 & 316 & 0.60 & \\
\hline Masking strength $\times$ Prime-mask SOA & 6.60 & 1,11 & 41 & $<.05$ & $*$ \\
\hline Semantic congruency $\times$ Mask-target SOA & 0.27 & 3,33 & 229 & 0.84 & \\
\hline Masking strength $\times$ Mask-target SOA & 1.86 & 3,33 & 268 & 0.15 & \\
\hline Prime-mask SOA $\times$ Mask-target SOA & 1.27 & 3,33 & 226 & 0.30 & \\
\hline $\begin{array}{l}\text { Semantic congruency } \times \text { Masking strength } \times \\
\text { Prime-mask SOA }\end{array}$ & 0.33 & 1,11 & 116 & 0.58 & \\
\hline $\begin{array}{l}\text { Semantic congruency } \times \text { Masking strength } \times \\
\text { Mask-target SOA }\end{array}$ & 1.22 & 3,33 & 257 & 0.32 & \\
\hline $\begin{array}{l}\text { Semantic congruency } \times \text { Prime-mask SOA } \\
\times \text { Mask-target SOA }\end{array}$ & 1.16 & 3,33 & 178 & 0.34 & \\
\hline $\begin{array}{l}\text { Masking strength } \times \text { Prime-mask SOA } \times \\
\text { Mask-target SOA }\end{array}$ & 0.40 & 3,33 & 178 & 0.76 & \\
\hline $\begin{array}{l}\text { Semantic congruency } \times \text { Masking strength } \times \\
\text { Prime-mask SOA } \times \text { Mask-target SOA }\end{array}$ & 0.19 & 3,33 & 173 & 0.90 & \\
\hline
\end{tabular}

Note. GG $=$ Greenhouse-Geisser correction was applied, if Mauchly's sphericity test indicated a violation of the sphericity assumption. '***' $p<0.001$; '**' $p<0.01$; '*’ $p<0.05$; '.' $p<0.1$. 
Table D12.1

ANOVA on Errors of Experiment 4.3B (semantic task level) with perceptual congruency as a factor

\begin{tabular}{|c|c|c|c|c|c|}
\hline Effect & $\boldsymbol{F}$ & $d f$ & $M S_{e}$ & \multicolumn{2}{|c|}{$\boldsymbol{p}$ (p.r.n. GG) } \\
\hline (Intercept) & 35.92 & 1,11 & 1.326 & $<.0001$ & $* * *$ \\
\hline Perceptual congruency & 1.21 & 1,11 & 0.016 & 0.29 & \\
\hline Masking strength & 0.04 & 1,11 & 0.016 & 0.85 & \\
\hline Prime-mask SOA & 1.53 & 1,11 & 0.011 & 0.24 & \\
\hline Mask-target SOA & 1.57 & 3,33 & 0.020 & 0.21 & \\
\hline Perceptual congruency $\times$ Masking strength & 0.01 & 1,11 & 0.027 & 0.92 & \\
\hline Perceptual congruency $\times$ Prime-mask SOA & 10.34 & 1,11 & 0.021 & $<.01$ & $* *$ \\
\hline Masking strength $\times$ Prime-mask SOA & 0.0004 & 1,11 & 0.020 & 0.99 & \\
\hline Perceptual congruency $\times$ Mask-target SOA & 0.80 & 3,33 & 0.023 & 0.50 & \\
\hline Masking strength $\times$ Mask-target SOA & 0.82 & 3,33 & 0.022 & 0.49 & \\
\hline Prime-mask SOA $\times$ Mask-target SOA & 0.19 & 3,33 & 0.033 & 0.90 & \\
\hline $\begin{array}{l}\text { Perceptual congruency } \times \text { Masking strength } \\
\times \text { Prime-mask SOA }\end{array}$ & 0.73 & 1,11 & 0.020 & 0.41 & \\
\hline $\begin{array}{l}\text { Perceptual congruency } \times \text { Masking strength } \\
\times \text { Mask-target SOA }\end{array}$ & 0.99 & 3,33 & 0.025 & 0.41 & \\
\hline $\begin{array}{l}\text { Perceptual congruency } \times \text { Prime-mask SOA } \\
\times \text { Mask-target SOA }\end{array}$ & 0.33 & 3,33 & 0.013 & 0.80 & \\
\hline $\begin{array}{l}\text { Masking strength } \times \text { Prime-mask SOA } \times \\
\text { Mask-target SOA }\end{array}$ & 0.74 & 3,33 & 0.022 & 0.54 & \\
\hline $\begin{array}{l}\text { Perceptual congruency } \times \text { Masking strength } \\
\times \text { Prime-mask SOA } \times \text { Mask-target SOA }\end{array}$ & 1.70 & 3,33 & 0.022 & 0.19 & \\
\hline
\end{tabular}

Note. $\mathrm{GG}=$ Greenhouse-Geisser correction was applied, if Mauchly's sphericity test indicated a violation of the sphericity assumption. '***’ $p<0.001$; ‘**’ $p<0.01$; '*’ $p<0.05$; ‘’ $p<0.1$.

Table D12.2

ANOVA on RTs of Experiment 4.3B (semantic task level) with perceptual congruency as a factor

\begin{tabular}{|c|c|c|c|c|c|}
\hline Effect & $F$ & $d f$ & $M S_{e}$ & $p$ (p.r.n & JG) \\
\hline (Intercept) & 4273.03 & 1,11 & 19136 & $<.0001$ & $* * *$ \\
\hline Perceptual congruency & 1.30 & 1,11 & 123 & 0.28 & \\
\hline Masking strength & 36.44 & 1,11 & 132 & $<.0001$ & $* * *$ \\
\hline Prime-mask SOA & 1.21 & 1,11 & 124 & 0.29 & \\
\hline Mask-target SOA & 81.65 & 3,33 & 379 & $<.0001$ & $* * *$ \\
\hline Perceptual congruency $\times$ Masking strength & 0.22 & 1,11 & 126 & 0.65 & \\
\hline Perceptual congruency $\times$ Prime-mask SOA & 0.16 & 1,11 & 70 & 0.70 & \\
\hline Masking strength $\times$ Prime-mask SOA & 0.81 & 1,11 & 85 & 0.39 & \\
\hline Perceptual congruency $\times$ Mask-target SOA & 1.03 & 3,33 & 151 & 0.36 & \\
\hline Masking strength $\times$ Mask-target SOA & 4.06 & 3,33 & 91 & $<.05$ & $*$ \\
\hline Prime-mask SOA $\times$ Mask-target SOA & 2.41 & 3,33 & 87 & 0.08 & . \\
\hline $\begin{array}{l}\text { Perceptual congruency } \times \text { Masking strength } \\
\times \text { Prime-mask SOA }\end{array}$ & 0.40 & 1,11 & 49 & 0.54 & \\
\hline $\begin{array}{l}\text { Perceptual congruency } \times \text { Masking strength } \\
\times \text { Mask-target SOA }\end{array}$ & 1.66 & 3,33 & 146 & 0.20 & \\
\hline $\begin{array}{l}\text { Perceptual congruency } \times \text { Prime-mask SOA } \\
\times \text { Mask-target SOA }\end{array}$ & 1.97 & 3,33 & 83 & 0.14 & \\
\hline $\begin{array}{l}\text { Masking strength } \times \text { Prime-mask SOA } \times \\
\text { Mask-target SOA }\end{array}$ & 0.29 & 3,33 & 186 & 0.84 & \\
\hline $\begin{array}{l}\text { Perceptual congruency } \times \text { Masking strength } \\
\times \text { Prime-mask SOA } \times \text { Mask-target SOA }\end{array}$ & 0.38 & 3,33 & 83 & 0.77 & \\
\hline
\end{tabular}




\section{List of Figures}

\section{Chapter 2 - The Role of Visibility in Masked Priming:}

\section{A Model and a Meta-Analysis}

Figure 2.1 Psychological model of moderators.

Figure 2.2 Flow diagram of study selection.

Figure 2.3 Effect sizes for different task levels.

Figure 2.4 Schematic depiction of a possible interference of moderators

with an impact on the visibility measurement.

Figure 2.5 Funnel plot with estimated summery effect and standard error. 60

Figure 2.6 Funnel plots with illustrated levels of three moderators. 61

Figure 2.7 Histogram of the frequencies of included $d$ '-values. 62

\section{Chapter 3 - Prime Visibility in Perceptual and Semantic Priming}

Figure 3.1 Stimuli applied in Experiment 3.1.

Figure 3.2 The sequence of a trial used in Experiments 3.1 - 3.3. 82

Figure 3.3 Priming effects and $d$ '-values in Experiment 3.1. 86

Figure 3.4 Reaction times in Experiment 3.1. 88

Figure 3.5 Ratings of subjective prime visibility in Experiment 3.1A. 90

Figure 3.6 Ratings of subjective prime visibility in Experiment 3.1B. 91

Figure 3.7 Stimuli applied in Experiments 3.2 and 3.3. 94

Figure 3.8 Priming effects and $d$ '-values in Experiment 3.2. 97

Figure 3.9 Reaction times in Experiment 3.2. 98

Figure 3.10 Ratings of subjective prime visibility in Experiment 3.2. 100

Figure 3.11 Priming effects and $d$ '-values in Experiment 3.3. 104 
Figure 3.12 Reaction times in Experiment 3.3. 106

Figure 3.13 Ratings of subjective prime visibility in Experiment 3.3. 109

Figure 3.14 Reduction of prime visibility with pattern and metacontrast masks. 122

\section{Chapter 4 -Temporal Parameters in Perceptual and Semantic Priming}

Figure 4.1 Distributions of applied SOA in priming studies. 132

Figure 4.2 Stimuli used in Experiments 4.1 - 4.3. 136

Figure 4.3 Sequence of a trial used in Experiments 4.1 - 4.3. 138

Figure 4.4 Priming effects and $d$ '-values in Experiment 4.1. 141

Figure 4.5 Reaction times in Experiment 4.1. 143

Figure 4.6 Ratings of subjective prime visibility in Experiment 4.1. 147

Figure 4.7 Priming effects and $d$ '-values in Experiment 4.2. 154

Figure 4.8 Reaction times in Experiment 4.2. 156

Figure 4.9 Ratings of subjective prime visibility in Experiment 4.2. 159

Figure 4.10 Priming effects and $d$ '-values in Experiment 4.3. 168

Figure 4.11 Reaction times in Experiment 4.3. 170

Figure 4.12 Ratings of subjective prime visibility in Experiment 4.3. 173

\section{Chapter 5 - Perceptual and Semantic Priming without Response Association: Electrophysiological differences}

Figure 5.1 Stimuli applied in Experiments 5A and 5B. 192

Figure 5.2 The sequence of a trial used in Experiments 5A and 5B. 194

Figure 5.3 Occipital, centro-parietal and temporal electrode clusters. 196

Figure 5.4 Priming effects on reaction times in Experiments 5A and 5B. 198

Figure 5.5 Objective and subjective prime visibility in Experiment 5. 199 
Figure 5.6 Modulations of grand avarage event-related potentials through task relevance and prime-mask SOA. 201

Figure 5.7 Voltage maps with topographical distributions for priming effects. 202

Figure 5.8 Modulations of grand avarage event-related potentials through effects type. 203

Figure 5.9 Correlations between behavioral and ERP priming effects. 206 
V | Approaching Consciousness with Masked Priming 


\section{List of Tables}

\section{Chapter 2 - The Role of Visibility in Masked Priming:}

\section{A Model and a Meta-Analysis}

Table 2.1 Moderators with a potential Influence on the Effect sizes or/and the visibility measures

Table 2.2 Test Statistics for estimated Models with Single Moderators

Table 2.3 Test Statistics for estimated Models with Single Moderators and

their Interaction with d' of the first visibility measurement 38

Table 2.4 Test Statistics for estimated Models with Single Moderators and their Interaction with d' of the last visibility measurement

Table 2.5 Pearson Correlations with p-Values among Moderators

Table 2.6 Variance Inflation Factors among potential Moderators

Table 2.7 List of calculated Model with their components 46

Table 2.8 Test Statistics for all estimated Models 47

Table 2.9 Fitted third-order Model including 'Masking Method' 50

Table 2.10 Fitted third-order Model including 'Depth of Prime/Target Processing' 51

Table 2.11 Correlations between characteristics of visibility measurements, moderators of the comprehensive models and of prime visibility

\section{Chapter 4 - Temporal Parameters in Perceptual and Semantic Priming}

Table 4.1 Meta-analytic model with effect type, prime-mask and mask-target SOA 132

\section{Appendix I}

Table A Included Studies with their Characteristics and Effect Sizes 275 


\section{Appendix II}

Table B1.1 ANOVA on Errors of Experiments 3.1A and 3.1B

Table B1.2 ANOVA on RTs of Experiments 3.1A and 3.1B 297

Table B1.3 ANOVA on objective prime visibility of Experiments $3.1 \mathrm{~A}$ and $3.1 \mathrm{~B}$ 298

Table B1.4 ANOVA on subjective prime visibility of Experiments $3.2 \mathrm{~A}$ and $3.2 \mathrm{~B}$ 298

Table B2.1 ANOVA on Errors of Experiments $3.2 A$ and $3.2 B$ 299

Table B2.2 ANOVA on RTs of Experiments $3.2 A$ and $3.2 B$ 299

Table B2.3 ANOVA on objective prime visibility of Experiments $3.2 \mathrm{~A}$ and $3.2 \mathrm{~B}$ 300

Table B2.4 ANOVA on subjective prime visibility of Experiments $3.2 \mathrm{~A}$ and $3.2 \mathrm{~B}$ 301

Table B3.1 ANOVA on Errors of Experiments $3.3 A$ and $3.3 B$ 301

Table B3.2 ANOVA on RTs of Experiments $3.3 A$ and $3.3 B$ 302

Table B3.3 ANOVA on objective prime visibility of Experiments $3.3 \mathrm{~A}$ and $3.3 \mathrm{~B}$ 302

Table B3.4 ANOVA on subjective prime visibility of Experiments $3.3 \mathrm{~A}$ and $3.3 \mathrm{~B}$ 303

Table B4.1 ANOVA on Errors of Experiments 3.2A, 3.2B, 3.3A and 3.3B 303

Table B4.2 ANOVA on RTs of Experiments 3.2A, 3.2B, 3.3A and 3.3B 304

Table B4.3 ANOVA on objective prime visibility of Experiments 3.2 and 3.3 305

Table B4.4 ANOVA on subjective prime visibility of Experiments 3.2 and 3.3 305

\section{Appendix III}

Table C1.1 ANOVA on Errors of Experiments $4.1 \mathrm{~A}$ and $4.1 \mathrm{~B}$ 307

Table C1.2 ANOVA on RTs of Experiments $4.1 \mathrm{~A}$ and $4.1 \mathrm{~B}$ 308

Table C1.3 ANOVA on objective prime visibility of Experiments $4.1 \mathrm{~A}$ and $4.1 \mathrm{~B}$ 309

Table C1.4 ANOVA on subjective prime visibility of Experiments $4.2 A$ and $4.2 B$ 309

Table C2.1 ANOVA on Errors of Experiments $4.2 A$ and $4.2 B$ 310

Table C2.2 ANOVA on RTs of Experiments $4.2 A$ and $4.2 B$ 311 
Table C2.3 ANOVA on objective prime visibility of Experiments $4.2 A$ and $4.2 B$ 312

Table C2.4 ANOVA on subjective prime visibility of Experiments $4.2 \mathrm{~A}$ and $4.2 \mathrm{~B}$ 312

Table C3.1 ANOVA on Errors of Experiments $4.3 A$ and $4.3 B$ 313

Table C3.2 ANOVA on RTs of Experiments $4.3 A$ and $4.3 B$ 314

Table C3.3 ANOVA on objective prime visibility of Experiments $4.3 \mathrm{~A}$ and $4.3 \mathrm{~B}$ 315

Table C3.4 ANOVA on subjective prime visibility of Experiments $4.3 \mathrm{~A}$ and $4.3 \mathrm{~B}$ 315

Table C4.1 ANOVA on Errors of Experiments 4.1A, 4.1B, 4.2A and 4.2B 316

Table C4.2 ANOVA on RTs of Experiments 4.1A, 4.1B, 4.2A and 4.2B 318

Table C4.3 ANOVA on objective prime visibility of Experiments 4.1 and 4.2 320

Table C4.4 ANOVA on subjective prime visibility of Experiments 4.1 and 4.2 321

\section{Appendix IV}

Table D1.1 ANOVA on Errors of Experiment 3.1A with semantic congruency 323

Table D1.2 ANOVA on RTs of Experiment 3.1A with semantic congruency 323

Table D2.1 ANOVA on Errors of Experiment $3.1 B$ with perceptual congruency 323

Table D2.2 ANOVA on RTs of Experiment 3.1B with perceptual congruency 324

Table D3.1 ANOVA on Errors of Experiment 3.2A with semantic congruency 324

Table D3.2 ANOVA on RTs of Experiment 3.2A with semantic congruency 324

Table D4.1 ANOVA on Errors of Experiment 3.2B with perceptual congruency 325

Table D4.2 ANOVA on RTs of Experiment 3.2B with perceptual congruency 325

Table D5.1 ANOVA on Errors of Experiment 3.3A with semantic congruency 325

Table D5.2 ANOVA on RTs of Experiment 3.3A with semantic congruency 326

Table D6.1 ANOVA on Errors of Experiment 3.3B with perceptual congruency 326

Table D6.2 ANOVA on RTs of Experiment 3.3B with perceptual congruency 326

Table D7.1 ANOVA on Errors of Experiment 4.1A with semantic congruency 327 
VI | Approaching Consciousness with Masked Priming

Table D7.2 ANOVA on RTs of Experiment 4.1A with semantic congruency 327

Table D8.1 ANOVA on Errors of Experiment $4.1 B$ with perceptual congruency 328

Table D8.2 ANOVA on RTs of Experiment 4.1B with perceptual congruency 328

Table D9.1 ANOVA on Errors of Experiment 4.2A with semantic congruency 329

Table D9.2 ANOVA on RTs of Experiment 4.2A with semantic congruency 329

Table D10.1 ANOVA on Errors of Experiment 4.2B with perceptual congruency 330

Table D10.2 ANOVA on RTs of Experiment 4.2B with perceptual congruency 330

Table D11.1 ANOVA on Errors of Experiment 4.3A with semantic congruency 331

Table D11.2 ANOVA on RTs of Experiment 4.3A with semantic congruency 331

Table D12.1 ANOVA on Errors of Experiment 4.3B with perceptual congruency 332

Table D12.2 ANOVA on RTs of Experiment $4.3 B$ with perceptual congruency 332 


\section{List of Abbreviations}

AIC

ANOVA

Amp

COL

ER

ERP

GG

GNW

M1

M2

MAM

ML

MOD

MTS

$\mathrm{N}$

NOT

PAS

PDU

PE

PER

PMS

PN

PTR2/5 Prime-target relation (2 levels or 5 levels)

RA

Akaike information criterion

Analysis of variance

Amplitude

Systematic differences in color

Error rate

Event-related potentials

Greenhouse-Geisser correction

Global Neuronal Workspace

First visibility measurement

Last visibility measurement

Masking method

Maximum likelihood estimation

Systematic differences in modality

Mask-target SOA

Sample size

Systematic differences in notation

Perceptual Awareness Scale

Prime duration

Priming effect

Systematic differences in perspective

Prime-mask SOA

Prime novelty

Response association 
VIII Approaching Consciousness with Masked Priming

REML Restricted maximum likelihood estimation

RT Reaction time

RTY Response type

SIZ Systematic differences in case or size

SHA Systematic differences in shape

SOA Stimulus onset asynchrony

TL Task level

VIF Variance inflation factors 